



\section{Allgemeine}

\section{Botanische Zeitschrift}

für

Systematik, Floristik, Pflanzengeographie ete.

Referierendes Organ des bot, Vereilis der Provinz Brandenburg.

der kgl, bot. Gesellschaft zu Regenshburg, des Preass. bot. Vereins in Königsberg.

und

Organl der Botan, Vereinioung in Würzburg

und des Berliner bot. Tauschrereins.

\section{Litterarische Beiträge}

von

Abromeit; Klonski, Franz; Blümml, E. K.; Buser, R.; Eggers; Figert, E.; Geisenheyner, L.; Goldsehmidt, M.; Gross, L.; Hellwig, Th.; Kükenthal, G.; Kuntze, Otto; Lackowitz, W.; Laurell, J. G.; Magnus, P.; Mareowiez, B.; Matouschek, Franz; Murr, J.; Ortlepp, Karl; Palla, E.; Petunnikov, A.; Post, Tom von; Pöverlein, H.; Reineck, Ed. Martin; Schmidle, W.; Seemen, Otto von; Spiesen, von; Trautschold, H. v.;

Warnstorf, C.; Will, Otto.

Herausgegeben

von

A. Kneucker.

Jahrgang 1900.

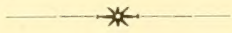

\section{KarIsruhe.}

Druck und Verlag von J.J. Reiff. 



\section{Inhaltsverzeichnis der Zeitschrift.}

\section{Originalarbeiten:}

Blonski, Dr. Franz, Ueber das Prioritätsrecht von Klukia Andrz. 1821 von Chamaeplium Wallr. 1822 .

- Zur Chronik der preussischen Flora

177. 205

Blü um l, Dr. E. K., Beiträge zur Flora von Niederösterreich

24. 105

Buser, R., Ueber Alchimilla pubescens Koch, truncata Rchb. u. eine neue verwandte Art aus den Tiroler Alpen (A. acutata)

Eggers, Botanische Beobachtungen auf meiner Reise nach dem Orient 1899 101. 128

Figert, E., Botanische Mitteilungen aus Schlesien . . . . . . . . . . 37

Goldschmidt, M., Die Flora des Rhöngebirges. I. . . . . . . . . 238

Gross, L., Anemone trifolia f. biflora . . . . . . . . . . . 177

- - Ist Draba Thomasii Koch eine gute Art? . . . . . . . . . . 55. 80

Gross, L. u. Kneucker, A., Unsere Reise nach Istrien, Dalmatien, Motenegro,

der Hercegovina und Bosnien im Juli und August 1900 . . 218. 236

Hellwig, Th., Florenbild der Umgegend von Kontopp im Kreise Grünberg in Schlesien . . . . . . . . . . . . . 3. 35. 104. 135

Kneucker, A., Bemerkungen zu den ,Carices exsiccatae“ VII. Lief. . . . 7. 43

- Bemerkungen zu den „Cyperaceae (exclus. Carices) et Juncaceae exsiccatae" I. Lief. 1900

- Bemerkungen zu den „Cyperaceae (exclus. Carices) et Juncaceae exsiccatae" II. Lief. 1901

- - Bemerkungen zu den „Gramineae exsiccatae" I. u. II. Lief. 1900

- Bemerkungen zu den „Gramineae exsiccatae“ III. u. IV. Lief. 1901

Kükenthal, G., Carex Canariensis nov. spec.

Kuntze, Otto u. Post, Tom von, Nomenklatorische Revision höherer Pflanzengruppen und über einige Tausend Korrekturen zu Englers Phanerogamen-Register

110. 148.179

L a urell, J. G., Ueber einige Carex-Hybriden aus Schweden

Magnus, P., Bemerkungen zum Berichte über die Sitzung des bot. Vereins für die Provinz Brandenburg vom 9. Febr. 1900 .

Marcowicz, B., Lappa Palladini n. sp.

Murr, Dr. J., Nachwort zu meiner Abhandlung „Ueber einige kritische Chenopodium-Formen "

- - "Griechische Kolonien" in Valsugana (Südtirol) .

1. 20

- Phaenologische Plaudereien aus der Innsbrucker Flora

81. 108

Ortlepp, Karl, Abnormität in der Blütenstelluag zweier Orchideen

Palla, E., Die Gattungen der mitteleuropäischen Scirpoideen

Petunnikov, A., Ueber den Wert anatomischer Merkmale zur Unterscheidung der Abies-Arten.

Reineck, Ed. Martin, Floristisches vom Strande von Bahia Blanca (Prov. Buenos Aires, Argentinien

Schmidle, W., Algologische Notizen

Seemen, Otto von, Mitteilungen über die Flora der ostfries. Insel Borkum

Spiesen, Freiherr v., Das Süskenbruch bei Dülmen in Westfalen

- - Die Wieselsheimer Salzwiesen in der Wetterau.

Warnstorf, C., Weitere Beiträge zur Flora von Pommern III. .

Will, Otto, Uebersicht der bisher in der Umgebung v. Guben in der Niederlausitz beobachteten Leber-, Torf- und Laubmoose . 82. 109. 143. 207 


\section{- IV -}

\section{Botanische Litteratur, Zeitschriften ete.}

\section{a. Eingehendere Besprechungen von selbständigen Werken, Aufsătzen etc.}

Appel, Dr. Otto u. Loew, Dr. E., Mitteilung blütenbiolog. Beobachtungen (Bitte)

Ascherson, P. u. Gräbner, P., Synopsis der mitteleurop. Flora (Ref. v.

$$
\text { A. K.) . . . . . . . . . . . . . - 45. } 166.245
$$

Bl ücher, H., Praktische Pflanzenkunde (Ref. v. A. K.) . . . . . . . . 209

Bubani, P., Flora Pyrenaea (Ref. v. A. K.) . . . . . . . . . . . . 165

Christ, H., Die Farnkräuter der Schweiz (Ref. v. A. K.) . . . . . . . . 245

Cryptogamae Japonicae iconibus illustratae (Ref. v. A. K.) . . . . 229

Dalla Torre, Dr. C. G. v. u. Harms, Dr. H., Genera Siphonogamarum ad systema

Englerianum conscripta (Ref. v. A. K.) . . . . . . . . . 95. 228

Eine Flora des Schwarzwaldes (Ref. v. A. K.) . . . . . . . . . . 70

Fedtschenko, Boris, Die im europ. Russland in d. Krim u. im Kaukasus vor-

kommenden Arten der Gattung Hedysarum (Ref. v. H. Trautschold)

Fed t s c he nk o, O.u.B., Ranunculaceen d. russisch. Turkestan (Ref. v. H. Trautschold)

Fonck, Leopold, S. J., Streifzüge durch die biblische Flora (Ref. v. A. K.)

Fritsch, Dr. K., Schulflora für die österr. Sudeten u. Alpenländer (Ref. v. A. K.)

Ga ucheri, Paul, Untersuchungen über den "Nanismus ${ }^{\star}$ der Pflanzen (Ref. v. Matouschek)

Giesenhagen, Dr. K., Unsere wichtigsten Kulturpflanzen (Ref. v. A. K.)

Gradmann, Dr. Rob., Das Pflanzenleben der schwäb Alb (Ref. v Dr. Pöverlein u. Dr. Murr) .

Halácsy, Dr. E. v., Conspectus florae Graecae (Ref. v. A. K.) .

Hervey, E. Williams, Observation on the colors of flowers (Ref. v. A. K.) . 96

Jaennicke, Fried r., Studien über d. Gattung Platanus L. (Ref.v. L. Geisenheyner) 208

Klein, Dr. Ludwig, Die Physiognomie der mitteleurop. Waldbäume (Ref.v. A. K.) 44

Kronfeld, Dr. M., Studien über die Verbreitungsmittel der Pflanzen (Ref. v. A. K.) 246

Kükenthal, Georg, Die Carexvegetation des aussertropischen Südamerikas (Ref. v. A. K.)

Kull, Albertu. Lutz, Dr. K. G., Bilder aus der heimatl. Vogelwelt (Ref. v. A. K.) 246

Lackowitz, W., Flora von Berlin u. der Prov. Brandenburg (Ref. v. A. K.) . 209

Lang, Dr. G., Yon Rom nach Sardes (Ref. v. L. Gross) . . . . . . . , . 245

Newberry, L. C., The later extinct floras of North-Amerika (Ref. v. H. Trautschold)

Pax, Dr. Ferd., Prantl's Lehrbuch der Botanik (Ref. v. A. K.)

Phanerogamae et Pteridophytae Japonicae iconibus illustratae

$$
\text { (Ref. v. A. K.) }
$$

Rus ch a u pt, Dr. G., Bau unb Leben der Pflanzen (Ref. v. A. K.) . . . . 166

Schinz. Dr. Hans u. Keller, Dr. Rob., Flora der Schweiz (Ref. v. A. K.) . 165

$\mathrm{S}$ chwarz, A ug. Friedr., Phanerogamen- u. Gefässkryptogamenflora der Umgegend von Nürnberg-Erlangen (Ref. v. Dr. Pöverlein) . . . . . 93

J. sturm's Flora von Deutschland (Ref. v. A. K.) . . . . . . . . . . 71

W e ig e I, Ott o, Botan. Lagerkatalog . . . . . . . . . . . . . . 167

W i e s b u r, J. B., Unsere Misteln und ihre Nährpflanzen (Ref. v. A. K.) . . . 166

W i n k l e r, W., Sudetenflora (Ankündigung' . . . . . . . . . . . . . 96

- - Sudetenflora (Ref. v. A. K.) . : . . . . . . . . . . . . 165

W i l demann, E. de et D u rand, Th., Plantae Thonnerianae Congolenses etc.

(Ref. v. A. K.) . . , . . . . . . . . . . . . . . . . 164

W ti ns che, Dr. Otto, Die verbreitetsten Pflanzen Deutschlands (Hef. v. A. K.) 166 


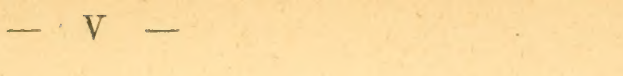

B e richte der bayr. bot. Gesellschaft

28. 72.246

B e rich t e der deutsch. bot. Gesellschaft

45. 72. 121. 167. 193. 209. 246

B erichte der schweizerischen bot. Gesellschaft . . . . . . . . 210

Bolletino del R. orto bot, di Palermo . . . . . . . . . . 122

Botanical Gazette. . . . . . 10 28. 46. 72. 122. 193. 210. 230. 246

Botanisches Centralblatt . 10.28. 45. 71. 97. 121. 167: 193. 210. 229. 246

Botaniska Notiser. . . . . . . . 10. 46. 72. 122. 210. 230

Bulletin de l'acad. internat de géogr. botanique 10. 28. 46. 121. 168. 194. 210. 247

Bullet in de l'association Française de botanique . . 10. 46. 121. 168. 194. 230

Bulletin de l'association Pyrénéenne . . . . . . . . . 194

Deutsche bot. Monatsschrift . . . 9 9. 27. 45. 71. 97. 120. 167. 193. 209

Jahresbericht des Preuss. bot. Vereins . . . . . . . 97. 210

La Nuova Notarisia . . . . . . . . . 10. 122. 194

Missouri Bot.Garden . . . . . . . 168

Mitteil un gen der bayr. Gesellschaft . . . . . . . . . . . 121

Mitteilungen des badischen bot. Vereins . . . . 45. 71. 246

Mitteilungen des thüringischen bot. Vereins . . . . . . . . 45

Oesterreichische bot. Zeitschr... . . 9. 27. 45. 96. 120. 167. 192. 210. 229

Verhandlungen des bot. Vereins der Prov. Brandenburg . . . . . . 27

Verhandlungend. k.k. zool.-bot. Gesellschaft in Wien . 28. 45. 121. 168. 193. 246

Zeitschrift der bot. Abteilung des naturw. Vereins der Prov. Posen . 72. 167. 246

$\mathrm{Z}$ eits ehrift für Gewässerkunde

97

c. Eingegangene Druckschriften

45. 168.210 .230

\section{Botanische Anstalten, Vereine, Tauschvereine, Exsiccatenwerke, Reisen etc.}

a. Botanische Gesellschaften, Vereine, Anstalten etc. (Sitzungsberichte etc.)

Botanic Gardens Sydney

Bot. Verein für die Provinz Brandenburg - . 10. 28. 47. 73. 98, 123. 230. 247

Deutsche dendrol. Gesellschaft, 8. Jahresversammlung . . . . . 172

Kgl. bot. Gesellschaft zu Regensburg . . . 11. 29. 48. 99. 169. 211

Internationales bot. Institut in Amazonas . . . . . . . . 75

Internationaler bot. Kongress in Paris . . . . . . 75

Pflanzengeographisehe Durchforschung Badens. . . . . . . 74

Preisausschreibung . . . . . . . . . . . . . 124

Preussischer bot. Verein in Königsberg i. Pr. 13. 29. 51. 72. 97. 122. 248

72. Versammlung deutscher Naturforscher u. Aerzte zu Aachen . . . . 75

\section{b. Botanische Tauschvereine und deren Kataloge, selbständige Exsiccatenwerke, Sammlungen etc.}

Allescher, A. u. Schnabl, J. N., Fungi Bavarici exsiccati

Arnold, F., Lichenes exsiccatae

- Lichenes Monacenses exsiccati.

Association Pyrénéenne

Bänitz, Dr. C., Herbarium Europaeum . . . . . . . . 251

Bauer, E., Bryotheca Bohemica . . . . . . . . . 100

Becker, W., Violae exsiccatae . . . . . . . . . . 212

Berliner bot. Tauschverein . . . . . . . . . . 14. 251

Botan. Tauschverein in Arnstadt . . . . . . . . . 232

Collins, Fr. Sh., Holden, J. and Setchell, W.L., Phycotheca boreali Americana 194

Cusick, Wm. C., The Eastern Oregon and Western Idaho Flora . . . . 194 
Delectus plantarum exsiccatarum quos anno 1899 permut. offert. Hortus Bot. Univ. Jurjevensis

Flora exsiceata Bavarica.

32. 100

Goldschmidt, M., Pflanzenverkauf.

Haglund, Arv.u.Källström, Joh., Katalog getrockneter Pflanzen a. Skandinavien 252

Herbarium Böckeler . . . . . . . . . . . . . . . . 76

Herbarium normale . . . . . . . . . . . . . . . 251

Herbar-Versteigerung . . . . . . . . . . . . . . . . 52

Hofmann, H., Plantae criticae Saxoniae . . . . . . . . . . . . 31

Jaczewski, Komarov, Tranzschel, Fungi Rossiae exsiccati . . . . 232

Karo, F., Plantae Amurenses . . . . . . . . . . . . . . . . . . 16

Krieger, W., Fungi saxonici. Fase. XXX u. XXXI . . . . . . . . . 76. 194

Ortlepp, Gesuch um Uebersendung von Labiatensamen . . . . . . . . 252

Raciborski, M., Cryptogamae parasiticae in insula Java lectae exsiccatae . . 124

Reineck u. Cermak, Plantae Brasiliae meridionalis . . . . . . . . . . 76

Reverchon, Elisée, Catalogue de 1900. . . . . . . . . . . 252

Schulz, Paul, Tauschvermittlung für Herbarpflanzen . . . . . . . 194. 251

Siegfried u. Sickenberger, Herbarien . . . . . . . . . . . . . 232

Simmer, Hans, Kryptogamen des Kreuzeckgebietes . . . . . . . . . 15

The Botanical Exchange Club of the British isles . . . . . 31. 172

Thüringischer botan. Tauschverein . . . . . . . . . . . . . 251

Treffer, Georg, Getrocknete Herbarpflanzen . . . . . . . . . . . 16

Vestergreen, Tycho, Micromycetes rariores selecti . . . . . . . . 194

Wetzsteiu, Exsiccaten aus Ohio . . . . . . . . . . . . . 172

Wi ener bot. Tauschverein . . . . . . . . . . . . . . 15

Wirtgen, F., Pteridophyta exsiccata . . . . . . . . . . . . . . . 32

\section{c. Botanische Reisen.}

A m d r u p, C. G., Ostgrönländische Expedition . . . . . . . . . . . . 195

B ornmüller, J., Bot. Forschungsreise nach den canar. (nicht canad.) Inseln 212

Botaniker, tropendiensttaugliche . . . . . . . . . . . . . . 2 232

Botan. Reis e nach Transkaukasien . . . . . . . . . . . . . 124

C a 11 i e r, Neue bot. Sammelreise in die Krim . . . . . . . . . . . . 16

Diels, Dr. L., Bot. Forschungsreise nach Südafrika und Australien . . . . 195

Gross, L. u. K n e u c k e r, A., Bot. Reise durch Istrien, Dalmatien, Montenegro, die Hercegovina und Bosnien . . . . . . . . . . . . . . 172

Palla, Dr. Ed., Reise nach Java . . . . . . . . . . . . . . . . . 212

Pa 1 s en, Ove, Centralasiatische Reise . . . . . . . . . . . 195

Pritze 1, , Dr. Ernst, Bot. Reise nach Westaustralien . . . . . . . . 194

Sch midt, Johs., Reise nach Siam . . . . . . . . . . . . 195

Sinten is, P., Bot. Reise nach. Transkaspien etc. . . . . . . . . . . 195

Sintenis, P., Bot. Reise nach Turan und Persien . . . . . . . . . . 52

Personalnachrichten $\quad 16.32 .52 .76 \quad 100.124 .172$.

195. 212. 232. 252

Corrigenda

Zur Nachricht 172. 196. 252 (auf d. Umschlag). Anfrage 


\section{Generalregister der Pflanzennamen

\section{„Allgemeinen Botanischen Zeitschrift“ Jahrgang VI. 1900.}

Die neu beschriebenen Arten, Formen etc. sind cursiv gedruckt, ausserdem wurden in dem nachstehenden Verzeichnis nur solche Pflanzen anfurnommen, bei denen kritische Bemerkungen etc. zugefügt sind. Die mit * versehenen sind abgebildet.

Seite

Abies-Arten 126. 127

Acer Monspessulanum L. v. Liburnica Pax

Aconitum telyphonum Rchb.

Aera capillaris Host $\alpha$, genuina Gren. et Godr.

- caryophyllea L.

- praecox L.

Agropyron cristatum P. B.

- repens P. B. var. Vaillantiana (Schreb.)

- r. P. b. $\alpha$. vulgare Doell

Agrostis Reuteri Boiss

- trumculata Farl.

Alchimilla acutata Buser

- acutidens Buser.

- alpestris Schmidt

- colorata Buser

- flabellata Buser

- pubescens Koch

- saxatilis Buser

- truncata Rehb. non Tausch. 25, 42,57

- trunc. Tausch.

Aldrovandia vesiculosa $\mathrm{L}$.

Alectorolophus serotinus G. Beck.

- stenophyllus Sterneck

- Vollmanni Poeverlein

Alopecurus pratensis L.

Ammophila arenaria Lk

- ar. $\chi$ Calamagrostis epigeios $\mathrm{R}$ th.

(Lk.) f. subarenaria Marss.

Anabaena Hansgirgi Schmidle

Anemone nemorosa L. o. hirsuta

- trifolia L. f. biflore

Andropogon contortus L. v. glaber Hack. subv. Allionii Hack.

- distachyus $\mathrm{L}_{\text {. }}$.

- Halepensis Brot. v. genuinaHack.

- hirtus L. v. genuina Hack.

- h. L. f. inter v. genuinam Hack. et v. pubescentem ( $V$ is.) Hack.

- hirt. v. pubescens. (Vis.)

Anthemis arvensis $\mathrm{L}_{\text {i. }} \mathrm{f}$. arenosa $\mathrm{A}$. Schwarz

- Austriaca $>$ tinctoria $\mathrm{L}_{\text {. }}$.

- tinctoria L.

Anthoxanthum aristatum Boiss.

- odoratum L.

- od. L. f. villossa Lois.

- Puellii Lec. et Lam.

Apera spica venti P. B.

Arabis alpina L monstr

Argemone Mexicana L.
Aristida coerulescens Desf.

Seite

- Forskåhlei 'Tsch.

83

Asperula cynanchica L. v. scabrida Freyn

Astragalus arenarius $L_{\text {. }} \gamma$ angustifolius - ar. . latifolius

Atriplex patulum L.

- pat. L. v. angustifolia Sim.

- pat. L. v. erecta Huds.

- pat. L. v. integrifolia Beck.

- pat. L. v. latifolia Vollmann

- pat. L. f. macrotheca Beck.

- pat. L. f microtheca Beck.

Avena pratensis $\mathrm{L}$.

- pubescens Huds.

- versicolor Vill.

Beckmannia erucaeformis Host

Bellis perennis $L$.

Bidens connatus Mhllog.

- frondosus L.

Blysmus Panz

Brachythecium polygamum II arnst.

Briza maxima I.

- media L.

Bromus albidus M. B. fintermedia Hack. 91

Cacoma deformans . . . . 98

Calamngrostis lanceolata Roth . . 86

- littorea P. B.

- litt. f. inter f. typicam et laxam (Host) Hackel

- litt. v. laxa (Host)

Calluna vulgaris Salisb.

Calothrix Hansgingi Schmidle

Campanula cervicaria $\mathrm{I}$. $>$ glomerata $\mathrm{L}$. ?

Campylonema Schmidle

Cardamine pratensis L. f.

Carduus litoralis Borloás

Carex

- Buekii Wimm.

- B. Wimm. $\times$ gracilis Curt.

- B. W. $\times$ vulgaris Hries (Figert)

- Canariensis Kükenthat .

- caespitosa L.

- capillaris L.

- cap. L. f. minima Beck.

- Davalliana Sm. $\times$ dioica $L$

- ferruginia Scop.

- fer. Scop. v. capillarioides MIurr

- fer. Scop v. Kerneri (Kohts)

86 - fer. Scop v. tenerrima Murr

- fer. Scop. f. transiens

29) - filiformis L. $\times$ riparia Curt
6

89

89

91

86

85

85

86

4

35

95

233

123

220

216

39

37

37

235

38 
Carex frigida All.

- frigida All. f. flavescens Christ.

- gracilis Curt.

- Lignicensis Figert

- refracta Schkr.

- riparia Curt. $>$ rostrata With

- rip. Curt. $\chi$ vesicaria $L$.

- sempervirens Vill. f. coarctata Huter .

- sempervirens Vill. f. semipallescens Kneucher.

- tristis M. B.

- Vratislaviensis Higert

- vulgaris Fr.

Cariceen

Cecropia

Centaurea jacea L. v. linearifolia Klinggr.

- jac. L. $>$ Rhenana Bor.

- rupestris L. V. armata Koch

- rup. v. subinervis Koch

Centritractus Lemermann

Chamneplium Wallr.

- officinale Walls.

Chantransia pulvinata Schmidle

Chenopodium album

- album L. $>$ ficitolium Sm.

- alb. I. v. hastata Klinggr.

- alb. L. $>$ opulifolium Schrad. $v$. betulifolia Murr

- alb. L. v. spicata Koeh .

- alb. L. v. viridescens St. Amans

- alb. L. v. viridis L.

- bonus henricus L.

- ficifolium Sm.

- ficif. Sm. X opulifolium Schrad.

- fic. Sm. $>$ striatum (Kraš.) Vurr

-- glaucum L.

- opulifolium Sehrad.

48. 49. 204

- opul. Schrad. × striatum (Kraš.)

- polyspermum L.

- polysp. L. v. acutifolia Sm.

- polysp. L. v. cymosa Chevall.

- pseudopulifolium Murr

- striatum (Krǎ̌.) Murr

- urbicum L.

Chroococcus Hansgirgi Schmidle . . $\quad 79$

Chlorocypereen Rikli

Chlorocyperus likikli

- badius Palla

- globosus Palla

- glomeratus Palla

- laevigatus Palla

- rotundus Palla

- serotinus Palla

Cichorium intybus L. f. alba Blümml

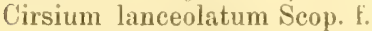

- nemorale Rehb.

- (oleraceum $>$ acaule) $\gamma$.giganteum

Cladium Schrad.

Clathrus cancellatus 'Tourf.

Clematis recta $\mathrm{L}$.

Conferva amoena v, crassior Hansgirg
49. 202

48

Soite
Conferva Raciborskii Gutwinsky

Corydalis solida $\beta$. multifida

Cosmurium bifurcatum Schmidle*

Crueigenia Morren

Cynosurus echinatus L. . . . . 22. 49

Deschampsia Bottnica Trin. . . . 87

- caespitosa P. B. $\gamma$. setifolia Bisch. 87

Dichostylis Beauv.

201. 217

Micheliana Nees.

Digitalis Iaevigata W. \& K. . . . 237

Draba confusa Ehrh. . . . . . 55. 81

- incana L. . . . . . . . 55.80

- legitima Lindbl. . . . . . 80

- Thomasii Koch . . . 55. 80

Echium vulgare $L_{\text {. }}$. . . . . . 5

Elatine hexandra DC. $\beta$. erecta . . 94

- hex. $\alpha$. fivitans . . . . . 94

- hex. $\gamma$. prostrata. . . . . 94

- orthosperma v. Dïben . . . 29

Elodea Canadensis R. \& Mx. . . . 247

Eiymus arenarius L. . . . . . . 92

Elyna Schrad. . . . . 216

Limpetrum nigrum L. . . . . . 4

Endoderma immane Schmidle. . . 17

Epipactis sessilifolia Peterm. . . 250

Erianthus strictus Bluff \& Fingerh. . 240

Eriophorum I. . . . . 214. 217

Ervum tetraspermum. L. ß. tennue . 94

Erysimum strictum El. W ett. $\beta$. patens 93

- strict. $\alpha$. typicum . . . . . 93

Furstrum Hansgirgi Schmidle* . . 34

Eucypereen Rikli . . . . 213. 216

Eucyperus Rikli . . . . 214. 217

Euphrasia stricta v. Wettst. . . . 31

Festuca myurus L. . . . ‘. . . 90

- ovina L. v. mupicaprina Hack. 99

- spadicea L. v. Durandii Hack. 990

Fimbristylis Vahl . . . . 201. 217

- dichotoma Vahl . . . . . 222

Frangula alnus Mill. . . . . . . 5

Gagea spathacea Salisb. . . . . 98

Galilea Parl . . . . . 201. 216

- mucronata Parl. . . . . . 61

Galium elatum Thuill. . . . . 23

- Podlachicum Kluk . . . . . 23

- saxatile L. . . . . . . . 249

Genista silvestris Scop. $\alpha$. typica Posp. 219

Gentiana pneumonanthe L. f. latifolia Scholler. . . . . 73

Geranium pusill. L. $>$ Pyrenaicum L. 94

Gloeotrichia Indica Schmidle . . . 35

Ty aynaldia villosa Schur . . . . 95

Hedera helix I. $\delta$. acerifolia . . 91

Heleocharis R. Br. . . . 215. 217

- acicularis $\mathrm{R}$. Br. . . . . . 63

- Carniolica Koch . . . . . 224

- ovata R. Br. . . . . . . 224

- palustris R. Br. . . . . . . 62

- uniglumis Schult. . . . . . 62

- alopecuroides Host . . . . . 84

Hierochloë alpina R. \& Sch. . . . 243 
Hierochloë australis R \& Sch

Holoschoenus Lk. . . . . . 214. 217

- anstralis Fritsch . . . . . 61

Hypnem Madüense Warust. . . . 19

Hypocrea fungicola Karst. . . . . 47

Impatiens noli tangere L. $\beta$. albiflora 94

Isolepis R. Br.

215. 217

- Savii Schult.

223

223

Juncus anceps J. de Laharpe v. atricapillus Fr. Buchenau

- anc. J. d. L. v. atric. $>$ lampocarpus Ehrh. (Buchen.)

- areticus Willd.

- atratus A. Krocker.

- Balticus Willd.

- Balt. $>$ filiformis L. (Buchenau)

- buf nius $\mathrm{L}$.

- effusus $\mathrm{L}_{\text {. }}$.

- eff. L. $\times$ glaucus Ehrh. (Buch.)

- flliformis L.

- glaucus Ehrh.

- maritimus Lam.

- squarrosus L.

- subulatus P. Forskal

- tenuis IVilld.

- trifidus L. f. parva unifiora

Klukia Andrz.

-n officinalis Andrz.

226

226

226

225

225

225

64

64

226

64

64

225

63

63

225

23

23

Knautia-Arten u.-Formen . . 1. 70. 171

- arvensis Coult. ठ. pinnata . . 95

- arv.Coult. $>$ silvatica Dub. . . 95

Kobresia Willd.

Koeleria cristata Pers. $\propto$. genuina Gren. et Godr.

- cristata Pers. v. gracilis (Pers.) Gren. et Godr.

Lappa Palladini Marcowicz

Lathyrus silvester L. v. capillaceus Scholz

Lepidium campestreR.Br". $\gamma$ subglabrum 93

Leptochaete Hansgirgi Schmidle

Lilium martagon L. f. alba Blïmml

Listera ovata L.

Lolium rigidum Gaud.

Luzula campestris DC. v. congesta Fr. Buchenau

- camp. v. multiflora Celak.

- camp. v. Sudetica Celak.

- camp. DC. v. vulgaris Gaud.

- camp. DC. v. vulgaris Gaud. f. collina G. F. W. Meyex

- confusa C. J. Lindebg. .

- Forsteri DC.

- Iutea DC.

- nemorosa E. Mey.

- nem. v. rubella Gaud.

- nivea DC

- nutans J. Luv. Jouve

- Pedemontana Boiss. et Reut.

- pilosa Willd.

- purpurea Mass.

- silvatica Gsud.

66

67

67

66

227

66

226

65
Lycopodium annotinum $I_{\text {. }} v$. pungens Desv.

-.- selago L.

Mastigocladus flagelliforme Schmidle 53.233

- Hansgirgi Schmidle . . . . 54

Medicago declinata Kit. . . . . . 2.36;

- prostrata Jcq. v. glandulifera Urb. 286

Melica ciliata I. $\alpha$. Linnaei Hack. .

- nutans L.

- uniflora Retz.

Melilotus parviflorus Desf.

- Tommassinii Jord.

Metosaenium Hansgirgi Schmidle . 1

Mibora verna Beauv.

Microspora amoena f. crassior Wille 234

- am. v. crassa Schmidle . . . 234

- de Toniana Lagerheim . . . 234

Nilium effusum L. . . . . 81

Myricaria Germanica Desv. . 178. 20.5

Nostochopsis Hansgirgi Schmidle . 77

Genothera biennis L. b. parviflora A. Gray

Ononis proccurens Wallr. ठ. Hagelliformis

Orchis militaris L. f.albiflora Blïmml

Oryzopsis coerulescens Hackel

- miliacea Aschs \& Schweinf. .

- virescens Beck

249

Panicum crus galli L. v. breviseta Döll

- er. g. v. longiseta Trin.

- glaucum L.

- lineare Krocker

- Numidianum Lam.

- sanguinale $\mathrm{L}$. v. ciliaris (Retz.)?

- sang. f. inter subv. Aegyptiacam (Retz.) et v. vulgarem Döll (Hackel) .

- sang. v. vulgaris Döll

- viride L.v. Weinmanni (R.\& Sch.)

Papaver rhoeas L. o. albiflorum

Pastinaca sativa L. $\beta$. montana

- sat. o. sativa

Peucedanum palustre Nnch. $\beta$. simplex

Phalaris Canariensis L. .

Phleunı arenarium L.

- Boehmeri Wib.
- panieulatum Huds.

Phylloplax purpureum (Racib.)

Phormidium Hansgirgi Schmidle

Physalis Peruviana L.

Picea alba Lk.

-- excela Lk.

Pilinia stagnalis G. S. West

Pinus montana Mill.

- mont. Mill. v. uncinata Rehb.

- m. Mill. unc. Rehb. f. rostrataAnt.

- m. M. v. unc. Reh. rotundata Ant.

- m. Mill. v. pumilio Hnke.

- silvestris L.

Pirola Graebneriana v. Seemen

- minor $\mathrm{L}$.

- rotundifolia $\mathrm{L}_{\text {. }}$

Pithophora pachyderma Schmidle
94

105

244

244

244

242

242

243

242

242

241

242

241

24:3

$9: 3$

$9 \frac{4}{4}$

9.4

94

$24:$

84

54

84

234

(i)

107

บ

4.

3)

14

14

14

14

14

$1: 3$

5

$\therefore$

17 
Plantago major L. f. rubra Bliumml . 107

Pon Badensis Hünke. . . . . . 252

- bulbosa L. . 90

- bulb. L. v. vivipara L. . . . . 90

- palustris L. . . . . . 90

P'olygala vulgaris L. B. coerulea . $\quad 93$

- vulg. $\alpha$. rosea. . . . . . 93

Polyporus betulinus . . . . . 47

- fomentarius . . . . . . 14

Eadiofilum apiculatum IV. \& 1 . Went 2:31

- conjunctivum Schmidle . . . 234

Raphanns Landra Moretti . 21

Rhynchospora Vahl . . . . . . 216

- alba Vahl . . . . . . . 63

Rhynchosporeen . . . . . 216

Ribes grossularia L. $\beta$. pubescens forma montana

Rivularia Hansgirgi Schmidle

Salix purpurea L. cht formae

- purp. L. $>$ viminalis L. et formae

Saxifraga decipiens Ehrh. ठ. crassifolia

- decip. E. polydactyla A. Schwarz

- decip. 3. spatulata

Scabiosa australis Wulfen.

- inflexa Kluk

Schoenoplectus Palla

- supinus Palla.

- sup. f. monostachya

- Tabernaemontani Palla

Schoenus L.

- ferrugineus $I_{\text {. }}$.

- nigricans $I_{\text {. }}$.

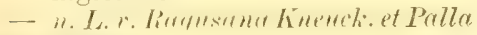

sicirpuidee'n

Scirpus L.

214

- caespitosus L.

- masitimus L.

- marit. f. (wimpacta (Hoffm.)

-- parvulus R. Sich.

- pauciflorus Lightf.

Sclerochloa dura P. B.

Scleropoa rigida Griseb.

Scytonema Hansgirgi Schmidle

- maculiformis Schmidle

seneciu subalpinus koch formae

S'esleriacuerulea Ard. v. calcarea(Opiz)

Sherardia arvensis I. f. erecta
Spirogyra rupestris Schmidle.

Seite

Stachys annua L. f. alba Blïmml 107

Stellaria nemorum L. ß. ciraeoides . 98

Stigonema Indica Schmidle . . . 54

Stupa aristella L. . . . . . . . 243 gigantea Kagr. v. Lagascie Hack. 84

- juncea L. . . . . . . 244

- pennata L. ssp. Gallica Celak. . 244

- penn. ssp. Joannis Celak f. inter Joannem et ssp. Tirsam ster. Bull.

24:)

- penn. ssp. pulcherima (C. Kóch) v. lirisuta (Vel.) f. villifolia Sink. 243

- Redowskii Trin. . . . . . 84

Thlispi montanum $I_{\text {. }}$. angustifolium 93

mont. $\alpha$ typicum . . . 93

Tolypothrix Ceylonica Schmidle . . 78

Tragus racemosus Desf. . . . . 241

Trenteroletiremonitia De Ir ild. f. Thyalina 18

Trichophorum Pers. . . . 214. 217

- Austriacum Palla . . . . 73. 222

- Germanicum Palla . . . . 223

Trifolium alpestre $\mathrm{L}_{0}>\operatorname{medium} \mathrm{L}$. . 94

— incarnatum L. $\gamma$. scandens . .94

- nigrescens Viv. . . . . . . 21

- Panormitanum Presl. . . . 21

Trisetum Gaudinianum Boiss. ... 87

- ovatum Pers. . . . . 87

Triticum eylindricum Ces. . . . . 92

- ovatum Gren. et Godr. . . . . 92

Clex Europaeus L. . . . . . . 4

Uncinia Pers. . . . . . . . 216

Lromyces truncicola. . . . . . 98

- accaria parviflora Mnch. 3. unhilis 93

- parc. $\propto$. typica .. . . . . . 93

Vaccinium myrtillus $L \cdot \gamma$. cordifolium 95

Ventenata dubia F. Schultz . . 88

Veronica chamaedrys L. f. albiflora Blümml . . . . . 107

- Dillenii Crantz . . . . . 98

Vicia glabrescens Koch . . . . . 21

- varia Host . . . . . . 21

Viscum album L. . . . . . . . 122

Veingaertneria canescens Bernh. . 87

WVeneda purpurea Racib. . . . . 234

Zacyntha verrucosa Gaertn. . . . 22 


\section{Verzeichnis der unter der Rubrik „Personalnachrichten“ vorkommenden Botanikernamen.}

\begin{tabular}{|c|c|c|c|c|c|}
\hline & selt & & & & \\
\hline meit, Dr. & 76 & Hooker, J. D. & 172 & 5. Carl . . & \\
\hline les, Dr. V. & 21 & Charles & 52 & rski, Dr. M. & \\
\hline Ilen, Grant . & 5 & ewezky, B. B. & 76 & athay, Emmerich & \\
\hline ltum, Bernard & 52 & , Н. Н. . . & 52 & Rawson, William. & \\
\hline Imbronn, Dr. & 52 & Jepson, W. J. . & 52 & Richter, Dr. Aladar 19 & \\
\hline r, E. A. N. & 76 & J urišič, Zivojn J. & 16 & Robinson, Benj. Linc. & \\
\hline ens, Dr. J. & 195 & dy, P.Reveridge & 232 & Rosenvinge, Dr. L.Kol- & \\
\hline ese, A. N. . & 52 & ck, Ernst. . & 124 & derup. . & \\
\hline esen. F. - & 195 & koll, Hjalmar 124 & t. 196 & Rothert. Dr. W. & \\
\hline czow, N. J. & 76 & & 196 & Roze, Ernest . & \\
\hline u, Dr. G. v. . & 212 & nn, Dr. F. R. & 32 & kin, John . & \\
\hline Dr. W. & 76 & y, Mary $\mathrm{H}$. . & 196 & rdo,Dr Domenico & \\
\hline , J. B. . & 52 & p Rosenvinge, & & er, Dr. V. . . & \\
\hline ers. Dr. J. & 76 & & 212 & kow. & \\
\hline a, F. . . . & 52 & z, Dr. . . . & 172 & rendener & \\
\hline , Dr. Adolf & 196 & ski, Dr. Serg. & & , Walter. & \\
\hline in, Georges & & & 252 & rt, H. . . & \\
\hline d, Dr. 1 & 232 & , Dr. Fr. & 212 & h, Charles E. & \\
\hline & 5 & p, Dr. Alfr. & 76 & Dr. Julia W. & \\
\hline , George & 16 & & 52 & der, Dr. . . & \\
\hline Dr. O. & 124 & h. Dr. & 32 & ck, Dr. J. v. & \\
\hline yer, Dr. & 8 & Dr. . . . & 52 & res, G. G. . . & \\
\hline Dr. A. & 124 & n, Fanny E. & 74 & , Dr. Lawson . & \\
\hline $\mathrm{r}$ & 5 & Anders. & 124 & e, Dr. R. . & \\
\hline etland & & Ir 195 & & r, Dr. G. & \\
\hline Boris & & Joser & & J. W. . & \\
\hline ga & 195 & Gust. & 212 & Dr Rodney H. & \\
\hline & 3 & & 196 & ikl, C. . . & \\
\hline ruges & & W. & & Dr. A. A. & \\
\hline & & 0. . & 5 & Pr. . & \\
\hline Dr. Ed. & 19 & Dr. & 172 & Hugo de & \\
\hline & 52 & , Wm. R. & 32 & er, Dr. & \\
\hline b. Bernh. & 232 & . . . & 232 & Dr. R. . & \\
\hline ale. & 5 & h, Dr. K. & 212 & , Alexander & \\
\hline 100 & 196 & C. A , & 213 & & \\
\hline Dr. Aug. . . & 32 & , Dr. A. & & , Dr. R. 1 & \\
\hline & 172 & & 23 & ead, John. & \\
\hline Dr. Aug. & 76 & & 172 & , Dr. Arved & \\
\hline$r$. & 52 & Erik OlofAug. & 212 & er, Dr. J. . & \\
\hline eorge L. & 195 & & 195 & $\mathrm{r}, \mathrm{J}$ & \\
\hline 1. W. & & & 76 & Winslow, Andr. Pet- & \\
\hline erick 0. & & & & & \\
\hline & 232 & , Arthur & 52 & altmann, Dr. & \\
\hline & 32 & Pfeffer, Dr. . . & 124 & $\mathrm{p}, \mathrm{R} . \mathrm{H}$. & \\
\hline & 195 & Planander, Johannes & & n, Hermann & \\
\hline n, Dr. Walt. J. & 52 & & & Zittel, Dr. v. & \\
\hline & 196 & Polack, & 196 & Zukal, H. . & \\
\hline
\end{tabular}

Hofmann, Dr. Ottmar 52 



\section{Allgemeine \\ Botanische Zeitschrift \\ für Systematik, Floristik, Pflanzengeographie ete. \\ Referierendes Organ}

des hot. Vereins der Provinz Brandenburg, der $\mathrm{kgl}$. hot. (iesellschaft zu liegenshurg. des Preuss. bot. Vereins in Königsberg,

und Organ der Botan. Vereinigung in Würzburg, des Berliner und schlesischen bot. 'Tauschvereins.

Unter Mitwirkung hervorragender Fachmänner herausgegeben

von . Héneker. Werderplatz 4.8 in Karlsruhe.

Verlag von J. J. Reif in Karlsruhe.

\begin{tabular}{|c|c|c|}
\hline 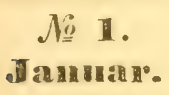 & $\begin{array}{l}\text { Erseheint am 15. jeden Monats. - } \\
\text { Preis: vierteljährl. } 1.50 \mathrm{Mk} \text {. bei freier Zusendung. }\end{array}$ & $\begin{array}{l}\text { 1900. } \\
\text { VI.Jahrgang. }\end{array}$ \\
\hline
\end{tabular}

Inhalt

Opiginalarbeiten: Dr. Jos. M urr, , rriechische Kolonien" in Valsugana isüdtirol). - Th. Hellwig. FlorenbiId der Ungegend von Kontopp im Kreise Grünberg in Schlesien. - Otto v. Seemen. Mitteilungen über die Flora der ostfries. Insel Borkum. - C. W arnstorf, Weitere Beiträge zur Flora von Pommern. III. (Forts.). A. Kneucker, Bemerkungen zu den "Carices exsiccatae" Lief. VII. (Forts.)

Bot. Litteratur, Zeitschriften ete. H. 'Tra utscholi, The later extinet floras of North-America (Ref.). - Inhaltsangabe verschied. bot. Zeitschriften.

Bot. Anstalten, Vereine, Tausehvereine, Exsiceatenwerke, Reisen etc.: Bot. Ver. d. Prov..Brandenlurg (Ref.), - Korl. hot. ('esellschaft zu Regenshurg (Ref.). Preuss. bot. Verein (Ref.). - Berliner bot. Tauschverein - Wiener bot. Tauschanstalt. Simmer, Hans, Cryptogamen des Kreuzeckgebietes (Ref.). - Treffer, Georg, Getrocknete Herbarpflanzen. - Karo, F., Plantae Amurenses. - Calli er, Neue bot. Sammelreise in die Krim.

Personalnachrichten.

\section{„Griechische Kolonien“ in Valsugana (Südtirol).}

Von Dr. Jos. Murr (T'rient).

Gelegentlich rines hotanischen Austluges nach dem Caldonazzosee am 10. Juni d. J. bemerkte ich an der Haltestelle s. (Thristoforo mmittelbar vor dem Einsteigen etliche mediterrane Arten und ebenso im weiteren Verlaufe der Rückfaht bei der Station Pergine noch andere verdächtige Gestalten zwischen veruäterischen Büschen von Titirmm rillosmm M.B. und r'ynosmbis rhimutus L. Bereits am folgenden 'Tage untersuchte ich demn auch die nächste Ungebung der Station L'rgine mit reichem Erfolge, am 16. Juni den Bahndamm zwischen der Haltestelle Castelnuovo und Borgo; am 23., resp. :30. enteleckte ich reiche Tiolonien von Ausländern unfern der Haltestelle l'ovo und in ,Alle Ghiaie" bei Trient, am 5. Juli sammelte ich an der Station lioncegno, am 9. wurde noch eine Rekognosciermongaht bis Grigno (nahe der Reichsurenze) unternommen, die Strecke Roncegno-Baco-Levico-Calceranica abgegangen und die besonders ergiebige Dammpartie gegens. 
Christoforo hin nochmals ausgebentet; an demsellen Punkte, wie auch in Pergine, hielt ich now nach der Riückkehr von den Ferien am 26. September eine kleine Nachlese.

Die P'flanzen waren. wie man sofort ersehen musste und wie auch eine Rücksprache mit dem mir befreundeten Herrn Betriebsleiter der Talsnganahahn, I. Bachler, welchem ich an dieser stelle den wärmsten Dank für die fremdliche Förderung meiner Studien ausspreche, noch weiter erhärtete, infolge der seit 1897 vorgenommenen wiederholten Bestockung entsprechender meist mehr weniger lockerer oder schoderiger stellen des Bahnkürpers mit ausländischen Grasund Luzernesamen hier aufgegangen. Auf meine Anfrage bei dem Lieferanten der sänereien. Hrn. Voltolini in Pergine, erhiclt ich die Auskunft, dass die simen ron Griechenland bezogen wurden, wozu auch im allgemeinen die von mir gefundenen Arten stimmen. Näheren Aufschluss konnte ich nicht erlangen; aus einzelnen species liesse sich vielleicht Corfu als encere Heimat unserer Einwanderer remuten.

Der Zustand der einzelnen "Kolonien" war ein sehr verschiedenartiger. Während gewisse kleinere, sehr sterile und augenscheinlich erst 18.98 mit einigem Erfolg hesiete stellen in Ghiaie hei Trient. PovoVillazano, Pergine, S. Christoforo u. s. w. sich, weil ron der sense noch völlig unherührt, in frischer Trsprünglichkeit repräsentierten und eine grosse Ilannigfaltigkeit ron offer's nur in einzelnen Exemplaren vorhandenen Arten aufwiesen, waren an den meisten anderen Punkten infolge eimmaliger oder öfterer Hahd augenscheinlich viele einjährige Arten. die anch schwerlich Zeit gehaht hatten, samen anzusetzen, bereits völlig rerschwmden; an mehreren offenbar schon 1897 besäcten stellen hate der üppige Sachwuchs ron Gras und Luzerne ("r.tu spryna", oder auch die einer geschlossenen Heeressäule gleich unerbittlich vordringenden Hasse von C'idrorium, Picris u. dgl. im grausamen Lampfe um die Herrschaft alles his anf wenige besonder's rermehrungsund widerstandsfähige Arten erstickt. Am interessantesten waren in dieser Hinsicht die Verhältnisse unter Roncegno, wo sich hereits nur mehr wenige Arten, aher die Mehrzahl davon, vormehmlich Triticum

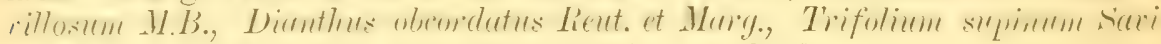
und pallidum $W . K$. in dichten Beständen vorfanden.

Zu bemerken ist. dass sich noch keine einzige aus den zahlreichen aufzuzïhlenden suecies von der unmittelbaren Nähe des Bahnkörpers entfernt hat. wozu wohl auch zu kurze Zeit seit der ersten Aussaat verstrichen ist.

Nur etwa ein halles Dutzend Arten, wie ausser dem bereits wiederholt genamntern Tritirnm illsism, welches an einer Stelle bei Roncegno rine etliche Meter lange Miniatursteppe bildet, und dessen ständigem Begleiter Dimethr: olumetutus, der bei Povo und Roncegno in dichter rosiger Saat den vorüherfahrenden Pflanzenfreund begrüsst, finden sich längs der canzen strecke auch noch P'icris symenyerimu Lom., I'iciu rum

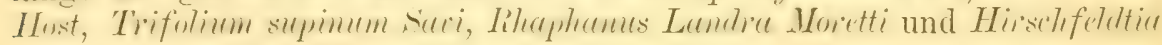
mehr weniger häufig.

Im ïbrigen ist die Gruppierung der Arten an allen Stationen ziemlich verschieden. Die richtige mediterrane Mischung zeigte sich infolge gleichmässigerer Vertretung der Grumineen, Limbellifiven, Pripilimucen, Hulcuceen, Cruciforen etc. in Pergine; auf einem äusserst sterilen 
Gehänge bei Povo dagegen setzt sich die Gerellschaft der Einwanderer

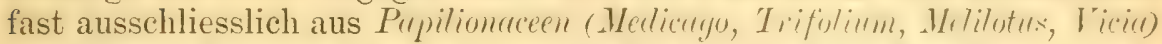
zusammen; Species, die an einzelnen Punkten zahlreich auftraten, wie

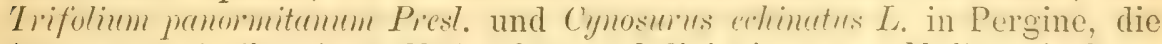
Arten von Aeyilops in $s$. Christoforo und diejenigen von Mrrlirming in P'ovo fanden sich an anderen Stellen gar nicht oder nur spärlich vertreten.

Was num die Zukunft unserer Kolonien betrifft, so diurfte trotz so vieler widriger Umstände immerhin eine beträchtliche Anzahl von Arten, in erster Linie die oben als allgemein verbreitet genannten, unserer Flora erhalten bleiben. Viele ron den Ankömmlingen, auch solche, welche jetzt durchaus nur vereinzelt auftreten, besitzen ehen duch bereits allzu zahlreiche Standorte und mögen sich unter günstigen L'mständen an einem derselben dauernd festretzen. Was etwa im höher gelegenen Valsugana abstirl,t, kann sich an der somnigen Lehne des Etschthales bei den ersten Stationen der Valsuganabahn, Villazano und Povo, akklimatisieren.

(Forts. folgt.)

\section{Florenbild der Umgegend von Kontopp im Kreise Grünberg in Schlesien.}

Von Th. Hellwig.

(Fortsetzung.)

\section{Das Haidebild.}

Es ist ein hüchst vielgestaltiges, ahwechslungsreiches. Zahlreich sind an tieferen, sumpfigen Stellen gleichsam als grössere oder kleinere Augen der Landschaft bald ein Ericetum, bald ein Caricetum oder ein sphagnetum, oder eine Terbindung unter den dreien eingeschoben.

Ausser den Waldbäumen sind hier. Typen: Osmunda regalis L. (mit var. c. interrupta Milde und $\because$ pumila Milde), Blechnum Spicant (L.) With., Aspidium cristatum (L.) Sw., Andromeda poliifolia L., Oxycoccus palustris Pers., Ledum palustre L., Rhynchospora alla (L.) Vahl, Drosera rotundifolia L., D. anglica Huds., D. rotundifolia > anglica Schiede, Arctostaphylos officinalis W. u. Gr. (bei Josephshof), Equisetum hiemale L. (mit var. Schleicheri Milde und var. minus A. Br.), Calluna vulgaris L. var. hirsuta Presl, Scorzonera humilis L., Tiola arenaria DC., V. silvatica Fr., T. arenaria $x$ silvatica Focke, Pulsatilla patens (L.) Mill., P. rernalis (L.) Mill., P. pratensis (L.) Mill., Dianthus Carthusianorum L., D. arenarius L., D. Carthusianorum $>$ arenarius C. Lucas, Geranium sanguineum L., Rubus saxatilis L., Scahiosa suaveolens Desf., S. Columbaria L., Teronica spicata L. und Potentilla reptans L. var. pubescens Fiek.

Beginnen wir hier mit den den Rahmen abgebenden Bäumen, und zwar mit Pinus silvestris. An letztere lehnt sich eine artenreiche Schmarotzerflora an. Es sind aus den Pilzen: Coleosporium Senecionis (Pers.) Form 2., Dacryomyces abietinus Pers., Corticium gigantem (Er.), Stereum Pini (Fr.), Sistotrema fusco-violaceum (Schrad.), Polyporus amorphus (Fr.), Crucibulum vulgare Pul., Belonium pineti (Batschy, Dasyseyphia calycina (Schum.), Cenangium Abietis (Pers.) (C. ferrugineum Er.), I'ezicula encrita (Karst.), Coceophacidium Pini (Alb. et Schw.), Lophodermium pinastri (Schrad.), Hysterium alneum (Ach.), Lophium mytilinum (Pers.), Nectria Cucurbitula (Tode), Sacidium Pini und eine wahrscheinlich neue Monilia sp. 
Von den Flechten sind dazu zu verzeichnen: Lecanora effusa (Pers.) f. raviosa (Hoffm.), l'sora ontreatir (Hoffm.), Bacidia rubella (Ehrh.), und an morschen kiefernen \%ambrettern Acolium Notarisii Nyl. (neu für Schlesien).

Am Fusse (auf Wurzelkïpfen) siedelt sich mit Vorliehe ein Laubmoos: Weisia cirrhata Hedw. an.

Der Epiphyt, Viscum album L. (auch V. laxiflorum Boiss. wurde gefunden), bot Gibberidia Visci Fuck. (Diplodia (Sphaeria) atrovirens (Alb. et Schw.) und D. atra?).

Juniperus communis trägt Lophodermium juniperinum (Fr.) und Apiosporium pityophilum (Nees).

An Alnus wurden beobachtet: Stereum rugosum (Pers.), Cryptosporium Neesii, Hypoxylon fulrum und H. crustaceum, Aegeria candida; von Flechten: Bacidia albescens Ach., Buellia schaereri de Not.; als Laubmoos: Fontinalis hypmoides llartm. und das Lebermoos Chiloscyphus polyanthus (L.) Corda.

Alnus glutinosa speziell ergab: Exoascus Tosquinetii (Westend.) (E. Mlni), Taphria Sadebeckii Johans., Phyllactinia suffulta (Rabenh.) und Guignardia (Laestadia) alnea (Fr.).

An Betula fand sich: Polyporus betulinus (Bull.), P. elegans (Bull.) and P. hirsutus (Wult); eirens an B. verrucosa: Fxoascus Betulae Fuck, Microsphaeria Alni (DC.) und Venturia ditricha (Fr.), sowie von Cecidien: Hieron. Nr. 56, Cephaloneon betulinum Bremi (Hier. Nr.57) und Hier. Nr. 58.

Quercus-Stöcke werden von I)acryomyces deliquescens (Bull.) zerstört. Als Flechten-Ansiedler wurden an kichen-Stümpfen gefunden: Buellia myriocarpa DC. ж. punctiformis (Holfm.), Coniocybe pallida (Pers.) \%. stilbea (Ach.) (C. leucocephala Wallr. Khr.), Pragmapora Lecanactis Mass.

(F'ortsetzung folgt.)

\section{VIitteilungen über die Flora der ostfriesischen Insel Borkum.}

Von Otto von Seemen in Berlin.

IV.

Als ich in diesem Jahre (1899) am 11. Juli nach Borkum kam, traf ich dort den Lehrer Messinger aus Frankfurt a. M., der sich sehr eifrig mit der Flora beschäftigte und auch bereits manches Neue festgestellt hatte. So hatte er gefunden, dass von Empetrum niqum L., von welchem bisher nur ein Exemplar in ter Langendelle (Viiirnloppens) hekannt war, an demselhen standorte noch melrere andere Exemplare sich befinden, welche in den dichten Polstem von Calluna vulgaris Salisb. stehen und diese kaum überragen. Ausserdem fand er noch zwei andere selur starke Exemplare davon in dem westlichsten Teile der Kievietsdelle nach dem elektrischen Leuchtturme zu. An dieser Stelle entdeckte er auch einen kräftigen Busch von Ulex europaeus L., welche Pflanze bisher ebenfalls nur in der Langendelle (Vüürgloppen) und zwar in der früheren (jetzt bereits ganz rerschwundenen) Kiefernuflanzung hekannt war. Oh diese neuen Standorte von Empotrum und Ulex in der Kievietsdelle vielleicht durch die hier in den letzten Jahren vieltach vorgenommenen Anptlanzunen entstanden sind, liess sich nicht sicher feststellen, wurde von den Einwohnern aber für möglich gehalten.

bei mmern gemeinsamen hotanischen Wanderungen entdeckte damn Herr Messinger noch auf der Woldendüne Lycopodium Selago L., das bis jetzt wohl anf Juist, Nonderney, Lamgeoog, Spiekeroog und Wangeroog, aber nicht 
auf Borkmm und Baltrum hekannt war (Franz linchenan: Flora der astfieninchen Inseln, III. Aufl., S. 44, 45). Später konnte ich feststellen, dass diese P'llanze an mehreren Stellen auf der Woldendine vorhanden war.

Von meinen eigenen Beobachtungen wäre noch hervolzuheben:

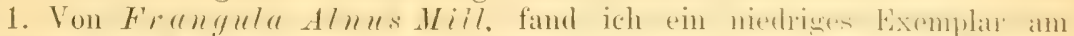

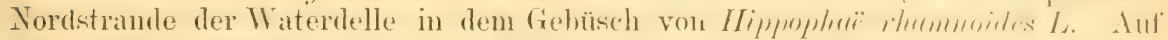
den ostfriesischen Inseln wal dieses (iehölz bisher nicht bekannt; aul dem feestlande ist es aber vielfach, namentlich auf feuchtem Boden der Geest und des

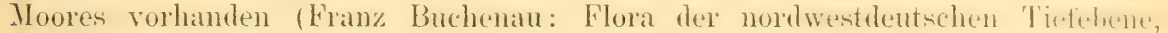
S. 343). Es ist wohl anzunehmen, dass hierbei in gleicher Weise wie bei Pinus ancuparia Gärtn. Beeren durch Vögel verschleppt worden sind.

2. Echium vulgave L., von dem ich im Jahre 1896 ein Exemplar auf dem Nachtwaichter (irundstacke hei Uphohn fand, steht jetzt anch an der Fisenbahn auf den östlichen Ausläufern der Woldendüne.

3. Dem Bastard Pirola minor $\times$ rotundifolia $=$ P. Graebneriana

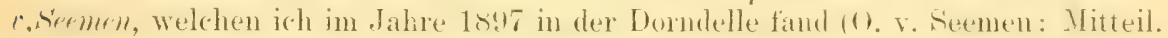
über die Flora der ostfries. Insel Borkum, III.; ,Allg. bot. Zeitsehr. Nr. 7/8, Jahrg. 18!8), wandte ich hesondere Autmerksmbeit zu. Lis fimden sich in einer Delle der Woldendüne zwischen den ummittelhar bei einander stehenden stammeltern melnfach Exemplare, die für diesen Minchling gohalten werden mussten. schon in ihrem Hahitus zeigten sie die Wittelforn zwischen der langen, lockeren Blütentraube der $P$. rotundifolia $L$. und der kuxzen, gedrungenen der $P$. minor $L$. Die Blüten neigen der geschlossenen, kugeligen Form von $P$. minor zu, und ebenso liegen auch die Kelchzipfel wie bei dieser Art mehr oder minder flach der Blumenkrone an, während sie bei der $P$. rotundifolia an der Spitze-meistens abstehen. Der Griffel ist kürzer als bei dieser Art und meistens ganz gerade. Der Ring an der Narbe fehlt entweder wie bei $P$. minor ganz, oder ist nur sehr schwach vorhanden. Die Ntaubhlitter liegen teils gerarte am diritide teils sind

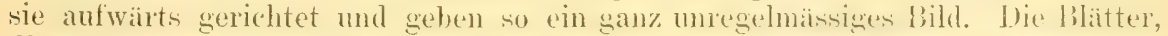
die schon bei den beiden Stammeltern sehr schwer von einander zu unterscheiden

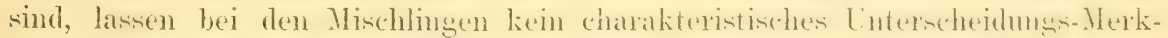
mal erkennen. Der unmittelbare Vergleich der lebenden Pflanzen aui ihrem

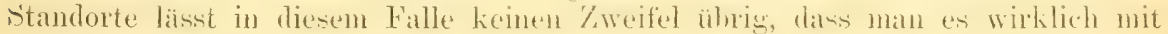
Bastarden zu thun hat, die bald mohı die Nerkmale der einen, bald die der anderen Stammart besitzen.

4. Anthoxanthum aristatum Boiss. = A. Puellii Lec. et Lam., das im Jahre 1894 von E. Wirteren an der Wasserstation in der Kievietselle funden wurde (Franz Buchenau: Flora der ostfries. Inseln, III. Aufl., S. 55), stand in diesem Jahre massenhaft in der Delle nördlich vom elektrischen Lenchtturme, also westlich von der Kievietsdelle. Die Pflanze zeichnete sich durch auffillend hohen Wuchs und eine gedrumgene Aehe ans mol stimmt genau mit einem im Herbar von K. F. W. Jessen enthaltenen Exemplar überein, das auf der nordfriesischen Insel Amrum gesammelt ist. Dr. Paul Knuth sagt zwar in seiner "Flora der nordfries. Inseln", S. 140, dass A. odoralum L. f. villossum Lois., welche Form auf Röm, Sylt und Föhr vorkommt, oft mit d. Prellii Lec. et Lam. verwechselt werde, es konnte aber gleich in dem Jessen'schen Herbar durch Vergleich mit einem dort ebenfalls vorhandenen Exemplar von A. odorutum $L$. festgestellt werden, dass ein solcher Irrtum bei der Anrumer Pflanze ausge-

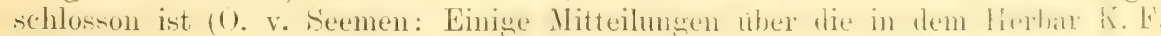
W. Jessen enthaltenen Pflanzen von der nordfries. Insel Amrum "; Engler's Bot. Jahrb., 27. B. 1. Heft 1899, Beiblatt Nr. 62). Anch bei der vorliegenden Ptianze von Borkum erscheint eine solche Verwechslung mit A. odoratum L. f. cillosum Lois. ausgeschlossen. 


\section{Weitere Beiträge zur Flora von Pommern. III. \\ Von C. IV arnstorf. \\ (Fortsetzung.)}

\section{Br. polygamum II arnst. n. sp.}

Erdmoos; in dunkelgrünen, dichten, mattglänzenden Rasen: habituell noch am meisten an Br. salebrostm erimernd. Stengel kriechend, unregelnässig mit verbogenen, bis $2 \mathrm{~cm}$ langen, nach der Spitze verdünnten, locker behlätterten, einfachen oder secundär verzweigten Aesten, durch zahlreiche Rhizoidenbüschel dem Boden fest angeheftet, weder Stamm- noch Astspitzen stoloniform, mitunter auch die Aeste in ihrer unteren Hälfte wurzelnd.

Stengelblätt er breit-lanzettlich, flachrandig, in der oberen Hälfte bald stärker, bald schwächer gesägt, mit einer starken oder mehreren schwachen Längsfalten; Rippe dünn und etwa $3 / 4$ des Blattes durchlaufend, aussen am Grunde häufig mit Rhizoiden; Zellen eng, 8-12mal so lang wie breit, nach der Basis allmählich kürzer und weiter, am Grunde rechteckig, an den nicht herablaufenden Blattflügeln quadratisch und kurz rectangulär, nicht verdickt und nicht oder kaum getüpfelt; sämtliche Zellen mit grobkörnigem Chlorophyll angefüllt, ohne oder mit gewundenem, erkennbarem Primordialschlauch. Astl)lïtter locker gestellt, trocken flatterig abstehend und mit zumteil gedrehten Spitzen, öfter nur gegen die Spitze deutlich gesägt, im übrigen nach Grösse und Form von den Stammblättern kaum verschieden.

Blüten polygam; die stengelständigen ơ Blüten dick knospenförmig und mit zahlreichen, von Paraphysen überragten Antheridien, die astständigen kleinen mit nur wenigen Antheridien; Hüllblätter aus eiförmigem Grunde in eine längere oder kürzere, meist ganzrandige Spitze ausgezogen und ohne Rippe. o und Zwitterblüten stammständig, mit einer geringen Zahl Geschlechtsorgane, Hüllblätter beider ebenfalls rippenlos. Perichaetialblätter scheidig, plötzlich lang pfriemenförmig, rippenlos oder zart gerippt. Kapsel auf kurzer, wlatter Suta übergeneigt; Epidermiszellen der Urne kurz rectangulär, quadratisch und polygonal. Zähne des äusseren Peristoms unten rotbraun, nach oben gell, und in der Spitze weiss, lreit treppenförmig gesäumt, aussen mit medianer Zickzacklinie und quergestreift, oben grob papillös; inneres Peristom blassgelb, Fortsiitze breit klaffend, Wimpern sehr fein, meist mit Anhängseln. Sporen olivengrün, $15-25 \mu$ diam.

Buslar: Pfarrgarten unter Gesträuch auf fettem Boden. Von Bi. weldersum hamptsïchlich durch polygame Blüten und schwachfaltige Blätter verschieden.

$B r$. relutimum Br. Inr. In reichfruchtenden dichten Rasen auf einer alten

Kopfweide am Wege nach Klützow.

Br: thlareusum Br. cmir. Buslar: Mergelgrube am Wege nach Klützow ster. Br. albicans Br. eur. Ebendort.

Hypmum chrysophylum Briel. ?. Buslar: In einer alten Mergelgrube rechts vom Wege nach Margaretenhof.

H. stellatum sichly. Carolinenhorst: Auf Moorwiesen zwischen Carices.

H. polygamum Sclipn. var: fallaciosum Ju: c. fr: Gr. Küssow: Innerhalb der Phragmitisregion im Madübette.

II. Knciffii Schy". Carolinenhorst: In verlassenen Torfgräben schwimmend. (Schluss folgt.) 


\title{
Bemerkungen $\mathrm{zu}$ den ,Carices exsiccatae“
}

\author{
von $A$. $K n$ e $u$ c $k$ e r.
}

VII. Lieferung 1900.

(Fortsetzung.)

Nr. 202. Crovex firgida All. fl. ped. II. p. $270(1785)=$ (. Hetration Honck. syn. I. p. 375 (1792) = C. spadicea Schlkr. Car. I." p. 90 $(1801)=C$. geniculata Host gram. IV. tab. $94(1809)=C$. sphaerica Lap. abr. p. 579 (1813) = C. caespitosa 'T'en. viagg. in Cal. p. 129 (1827). [Die Synonyme sind aus „K. Richter, Plantae europ. Tom. I. 1890 p. 162 entnommen.]

An 2 Stellen in der Schweiz auf Urgestein, an feuchten, quelligen Orten.

1. Am Rande der Gotthardstrasse, etwa in der Mitte zwisch. Hospenthal und Gotthardpass im Kanton Uri. Begleitpflanzen: Carex grypus -Schlrr., irrigua Sm., Persoonii O. F. Lang, Aspidium Lonchitis Sw., Pedicularis recutita $L$. etc.

Ca. $1800 \mathrm{~m}$ ü. d. MI.; $46^{0} 35^{\prime}$ n. Br. u. $26^{\circ} 2^{\prime}$ östl. v. F.; 1. Aug. 1899,

2. An feuchten, von Wasser überrieselten Felsen hinter dem Dorfe Binn im Kanton Wallis unter Gebüsch von Alme viridis $D C$,

Ca. $1400 \mathrm{~m}$ ü. d. M.; $46024^{\prime}$ n, Br. u. $25^{0} 1^{\prime}$ östl. v. F.; 11. Aug. 1899.

Der Standort, von dem jeweils die ausgegebenen Pflanzen stammen, ist unterstrichen.

leg. A. Kneucker.

Eine sehr interessante und sehr seltene Form, Carex friqida All. $f$. flunespus Christ, fanden Herr Kükenthal und ich am 3. Aus. 18!9 an fenchten Felsen der Rhonethalstrasse unterhalb des Hotels Gletsch und der Rhonebrücke am rechten Strassenrand in einer Höhe von etwa $17.25 \mathrm{~m}$ ï. d. M. Die f Aehren (Deckspelzen und Schläuche) haben ein vollstandig gelbes Kolorit. 6 'Tage später wurde dienelhe Form von Kükenthal etwas oberhalh dieser ritelle nochmals aufgefunden.

A. K.

Nr. 203. Cruex ferruginer Scop fl. carn. ed. II. p. $245(1772)=($ ". Scopoliana Willd. Sp. IV. p. $292(1805)=$ C. Mielichhoferi'Schkr. Car. II. p. 66 $\left(1806^{\circ}\right)=$ C. brecifolic Host gram. IV.p. $50(1809)=$ C. spadicea Host gram. IV. p. $51(1809)=$ C. cristata Clairv. man. p. $291(1811)=$ C. Scopolii Gaud. agr. II. p. $168(1811)=$ C. erecta DC. fi. fr. VI. p. $292(1815)=$ C. maciostachys Bert. am. ital. p. $421(1819)=$ C. gracillima Steud. et Hochst. en. pl. p. $132(1826)=$ C. manostachys Spr. syst. III. p. $824(1826)=$ C. scotica Spr. syst. III. p. 819 (1826). [Die Synonyme sind aus ${ }_{n}$ K. Richter, Plantae europ. Tom. I. 1890 p. 162 entnommen.]

Auf Kalkfelsen am Monte Autaretto in den Seealpen. Begleit. $\mathrm{p}$ fla $\mathrm{n}$ z en: Rhododendron fermineum. L., Anemone nurcissiflor L., Geum montamum L., Geranium silvaticum L., Alchemilla alpina L., A. vulgaris L., Bartsiu alpina L., Homogyne alpina Cass., Trollins enropaens L., Pedicularis incarnuta Jacq., Luzula siliatica Gand., Silene acaulis L., Teratrum album L., Bellidiastrum Michelii Cass., Pedicularis comosa L., Hedysarum obscurum L., Pluntago fuscescens Jord., Astrantia minor $L$.

Ca. $1800-2000 \mathrm{~m}$ ü. d. MI.; ca. $44^{0} 34^{\prime}$ n. Br. u. $24^{0} 37^{\prime}$ östl. v. F'; 20. Juli 1899. leg. E. Ferrari ('Turin). 


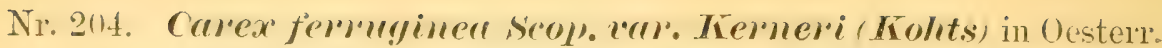
bot. Zeitschr. p, 64 (1870). Vergl. „Dr. J. Murr, Die Carexarten der Innsbrucker Flora" in Oesterr. bot. Z. 1891 p. 125. $=C$. alpigena Kemer sine descr. $(1870)=$ C. brachyrihneha Gsaller in „Oesterr. bot. Zeitschr" p. 199, 292 (1870).

1. Auf der Höttinger Alpe bei Innsbruck in Tirol; Kalk- und feiner Geröll b od en. B egleitpflanzen: Carex glanca Murr., fermginea'Scop. in typischer und subtypischer Gestalt, sempervirens Vill. Saxifraga

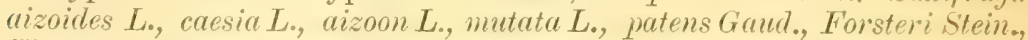
Hieracium subspeciosum Naeg., villosum L., glabratum Hoppe, glabratiforme Murr, Pedicularis rostrata L., Aster alpinus L. etc. Gegen die Frau Hitt hin gesellte sich zu Co. Kerneri Kohts auch Avenc Hostii Bss. und bei Matrei, wie auf der hohen Saile bei Innsbruck, Festuca Schenchzeri Gaud. 29. Juli 1899.

Ca. $1600 \mathrm{~m}$ ï. d. M.; $46028^{\prime}$ n. Br. u. $2901^{\prime}$ üstl. v. F.; Juli 1895 und

2. Auf dem Salzberg bei Hall in Nordtirol; ca. $1600 \mathrm{~m}$; Juli 1895. (Bei den wenigen Exemplaren, welche von diesem 2. Standort stammen, ist diese letztere Standortsangabe blau angestrichen.)

leg. Dr. J. Murr.

Nr. 205. Caren fermginea scop. f. transiens. (Siehe die Bemerkung unter Nr. 205 a.)

Auf steinigem und grasigem Boden bei Stuben am Alfenzbach in Vorarlberg. Begleitpflanzen: Vereinzelt Carex ferruginea Scop. v. Kerneri Kohts, glanca Murr. Hieracium Jurassicum Griseb., subsperiosum Naeg. subsp. melano-

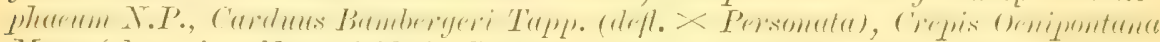
Nurr (alpestris $\times$ blattarioides), Cirsium acaule $\times$ oleraceum, spinossisimum $\times$ oleraceum.

Ca. $1400 \mathrm{~m}$ ü, đ. M.; $47^{\prime} 8^{\prime}$ n. Br. u. $27^{0} 30^{\prime}$ östl. v. F.; 2. Aug. 1899. leg. Dr. J. Мг и r.

\section{Nr. 205a. Carer fermuinea Scop. f. transiens.}

An dem felsigen und dicht berasten 1. Ufer des Längthalbaches, etwas

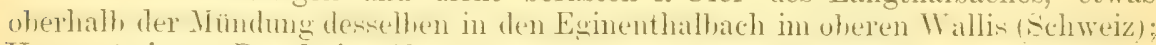
Urgestein. Begleitpflanzen: Carex glanca Mur.f., vulgaris Fr. F., frigida All., Kerneri Kohts, Senecio doronicum L., Hieracium glaciale Lach.

Ca. 1950 m ü. d. MI.; $46^{\circ} 28^{\prime}$ n. Br. u. $2602^{\prime}$ östl. v. F.; ว̆. Aug. 1896 und 7. Aug. 1899.

leg. A. Kneucker.

An dieser Stelle sei erwähnt, dass das Material der ausgegebenen Formen von C. ferruginea meinem Freunde $G$. Kükenthal zur Determination vorlag. Nr. 204 hingegen ist von Hrn. Dr. J. Nurr, welcher auch die Pflanze sammelte, bestimmt worden. In der "Oesterr. bot. Zeitschr." 1891, p. 125 wird nun von Murreine C. ferruginea var. capillarioides und von MIurr und Appel eine C. tenerrima aufgestellt. Dr. Murr hält dort die Möglichkeit offen, dass die C. tenerrima sich zu Carex Kerneri Kohts verhalte, wie cupillarioides zum Typus. Kükenthal äusserte mir gegenüber diese Ansicht auch brieflich, indem er $C$. Kerneri Kohts als Varietät der C, fernuginea ansieht, C. fermgineu var.capillarioides wäre alsdann eine gracile Form des Typus, während $C$. tenervima als

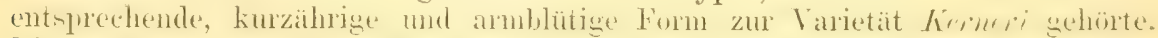
Diese Formen und Varietiten sind durch vielfache Teherginge mit einander verknüpft. So ist z. B. auch die als f. transiens unter Nr. 205 n. 205 a bezeichnete Form als ein Uebergang zwischen dem Typus und var. Kemeri anzusehen. (Schluss folgt.) A. K. 


\section{Botanische Litteratur, Zeitschriften etc.}

The later extinet floras of North-America, a posthumous work of L. C. Newberry, edited by Arthur Holliek. Washington 1898. Monographs. of the United States geological survey. Text 149 p. 68 plates.

Newberry's nachgelassenes Werk über die späteren erloschenen Floren. Fordamerikas, herausgegehen ron $\mathrm{A}$. Hollick, ist schon $18 \mathrm{il}$ angetinsern mut 1878 von dem verstorbenen Verfasser fortgesetzt. Es ist unvollständig, da Original-Exemplare abhanden gekommen sind, das Mamukript matulihe duklamsungen anfweist und Bestimmungen Newherry"s, die heute nicht mehe antrecht erhalten werden können. Das Werk enthält indessen so viel Wertvolles und Neues, dass seine Veröffentlichung notwendig' erschien. - Von den 68 Tafeln enthalten 21 Kreide- die übrigen 'T'ertiarpllanzen. Beschrieben sind 17t Arten:

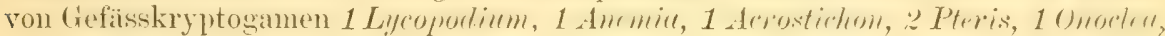
1 Lastrea, 1 Aspidium, 1 Phegopteris, 1 Sphenopteris, 4 Equistum; von Gymnospermen eine Cycadee, Nilsona; von Coniferen 1 Araucuria, 1 Abietites, 5 Sequoia, 2 Taxodium, 1 Glyptostrobus, 1 Thuja; von. Monocotyledonen 1 Phragmites, 4 Sabal, 1 Hanicaria, 1 Smilax, 1 Iris; von Dicotyledonen 2 Juglans, 2 Carya, 1 Wurica, 18 Populus, 6 Salix, 1 Carpinus, 4 Corylus, 3 Betula, 4 Alnus, 1 Fagus, 17 Quercus, 1 Ulmus, 5 Planera, 1 Celtis, 6 Ficus, 1 Piotoficus, 1 Aristolochïu, 2 Cabomba, 1 Brasaenia, 4 Magnolia, 2 Liriodendron, 1 Berberis, 2 Sassafras, 1 Cinnamomum, 2 Liquidambar, 5 Platanus, 1 Pyrus, 1 Amelanchier, 1 Crataegus, 1 Prumus, 1 Cassis, 1 Rhus, 1 Acer, 1 Negundo, 2 Sapindus, 3 Rhommus, 1 Zizyphus, 1 Vitis, 3 Aralia, 1 Comus, 2 Nyssa, 1 Sapotacites, 3 Fraximus, \& V'ibumum, 3 I'rotophyllum, 1 I'terospermites, g problematische I'hyllites, Carpolithes, Calycites und Nordenshijöldia.

Alle diese Pflanzen sind über ein weites Areal der Staaten New-Jersey, Tansas, Tehraska, Ltah, New-Mlexiko, Dakota, Tanconver-Insel, Washineton,

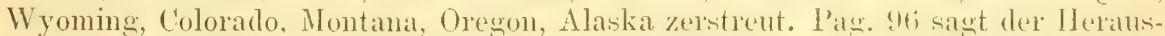
geber mit Recht: alle dirse Pflanzenaten lehren uns die interessinte It ahrheit, dass Liriodentron, Sassafras, Magnolia, Quercus, Salix, Platanus, Populus und viele andere lebende dattmenen lange vor der 'Tertianeriode in Nordamerika erschienen sind und trotz der langen Zeiträume, die sie durchlebt, sich unverandert erhalten haben, und noch in unseren Wälern die Hamptrertreter jener oft an Arten so reichen Gattungen sind.

Zugleich weist die weite Verbreitung dieser Flora darauf hin, dass schon während der Kreideperiode Nordamerika ein grosses Festland darstellte, welches allmählich von dem Kreide- und 'Tertiär-Meer' verlassen wurde und die Gestalt annahm, welche sie uns auf unseren Landkarten zeigt. Anch ist die Amnahme ron der Hand zu weisen, dass dieser gesamte Kontinent seine Form einer Hebung zu verdanken hahes. Diese kam nur eine jartielle anf eincelue (lote heschrankt

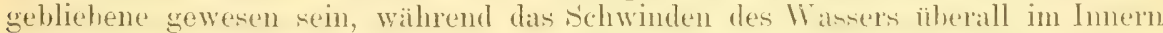
des Limbes durch Linien des früheren Niveaus in frecerocken und Flussthailerm bezengt wird.

H. 'Trautsehold.

Oesterreichische bot. Zeitschrift 1899. Nr.12. Wrettstein, li. V., Dio weibliche Blüte von Gingko. - Fritsch, K., Zur Systematik der Gattung Sorbus. - Dalla 'Torre, K. v., Historisch kritischer Beitrag zur Flora von Oberösterreich. - Richeu, G., Nachträge zur Flora v. 'Tirol und Liechtenstein.IV a isbecker, A., Beiträge zur Kenntnis der Gattung Odontites. - K a r asek. A., Standortsangaben aus Mähren. - Litteratur-Uebersicht.

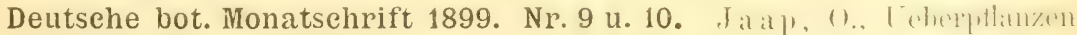
bei Bad Nauheim in Oberhessen. - Murr, Dr. J., Beiträge zur Flora von Tirol und Vorarlberg. - Winkelmann, Dr. J., Ein Ausflug nach Bornholm. 'Tscherning, Dr., Ueber Pinus silvestris I. f. Bänitzii. - Scholz. Jos. B., Der Formenkreis von Anemone ranunculoides und nemorosa. - Höck, Dr. F'. Allerweltspflanzen in unserer heimischen Phanerogamen-Flora. 
Bot. Centralblatt 1899. Nr.50. Ludwig, Dr. F., Weitere Beobachtungen über die Biologie von Helleborus foetidus. - Leisering, Bruno, Ueher die lintwickelungsgeschichte des interxylïren Leptoms bei den Dicotyledonen. - Nabokich, Dr. A., Ueber die Funktionen der Luftwurzeln. - Nr. 51. We hmer, C., Ueber einige neue Aspergillus-Arten. - Th o m a n n, Dr. J., Ueber die Bedentung des Atropin in Daturasamen. - Leisering, Bruno, Wie in vor. Nr. - Nabokich, Dr. A., Wie in vor. Nr. - Nr. 52. Leisering, Bruno, Wie in Nr. 50. - Nabokich, Dr. A., Wie in Nr. 50. - $1900 \mathrm{Nr}$. 1. Táliew, Dr. W.. '/um Bestauhungsmechanismus von Borago officinalis L. ete. - Seitel, Rud., Beiträge zur vergleichenden Anatomie der Laubbläter bei den Campanulaceen der Capflora. - Dalla Torre, Dr. K. W. v. und Sarnthein, Graf von, Die Verbreitung der Angelica verticillaris L. in I'irol.

Bulletin de l'académie internationale de géographie botanique. 1899. Nr. 121. Re ynier, A., Variation morphologique de la Ballota foetida (Imk.). Capoduro, M., De la Concrescence en botanique et en tératologie végétale. Marcailhou-D'A ymeric., Aperçus généraux sur la Flore du Japon. It onguillon, E., Catalogue des Lichens du département de la Sarthe.

Bulletin de l'association Francaise de botanique 1899. Nr. 24. Sudre, M. H., Excursions batalogiques dans les Pyrenées. - Olivier, abbé H., Exposé systematigue et description des Lichens de louest et du Nord-Ouest de la France. 1900 Nr. 25. 'T'hériot, M.J., Aperçu sur la Flore hryologique de Tunésie. Brachet, M. Flavien, Excursions botaniques, de Briançon aux sources de la Clarée et de la Durance (Hautes-Alpes). - Belèze, M arguerite, A propos de j'Heleocharis ovata h.Br. - Basset, Contribution a l'étude de l'Heleocharis ovata R.Br. Sudre. H., Excursions batalogiques dans les Pyrenées (suite). Deysson, J. et Cassat, A., Le Paspalum dilatatum Poir. à Bordeaux.

Botaniska Notiser. 1899. Heft 6. Fries, Roh. E., Polysaccum crassipes en för Sverige ny Gasteromycet. - Svedelius, Nils., En algologisk undersökning frăm svenska kusten af Oestersjön. - Brühn, N., Descriptio muscorum duorum Norvegicorum. - Holmboe, Jens, En fjeldform af Capsella bursa pastoris. - Nordstedt, O., Algologiska småsaker.

La Nuova Notarisia. Januar 1900. p. 1-48. De T oni, G., Commemorazione del conte ab Francesco Castracane degli Antelminelli. - Forti, Achille, Contributo 3. alla conoscensa della florula ficologica Veronese. Borese, ()., I teber:icht der neu erscheinenden Desmidiaceen-Litteratur. - Litteratura Phycologica.

Batonieal Gazette 1899. Oktober. Stevens, F. L., The compound oosphere of Albugo Bliti. - Derick, Carie All., Notes on the development of the holdfasts of certain Florideae.

\section{Botanische Anstalten, Vereine, Tauschvereine, Exsiccatenwerke, Reisen etc.}

Botanischer Verein für die Provinz Brandenburg. Die Nonatssitzumg am 8. Dezember eröffnete der Vorsitzende Prof. Schumann mit einigen ge. schäitlichen Mlitteilungen, unter denen die Vorlage des Prospektes einer nenen Tauschvermittlung für Herbarpflanzen, welche von dem Lehrer Paul Schulz in Berlin (Virchowstr.9) ins Leben gerufen worden ist und von der inzwischen auch schon ein erstes reichhaltiges Verzeichnis zur Versendung gelangte. Danach legte zunächst Dr. Lindau den zweiten Band des Bakteriensystems von W. Migula vor, mit kurzen Bemerkungen, welche den allgemeinen Wunsch erregten, dass er darüber demnächst doch einen eingehenderen Vortrag halten möchte, was er denn auch freundlichst in Aussicht stellte. - Prof. Sorauer legte eine Kollektion von Käfern vor, meist Cerambyxarten, welche als gefiahliche 
Fende der Kulturflanzungen in Brasilien anftreten. Danach hielt dersellhe einen Tortrag äber Beohachtungen, welche er in den Bolleschen Obstkulturen boi Köpenick in der Nähe von Berlin gemacht hat. Lr fand nämlich in diesen,

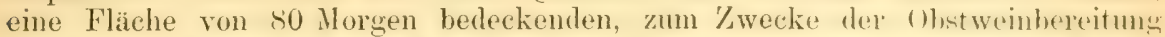
angelegten Kulturen ganze Komplexe von Johamesbeerstrïuchern, deren Blitter durch einen Pilz vollständig zerstört waren, wihrend dazwischen ginz gesunde Sträucher standen, die sich in keiner Weise angegriffen zeigten. Die nähere Untersuchung ergah, dass die heiden, sich gegen den Pilz so autfillend verschieden haltenden Sträucher zwei verschiedenen Beerensorten angehörten, und dil fortragender analoge Beobachtungen schon an Kirschen u. a. Obstbäumen genacht hat, so glaubt er folgern zu dürfen, dass gewisse Pilze nur gewisse Sorten von Pflanzen für ihre verheerenden Wucherungen auswählen und anch diese nur für hestimmte Lokalitaiten, da auch z. B. der feuchtere oder somnigere standort mit dazu beizutragen scheint. - Prof. Ascherson legte zwei neue Werke: Flora von Nürnberg von A. Fr. Schwarz als Muster einer Lokalflora, und das Pflanzenleben der schwähischsn Alh, von Dr. Rob. Gradmann vor, charakterisierte dieselben und stellte den Antrag: die Herren Verfasser zu korrespondierenden Mitgliedern des Vereins zu ernennen, welchem Antrage einstimmig entsprochen wurde. - Kurze Bemerkungen machten: Prof. Beyer ïber Nisshildungen an Cyclamen und über lioss' Flora von Sicilien; Dr. Diels über den l'arasitismus von Viscum und Loranthus; P. Hennings über einen neuen Pilz, der den Namen Nolanea hiemalis erhalten hat. - Den Schluss der Sitzung hildete ein Tortrag des Prof. Sch umann über die Kolanuss, jenes seit Jahrhunderten von den Negern viel begehrte Kau- und Genussmittel. In eingehender Weise sprach der Tortragende üher den Kolahaum, der in Westafrika von Senegambien his Angola seine Heimat hat, durch die Neger aber auch nach Westindien, Brasilien und Mexiko veryflanzt worden ist; über verschiedtene Arten von Kolanüssen, deren Wirkungen, Tertrieb durch den Handel und symbolinche Bedentung bei den afrikanischen Völkern.

W. La a ckow it z.

Kgl. bot. Gesellschaft zu Regensburg. Floristische Sitzungen: 11. Oktober 1899. Dr. Poeverlein sprach über die graphische Dar st ellung p flanzengeographisc her Result a te unter hesonderer Berïcksich. tigung der dahinzielenden Bestrehungen im leutschen Reiche. Die ilteste, aber keineswegs schlechteste Nethode, die Verbreitmg der Arten immerhalb eines beschrä n k t e n Florengebietes darzustellen - im viegensatze zu den in kleinerem Massstabe gehaltenen Areal- und Verbreitungskarten, wie sie sich hei A. P. De Candolle, Grisebach, Engler, Drude, Schimper und neuerdings in den Werken von Kenner und II'ettstein finden - ist die von $\mathrm{H}$. Hoffmann $\mathrm{H}$ seinen Beiträgen zur Flora des Mittelrheingebietes (Berichte der Oberhessischen Ciesellschaft für Natur- und Heilkumde, Bd. 6-13, 18-26. Giessen 1857-1859) angewandte. Hoffmann teilte eine Karte des von ihm bearheiteten Gebietes in lauter gleich grosse Quadrate, die or an der einen seite durch Zahlen, an der anderen durch Buchstaben nummerierte (ïhnlich dem bei den Stadtplänen und Schathproblemen in der Regel angewandten Terfahren). Entsprechend dieser Quadrierung giah, er jeder Artheschreibung ein in möglichst kleinem Massitahe gehaltenes Verbreitungskärtchen hei, auf welchen das Vorkommen der betreffenden Art innerhalb der einzelnen Quadrate durch schwarze Fiabung derselhen angedentet war. Sellsstverständlich kömnen diese Kärtchen - namentlich von Ungeühten - nu unter gleichzeitiger Benützung der in grösserem Massstahe gehaltenen Karte gelesen werden, bieten aler dafür den grossen Vorteil, dass die Verhreitung anch ol ne Beihilfe der kleinen Kïrtchen durch Angabe der Quadrate, \%. B. al 1, f 10. h 5 ausgedrückt werden kann.

Eine davon grundverschiedene Methode ist die von der Bay erischen Botanischen Gesellschaft zur Erforschung der heimischen Flora

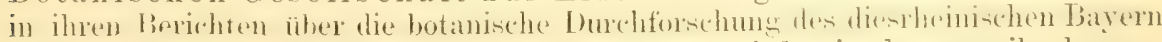
(Berichte Bd. I p. $1 \mathrm{ff}$., II p. $1 \mathrm{ff}$.) und neuerdings wieder in den von ihr heraus- 


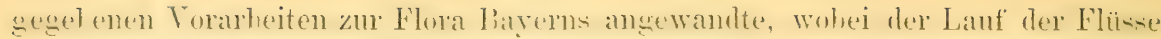

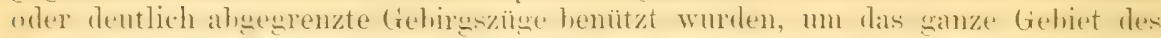

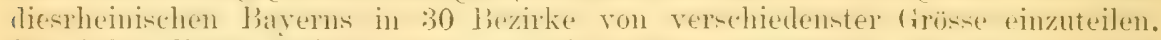

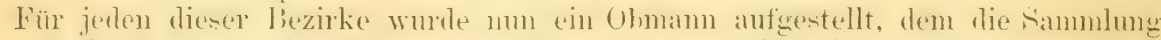

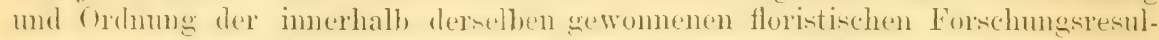
tate und deren Einsendung an die Zentralleitung der Gesellschaft, sowie die

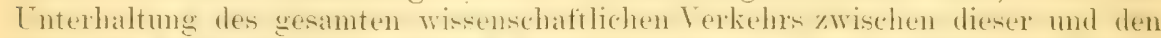

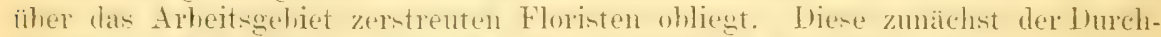

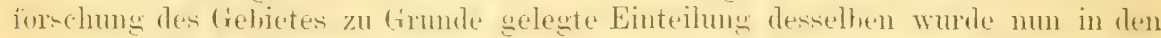

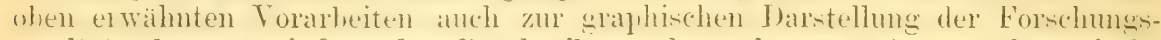
yesultate benützt, indem der Beschreibung der selteneren Arten schematische Kartchen beigegehen wurlen, anf denen die mehr oder minder gronse Häntigket des Vorkommens durch $\mathrm{v}^{1-5}$ bezeichnet wurde. Diese Methode zeichnet sich vor der einfachen Quadrierung zwar dadurch vorteilhaft aus, dass an Stelle des

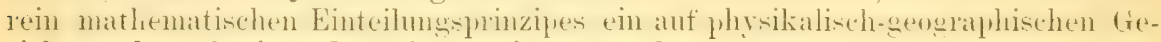
sichtspunkten basierendes tritt, weist aber dem gegenüber auch mehrere sehr stark ins Gewicht fallende Nachteile auf: vor allem macht die zumteil recht

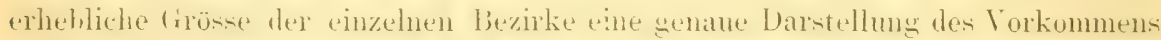

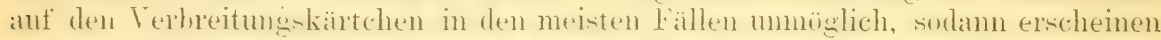

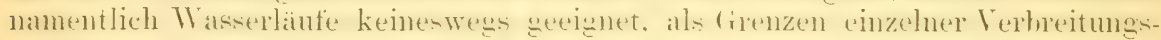
bezirke zu dienen, da ja dieselben bekanntermassen nur in den seltensten Fällen trennend, in der Regel sogar verbindend - sei es zwischen beiden Ufern, sei es zwischen weiter oherhalh mo unterhalh, gelegenen stellen derselhen - wirken.

Einen weit grösseren Massstab als diese Verbreitumgskärtchen besitzen die von meinem hochverehrten Freunde A. F. Schwarz, dem die pflanzengeographische Forschung miseres engeren Vaterlandes schon mehrere hervorragende Arbeiten verdankt, entworfenen und gezeichneten, im Verlag von U. E. Sebald in

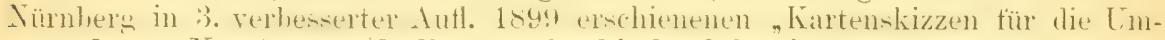
gegend von Nürnberg und die sog. fränkische Schweiz zum Zwecke von Ein-

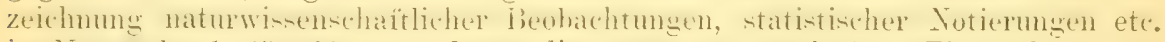

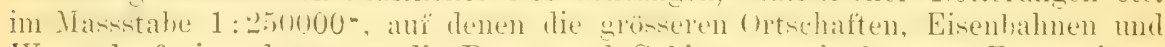
Wasserläufe in schwarzer, die Berge und Gebirgszüge in brauner Farbe eingezeichnet sind. Die Einzelehnmg der Verheitung genchieht durch holoriermug der Standorte, am besten mit roter Farbe. Diese Methode ermöglicht eine Darstellung der VerbreitungsverhäItnisse bis in die kleinsten Details, die freilich an solchen Geländestellen, die auf der Karte weder durch eine Ortschaft, noch eine Eisenbahn, eine Bodenerhebung oder einen Wasserlauf markiert sind, an Genauigkeit zu wünschen übrig lassen dürfte.

Neuestens hat nun Gradmann, der rühmlichst bekannte Verfasser des P'flanzenlelens der schwalischen Alh, in den Jahresherten des Verems für vaterl.

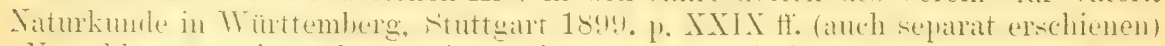

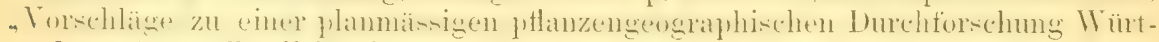
tembergs" veröffentlicht, in denen er - ausgehend von der unleugbaren Thatsache, dass die einzig befriedigende Form der Veröffentlichung die Karte ist zunächst die Anlegung von handschriftlich hergestellten Arealkarten - etwa im Massstabe 1:350000 - empfiehlt, zu denen das Material - ähnlich wie in der Bayerischen Bot. Gesellschaft - durch Vertrauensmänner gesammelt und

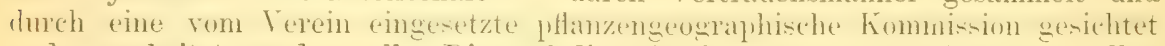
und verarbeitet werden soll. Die auf diese Weise gewomnenen Resultate sollen dann seinerzeit ebenfalls in kartographischer Form veröffentlicht werden. So sehr ich nun die von Gradmann gegebene Anregung mit Freuden begrüsse und

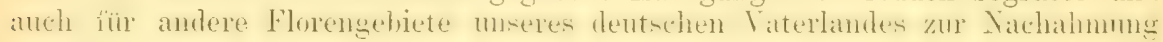
empfehle, muss ich doch in zwei nicht unwesentlichen Punkten mich auf eimen abweichenden Standpunkt stellen. Gradmann will nämlich als niederste topographische Einheiten die Ortsmarkungen benützt wissen. Die Vorteile eines solchen Verfahrens sind nun augenscheinlich, indem eimmal das Auffinden der ()rt-chaften mit Hilfe der in newerer Zeit immer mehr in Anwembung kommenden 
Shrtslexika unschwer selingt, während für die ortsüblichent Namen dex locken-

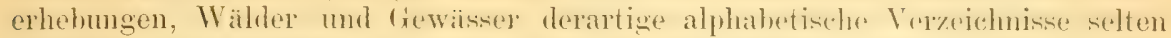
existieren und diesthen oft nicht eimmal ans den Karten zu arsehen sind, andrerseits auch bei Ortsnamen die Schreibweise in der Regel eine viel konstantere

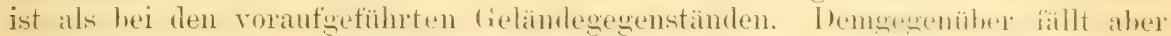
sehr ins Gewicht, dass die Grenzen der einzelnen Ortsmarkungen - wenigstens bei uns in Bayern - den Wenigsten, auch unter den lokalkundigen Leuten,

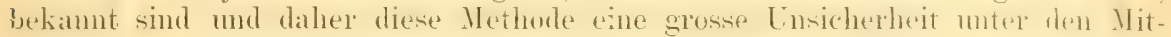
arbeitern im Gefolge hätte, die gar leicht die Quelle vieler Unrichtigkeiten werden könnte. - Ein zweiter Punkt, in dem ich Gradmann nicht beipflichten kamm, ist die von ihn rorgenchlagene Beschrönkung der Forschung anf rinzehne charakteristische und leicht bestimmbare Arten. Wenn ich ihm auch darin Recht geben muss, dass eine Ausdehnung der Arbeit auf unsere sämtlichen Getäspllanzen wenigstens für die nächste '/ulinnt mmöglich sein dürfe, so eracheint mir doch die Einheziehung wenigstens einzelner kritischen battungen moumgänglich notwendig; denn gerade dadurch wärde die Arbeit nicht nur einen nenen Reiz, sondern auch eine um vieles erhöhte wissenschaftliche Bedeutung erhalten, indem ihre hesultate anch der phylogenetischen Forschung direkt zugute kïmen; andrerseits bieten gerade die meisten kritischen Formen in ihrer Verhreitumg weit mehr Interestutes als die Mehrzahl der scharf mol dentlich ahgegrenzten Arten.

Ich möchte unter Würdigung aller voraufgeführten Gesichtspunkte eine

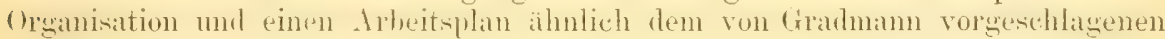
empfehlen, würde aber zur Benützung ähnlicher Verbreitungskarten, wie sie Schwarz bereits verwendet, raten; diese könnten jedoch dadurch noch eine wesentliche Verbesseruug erfahren, dass sie mit einem andersfarbigen Grad-, bezw. Quadratnetz durehzogen würden, da dieses seitens der Mitarbeiter ant jede heliehig gromse Karte ühertragen werden kïnnte und daduch das $A$ blesen und genaue Auftragen selbst der kleinsten Details ermöglichen würde.

Es ist wohl am Platze, bei dieser Gelegenheit dem Wunsche Ausdruck zu verleihen, es möchten für ein derartiges Unternehmen - ähnlich wie dies bisher für geologische und metereologische Zwecke vielfach geschehen - seitens des

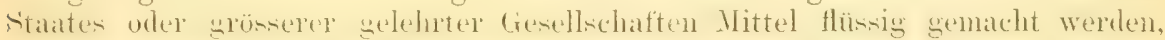

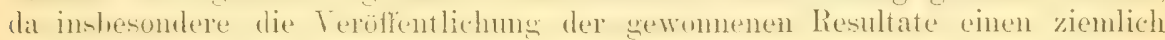
erheblichen Kostenanfwand notwendig machen würde.

(Forts. folgt.)

Preussischer Botaniseher Verein.*) I I. sitzung Känigsherg i. I'r., 18. Dezember. Es erfolgten durch Dr. Abromeit einige Vorlagen aus der neneren Fachlitteratur, 11. a. anch die lenk-chrift äber die Entwickelumg Kamerums im Jahre 1897.98, die dem Verein von der Kolonial-Abteilung des Auswärtigen Amtes im Juni d. J. zugegangen war. Darin beanspruchte das meiste Interesse der Jahresbericht über den botanischen Garten und die Versuchspflanzung in

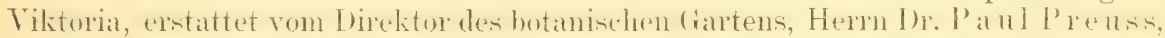
unserem hocherechitzten Vitgliede und ehemaligen Kommilitomen der Alhertina,

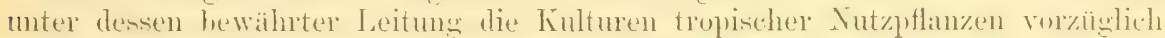

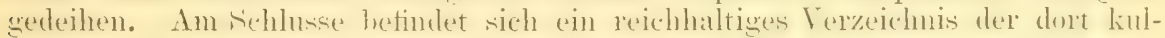
tivierten P'flanzen mit Angaben ïher Hermat und Bemerkungen ühor Verwendbarkeit unter Anführung volkstümlicher Namen ete. Sodamn erfolgte unter Demonstrationen ein Vortrag über Abänderungen einiger Arten der Gattung

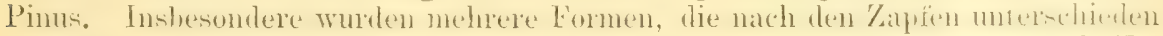
werden, von Pinus silvestris, sowie der auf den Dünen der Nehrungen mit Es:-

*) Korrektur zum vorigen Bericht: p. 291 Zeile 10 von oben Zü chtumgaversuche statt Züchtigungsversuche, Zeile 25 von unten Postverwalter statt Postverwaiter. In den hergestellten separatabdrïcken finden sich dieselben lehler und allswerdem moh auf der ersten Seite durch das Umbrechen des Satzes veranlasst: Zeile $\mathscr{y}$ von unten „blühend an" statt auf, und Zeile 7 von unten "Hierauf" statt Hieran. 
folg kultivierten P. montana Mill. eingehender behandelt. Von letzterer wird hesonder's die Form a) meinata Rchh., „Hakenkiefer" viel angetroffen, auch in den Unterformen rostrata Ant., rotundata Ant. und I'ehergiangen zur Form b) Pumilio Haenke. Letztere findet sich in den Inumenkulturen chenfills, aher nur eingesprengt. Die Höhe der Haken-, hezw. Zwergkiefer weehselt je nach der Lage des fitandortes. Während sie an exponierten Sitellen auf der hohen Düne nur niedrig bleiht, wird sie an geschützteren fitellen in Dünenwaldungen hei Memel, Nidden, Cranz, Neuhäuser und auf der frischen Nehrung ein kleiner Baum von mehreren Metern Höhe und oft sehr breitem Umfang der Krone. Hierauf wurlen zwei cigenartig entwickelte, verbildete fruchtträger von Hymenomyceten vorgelegt, die höchst wahrscheinlich zu Polyporus fomentarius gehörten. Das eine Exemplar hesast die Form mul Grösse einer schlanken bramen Hamb mit 5 teilweise getrennten fingerformigen Fortsätzen, während das andere einem schwach gekiümmten, vielfach wulstigen Lnterarme glich. Das letzteres stück zeigte an einigen stellen hereits die Anfänge der liöhrenschicht, wiihrend das ersterwähnte völlig steril war. Sie sind von einem Arbeiter an Stämmen im Wehlauer Stadtwalde entdeckt und von Herrn Lehrer Baenge in Wehlau eingesandt worden. Monströse Bildungen von Hymenomyceten, meist aus den Gattungen P'olyporus mol Lentinus, wurlen im Vereinsgelpet bereits von Loesel (1654) beobachtet und beschrieben. In Loesels Flora Prussica (ed. Gottsched Regiom. 176:3) befinden sich auf 'Tah. 17 zwei derartige Monstrositaten, aus Bienenstöcken herrührend, abgebildet und werden auf S. 99 beschrieben. Auch in $\mathrm{Hel}$ wing grs Flora quamodogenita 171: und in supplomentum 1726 werden einige monströse Bildungen von Pilzkörpern neist ausführlich heschrieben, doch ist es wohl kaum möglich, aus den Beschreibungen etwa die Pilzart zu erkennen. schon vol mehreren Jahen wurde ant einer Terein-mitzung ein geweihartig entwickeltes Mycel von Lentinus lepideus demonstriert, das ans einem unterirdischen Kelleramm hierselhst stamme. S'ehr wahrscheinlich limmen derartige monströre Bildungen offter vor, doch gelangen sie nur in selteneren Fällen zur Kenntnis. Herr Oberlehrer Vogel referierte sodann über eine Arbeit Angström's über finnische Hoore und deren subfossile Flora. Schliesslich machte Herr Lehrer

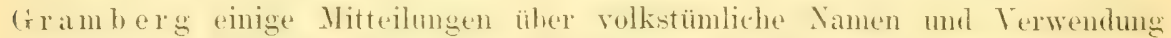
eimheimischer P'flanzen (Krenzdorn, Schöllkiant, Harthen oder Jesuwundenkrant als "Arnika", Sarothamnus scoparius „Gehrkestrauch" bei Putzig etc.).

Dr. A bromeit.

Berliner botan. Tauschverein. Vor ca. 4 Wochen wurde von Seminarwherlehrer (Ot to Leon ha rdt in Nossen, Kgr. Sachsen, das Doubletten-Verzeichnis des :31. Tanschjahres versandt. Dasselbe enthält a. (i:300 Nummern P'hanerosamen und Kryptogamen und dünte somit der reichlaltigste Katalog sem, den dieser Verein bis jetzt ausgab. Ausser Europa enthält die Liste noch Pflanzen ans syrien, Kileinasien, Persien, Nordafrika (Algier', 'Tunis) und zum ersten Male in grösserer Zahl auch aus Egypten und Tordanerika. Kryptogamen aus NordAmerika mussten nath Mitteilung des Tauschleiters vorerst yon der Aufnahme ausgeschlossen werden, weil sonst der Katalog zu umfangreich geworden wäre. Enter den Kryptosinnen flnten sich hesonders hochmordische und hochalpine Raritäten, auch solche aus der deutschen Kolonie Kamerun. Unter den Raritäten sind besonders zn nemen: Arctophila Lapjonica, Aster Silicicus, Chrysanthemum arcticum, Paconia anomala, Pyrethrum bipinnatum, Ranunculus Pallassii, Centaurea lianatica, macrorrhiza, ('yperus polystachys, Euphorbia (iaillardoti, Gaylussacia ursina, Hydrangea quercifolia, Leiojhyllum huxifolium, Najas tenuissima, Trillium stylosum, grandiflorum, Shortia galacifolia, Rhododendron arborescens, Vaseyi, punctatum, Menziesia globularis etc. etc. In den einzelnen (iruppen enthailt der Katalog 440 Fungi, 90 Algae, 200 Lichenes, 60 Characeae, 80 Musci Hepatici, 520 Frondosi, 150 Pteridophytae und 4800 Phanerogamae. Bei einer solchen lieichhaltigkeit dürfte wohl auch jeder Spezialist interessante Dinge zur Auswahl finden. 
Wiener bot. Tauschanstalt. Num ist auch der Jahreskatalue der Miemen.

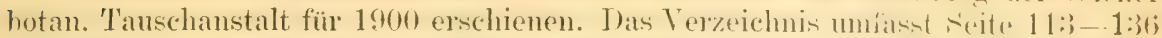
und enthät ca. 4000 Phanerogamen und P'teridophyten ans 101 verschientenen Ländern und Landesteilen in ti Wertgruppen eingeteilt mal in denselhen alphatbethisch geordnet. Die Pflanzen der ersten Gruppe sind zu 3, die der 2. zu 4, die der 3. zu 5, die der 4. zu 6 und die der 5. zu 8 Einheiten (die Einheit zu $5 \mathrm{Pfg}$.) bewertet. Die Pflanzen der 6. Gruppe sind die seltensten und wert. vollsten. In dieser Abteilung ist jeweils die Vertziffer beigesetzt, mad wir treffen dat Raritaten, welehe sogar mit 200 Linheiten, also mit 10 Hark pro Exemplar bewertet sind. En sei gestattet, nach dem Werte geordnet, aus dieser 6. (ipmpin, die ca. 1000 Phanerogamen enthält, eine kleine Blütenlese zu veranstalten. "/n je 200 Werteinheiten werden angehoten: Achullea Aegyptica L., Cirsmun acanle montanum, oleraceum $>$ pauciflorum, (hrehis sancta L. (aus Naxos) und Ranun(ulus lacerus Bell.; zu 150 Einheiten: Arabis I)örfleri Hal. und Koniga Py'enaical Nym., zu 100 Einheiten: Arabis ochroleuca B.H., Cirsium montanum $\times$ heterophyllum, pauciflorum $>$ palustre, fientiana lutea $\times$ Burseri, Neapolitana firol., Helichrysum Amorginum Boiss. Malcolndia cymbalaria H.S.?, Prephellus declinatus Nym., Reilla Mesmeniaca Boiss. Statice I)örfleri Hal., (Cheilanthes Hispanica Mett., zu so Einheiten: Allium gomphrenoides B.H., Andrzeiowskia Cardamine Richł., ('entaurea Guicciardii Bss. v. lineariloba Hal. Dörfl., Tuntasia Heldr., Chaerophyllum Bycantinum Bss. v. glabrescens Azn., ('irsinm Erisithales $\times$ paticiflorum, hypoleucum DC., Convolvulus Persicus L., Draha arctica Tahl., Hammatolobium Graecum Heldr. und 'Thalictrum orientale Bss. $1 / 4060$ Einheiten sind 133 und zu bu Einh. ca. 30 Pflanzen hewertet. Der Wert der ïhrigen Nummern beweat sich zwischen 10 und 50 Einheiten. Recht wertvoll sind auch die zahlreichen kritischen Fusinoten, welche sich auf eine Reihe besonders interessanter Gewächse beziehen. Der Katalog ist von dem Leiter der Wiener bot Tauschanstalt. J. Dörfler in Wien III, Barichgasse 36, gegen Einsendung zweier Ansichtsmostkanten, anf denen der Katalog verlangt wird, gratis und franko zu beziehen.

Simmer. Hans, Cryptogamen des Kreuzeekgebietes. Museineae, Fase. I. Nr. 4001-4050. Ton diesem schon l). 135 11. 168 des vor. Tahreange angekiundigten Exsiccatenwerk sind nun 4 Fascikel erschienen und zwar 1 Fascikel Noose, 1 Fascikel P'ilze und 2 l'ascikel Flechten. Die Einrichtung der Sammlung ist ganz vorzüglich. Die in ('ouverten eingeschlossenen und mit gedruckten Etipuetten versehenen l'fimzen liegen in gebeizten Holzschachteh im Lormat von $3: 2 / 34 \mathrm{~cm}$, welche mit hïhsch überzogenen Pappdeckeln verschlossen sind. Jedem Faticikel ist ein hektographiertes alphabetisches Inhaltsverzeichis beigefügt, mol ilen später erscheinenden Lieferungen sollen anch Separatahdrücke üher die in der "Allg. bot. Zeit-chrift" erschienenen Berichte der bot. Durchforschung der Krenzeckgrujpe beigelegt werden. Für je 2 Fascikel wird der äusserst mässige l'reis von $9 \mathrm{fl}=15$ Mark berechnet. Ich beschränke mich darauf, in der Folge aus jeden Fascikel nur eine leihe von interessanten Arten namhaft zu machen: Anthelia nivalis Lindbg., P'lagiothecium Silesiacum Br. etr., Achistidium gracile Limpr., Thuidium Philiberti Limpr., Catharinea Haussknechtii Limpr., Riccial sorocarpa Bisch.

Fungi, Fase. I. Nr. 2001-2050. Abrothallus Parmeliarum (Sinf.) Nvl. und Abroth. Smithii f. Usneae Stein, Gelidium varium (Tul.) Rehm., Coleo-jormm Campanulae (Pers.) Wint., Cronartium flaccidum (Alb. et Sch.) Wint., Emblophyllum Sempervivi (A. et S'ch.) Wint., Lamproderma violaceum Rost v. ('arestiate, l'uecinial alpina Fuck., Puce. conglomerata Schm. et Kz., Puce. Veronicale (s'chw.) Wint., Trichia Botrytis Pers., 'Tympanis alnea (Pers.) Fr., Tromyces Aconit i lacoctoni (DC.) Wint., Ur. Cacaliae (DC.) Ung* und Ur. Primulae integrifoliate (D) IV Int.

Lichenes, Fase. I u. II. Nr. 3001-3100. Bacidia atrusinguinea v. muscorum Fr., Blastenia leucorea Th. Fr., Buellia myriocarpar v. chloropolia Khr., Cladonia cyanipes 'Th. Fr., Clad. degenerans v. cladomorplia Ach., pycidata v. 


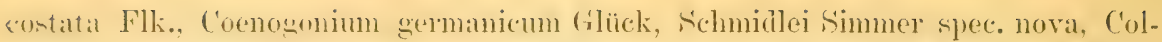

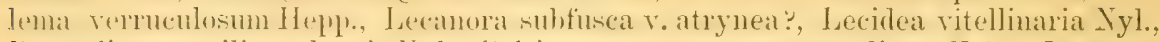
P'armelia saxatilis v. lateris Nyl., l'eltigeral scutata v propragulïera Krh.:, Psoroma

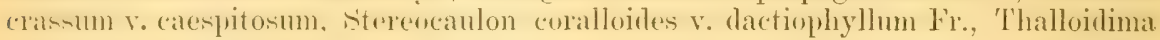
vesiculare v. dispersa Nyl., Thelotrema lepadinum v. saxicola Oliv., Verrucaria hyasceus Nyl.

Der Herausgeber Hans Simmer wohnt zur Zeit noch in Dellach im (H)erlranthale in Karnten. wirl aher in Bailde als Stationsehef nach Niklaselorf in Obersteiermark übersiedeln.

A. K.

Treffer, Georg, Getrocknete Herbarpflanzen. (i e o r g 'T reffer in Luttach, T'ost Sand in 'Tirol, versanulte küzlich sein dienjahriges (20.) Terzeichnis. Wie schon früher hervorgehoben wurde, zeichnen sich die Treffer'schen Exsiccaten durch sanz ansgezeichnete Präparation, sehr reichliche Auflage und glose billigheit aus. J)ie ca. 1000 angehotenen P'flanzen sind in 4 (rruplen zu je 10, 12, 14 u. $20 \mathrm{Pfg}$. eingeteilt. Die letzte, 25 Nummern starke Gruppe enthält meist Vertreter der Genera Cirsium, Hieracium und Sempervivum.

Karo, F., Plantae Amurenses. Die in d. J. 1897 u. 98 von F. Karo in dex. Lmgebung rom Blagowjeschtschensk (Amurgehiet in Ostsibirien) gesammelten Pflanzen sind in tadellos schöner Präparation bei J. Dörfler in Wien III, barichgasse 36 eingetroflen. Kollektionen dieser Ausbeute dürten im Frühjahr 1900 zur Tersendumg kommen. Reflektanten kömen sich jetzt schon vormerken lassen. (Aus Dörflers 'Tauschkatalog 1900.)

Callier, Neue botan. Sammelreise in die Krim. Der durch seine prächtigen tandischen Summlungen wollbekannte Botaniker A. Calli er unternimmt abermalis eine anf eine ganze Salson berechnete sammelreise in die Krim. Callier

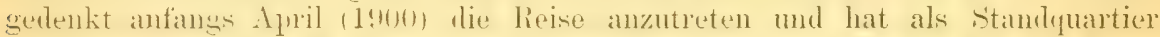
die steple zwischen simitropol und harazulasar gewählt. Da ein Teil der lieisekosten ilurch substiption anfgehracht werden miss, werden Interessenten, die ich sammlungen dienel Aushente gu einem bedeutend ermässigten substiptions. preine sichern wollen, erucht, ihre Wünsche haldigst an J. Jörfler in Wien III,

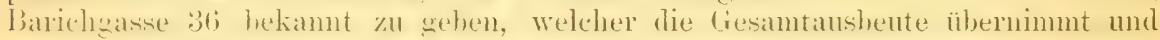
durch die Wiener bot. Tauschanstalt zur Verteilung bringen wird. Callier ist anch bereit, Wünache nach samen, Knollen und \%wiebeh für Kulturzwecke zu berücksichtigen. (Aus Dörflers Tauschkatalog 1900.)

\section{Personalnachrichten.}

Ernennungen etc.: Dr. V. Schiffner w. d. Ritterkreuz des holl. Ordens von Oranien-Nassau velliehen. - Prof. Zivojn J. Jurišić ist z. Honorar-Professor der Hochschule und stellvertretendem Direktor des bot. Gartens zu Belgrad ernammt worden. - Dr. J a k. v. Ster ne $\mathrm{ck}$ ist nach Trautenau in Böhmen verzogen. H. Sievert, im bot. Garten zu Berlin, tritt v. 10 Dez. 99 an in d. Dienst der Kamerun-Land- und Plantagen-Gesellschaft. - Prof. J. Wiesner, an d. Wiener

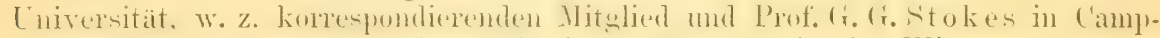
bridge (Lngland) z. answirtigen Mitglied dex Akademie der Wissenschaften zu Berlin ernannt.

Todesfälle: George Dowker am 22. Sept. - A lexanderWallace am 7. Oktober. - William Pamplin am 9. August. - Thomas Bruges Flo wer am 7. Oktober.

Druck von J.J. Reiff in Karlsruhe. 


\section{Allgemeine}

\section{Botanische Zeitschrift}

für Systematik, Floristik, Pflanzengeographie ete.

Referierendes Organ —-

des bot. Vereins der Provinz Brandenburg, der kgl. bot. Gesellschaft zu Regenshurg, des Preuss. bot. Vereins in Königsberg,

und Organ der Botan. Vereinigung in Würzburg, des Berliner und schlesischen bot. 'T'auschvereins.

Unter Mitwirkung hervorragender Fachmänner herausgegeben von A. Hewe-1ren', Werderplatz 48 in Karlsruhe. Verlag von

\begin{tabular}{|c|c|c|}
\hline $\begin{array}{l}\text { Je } 2 . \\
\text { Felonar. }\end{array}$ & $\begin{array}{l}\text { Erscheint am } 15 . \text { jeden Monats. - } \\
\text { Preis: vierteljährl. } 1.50 \mathrm{Mk} \text {. bei freier Zusendung. }\end{array}$ & $\begin{array}{l}\text { ISBO. } \\
\text { VI. Jahrgang. }\end{array}$ \\
\hline
\end{tabular}

\section{Inhalt}

Originalarbeiten: W. Sch mid le, Algologische Notizen. - C. W arnstorf, Weitere Beiträge zur Flora von Pommern. 11I. (Schluss). - Dr. Jos. M urr, , Griechische Kolonien" in Valsugana (Südtirol). - Dr. Franz Blonski, Ueber das Prioritätsrecht von Klukia Andrz. 1821 vor Chamaeplium Wallr. 1822. - Dr. E. K. Bliimml, Beiträge zur Flora von Niederösterreich. - F. Buser, Ueber Alchimilla pubescens Kioch, A. truncata Rchb. und eine neue verwandte Art aus den Tiroler Alpen (A. acutata).

Bot. Litteratur, Zeitsehriften ete.: A. Kneucker, Kükenthal. Georg, Die Carexvegetation 'des aussertropischen Südamerikas, ausgenommen Paraguay und Südbrasilien (Ref.). - Derselbe, Giesenhagen, Ir. K., Unsere wichtigsten Kulturuflanzen (Ref.). - Inhaltsangabe verschied. bot. Zeitschriften.

Bot. Anstalten, Vereine, Tausehvereine, Exsiceatenwerke, Reisen ete.: Bot.Verein der Prov. Brandenburg (Ref.). - Kgl. bot. Gesellschaft zu Regenburg (Ref. Schluss). - Preuss. bot. Verein (Ref.). - A. K n e u ck e r, Botanic Gardens Sydney (Ref.). The Botanical Exchange Club of the British isles. - Hofmann, H., llantae exiccatae Saxoniae. - Wirtgen, F., Pteridophyta exsiceata. Lief.iV. - Flora exsiccatae Bavarica.

Personalnachrichten. Glumaceae exsiceatae (auf dem Umschlag).

\section{Algologische $\mathrm{Notizen.}$ \\ Von W. Schmidle (Mannheim).}

XIV.

Einige neue von Professor Dr. Hansgirg in Vorderindien gesammelte Süsswasseralgen.

1. Pithophove prechyelerma Schmidle n. sp. Hauptstamm $60-80 \mu$ dick, mit Verzweigungen bis zum 2. Grade, die meist cinzeln abgehen. Reife Sporen end- oder mittelständig, fast kugelrund, 88-147 $\mu$ dick, mit dicker $(10-20 \mu)$, geschichteter, hyaliner '/chlliatut und braunrotem Inhalte. Doppelsporen nicht selten, gleichigestaltet. Sulspporale Zweige nicht selten, Rhizoiden und helicoide Zhweige fehlen.

Bei Mahalakshmi (Vorderindien); 14.XI.95.

2. Endodema immane schmiale n. sp. Anf obiger Pithophora sitzend. Zellen $20-40 \mu$ dick, stark torulös, mit $3-4 \mu$ dicker, geschichteter, hyaliner \%ellhaut, wenig verzwoigt mul fist 
nie eine geschlossene Fläche bildend, meist unverzweigt, ohne Haare, mit vielen Pyrenoiden. An den Enden gehen die Fäden meist plötzlich in $16 \mu$ breite und $40-60 \mu$ lange, fast rechteckige, wenig oder nicht torulöse Zellen aus.

Trentepohlia monilia De Hild.f. hyalina. Bildet ausgebreitete Räschen von gelbgrüner Farbe an Baumrinde. Stämmchen reich verzweigt, die Zweige gleichen dem Hauptstamme, nach aufwärts nicht verschmälert, die Zellen sind lang rhombisch-elliptisch mit stark verschmälerten Enden und hyaliner, dünner Zellhaut, ca. $16 \mu$ breit und $1^{1 / 2}-2^{1 / 2}$ mal so lang. Die Sporangien sind $\mathrm{k} u \mathrm{~g}$ elru $\mathrm{nd}$, entweder (selten) endständig sitzend oder (meistens) seitenständig der Mitte einer Fadenzelle aufsitzend.

Im Palmenwalde bei Mahim (Bombay) 10.XI.95.

3. Spirogyra mpestris Schmidle $\%$. sp. Die Zellen sind $33 \mu$ breit, $40-80 \mu$ lang, cylindrisch, nicht eingefaltet, mit 2-3 Chromatophoren von 3 Umgängen und vielen Pyrenoiden. Die Zygosporen in der angeschwollenen Mutterzelle sind $40 \mu$ breit, in der Länge sehr variabel und $48-90 \mu$ lang, mit dunkelbraunem glattem Epispor, oval oder bei grosser Länge fast cylindrisch mit breit abgerundeten Enden.

An feuchten Felsen zwischen Neral und Matheran. 18.XI.95.

4. Mesotaenimm Hansyirgi Schmiale n.sp. Die Zellen liegen in kleinen mikroskopischen Gallertkugeln oft gloeocystisartig eingeschachtelt. Die äusseren Hüllen sind hyalin, die inneren stahlblau oder (meistens) violett. Die Zellen sind $8 \mu$ breit, und $12-16 \mu$ lang, cylindrisch oder elliptisch mit breit abgerundeten Enden und grünem Chromatophore.

An alten Mauern unter Algen vereinzelt bei Igatpuri. 3.XI.95.

5. Cosmarium Hansgivgianum Schmidle n. sp. Die Zellen sind $24 \mu$ lang und $22 \mu$ breit mit gerader, enger, tiefer Einschnürung. Die Halbzellen sind fast halbkreisförmig mit abgerundeten unteren Ecken und abgestutztem und etwas vorgezogenem Scheitel. Die konvexen Seiten besitzen 3-4 an der Spitze abgerundete bis abgestutzte Ausbuchtungen, von welchen die scheitelständigen klar ausgebildet, die basalen allmählich verschwindend sind. Im Innern ist je 1 Pyrenoid. Die Zellh aut ist glatt, die Scheitel- und Seitenansicht nicht tumiert.

Die Zygoten sind rund, $20 \mu$ im Durchmesser gross und mit kurzen, breit abgestutzten Prominentien bedeckt, welche auf der abgestutzten oberen Seite mehrere spitze Granula tragen.

In kl. Sümpfen am Ufer des Meeres zwischen Cumballa Hill und Volkeshwar bei Bombay, 15.XI.95, unter Enteromorphen (Salzwasser!)

6. Cosmarimm mirificum schmidle m. sp. Fig. 1, 2, 3, 4. $\left.{ }^{*}\right)$ Die Zellen sind ca. $42 \mu$ breit und $65 \mu$ lang mit tiefer, gerader Einschnürung. Die Halbzellen besitzen an den unteren Enden je einen etwas abwärts gerichteten, zugespitzten und an der Spitze abgerundeten Lappen und einen ebensolchen aufwärts gerichteten an den oberen. Die Seiten sind fast gerade aufsteigend (etwas konvergierend), in der Mitte stark konkav, der Scheitel breit und flach gewölbt, jedoch stets durch 3-4 grosse kegelförmige Tumoren mit abgerundeter Spitze, die unterhalb desselben in fast horizon-

*) Die Figur wird, da sie auch andere Arten enthält, an einer an Jern Stelle eingefügt. 
taler Reihe stehen, bedeckt. Die Zellhaut der Tumoren (auch der seitlichen und basalen) ist granuliert, im übrigen glatt, bis auf einige Granula, die in horizontaler Reihe über dem Isthmus stehen. Die Scheitelansicht ist elliptisch, über dem Isthmus schwach beiderseits tuniert, diese seitlichen Tumoren jedoch durch die 8-10 apikalen, grossen, kegelförmigen Prominentien verdeckt. Die Zygoten sind ohne Stacheln $32 \mu$ dick und mit $18 \mu$ langen, nicht selten dichotom geteilten und an den Enden zweizinkigen Stacheln versehen.

Mahableshwar an Baumrinde unter Trentepohtia aurea var. acutata nob. 24.X.95.

(Forst. folgt.)

\title{
Weitere Beiträge zur Flora von Pommern. III.
}

\author{
Von C. Warnstorf.
}

(Schluss.)

\section{H. madüense Warnst.}

Manchen schwächlichen Formen des $H$. aduncum oder $H$. polycarpum ähnlich und in dichten niedergedrückten Rasen. Stengel niederliegend oder aufsteigend, häufig am Grunde der Blätter a ussen mit büschelförmigen Rhizoiden, einfach oder oberwärts geteilt und unregelmässig mit kürzeren oder längeren, teilweise fiederig angeordneten dünnen Aestchen besetzt, am Gipfel niemals sichelförmig gebogen, sondern $d u r c h$ die zusammengewickelten Blätter geradspitzig; Aeste nach der Spitze $\mathrm{zu}$ verdünnt und nur selten hier schwach sichelförmig. Stammquerschnitt rundlich-polygonal, Centralstrang armzellig, Grundgewebe farblos und dünnwandig, gegen den Umfang hin 2 Reihen wenig kleinere, gelb- und dickwandige Zellen, die beiden peripherischen Rindenzellenreihen sehr verdickt, gelb und substereid. Stengelblätter aufrechtstehend, trocken: an der Spitze mehr oder weniger gedreht, meist deutlich längsfaltig, die oberen kürzer, aus ovaler Basis rasch in eine verhältnismässig kurze, hohle Pfrieme auslaufend, die unteren länger, breitlanzettlich, sämtlich ganzrandig, Rippe dünn, gelb, über der Mitte der Lamina erlöschend. Zellen eng, in der Blattmitte 8-10, gegen die Spitze 12-15mal so lang wie breit, am Grunde zu beiden Seiten der Rippe mit kurzen, rechteckigen, stark getüpfelten, gelben, und an den Blattflügeln mit einer ausgehöhlten Gruppe grosser, rechteckiger u. polygonaler, gelblicher oder zumteil hyaliner, getïpfelter, die Rippe nicht erreichender Zellen, welche nach oben nicht immer scharf abgegrenzt sind. In den Achseln der oberen Stammblätter mit kugeligen oder ovalen kleinen Bulbillen, welche aus rundlichen, eiförmigen, rippenlosen Blättern mit rhomboidischem Zellnetz bestehen. Astblätter viel kleiner, meist sparrig abstehend, seltener teilweis etwas sichelförmig einseitig gebogen, mit gedrehten Spitzen, am Astende zu einem geraden oder hakigen Spitzchen zusammengewickelt. Zweihäusig; \& Blüten mit zahlreichen Archegonien und wenigen lanzettlichen, rippenlosen. Hüllblättern. ơ Blüten dick knospenförmig, mit zahlreichen von Paraphysen überragten Antheridien; Hüllblätter eiförmig, plötzlich kurz zugespitzt, sehr hohl und rippenlos. Sporogone unbekamnt. 
Gr. Küissow: Auf sandigem Schlickboden am Grunde von Rohrstengeln.

Mit Iypm. Kuneiffii rar. pungens und $H$. psento-fluitrms zu vergleichen.

H. cupressiforme $L$. var. eriectorum $\mathrm{Br}$. eur. Carolinenhorst: Mooriger Kiefernwald.

H. cordifolium Heduv, Carolinenhorst: In Torfgräben.

b. Sphagna.

Sipharmum cuspidatum (Ehrh.) rar. phumosum f. seviratu (Schliegh.). Sph. papillosum Lindb. var. sublaevis Limpr.

Beide bei Carolinenhorst in Hochmoorsümpfen.

c. Lebermoose.

Riccia fluitans $L$. Tümpel bei der Klützower Ziegelei mit Lemm-Arten. Aneura pinguis Dmrt. Buslar: In einer alten Mergelgrube am Wege nach Klïtzowi.

Pellia endivicefolia Dmrt. Mit voriger Art.

Frullumia ditatata Nees. Schafwäsche bei Margaretenhof an alten Weiden.

Neuruppin, im August 1899.

\section{„Griechische Kolonien“ in Valsugana (Südtirol).}

Von Dr. Jos. Murr (Trient).

(Fortsetzung.)

Die Namen derjenigen Arten, deren Bestand mehr weniger gesichert scheint, sind in der Aufzählung durch gesperrten Druck und Standorte, an denen eine Art besonders zahlreich auftrat, durch Ausrufszeichen hervorgeholen, solche Species dagegen, die joh nur in ganz vereinzelten Stiicken auffand, durch ein vorgesetztes $\dagger$ bezeichnet. Solche Arten, welche bereits als in Südtirol wild oder verwildert gefunden publiziert wurden, sind in Klammer gesctzt. Die von Herrn Baurat Freyn, dem hochverdienten Erforscher und Kenner der Mediterranflora, guitig-t determinierten Species sind durch Asteriscus kenntlich gemacht. Für die einzelnen Stationen der Linie Trient bis Castelnuovo - die Grenze bei Tezze, welche noch einiges bieten dürfte, konnte ich hislang nicht untersuchen - mögen folgende Abkürzungen angewendet werden:

G (Ghiaie hei Trient). V (Villazano), Pv (Poro), Pr (Pergine), Ch (S. Christoforo-Calceranica), B (Barco), R (Roncegno), Cn (Castehnovo).

Nun zur Aufzählung!

* Ranunculus sardous Crntz. var. laevis Celak. Pr, Ch, Cn.

†Delphinium ajacis L. R.

Nigella damascena $L$. Pr, B.

*Papaver rhoeas L. subsp.intermedium Becker, forma petalis nigromaculatis. $\mathrm{Pr}, \mathrm{Ch}, \mathrm{Cn}$.

Hir schfeldtia adpressa Moench

*forma glabrata Freyn. G!, Pv, $\mathrm{Pr}$, Ch! ete.

[Lepidium draba L.] Ch.

[L. campestre R. Br.] Pr, Ch ete.

${ }_{\dagger}^{*}$ Neslea paniculata Desv., gegen $N$.Thracica Telen. neigend. Ch.

†Calepina Corrini Desv. $\left.{ }^{1}\right) \mathrm{Pr}$.

Bunias erucago L. var. macroptera Vis. Freyn G!, Ch, R.

[Rapistrum migosum All.] gemein an der ganzen Strecke.

1) Nach Mitteilung Gelmi's von ihm vor Jahren einmal bei Trient verschleppt gefunden. 
*Rhaphanus Landra Morett $i^{1}$ ) G, V, Pr, Ch!, B; mit Vorliebe auch an schattigen, etwas feuchtenStellen unter den Bahndurchlässen.

Dianthus obcordatus Reut. et Marg. G, Pv!, Pr, Ch!, R!, Cn!; tvar, albiflora Pv.

[Saponaria vaccaria Med.] var. grandiflora Fisch. Pr; trar. albiflora Pr.

tSilene remotiflora Vis. G.

S. trinervia S. et M. G, Ch, R.

+ [S. Gallica L.] G, Pv.

S. Graeca B. et S. Pr, Cn.

* Malva moschata L. Pr.

[Althaea hirsuta L.] Pr, Ch.

Lavatera punctata.All. Pr, Ch.

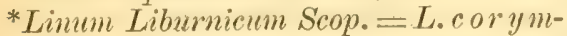

bulosum Rchb. sec. Freyn! G, $\mathrm{Pv}$, Ch.

L. angustifolium Huds. $\mathrm{Pv}, \mathrm{Pr}, \mathrm{Ch}, \mathrm{B}$. [Medicago orbicularis All.] $\mathrm{Pv}, \mathrm{Pr}$.

[M. Gerardi II.K.] Pv.

$\dagger$ M. tribuloides Desr. Pv.

11. tuberculate W. G, Pv. Pr.

[M.hispida (Gärtn.ampl.) Garcke] Pv,Pr.

$\dagger$ M. apiculata Wlld. Pv.

M. minima Desr. var. longiseta $D C$., Vis. Pv.

Trigonella corniculata L. Pv, Pr, Ch, R, Cn.

$\dagger T$. Monspeliaca L. Ch.

* Melilotus maximus Legr. Pr.

M. parviflorus Desf. ${ }^{2}$ ) G, Pv, Pr,Ch. $\dagger$ M. sulcatus Desf. Pr.

$\dagger$ Trifolium angustifolium L. Pv.

*T. Panormitanum Presl. $\left.{ }^{3}\right)$ G, $\mathrm{Pv}, \mathrm{Pr} !, \mathrm{Ch}$.

T. supinum Savi G, V,Pv, $\mathrm{Pr}, \mathrm{Ch}, \mathrm{R} !, \mathrm{Cn}$.

†T. Dalmaticum Vis. $\mathrm{Pv}, \mathrm{Pr}, \mathrm{Ch}$.

T. pallidum W.K. G, V, Pv, Pr, R!

T. la pрас еитL. G, Pv, Pr, Ch, R!,

1) Die von mir gefnndene Pflanze besitzt durchwegs hellg oldgelbe Kronenblätter; Frits ch (Exkursionsflora S. 242) führt nur die seltenere Form mit helllilafarbenen violett geaderten Kronenblättern an.

${ }^{8}$ ) Die hiesige Pflanze scheint der echte 1. parviflorus Desf., nicht der bei Freyn Fl. v. S.-Istr. [S. $308 \mathrm{f}$.] beschriebene $\boldsymbol{M}$. Tommasinii Jord. zu sein, wenigstens sind die obersten Blätter nicht keilförmig.

$\left.{ }^{3}\right)$ Die hiesige Pflanze blüht durchaus gelblichweiss, während in den Floren mehrfach (z.B bei PospichallI S. 381) die Blittenfarbe als rosenrot oder rötlichweiss bezeichnet wird.
†T. nigrescens Viv. ${ }^{4}$ ) $\mathrm{Pr}$.

$\dagger T$. vesiculosum Savi. Pr.

T. resupinatum L. G, $\mathrm{Pv}, \mathrm{Pr}, \mathrm{Ch}$.

+Psoralea bituminosa L. Pr.

Bonaveria Securidacea Rchb. G, Pr!, Ch.

+[Coronilla scorpioides Koch] $\mathrm{Pr}$.

*Ervum gracile DC. Pr, Ch.

*Vicia varia Host ${ }^{5}$ ) Pv!, Pr!, Ch!, Levico.

V. Bithynica L. G, Pv, $\mathrm{Pr}$, Ch.

$V$. grandiflora Scop. (vera) Pv.

[V. (lutea L. var. B.) hivta Balb.]

G, Pv, Pr!, Ch.

$+\left[V\right.$. peregrina $\left.L_{0}\right] \mathrm{Pr}$.

[Lathyrus aphaca L.] Pr, Ch etc.

[L. hirsutus L.] Pv, Pr, Ch.

+* $L$. sativus $L$.? In $\mathrm{Pv}$ ganz vereinzelt eine sehr kritische Form mit beträchtlich kleineren Nebenblättern, welche nur ein gutes Drittel der Blattspindel erreichen. Infolge Mangels entwickelter Hülsen konnte die Pflanze nicht mit Sicherheit bestimmt werden.

* Orlaya grandiflora Hoffm., in der Form der obersten Blätter der O. Daucorlaya Murb. entsprechend. Cn etc.

$\dagger^{*}$ Ammi mains L. $\gamma \cdot$ glaucifolium Noulet + A. visnaga Lam. Pr. [Pr.

Bupleurum protractum $H$. et L. G, Pr, Ch!, Cn.

[Torilis nodosa Gaertn.] G,Pv, $\mathrm{Pr}, \mathrm{Ch}, \mathrm{R}$. [Turgenia latifolia Hoffm.] Ch, Cn.

[Asperula arvensis L.] Pr.

$+[$ Galium tricorne With.] Pr.

[G. Parisiense L.] Cn.

[Valerianella rimosa Bast. (V. auricula DC.)] $\mathrm{Pr}$, Ch.

$+[V$. curonata DC. $] \mathrm{Ch}$.

4) I. nigrescens Viv. wird bei Fritseh Exkursionsflora S. 327 von Südtirol angegeben: mir ist kein ailterer Tiroler Standort bekannt geworden.

$\left.{ }^{5}\right)$ Die Trauben dieser Art werden in den Floren als 6-14-blütig angegeben; auch an unserer Pflanze sind sie wie beim 'I'ypus tiefpurpurn, locker und öfter armblütig: doch finden sich häufig auch ungewöhnlich üppige, meterlange Exemplare mit zumteil verlängerten, bis 25-blütigen Trauben. Neuestens (Oest. bot. Zeitschr. 1899 S. 405) hat Dr. F. S a u ter die $V$. varia Host von Bozen und Lienz angegeben; doch ist dies sicher nur eine Wiederholung der irrigen Gleichsetzung dieses Namens mit V. glabrescens Koch bei Hausmann. 


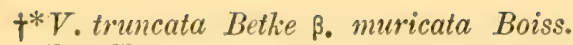
$\mathrm{Pr}, \mathrm{Ch}$.

†Tnautia integrifolia (L.) Bert. G, Ch. †[Anthemis cotula L.] Pr.

A. altissima L. Pv, Pr.

A. tinctoria L. ${ }^{3}$ ) V, Ch.

Chrysanthemum segetum L. Pv, Pr, Ch.

†*Notobasis Syriaca DC. (forma reducta) Pv.

*Carduus a cicularis Bert. G!, Pv, $\mathrm{Pr}, \mathrm{Ch}$.

+Tyrimnus leucographus Cass. G.

Scolymus Hispanicus L. ${ }^{2}$ ) G, Ch.

*Sonchus glaucescens Jord. Ch,Cn. [Crepis setosa Hall.f.] gemein.

$C$. neglect a $L$. mit ganzrandigen oder fiederspaltigen Grundblättern Pr,Ch!, Cn!.

C. vesicaria L., eine Form mit ganzrandigen Blättern. Ch.

C. rhoeadifolia M.B. Pr. (In Tirol sonst nur $C$. foetida $L_{0}$ )

Zacyntha verrucosa Gaertn. ${ }^{3}$ ) $\mathrm{Pr}, \mathrm{Ch}, \mathrm{Cn}$ !; in $\mathrm{Pv}$ einzeln eine winzige, einköpfige Form.

[Helminthia echioides Gaertn.] ${ }^{4}$ ) G, $\mathrm{Pr}, \mathrm{Ch}$. Picris Sprengerian a. Lam. G!,Pv!, Pr, Ch!.

[Anchusa Italica Retz.] V, Ch.

Echium plantagineum L. Pr, Ch, an beiden Stellen in breit- $u$. schmalblättriger Form.

+[Cynoglossum pictum Ait.] Ch.

* [Linaria elatine Mill.] eine Form mit sehr verlängerten, zarten, kleinblätt. rigen Zweigen in $\mathrm{Ch}$.

†*L. lasiopoda Freyn. Pv.

1) Die Angabe bei Hausmann S. 456 Auf Hügeln in T'ridentinischen (Poll!) finde ich wie so viele andere Angaben Poll ini's nirgends bestätigt.

2) Nach Mitteilung Gelmi's von ihm vor Jahren einmal verschleppt bei Trient beobachtet.

$\left.{ }^{8}\right)$ Von dieser Pflanze fand ich bereits in den achtziger Jahren einen nichtblühenden Blattbüschel am Wege vor Schloss Weiherburg bei Innsbruck; der Same mag beim Transporte von Zierpflanzen dorthin geraten sein.

$\left.{ }^{4}\right)$ Von mir im Herbste 1897 und auch heuer massenhaft und anscheinend wild auf Wiesen am grossen Abzugsgraben ober Mezzotedesco gefunden. Vgl. D. bot. Monatschrift 1898 S. 64.
L. spuria Mill. Ch.

Vevbascum sinuatumL. Ch, R.

$\dagger$ †artschia viscosa $I_{1}$. Pv.

* Mentha mollissima Borkh. forma? (die Pfl. war noch zu wenig entwickelt) $R$.

Salvia verbenacea $L$. Ch.

†*S.amplexicaulis Lam.(salt.autt.hung.) R

S. horminum L. Pr, Ch, Cn; an letzterer Stelle einzeln auch die Form mit hellrosenroten Kronen und karminrotem Deckblattschopfe.

$S$. verticillata $L$. In Ch eine fremdartig aussehende Form mit dunkleren Kronen und dunkelgrünen, kahleren, oberseits fast glänzenden Blättern.

†Sideritis purpurea Talb. Pr.

Euphorbia stricta L. Pr.

[Sorghum halepense Pers.] Pr.

Phalaris aquatica L. (Ph. caemlescens Desf.) Pr!, Ch, R, Cn.

Ph. brachystachys Lh. $\mathrm{Pr}, \mathrm{Ch}, \mathrm{Cn}$.

$\dagger P h$. minor lietz. Pr.

$P h$. paradoxa L. Ch.

Cynosurus echinatus L. ${ }^{5}$ ) V,Pr!,Ch. †[Koeleria phleoides Pers.] ${ }^{6}$ ) G.

Avena barbata Brot. G.

†Hordeum bulbosum L. Cn.

Aegilops triuncialis L. Pr,Ch!,Cn Aeg. triaristata W. Pr, Ch!.

Triticum villosum M.B., längs der ganzen Strecke an zahlreichen Orten in Menge bei Pv!, Pr!, Ch!, R!; die forma purpurascens an den gleichen Orten öfter zahlreich.

T. monococcum L. G, $\mathrm{Pr}, \mathrm{Ch}$.

Brachypodium distachyon R. et $S$. $G$; Pv, Pr, Ch.

+Gaudinia fragitis Beauv. Pr.

[Lolium temulentum L. var. robustum Rehb.] Ch. var. oliganthum G.G. Pr.

$\left.{ }^{5}\right)$ Nach Mitteilung Ge l mi's in Pergine bereits einmal in neuerer Zeit auf $\mathrm{A}$ eck ern mit italienischen Getreidesämereien, die vom Consorzio agrario trentino bestellt worden waren, zahlreich eingeschleppt; ob sich die Art dortselbst auch in dieser Vorkommensweise erhalten hat, ist mir nicht bekannt.

$\left.{ }^{6}\right)$ Von Hellweger und mir in Riva wild gefunden. Vgl. D. bot. Monatscht 1899 S. 154. 
*[L. Boucheanum Kunth (= Italicum $A . B r)$.$] , mit meist sehr langer und$ schmaler Aehre, von der bei uns kultivierten Pflanze habituell sehr verschieden. $\mathrm{Pv}, \mathrm{Pr}, \mathrm{Ch}, \mathrm{Cn}$. $\dagger^{*}$ L. Gaudini Parl. (= multiflorum Koch). R.

Phleum tenue Schrad. Pr, Ch. [Alopecurus agrestis L.] Pr.

Die Anzahl der für Tirol bisher noch nicht angegebenen Arten beläuft sich demnach auf etwa 77, wovon jedoch nur gegen 50 von mir in grüsserer Individuenzahl beobachtet wurden.

Trient, am 30. November 1899.

\section{Ueber das Prioritätsrecht von Klukia Andrz. 1821 vor Chamaeplium Wallr. 1822.}

\section{Von Dr. Franz Blonski (Spiczynce, Ukraina).}

Der berühmte polnische Botaniker Anton Andrzejowski (178t-1868), durch seine langjährigen botanischen Forschungen in Volhynien, Podolien, Ukraina, im Cherson'sehem und Tauri'schem Gebiete, die bisher noch nicht erschöpfend durchforscht worden sind, hochverdient, stellte in seinem nicht zum Druck gelangten Werke über neue Cruciferen-Arten und -Gattungen, welches er als Manuskript dem Verfasser des ,Regni vegetabilis Systema naturale“,*) August Pyram De Candolle, zur Benützung, resp. Veroffentlichung übersandte, zu Ehren eines anderen polnischen Botanikers, des Pfarrers Christophor Kluk**) (1739-1796) eine neue Cruciferen-Gattung "Klukia" auf, welche das Scopoli'sche Sisymbrium officinale als Klukia officinalis Andrz. enthalten sollte.

A. P. De Candolle, der in dem 2. Bande des Syst. nat. (Parisiis, 1821) die Andrzejowski'schen neuen Arten und Gattungen von C'ruciferen veröffentlicht hat, stellte die Gattung Klukia Audrz, zu seiner Sect. I Velarum (von Sisymbrium L. 1. c. p. 459). Da aber De Candolle diesen von Plinius herrührenden Namen "Velarum" nur als Sectio, nicht aber als Gattungsbenennung in oben erwähntem Werke benutzte [derselbe wurde erst einige Jahre später (1828) in J. C. Mössler's gemeinnützigem Handbuche der Gewächskunde, 2. Auflage II. p. $1165 \mathrm{zum}$ Gattungsuamen genommen], bin ich der Ansicht, dass der Gattungsnamen Klukia Andrz. 1821 ein Prioritätsrecht vor I'elurum DC. 1821 (sect. von Sisymbrium L.) und noch mehr vor Velartum Mössler 1828 haben sollte.

Man kann sich nur wundern, wie Wallroth, dem das oben genannte De Candolle'sche Werk bald nach dem Erscheinen (I. vol. 1818, II. vol. 1821) bekannt geworden sein musste, seinen neuen Namen Chamaeplium Wallr. 1822 (Schedulae criticae de plantis florae Halensis selectis I. p. 377) aufstellen konnte, anstatt von dem Andrzejowski'schen Namen Klukia aus dem Prioritätsgrunde in seinen "Schedulae criticae" Gebrauch zu machen.

Aus oben erwähnten Gründen möchte ich allen Botanikern, die Prioritatsgesetzen streng zu folgen pflegen, Klukia officinalis Andrz. apud. DC. 1821 (Syst. nat. II, p. 460) anstatt Cham a e plium of ficinale W'allr. 1822 (Sched. crit. I, p. 377) empfehlen.

*) 2 vol. Parisiis $1818-1821$.

**) Verfasser mehrerer botanischer und zoologischer Werke, unter welchen das bedeutendste Dykcyonarz roilinny, 3 vol. Warschau, 1786-1788, die Beschreibung einiger nener Arten und zwar Scabiosa inflexa Kluk 1788 (nicht 1786, wie irrtiimlich von Dr. $G$. Beck in Flora von Niederósterreich p. 1144 angegeben ist) $=$ Sic. (unstralis Irulfen 1805, Galium Podlachicum Kluk $1787=$ G. elatum Thuill. 1799 u. a. enthält. 


\section{Beiträge zur Flora von Niederösterreich.}

Von Dr. E. K. Bl ü mml (Wien).

Unter diesem Titel sollen in zwangloser Folge Beiträge zur Flora des floristisch so interessanten Landes Niederösterreich erscheinen, deren Hauptzweck die Anführung neuer Standorte, wie sie sich aus der Durchsicht dieses und jenes Herbars, schriftlicher Aufzeichnungen und selbstgemachten Funden ergeben, sein soll, wobei hauptsächlich die selteneren Pflanzen ins Auge gefasst werden und nur ausnahmsweise auch verbreitetere und gemeinere Arten zur Besprechung gelangen und zwar nur dann, wenn sich dieselben entweder durch Abweichungen in der Blütenfarbe etc. besonders auszeichnen oder selten vorkommende Formen und Varietäten von ihnen vorliegen. Insbesondere werden jene Formen, bei denen Albinismus der Blüte vorliegt, eingehend in Betracht gezogen, wobei eine Menge ganz neuer Fälle aufgezählt wird, die in Beck's ausgezeichneter und insbesondere sehr detaillierter Flora von Niederösterreich nicht enthalten sind, also für Niederösterreich und manchmal überhaupt ganz neu sind. Auch die Pflanzen, die als zerstreut in Niederösterreich angegeben werden, kommen in Betracht und inbezug auf ihre Verloreitung werden nicht unwichtige Daten gebracht. Bezüglich der Nomenklatur, Abgrenzung der Arten etc. wird die „Flora Niederösterreichs" von Prof. Dr. G. Beck als Muster genommen, und es liegt mir nur noch die Pflicht ob, auch an dieser Stelle allen jenen Personen, die mich bei folgender Arbeit in liebenswürdigster Weise teils durch Ueberlassung von Daten über niederösterreichische Pflanzenfunde, teils durch Ueberlassung von Herbarmaterial, sowie überhaupt durch gütige Ratschläge unterstützten, meinen verbindlichsten Dank auszusprechen. Die Anordnung der gesamten Beiträge erfolgt ebenfalls nach Beck's Flora, wobei jene Arten, die in Beck's Flora nicht angegeben werden, als neu für Niederösterreich betrachtet werden, wobei natürlich auch die Litteratur von 1893 (erschien die 2. Abteilung des II. Bandes der Flora N.-Oest. von Beck) bis heute, d. h. die zahlreichen kleinen Mitteilungen in den Verhảndlungen der k. k. zoologisch-botanischen Gesellschaft zu Wien, in der "Oesterreichischen botanischen Zeitschrift" etc. berücksichtigt werden, sodass dadurch verhindert wird, dass etwa hier in den folgenden Beiträgen als für Niederösterreich neue Pflanzen solche veröffentlicht werden, die schon in der Litteratur von 1893-1899 als solche bezeichnet worden wären.

I.

Dieser erste Beitrag enthält beinahe durchwegs nur Standorte aus Klostermeuburg oder der nächsten Umgebung dieser Stadt und zwar aus jener Gegend, die etwa durch folgende besonders hervorstechende Punkte und Ortschaften umgrenzt werden kann: Klosterneuburg, Leopoldsberg, Weidling, Weidlingbach, Hameau, Sofienalpe, Vorderhainbach, Steinriegel, Kirchbach, Hintersdorf, Hagenthal, St. Andräa, Wördern und längs der Donau bis Klosterneuburg zurïck. Die Standorte aus dieser Gegend wurden durchweg vom verstorbenen Klosterneuburger Ordensgeistlichen Franz Sales Edler von Schreybers aufgefunden, von einem ïberaus fleissigen Botaniker, der uns noch in manchem Beitrage entgegentreten wird. Ueberall dort, wo in diesem Beitrage kein besonderer Sammler aufgefühnt wird, ist Schreybers als solcher zu betrachten, von dem auch 
einige Standorte aus dem Rohrwalde bei Stockerau hier enthalten sind, während die weiteren Standorte vom verstorbenen Lehrer $K r a ̈ t z$, sowie von den Herren Dr. Fr. Leithe und L. Huber, sowie von meinem lieben Freunde Fr. Höfer stammen, dem ich insbesondere für die gütige Ueberlassung der gesammelten botanischen Aufzeichnungen Schreybers und für die Erlaubnis, das Herbar des letzteren einsehen zu dürfen, $\mathrm{zu}$ grossem Danke verpflichtet bin.

Panicum miliaceum L. Donauufer und Kuhau bei Klosterneuburg. Verwildert.

Alopecurus geniculatus $L$. In Gräben zu Klosterneuburg.

Agrostis alba L. var. gigantea (Roth) f. decumbens Host. Bei Weidlingbach.

Arrhenatherum avenaceum P. B. var. precatorium P.B. Leopoldsberg. dorferau.

Hordeum sativum Jess. var. vulgure L. Verwildert in der Kritzen-

Cyperus fuscus $L$. Kritzendorferau.

Scirpus radicans Schkuhr. Kierling.

Carex nigra Beck. Feuchte Wiesen im Rohrwald.

Allium flavum $L$. Bisamberg (leg. Krätz).

Muscari comosum Mill. Kritzendorferau. Das Vorkommen in der $\mathrm{Au}$ ist bemerkenswert, da diese Pflanze sonst nur auf Bergen (Hügeln) vorkommt.

Ornithogalum umbellatum L. Siedersgraben bei Klosterneuburg.

(Forts. folgt.)

\section{Ueber Alchimilla pubescens Koch, A. truncata Rchb. und eine neue verwandte Art aus den Tiroler Alpen (A. acutata).}

Von R. Buser.

In der ersten Auflage seiner Synopsis ist von Koch eine neue Alchimillenart als $A . p u b e s e n s$ M.B. in die deutsche Flora eingeführt worden. Anfünglich bloss aus den österreichischen Alpen bekannt, hat sich die Art seitdem als eine in den central. und osteuropaiischen Gebirgen sehr verbreitete erwiesen: wir kemnen sie heute ans den Pyrenien, der Auvergne, den Vogesen, dem ganzen Alpen- und Karpatengebiet, der Balkanhalbinsel und rom bithynischen Olymp. Sie gehört zu den am leichtesten zu charakterisierenden Arten.

Anderwärts*) habe ich hinsichtlich dieser Art dreierlei nachgewiesen:

1. die alpine A. mubesens hoch und die gleichnamige Pflanze des Kaukasus und der pontischen Gebirge sind zwei von einander gut verschiedene Arten;

2. eine A. pubescens "M.B." existiert, genau besehen, nicht. Marschall von Bieberstein hat keine neue Art dieses Namens aufgestellt, sundern sich begnügt, die $A$. hybrida $L$. unter dem Umtaufenamen $A$. pubescens Lam. für sein Florengebiet anzugehen. Dass er für die Kaukasuspflanze die "differentia" verändert hat, ist für die Benennung belanglos;

3. die Benemnung der Kaukasuspflanze ist somit davon ahhängig, ob der ältere Name A. pubescens Lam. 1791 zur Anwendung kommt oder nicht. Bleibt er Synonym, so hat die Kaukasusart $A$. pubeseens 11 ' illl. (1808 oder 1809 Hort. Berol. II, t. 79) zu heissen, indem Willdenow fiast gleichyeitio mit Marschall von Bieberstein die gleiche Pflanze des Kaukasus und recht eigentümlicher Weise unter dem gleichen Namen mbescens, aber unahhüngig, als nene

*) Bus. Alch. crit. ou nouvelles, Grenoble 1891, p. 13 aus Bull. Soc. Dauphinoise, 2. Série, 1892, p. 102. - Magnier Scrinia fl. sel. p. 278. 
Art beschrieben hat, ohne offenbar von der äleren A. $\mu$ ubescens Lam. zu wissen.*) Für den Fall aber, dass A. pubescens $L a m$. zur Anwendung kommt, hat Reichenbach für die Kaukasuspflanze den Eventualnamen A. sericata vorgeschlagen (Iconographie I, 1823, p. 6 u. 95).

In jerlem Fall hat die Pflanze der europäischen Gehirge, d. h. A. pubescens $K$ och einen anderen Namen zu führen. Ich habe dafür (Notes etc., p. 12 1891) die Benennung A. flabellata in Vorschlag gebracht.

Durch die Guite des Herm Dr. J. W. Chr. Goethart, Konservator des Rijksmuseums in Leiden, ist es mir möglich geworden, von den Alchimillen des Koch'schen Herbars Einsicht zu nehmen. Was speziell A. pubescens betrifft, ergab das Herbar folgenden Befund. Es sind zwei Bogen da, der eine mit der Aussenaufschrift "pubescens Seisseralpe", der andere bloss "pubescens" aussen angeschrieben.

Der erste Bogen enthält in je 1 Exemplar:

1. eine Pflanze von der Seisseralpe, eingesandt von Zuccarini als A. $p$ ubescens M.B. - Das ist zweifellos das ursprüngliche Exemplar, das Koch besass, da Zuccarini schon früh, einer der ersten, die Seisseralpe bestiegen hat. Es zeigt auch, dass die Identifikation mit der Kaukasuspflanze von Zuccarini herrührt, Koch ist bloss gefolgt;

2. ein Fruchtstück einer Pflanze, eingesandt von Dr. C. H. Schultz, dem späteren Schultz-Bipontinus, mit der Etiquette: „A. vulgaris L. 26.VII.1832 Seyseralpe auf allen Triften." - Die Bestimmung dieses Exemplars als "p ubescens M.B." rührt von Koch her, wie sich aus Flora 1833, XVI, p. 632 ergiebt.**)

3. ein gutes Stück von Hoppe, namenlos, mit der handschriftlichen Bemerkung des Senders: „Diese seegrüne, behaarte, häufig auf der Pasterze wachsende Alchemilla halte ich für eigene Art." - Hoppe thut der Pflanze in Flora 1833, XVI, p. 687 Erwähnung („A. pubescens M.B. - nicht selten auf der Pasterze, an trockenen, sandigen Stellen - in niedrigeren Gegenden "), muss also offenbar für die in gleichen Jahre gesammelte Pflanze kurz vorher den Namen durch Koch erhalten haben;

4. ein kleines Stück, namenlos, aus den Fuscher Alpen, gesammelt von v. Spitzel.

Von diesen vier Pflanzen stellen Nr. 1, 3 u. 4 die A. flabellata Bus., Nr. 2 dagegen, die Schultz'sche Pflanze, die A. acutidens Bus. dar, letztere nicht typisch, sondern in einem gedrungenwüchsigen Exemplar mit stark abgeflachten Blattlappen. Nr. 1, 2, 3 bilden die Materialunterlage zur 1. Auflage der Synolsis (1835), während Nr. 4 erst in der 3. Auflage (1857) Erwähnung findet.

(Forts. folgt.)

\section{Botanische Litteratur, Zeitschriften etc.}

Kükenthal, Georg. Die Carexvegetation des aussertropischen Südamerikas, ausgegenommen Paraguay und Südbrasilien. (Sep. aus Englers bot. Jahrbüchern 1899. Bu. 27, Heft 4.

Terfasser führt in seiner 78 Seiten starken Arbeit in zusammenfassender Weise die 61 his jetzt bekamnten Arten des genamnten Florengebietes mit lateinischen Diagnosen versehen, teilweise unter neuen systematischen Gesichtspunkten,

*) Es ist das wenig auffällig, wenn man die durch die damaligen Kriegsläufe verursachte Erschwerung des Schriftenaustausches und die nationale Animosität bedenkt. In einer handschriftlichen Notiz, die DC. seinem Exemplar. Willdenow's Species plantarum vorgeklebt hat, schätzt er die Zahl der von Willdenow l. c. vernachlässigten Namen auf ca. 6600 und speziell die aus der nur selten citierten Encyclopädie Lamarck-Poiret's auf nicht weniger denn rund 1000 .

**) C. H. Schultz giebt zwar 1. c. an, dass die von ihm früher (op. c. 601) als A. montuna $W$. angeführte Pfanze von Koch als pubescens M.B. bestimmt worden sei. Koch's Herbar zeigt, dass dies nicht richtig ist, sondern dass das eingesandte Stück den Namen vulgaris trug. Schultz praecisiert den Standort folgendermassen: auf etwas feuchten Alpenweiden in der Nähe der letzten Alphütte beim Besteigen des Plattkofels. Einen feuchten Standort verträgt die xerophile $A$. flabellata nicht. 
welche in der Einleitung begründet werden, auf. Die von ihm neu aufgestellten Sektionen des Subgenus Eucarex sind: Bifurcatae und Mirrocarpae, bei den Tigneen Alatae und Capituligerae. Vom Verfasser werden folgendo neue Arten und Formen beschrieben: C'arex trichodes Steud. B. major, Gatjana. Drsu. B. rufa,

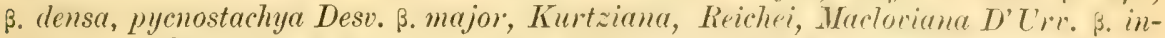

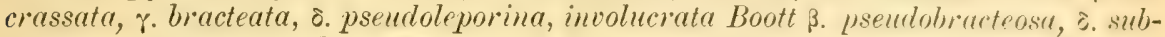
divulsa, $\varepsilon$. angustata, bonariensis Desf. o. remota, latibrarteolata, Bunlisii Buott $\beta$. distans, $f$. pallescens, $f$. brevifolia, o. abbreviata, decidua Boott $\beta$. minor, In minii

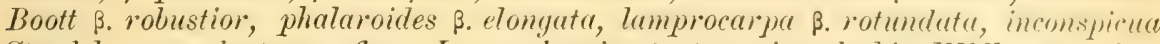
Steudel $\gamma$. pseudextensa, flaia L. ssp. brevirostrata, microglochin If hlby. ssp. fuegina, acutata Boott $\beta$. ciliata, $\gamma$. hirtisquama. Am Schlusse dieser sehr verdienstvollen Arbeit ist ein Schlüssel zur Bestimmung der Arten beigegeben. A. K.

Giesenhagen, Dr. K., Unserewichtigsten Kulturpflanzen. Mit zahlreichen Abbildungen im Text. (,Aus Natur und Geisteswelt." Sammlung wissenschaftlichgemeinverständlicher Darstellungen aus allen Gebieten des Wissens. 12 monatliche Bändchen zu je 90 Pf., geschmackvoll gebunden zu je 1.15 M., oder 54 wöchentliche Lieferungen zu je $20 \mathrm{Pf}$.) Verlag von B. G. Teubner in Leipzig.

Das vorliegende 114 Seiten starke Büchlein wurde an Bord eines Lloyddampfers während einer Reise nach Java geschrieben, ist populär abgefasst und enthält in 6 Vorträgen das Wichtigste über unsere hauptsächlichsten Getreide. pflanzen, über ihre Organe, ihre Entwickelung, ihre Ernährung, ihre Geschichte, ihre Krankheiten etc. Der Verfasser versteht, an der Hand von Beispielen in geschickter Weise die nötigsten allgemeinen botanischen Kemntnisse abzuleiten. Das Werkchen kann daher auch jedem gebildeten Laien als angenehme, leichtverständliche Lektüre bestens empfohlen werden.

A. K.

Verhandlungen des bot. Vereins d. Prov. Brandenburg. 41. Jahrg. 1899. Abhandlungen: Bolle, C., Eine Farbenvarietät der Armeria elongata. J a p, O., Aufzählung der bei Lenzen beobachteten Pilze. - W a rnstorf, C., Neue Beiträge zur Kryptogamenflora der Mark Brandenburg. - R uhland, IV., Ueber einige neue oder weniger bekannte Ascomyceten Deutschlands. - Hennings, P., Einige neue und interessante Ascomyceten aus der Tmgebung von Rathenow (Mit Abbildungen). - W $\mathrm{W}$ ise, A., Eine monströse Frucht von Citrus Aurantium (Mit Textfiguren). - Loeske, L., Bryologische Beobachtungen aus dem Jahre 1898. - Ascherson, P., Zur Chronik der Märkischen Flora. Fitting, H., Schulz, A. u. Wüst, E., Nachtrag zu August Garcke's Flora von Halle. - M agnus, P., Ueber A. Weise's monströse Frucht von Citrus Aurantium. - Loew, E., Die Kleistogamie und das blütenbiologische Verhalten von Stellaria pallida Piré. - Höck, F., Studien über die geographische V'erbreitung der Waldpflanzen Brandenburgs. V. - Spribille, F., Beitrag zur Kenntnis der Posener Rubi. - Ascherso 11, P., Uebersicht neuer, bez. neu veröffentlichter wichtiger Funde von Gefässpflanzen (Farn- und Blütenpflanzen) des Vereinsgebiets aus dem Jahre 1898.

Oesterreichisehe bot. Zeitsehrift 1900. Nr. 1. Jenčič, A., Untersuchungen der Pollens hybrider Pflanzen. - Richter, O., Ein neues Macerationsmittel für Pflanzengewebe. - Podpera, J., Ueber eine neve Art der liattung Fissidens. - Urumoff, J. K., Beiträge zur Flora von Bulgarien. - Dallia Torre v. u. Sarnthein, Graf v., Bedenkliche Miscellen. - Woloszczak, E., Bemerkungen zu der Abhandlung von A. Jenčič. - Litteratur-Uebersicht.

Deutsehe bot. Monatsehrift 1899. Nr.11 u. 12. II ur r, Dr. J., Beitriige zur Flora von Tirol und Vorarlberg. - Scholz, Jos. B., Der Formenkreis von Anemone ranunculoides $u$. nemorosa. - H a n e man $n$, J., Die F'loria des Frankenwaldes etc. - Ḧ̈ ck, Dr. F., Allerweltspflanzen unserer heimischen I'hanerogamenflora. - Kmet, A., Wie man botanische Monographien fubriziert: - 
I. eimhach, Dr. G., Die deutschen Tolksnamen der Orchideen. - 1900 Nr. 1. Murr, Dr. J., Wie in vor. Nr. - Leimbach, Dr., Wie.in vor. Nr. - Magnus, D1., Goldptlanzen. - Figert, E., Carex irrigua Sm. $>$ limosa L. nov. hybr.

Botan. Centralblatt. 1900 Nr.2. Popovici, Dr. P. Alexandru, Der Einfluss der Tegetationshedingungen auf die Jänge der wachsenden Zone. Feitel, R., Beiträge zur vergleich. Anatomie der Laubblitter bei den Campamulaceen der Kapflora. - Nr. 3. T'sw ett, Dr. M., Das Chloroglobin. - Fe itel, R., Wie in vor. Nr. - Nr.4. Feitel, R., Wie in vor. Nr. - Nr.5. Feitel, $\mathrm{R}$., Wie in vor. Nr. - Korshinsky, S., u. Monteverde, N., Bestäubungsversuche an Buchweizen.

Verhandlungen der k. k. zoologiseh-bot. Gesellsehaft in Wien 1899. Heft 9. Fritsch, Dr. C., Beiträge zur Flora der Balkanhalbinsel mit besonderer Berücksichtigung von Serbien. - Heft 10 enthïlt keine bot. Originalarbeiten.

Beriehte der bayerischen botanischen Gesellschaft. 1899. Band VI. Śolereder, Dr., Teber das Torkommen von Isoütes lacustris L. in S'ïdbayern. Naegele, Fritz, Cruciferen II. - Arnold, Dr. F., Zur Lichenenflora von Miunchen.

Botanical Gazette 1899. November. Clark, J. F., On the toxic effect deleterious agents on the germination and development of certain filamentous fungi. - Wiegand, Karl M., The development of the Microsporangium and Hicrospores in Convallaria and Potamogeton. - Dezember. Nelson, Aven, Some rocky mountain Chrysothamni. - Cla rk, J. F., Wie in vor. Nr. - Beadle, C. D., Studier in Crataegus.

Bulletin de l'académie internationale de géographie botanique. 1900. Nr. 122. (trelet, l'abbé, L'Ophrys aranifera et ses diverses formes dans le midi des Deux-sévres. - Claire, Ch., Les Centaures du nord-est de la France. Petitmengin, Marcel, Sur quelyues plantes rares et adventices en Lorraine.

\section{Botanische Anstalten, Vereine, Tauschvereine, Exsiccatenwerke, Reisen etc.}

Botanischer Verein für die Provinz Brandenburg. Die Sitzung am 12. Jamuar cröflnete ker Tors. Prof. Schumanm mit einer herzlichen Neujahrsbegrïssumg der anwerenden Mitglieder und dem Wunsche, dass der Verein im nenen Jahre die Ziele erreichen möge, die er sich vorgesteckt, zunächst die rüstige Fortführung einer Kryptogamenflora der Mark. Darauf begrüsste er das ans Brasilien zurückgekehrte Nitglied Dr. Pilger und machte Nitteilungen über die aut Reisen begriffenen Hitglieder Dr. Schlechter in Kamerun, Dr. Preuss in Südamerika und Prof. Tolkens, welcher glücklich auf den Karolinen-Inseln angekommen ist und wohl in Hochsommer zurtickerwartet werden dürfte. Nach Erledigung einer Anzahl geschäftlicher Dinge legte Custos Hennings ein gärtnerisches Buch in japanischer sjurache und auf dem bekamnten japanischen seiden. papier gedruckt vor, das ihm von dem anwesenden Prof. Shirai aus Japan mitgeteilt worden ist. Danselhe enthïlt in mehreren Teilen ca. dreitausend Abbildungen von P'flanzen mit lanachierten B Baittern, Twergformen u. dergl. aus den Gattungen Camellia, Thea, Ilex u. a.; es soll uralt sein, doch verrät ein Porträt am Schluss, wahrscheinlich des Verfassers, zweifellos neneren Lrsprung, so dass die Tremutung nahe liegt, das vorliegende Exemplar sei vielleicht nur ein Nendruck des alten Werkes. Darauf folgte noch ein kurzer Vortrag über einige neue Pilzformen aus Japan und Kamerun. - H. Kotzde sprach über die gross. artigen stadtischen Gewitchshäuser im Friedrich Wilhelms-(rarten zu Magdeburg nud legte eine gronse Anzahl schöner photographischer Aufuahmen aus denselben vor, woran l'rof. śchnma nu nähere Mitteilungen über diese, atus den ehemaligen Grusnn'schen Sammlungen hervorgerangenen Etablissements knüpfte, sowie 
namentlich über die prachtvollen, einzig in ihrer Art dastehenden carten, unter denen eine ganze Anzahl Unicas, wie kletternde species, ein fows 1/molomuldi mit $40 \mathrm{~cm}$ grossen Blüten u. a., eine spezialitit Grusons, der sogar seinen Obergärtner wiederholt nach Nexiko schickte, um besondere seltenheiten herbeizuschaffen, und so die berühnteste Cacteensammlung der Welt zusammenhrachte. Dr. Gilg hielt einen Vortrag über die afrikanischen Gattungen Monoles und Octolepis, deren Stellung zum System hisher noch zweifelhaft war. Er hat sich längere Zeit damit eingehend heschäftigt und alle Zweifel gehohen; die vorgelegten Exemplare von M. mannificus Grily sind von dem Mitgliede Giirtner (rïtze in Uhehe gesammelt, der leider am Nyassa dem mürderischen Klima erlegen ist. Den Schluss der Sitzung bildete ein höchst interessanter Vortrag des I'rof. Sch um an über die Meerkokos, die Jahrhumlerte hindurch mit geheimnisvollem Nimbus umgebenen Frïchte der Lodoiera Seychellarum, und üher deren Keimung im Berliner botanischen Garten.

W. Lackowitz.

Kgl. bot. Gesellsehaft zu Regensburg. Floristische Sitzungen. (Forts.) 25. Oktober 1899. Zur Vorlage gelangten die in diesem Jahre im Regensburger Florengebiete teils überhaupt neu, teils von neuen Standorten aufgefundenen Phanerogamen und Gefïskiryptogamen, von denen die folgenden besondere Erwähnung verdienen:*)

A'gemone Mexiecna Limmé adv. Westbahnhof in Regenshurg (Vollmann); Corydulis intermadia Patze, Meyer et Ellian, Laaberthal oberhalb Seubertshofen (Vollmann), Park von Fronberg;

Arabis auriculata Lamarek, auf der Brant bei Kelheim (Petzi);

Cardamine silvutica Link, Marienthal (Petzi);

Erophila majuscula Jordan, Prüfening (Petzi);

Cerastium glomeratum Thuillier, Marienthal (Petzi), zwischen Station Abbach und Saal (Petzi);

C. brachypetalum Desportes, Stöffling (Petzi);

C. glutinosum Fries, Keilstein (Petzi), Abensberg (Vollmann, Poeverlein);

C. arvense var: laricifolium, auf der Brant bei Kelheim (Petzi);

('ytisus luburmum Limme, qsu. Wald hinter dem Tergernheimerkeller (Petzi);

Trifolium arvense var. microcephalum Uechtritz, Burgberg: in Donaustauf (Vollmann);

Ervum silvaticum Petermann, Marienthal (Petzi);

Elatine orthosperma ron Dübem, in Botaniska Notiser $18: 39$ 1. 88, Schlamm am Rande eines Dunaualtwassers bei Donaustauf (die Exemplare stimmen im allgemeinen gut zur Diagnose dieser ausgezeichneten Art: "folii, petiolo brevioribus, floribus sessilibus octandris tetragyuis, capsula oblongal, seminibus leviter cygneo-areuatis," doch ist die capsula nicht, oblonga", sondern wie bei E. hydropiper Limé globosa-depressa");

Oenanthe fistulosa Linné, unterhalb Roith (Petzi);

Coriandrum satimum Linné, Sedanstrasse in Regensburg (Fürnrohr jun.);

Bifora radians Marschall von Bieberstein, ebendort (Fürnrohr jun.);

Cormus stolonifera Michaux, qsp. Nordseite des Oberen Wöhrd (Vollmann); Anthemis tinctoria Limé, (Form mit röhrigen Strahlenblüten) hinter dem Tegernheimerkeller (Petzi);

A. Austriaca Jacquin > tinctoria Linné, Etterzhausen (Petzi);

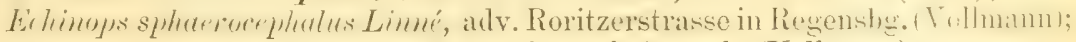
Cardus personata Jacquin, Donaufer bei Abbach (Vollmann);

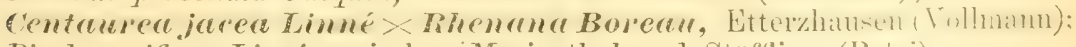
Pirola uniflora Limé, zwischen Marienthal und Stöffling (Petzi);

Gentiana pneumonanthe Linné, ein auffallendes Exemplar mit teilweise

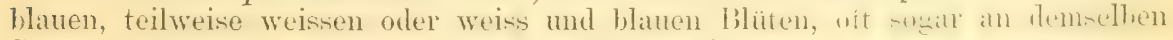
Stengel, Wolfskofen (Anton Mayer, 21. August 1899);

*) Vgl. Allg. bot. Zeitschrift 1896 p. 203; 1897 p. 182 f.; 1898 p. 186. 
Phacetia tanacetifotia Bentham, qsp. zwischen der Bahn und der neuen Kaserne in Regensburg auf Aeckern (Petzi);

Myosotis versicolor Smith, zwischen Marienthal und Stöfling (Petzi);

M. hispida Schlechtendal pat., Stöffling (Petzi), Wiesent (Vollmann);

M. sparsiflora Mikan, Wald am linken Nabufer, gegenüber Penck (Fürnrohr jun.);

Veronica montana Linné, Marienthal (Petzi);

T. opaca Firies, zwischen Abensberg und Sandharlanden (Vollm., Poeverl.);

T. triloba Opiz, Falkenstein (Petzi), Sandharlanden (Vollm., Poeverl.);

Pinguicula alpina Linné, Sippenauer Moor, auf tertiärer Unterlage (Lehrer Rieger in Kelheim);

Plantago lanceolata var. polystachys Schlechtendal, Grünthal (Familler);

Rumex limosus Thuillier (1799) = paluster Smith (1800), Oberer Wöhrd, Oberndorf, Abbach (Vollmann);

R. crispus Linné $\times$ hydrolapathum Hudson, Oberer Wöhrd (Vollm.);

R. maximus Schreber, Donaustauf, Oberer Wöhrd, Unterer Wöhrd, Schön$\operatorname{ach}($ Vollmann);

R. aquaticus Linné, Abbach, Oberer Wöhrd (Vollmann);

Ulmus montana Tithering, Douauschlucht zwischen Kelheim und Welten. burg (Vollmann, Poeverlein)*);

Orchis purpureus Hudson, Pielenhofen (Petzi);

O. mascula Limmé, Pielenhofen (Petzi), Eichhofen (Vollmann, Poeverlein);

Ornithogalum Bouchéanum Ascherson, Rasenplätze im Park von Fronberg;

Oryza clandestina A. Braun, Oberer Wöhrd (Vollmann);

Equisetum hiemale Limé, zwischen Gundelshausen und Oberndorf (Voll. mann), Donauschlucht zwischen Kelheim und Weltenburg (Vollmann, Poeverlein);

E. variegatum Schleicher, Oberer Wöhrd, Lausbuckel bei Roith (Vollmann);

Lycopodium chamapcyparissus A. Braun, zwischen Kronstetten u. Schwandorf;

Polystichum montanum Roth, Scheuchenberg (Petzi);

Asplemum viride Hudson, Kastlhäng bei Neuessing (Petzi), Höhlenweg bei Etterzhausen (Poeverlein).

Sehr grosses Interesse erweckten die von mehreren Mitgliedern vorgelegten Formen des im vergangenen Sommer für die Regensburger Flora neu aufgefun. denen Bastardes Cardamine amara Limé $\times$ pratensis Limé, die in der verschierlensten Weise die charakteristischen Merkmale beider Stammarten in sich vereinigen. Eine kurze Abhandlung darüber werde ich in der nächsten Nummer dieser Zeitschrift bringen.

Dr. Poeverlein.

Preussischer botanischer Verein. III. Sitzung, Königsberg i. Pr. 15. J anuar 1900. Der Schriftfïhrer des Vereins, Dr. Abromeit, gab einen ausführlichen Rückblick über die botanischen, speziell floristischen Leistungen im Tereinsgebiet während des verflossenen Jahrhunderts unter Vorlegung von leicht orientierenden Uebersichtskarten, auf denen er die botanisch erforschten Teile von Ost- und Westpreussen hezeichnet hatte. Trotz eifriger planmässiger Durchforschung des Geliets seit 1871 durch den Pr. hotan. Verein und durch den seit 1878 bestehenden Westpr. botanisch-zoologischen Terein bleibt noch viel zu thun übrig. I'm einen Teberblick über die bisherigen Ergebnisse zu erhalten, werden dieselben, wie bekannt, in Form einer Flora herausgegeben.

*) Ueber die von Vollmann und Poeverlein am gleichen Standort gefundenen Arten Bellidiastrum Michelii Cassini und Primula auricula Linné vgl. Allg. bot. Zeitschrift 1899 p. 182. 
Es bedarf jedoch noch rüstiger und unermüdlicher Thätigkeit geeigneter Forscher, um die noch bestehenden grossen Lücken zu füllen. Hoffentlich wird im neuen Jahrhundert, unter Voraussetzung für solche Forschungen günstiger Verhältnisse, das durch Caspary begonnene Unternehmen der Vollendung entgegengeführt werden. Sodann demonstrierte Herr stud. jur. Tischler einige bemerkenswerte Pflanzen aus der Umgegend von Losgehnen bei Bartenstein. Es waren darunter sehr schmalblättrige Formen von Centaurea Jacea, die schon durch v. Klinggraeff I als $v a r$ linearifolia bezeichnet worden sind; ferner eine Form des gemeinen Cirsium lanceolatum Scop. mit weit herablaufenden Blattrïndern und auffallend kleinen Köpfen, sowie ein anderes Exemplar mit fast weissen, kleinen Früchten und dadurch an $C$. nemorale $R c h b$. erinnernd, aber ohne weissfilzige Blattunterseiten. Der Vortragende legte ausserdem mehrere Formen von Euphrasia stricta v. Wettstein vor, die mancherlei Abweichungen zeigten. Vorgelegt wurde ferner eine kleine Kollektion exotischer Pflanzen, die Herr Oberlehrer Dr. Nanke gelegentlich eines Aufenthaltes in Oberitalien zur Weihnachtszeit in Anlagen von Gardone im Freien grösstenteils blühend oder fruchtend gesammelt hatte. Es waren darunter Laurus nobilis, Eriobotrya Japonica Linbg., Arbutus unedo, Tiburmu timus, Laurus camphora, Eucalyptus globulus und mehrere kultivierte Coniferen. Zum Schluss demonstrierte Herr Apotheker Roerdansz mehrere von ihm hergestellte photographische Aufnahmen von blühenden Obstbäumen und landschaftlich interessanten Partieen aus Litauen, speziell aus dem Memelgebiet. Eine Photographie zeigte einen starken, über $3 \mathrm{~m}$ hohen Birkenstamm als "Ueberpflanze* auf einer alten Weide, durch deren hohlen Stamm die Birke hindurchgewachsen war.

Dr. Abromeit.

Botanic Gardens Sydney. Die Direktion der botanischen Gärten in Sydney in Neu Süd Wales in Australien fragte bei dem Unterzeichneten über Preis, Umfang, Inhalt, Einrichtung einiger Exsiccatenwerke an, da die Anstalt die betr. Werke zu erwerben wünscht. Derselbe erlaubt sich, anbei die betr. Werke aufzuzählen, damit die Herausgeber derselben selbst mit dem bot. Garten in Sydney in Verbindung treten können. Verzeichnis der Exsiceatenwerke: B e cker, W., Plantae exsiccatae Dalmatiae et Violae exsiccatae; Flora Polonica exicc at a, herausgegeben von Prof. Dr. Woloszczak; Gandoger, Spanische Exsiccaten; Karó, J., Exsiccaten aus dem Amurgebiet; Mann, Gust., Farnexsiceaten aus Asien; Morawatz, M., Serbische Exsiccaten; Reineck u. Czermak, Plantae exsiccatae Brasiliae meridionalis; Siegfried, K., Exsiccatae Potentillarum; Wirtgen, Ferd., Pteridophyta exsiccata.

A. Kneucker.

The Botanieal Exchange Club of the British isles. List of desiderata for the years 1899 and 1900. Die von dem liebenswürdigen Sekretär des botanischen Tauschklubs, Herrn Charles Bailey in Manchester, eingesandte Desideratenliste enthält ausser den Tauschbestimmungen und der Mitglieder-Liste ca. 700 systematisch geordnete Pflanzennamen. Die Zahl der Mitglieder beträgt 45 .

Hofmann, H., Plantae eriticae Saxoniae. 5. Fascikel. Der Herausgeber H. Ho fm a n n, in Grossenhain, Kgr. Sachsen, versandte kürzlich den 5. Fascikel seiner kritischen Exsiccaten, enthaltend die Nr. 101-125. Preis pro Lief. mit Mappe 6 und ohne Mappe 5 M. Ueber die schöne Ausstattung der Exsiceaten wurde schon wiederholt berichtet. Der 5. Fascikel enthilt: Rubus acanthode's nor. sume, flaccidifolius P.J. Mäller, serpens Weihe non. I. scabriformis, Rosa tomentoria s'm. i. dimorpha (Bess.) Grenier, n. renusta (Schentz) anct. f. Geisingensis ITicshaner, tomentella Lem., e. scalnata Corp. f. Missnimsis sichlimp., canime L. I. Andegacensis Bast., dumetorum Thuill. r. Thuillievi Christ, r. pilosa Opiz (1m. sp).) f. peracutu.

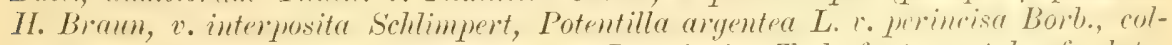

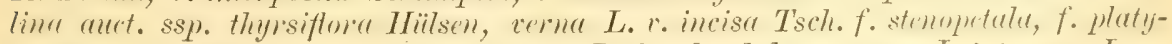
petala, v. Neumanniana Rchbch., opaca L. f. eglandulosa, opaca L. × verna $L$. $v$. aurulenta Gremli f. eglandulosa, arenaria Borkh. $f$. glandulose; supreverna $L_{0}$ - 


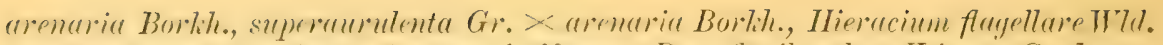
ssp). flagellar a. yemuimum 1. normale Naeg. et Pet., floribundum II im. et Grab. ssp.

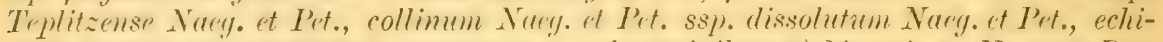

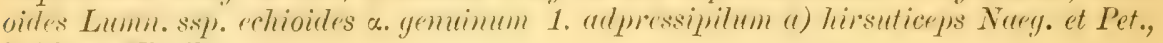
bifidum Kitaib.

Flora exsiceata Bavarica. Fase. III dieses in der "Allg. hot. \%." schon früher genamnten Exsiccatenwerkes soll demüichst in der Stärke von 100 Nummern zur Ausgahe gelangen. Teber den wertvollen Inhalt des II. Fascikels wurde p. 87 des vor. Jahrgangs ausführlich herichtet. Das Exsiccatenwerk ist auch, soweit der Vorrat reicht, käuflich zu beziehen. (Siehe das Inserat auf dem Umschlag dieser Nummer.)

Wirtgen. F., Pteridophyta exsiceata. Lief. V. Die kürzlich versandte 5. Lieferung enthält die Nr. 213-275. Der vorliegenden Lieferung sind 2 Separatabdrücke von einschlägigen Arleiten der Herren Dr. Chr. Luerssen und J. S. Kaulfuss beigegeben. In dem 5. Fascikel sind vertreten die Genera Hymonophyllum, Alhyrimm, C'ystopteris, Axpidium, Hoodsia, Blechmm, Scolopendrium, Asplemum, I'teridium, Nottolarna, Polyportium, Osmumla, Botiychium, Equisetum, Lypopodium und Suluginella. Viele Formen wurlen von 2 und mehr Standorten ausgegeben. Von ausserdentschen Mitarbeitern ist hesonders B. Marcowicz zu nemmen, welcher aus dem Kaukasus Woorlsin frofilis Trovin. geliefert hat. Sehr zahlreich sind die anflegenden Equisetum- und Blechmm-Formen. Möchten sich, wie schon früher erwähnt wurde, vor allem auch recht viele Floristen des Auslandes an dem verdienstvollen Werke beteiligen. Die Adresse des Herausgebers ist: Apotheker F. Wirtgen in Bonn a. Rhein.

\section{Personalnachrichten.}

Ernennungen etc.: Dr. Th. M. Fries, Prof. d. Bot. an d. Univ. Upsala, ist in den Ruhestand getreten. - Dr. A. Nabokich w. Assistent des mikrobiolog. Laboratoriums des k. russ. technolog. Tnstituts in st. Petersburg. - Dr. s'o lereder, Privatdozent und Custos am Herbarium zu München, w. z. a.o. Prof. ernannt.I'rof. Dr. W. Rothert in Charkow w. z. korresp. Mitglied der Akarl. der Wissenschaften in Krakiu (mathem. naturwissensch. Klasse) gewählt mol wird das nibchste Halbjahr zu wissenschaftl. Zwecken in Leipzig am hot. Institut zuhringen. - Apotheker fichlockow in Breslau w. Assistent am agrikultur-chemisch. bakteriol. Instit. d. Universität Breslau. - Dr. F. R. Kje $11 \mathrm{mann}$ in Upsala w. Prof. der Botanik. - Dr. Arved Wieler, Dozent der Bot. an d. kgl. techn. Hochschule zu Aachen, w. Professor. - Prof. Dr. Ebermeyer in Nünchen tritt in den Ruhestand. - Prof. Dr. W o h ltman $n$ in Bonn w. zo kais. Geh. Regierungsrat ernannt. Dr. A. A. Ty Jer w. Assist. d. Bot. an d. Univ. von Arizona. - Wm. R. II axon w. Assist. der Kryptogamen-Botanik an L. S. National Herbarium. - Dr. (ieo. W. Martin w. Prof. der Biologie an der Vanderbilt-Universität. - Prof. Dr. Aug. Garcke w. z. Geh. Regierungsrat emannt. - Prof. Dr. Kulisch zu Geisenheim a. Rh. w. vo Ministerium für Elsass-Lothringen als Direktor an der Kais. Versuchsstation nach Kolmar berufen. - Prof. Dr. P. Ascherson (Berlin) erh. den roten Adlerorden. - Prof. Dr. A m bronn w. z. a.o. Prof. a. d. Universität in Jena ernannt.

Todesfälle: John Witehead am 2. Juni in Hoi-Kow, China. - F. Guthrie im Oktober in Claremont, Cape Jowa. - Dr. Law son T'a it am 13. Juni in London, $55 \mathrm{~J}$. alt. - Karl Erdinger, Domprobst in St. Pölten in Niederöstereich am 14. Dez. $1899 \mathrm{im}$ Alter von 77 Jahren. 


\section{Allgemeine}

\section{Botanische Zeitschrift}

für Systematik, Floristik, Pflanzengeographie ete.

Referierendes 0rgan

des bot. Vereins der Provinz Brandenburg, der kgl, bot. Gesellschaft zu Regensburg, des Preuss. bot. Vereins in Königsberg,

und Organ der Botan. Vereinigung in Würzburg, des Berliner und schlesischen bot. 'T'auschvereins.

Unter Mitwirkung hervorragender Fachmänner herausgegeben von . Hencher. Werderplatz 48 in Karlsruhe.

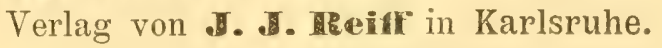

\begin{tabular}{|c|c|c|}
\hline 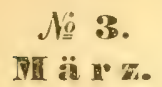 & $\begin{array}{l}\text { Erseheint am } 15 . \text { jeden Monats. - } \\
\text { Preis: vierteljährl. } 1.50 \mathrm{Mk} \text {. bei freier Zusendung. }\end{array}$ & $\begin{array}{l}\text { 1904. } \\
\text { VI.Jahrgang. }\end{array}$ \\
\hline
\end{tabular}

\section{Inhalt}

Originalarbeiten: IV. Schmidle, Algologische Notizen (Forts.). - 'Th. Hellwig, Florenbild der Umgegend von Kontopy im Kireise (rrïnherg in Sehlesien (Forts.) E. Figert, Botanische Mitteilungen aus Schlesien. IV. - F. Buser, Ueber Alchimilla pubescens Koch, A. truncata Rchb. und eine neue verwandte Art aus den Tiroler Alpen (A. acutata) (Forts.), - A. Kneucker, Bemerkungen zu den „Carices exsiccatae* Lief. VII (Schluss).

Bot. Litteratur, Zeitsehriften ete.: A. Kneucker, Klein, Dr. Ludwig, Die Physiognomie der mitteurop. Waldbäume (Ref.?- D erselbe, Ascherson, P., u. Graebner, P., Synopsis der mitteleurop. Flora (Ref.). - Inhaltsangabe verschied. bot. Zeitschriften. - Eingegangene Druckschriften.

Bot. Anstalten, Vereine, Tauschvereine, Exsiceatenwerke, Reisen etc.: Bot.Verein der Prov. Brandenhury (lief.). - Kigl. bot. Gesellschaft zu Regenhurg (Ref.) Preuss. bot. Verein (Ref.). - Goldschmidt, MI., Pflanzenverkauf. -- Herbarversteigerung. - Sintenis, Paul, Botan. Reise nach Turan u. Persien-

Personalnachrichten. Glumaeeae exsiceatae (auf tem Umschlas).

\section{Algologische Notizen.}

Von W. Schmidle (Mannheim).

XIV.

(Fortsetzung.)

Der Standort dieser eigentümlichen Desmidiacee ist sehr merkwürdig. Ich kamn jedoch nicht behaupten, dass dieser Trentepohliarasen, in welchem die Alge nebst den folgenden Desmidiaceen häufig vor'kommt, der ursprüngliche Standort ist, weil ich nur leere Exemplare gesehen habe. Die Zygoten dagegen hatten stets Chlorophyll. Es ist die Möglichkeit nicht ausgeschlossen, dass die Alge durch irgend einen Zufall hierherkam; andrerseits kann man sich nicht wundern, bei dem feuchten tropischen ílima auch an Baumrinden Desmidiaceen zu treffen, welche in der Regenzeit dort vegetieren, und nachher gestorben sind oder in Ruhezustand übergingen. Merkwürdig waren alle Formen, die ich hier sah, und die im folgendem beschrieben sind. 
\%. Iurstum Mrmsgingi Schmille m. sp. Fig. 5-8. Die im gamm'n rechteckignen Zellen sind 3is y lang und $2+\mu$ breit, mit enger gerader Einschnirung. l)ie Ilalhzellen sind e fronte rechteckig mit rechtwinkligen nutcen Enden wind im ganzen gerade aufsteigenden Seiten, welche im ersten Drittel der Höhe auf jeder Seite einen abgerundeten seitlichen Lappen bilden, über demsclben cine ziemlich enge ahgerundete Einbuchtung tragen und sich dam wieler zu dem horizontalen, algerundet'n Endlappen verbreitern. Der Schoite] ist gerade, ohne Einschnitt. Die \%ellhaut ist glatt und trägt in der Mitte nuerhalb des Scheitels zwei kleine Tumoren. Die Scheitelansicht ist rechteckig, die Seitenansicht nach oben verbreitert. Die Sitze der suthlichen Liappen hat ein Grübchen, ein anderes ist oft unterhall, der heiden scheitelständicren 'lumoren (e fronte). Ueber der Einschnürung befinden sich ebenfalls oft 2 kleine Tumoren.

Mit obiger Alge.

8. Cosmurum (Plenotreniopsis) bifurentum. Schmidle 2. $\$ 1 \%$ Fig. 9, 10. Die /elle ist cylindrisch mit schwacher Mitteleinschniirung und alogermateten Enden. U eber dem Isthmus trägt sie jederseits zwei Horizontalreihen kurzer zweizinkiger Dornen, und in etwas weiterem Abstand 4 weitere ebenso gelagerte mit ebenfalls kurzen zweizinkigen Dornen. Die Scheitclansicht ist genau rund. Der scheitel selbst tragt ein kleines kreisrumdes glattes Feld. Die 7ellen sind $5 \pm \mu$ lang und $24 \mu$ breit.

Mit obiger Alge.

Drei weitue C'osmaricnarten dieses Standortes müssen noch genauer untersucht werden.

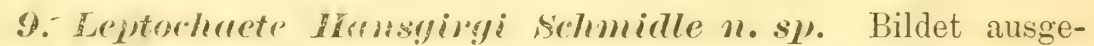
breitef Räschen anf Bammb]aittern. Das horizontale dïnne Lager ist vielschichtig und hestelst aus $3-4 \mu$ grossen blaugrimen Zellen (die oft zu 4 genähert sind). Die dichtstehenden aufsteigenden Fäden sind 1,7--2 2 breit, blaugrün, mit hyaliner, am Grunde stets gelblicher scheide, nach aufwarts nicht verschmälert, 200 u lang, ohme sichthare Scheidewinde und mit sehr kurzen rechteckigen Zellen. In Alter verbreitern sich diese Faden an der Basis, die Zellen werden hier zugleich etwas torulös, sie erscheinen dann nach aufwärts allmählich verschmälert.

\section{Im Victoria-Garten in Bombay.}

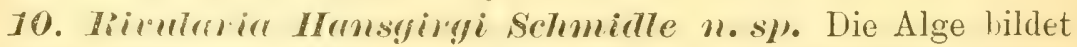
ausgebreitete, wallertice, schwarzbraune pajierdüne, feste Massen. Die Fäden sind horizontal, meist verworren, dicht gehäuft, mit 1-2 basalen, kugeligen, hy alinen, ca. $8 \mu$ dicken Grenzzellen, meist unverweigt, die seltenen Zweige sind, wie der Hauptfaden, mit denselben hasalen Grundzellen, sich langsam verschmälernd, auf weite strecken ca. $6 \mu$ dick, und meistens in $2-3$ ca. $4 \mu$ breiten torubisen, hyalinen Ztllen endjgend, hinter welchen man nur selten ein meist kurzes farbloses Har sieht Die Scheiden sind schr dünn, hyalin oder schwach gelblich, enge anliegend. Die Zellen sind blaugrün, körnig, rechteckig oder sehr schwach torulös, quadratisch, oder kürzer als lang, die Scheidewand meist schwer sichtbar.

Igatpuri (Indien) 3.XI.95. 
11. Glocotrichia Indica Schmialle n. sp. Bildet $1-2 \mathrm{~mm}$ grosse weiche, hohle (?) Kugeln mit radialen Fäden; diesclben sind $260 \mu$ lang, und bestehen a usser den $60-70 \mu$ langen, und $16-20 \mu$ bre $\mathrm{i}-$ ten (ohne Epispor) Sporen bloss aus 2-3 torulösen und ca. $8 \mu$ breiten Zellen und einem langen, dünnen Haare mit cylindrischen Zellen. Das Epispor ist dick, geschlossen, anliegend, nicht zerfasert, zunächst hyalin, im Alter braungelb und aussen fein punktiert und granuliert.

\section{In einem See bei Igatpuri 3.XI.95.}

12. Calothrix Mansgingi Schmialle n. sp. Aeusserst klein, nicht mit Kalk inkrustiert, heerdenweise an anderen mikroskopischen Pflanzen (Pithophora) sitzend, unverzweigt, senkrecht abstehend, ohne Grenzzellen, nach aufwärts verschmälert und in ein kleines Haar ausgehend, oft gekrün:mt, oft gerade, und am Grunde oft zu zweit verwachsen. Die Scheiden sind zart, hyalin, nicht zerfasert, anliegend, die Zellen nicht torulös, schr kurz, am Grunde ca. $4 \mu$ breit, ihre Scheidewände schwer sichtbar, ihr Inhalt violett. Die ganze Pflanze ist nur $60-80 \mu$ lang und bildete trotzdem schon Hormogonien.

Im See bei Danger-Point, Indien.

(Forts. folgt.)

\section{Florenbild der Umgegend von Kontopp im Kreise Grünberg in Schlesien.}

Von 'Th. Hellwig.

(Fortsetzung.)

An Rotbuche (wie auch an Eiche, Weide, Linde) ist Lecidella sabuletorum Schreb. $\gamma$. entcroleuca (Fr.) nicht selten, an Hainbuche Callopisma cerinum (Ehrh.) verbreitet. An letzterem Baum findet sich Cecidomya Carpini Fr. Löw (Hier. Nr. 404). An Buchen überhaupt wächst gern Pyrenula nitida (Schrad.).

Rhamnus cathartica ist reich überwuchert mit dem Aecidium von Puccinia coronata Corda, desgleichen Frangula alnus (mit der Form Puccinia sertata Prenss). Rh. cathartica besitzt auch Microsphaera Alni (DC.) (Erysiphe penicillata Dub.), Frangula aln. dagegen Pezicula versiformis (Alb et Schw.) (P. Frangulae) und Microsphicera divaricata (Wallr.). Die Galle von Trioza Walkeri (Frst.) F. Löw (Hieron. Nr. 300) gehört Rh. cath. an.

Prunus padus weist Hysterographium curvatum (Fr.) auf und die Galle Erineum Padi Rebent. (Hieron. Nr. 184), P. spinosa Sclerotinia cinerea (Bon.) (Monilia cin. Pers.) und die Gallen Cephaloneon molle Bremi (Hier. Nr. 187), sowie Diplosis marsupialis F. Löw (Hier. Nr. 4\&8).

Ribes rubrum bringt Cronartium ribicola Dietr. hervor, IR. nigrum Aecidium Grossulariae Pers. und hat die Galle Aphis Ribis L (Hieron. Nr. 355).

Rubus fruticosus zeigt die schöne Chrysomyxa albidic J. Kïhn, R. plicatus und $\mathrm{R}$ idaeus die Galle Phyllerium Rubi Fr. (= Erineum rubeum Pers.) (Hieron. Nr. 203 u. 201).

Viburnum opulus wird von Microsphaera Alni I)C. (Erysiphe penicillata Dub.) befallen. 
An Osmunda regalis findet man Leptostroma filicinum, Mycosphaerella Filicis (Auersw.) (Sphaerella Osmundae Schroet.) und Cladosporium; desgleichen eine unverzeichnete Galle.

Zwischen faulendem Pteris aquilina siedelt sich Hypochnus mucidus n. sp. (Schroet.) an; auf Pt. aquil. Dasyscypha (Trichopeziza) Pteridis (Alb. et Schw.), Cryptomyces Pteridis (Rebent.) und Ascochyta Pteridis.

Athyrium filix femina enthält als Galle: Anthomyia spec. Trail Liebel (Hieron. Nr. 385).

Ledum palustre ist öfters von Uredo zu Chrysomyxa Ledi Alb. et Schw. befallen. Andromeda polifolia ist Nährpflanze von Rhytisma Andromedae (Pers.), Coleroa Andromedae (Rehm.) und Trichosphaeria Andromedae.

Oxycoccus palustris ist bewohnt von Mlelampsora Vacciniorum (Lk.), Exobasidium Vaccini (Fuck.) und Leptostroma Oxycocci (Fr.).

Schr reichlhaltig ist die Besiedelung von Arctostaphylos officinalis, wenn auch nicht die hewohnenden Arten bei Kontopp gesammelt wurden: Melampsora Vacciniorum (Lk.), Exobasidium Vaccini (Fuck.), Lachnum pulverulentum (Lih.) und L. niveum, Podosphaeria myrtillina (Kunze), Dimerosporium Arctostaphyli und Gloeosporium sp.

Melampsora Pyrolae (Gmel.) kommt auf Pyrola rotundifolia, P. minor und $\mathrm{P}$. secunda vor.

Vaccinium vitis idaea ist befallen von Melampsora Vacciniorum (Lk.), Calyptuspora Goeppertiana J. Kühn, Exobasidium Taccini (Fuck.) und Sclerotiana baccarum Schroet.

Juncus Leersii bietet Lachnum pudicellum (Quel.), Mollisia juncina (Pers.) und Mycosphaerella pheidacea n. sp. (Schroet.).

Eriophorum polystachym L. (z. t.) (E. angustifolium Rth.) und Carex Goodenoughii erzengen das seltene Lachum callimorpha (Karst.); ähnlich E. latifolia und Carex-Arten die Nollisia emparaphysata n. sp. (Schroet). Carex rerma hat Leptosphaeria Caricis Sichroet., (C. elongata Trac.va ignobilis Karst., C. ericetorum Schizonella melanogramma (D.C.), Puccinia silvatica Sihroet. und Naevia diminuens (Karst.), C. hirta Puec. Caricis (Schum.), C. praecox (Schreberi) und C. arenaria Ustilago Caricis (Pers.), letztere auch Lophodermium caricineum (lioberge) und Cladosporium, C. leporina $>$ arenaria Pucc. silvatica Schroet.

Die (aalle von Hormonyia Fischeri Frauenf (Hieron. Nr. 399) zeigt sich an Carex arenaria und C. hirta.

Auf Luzula pilosa schmarotzt Puccinia ohlongata (Lk.), an Panicum crus galli Tolyposporium bullatum (Schroet.).

Gallen bieten: Agrostis vulgaris Tylenchus sp. (Hieron. Nr. 5) und A. canina 'Tylenchus sp. (Hieron. Nr. 4).

Agrostis sp. lieferte Puccinia graminis Pers., Agrostis alba und Poa compressa lieferten Epichloë typhina Pers.

An Calamagrostis lanceolata wachsen Puccinia graminis Pers. und Pleospora (Pyrenophrora?) trichostona (Fr.), an ('. lanc. und C. epigeia Purc. coronata Corda, an C. arundinacea Lophodermium arundinacea (Schrad.).

Letztgenannten Pilz sieht man auch an Koeleria cristata und Sieglingia dectimliens. Holinia coerulea crgab) Leptosphateria culmorum Aluersw. 
Festuca rubra und F. elatior fructificieren das Sclerotium von Claviceps purpureum (Fr.).

An Dianthus arenarius erscheinen Peronospora Dianthi de Bary, Ustilago violacea (Pers.), Sorosporium Saponariae P'ers, Septoria dianthicola und Phoma.

An Silene nutans und S. Otites zeigt sich Uromyces Silenes (Schleichtend.), an Cerastium triviale Melampsorella Cerastii (Pers.).

Viola canina hat Puccinia Violae (Schum), V. silvatica ausser Septoria Violae Lachnum agaricinum Retz. (L. virginea Karst.); letzteres auch auf Carex ericetorum.

Beide Viola-Arten besitzen die Galle von Cecidomyia affinis Kieff. (Hieron. Nr. 602, 603)

Lathyrus montanus (Orobus tuherosus) ist mit Uromyces Viciae (Pers.) hehaftet, Astragalus glycyphyllos mit Uromyces Astragali (Opiz) und Erysiphe Astragali (DC.).

Trifolium aureum bringt eine Galle hervor (Vgl. Hieron. Nr. 802), ebenso T. filiforme eine nicht verzeichnete.

Potentilla arenaria und P. argentea sind mit Phragmidium Potentillae (Pers.) geschmückt.

(Forts. folgt.)

\section{Botanische Mitteilungen aus Schlesien.}

Von E. Figert.

IV.

Das ungünstige $\mathbb{W}^{\top}$ etter im vorigen Sommer hat mich, und sicherlich manchen andern Botaniker, oft an grösseren Exkursionen verhindert; ganz hesonders aher zeichneten sich die Monate Mai und Juni durch viele Regentage aus. Da nun aber die meisten Wiesen gerade in dieser Zeit zum ersten Vale gemäht werden, so war das Beobachten und Einsammeln mancher Gramineen und Cariceen in diesem Jahre schwierig, wenn nicht ganz ummöglich. Aber wenn man immer und immer wieder durch Regenwetter von seinem Vorhaben abgehalten wird, so wird man schliesslich gleichgiltig und macht auch wohl bei einem herannahenden Gewitter einmal eine Exkursion, und umso eher, weil damn eine grössere "Sicherheit" für den vom Wege abschweifenden Botaniker damit verbunden ist. -- Gewöhnlich ist es nicht ratsam, kurz vor dem Grasschnitt eine mit üppigem Grase bewachsene Wiese nach allen Richtungen zu durchkreuzen oder gar Pflanzen in grösserer Menge für den Tausch oder ein Exsiccatenwerk mit den Wurzehn auszusterhen. Man kamn es dem Grumdhesitzer nicht verargen, wenn er den rücksichtslosen Maulwurf wegfüngt, der ihm beim Verwüsten der Fluren sogar noch einigen Nutzen erweist, wieviel weniger, wenn er einen Botaniker auf die erlaubten Wege verweist!

Es war am 10. Juni v. J., an einem Sonnabend, wo für mich wegen der angedeuteten, Sicherheit" der 'Tag günstig schien. Ich hatte die Absicht, die im vorigen Jahre von mir bei Parchwitz (in der Nähe von Liegnitz) beobachtete Carex Buelii $x$ gracilis aufzusuchen und für Kneuckers „Carices exsiccatae" in grösserer Menge zu sammeln, fand aber leider den grössten Teil der betreffemlen Wiese hereits abgemaht. Dagegen hemerkte ich in einiger Entfernung daron sowohl am Kitzhachufer als auch anf der anstosichnden Wiese den Bastard:

\section{Carex Buelii Wimm. $\times$ vulgaris $F r . n$. hybr.}

Es war nicht schwer, die Pflanze sofort richtig zu deuten, da $C$. vulgaris auf der ganzen Wiese vereinzelt und mit $C$. Buelii vermischt whehs. Auch der Gesamthabitus der Pflanze liess eine andere Deutung nicht zu. Schon seit 
einigen Jahren fahndete ich sowohl in der Breslauer als auch in der Liegnitzer Flora nach dieser Kreuzung, konnte aber beide Stammarten niemals zusanmenstehend finden. Die C. vulyaris auf der betreffenden Wiese an der Katzbach ist nun aber eine wirkliche vulgaris Fr. und ganz typisch, d. h. sie hat etwas eingerollte Blaitter von graugrüner Farbe, Rhizom lange unterirdische Ausläufer treibend, nicht rasenförmig, mit nur einem of Aehrchen, unteres Traghlatt den Blïtenstand nicht üherragend, Halm nur unter dem Blütenstande rauh etc. Alle diese Nerkmale will ich bei einer echten $C$. vulyaris $F r$. veremigt sehen. - Zwischen C. vulgaris Fr. einerseits und der C. gracilis Curt. und $C$. cuespitosa $L$. andererseits giebt es so uiberaus viele Varietäten, Formen und Bastarde, dass es fast unmöglich ist, sich zurecht zu finden. Es wäre deshalb dringend wünschenswert, wemn innerhalh der genannten 3 Arten: C. mlyaris $F r$., C. yracilis Curt. und $C$. causpitosa $L$. (vielleicht wäre auch noch $C$. strirtco Good. in Erwägung zu ziehen), die streng von einander abgegrenzt werlen müssten, vielleicht $2-3$ als eigene, gute Arten a usgeschieden würden; die gegenwärtige Verwirrung wäre mit einem Male erheblich vermindert, wenn nicht ganz aufgehoben.

Auf der erwähnten Wiese an der Katzbach bei Parchwitz stand auch die andere Kreuzung C. Buel $i$ i $x$ gracilis in einigen Exemplaren und rermutlich auch $C . g r a c i l i s \times v u l g a r i s$. Letztere habe ich an diesem Tage nicht mitgenommen und später war alles abgemäht. -

Da die Flora von Liegnitz seit langer Zeit in der botanischen Litteratur einen guten Ruf hat, weil sie mehrere sowohl für Schlesien als auch für ganz Dentschland seltene Pflanzen aufweist, unter denen namentlich einige selır selteneCarex-Hy bride bemerkenswert sind, so will ich den Namen ,Liegnitz“ (Lignicia) henützen, und die nene Carex-Hybride aus dieser Flora: Carex Buelii Wimm. $\times$ vulgaris $F r$. hiermit als

\section{Carex Ligmiciensis $m$.}

benennen. Es steht zwar sicher zu erwarten, dass die genannte Pflanze auch an andern Orten im Bereich der Oder wird aufgefunden werden. Einen zweiten Standort habe ich hereits am 10. September 1. J. im henachbarten Steinauer Kreise (Jürtscher See), wo die Pflanze nach dem Abmähen um diese Zeit allerdings nur Blätter zeigte, sicher in Aussicht genommen.

Ich beschreibe diese neue Hybride kurz, wie folgt: $\mathrm{Rhizom} \mathrm{mit} \mathrm{zahl-}$ reichen, hindfadenartigen, nach unten gehenden Wrurzeln, gegen die Stengelhasis dichtfilzig, mit einigen wenigen längeren oder kürzeren unterirdischen Ausläufern; Wuchs fast rasenförmig, kleine Stöcke mit 2-10 fertilen mud ungefähr ebensoviel sterilen Stengeln. Wegen der unterirdischen Ausläufer entwickelten sich meist kleinere oder grössere Gruppen der Pflanze, die einen grossen Teil der Wiese bedecken; Stengel $25-30 \mathrm{~cm}$ hoch, also nicht viel höher, als bei $C$. vulyaris $F r$. Die sterilen Stengel überragen mit ihrem hohen und kräfigen Blattwerk die fertilen hedeutend, und daher mag auch wohl die I'flanze hisher immer ühersehen worlen sein. Die fertilen Stengel sind verhälnismässig dünn, am Grunde von den Resten der abgestorbenen und wahrscheinlich zweimal ah)gemähten vorjihlrigen Blïter umgeben, ziemlich scharfkantig, aber nur bis ungefähr zur Mitte herab) rauh. Die unteren Scheiden sind stark netzfaserig, schmutzig-rotbram bis purpurn gefärbt, namentlich an den sterilen Stengeln. Blätter starr und rückwärts sehr rauh, mässig breit, etwa $3-4 \mathrm{~mm}$, sehr lang zugespitzt, in eine feme horstenfömige Fpitze ausgehend, ziemlich flach, oberseits mit ¿ etwas hervortretenden Seitennerven, daher erscheinen sie unter" seits mit zwei und oherseits mit einer seichten Längsinne, dunkelgrün mit einem Stich ins Blänliche, die der fertilen Stengel den Blütenstand nicht erreichend, die der sterilen Stengel dieson weit überregend. Das untere Tragblatt blattartig, aber über die Nitte des $\sigma^{7}$ Aehrchens nicht hinausgehend; das

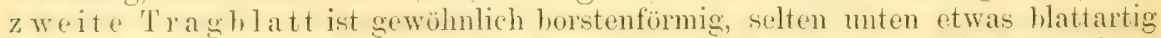
verheitert und von der Länge des dazugehörigen $Q$ Aehrehens. P A ehrehen 
3-4 (meist 3), duinn, kurz-walzenförmig, gegen die Spitze wenig verdickt, genuihert, so dass das unterste mit seiner Spitze etwa die Mitte des zweiten, dieses wieder die Mitte des dritten erreicht. Ebenso ist das Grössenverhältnis der q Aehrchen; das unterste ist meist etwas gestielt, unterwärts lockerfrüchtig und etwa $3 \mathrm{~cm}$ lang, das zweite etwa $2 \mathrm{~cm}$ und das dritte etwa $1 \mathrm{~cm}$ lang. $\mathrm{O}^{7} \mathrm{~A}$ ehrch en 1-2, länglich-walzenförmig. D e ckschuppen der $q \mathrm{Bl}$ üt en eiförmig, stumpf, dunkelbraun, kürzer als die Schlïuche. D e ckschuppen der o Blüten stumpflich, schwarzbraun bis schwarz, mit hellerem Mittelstreif. Schliuche klein, eiförmig, nervenlos, grün, niemals ausgebildet, also vollkommen steril, nicht so zeitig abfallend, als bei C. Buelii Wimm.

Aus der gegebenen Diagnose geht klar hervor, dass C. Buekii $\mathrm{W}$ imm. bei dem Bastarde sehr stark beteiligt sein muss, und wäre die Pflanze nicht in allen ihren Teilen so klein, die Stengel so niedrig und dünn, die Blätter so schmal ete., man könnte sie sonst für eine diesen Merkmalen angepasste Form. der C. Buekii halten. Indessen ist die Beteiligung der C. vulyaris $\mathrm{Fr}$. neben den angegebenen Merkmalen auch schon deshalb ausser allem Zweifel, da diese Art am Standort mit der $C$. Buekii sich mehrfach berührt und eine andere verwandte Art, etwa C. strick Good., oder C. gracilis Curt. a'ıs vielen Gründen gänzlich ausgeschlossen werden muss. C. caespitosı $L$., die allenfalls in Frage kommen könnte, wächst aber suwohl am Standort als in der ganzen Parchwitzer Gegend nicht. -

Da die $C$. vulgaris $F$. nach der bisher üblichen Ansicht so überaus formenreich ist, so dürfte auch der Bastard an anderen Orten diesen Formen entsprechend von der ehen heschriebenen Parchwitzer Pflanze in mehrfacher Hinsicht abweichen, wie ja auch die andere Kreuzung der C. Buckii I'imm., nämlich C. Butiii $\times$ yracilis, von den drei mir bisher sicher bekannten Standorten bei Breslau (Ohle-Niederung), Li egnitz (Parchwitz) und II altsch (alte Oder am Regnitzer Damm) auch nicht völlig übereinstimmt. Am letzteren Standorte sammelte sie im vorigen Sommer mein Freund, der Herr Apotheker C. Scholz aus Maltsch. Leider waren die Exemplare von dort infolge andauernden Hoch. wassers vollständig verschlammt und beinahe unkenntlich; sie dürften indes mit denen von Breslau ziemlich übereinstimmen.

Da ich diesen Carex-Bastard zuerst in der Gegend von Breslau fand, und gerade diese Stalt mit ihrer Ungebung für viele Floristen und namhafte Botaniker so überaus hedentungsvoll geworden ist, so halte ich es für angemessen, dass der Name Breslau (Vratislavia), der Hauptstadt unserer so pflanzenreichen Provinz, auch in der botanischen Litteratur eine formelle Auszeichnung erfährt. Deshalb benenne ich die eben erwähnte Carex Buekii Irimm. $\times$ gracilis Curt. als

\section{Carex Vratislaviensis $m$.}

Bereits in Nr. 1 der "Allg. bot. Zeitschrift" IV. Jahrg. p. 4 habe ich die Pflanze aus der Breslauer Flora erwähnt, wegen der mangelhaften Beschaffenheit der Exemplare aher von einer genauen Beschreihuug Abstand genommen. Unterdessen labe ich dieselbe Kreuzung in ziemlicher Menge an der Katzbach bei Parchwitz und, wie bereits erwïhnt, mein Freund Scholz in der Nihe von Maltsch gefunden. Die folgende Beschreibung bezieht sich zuniichst auf die Parchwitzer Pflanze, da ich diese in verschiedenen Zeiten zu beobachten und in tadellosen Exemplaren einzulegen Gelegenheit hatte.

Beschreibung: Rhizom fast rasenförmig mit 1-2 langen, starken unterirdischen Ausläufern. Wuehs einzelne von einander ziemlich weit entfernte kleine Stücke bildend, die aus einem oder mehreren fruchtbaren und sterilen Blattrieben zusammengesetzt sind. Gewöhnlich ist in einem solchen Stocke nur ein ährentragender Stengel mit 2-3 Blattrieben vereinigt; oft sind aher nur Blattriebe zu kleinen Stücken vereinigt, die im ersten Jahre durch die Ausläufer erzeugt werden. Stengel $50-70 \mathrm{~cm}$ hoch, unten stumpf-, oberwärts scharf-dreikantig und weit herab raul. Die fertilen stengel sind ziemlich dünn nnd schlank, an Grunde von den vorjährigen abgestorbenen Blattresten umgeben, 
nur im unteren Drittel beblättert. Die sterilen Stengel sind nur am Grunde vor einigen kurzen, zugespitzten, dumkelrotbraunen Scheiden umgeben; die darüber stehenden neigen bald zur Blattbildung, da sie in eine mehr oder weniger verlïngerte, hellbräunliche bis weisslichgrüne, blattartige Spitze ansgehen, his sie schliesslich als vollkommene kurze Stengelblätter erscheinen. Die unteren und mittleren Scheiden sind stark netzfaserig; das Fasernetz ist aber hedeutend heller, als bei $C$. Buclii. Der weisshäutige 'Teil an den Scheiden der Stengelh]aitter hat oben zum Alıschluss einen dumklen Rand. Die Anordnung der blattlosen Scheiden zur Dreikantenbildung, wie sie bei $C$. Buclii besonders scharf hervortritt, ist hier insofern etwas abgeschwächt, als dieselhen weniger scharf gekielt sind. Blätter bis $5 \mathrm{~mm}$ breit, flach, rauh, weniger starr als bei $C$. Buelii, grasgrün (im trockenen Zustande etwas ins Blïuliche ïbergehend), an den fertilen Stengeln stets kürzer als der Blütenstand, an den sterilen diesen weit übernagend und in eine lange, feine Spitze ausgehend. Das untere Tragblatt ist meist etwas kürzer als der Gesamtlüutenstand, selten die Spitze des ohersten männlichen Aehrchens erreichend; die andern Traghlätter sind gewöhnlich kaum blattartig bis kurz borstenförmig. Weibliche Aehren 3-4 (meist 4), mittelmässig dick, ziemlich lang-walzenförmig, gegen die Spitze etwas verdickt, unterwärts oft lockerfiüchtig (namentlich die unterste), unterste meist von den andern entfernt, lang gestielt, die oberen genihert und entsprechend kürzer. II ïnn liche Aehren 1-2, meist 2, genähert, eine längere walzenfürmige oben und, eine sehr kurze (oft nur angedentet) darunter, schwarzbraun, mässig dick. Deekschuppen der weiblichen blïten eilanzettlich, spitz, dunkelrothram bis schwarz, mit einem schmalen, helleren Mittelstreif, so lang oder wenig linger als die Schläuche. Deckschuppen der männlichen Blüten verkehrtlänglicheiförmig oder spatelförmig, schwarzbram mit hellerem Iittelstreif. Schläuche maissig gross, elliptisch mit keiförmiger Basis, aber doch nicht gestielt, beiderseits schwach gewölht, glatt, hellgrïn oder oben an dem von den Deckschuppen nicht hedeckten Teile schwach brïunlich, in einen deutlichen Schmabel ausgehend, vollkommen leer und daher zeitig zusammenschrumpfend.

Die Pflanzen aus der Breslauer Gegend sind im allgemeinen kräftiger, die Stengel höher, die Blätter breiter und die weiblichen Aehrehen länger, auch an der Spitze zuweilen $\sigma^{7}$. Das Fasernetz an den unteren Basalscheiden ist nicht so deutlich als hei den Parchwitzer l'flanzen. Wahrscheinlich ist dies. unter der mechanischen Einwirkumg des lange andauernden Hochwasser's der Oder und Ohle zerstört worden. -

Bei dieser Gelegenheit will ich hervorheben, dass ich in Beziehung auf die Erhaltung des Fasernetzes beim Ausstechen, Reinigen und Einlegen der Burliz-Hybride aus der Parchwitzer Gegend besonders vorsichtig verfahren bin und meist einen 'Teil der daran hängenden Erele nicht mit peinlicher sorgfalt entfernt habe. Wer also von meinen Händen aufgelegte Exemplare davon erhält, wolle den genannten Mangel mit dieser meiner wohlgemeinten Absicht entschuldigen. - Ganz ähnlich verhält es sich bei dem ebenfalls aus der Liegnitzer Flora stammenden Carex-Bastard: C. Davalliana $\times$ dioica. Hier handelt es sich aber nicht $u m$ ein Fasernetz, sondern um die Erhaltung der Entwickelung und Ausgestaltung des Rhizoms. Ich habe meist nicht nur Stunden, sondern ganze Tage zugebracht bei der äusserst schwierigen Arbeit, die lockeren hasen des genannten bastardes von Moorboden und allerhand fremdem Wurzelwerk zu säubern, ohne die überaus zarte, fadenförmige Verästelung zu zerstören, die zum sicheren Erkennen der Pflanze unbedingt nötig ist. - Wenn man instruktive Exemplare erhalten will, düfen die charakteristischen Merkmale auch an den unterirdischen Teilen einer Pflanze nicht zerstört werden.

Liegnitz, im Dezember 1899. 


\section{Ueber Alchimilla pubescens Koch, A. truncata Rehb. und eine neue verwandte Art aus den Tiroler Alpen (A. acutata).

\author{
Von R. Buser.
}

(Fortsetzung.)

A. flabellata (Nr. 1, 3, 4) ist eine ungemein einförmige, konstant pubescierende Art, während A. acutidens (Nr. 2 Schultz) so schwach behaurt ist, dass sie auf den ersten Blick als kahl erscheint. Dass letztere Ptlanze aber, obwohl Synopsis edit. 1 nicht ausdrücklich citiert (wohl nur deshalh, weil sie Koch von der gleichen Lokalität duch einen älteren Simmler, Zuccarini, besass), doch wesentlich zur pubescens $\mathrm{H}$ och mitgehört, ergebt sich nicht bloss aus der Stelle Flora XVI, 632, sondern aus der Koch'schen Beschreibung selbst. Koch's Bemerkungen unter A. ul $_{\text {y }}$ a $r$ is und fis a zeigen deutlich, dass er der Ansicht war, dass alle Alchimillen-Arten inbezug auf Beharmo starken Schwankungen unterliegen, von kahl alle Stadien bis zottig durchlaufen können. Wenn er nun bei seiner pubescens jede Angabe über das Indument weglasst, mnd bloss der Name noch vag an eine pubescente Pflanze erimert, so ist das offenbar die Folge des Einbezugs der fast kahlen Schultz'schen Pllanze (Nr. 2). Auch die Angabe „foliis acute dentatis" fïhre ich in erster Linie auf das B.hultz'sche Exemplar zurück, denn gerade A. flabellata ist durch eine stumpfe, kurze, fast zehenförnige Blattzahnung ausgezeichnet. In Koch's Augen waren für pubescens bloss die vorne gestutzten, seitlich \pm ganzrandigen Blattlappen massgebend, das Indument unwesentlich.

Im zweiten Bogen, mit der Aussenaufschrift ,pubescens" finden sich zwei Pflanzen vor, beide ans den Kitzhühelex Alpen und von Traunsteiner gesammelt. Die Etiquette der ersten lautet: „n. 79. Alchimilla rulyaris $x$ montana? (darüber "pubescens" von Koch's Hand gesehrieben) auf dem kleinen Röthenstein 700u' neben und unter Alch. fissa. Traunsteiner." Dazu gehört ein dürftiges Exemplar, allerdings der $A$. flabellata ungemein ähnlich, umsomehr, als es offenbar nicht normalwüchsig ist, sondern eine $f$. subtruncata darstellt, das aber trotzdem mit $A$. flabellata nicht identificiert werden kann und unten als $A$. a cut at a beschrieben werden soll. - Die 2. Etiquette lautet: „Nr. 27. Alchemilla pubesens M.B. Alpen um Kitzbühel 6 - 7000' - Juli 1840 - legit Tramsteiner. ExHerbario Musei l'irolensis" und damit gehen 2 normalwächsige, gute Exemplare der gleichen a cutata. - Koch hat offenbar die Pflanze zuerst von Traunsteiner selbst als montana? erhalten, seinerseits als A. pubescens M.B. bestimmt und sfuater dann unter diesem lamen cinen besseren Bogen, wohl durch Hansmann, aus dem Ferdinandenm, erhalten. Dieser L'Hanzen hat Koch in der 2. Auflage der Synopsis (1843) Erwähnung gethan.

Es ist somit A. pubescens Koch: 1. im wesentlichen = A. flabellata Bus. Standorte der 1. Aullage und "Fuscheralyen" der. 3. Aullage der bynopsis 2. = A. acutata Bus. die Traunsteiner'schen Pflanzen der 2. Auflage; $3 .=A$.

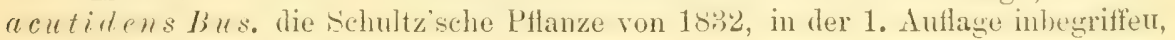
obwohl nicht ausdrücklich citiert.

In den Herbarien findet man $A$. flabellata nicht selten unter dem durch Reichenbach in Aufnalime gehrachten und hauptsachlich durch Reuters Bestimmungen ad amicos verbreiteten Namen: "A. truncata Tausch". Damit hat es folgende Bewandtnis. Tausch hatte im Index pl.hort. Canal. (1821) eine $A$. truncata namhaft gemacht als nomen nudum, aber sellst niremes beschrietren. In die Litteratur wurde diese truncata rechtsgültig 1827 durch Wimmer und Grabowsky nach einem authentischen Exemplar als vulgavis $\delta$. truncat a eingreführt (Fl. sil. I, 135): glahriuscula, foliis semiorliculatis, hani subcortatis truncatis. Riesengebirge. - Koch (Syn. ed. 1. 18:5, 231) wwihnt ihrer eluen falls unter $v u \lg a r i s$. Variat.... toliis basi minus cordatis truncatis. 
Was ist nun diese A, truncata $T$ a $u s c h$ ? Nach Ausweis des Herbariums Tausch nichts anderes als eine Hungerform der A. alpestris Schmidt (1794), $\left.{ }^{*}\right)$ d. h. wohl einer der verbreitetsten und gemeinsten, grossen, fast kahlen Arten der Gattung. Ich habe mich über diese Hungerformen, wie sie bei jeder Alchimillenart vorkommen können, über die Art ihrer Entstehung, ihre morphologische Bedleutung im Bulletin de l'Herbier Boissier II, 1894, 39-42 ausführlich geäussert, worauf ich verweise. Die Unhaltbarkeit seiner A. truncata und deren Abhängigkeit von alpestris ist übrigens von Tausch später selbst erkannt worden: in seinem Herbar hat er den Namen erst in A. glabra $\beta . t r u n c a t a$ Tausch und in der Folge in vulgaris var.truncata abgeändert.

Als Reichenbach in der Flora excursoria 1832 den Versuch machte, die Alchimillenformen seiner Vorgänger in ein System zu bringen, machte er aus der A. truncata Tausch eine der drei Hauptformen, die er im Rahmen der $A$. vulgaris L. unterschied. Seine Vorstellung ist recht eigentümlich, doch hat sie heute nur noch historisches Interesse. "Distinguas formas tres": a. ac ut $i$ loba: lobis ovatis, $\left.{ }^{*}\right)$ B. rotundata: lobis brevibus rotundatis, $\gamma_{\text {. }}$ runcata (Tausch ntspec.): lobis truncatis. "Diese drei Formen unterscheiden sich inmer," sagt Reichenbach; innerhalb jeder unterschied er, ohne sie indessen zu benennen, die Namen der äIteren Autoren bloss als Synonyma anführend, eine \pm kahle und eine pubescente Modifikation, seiner Ansicht nach blosse Standortsprodukte. ( Die Behaarung nimmt mit der Trockenheit des Standortes zu“ l. c.). Es überrascht einigermassen, solche Vorstellungen gerade bei dem Autor zu treffen, der seiner Zeit der berufenste Vertreter der analytischen Richtung in der Floristik war, wenn sie sich auch aus dem Fehlen jeder praktischen Erfahrung in der Gattung Alchimilla natürlich und genügend erklären. Es entsprechen diese drei Formen nichts konkretem, sondern sind blosse schematische Abstractionen. In der. Unterbringung der einzelnen Arten der früheren Autoren in seine drei Hauptformen verfuhr dam Reichenbach recht willkürlich und $A$. $t$ r une at a speziell wurde von ihm in einer Art und Weise diagnostiziert, die mit der 'Tausch'schen nichts melr gemein hat. Demn während Tausch's A. truncat $a$ an der Basis abgestutzte Blätter hat, schreibt Reichenbach derselben vorne abgestutzte Blattlappen zu. Und das ist etwas ganz anderes.

Reichenbach hat später seine Ansicht über A. truncata (non Tansch) modifiziert und die Pflanze $18: 38$ in der Flora germanica exsiccata unter Nr. 1476 als selbstandige Art ausgegelien, unter ausdrücklicher Citierung der vulgaris $\gamma$. truncata Fl. excurs, als Synonym. Das Material zur Nummer stammte vom kleinen Röthenstein bei Kitzbühel und war von Traunsteiner gesammelt. Es war somit die gleiche Pflanze, deren Koch 5 Jahre später (1843) in der 2. Auflage der Synopsis unter pubseens Erwähnung that. Die Reichenbach'sche Numnier ist indessen nicht einheitlich: in den einen Herbarien (z. B. Herb. Döll) stellt sie A. flabellata, in anderen (z. B. Herb. Boissier) A. acutata vor. Es kommen bei Kitzhühel die beiden Arten offenbar mit einander, im Gemenge, vor und es ist wohl nur Zufall, dass Koch gerade nur Exemplare der acut a ta erhalten hatte.

In diesem Sinne sind somit nach Standort und Auffassung des Autors $A$. pubescens fioch (non II.) und A. trumeata Richb. (non Tausch) genau identisch: bei beiden Autoren die gleiche Termengung von A. flabellata und acutata und

*) Syn. A. glabrata Tausch 1821, A. glabra Neygenfind 1821, A. vulgaris var. glabra Nert. et Koch 1823. Wimm. u. Grab. 1. c. 1827.

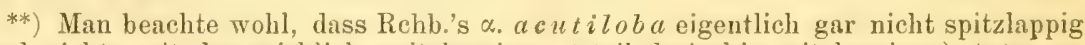
ist und nichts mit den wirklich spitzlappigen (steil dreieckig-spitzlappigen) Arten zu thun hat, die wir heute kennen (A. straminea, acutangula, tredecimloba Bus.). Vie Worte lobis ovatis zeigen, dass es sich bloss um Formen mit etwas gestreckterer Lappenform im Gegensatz zu den lobi breves rotundati der $\beta$, votundata handelt. Die beiden unter $a$ acutiloba gestellten Formen künnen nach Rchb's Diagnosen mit A. alpestris Schmidt und A. vulgavis L. (A. pastoralis Bus.olim) identificiert werden, d. h. mit den 2 vielleicht gemeinsten Arten der Gattung. 
die gleiche Beimischung fast kahler Pflanzen (A. acutidens Bus. bei Koch; "A. conglomeratu und alpestris Schm.? " unter $\gamma$. truncata in Rehb.'s Fl. excurs.).

Wie bei Kitzbühel nach den Tramsteiner'schen Exsiccaten A. Habellata und acutata durcheinander wachsen, so ist dies auch auf den beiden andern Standorten, wovon mir A. acutata bekannt geworden, der Fall. Beide Arten sind xerophil und haben offenbar genau das gleiche Trockenheitsbedürfnis. - In den $80 \mathrm{er}$ Jahren ist von Herrn Apotheker Woynar in Rattenberg A. flablluta sehr ansgiehig am Sonnwendjoch gesammelt worden; man trifft die instructiven Exemplare in fiast allen Sammlungen an und darunter von Zeit zu Zeit ein Exemplar der acututu, zum Beweis, dass sie anch hier unter flabellatu eingestreut vorkommt Im verflossenen Sommer hat Georg Treffer in Luttach die A. flabrllata ebenfills in Menge aufgelegt und entfielen in seiner Aufsammlung, die ich durchzusehen Gelegenheit hatte, auf ca. 200 stiick der flabrllata noch nicht gauz 2 Dutzend Stücke der acutata. Diese letzteren, prïchtig entwickelt und gut präpariert, haben der nachfolgenden Beschreibung zur Grundlage gedient.*)

\section{Bemerkungen zu den „Carices exsiccatae“}

von $A$. K n e u c $\mathrm{k}$ e r.

VII. Lieferung 1900.

(Schluss.)

Nr. 206. Carex refrecta Schlir. Car. II. p. 62 (181)6) $=$ C. $\operatorname{ten}(1, x$ Renter in Act. Soc. Hall. sec. Christ in Journ. of Bot. p. 263 (1885).

Auf herastem, felsigem 'Terrain und unter Gehüsch auf dem Berge "Che gu l" bei Trient in Südtirol; Kalk. Begleitpflanzen: Aronia rotundifolia Pers., Cytisus purpureus Scop., Daplne alpina L., Carex aligitata L. ssp. subnitalis (A.-T.) ete.

Ca. $1000 \mathrm{~m}$ ü. đ. M.; 460 n. Br. u. $29047^{\prime}$ östl. v. F.; 13. Juni 1899.

leg. Enrico Gelmi.

Nr. 207. Carex tristis M.B. H. t. c. III. p. 615 (1819).

Auf der transsylvanischen Alpe Peatra mare (Nagy Köhavas, Hohenstein) bei Kronstadt in Siebenbürgen auf Geröll und anstehendem Felsen des Jurakalks. Begleitpflanzen: Asperula capitate Kit., Achillea Schurii Simk, Valcriana montana L., Saxifraga aizoon L., Melissa Bamgartenii Simk, Ranunculus Carpaticus Grisb., Dryas octopetala L., Dianthus temufolius Schur, Erysimum Wahlenbergii Asch. et Engl, Draba aizoon Whhlb.

Ca. $1600-1700 \mathrm{~m}$ ü. d. M.; $45^{0} 33^{\prime}$ n. Br. u. $43018^{\prime}$ östl. v. F.; Juli u. Aug. 1896 u. 1899.

leg. J. Römer.

Nr. 208. Carex sempervirens Vill. f. courctutu Huter. in sched.

Auf Weiden am Monte Nota in Tüdtirol; Kalk. Begleitpflanzen: Carex digitata $L$.

Ca. $1000-1100 \mathrm{~m}$ ü. d. M,; ca. $45^{0} 52^{\prime}$ n. Br. u. $26^{\prime}$ östl. v. F.; Jumi 1899. leg. Pietro Porta.

Die vorliegende, habituell auffälige Form mit längeren Halnen und hreiteren Blattern wurle nach hrieflicher Mitteilüng P. Porta is s. Zt. ron R. Huter mit Rücksicht aü das beschrankte Areal ihrer geographischen Terbreitumg als C. sempervirens Vill. $f$. coarctata bezeichnet

*) Ich möchte hier die Hoffnung aussprechen, dass Herr Treffer nächstes Jahr nicht bloss A. fabellata, sondern ganz besonders A. acutata und die von ihm im Consortium aufgefundene $A$. exigua Bus. für seine Tauschanstalt auflegen möge. $A$. exigua, in 'Tirol weit verbreitet und gewöhnlich mit pubescens $K$. (flabellata Bus.) verwechselt, unterschedet sich hievon auf den ersten blick durch answen kahle Früchte. 
Bei dieser Gelegenheit sei noch erwähnt, dass ich in Gesellschaft von Hrn. Kükenthal am 11. Ausust 1899 am oheren 'Teile der l. 'Thalseite des Fleschenbachthals, eines l. Seitenthales des Binnenthals im Wallis (Schweiz), in einer Höhe von ca. $2200 \mathrm{~m}$ ü. d. M. eine ziemlich niedere Form der $C$. sempervirens rill. mit gelblichen $Q$ Aehren fand; ich erlaube mir, dieselbe mit dem Namen C. sempervirens Vill. f. semipallescens zu bezeichnen. A. K. Nr. 209. Carex capillaris L. Sp. pl. ed. I. p. $977(175.3)=$ C. pendulu Geners. el. Nr.871 (1798) $=$ C.plena Claivv. man. p. 292 (1811).

Auf einer feuchten, aus Kies und sch lam m igem All uvium bestehenden ebenen Fläche des mittleren Teiles vom Eginenthal im oberen Wallis (Schweiz), welche meist mit herahgeschwemmten Alpenpflanzen hewachsen ist, in der Nähe der 2. Brücke, auf dem 1. Ufer des Baches, bei "Galnern“. Begle itpflanzen: Carex bicolor All., frigida All., vulgaris Fr. f., Phleum alpinum L., Scirpus pauciflorus Lightf., Saxifiaga aizoides L., Polygonum viviparm L. ete.

Ca. $1650 \mathrm{~m}$ ü. d. H.; $46^{\circ} 29^{\prime}$ n. Br. u. $26^{\prime} 2^{\prime}$ östl. v. F.; 7. Aug. 1899. leg. A. Kneucker.

Nr. 210. Carex cavillaris L.f: mimimaBecl: Fl. v. Nied.-Oesterr. p. $144(1890)$.

Auf Kalkschiefer am Ringelstein im 'Tristenbachthal, einem Seitenthal des Weissenbach-Thales, nordwestl. von Bruneck im Pusterthal in Tirol; Be gleitpflanzen: Carex omithopodioides Hsm., Diryas octopetala L.

Ca. $2000-2500 \mathrm{~m}$ ï. đ. M.; $46056^{\prime}$ n. Br. u. $29^{\prime \prime} 30^{\prime}$ östl. v. F.; 25. Aug. 1897.

leg. Georg 'Treffer.

Nr. 210 a. Carex capillaris L. f. minima Becl.

Auf kurzberasten und trockenen Alptriften hinter den Abstürzen des Ofenhorns im hintersten T'eile des Binnenthals im Wallis (Schweiz); krystallin, Marmor. Begle itpiflanzen: Carex omithopodioides Hsm., fermginea Scop.f., nigra All., firma Most, Saussurea discolor DC., Gnaphalium leantopodium Scop., Carpathicum Thhlbg.

Ca. $2300 \mathrm{~m}$ ü. d. .I.; $46023^{\prime}$ n. Br. u. $25^{\prime \prime} 57^{\prime}$ östl. v. F.; 12. Aug. 1899.

Die zieml. spärlichen Exemplare dieses Standortes sind zur Unterscheidung der Exemplare des Standortes Nr. 210 auf Papierstreifen aufgeklebt.

leg. A. Kneucker.

Die Ausgabe einiger sehr seltener und kritischer Formen konnte nur durch sparliche Anflage dereelhen ermöglicht werten. Als reichliche Entschädigung dieses Ausfalles darf wohl die Gratisausgabe der Nummern 183 a, 187 a, 190 a, 205 a und 210 a gelten.

A. K.

\section{Botanische Litteratur, Zeitschriften etc.}

Klein, Dr. Ludwig. T)i, Plysiognomie der mittelenropäischen Waldhäume. (Festrede zur Einweihungsfeier des neuen bot. Instituts der techn. Hochschule zu Karlsruhe am 18. Nai 1899.) Mit 10 Tafeln in Tichtdruck nach Originalaufnahmen des Verfassers. Verl. von W. Jahraus in Karlsruhe. $26 \mathrm{~S}$. Preis $2.40 \mathrm{MI} .^{*}$ )

Wie schon der 'litel sagt, handelt es sich hier um keine kritisch-systematische Arbeit, sondern es sollen, um die Worte des Verfassers zu gebrauchen, , die Ursachen der Baumgestalt an unseren wichtigsten waldbildenden Bäumen innerhalb und ausserhalb des Waldes erörtert werden." Verfasser unterseheidet scharf 2 Reihen von Faktoren, von welchen die Baumgestalt abhängig ist. Die Faktoren, welche den die betr. Art charakterisierenden Hahitus bedingen, werden

*) Interessenten seien hiermit auch auf eine einschlägige Arbeit von B. Eblin, betitelt: "Waldreste des Averser Oberthals", em Beitrag zur Kenntnis unserer alpinen Waldbestände, im Heft V der Berichte der schweiz. bot. Gesellschaft 1895, p. 28-64, aufmerksam gemacht, welche 6 prächtige Tafelu enthält. 
hier nicht berücksichtigt, und nur diejenige formbestimmende Faktorenreihe wird in den Kreis der Betrachtung gezogen, von welcher die P'hysiognomie des Individums abhängig ist. Ausser den Hauptfaktoren Licht, Warme und Wasser kommen u. a. noch die verschiedenartigen mechanischen Verletzungen bei der Bildung der Baumgestalt in Betracht. Jit Vorliehe beschättigt sich der Autor mit den verschiedenartigen Wetterformen der Hochgebirgsbämme, von denen en. eine Reihe ehrwürdiger Gestalten uns im Bilde vorführt, nebst einer Anzahl einzeln gewachsener wundervoller Baumformen niederer Lagen. Das Werkchen ist nicht nur für den Fachmann, sondern auch für jeden Naturfreund empfehlenswert, der durch dasselbe bei eigentümlichen baumformen zum Nachdenken über ihre Entstehungsursache angeregt wird.

A. K.

Aseherson, P., u. Graebner, P., Synopsis der mitteleurop. Flora. \&. u. 9. Lief. Verlag von W. Engelmann in Leipzig. 1899. 8" 1\%. Bd. p. 145-304. Preis jeder Lieferung $2 \mathrm{M}$.

Die vorliegenden Lieferungen 8 u. 9 dieses ausgezeichneten Werkes enthalten die Genera: Phteum, Lagurus, Cimna, Polypogon, Gastridium, Chaeturus, Sporolobus, Agrostis, Calamagrostis, Holcus, Avena, Trisetum, Ventenata, Aera, Antinoria, Periballia, Aeropsis, Weingaertneria, Sieglingia, Danthonia. A. K.

Oesterreichische bot. Zeitschrift 1900. Nr. 2. Polatck, Ur. Joh a nn II a ria, Untersuchungen über die Staminodien der Scrofulariaceen. - J enčič, A., Untersuchungen des Pollens hybrider Pflanzen. - Fleischer, R., Zwei neve Kompositen-Bastarde. - Scholz, J. B., Studien über Chenopodium opulifolium etc. - Murr, J., Beiträge zur Kenntnis der Hieracien von Kärnten und Steiermark. - Litteratur-Uebersicht.

Deutsche bot. Monatsehrift 1900. Nr.2. Usteri, Beitrige zu einer Monographie der Gattung Berheris. - Zschacke, Beiträge zur F'lora Anhaltina VII. L eimbach, Die Volksnamen unserer heimischen Orchideen. - Hanemann, Die Flora des Frankenwakles etc. - Suksdorf, Washingtonische Pflanzen. $\mathrm{Kmet}$, Wie man botanische Monographien fabriziert?

Botan. Centralblatt. 1900. Nr.6. II üller, Karl, Bryologische und hepaticologische Fragmente. -I. - Kra use, Ernst H. L., Floristische Notizen. Nr.7. Lemmermann, E., Spirodiscus Eichwald oder Ophiocytium Nägeli? K rause, Ernst H. L., Floristische Notizen. - Nr. 8. Rassmann, Moritz, Eine bisher nicht beobachtete Minshildumg bei Stachys Germanica L. - Müller, $\mathrm{K}$ a rl, Zusammenstellung der Lehermoose aus dem Reichslande Elsass-Lothringen.Nr. 9. Müller, Karl, Wie in vor. Nr.

Mitteilungen des thäring. botanischen Vereins. 1899. Heft XIII u. XIV. (Neue Folge). Bornmüller, J., Pysoptychis Haussknechtii Bornm. - Jacobasch, E., Teratologische Mitteilungen. - Derselhe, Mykologische Hitteilungen aus d. Hlora von Jena. - Derselbe, Ist Cirsium silvaticum 'I'sch. Art oder Varietä? - Blied ner, A., Weitere Beiträge zur Flora von Eisenach. - H a ussknecht, C., Symbolae ad floram graecam (Schluss). - J a cobasch, E., Einige mykologische Absonderlichkeiten. - Marbach, F., Beitrïge zur Flora des Orlathales. - Koch, E., Neue Beiträge zur Kenntnis der deutschen Pflanzenwelt. Berichte über die Hauptversammlungen.

Mitteilungen des bad. bot. Vereins. 1900. Nr. 169 u. 170. I a ck. Lr. Jos. B., Zu den Lebermoosstudien in Baden.

Verhandlungen der k. k. zoologisch-bot. Gesellsehaft in Wien 1900. Heft 1. Warnstorf, C., Beiträge zur Kenntnis der Moosflora von Südtirol.

Berichte der deutschen bot. Gesellschaft. 1900. Heft 1. ('olakorisy, L. J., Ueber die Emporhebung von Achselsprossen. - Schroeder, Bruno, Cosmocladium Saxonicum De Bary. - Lemmerm ann, E., Beiträge zur Kenntnis der Planktonalgen. - Z opf, W., Oxalsäurebildung durch Bakterien. 
Botaniska Notiser. 1900. Heft 1. Borge, O., Schwedisches Süsswasserplankton. - Vestergren, 'T'., Terzeichnis nebst Diagnosen und Bemerkungen zu meinem Exsiccatenwerke "Hicromycetes rariores selecti“. Fasc. VII-X.

Botanical Gazette 1900. Vol. XXIX. Nr.1. Pollock, James B., The mechanism of root curvature.

Bulletín de l'aeadémie internationale de géographie botanique. 1900. Nr.123. Parmentier, P., Une nouvelle fougère hybride. - Maire, R., Un parasite d'Encelia tomentosa. - Claire, Ch., Le's Centaurées du nord-est de la France. - Feret, A., Les plantes des terrains salés. - Spalikowski, Dr. Ed., Sur la croissance du Gui en Normandie. - Petitmengin, Marcel, Sur quelques plantes rares et adventices en Lorraine. - Monguillon, E., Catalogue des Lichens du departement de la Sarthe. - Nr. 124. Parmentier, P., Inclinomètre Parmentier. - Claire, Ch., Wie in vor. Nr. - Etoc, l'ab bé G., Les plantes de la Bible. - Petitmengin, Marcel, Wie in vor. Nr. - Monguillon, E., Wie in vor. Nr. - Castanet et Léveillé, Les plantes utiles de la Mayenne.

Bulletin de l'association Française de botanique 1900. Nr.26. Gillot, Dr. X., Les Menthes hybrides d'après les travaux de Ern. Malinvaud. - Le Grand, Ant., Coup d'oeil sur la récente publication de M. Husnot: Graminées. B lanchard, 'Th., Liste de noms patois de plantes aux environs de Maillezais. Sudre, H., Excursions batologiques dans les Pyrenées. - Olivier, l'a b bé H., Exposé systématique et description des Lichens de l'Ouest et du Nord-Onest de la France. - Brachet, Fl., A propos du Paspalum dilatatum Poir.

Eingegangene Druckschriften. Mïller, Karl, Moosflora des Feldberggebietes (Sep. aus d. ,Allg. bot. Z.“ 1898 u. 99). - Dybow sky, Dr. W., Scorowidz do zielnika flory Polskiej (aus Kosmos 1899). - Podpera, J., Bryologische Beiträge aus Südböhmen (Sep. aus Sitzungsberichte d. kgl. böhm. Ges. d. Wissenschaften, mathem. naturw. Klasse. Prag 1899). - Ascherson, 1)r. P., u. Graebner, Lr. P.. Synopsis der mitteleurop. Flora. 8. u. 9. Lief. 1899. Verl.v. W. Engelmann, Leipzig. - Orzesk o, N., Coupe des Glumacées (Revue bryologique Nr. 1, 1900). - Hervey, Williams, Observations on the Colors of Flowers. E. Anthony \& Sons, New Bedford 1899. Wa isbecker, Dr. A., Beiträge zur Kenntnis der Gattung Odontites (Eep. aus der "Oesterr. bot. Z." 1899 Nr. 12) - Richen, Gottfr., Prof. s. J. Nachträge zur Flora von Vorarlberg und Liechtenstein (Sep. aus d. ,Oesterr. bot. Z." $1899 \mathrm{Nr} .12$ ). - Ja a p, Otto, Ueberpflanzen bei liad Nauheim in Oberhessen (rep. aus „Deutsche bot. Monatschrift 1899 Nr. 9 10). - Klein, Dr. L., Prof., Die Physiognomie der mitteleuropäisch. Waldbäume (mit 10 Tafeln in Lichtdruck), Verl. v. Wilh. Jahraus in Kalsruhe 1899. Kükenthal, Georg, l lie Carexvrgetation des aussertropischen Südamerika, ausgenommen Paraguay und Siidbrasilien (sep), aus „Engler's bot. Jahrbüchern * 27. Bd., 4. Heft 1899. - Cryptozamae japonicae iconibus illustratae. Tókyó 1899 Nr. 5 - 7. Phanerogama e et Pteridophytae japonicae iconibus illustratae $1899 \mathrm{Nr} .5-7$. Jack, Dr. Jos. B., Zu den Lebermoosstulien in Baden (Sep. aus d. Mitteil. des bad. bot. Vereins 1900). - Schinz, Dr., lier bot. Garten u. d. bot. Museum d. Universität Zürich im J. 1899. - M üller, K., Revision der Hepaticac in Mougeot-, Nestler- u. Schimper Stirpes hryptoganae Voges. Khenanae : \$10-1860 (Sep. aus Ném. de l'herb Boissier. Bulletin Nr.6 1900). Kamienski, Fr., Sur une espèce d'Utricularia nouvelle pour la flore du pays (Galicie). (Sep. aus, Bullet. de l'académ. des sciences de Cracovie. Dez. 1899). - Höck, Dr., Studien über die geogr. Verbreitung der Waldpflanzen Brandenburgs (Sep. aus d. Abhandl. d bot. Ver. d. Prov. Braudenb. 1899). - Dersel be, Zahlenverhältnisse in der Pflanzenwelt Norddeutschlausts (Sez. aus d. dbhandl. des bot. Fer. d. Prov. Brandenburg. 1899). - Derselbe, Allerweltspflanzen in unserer heimischen Phanerogamen-Flora (Sep. aus ,Deutsche bot. Monatschr." 1899 Nr. 9-12). - Dalla 'Torre, Dr. C. G. v., u. Harms, Dr. H., Genera syphonogamarum. Fasc. I. Leipzig. Verl. v. W. Engelmann. 1900.

Oesterreichische bot. Zeitschrift 1899 Nr. 12 und $1900 \mathrm{Nr}$. 1. - Botanical Magazine, Tōkyō 1899 Nr. 153 u. 154. - Bulletin de l'académie internationale de géographie botanique $1899 \mathrm{Nr} \cdot 121-124$. - Botanical Gazette 1899 Bd. XXVIII Nr.4-6u. 1900 Bd. XXIX Nr. 1 u. 2. - Z e its ch rift f. angew. Mikroskopie V. Bd. Nr. 8 u. 10. - La Nuova Notarisia 1900 Januarheft. - Bulletin de l'association Fran- 
clise 1900 Nr. 25-27. - Botaniska Notiser 1899 Nr. 6 u. 1900 Nr. 1. - Deutsche. bot. Monatschrift $1899 \mathrm{Nr} 9$ u. 10, $1900 \mathrm{Nr} .1$ u. 2. - Berichte der bayer. bot. Gesellsch. Bd. VI. 1899. - Mitteilungen der bayer. hot. Gesellsch. Nr. 1-14 (1892 bis 1900). - Verhandlungen d. k. k. zool. bot. Gesellsch. in Wien 1899 Nr. 9 und 1900 Nr. 10 u. 1. - Berichte d. deutsch. bot.Gesellsch. Bd.X VII Heft 1 - 10 u. 1900 Nr. 1. Glasnik hrvatskoga Naravoslovnoga Društva. $1898 \mathrm{Nr} .1-5$ und $1899 \mathrm{Nr}$. 6. - Le monde des plantes 1900 Nr. 5. - Verhandlungen d. bot. Ver. d. Prov. Brandenb. 41. Jahrg 1899. - Mitteilungen d. thür. bot. Ver, 1899. N. Folge Hoft XIII u. XIV. Mitteilungen d. bad. bot. Ver. 1900 Nr. 169 u. 170. - Schedae zum V. Fascikel der "Plantae crit. Saxoniae" Nr. 101-125. - Botanical exchange club of the British isles. List of desiderata 1899/1900. - Herbarium europaeum. Prospect 1900. Schulz, Paul, Tauschvermittlung für Herbarpflanzen, Katalog 1899. - W iener bot. Tauschanstalt, Katalog für 1900. - B e rl in er bot. Tauschverein, Katalog des 31. 'Tauschjahres. - Treffer, Georg, Katalog getrockneter Herbarpflanzen. - B ern. Seeber, Bücherkatalog. Firenze 1899. - Rousset, Jules, Bücherkatalog. Paris 1899. Jordan Richard, Bücherkatalog. München 1899.

\section{Botanische Anstalten, Vereine, Tauschvereine, Exsiccatenwerke, Reisen etc.}

Botanischer Verein fü die Provinz Brandenburg. Die Sitzung am 9. Februar eröffnete der Vorsitzende Prof. Schumann mit der Mitteilung, dass das Ver.-M. Justizrat Julius Ritschl in Stettin, ein Sohn des älteren Posener Floristen Georg Ritschl, gestorben sei, und Prof. As cherson widmete dem Verstorbenen einen ehrenden Nachruf. Nach einigen weiteren geschïftlichen Anzeigen besprach Dr. No ewes die von Prof. Hansen in Giessen herausgegebenen "Pflanzengeographischen Tafeln", ein äusserst willkommenes Anschauungsmaterial für den Unterricht, umso mehr, als dieser Gegenstand durch die Hervorhebung der physiologischen und biologischen Gesichtspunkte gegenwärtig ein allgemeineres und lebendigeres Interesse gewinnt. Der Herausgeber kam zu der Ueberzengung, dass nur die fortgeschrittene photographische Technik, mit Ausschluss der Farbe, da diese erfahrungsgemäss von der Betrachtung der Form ablenkt, im Stande sei, geeignete scharfe Bilder zu liefern, und nach grossen Torbereitungen wird das von der Neuen photographischen Gesellschaft in Steglitz hei Berlin herge. stellte Werk 20 Tafeln in 4 Lieferungen, jele zu 40 Mlk., umfassen. Die vorgelegten Proben: aus dem tropischen Asien Baumfarne in den Gehirgswäldem Ceylons und aus dem Wüstengebiete Nordafrikas Dattelpalmen in einer Oase bei Tripolis, entsprachen völlig den ihnen von dem Vortragenden gespendeten Lobe. Ferner legte Dr. Moewes ein Stammstück von einer Cecropia aus Brasilien vor, dessen Hohlräume von einer kleinen Ameisenart zur Anlegung von Kolonien henützt wird; zugleich finden die T'ierchen in den sogenannten , Hüller'schen Körjerchen ", kleinen, am Grunde der Blattstiele entstehenden, Insekteneiern gleichenden Gebilden, eine ihnen sehr zusagende Nahrung. - Custos P. Hennings machte Nitteilung über den Gitterschwamm, Clathrus cuncellutus Tourf., einen l'ilz, welcher zum erstenmale in der Mark und zwar auf einem Palmenkühel im Haak'schen Wintergarten in Steglitz aufgetreten ist, hisher in Deutschland üherhampt noch nicht gefunden, wohl aber in Italien, Südtirol und in der steiermark beobachtet wurde. - Sehr interessante Kulturversuche hat Dr. Ru hland mit IInpucrea fungicola Karst. gemacht, einem l'ilz, der zwar auf totem Jolze liemte, sich aber nicht weiter entwickelte, wähend er in Hutpilzen, namentlich in I'olyfurus betulims ein ihm völlig zusagendes Wohnmess- and Nährmedium findet. - I'rof. Ascherson legte ein von P'rof. Conwentz, linektor des I'rovinzialmusemms in Danzig, auf Veranlassung des landwirtschaltl. Ministeriums verfasstes ,Forsthotanisches Merkhuch" vor. In demselben simt, z. t. auch mit klemen Abhildungen, alle in den verschiedenen Verwaltumgshezirken der l'rovitz Mestprensisen noch vorhandenen, durch Alter, Grösse, eigenartige Wuchsverhältnisse, durch historische Ereignisse oder anderweite Eigenschaften merkwiirdige baimuc, suwie seltene 
Baumarten und noch urwüchsige Waldpartien vorgefüht, deren Erhaltung von höchstem kulturhistorischen Interesse sein dürfte. Es ist hoch anzuerkennen, dass die Staatshehörden, wohl infolge einer Anregung seitens des Reichstagsabgeordneten Wedekamp i.J. 1898, gewillt zu sein scheinen, derartige Denkmäler der Natur unter ähnlichen staatlichen Schniz zu stellen, wie ihn die Kunstilenkmäler geniessen. Wie Geh.-R. l'rof. W it tmack im Anschluss daran mitteilte, ist an ihn bereits die Aufforderung ergangen, ein dahin zielendes Gutachten abzugeben, und er bat die Mitglieder des Vereins, ihn bei der Sammlung des Materials möglichst vielseitig unterstützen zu wollen; dem wenn für alle Provinzen des Staates dergleichen Merkbücher ins Leben gerufen werden sollen, so erfordert das eine jahrelange Arbeit, die von einem Einzelnen kaum zu überwältigen sein dürfte, während es die vereinten Kräfte einer ganzen Körperschaft recht wohl möglich machen könnten. Prof. íchumann schloss daran Mitteilungen üher Reste alter Urwallbestande in Böhmen und Oldenburg, deren Erhaltung sehr wünschenswert erschiene, wie aber auch ausserdem etwas geschehen müsste, um beispielsweise die so hochinteressanten Fenns im (irmewalde bei Berlin vor ferneren Eingriffen der Villenkoloniemanie zu schützen. Prof. B eуer findet es gleicherweise thunlich, anch die für unsere Mark Brandenburg so charakteristischen herrichen Seen in ihrer Ursprünglichkeit zu erhalten mnd in die gemeinsame Arbeit mit einzuschliessen. - Zum Schluss der Sitzung nahm Dr. We isse die Deutung einer monströsen Apfelsine, wie er sie vor längerer Zeit dem Verein vorgelegt, noch einmal auf, und wies die inzwischen erfolgte andere Deutung seitens des Prof. Magums, die klar erkennen lasse, dass derselbe das corpus delicti weder gesehen, noch untersucht hahe, ziemlich energiseh zurück.

IV. L a ckowitz.

Kgl. bot. Gesellschaft zu Regensburg. Floristische Sitzungen: 8. November 1899. Herr Prof. Dr. Vollmann besprach unter Vorlage ansgiehigen Herkarmateriales die mm Regenshmeg vorkommenden drten mud formen der Gattungen Chenopodium und Atriplex. Nach einem gedrängten Ueberblicke über die wichtigste Litteratur (Moquin-Tandon, Winkler, Krašan, Murr) und die Einteilung der Chenopodiaceen kam der Vortragende auf die einzelnen Arten zu sprechen und gelangte dabei zu manchen beachtenswerten Ergehnissen, die im Folgenden kurz zusammengefasst werden mögen:

Bei Ch. polyspermum Limné betonte er die unlösbaren Widersprüche, die sich in den Definitionen der Formen dieser Art bei G. Beck (Flora von NiederOesterreich. I. p. 330) und bei Ascherson-Graebner (Flora des Nordostdeutschen Flachlandes. p. 279) finden. Indem sich Vortr. den letztgenannten Autoren anschloss, bemerkte er zugleich, dass die sämtlichen Merkmale von var. cymosum Chevallier und var. acutifolium. Smith in den mannigfachsten Kombinationen sich finden.

Chenopodium urbicum Limné - in đen Lokalfloren des hiesigen Gebietes (Fürnrohr, Singer) als nicht gemein * bezeichnet - wurde in neuerer Zeit in demsethen nicht mehr heolachtet; die im Gesellschaftsherbar vorhandenen Exemplare stammen von Irlbach bei Straubing.

Die Vielgestaltigkeit des Chenopolium album Limné hat zu einer grossen Ferschiedenheit in der lartellung und Einteilung seines Formenkreises bei den verschiedenen Autoren gefülut. Als beste Darstellung ist die bei AschersonGrachner (1. c. 1, 280), rehom aus dem Grmele zu bezeichnen, weil hier die Prioritat der - in ihrer Bedentung allerdings nicht immer numstrittenen - Varietätsnamen am besten gewahrt erscheint. Nur mit der Wahl des Namens viridescens St. Amans für die Uebergangsform der var. viride Linné zur var. spicatum Koch konnte sich Vortr. nicht einverstanden erkliren, da sich die Benchreilumg dieser Form bei St. Amans im Ganzen mit der von Ch. viride Linné deckt und ihre Aulstellumg nur dafurch veranlans erscheint, dass mehrere fianzösische Antoren vor Moquin-'Tandon (der bei De Candolle, Prodromus. XXIII, 2. p. 68 den Irrtum aufklärt) das Linnésche $C h$. viride mit $C h$. opulifolium Schrader identifizierten. Die Einteilung bei G. Beck (1. c. p. 332) leidet an dem Fehler, dass sie die 
mehlige Bestaubung zu wenig berücksichtigt, während Kraśan ( Fragmente aus der Flora von Steiermark" in Nitteilungen des Naturw. Vereines für Steiermark. Jahrg. 1893. p. 253 ff.) der bisherigen Nomenklatur nicht genügend Rechnung trägt.

In der Regensburger Flora wurden von hierher gehörigen Virictiten umd Formen bisher beobachtet: die var. spicatum Koch, seltener in der forma ylomerulosum Reichenbach, am häufigsten aber die var. viridr Lime, anch in der f. payanmm Reichenbach (= virideserns Ascherson-Graebner, nm. St. Amms), daneben noch die rar. lanceolatum Miihlenberg nicht gerade selten.

Auch das von Krašan (1. c.) als Varietät von C. album aufgestellte, nach Nurr ( Ueber einige kritische Chenopodinm-Formen" in Dentsche but. Honatsschrift 1896. \%. $32 \mathrm{ff}$. t. I, II) als Art anzusprechende Ch. striatmm findet sich, wie bereits an frïherer Stelle*) mitgeteilt, gleichfalls im (Gehiete. Die von MInr**) geäusserten Zweifel, ob die damals vorgelegenen Exemplare mit seiner "Art" identisch seien, erwiesen sich inzwischen, nachdem Vortr. sich in den Besitz Nurr'scher Originalexemplare gesetzt, als unbegrïndet. Trotzdem kann sich Vortr. auch jetzt nicht von dem Artcharakter des Ch. striatum überzeugen, da sich sïmtliche als für dieses charakteristisch angegebenen Nerkmale (st ump fe, schwach aukgehissen-gezähnte, oberseits sehr glatte und zuweilen fast spiegehde Blätter, parallel mit dem Rande verdanfende Blattnerven, kleine Blütenknäuel, breite gelbliche Berandung der Kelchsegmente, violette Streifung des Stengels) auch bei anderen Formen des $C h$. album - wenn auch oft in anderer Zusammenstellumg - finden. Inshesondere ist gerade die Streifung des Stengels ebenso wie die rote Besinumung der Blätter, ähnlich wie das bei mehreren Arten der (Gattung Rumex der Fall ist,***) auf Wittermoscinflüsse zurückzuführen um erscheint gerade deshalh, auch die Benennung der Form nach diesem Herkmale wenig angezeigt. Vortr. glaubt beobachtet zu haben, dass gerade bei den Exemplaren, die in einer späteren Vegetationszeit zur Entwichelung gelangen, die rote Streifung starker ausgenright ist und anch die Teigung zur Bildung stumpfer Blätter vorherrscht.

Ch. opulifolium Schrader wird von Krašan (1. c.) als Varietät zu Ch.album gezogen, ohwohl er für die Unterscheidung Beider ein in der liegel zutreftendes Nerkmal angieht: Kelchsegmente bei Ch. opmlifolium am Rï̈lien flügelig, bei Ch. allum stumpfgekielt. Vortr. möchte als charakteristisch für erstere Art noch den spezifischen Geruch und die im Gegensatze zu Ch. album längere Tegetationsda uer bezeichnen, $\dagger$ ) Die $v a r$. mucronulatum Bech (=? betulifolium Murv) wurde im Gebiete mehrfach an Strassenrändern und auf Kulturboden konstatiert.

Ch. glaucum Linné kommt sowohl in der typischen Form als auch in den

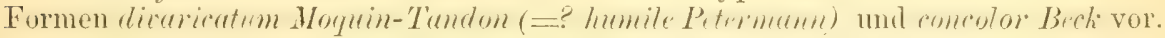

Von Ch. Bonus Henricus findet sich neben der typischen Form auch die var. erosum Moquin-Tandon.

Atriplex hortense Limé wurde verwildert an zwei Stellen in der Nähe der Stadt angetroffen.

Von Atriplex patulum Limné ist die rar. angustifolium Smith und zwa. in der f. integrifolium Becl: selten, häufig dagegen die vai. erectum IIudson. Für eine auf Bahnschutt aufgefundene Form der Letzteren mit sehr breiten, im unteren Teile beiderseits mit grossen, vorgezogenen Lappen versehenen Blättern stellt Vortr. den Namen la tifolium auf, da die von G. Beck (1. c. p. 335) in Anlehnung an anyustifolia Smith gewählte Bezeichnung einerseits wegen der Blattform wenig passend, andererseits schon deshalb unrichtig erscheint, weil die var. angustifolia Smith identisch mit macrotheca Bech: ist, während Beck seine forma angustifolia unter den Formen seiner rar, microtheca auffiihtht.

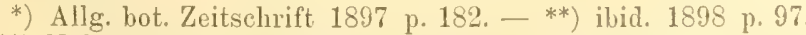

***). Vgl. Rechinger in Schedae ad Flo1 am exsiccatam Austro-Hungaricam VIII p $75 \mathrm{f}$.

†) Vgl. neuestens J. B. Scholz "Studien über Chenopodium opulifolium Schrad., C. ficifolium Sm. und album L." in Oesterr. bot. Zeitschrift 1900. p. $49 \mathrm{ft}$. 
29. November 1899. Herr Prof. Petzi legte die Belegexemplare für die von ihm im vergangenen Sommer im bayerischen Walde gemachten wichtigeren Funde vor, von denen die folgenden besondere Erwähnumg verdienen:*)

a. Arten, welche bisher (nach Sendtner, Die Vegetationsverhältnisse des bayerischen Waldes) im bayerischen Walde nur in geringerer Neereshohe gefunden wurden:

Impatiens Noli tangere Linné $1150 \mathrm{~m}$ (nach Sendtner bis $942 \mathrm{~m}$ );

Trifolium hybridum Linné $830 \mathrm{~m}$ (S. $689 \mathrm{~m}$ );

Sanguisorba officinalis Limné $830 \mathrm{~m}$ (S. $740 \mathrm{~m}$ );

Heracleum Sphondylium Linné am Plattenhausen $1250 \mathrm{~m}$ ! (S. $705 \mathrm{~m}$ );

Lysinachia vulgaris Limé $740 \mathrm{~m}$ (S. $568 \mathrm{~m}$ );

Chenopodium Bonus Henvicus Linné beim Arberschutzhaus

$1400 \mathrm{~m}$ ! (S. $1134 \mathrm{~m})$

Gymnadenia conopea Robert Brown am Lusengipfel $1372 \mathrm{~m}$ ! (S. $822 \mathrm{~m}$ ).

b. Neue Standorte für seltene oder weniger häufige Pflanzen:

Cardamine amara Linné im Rachel-Lusengebiet;

Comarum palustre Linné am Rachelsee (1087 m);

Circaea intermedia Ehrhart mehrere Standorte in einer Höhe von 800 bis $1000 \mathrm{~m}$ am Südabhange des Gebirgszuges Rachel-Lusen;

$\left.\begin{array}{l}\text { Pirola uniflora Limné } \\ \text { Ramischia secunda Garche }\end{array}\right\}$ an Arber (ca. $900 \mathrm{~m}$ );

Veronica opaca Fries Guglöd (840 m);

Teucrium Scorodonia Limmi zwischen Falkenfels und Straubing (westlichster Standort!) ;

Gymmatemia albida Richard am Arber! (bisher nur für den Rachel angegeben);

Listera cordata Robert Brown Hochberg bei Eisenstein, Zirkelfilz am Plattenhausen ;

Juncus filiformis Linné am Rachelsee;

Phlemin uljimum Limme am Lusen! (bisher nur am Rachel u. am Plattenhausen);

Lycopodium inundatum Limné am kleinen Arbersee.

c. Arten und Formen, welche bisher im bayerischen Walde noch nicht gefunden wurden:

Aconitum Stoerlianum Reichenbach, non Sendtner am Rachel; daselbst auch die var. versicolor Reich mbach.

A. Telyphonum Reichenbach Lichtenwald bei Donaustauf, bisher als A. Lycoctomum Limm hestimmt (bei der Lntersuchung dieser Art hat sich auch ergeben, dass das bisherige $A$. Lycoctomm des Regensburger Juragebietes $A$. rulparia lifichenbach ist, wozu wohl anch die im imneren bayerischen Walde gefundenen Exemplare gehören, während das gleichfalls für das Regensburger Florengebiet aus dem Penckerthal konstatierte ,A. variagatum" A. Cammarum Jacquin darstellt, zu welchem auch am Plattenhausen gefundene Exemplaro zu zählen sind);

Senecio subalpinus Koch kommt im bayerischen Walde in drei Formen vor:

1. die typische Form mit dreieckigen Blättern (Länge: Breite $=1: 1$ );

2. eine Form mit verlängerten Blättern (Länge: Breite $=2: 3-4$ );

3. eine Form mit leierförmigen Blättern (entsprechend dem S. cordatus Koch var. sublyratus).

Salix grandifolia S'eringe am Südabhange des Rachel (1200 m) in 12-15 Stöcken 37, q und androgyn in Formen, welche sich der forma lancifolire Wimmer und fagifolia ITimmer nähern. Ausserdem in einigen Exemplaren

*) Vgl. Petzi, F., Floristische Notizen aus dem bayerischen Walde" in Denkschriften der Kgl. bot. Gesellschaft in Regensburg. VII. Band (N. F. I. Band), p. $109 \mathrm{ff}$.

Peter, A., "Ein Beitrag zur Flora des bayerisch-böhmischen Waldgebirges" in Oesterr. bot Zeitschrift 1886 p. $11 \mathrm{ff} .$, p. $41 \mathrm{ff}$.

Schorler, B., "Ein Beitrag zur Flora des Böhmerwaldes“ in Sitzungsberichte der naturw, Gesellschaft Isis in Dresden 1897. p. $71 \mathrm{ff}$. 
im Risloch am Arber. Das Vorkommen dieser Art an beiden Standorten ist umso interessanter, als dieselbe in Bayern bisher nur aus den Alpen und von - einigen Stellen der oberen Hochebene bekannt war.

Eine den Blättern nach der Bastardverbindung $S$. auvita $>$ grandifolia entsprechende Form sammelte Herr Lehrel A. Mayer an der Strasse von Ludwigsthal nach Eisenstein; doch ist eine definitive Entscheidung äber diese Exemplare erst nach dem Einsammeln der Blütenzweige möglich.

Lycopodium annotimu Limé am Lusen in einer Form, welche sich durch die $5 \mathrm{~mm}$ langen, fast ganzrandigen, starren Blätter als var. pungens Desvaux charakterisiert. (Nach Ascherson-(irather, synopisis der mittelenropaischen Flora wurde diese Varietät bisher nur in 'Tirol, Ostpreussen und Mähren gefunden.)

Dr. Poeverlein.

Preussischer botaniseher Verein. IV. Sitzung, Königsberg i. Pr., 19. Fe bruar 1900. Herr Lehrer (iramberg demonstrierte mehrere bemerkenswerte Pflanzenfunde und monströse Bildungen, die er auf vereinzelten Ausflügen in vergangenen Sommer m Künigsherg, Janzig und Thorn beobachtet hatte. Er legte u. a. vor Phleum pratense mit doppelter Rispe, Carum Carvi mit deutlich entwickelten Hïllb]ätern, ferner die haitig kultivierte Zierpflanze C'ampamula fyramidalis mit lauhartig verbildetem Kelch, sowie Vergrünumgen der Aehrehen von Derlylis glomeratu und spratch über die in Ostpreussen seltene Hypochoeris glebra, die er bei Danzig sesammelt hatte. Herr Apotheker Roerdansz hielt einen Vortrag über die chemischen Vorginge während der Keinung einiger Samen unter Bezugnahme auf seine im pharmacentisch-chemischen Laboratorium angestellten Versuche, von denen er einige wälnend seines Vortrages wiederholte. Herr (Hherlehrer Vogel legte newere Fachlitteratur vor und demonstrierte ein Exemplar von Papacer bracteatum, bei dem bekanntlich oft nahezu simtliche Staubblitter in Pistille umgewandelt werden. Die oberwärts in Fruchtknoten verwandelten Filamente hängen an der Basis zusammen. Auch demonstrierte dor Vortragende eine Monstrositiit von Cyclaminus Coum mit beblätterten Blütenstielen und teilweise verbildeten Blüten. Herr Apotheker Perwo hielt einen Vortrag über die Flora der Nordsecinseln, die er gelegentlich eines Ausfluges kennen gelernt hatte. Inshesondere schilderte der Vortragende die Pflanzenwelt des Watts und der Geestwiesen und legte verschiedene Formen von Salicornia hrbacen vor, die er nicht selten durchemander wachsend angetruffen hat; ferner Cbione fortulacoides meist in halistrauchigen Exemplaren an den Gräben in Watt. Einen grossen Schmuck gewahrt jenem Gebiet die stattliche, oft in grosser Menge vorkommende Statice Limonium, die ganze Strecken durch ihre ruten Blüten kennzeichnet. Glyceria maritima findet sich in ungeheurer Nenge auf dem Watt und bildet auf manchen Halligen den hauptsächlichsten Graswuchs. Auch Glau.r maritima und Armeria vulgaris, letztere in einigen Formen, sind echte Wattpflanzen. Die wilde Sellerie, Apium graceolens, findet sich besonders in Junceten der Halligen und ist ebentalls nur auf dem Watt zu bemerken. Plantayo maritima wächst dort in schmal- und breitblättrigen, zwerg- und riesenhaften Exemplaren mit teils ganzrandigen, teils vereinzelt gezähnten Blattrandern. Während diese Wegerichart bezüglich des standorts weniger wählerisch ist, komnte Pl. Coronopus stets nur aut niedrigen Ameisenhügreln beobachtet werden. Atriplex litoralis, Artemixia maritima und Epipactis palustris kommen nicht selten vor, desgleichen Pirola minor und $P$. rotundifolia, nebst ihren nur von den ostfriesischen Inseln bekannten Abänderungen. Narthecium ossifragum wurde nur einmal bemerkt auf einer Geestwiese. In der nördlichien Vogelkoje aut der nordfriesischen Insel fand der Vortragende, wahrscheinlich durch Tögel dorthin verschleppt, vor: Polygonatum multiflorum, Osmunla regulis und Lonicera Caprifolium. Neu für Amrum ist Centanrea Jacea und für Führ Sparyanium neglechm, letzteres in einigen Grïben entdeckt. Zum Schluss legte Dr. A b romeit das neuerdings bei Borntraeger in Berlin erschienene forstbotanische Merkhuch von Herm Prof. Dr. Conwentz vor. Das in handlichem Taschenbuchformat erschienene Werk wird auf Veranlassung des Hinisters für 
Landwirtschaft, Domainen und Forsten herausgegeben und heriicksichtigt die in Westureussen beobachteten selteneren oder benerkenswertesten Holzuflanzen, von denen eine Anzahl wohlgelungener charakteristischer Abbihlungen dem Text heigegehen werden. I)er Wert dieser erwünschten Zusammenstellung wird dadurch erhöht und wird hoffentlich zu weiteren Nachforschungen in dieser Richtung auch in weiteren Kreisen anregen.

Abromeit

Goldschmidt, M., Pflanzenverkauf. M. Goldschmidt in Geis a in. Thüringen bietet gut aufgelegte Herbarpflanzen aus spanien, Algier, Frankreich, Korsika und Sardinien zum Preise von $12 \%$ Pfg. pro Nummer an. Liste steht z.u Diensten. Die Pflanzen sind von bewährten Kennern bestimmt. Da die Limmmern meist nur e inmal vorhanden sind, wird um gef. baldige Tachfrage und event. Bestellung gebeten.

Herbarversteigerung. Am 23. Mïrz wirl in Planegg bei München ein sehr unfanereiches Herbar, welches l'flanzen aus allen Welteilen enthät, versteigert. (Näheres siehe auf dem Umschlag dieser Nummer.)

Sintenis, Paul, Botan. Reise nach Turan u. Persien. Wie aus dem Anzeigeteil der Nr. 2 dieser Zeitschrift zu ersehen ist, gedenkt Herr Paul sintenis aus Kupterberg in Schlesien eine grüssere Sammelreise in die oben genannten Gehiete zu untermehmen und ist bereits Ende Februar nach Odessa abgereist. Von hier aus wird er die Reise über Batum, 'Tiflis, Baku nach Krasnowodsk jenseits des kaspischen Meeres fortsetzen, die transkaspische Bahn bis Aschabad henïzen und von dort aus nach dem persischen Grenzgehirge gehen. Die Bestimmung der Ausbeute wird Herr Baurat J. Freyn in Schmichow-Prag gütigst ühernehmen, welcher auch bestellungen und Anzahlungen entgegennimmt. Preis pro Centurie 40 Mark, bei $50 \%$ Anzahlung 32 Mark.

\section{Personalnachrichten.}

Ernennungen etc.: Prof. F. Cacara in Vallombrosa w. a.o. Prof. d. Bot. und Direktor d. bot. Gartens in Cagliari. - Prof. A. N. Berlese vom R. Liceo Minghetti in Bolognat w. a.o. Prof. d. Bot. u. Direktor d. bot. Instituts in sassari. Prof. Dr. O. Il at tirolo, Ordinarius der Botanik am R. Instituto di studi super. in Florenz, w. o. Prof. d. Bot. an der Universität Turin. - Benjamin Lincoln Robinson w. L'rof. der Bot. an der Harward Lniversity. - Dr. Küster in Munchen hat sich in Halle a. S. habilitiert. - L'rof. Kruis, Locent der Gührungschemie an d. techn. Hochschule in Prag, w. a.o Professor. - W. L. L e p son w. Prof. der Bot. an d. Lniv, of California. - H. H. H u me w. Assist d. Bot. an Florida Agricult. College zu Lake City. - Dr. Ambronn in Leipzig w. a.o. Prof. a. d. Univ. Jena. Dr. Boris Fedtschenkow. Conservator am bot. Garten in St. Petersburg. Dr. R. II a gner, Assistent am hot. Garten der techn. Hochschule in Karlsruhe, w. Assist. am bot. Garten u. bot. Institut der Univ. Wien.

Todesfälle: Dr. Ad. Franchet, einer der besten Kenner der ostasiatischen Flora und bedeutender Caricologe, im Alter v. 66 Jahren. - Dr. Ottmar Hof mann, kgl. Regierungs- und Kreismedizinalrat in Regensburg, langjäh. Direkt., bezw. Torstand der kgl. bot. Gesellsch. und des naturwissensch. Tereins in Regensburg. - bernard A lt um, langjähr. Lehrer d. Zoologie u. d. Forstakademie Eberswalde, Prof. u. Geh. Regierungsrat. - Grant Allen am 25. Okt. 1899. - A u g. Crié in Mans, $81 \mathrm{~J}$. alt. - Arthur Pellerin, Direktor d. bot. Gartens in Nantes. - Prof. J. B. Carnoy am 6. Sept. in Schuls in Engadin. - Dr. A. Ernst, Direktor des Nationalmuseums in Caracas in Vellezuela. - Pasquale Freda, Direktor der landwirtschaftl. Versuchsstation in Rom, am 4. Juli. - Charles $\mathrm{H}$ owie, Bryologe in St. Andrews in Scotland. - Dr. Walt. J. Hoffmann in Reading, Pa., am 8. Nov., 53 J. alt. - IV alt he r Goetze, bot. Forschungsreisender, von dem bot. Nuseum in Berlin nach dem Nyassasee entsandt, ist aul dieser Expedition am 9. Dez. 1£99 zu Langeuburg (Deutsch-Ustafrika) dem Schwarzwasserfieber erlegen.

Druck von J.J. Reiff in Karlsruhe. 


\section{Allgemeine}

\section{Botanische Zeitschrift}

für Systematik, Floristik, Pflanzengeographie etc.

Referierendes Organ

des bot. Vereins der Provinz Brandenburg, der kgl. bot. Gesellschaft zu Regenshurg. des Preuss. bot. Vereins in Königsber@.

tnd Organ der Botan. Vereinigung in Würzburg und des Berliner bot. Tauschvereins.

Unter Mitwirkung hervorragender Fachmänner herausgegcben

von A. Hácucicer. Werderplatz 48 in Karlsiulne.

Verlag von J. J. Hein in Karlspuhe.

\begin{tabular}{|c|c|c|}
\hline 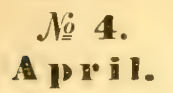 & $\begin{array}{l}\text { - Erseheint am } 15 . \text { jeden Monats. - } \\
\text { Preis: vierteljälnl. } 1.50 \mathrm{Mk} \text {. bei freier Zusentume. }\end{array}$ & $\begin{array}{l}\text { 1 DABP. } \\
\text { VI.Jaligang. }\end{array}$ \\
\hline
\end{tabular}

Inhalt

Originalarbeiten: W. Schmidle, Algologische Notizen (Forts.). - L. GrossNürnberg, Ist Draba Thomasii Koch eine gute Art? - F. Buser, Ueber Alchimilla pubescens Koch, A. truncata Rchb. und eine neue verwandte Art aus den l'iroler Alpen (Schluss). - P. Magnus, Bemerkungen zum Berichte über die Sitzung des Bot. Ver: f. d. Prov. Brandenburg vom 9. Febr. 1900. - A. Kneucker, Bemerkungen zu den "Cyperaceae (exclus. Carice;) et Juncaceae exsiccatae

Bot. Litteratur, Zeitsehriften ete. : 1r. H. Poeverlein u. J. Al ur r, Gracmann, Dr. Robert, Das Pflanzenleben der schwïb. Alb etc. (Ref.). - A. K neucker, Halácsy, Dr. E v., Conspectus florae Graecae (Ref.). - Derselbe, Fritsch, Dr. Karl, Schulflora (Ref.) - H. v. Trautsehold, Fedtschenko, O. u. B., Ranunculaceen etc. (Ref.). - Derselbe, Fedtschenko, B., Die im europ. Russland etc. vorkomm. Arten v. Hedysarum (Ref.). - A. Kneucker, Phanerogamae et Pteridophytae Japon. (Ref.). Derselbe, Eine Flora des Schwarzwaldes. - J. Sturms Flora v. Peutschland A p pel u. Loew, Blätenbiolog. Beohachtungen. - Inhaltsangabe verschicd. bot. Zeitschr.

Bot. Anstalten, Vereine, Tausehvereine, Exsiceatenwerke, Reisen etc.: Preuss. bot. Ver. (Ref). - Bot. Verein f. d. Prov. Brandenburg (Ref.). - Pflanzengeogr. Durchforschung Badens. - 72. Versamml, deutscher Naturforscher u. Aerzte. - Internationales Institut in Amazonas. - Internationaler bot. Kongress in Paris. - A rnold. F., Lichenes exsiccati. - D e rs elbe, Lichenes Monacenses. - Kr i g e r, Fumgi saxonici. - Herbarium Böckeler. - Delectus plantarum exsicc..... univ. Jurjevensis. Reineck u. Czermack, Plantae Brasiliae merid.

Personalnachrichten.

Algologische $\mathbb{N}$ ot ize n.

XIV.

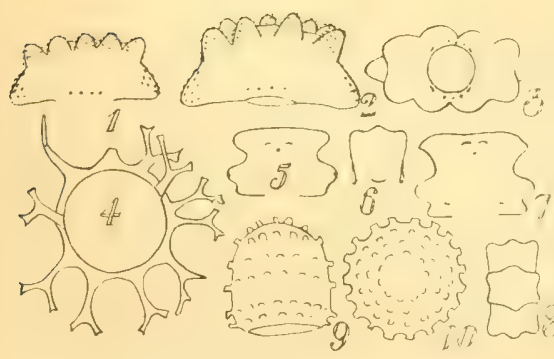

13. Marstigocludus flargelliforme Schmidle m. sp. Die Alge bildet schwarzgrüne, feine, sehr kleine Geflechte. Die kriechenden Fäden sind bis $10 \mu$ dick, tortulö:, mit feiner, hyaliner, selten etwas gelblicher Zellhaut und homogenem, blaugrünem Zellinhalt, elliptischen oder runden Zellen, welche so lang als breit, oder etwas kürzer oder

"Leider wurde aus Versehen das vorstehende l'extbild mit den 10 Figuren his zur Nr. 4 zurückgestellt. Dasselbe bezieht sich auf die Diagmosen N1. 7 u. 8 auf 1).84 in Nr. 3, und auf die Diagnose Nr. 6 auf p. 18 Nr. 2." 
lïnger sind, und meist im Faden einfach, selten zu zweien nebeneinander stehen. I)ie Zweige (aufsteigende Fäden?) stehen einseitig, sind meist kur\%, selten 300-500 / lang. Am Grunde ca. (j y dick und a us einigen torulösen Zellen bestehend, verschmälern sie sich nach a f färts sehr rasch und beträchtlich, die Yellen sind damn rechteckig, bei langen Fïden auf weite Strecken ca. 4 " breit, und am Ende allmählig a uf 2 u verschmälert, die 7elllänge nimmt jedoch kaum zu, und der Yellinhalt ist zuletzt ziemlich farblos. Nicht selten verdicken sich diese Fäden nach aufwärts wieder bis zur Breite der grundständigen Fäden, werden torulös, um sich wieder zu verschmälern. Sie tragen sehr selten lange, etwas elliptische Grenzzellen.

Auf feuchten Felsen zw. Neral u. Matheran; 18.X.95.

Die Alge geht in einen Anabaenazustand über, und zerfällt, wie Nostochopsis lobatus in einzellige Coccen.

1t. Stigomema Imatra Schmidle $\boldsymbol{n}$. sper. Die Pflanze bildet krause, 1-2 $\mathrm{mm}$ dicke, sehr lockere Räschen zwischen Lebermoosen vou gelblichblauer bis weissgelber Farhe. In unteren Teile bestehen sie aus farblosen, verklebenden, leeren, aufsteigenden scheiden, welche oben sich mit Inhalt füllen. Meist werden dort die Scheiden doppelt und hestehen aus einer äusseren hyalinen, schleimigen und einer festen, braungelben, gelllichon, oder dunkelbrannen, diumen, anliegenden, inneren Schicht. Das 'Trichom ist gellolich oder gellowaun, mit rechteckigen, isodiametrischen oder etwas längeren, oder kürzeren Zellen mit grobkörnigem Inhalt und kaum sichtbaren Scheidewänden. Nicht selten ist es aber in den unteren Teilen stark torulös und dann meist reichlich echt verzweigt. Die Zweige gehen beiderseits ab, verdicken sich gegen die Spitze zu und gleichen den Fäden mit dem cylindrischen Trichome. Sie enthalten ziemlich hïufig rechteckige oder runde Grenzzellen.

An den oberen Enden schnïren sich nun succesive die obersten 4-18, etwas torulös gewordenen Zellen mitsamt d er Scheide ab und bilden wenigzellige, bescheidete Pseudohormogonien. Dieselben wa chsen beiderseits a us, erhalten eine mediane Grenzzelle, und dadurch, dass sie sich beiderseits nach a uf wäts krümmen, kommt diese Grenzzelle in den tiefsten Teil des Biischels zu liegen. Rings um dieselbe werden die Zellen torulös, verzweigen sich, und dadurch, dass damn diese Zellen zu Grunde gehen, kommen die anfangs geschilderten aufsteigenden Fäden zustande. Mit diesen kurzen, sprossenden, gekrümmten lïaden sind die Büschel oben dicht bedeckt.

Im Palmenwalde bei Mahim bei Bombay; 10.XI.95.

15. Mastigocladus Hamsgingi schmialle n. s1). Die Alge bildet papierdiune, fast einschichtige, schön hlaugriune Teberziige. Die Fäden sind unregumassig verflochten und gekrummt oft auch fast parallel), cylindrisch, mit seltenen lïnglich-ovalen oder rechteckigen, in der Länge sehr variabelen, hyalinen Grenzzellen, die meist breiter als das Trichom sind. Die F i den sind $8 \mu$ di $\mathrm{ck}$, verschmälern sich jedoch allmählich bis zu $3 / \mu$, sie sind meist zweiglos, sehr selten scytonenaatig verzweigt, mit sehr kuizen, scheidelosen Zweigen. Die Scheiden sind hyalin, verschleimend, anliegend und Phormidinm-artig ein 
schleimiges Lager bildend. Die Triclome sind hlangriun, meist $6-8$ p b reit, die Zellen isodiametrisch oder bis um die Hälfte länger oder kürzer, mit etwas granuliertem Inhalt, in alten Fïiden schwach torulïs, in jüngeren (oder am Ende) rein eylindrisch. An wanz alt('n Fïiden werden sie gelbbraun, runden sich innerhalb der thellhaut ab, bei einer Breite von 8-10 u umgeben sich mit einer nenen \%ellhaut und bilden Conidien. Da dieses an allen Zellen zugleich stattfindet, so erhält dann die Alge ein Anabaena-artiges Aussehen.

In Sïmpfen bei Matonga nächst Bombay; 20.XI.95, und bei Parel; 10.XI.95.

Obwohl ich keine echte Verzweigung noch gesehen habe, zeigt der ganze Habitus, dass ein Mustigocludus vorliegt. Anch bei $1 \%$. lumimosus Coln sucht man oft vergebens nach solchen.

(Forts. f.)

\section{Ist Draba Thomasii Koch eine gute Art?}

Von L. Grose-Nürnberg.

Wer die in den Koch'schen Floren gegebenen Diagnosen von Drubu incana L. und Draba Thomasii Koch mit einiger Aufmerksamkeit prüft, bemerkt eine Unsicherheit des Trteils, welche bei dem grossen Erlanger Systematiker geradezu befremdlich erscheint. Und wer gar einmal beide "Arten“ in grösserer Menge sammelte und zu Hause eine reinliche Scheidung derselben versuchte, dürfte dabei leicht in die Brïche geraten sein. Mir wenigstens ging es so und zwar mit Exemplaren vom vielbesuchten Schlern bei Bozen, bis sich mir nach und nach die Teberzeugung aufdrängte, dass Drubu. Thomusii Korh, wenigstens als Art, fallen zu lassen ist. Ich wïrde kaum wagen, diese Teberzeugung hier auszusprechen, wenn ich nicht einen ausgezeichneten Gewährsmann anführen kömnte, der weiter unten das Wort haben soll, nämlich Frhr'v. Hausmann, den Verfasser der Flora von Tirol.

Die Koch'schen Werke, auf welche ich mich in der Folge beziche, sind dessen Synopsis der Deutschen und Schweizer Flora, 1. Aufl, Frankfurt a. M. 1838, dessen Taschenbuch der Deutschen und Schweizer Flora, 1. Aufl, Leipzig 1844 und dessen Synopsis, 2. Aufl., Leipzig 1816. Sie seien kurz mit $\mathrm{S}_{1}, \mathrm{~T}$ und $\mathrm{S}_{2}$ bezeichnet.

In $S_{1}$ ist Dirabe Thomasii Koch noch gar nicht anfgeführt, sonder'n dafür Irabe confiese Ehrleart, angeblich infolge einer schon von Hopper

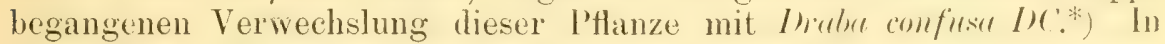

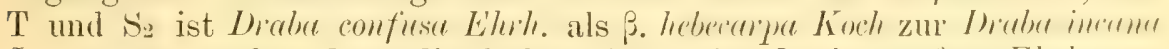
$L$. gezogen, während an die frühere Stelle der Drobu confusa Ehilh. nun Draba Thomasii Koch getreten ist. Die Diagnose der letzteren lantet

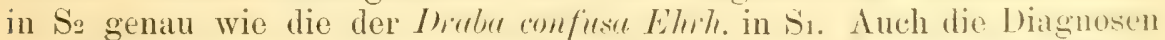

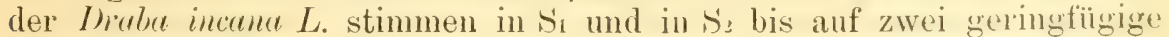
Unterschiede ïberein. Um so auffallender sind die Aendermang beider Diagnosen in T. 'Zur bequemeren Vergleichung stellen wir die Koch'schen Texte in $S_{1}$ und $S_{2}$ einerseits und in $T$ andererseits einander

*) Vergl. darïber Koch, Synopsis 2. Aufl. unter Diabo Thomasii SchlechtendalHallier, Flora von Deutschland, Jubiläumsausgabe, Band 14, S. D.t und J. Sturm, Deutiehlands Flora in Abbildungen nach der Natur, Heft 60, l'afel 16. 


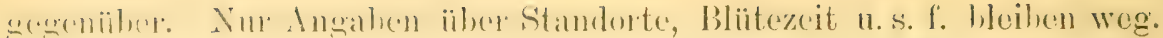
Was koun markierte, ist auch hier durch gesperten Druck gekemnzeichnet.

\section{$\mathrm{S}_{1}$ und $\mathrm{S}_{2}$}

Draba incana L. - St. vielblittrig: B. ganzrandig oder gezähnt, die wurzelst. rosettig.*) lanzettl., die oberen stenyelst. eifömig: Blb. mehr als noch e inmal so lang, als d. K.; Schötch. länger als d. Stielch., schief gedrehet, kahil oder flaumig. - Dauert wegen vorhandener micht bliihender Wurzelrosetten oft mehrere Jahre. Schötchen meist kihl. ***)

Draba confusa $E h r$. in $\mathrm{S}_{1}$.

Draba Thomasit Koch in S2.

St. vielblattrig; B. ganzrandig orler gezihnt, die wurzelst. rosettig, lanzettl., die oberen stengelst. ]äingl.-lanzettl.; B Bl). anderthalbmal so lang als d. K. schötch. länger als d. Bthstielch., flach, flaumh. - St. oft von der Wurzel an ästig; nichtblühende IV urzelrosetten fehlend, Ilaum kïrzer, Bth. um die Hälfte kleiner, als bei der vorhergehenden.
T

Draba incana L. - St. vielblattrig, an der Spitze ästig od. einfach; W. nichtblühende Rosetten trag.; 13. ganzrandig od. gezähnt, die wurzelst. dichtrosettig, lanzettl., die oberen stengelst. eif.; Schötchen länger als das Bthstielchen, kahl oder flaumig.

\section{Draba Thomasii Koch.}

St. vielblättrig, v. der Basis an istig od. einfacb; nichtblüliende Rosetten fehlend; B. ganzrandig od. gezähnt, die wurzelst. rosettig, lanzettl., die oberen stengelst. längl.-lanzettl.; Schötchen länger als das Bthstielchen, Haumh. Der Uf. zuweilen ziemlich lang, zuweilen fast fehlend.

*) In is dic t-rosettig.

*) Ist in $\mathrm{S}_{2}$ soestrichen.

Wir sohen, lass in T nerkwiirdiger. Weise gerade jene Diagnosenbestandteile fehlen, welche in $S_{1}$ und in $S_{\text {s }}$ besonders stark hervortreten, während in $\mathrm{S}_{2}$ fehlt, was in ' $\mathrm{I}$ wichtig war. Was mag aber

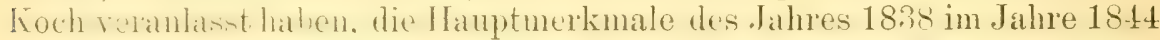
zal streichen, $n m$ sie 1816 wieder aufzunchmen, während er andererseits

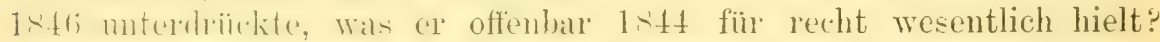
Jin cinfincher lensehen hei den lenderungen in $\mathrm{T}$ ist ausgeschlossen, weil somst schmolich koresumdierende Liir licu in beiden Diagnosen

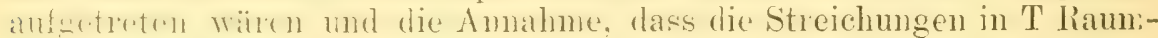

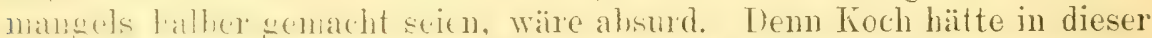

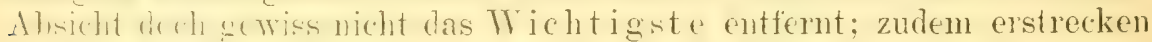
sich firizmenen an keiner anderen stelle des Taschentuchs auch auf dic l)julfonce. Koch hatte also offenhat $184 t$ iiber unsele l'flanzen eine andere Ansicht als 1838**) Wer begreift aber, weshalb er $1846 \mathrm{zum}$ zweiten Mal sein Urteil änderte? Oder hat er es gar nicht zum zweiten Male geändert? Hat er sich etwa bei der Niederschrift von Si auf die Angaben anderer Autoren verlassen, 6 Jahre später mit

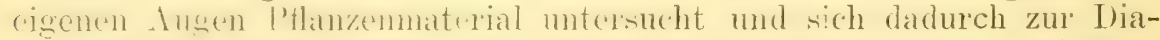

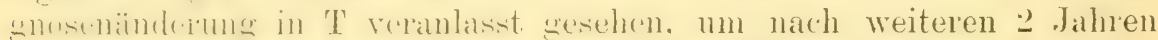

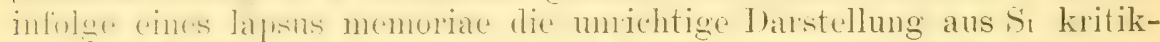

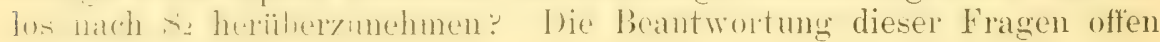
lascend, wollen wil nun einmal sehen, wie die Koch'schen Angaben,

*) L's wurde hier wie weiter oben davon abgesehen, dass diese Ansichtsverschielungun, genau betrachtet, schon etwas früher eintraten, 
die in $\mathrm{S}$ sowohl, wie jene in $\mathrm{T}$ mit den thatsächlichen Verhältnissen iibereinstimmen. Zunächst lïsst sich da eine Verschicdenheit in der Grösse der Blüten, bezw. im Grössenverhältnis der Kron- und Kelchblätter an meinen Schlernpflanzen nicht erkemnen und Exemplare, welche ich von der Forka im Contrin (leg. Rodler) besitze und anderwärts zu besichtigen Gelegenheit hatte, unterscheiden sich auch in dieser Hinsicht nicht von den Schlernpflanzen. Auch trifft nicht immer zu, dass del Draba Thomasii Koch die nichtblühenden Blattrosetten fehlen. Diesen P'unkt stellte schon Hausmann richtig, denn er schreibt:*) „Einzehne nicht blühende Blattrosetten beobachtete ich am Schlern, wiewohl selten auch an dieser Art." Umgekehrt dürfte Draba incena L. auch o hn e Blattrosetten vorkommen; denn mein Freund Gugler-Nördlingen teilt mir brieflich mit, Herr Hoock-Nördlingen besitze vom Schlern sicher ein solches Individuum. Während eines achttägigen Aufenthalts auf dem wiederholt erwähnten Bergplateau im Juli 1899 schenkte ich diesem Umstand leider keine Aufmerksamkeit. Was ferner die stïkere graue Behaarung der Draba incama $L$. betrifft, so hält sich anch diese nicht konstant. Ich selbst besitze ein sonst typisches incana-Exemplar, welches völlig gr ü $n$ ist, und in der Jubiläums-Ausgabe der Flora von Deutschland von Schlechtendal-Hallier lese ich:**) "Auch dicser Name ist nicht passend gewiihlt, denn die Behaarung ist unbeständig." Machen wir nun den Versuch, mit Hilfe des Gegensatzes "Schötchen schief gedreht" — "Schötchen flach" Ordnung zu schaffen, so werden wir gar bald aufs Neue daran erinnert, dass manche (ruciferen Freunde der Ahwechslung: sind. Nicht besser gehts mit der Verästelung; denn neben unverästelten Individuen finden sich hei beiden "Arten" häutig solche, bei welchen der Stengel schon von Grund an ästig ist; die unteren Aeste steigen dann gewöhnlich bogig auf, wodurch ein Habitus entsteht, welcher demjenigen vielstengeliger stücke gleicht, die ebenfalls nicht selten sind.

(Schluss folgt.)

\section{Ueber Alchimilla pubescens Koch, A. truncata Rchb. und eine neue verwandte Art aus den Tiroler Alpen (A. acutata).

\author{
Von R. Buser.
}

(Schluss.)

Llchimille acutata Bus. sp. nov.

Syn. A. pubescens Koch Syn. ed. 2 (18t3) p. 256 p. p. (pl. Traunsteinerianu) non W. nec Lam.

A. truncata Rehb. Fl. germ, exs. no. 1476 (1838) p. p. (e. g. in Herb.Boiss.) non 'Tausch.

Untere Wurzelhaitter rundlich-nierenfömig mit schmilem Anschnit über Stiel, obere rundlich mit über Stiel sich berïhrenten Endlaynen, - - his mowilkommen 9-lappig. Latppen bogig gerundet bis bogig truncat, an den Lirstlingsb)iittern seicht, ca. '/4 Radius und seitlich alle, wenn auch mit schmalen sivalt, ron einander abgesetzt; an den Hochsommerblittern tiefer gehend, l, in 2':5 Radius und truncat und hier nur die äussern, dem Stiel nächsten, seitlich mit \pm keil-

\footnotetext{
*) v. Hausmann, Flora von Tirol, 1. Bändchen, pag: 77.
}

**) Band 14, pag. 36, 2. Fussnote. 
förmigem Einschnitt abgesetzt, die medianen Lappen mit stark gerundetem Eckenzahn seitlich über einander reitend und in sicco kleine Falte bildend. Zähne jederseits 4-5, ziemlich klein, schief eiförmig, vorgestreckt, an den unteren Blättern stumpflich, an den oberen länger, pinselig zugespitzt. Erwachsene Blätter derblich, mit Ausnahme der geringen Lappenfalten plan, die Falten der Knospenlage als Radiallinien erkennen lassend, oben blaugrün, unten trübgrün, beider. seits (bald gleichmässig, bald in den Falten stärker) die unteren Blätter von relativ kurzen und mehr abstehenden Haaren, die späteren Blätter von doppelt längeren, derberen und (ausser auf den Nerven der Unterseite) mehr anliegenden Haaren locker anliegend-zottig, die letzten Hochsommerblitter stark zottig und oft schon fast seidig glänzend. Stengel (und Stiele) gerade, oft schnurgerade, steif aufrecht, sehr schlank, abstehend zottig, in der Sonne braunrot sich fürbend. Oberste Stengelblätter tief eingeschnitten, mit spreizenden, spitzzähnigen Ab. schnitten. Blüten relativ gross, grünlichgelb, in pseudodoldigen Büschelchen und diese corymbös genähert. Kelchröhren (halbreif) schmal kreiselförmig, an Basis sich verschmälernd, samt den gleichlangen oder um die Hälfte längeren, straff aufgerichteten Stielchen dichtzottig. Sepala verlängert eiförmig-dreieckig, seitlich ziemlich gerade begrenzt, aussen wenighaarig, von nach der Spitze pinselig zusammenneigenden Haaren zugespitzt erscheinend, innen an Spitze mit grossem Schliesshaarbüschelchen. Aussenkelch gross entwickelt, $2 / 3-3 / 4-$ oft gleichlang mit Kelch, mit schmal lanzetten bis sublinealen, spitzlichen, wie die Sepala steif schief-aufgerichteten Blättchen.

Blätter $20-36 \times 17-37 \mathrm{~cm}$. Stengel $7-16 \mathrm{~cm}$. Blüten $3-4 \mathrm{~mm} \mathrm{lang}$, $31 / 2-5$ breit. Kelchröhren $11 / 4-1 \%$, Sepala $11 / 2-2$, Blütenstiele $1 \frac{1}{2}-3 \mathrm{~mm}$.

Hab. Nordtirol: Amtmoos und Rafan am Sonnwendjoch (Woynar, Juli 1885). Kitzbüheler Álpen: am kleinen Rüthenstein $7000^{\prime}$ (Traunsteiner, 1837 bis 1840). - Centraltirol: Luttach bei Sand, „im Bach * 10-1500 m, am Weitfeld $21-2300 \mathrm{~m}$ (G. Treffer, 1898/99). - Ueherall in Gesellschaft von A. flabellata Bus.

In den allgemeinen Grössendimensionen, in der Tracht stimmen A. acutata und fabellatu völlig miteinander überein: der gleiche steifaufrechte, gracile Wuchs, die relativ kleinen Blätter mit lange sichtbar bleibenden Faltenlinien. Die besten Unterschiede liefern die Lappen und Zähne der Blätter, sowie die Form der Blüten, resp. Früchte. Bei Hubellata sind die Lappen der mehr nierenförmigen Blätter kürzer, gestutzt, oft vorne fast geradlinig begrenzt, seitlich ganzrandig oder selbst wenn, wie dies an den oberen Blättern oft der Fall ist, die Lappen mehr zugerundet sind und die Zähne seitlich in die Buchten hinabzugleiten scheinen, sind doch immer alle Lappen von einander spreizend abgesetzt und die kurzen Zähne eiförmig, zehenförmig, stumpf. Bei acutata dagegen decken sich wenigstens an den (runden) Hochsommerblättern die mittleren Blattlappen seitlich und maskieren den bogig gekrümmten Einschnitt, die Zähne sind grösser, spitz verlingert. Bei fabellata sind die Kelchzipfel breit herz-eiförmig, abgerundet, wellig-offen, die Kelchröhren kurz kreiselförmig bis fast halbkugelig. Bei arutata dagegen, deren grosse Kelchentwickelung fast an die Calicinx erinnert, sind Kelchröhren und -Zipfel verlängert, verschmälert, steif schief aufgerichtet. Vor allen Terwandten zeichnet sich flabellata durch bleiche, seegrüne Blätter (heller fast noch als Sibbalifi jrocumbens) und + citrongelbe Blüten aus. Soweit sich Coloritverhältnisse an trockenem Material beurteilen lassen, ist das Laub der acututa dunkler (aber rein) blaugrün, die Blüten grünlicher. An gut entwickelten Exemplaren der flabellata sind die knäueligen Teilinflorescenzen gut von einander entfernt und pseudospicat über einander gereiht, ähnlich wie dies unter den Alpinen bei A. saxatilis Bus. der Fall ist, während durch die Streckung der successiven Axen acutatı (wie colorata!) einen mehr corymbösen Gesamtblütenstand gewinnt. Bei flabellata sind Blattstiele und Stengel dichter zottig, besonders im Blütenstand, obwohl das einzelne Haar kürzer und dünner ist, die 
Kelchröhren (besonders unterhalb der Sepala) meist schwächer zottig, die Blütenstiele kürzer und die Blütenknäuel so kompakter, die Stengelhochblätter weniger tief eingeschnitten, dafür deren Stipelscheiden entwickelter.

Von A. colorata Bus., der die acutata in allen Stücken sehr nahe kommt, so nahe, dass man sie ihr als Subspecies unterordnen könnte und die sie nur in der grossen Entwickelung der Kelche übertrifft, unterscheidet sich unsere Art schon schwieriger. Bei der derberen coloruta sind die Blätter stark wellig, ohne bleibende Faltenlängslinien, gerundeter, mit stärker abstehendem Indument, die Stengel typisch bogig-aufsteigend, die Blütenstiele bloss dümnbehaart bis kahl, deren untere in Frucht schon deutlich herabgebogen, die Kelch. röhren und -Zipfel breiter, kürzer, letztere wellig offen und liegt über der ganzen Pflanze ein trüber, grauer Farbenton. Bei colorata sind im Fruchtstadium die Kelche, der Discus, kurz die ganze Blüteninnenseite dunkelbraunpurpurn gefärbt; ob dies auch bei arutata der Fall ist, kann ich nicht sagen, da ich das entsprechende Stadium noch nicht gesehen, doch ist es wahrscheinlich, nur dürfte die Färbung eine hellere und reinere sein.

\title{
Bemerkungen zum Berichte über die Sitzung des Botanischen Vereins für die Provinz Brandenburg vom 9. Februar 1900.
}

\author{
Von P. Magnus.
}

In diesem Jahrg. dieser Zeitschrift steht S. 48 in dem in der Ueberschrift genamnten Berichte: Dr. Weisse.... wies die inzwischen erfolgte andere Deutung seitens des Prof. Magnus, die klar erkennen lasse, dass derselbe das corpus delicti weder gesehen, noch untersucht habe, ziemlich energisch zurück!

Sollte Herr Dr. Weisse, der recht wohl wusste, dass ich seine Apfelsine nicht gesehen hatte, da ich sie nicht von ihm verlangt hatte, wirklich dieses als Grumd gegen meine Ausführung mit angeführt haben, so stellt er sich und seiner Arbeit damit ein seltenes A rmutszeugn is aus, worauf ich hiermit express hinweisen möchte. Denn er sagt damit direkt, dass man aus seiner Beschreibung und seinen Abbildungen nicht iiber das Objekt urteilen kann, d.h. dass seine Beschreibung und Abbildungen keinen wissenschaftlichen Wert haben, und ohne Ansicht des von ihm beschriebenen Objektes nicht wissenschaftlich verwertet werden können. Etwas anderes vermag ich aus diesem Vorwurfe nicht herauszulesen.

Es ist genau dasselbe, als wenn Jemand eine neue Art beschreibt und ein Anderer nach der Beschreibung ein Urteil über dieselbe abgiebt, ohne das Exemplar gesehen zu haben. Mir ist auch schon passiert, dass man mir daraus einen Vorwurf machen wollte. Ich konnte dam nur erwidern, dass der die Art beschreibende Autor selbst anerkennt, dass man nach seiner Beschreibung seine Art nicht bestimmen und erkennen kann, was oft zutreffen mag. Ich habe schon an anderem Orte angeführt, dass man die mit unkenntlichen oder falschen Beschreibungen veröffentlichten Arten nicht anerkennen sollte.

Ueber die sachliche Differenz mit Herrn Dr. Weisse werde ich das Erscheinen seiner Replik abwarten und mich dann über dieselbe äussern oder auch nicht äussern. Jetzt muss ich nach seiner Beschreibung und seinen Abbildungen das unter der Oeffnung liegende achtteilige, sternfürmige Gebilde noch für einen inneren Carpellkreis erklären, wie ich das in den Abhandlungen des Botanischen Vereins der Provinz Brandenburg, Bd. XLI (1899) S. 166-168 des Näheren ausgeführt habe. 


\title{
Bemerkungen zu den „Cyperaceae (exclus. Carices) et Juncaceae exsiccatae"**)
}

\author{
von A. Kneucker.
}

I. Lieferung 1900.

Nr. 1. Chlorocyjerus globosus Pulle = (yperus ylobosus All. Auctor. ad fl. Ped., p. 49 (1789).

In Sümpfen am Flusse Roja bei Ventimiglia in Ligurien (Oberitalien); Alluvinm. Begleitpflanzen: Chlorocyperus serotinus Pall., flavescens Rikli Juncus lampocarpus Ehrh. ete.

Ca. 5-10 m ü. d. M.; Sept. 1899.

leg. Luigi Pollini.

Die Gattung Chlorocyperus ist von M. Rikli in seiner Arbeit, „Beiträge zur vergleichenden Anatomie der Cyperaceen, mit besonderer Berïcksichtigung der immeren Parenchymscheide", Jahrb. f. wiss. Botanik, Bd. 27, 1595, S. 485-580) aufgestellt und hegrïndet worden. Sie umfasst alle jene Cyperaceen der Sammelgattung. "Cy p erus", bei denen das Assimilationsgewebe die grönen Zellen des Blattes und des Stengels) in (querschnitte kranzförmig in einer einzigen Zellschicht um die Ciefisshiundel hexum angeordnet erscheint; un die kleinen Gefïshündel von kreistumler Gestalt verläuft der Kranz meist vollkommen geschlossen, an den grösseren, die eine ovale oder elliptische Gestalt anfweisen, erscheint er wenigstens an der (trenze zwischen sieb. und Holzteil unterhochen, im Stengel an den von der Peripherie weiter weg entfernten Bündeln nur am Siebteil angedentet oder ganzlich fehlend. Ausser diesem ausseren Kranz von Assimilationszellen findet sich innerhalh des Gefiisshündels selbst, von der trehutzscheide umgehen, ein immerer einschichtiger Kranz von grünen Zellen, welcher bezüglich seiner vollstandigen oder unvollstandigen Ausbihlung meist genau dem äusceren entspricht. Die übrigen "Cyperus"-Arten, welche die eben angedenteten Terhältuisse nicht zeigen, fasst Rikli in der Gattung E u c y perus zusammen.

Mit Chlorocyperus stimmen in den für die Gattung charakteristischen anatonischen Merkmalen von den C'yperaceen, bei denen die Traghläter gleichfalls nach der $1 / 2$ Stellung angeordnet sind, noch folgende Gattungen ïherein: Kyllingia liotth., Dichostylis Beauv. [hei der einhemischen D. Micheliana(L.) Nees Traghlatter schrauhig gestellt\} und Gialilea Parl. Ton diesen unterscheiden sich von ('hlorocylerus Kyllingia durch die einblïtigen Aehrchen, Dichostylis durch das Vorhandensein von (in der Regel) nur 1 Staubgefäss und durch die an der meist gramnenartigen Suitze mehr oder weniger zurückgokrümmten Tragblätter, Cxalilea durch die rerophile Ausbiling des ritengel- und Blatterewebes. Hiezu ist aber ausdrücklich zu hemerken, dass erst kïnftige Cntersuchungen uns ïher dir phylogenetishen Jieziehungen einerseits der Chlorocyperus-Arten unter cinander, andererseits von Chlorocyperus zu Kyllingia, Dichostylis und Galilea aufklären können und dass vielleicht die.eine oder andere der drei zuletzt genamuten (iattungen zu Chlorocyperus gezogen oder ïherhaupt eine andere Gruplerung der hierher sehörigen Arten vorgenommen werden muss; so lange aber eingehende Arheiten ïher diese Cyperaceen nicht vorliegen, empiehlt es sich, vorderhand an diesen vier Gattungen festzuhalten. Die Eucyperus.Arten

*) Die Revision, bezw. Bestimmung des ausegehenen Materials und die Zusammenstellumg der Litteraturnachweice wude hei den (yperaceen von H. Dr. E. Palla in Graz

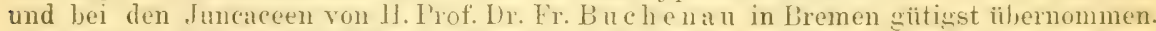
Ausserdem wirkte noch Herr Schriftsteller W. Lackowitz bei Durchsicht der Korrekturabzüge freundlichst mit. [ife schedae sind durch den Herausgeber selbst zusammengestellt. Da der unterzeichnete Herausgeber die Druckkosten für die Schedae trägt, so werden letztere in der Weise den Abonnenten der "Allg. bot. Zeitschr." zur Terfügung gestellt. dass die ursprüugliche Grösse der Zeitschrift sich um den Umfang der betr. Schedate erweitert. Für Melnporto und Yapier wird event. ein ganz geringer Betrag in Anrechnung gebracht werden.

A. Knencker. 
hingegen, zu denen aus unserer Flora Eucyperus fuscus (L.) Rikli gehört, dürfen selbstverständlich auf keinen Fall mehr mit Chlorocyperus-Arten in derselben. Gattung belassen werden.

Palla.

Nr. 2. Chlorocypems badius Palla $=$ Cyperus badius Isf. Fl. Atl.'I., p. $45(1798)=$ C. brachystachys Pr. Cyp. et Gram. sic., p. $15(1820)=$ C. thermalis Dum. Fl. belg., p. 145 (1827).

An fenchten Stellen am Flusse Roja bei Ventimiglia in Ligurien (Oboritalien); Alluvium. Begleitpflanzen: Juncus obtusiflorus Ehrh. etc.

$5-10 \mathrm{~m}$ ü. d. M.; 31. Mai 1899.

leg. Clarence Bicknell.

Nr. 3. Chlorocyperns rotumdus Palla $=$ Cyperus rotundus L. Sp. pl, ed. I., p. $45(1753)=$ C. olicuris Targ. Tozz. in Mem. d. soc. it. d. sc. XIII, 2, p. 338.

An Wegen, an kultivierten und unkultivierten Orten bei Bordighera in Oberitalien (Ligurien); Sand-u. Thonboden. Begleit p flanzen: Amarantus deflexus L., patulus Bert., Chenopodium album L., Cyperus aureus 'Ten.

$5 \mathrm{~m}$ ü. d. M.; Sept. 1899.

leg. A. Bianchi.

Nr. 4. Chlorocynems glomerutus Palla $=$ Cypens glomeratus $L$. Am. Ac. IV., p. $301(1759)=$. cimamomens Kietz Obs. IV., p. $10(1779-91)=$ C. australis Schrad. Fl.Germ. I., p.116(1806).

An nassen und sandigen Stellen, Wegrändern und in Gräben bei KunFélegyhiza in Centralungarn. Begleitpflanzen: Jumcus lampocarpus Ehrh., Carex riparia Curt., Polygonum mite Schrk., persicaria L., Verhena officinalis L., Althaea officinalis L., Bidens tripartitus L.

Ca. $100 \mathrm{~m}$ ü. d. M.; 5. Okt. 1899.

leg. Prof. J, Wagner.

Nr. 5. Galilea mucromata Prrl. in Fl. pal. I., p. 299 (1845) = Schoenus mucronatus L. Sp. pl. ed. I., p. 42 (1753) $=$ Cyperus capitatus Vand. f., p. $5(1771)=$ C. Aegyptiacus Glox. Obs: bot., p. ${ }^{20}(1785)=$ C. schoenoides Gris. Spic. II., p. $421(1844)=$ Chlorocyperus Aegyptiacus Rikli in Jahrb. f. wissensch. Bot., Bd. 27, p. 564 (1895).

An sandigen Stellen in der Nähe des Meeres bei Bordighera in Oberitalien (Ligurien); Meersand. Begleitpflanzen: Medicago marina L., Euphorbia paralias L., Silene sericea ete.

Am Meeresstrand ca. $1 \mathrm{~m}$ ü. d. M.; Mai 1899. leg. Clarence B icknell.

$\mathrm{Nr}$. 6. Holoschoemus australis Fritsch in Excursionsfl. f. Oesterreich, p. 80 (1897) = Scirpus australis L. Syst. ed. XIII., p. 85 (1774).

Auf etwas sumpfigen Wiesen und Weiden bei Riva in Südtirol; Kalk boden. Begleitpflanzen: Calamintha officinalis Mnch.

Ca. 78-100 m ü. d. M.; Juli 1899.

leg. Porta.

Die Kenntnis der Arten der Gattung Holoschoenus, die sich um Holoschoenus rulgaris Lk. gruppieren, liegt derzeit sehr im Argen; withrent vielfach uberhaupt nur eine einzige Art angenommen wird, der $\mathrm{H}$. vulgaris Lk. (- Scirpus Holoschoenus L.), ist andererseits eine grossere Anzahl von Arten unterschieden worden, die hauptsächlich auf der Grösse und Anzahl der Blütenköpfchen basieren; eingehende neue Untersuchumgen wären deshalh sehr wünschenswert. Palla.

Nr. 7. Schoenoplectus Trbenucuenomtumi Prill in Botan. Jahrb. f. Systematik, X., p. 299 (1888) = Scirpus 'Tabernaemontani Gmel. Fl. Bd. I., p. 101 (1805) = Scripus glaucus Sm. Engl. Bot. t. 2321 (1812). 
An feuchten Stellen der Knielinger Schweinsweide zwischen Knielingen und Neurenth in Baden; Alluvium. Begleitpflanzen: Carex Oederi Ehrh. v. canaliculata Callmé, teretiuscula Good., stricta Good., vulgaris Fr., stricta $>$ vulgaris, disticha Huds., rostrata With. ete.

Ca. $110 \mathrm{~m}$ ü. d. M.; 19. Juni 1899.

leg. A. Kneucker.

Weder Schoenoplectus lacustris noch Sch. Tabernaemontani sind einheitlich, sondern nur Gruppen engverwandter Arten; künftige eingehende Untersuchungen, die sich aber nicht in der bisher üblichen Methode darauf beschränken dürften, bloss einige äussere Merkmale wie die 2- und 3-Narbigkeit, die reinoder blaugrüne Färbung des Stengels u.s.w. in Betracht zu ziehen, dürften noch sehr interessante Resultate ergeben.

Palla.

Nr. 8. Schoenoplectus supimus Palla in Botan. Jahrb. f. Systematik, X., p. 299 (1888) = Scirpus supimus L. Sp. pl. ed. I, p. 49 $(1753)=$ Isolepis supina R. Br. Prodr. I, p. 77 (1810).

An sumpfigen Stellen, besonders am Rande von Wasserlöchern auf der Schweinsweide bei Aua. Rh. in Baden; Alluvium. Begleitpflanzen: Heleocharis acicularis R. Br., Isolepis setacea R. Br., Ranunculus paucistamineus Tsch., Marsilia quadrifolia L., Limosella aquatica L., Myriophyllum alterniflorum DC. etc. Ca. $120 \mathrm{~m}$ ü. d. M.; 3. Juli 1899 u. 17. Aug. 95. leg. A. Kneucker.

\section{Nr. 9. Schoenoplectus supinus Pulla f: monostachya.}

Standort und Datum des Einsammelns wie bei vorhergehender Nummer. leg. A. Kneucker.

Eine geringwertige, meist einährige Standortsform des schlechteren Sandbodens, während die typische Pflanze an fetteren Stellen wächst. A. K.

Nr. 10. Heleocharis palustris $\boldsymbol{R}$. Br. in Prodr. I, p. $80(1810)=$ Scirpus palustris L. Sp. pl. ed. I, p. 47 (1753).

An schlammigen Stellen der Knielinger Schweinsweide zw. Knielingen und Neureuth in Baden; Alluvium. Begleitpflanzen: Carex pseudocyperus L., gracilis Curt., acutiformis Ehrh., Scirpus silvaticus L., Galium uliginosum L.

Ca. $110 \mathrm{~m}$ ü. d. M.; 19. Juni 1899.

leg. A. Kneucker.

H. palustris ist sicherlich schon in Europa keine einheitliche Art und dürfte mit der Zeit in eine ziemlich beträchtliche Anzahl äusserlich nicht leicht von einander unterscheidbarer Arten zerlegt werden; doch muss, so lange nicht. diesbezüglich umfassende Untersuchungen vorliegen, vorläufig der Sammelname palustris angewendet werden.

Palla.

Nr. 11. Heleocharis unighumis Schult. in Mant. II, p. $88(1824)=$ Scirpus uniglumis Lk. in Jahrb. d. Gew. I, 3, p. 77 (1818).

Auf sandigem und feuchtem Alluvium am Altrhein bei Leopoldshafen in Baden. Begleitpflanzen: Deschampsia caespitosa Beauv. $\gamma$. setifolia Bisch., Heleocharis palustris R. Br., Carex panicea L.. riparia Curt., glauca Murr., flava L., vesicaria L., Equisetum trachyodon A. Br. etc.

Ca. $110 \mathrm{~m}$ ü. d. M.; 24. Juni 1899.

leg. A. Kneucker.

$H$. uniglumis wird häufig mit $H$. palustris verwechselt oder überhaupt nicht von ihr unterschieden. Das ausgezeichnete Merkmal, dass die unterste Deckschuppe das Aehrchen mit ihrer ganzen oder nahezu ganzen Basis umfasst nur selten kommen vereinzelte Ausnahmen vor, während umgekehrt ganz ausnahmsweise bei $H$. palustris die unterste Deckschuppe sich wie $H$. uniglumis verhält - charakterisiert allein schon hinreichend diese Art; auf die reingrüne Farbe der Halme ist allerdings weniger zu geben, da auch gewisse $H$. „palustris" rein grün gefärbt sind. Die "Ueberginge" von $H$. uniglumis in $H$. palustris oder umgekehrt, von denen in manchen Floren die Rede ist, beruhen wohl nur auf unzureichender Beobachtung der betreffenden Autoren.

Palla. 
Nr. 12. Heleocharis acicularis R. Br. in Prodr. I, p. $80(1810)=$ Scirpus acicularis L. Sp. pl. ed. I, p. 48 (1753).

An schlammigen Stellen, besonders am Rande von Wasserlöchern auf der Schweinsweide bei Au a. Rh. in Baden; Alluvium. Begleitpflanzen: Schoenoplectus supinus Palla, Isolepis setacea R. Br., Ranunculus pancistamineus Tsch., Marsilia quadrifolia L., Limosella aquatica L., Myriophyllum alterniflorum DC. ete.

Ca. $120 \mathrm{~m}$ ü. d. M.; 3. Juli 1899.

leg. A. Kneucker.

Nr. 13. Rhynchospora albe Vahl in Enum. II, p. $236(1806)=$ Schioenus albus L. Sp. pl. ed. I, p. 44 (1753).

Auf sandigem Diluvium des Exerzierplatzes bei Weissenburg i. E., an vorwiegend feuchten Stellen. Begleitpflanzen: Carex echinata Murr., Oederi Ehrh., pilulifera L., vulgaris Fr., gracilis Curt., Lycopodium inundatum L., Dro. sera intermedia Hayn., Pedicularis silvatica L。 etc.
Ca. 132 m ü. d. M.; 25. Juli 1899.

Nr. 13a. Rhynchospora alba Vahl.

Moorsümpfe unweit Salvan im Kanton Wallis in der Schweiz; Unterlage krystallinisch. Begleitpflanzen: Salix aurita L., repens L., Lythrum hyssopifolia L., Pinguicula alpina L., Menyanthes trifoliata L., Pteridium aquilinum Kuhn, Blechnum spicant Sm. etc.
Ca. $1091 \mathrm{~m}$ ü. d. M.; 26. Aug. 1899.
leg. Prof. F. O. Wolf.

\section{Nr. 13 b. Rhynchospora alba Vahl.}

In den Wasserrinnen der Torfsümpfe bei Viomnaz im unteren Wallis, Schweiz. Begleitpflanzen: Erigeron annuus Pers., Aster brumalis Nees, salicifolius Scholl., Erigeron angulosus Gaud., Inula Britannica L., salicina L., Vaillantii Vill., semianplexicaulis Reut., Bidens cernua L., Carpesium cernuum L., Serratula tinctoria L., Gentiana cruciata L., pneumonanthe L., Erythraea centaurium Pers., Pedicularis palustris L., Carex rostrata With., filiformis L., Aspidium thelypteris Sw., cristatum Sw. ete.

Ca. $384 \mathrm{~m}$ ü. d. M.; Sept. 1899.

leg. Prof. F. O. Wolf.

Nr. 14. Juncus subulatus P. Forsticil Fl. aegypt.-arab., p. 75 (1775); v. Fr. Buchenau, Monogr. Junc., p. $171(1890)=J$. multiflorus Desf. Fl. atlant. I, p. 313 (1798).

Auf alluvialem Sand- und Kalkboden bei Bougie am Cap Aokas in Algerien (Afrika). Begleitpflanzen: Scirpus maritimus L., Heleocharis palustris R. Br., Juncus maritimus Lk., Agrostis Reuteri Boiss.

Am Meeresufer; August $1898 . \quad$ leg. E. Reverchon.

Nr. 15. Juncus tenuis II illd. Linn. Spec. plant., II, p. 214 (1799); v. Fr. Buchenau, Monogr. Junc., p. $193(1890)=J$.gracilis J. E. Smith, Comp. fl. brit., p 55 (1800).

An einem Fusswege zw. Erlen- und Haselgebüsch am r. Ufer der Triebisch bei Herzogswalde im Bezirk Dresden (Kgr. Sachsen); Alluvium a uf Phyllit. Die Pflanze wächst meist allein in kräftigen Büschen oder in Gesellschaft von Holcus lanatus L.

Ca. $200 \mathrm{~m}$ ü. d. M.; 15. Juli u. 18. Aug. 1899.

leg. P. Seurich.

Nr. 16. Juncus arcticus Willd. Linn. Spec. plant. II, p. 206!(1799); v. Fr. Buchenau, Monogr. Junc. p. $222(1890)=J$. effusus car. ๙., L. Spec. plant. ed. I, I, p. $326(1753)=J$. effusis var. $\beta . ~ L$. F]. suec. ed. II, p. $111(1755)=J$. Jacquini J. E. Smith in Linné, Fl. lapp., ed. II, p. $91(1792)=J$. pauciforus C. Mïnch in J. C. Schleich., Catal. plant. in Helvetia cis- et transalp. sp. 
nasc., p. $57(1799)=$ J. acuminatus J. B. Balbis Add. ad Fl. Pedem. in Römer Archiv f. d. Bot., III, p. 129 (1803).

Auf angeschwemmtem Gletschersand im Findelenthal ob Zermatt (Westschweiz) am Rande iles blawen Gletschersees, Stellisee". Dieser See billete vor 50 Jahren die äusserste Zunge des Findelengletschers, der jetzt eine starke halbe Stumde höher entfernt ist. In der Nïhe stehen Glimmer- und Chloritschiefer an. Begleitpflanzen: Trichophorum Austriacum Palla, atrichum Palla, Juncus triglumis L., Carex foetida Till., Persoonii O. F. Lang, grypus Śchk., Ranunculus glacialis L., Campanula cenisia L.

Ca. $2543 \mathrm{~m}$ ü. d. M., August 1899.

leg. Prof. F. O. Wolf.

N1. 17. Juncus effusus L. Sp. plant., ed. I, p. 326 (1753); pr. pte. v. Fr. Buchenau, Monogr. Junc., p. $228(1890)=J$. conglomeratus L. pro. pte. $=$ J. communis E. M. . effusus E. Meyer Junci Generis Monogr. spec., p. 20 (1819) et Synops. Junc., p. 12 $(1822)=$ "J. laevis Ger. em. 39" teste S. F. Gray, A natur. arrang. of brit. plants II, p. 161 (1821).

Fenchte und sumptige Stellen auf der Schweinsweide von Knielingen in Barlen unweit Karlssuhe; allu viale S S a d. Begleitp flanzen: Juncus lampocarpus Ehrh. glaucus Ehrh., effusus × glaucus, Cirsium palustre Ścop., Veronica anagallis L., Pulegium vulgare Mill. etc.

Ca. $110 \mathrm{~m}$ ü. d. M.; 19, Juli 1899.

leg. A. Kneucker.

\section{Nr. 17 a. Juncus effusus L.}

Auf Moorboden in der Nähe des Hohlohsees bei Kaltenbrom im nördlichen badischen Schwarzwald; C'nterlage Bundsandstein. Begleitpflanzen: Carex canescens L.. leporina I.. echinata Nurr., Juncus squarrosus L., tiliformis L.. Andromeda polifolia L., Enspetrum nigrum L., Taceinium axycoccos L., uliginosum L., vitis idaea L. ete.

$900-990 \mathrm{~m}$ ü. d. M.; 25. Aug. 1899.

leg. A. Kneucker.

N1. 18. Jumcus effusus L. $\times$ glaucus Ehr\%. $\because$. Fr. Buchenau Monogr.Junc, p. $231(1890)=$. . diffusus Hoppe Bot. Notizen, in Flora, p. $186(1819)=$ J. laevis Wallr. $\%$. diffusus Wallr. Schedulae criticae I, p. 142 (1822).

Feuchte und sumptige stellen auf der Schweinsweide von Knielingen in Baden unweit Karlsruhe; alluvialer Sand. Begleitpflanzen: Juncus cffusus L., glaucus Ehrh., articulatus L., Cirsium palustre Ścop., Veronica anagallis L., Pulegium vulgare Mill. ete.

Ca. $110 \mathrm{~m}$ ü. d. M,; 19. Juli 1899.

leg. A. Kneucker.

$\mathrm{Nr}$. 19. Nuncus glaucus $\mathbf{F} \boldsymbol{H}$. Elı Hart Calamariae, Graminae tripetaloideae exsicc. Nr. 85; v. Fr. Buchenau, Monogr. Junc., p. $243(1890)=J$. inflexus L. Spec. plant., ed. I, II, p.326 (1753); ed. II, I, p. 464 (1762) („J. filiformis" err. calami) $=J$. effusus J. A. Pollich Hist. plant. in Palat. elect. sponte nasc. I, p. 345 (1776) $($ non L.) $=J$. diaphragmarius $F$. A. Brotero flora lusitanica I, p. 511 (1804).

Fenchte und sumpfige Stellen auf der Schweinsweide von Knielingen in Baden unweit Karlsruhe; alluvialer Sand. Begleitpflanzen: Juncus effusus $\times$ glancus, lampocarpus Ehrh.. ('irsium palustre Scop., Veronica anagallis L., Pulegium vulgare Mill.

Ca. $110 \mathrm{~m}$ ü. d. M.; 19. Juli 1899.

leg. A. Kneucker.

Nr. 20. Jumrus maritimus Lam. Encycl. méth., bot., III, p. 264 (1789); v. Fr.Buchenau, Monogr. Junc., p. $256(1890)=J$. acutus var. . L. Spec. plant. ed. I, I, p. 325 (1753); ed. II, I, p. 464 
$(1762)=J$. spinosus Forskail Fl. aegypt.-arab., p. 75 (1775) (probab.!) = J. rigidus Desf. Fl. atlant. I, p. 312 (1798).

Auf den „Salzen-Wiesen“, sumpfigen Strandwi esen am Barsbecker Bimnensee hinter Stein an der Ostsee an Brackwassergräben. Begle it f f lanzen: Scirpus maritimus L., Rumex maritimus L., Erythraea linarifolia l'ers., Plantago coronopus L. ete.

$$
\text { 1-2 m ü. d. M.; 6. Aug. u. Okt. } 1899 .
$$

Nr. 21. Lusula lutea I)C. Fl. franç. p. $169^{\circ}(1805)$; v. Fr. Buchenau, Monogr. Junc. p. $90(1890)=$ Juncus campestris var. $\varepsilon$. L. Spec. plant. ed. II, I, p. $469(1762)=$ Junc. luteris All. Fl. pedem. II, p. 216 (1785).

Auf der Alpe de la Barma (Vallée de Dix) im Val d'Hérémence im Kanton Wallis auf Gneiss u. Glimmerschiefer. Begleitpflanzen: Carex aterrima Hoppe, nigra All., atrata L., clavaeformis Hppe, ferruginea Scop., Campamula thyrsoidea L., Hedysarum obscurum L., Scabiosa lucida Vill., Aster alpinus L.. Erigeron Villarsii Bell., Schleicheri Graeb., intermedius Schleich., Achillea moschata Wulf., Hieracium aurantiacum L., fuscum Vill. etc.

Ca. $2200 \mathrm{~m}$ ü. d. M.; Juli 1899.

leg. Prof. F. O Wolf.

N1. 22. Luala pedemontana Boiss. et Reut. Pugillus plant. Africae bor. Hispaniaeque austr., p. 115 (1852); v. Fr. Buchenau, Monogr. Junc., p. $96(1890)=L$. nemoiosa var. $\gamma$.gracilis E. Mey. Luzularum species, in Linnaea, XXII, p. 395 (1849).

An trockenen Stellen bei San Bartolomeo im Val Pesio in Piemont unter Kastanienbäumen auf mehr oder weniger $\mathrm{k}$ alkhaltigem Boden. Begleit. pflanzen: Phyteuma Michelii All., Trifolium elegans Savi, Melampyrum pratense L.

$750 \mathrm{~m}$ ï. d. M.; Juni 1899.

$$
\text { leg. Luigi Pollini. }
$$

Nr. 23. Lu:mla memorosa E. Meyer Luzularum spec. in Linn., XXII, p. 394 (1849); v. Fr. Buchenau Monogr. Junc., p. $93(1890)=$ Juncus pilosusvar. \&. L. Spec. plant. ed. I, I, p. 329 (1753); ed. II, I, p. $468(1762)=J$. nemorosus Pollich Hist. plant. in Palat. elect. sponte nasc. I, p. $352(1776)=J$. niveus Leers (non L.) Flora Herbornensis, p. 91 (1775):=J. angustifolius Wulf. Plant. rar. carinth. in Jacq. Collect., III, p. $56(1789)=J$. albidus Hoffm. Deutschl. Flora ed. I, p. 126 (1791) = Luzula albida DC. Fl. franc., III, p. 159 (1805) et aut. mult. $=L$. angustifolia Gurcle Flora v. Nord- u. Mitteldeutschl., 3. AuH., p. 348 (1854) et auct. mult.

Auf Sandboden im Durlacher Wald bei Karlsruhe in Baden unter Laub. mischwald. Begleitpil anzen: Luzula campestris DC. v. multiflora Celak.. nemorosa E. Meyer v. rubella Gaul., pilosa Willd., Carex pallescens L., pilulifera L., brizoides L., silvatica Huds., Anthoxanthum odoratum L., Melica uniflora Retz.. Milium effusum L. ete.

Ca. $117 \mathrm{~m}$ ü. d. M.; 27. Mai u. 28. Juni 1899. leg. A. Kneucker.

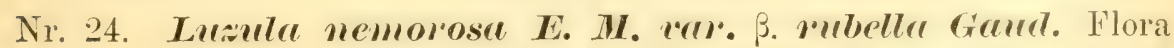
helv., I, p. 566 (1828); v. Fr. Buchenau, Monogr. Junc., p. 95 (1890). - Synonymia vide sub Luzula nemorosa forma rubella vix varietas esse videtur.

Auf Sandboden im Durlacher Wald bei Karlsruhe in Baden unter Laubmischwald. Begleitpflanzen wie bei Nr. 23.

Ca. $117 \mathrm{~m}$ ü. d. M.; 27. Mai u. 28. Juni 1899.

leg. A. Kneucker. 
Nr. 25. Luzula mivea DC. fl. franç., III, p. 158 (1805); v. Fr. Buchenau, Monogr. Junc., p. $100(1890)=$ Juncus pilosus var. $\eta$. L. Spec. plant. ed. I, p. 329 (1753) = Juncus niveus L. (Th. L. Nathorst). Flora monspel. in Amoen. acad., IV, p. 481 (1756).

Unter Lärchenwald bei Sion im Kanton Wallis (Schweiz); Glimmerschiefer. Begleitpflanzen: Echinospermum deflexum Lehm., Circaea alpina L., Geranium silvaticum L. ete.

Ca. $1400 \mathrm{~m}$ ü. d. M.; Juli 1899. leg. Prof. F. O. Wolf.

Nr. 26. Lusula confusa C. J. Lindeby. Resa i Norge $185+$ in Nya botaniska Notiser, p. 9 (1855); v. Fr. Buchenau, Monogr. Junc., p. $124(1890)=$ Juncus arcuatus var. $\beta$. Wahlenb. Fl. lapp., p. $88(1812)=$ Luz. hyperborea $R$. Br. et auct. multi pro parte.= Luz. arcuata Wahlenb. var. $\gamma$. E. Meyer Luzularum spec. in Linn. XXII, p. 195 (1849).

Auf trockener, verwitterter Glimmerschiefer-Erde des Gebirges Flöifjeldet bei Tromsö in Norwegen. Begleitpflanzen: Carex sparsiflora (Whlbg.), rupestris All., alpina Sw., rigida Good., Persoonii O. F. Lang. Festuca ovina L., Juncus trifidus L., Polygonum viviparum L. ete.

Ca. $600 \mathrm{~m}$ ü. d. M.; 26. Juli u. 20. Aug. 1899. leg. An dr. Notó.

Nr. 27. Lusula compestris DC.var.vulgaris Gaud. Fl. helv. II, p. 572 (1828); v. Fr. Buchenau, Monogr. Junc., p. 157 (1890)= Juncus campestris $\alpha . L$. Spec. plant. ed I, I, p. 329 (1753); ed. II, I, p. $468(1762)=$ L. campestris DC. fl. franç. III, p. 164 (1805) pro pte. $=L$. campestris DC. var. longistyla Celak. Prodr. der Flora v. Böhmen, p. 85 (1869) et p. $749(1881)=L$. vulgaris Fr. Buchenau Kritische Zusammenstellung d. europ. Juncaceen in Englers bot. Jahrbüchern, p. 175 (1885).

Auf dem sandigen Hochgestade zw. Mühlburg und Knielingen in Baden auf der rechten Rheinebene. Begleitpflanzen: Carex caryophyllea Latour., praecox Schreb., Vicia lathyroides L., Silene conica L., Teronica verna L., Cerastium semidecandrum L., Avena caryophyllea Web. ete.

Ca. $112 \mathrm{~m}$ ü. d. M.; 27. April $1899 . \quad$ leg. A. Kneucker.

Nr. 28. Lusula campestris DC. var. congesta Fr. Buchenau Monogr. Junc., p. $162(1890)=$ Juncus campestris L. Spec. plant. ed. II, I, p. 469 (1762)=Juncus congestus Thuill. Flore des env. de Paris, p. 179 (1799) = Luzula congesta Lejeune Flore des env. de Spaa I, p. 169 (1811) (exclus, auct. mult.) $=L$. multi. flora Lej. $\beta$. congesta Koch Synopsis flor. germ. et helv. ed. I, p. 734 (1837) (et auct. mult.) pr. pte.

Auf Noorwiesen bei Varel in Oldenburg. Begleitpflanzen: Carex pulicaris L., Nardus stricta L., Arnica montana L., Thalictrum flavum L. etc.

Ca. 2 m ü. d. M.; 6. Juni u. 13. Juni 1899. leg. Dr. Fr. Müller.

Mit der Species- oder V'arietäts-Bezeichnung , congesta der Luz. campestris ein ausserordentlicher Missbrauch getrieben worden, indem man jede Form der var. vulgaris, multiflora, Sudetica und sogar der var. pallescens, bei welcher die Stiele der Seitenküpfe wenig oder gar nicht entwickelt sind, ohne weiteres ,var. congesta" oder gar "Luzula congesta" namnte. Das sollte doch endlich aufhören! - Die hier vorliegende Pflanze ist die echte Luzula congesta Lejeune. Sie ist eine wenig verbreitete Form, da sie hüufiger nur in der norddeutschen Tiefebene und den angrenzenden Gebieten, sonst nur 
noch an einzelnen Stellen vorkommt. Sie liebt moorigen orler doch anmoorigen Boden. - Die Eigentümlichkeit des geknäuelten Blütenstandes besitzen noch zwei bemerklich verschiedene Varietäten der Luzula campestris, die var. tristachya Fr. Buchenau aus Chile und die var. capitata F. A. W. Miquel aus Japan (v. Fr. Buchenau, Monographia Juncacearum, p. 159-160, 1890). Fr. Buchenat.

Nr. 29. Lasula campestris DC.var. multiflora Celak: Prodr. d. Flora v. Böhmen, p. 85 (1869) et p. 749 (1881); v. Fr. Buchenau, Monogr.Junc., p. $161(1890)=$ Juncus campestris $\%$.L. Spec. plant. ed. II, vol. I, p. $469(1762)=J$. multiflorus Ehrh. Calamariae, Gramina et Tripetaloideae exsice. (ca. 1791) $=J$. intermedius. Thuill. Fl. des env. de Paris, p. 178 (1799) $=J$. erectus Pers. Syn. plant. I, p. $386(1805)=J$. nemorosus Host Icones et descriptiones graminum austriacorum III, Tab. 97, pr. pte. $(1805)=$ Luzula erecta Desv. Mémoire sur une monogr. du genre Luzula, in Journ. de botanique I, p. $156(1808)=L$. multiflora Lej. Fl. des env. de Spaa, I, p.169(1811). Forma parva.

Auf Moorboden neben der sogenannten Kieferweiche am Bugstein bei Georgenfeld und Zinnfeld (Bezirk Altenberg) im sächs. Erzgebirge sehr häufig. Begleitpflanzen: Carex Goodenoughii Gay und Sphagna.

Ca. 880 m ü. d. M.; 5. Juni 1899.

leg. Paul Seurich.

\section{Nr. 29 a. Lusula compestris DC. var. multiflora Celak.}

Auf S andboden im Durlacher Wald bei Karlsruhe in Baden unter Laubmischwald. B e gle itp fla n zen: Luzula nemorosa E. Meyer, pilosa Willd., Carex pallescens L., pilulifera L., brizoides L., silvatica Huds., Anthoxanthum odoratum L., Melica uniflora Retz., Milium effusum L. ete.

Ca. $117 \mathrm{~m}$ ü. d. M.; 5. u. 17. Mai 1899.

leg. A. Kneucker.

Nr. 30. Lusula campestris DC. var. Sudetica Celak. Prodr. d. Flora v. Böhmen, p. 749 (1881); v. Fr. Buchenau, Monogr. Junc., p. $164(1890)=$ Juncus campestris $\eta$. L. Spec. plant. ed. II, vol. I, p. $469(1762)=J$. Sudeticus Willd. Linn. Spec. plant., II, p. 221 (1799) = Luzula nigricans Desv. Mémoire sur une monogr. du genre Luzula in Journ. de botanique, I, p. 158 $(1808)=L$. campestris DC. var. alpina Gaud. Agrostol. helv. p. 247 (1811).

Feuchte, moorige Wiesen auf dem bewaldeten Gipfel des Keilberges im sächs.-böhm. Erzgebirge (Böhmen). Kommt zieml. selten allein an Wegrändern vor, oder in Gesellschaft von Juncus squarrosus L. Auf grasigen Stellen wächst die Pflanze vereinzelt mit Poa pratensis L. und Carex Goodenoughii Gay.

$1244 \mathrm{~m}$ ü. d. M.; 9. Juli 1899. leg. Paul Seurich.

\section{Nr. 30 a. Luala compestris DC. var. Sudetica Celaliovsliy.}

Elb- und Pantschewiese im Riesengebirge auf Moorboden. Begleitp flanzen: Rubus chamaemorus L., Archyrophorus uniflorus B. et F., 'Trichophorum alpinum Pers., Hieracium alpinum L., Pinus pumilio Hke., Lycopodium alpinum L., Juniperus nana Wlld, Nardus stricta L. ete.

Ca. $1300 \mathrm{~m}$ ü. d. M.; 15. Aug. 1899. leg. Jos. A nders.

Die hier gratis ausgegebenen Exemplare sind etwas spärlich, 11. ihre Priiparation lässt zu wünschen übrig.

A. $\mathrm{K}$. 


\section{Botanische Litteratur, Zeitschriften etc.}

Gradmann, Dr. Robert, Das Pflanzenleben der Sehwäbischen All, mit Berïcksichtigung der angrenzenden Gebiete Sïddentschlands. 2. Aufl. 2 Bände. Tübingen, Verlag des Schwäbischen Albvereins. 1900. Preis im Buchhandel y M., für Mitglieder des Verbandes deutscher Touristenvereine durch die Geschäftsstelle des Schwäb. Albvereins in Tübingen incl. Porto $6 \mathrm{M}$.

An diesem in so kurzer Frist bereits in zweiter Auflage vorliegenden Werke hat sich in schönster. Weise die von Drude an bchlusse seiner Einleitumg zu "Dentschlands Pflanzengeographie* (Erster Teil. Stuttgart 1896) ausgesprochene Hoffnung erfüllt, , dlass in Zukunft neben der Masse von grossen und kleinen Floren aus den mitteleuropaiischen Staaten auch solche Bücher zahlreicher hervorwachsen werden, welche - wie Christ's "Pflanzenleben der Schweiz" - auf dem Grund und Boden der Landesnatur stehend, mit heredten Worten die Lebenserscheinungen besprechen." Die Aenderungen, wehche das Werk in der neuen Autlage erfahren, sind, wie der Verfasser in Vorwort selbst sagt, mit Ausnahme der Beigabe von 8 weiteren - gleich den 42 älteren vorzüglich ausgeführten -Chromotafeln, nur muledentende. In der That ist auch namentlich der erste (allgemeine) Teil des Werkes - abgesehen von den Veränderumgen, welche die Fortschritte in der Wissenschaft notwendig mit sich bringen -. wohl kaum einer Verhesserung mehr fahis. Wir finden hier auf dem engen Raume von 101 Seiten zusammengedrängt eine üheraus reiche Fülle von biologischen und pflanzengeographischen 'Thatsachen verzeichnet, und zwar nicht etwa nur aus der Litteratur zusammengetragen, sondern nach sorgfältigem Studium und unter ausgiebigster Benützung derselben der heimischen Natur abgelaweht. Wem ich mich hier darauf heschranke, aus dem reichen Inhalte dieses 'Titels die prächtigen Vegetationshilder hesonder's hervorzuhehen, welche der Veriasser mit so viel Wärme und Hingebung von den Wäldern, von den Felsen mol Halden, den Gewässern und simmpten und schliesslich von den Kulturformationen des abwechslungsreichen Allggebietes entwirft, so geschieht es nicht deshalh, als ob der übrige Inhalt ihnen gegenüber in den Hintergrund träte, sondern allein in der Ueberzougung, dass beim Lesen dieser lebensvollen schilderungen nicht nur dem trockenen Systematiker, der hisher zufrieden war, wemn er nur für jeden seiner Funde einen wohlklingenden Namen gefunden hatte, die Reize und der wissenschattliche Gehalt unserer einheimischen Flora in einem ganz anderen Lichte erscheinen werdep, somlern auch mancher Naturfreund, dem hisher die Beschäftigung mit unserer wildwachsenden Pflanzenwelt als zu einförmig oder gar als minderwertig gegenüber anderen naturwissenschaftlichen Disziplinen erschienen, neu für dieselbe gewonnen werden wird.

Was den zweiten (besonderen) Teil anlangt, so gestattet mir der Raummangel hier zwar nicht, auf Einzelheiten einzugehen. W'enn ich aber einerseits die vorzüglichen, den strengsten wissenschaftlichen Ansprüchen genügenden Tafeln und Abhidungen und die nicht minder guten Bestimmungstabellen besondens anerkemend hervorhehen muss, so darf ich wohl damit eine Bitte an den Verfasser verhinden, deren Erfüllung ihm bei seiner reichen Begabung und seinem eisernen. Fleisse nicht schwer fallen und ihm andererseits namentlich die mehr systematischen Situdien huldigenden Botaniker zu grösstem Danke verpHichten wird: es mögen in der - hoffentlich recht bald notwendig werdenden dritten Auflage auch die in der zweiten etwas stiefmuitterlich behandelten kritischen

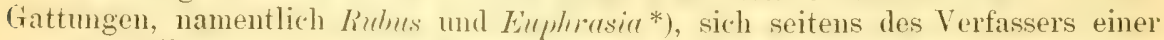
- ob verdienten oder unverdienten, mag ja dahingestellt bleiben - etwas liehevolleren Behandlung erfreuen.

Dr. H. Pöverlein.

*) Aus den übrigen kritischen Gattungen möchte ich nur erwåhnen, dass der "Alectorolophus angustifolius.", wenigstens nach mir aus der Gegend von Heidenheim vorliegenden Exemplaren (leg. Vollmann), recte A. serotinus G. Beck ist, und die auf S. 100 anfgeführte subsp. Sulix glabroides schenprle der st. glabia scopuli nicht hieher, sondern nach Originalexemplaren Schenerle's als rar. psendoglubru sichutz zu S. nigricans Smith zu ziehen ist. 
Der Unterzeichnete hat bereits auf ein ihm von der Redaktion des .Allgemeinen Litteraturblattes" (Wien) ïbermitteltes liecensions-Exemplar eine ansfïhrliche Besprechmo der Nenanflage dieses chenso inhaltlich tüchtigen wie reizend ausgestatteten Buches eingesandt. Da aher jenes Litteraturblatt vielen Lesern der Allg. bot. Zeitsch. nicht zugänglich sein dürfte, so mögen hier üher. 2 Punkte, nämlich die schreihmg der lateinischen Namen und die Illustrationen einige Bemerkungen Platz finden.

In ersterer Hinsicht steht die 2. Auflage auf dem fortgexchrittensten Standpunkte, ohne in Uebertreibungen hinsichtlich des Purismus auzuarten. Wir finden

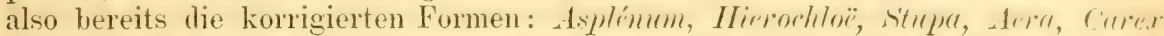

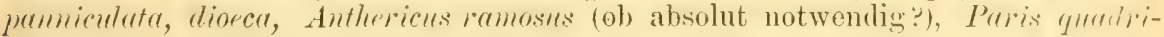
folius, Cypripedilum, Orchis purpureus etc., Corydallis, Polygala vulgare ete.,

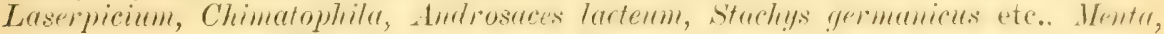
Alyssum calicimum, Tofieldia caliculata, Chenopodium hibridum u. s. w. Zu verbessem sind noch, zumteil mit Rücksicht auf meine Auseinandersetzunsm in der "Allg. bot. Z." $1897 \mathrm{Nr} .4$ u. 5, Formen wie Arhenaterum (à

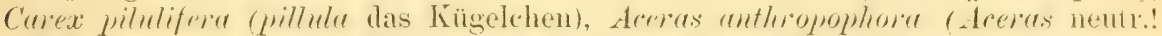

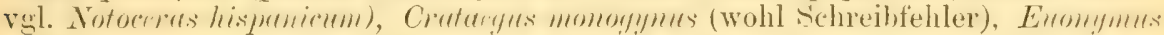
europaeus, Anthriscus nitida, Chaerophyllum (besser Chaerephyllum), Gléchoma

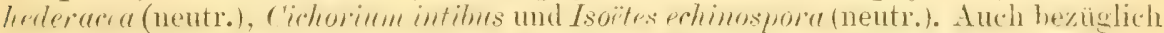
dess sächlichen Geschlechtes von squiranthes, Menyunthes, Pornunthes dürfte sich der Terfasser der vou mir a.a.(). vertretenen Anschaumng anzuschliessen vermoigen ;

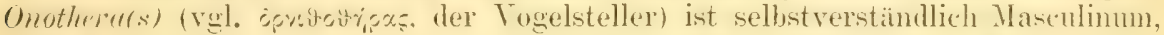
also 0 . muricatus, so sonderbar es für den Anfang klingen mag; dagegen hat wohl das ältere Galium Cruciata zu verbleiben. Nichts einzuwenden ist gegen

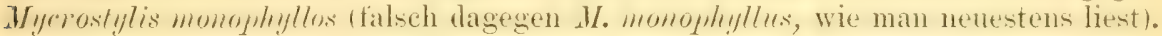
Als Druckfehler wäre nur zu erwähnen Carex ripraia, Teucrim bitrys. Hinsichtlich des illustrativen Teiles sind inshesondere viele der Chomotateln annerst gelungen, manche, wie Polygala chanaebuxus, Cotoneaster integerima, Lonicera alpigena, unübertreftlich. Von den Holzschnitten sind manche zu verbessern; als "Trifolium ribres" ist II S. 200 T. incernetum abgebildet, die Luterschriften von Veronica officinalis und chamaedrys (II S. 304) sind vertauscht.

\section{II u l r.}

Haláesy, Dr. E. v., Conspectus florae (iraecae. Verl. v. Wilh. Fngelmamn in Laipzig. 1900. Vol. I. Fasc. I. p. 1-224. Preis 5 M.

Nahezu 100 Jahre sind seit dem Erscheinen des "Prodromus florae Graecae" von sibthorp und snith und 16 Jahre seit Boissier's "Florat orientalis". welche anch die griech. Florat umfasst, verflossen. Seitden ist aber die hot. Durchforschung Griechenlands ausserordentlich forteseschitten. u. bot. 'T'auschvereine u. Exsiceatenwerke haben die interesmante Florar des Lamcles weiten Kreisen zuginglich gemacht. Es wurde daher das Bedürfnis nach einem Werke, das alle bis jetzt gemachten Forschungsergehnisse äber die griech. Flora zusiummenfasst, immer füblbarer. Jie durch Herausgabe botan. Litteratur rühmlichst bekannte Engelmann'sche Buchhandlung in Leipzig hat num zur liearheitung eines sulchen Werkes den um die Erforschung der griech. Flora hochverdienten Wienter butaniker Dr. E. v. Halácsy gewonnen. Die ganze Arbeit, zu welcher bereits das vollständige Manuskript vorliegt, soll im Zeitramm von 5-ti. Jahren in Lief. von je 10 Bogen zum (iesamtpreise von ca. 30 Al. zur Alusabe gelangen. derler Art (ausgenommen sind nur einige monotype, allgemein bekannte Arten) int eine lat. Diagnose heigegeben, die hauptsächl. die Enterscheidungsmerkntale von nalle vel'wandten Arten enthät. Ausser den Litteraturnachweisen, dencen leder keine Jahreszahlen beigefügt wurden, sind noch die standortsangahen berücksichtight, und artenreichen Gattungen wurden bestimmmesschlässel vorangestellt. 1)

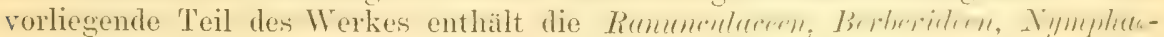
accen, Papaveraceen, Fumariaceen, Cruciferen, Cupparideen, Resedaceen, Cistaceen, Violaceen, Polygalaceen, Fiantieniaceen, Sileneen und einen Teil der Alsineen. 
Der gelehrte Verfasser wird sich durch Herausgabe dieses "Conspectus" ein unvergingliches Verdienst um die Wissenschaft und den Dank aller Botaniker erwerben, die sich mit der orientalischen und speziell der griechischen Flora beschäftigen.

A. K.

Fritsch, Dr. Karl, Schulflora für die österreich. Sudeten und Alpenländer (mit Áus. schluss des Küstenlandes). Verl. v. Carl Gerold's Sohn in Wien 1900. 387 S. Preis $3.60 \mathrm{M}$.

Die im Jahrgang 1897, p. 130 dieser Zeitschrift besprochene Exkursionsflora für Oesterreich von $K$. Fritsch hat auch dem Verfasser bei diesem 2. Werk als Muster gedient. Der Einrichtung nach schliesst sich das Werk genau der obengenamnten Flora an. Da das vorliegende Buch für den Schüler berechnet ist, sind in ihm im Vergleich zu der Exkursionsflora verschiedene Kürzungen vorgenommen worden, so wurden z. B. auch die Autorennamen nach Aschersons Torgang weggelassen. Auf diese Weise konnte der Preis des Werkes, welcher bei der Exkursionsflora auf 8 M. festgesetzt ist, auf 3.60 M. ermässigt werden, welcher Umstand für die Anschaffung der Flora an Schulen sehr günstig ins Gewicht fällt. Das Buch wird als Bestimmungsbuch für den botan. Unterricht an Mittelschulen unzweifelhaft recht gute Dienste leisten.

A. K.

Fedtsehenko, 0. u. B., Ranunculaceen des russischen 'Turkestan. (Sep. aus ,Engl. bot. Jahrbücher ${ }^{\star}$ 1899. Band 27. Heft 3.) Wilh. Fngelmann, Leipzig.

Der grossen 'Thätigkeit des Botanikerpaares 0 . u. B. Fedtschenko verdanken wir diese neue Bereicherung der Kenntnisse über die Flora Russlands. In der Arbeit sind 158 Arten beschrieben.

H. von Trautschold.

Fedtschenko, Boris, Die im europäischen Russland, in der Krim und im Kaukasus vorkommenden Arten der Gattung Hedysarum. (Sep aus „Bullet. der Nat. de Moscou 1899, Nr. 1.)

Die Arbeit stammt von Boris Fedtschenko allein, umfasst 18 Seiten und enthailt 3 Karten. Die Zahl der beschriebenen Arten beträgt 15. Sowohl dieser als der vorstehend genamnten Arbeit sind Bestimmungstabellen beigegeben und beide in deutscher Sprache geschrieben.

H. von Trautschold.

Phanerogamae et Pteridophytae Japonicae iconibus illustratae. Lief. 1-7. Tōkyō. 1899.

Die 7 vorliegenden Lieferungen enthalten die Ablildungen und Diagnosen von folgenden Arten: Daphne cammabina Wall.v. Kiusiana Mak., Edyerrorthia papyvifera sieb. Euce, Centhranthere Brunoniana Benth., Adianthum monochlamis Eat., Asplemm Wrightii Eat., Rhododrndron pentaphyllum Maxim., Polypodium ammifrons Mak., I'olypodium sesquipedale II all. f. leiopteris Mak., limeare Thunb., 1. r. Onof Mali., Taeniopluyllum aphyllum Mak., Rotala Hipmenis Mati., Heterosmilax. Japoniare Kunth, Trichomanes parvulum Poir., filicula Bory, acutum Mak., Hymenophylhum Wrightii I'. D. Bosch, flexile Mak., oligosormm Mak., barbatum Brak., Trichamanes Japonicum Fr. et Sac., auriculatum Bl., Hymenophyllum polyanthes Sir., Vittaria .Japonica Miq, Diplacium lanceum Presl., Antrophyum. Japonicum Mati., Drymoglossum carnosum Hook. v. subcordatum Bak., Polypodium hastatum Thumb., Engleri Lurss., Phegopteris Kram ri Fr. ot Sav., culgaris Mett., Polypodium Niponicum Mett., Burrgeriamum Miq., Gymnogramme salicifolia Mal., incoluta Ilook: Die trefflichen Abbildungen der einzelnen Arten füllen jeweils eine ganze Tafel. Die Diagnosen sind in japanischer Schrift und Sprache abgefasst.

A. $\mathrm{K}$.

Eine Flora des Schwarzwaldes. Nach Erscheinen des Gradmann'schen Werkes über die Flora der schwäb. Alb, wurde der Gedanke angeregt, auch eine ähnliche Arbeit über den Schwarzwald herauszugeben. Nachdem einer Publikation in Nr. 171 u. 172 der "Mitteilungen des bad. bot. Vereins" zufolge die finanzielle Beihilfe sowohl des bad. Schwarzwaldvereins, als auch der Regierung 
und einzelner Städte in Aussicht steht, scheint die materielle Seite des Unternehmens gesichert. Wie aus der Ankündigung zu entnehmen ist, soll ein ,populär und doch streng wissenschaftlich gehaltenes Buch über die Flora des Schwarzwaldes verfasst werden." Wir hoffen, dass unter ,populiir" wohl nicht die Ausserachtlassung der neueren und neuesten Forschungsergebnisse der Systematik auf dem Gebiete polymorpher Genera zu verstehen ist. Die Form möge also populär, der Inhalt aber streng wissenschaftlich sein. Es steht wohl ausser Zweifel, dass die verschiedenen Spezialisten gerne bereit sein werden, das im Lanfe der nächsten Jahre sich ansammelnde und in den grösseren bad. Herbarien sich vorfindende Material polymorpher Gruppen einer Revision zu unterziehen, aufgrund welcher dann die Bearbeitung solcher Abteilungen erfolgen könite. Möge es dem hochgeehrten Vorstande des bad. bot. Vereins, Herm Prof. Dr. Oltmanns in Freiburg i. B., der keine Mühe schent, dieses schöne Unternehmen zu fördern, und der sich um das Zustandekommen desselben schon so grosse Verdienste erworben hat, gelingen, in gleicher Weise nicht nur alle Mitglieder des bad. bot. Vereins, sondern auch alle Freunde der Botanik, welche den Schwarzwald lieben, zur ernsten und edlen Mitarbeit an dem Werke zu begeistern. A. K.

\section{J. Sturms Flora von Deutsehland.}

Der "Deutsche Lehrerverein für Naturkunde*, welcher sich Verbreitung naturwissenschaftlicher Kemntnisse als Ziel gesetzt hat, giebt in den Jahren 1900 bis 1905 neben dem Vereinsorgan „Aus der Heimat" (jährlich 6 Hefte) J. Sturm s „Flora von Deutschland in 12 Bädchen von je etwa 160 Seiten mit zusammen 600 farbigen und 120 schwarzen 'Tafeln heraus. Nicht nur die Abbildungen sind von seltener Schönheit und Genauigkeit, sondern auch der Text wird nach den neuesten Ergebnissen der Wissenschaft und mit weitgehender Berücksichtigung der Biologie bearbeitet. Die Mitglieder erhalten jührlich gegen den geringen Beitrag von 1.90 M., bezw. 1.60 M. die Heimathefte und 2 Bündchen dieser Flora zugestellt. Im Buchhandel wird dieses Werk 30-36 M. kosten; es werden deshalb Natur- und bes. Pflanzenfreunde auf diesen Verein und seine Veröffentlichungen aufmerksam gemacht. Anmeldungen nimmt der Schriftführer, Mittelschullehrer Bass, Stuttgart, Silberburgstr, $79 \mathrm{I}$ entgegen.

Appel, Dr. Otto, u. Loew, Dr. E., Mitteilung blütenbiologiseher Beobachtungen.

Nit den Vorarbeiten zur Herausgabe des IV. Bandes von Knuth's Handbuch der Blütenbiologie beschäftigt, bitten die Unterzeichneten alle Botaniker, welche im Besitze von blütenbiologischen Beobachtungen an aussereuropäischen Pflanzen sind, ihnen dieselben gütigst zur Verfügung stellen zu wollen.

Prof. Dr. E. Loew, Berlin SW., Grossbeerenstrasse 67, und Dr. Otto Appe 1 in Charlottenburg, Schlossstrasse 53.

(Bot. Centralblatt.)

Oesterreichische bot. Zeitschrift 1900. Nr. 3. Provazek, S., Synedra hyalina, eine apochlorotische Bacillarie. - Figdor, W., Zur Anatomie des Stammes der Dammarpflanze. - Rechinger, C., Ueber Lamium Orvala L. u. L. Wettsteini Rech. - Jenčič, A., Untersuchungen des Pollens hybrider Pflanzen. - Polak, J. M., Untersuchungen über die Staminodien der Scrophulariaceen. - Bornm üller, J., Ein never, bisher verkannter Bürger der europäischen Flora. - Scholz, J. B., Studien über Chenopodium opulifolium, ficifolium und album.

Botan. Centralblatt. 1900. Nr. 10. M üller, K arl, Zusammenstellung der Lebermoose aus dem Reichslande Elsass-Lothringen. - Kuntze, Otto, Spirodiscus: Ophiothrix: Ophiocytium, ein Nomenklaturbeitrag, - Nr. 11. Müller, Karl, Wie in vor. Nr. - Rost ow zew, Dr. S., Laboratoriumstisch für das Mikroskopieren. - Nr. 12. Müller, Karl, Wie in vor. Nr. - Nr. 13. Schmidle, W., Drei interessante tropische Algen. - Müller, Karl, Wie in vor. Nr.

Mitteilungen des bad. bot. Vereins. 1900. Nr. 171 u. 172. Herzog, Theodor, Standorte von Laubmoosen aus dem Florengebiete Freiburg. - Oltmanns, Pflanzengeographische Durchforschung Badens. 
Berichte der deutschen bot. Gesellschaft. 1899. Generalversammlungsheft II. Nachrufe über: J. H. Ch., Lange, Ferid. Cohn, I. Caruel, Otto Böekeler, Fr. Kuhla, W. F. R.Suringar, Axel Blytt. - 'T s wett, II., Ueber die Verknüptung des änsseren und inneren Leptoms der Solanaceen durch markstrahlenstandige Leptombiindel. - Neger, F. W., Beitrag zur Kenntnis der Gattung Phyllactinia. - Buchner; Ed., Ueber Zymosegährung. He in ic her, E., Zur Entwickelungsgeschichte einiger grüner Halbschmarotzer. Kolkwitz, R., Beiträge zur Biologie dex Florideen. - 1900. Heft 2. S'chulze, E, Ceber Eiweisszerfall und Eiweisshildung in der Pflanze. - Kny, L., Leber das angehlichie Vorkommen lebenden Protoplammas in den weiteren Lufträunen von Wasserpflanzen. - Steinbrinck, C., Zur Frage der elastischen Schwellumg von P'flanzengeweben. - Le win, L., Leber die toxicologische stellung der Raphiden. - M agnus, P., Beitragr zur Kenntnis der Neovossia Moliniate (Thu.) Koern.

Berichte der bayerisehen bot. Gesellschatt. Band VII. I. Abt. 1900. Arnold, F., Nachrufe üher William Nylander und Massalongo (mit Portraits). Nachrufe über J. N. Schnabl u. G. Woerlein (mit Portraits). - Neger, F. W., Einige mykologische Beobachtungen als d. Fichtelgehirge. - Kra enzle. J., Vachtrag zur Phanerogamen- und Gefisskryptogamenflora der Nünchener 'Thalehene von (i. Wverlein. - Arnold, Dr. F., Lichenes exsiccati 1601-1800 (Index).

Zeitschrift der bot. Abteilg. d. naturwiss. Vereins der Provinz Posen. VI. Jahrg. III. Heft. 1900. 'T orka, T.. Terfithmer und Fall des Lauhes im Herbste in der Umgegend von Paradies-Jordan-Schwiebus. - Derselbe, Beiträge zur Flora der Umgeg. v. Paradies-Jordan. - Schube, Neue Beobachtumgen im südl. Teile der Provinz. - Miller, Zur Flora der Umgegend von Tirschtiegel im Kreise Meseritz. - Spribille. Einige Aufzeichnmoen aus dem Kreise Kempen. - Bock, Die Flora Brombergs zu Kühlings Zeit und jetzt. -

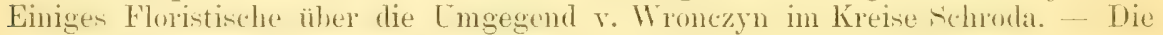
Riesen-Eiche von Büschkau im Kreise Schubin.

Botaniska Notiser. 1900. Heft 2. F́cottsherg. C.. Viola-formér fram Ösel. - Hellsing, G., Cassandra calyculata funnen in Sverige. - Neuman, L. M., Utricularia intermedia Hayne $x$ minor L. - Holm berg, O. R., Botaniska anteckningar. - Nordsteet, O., Sandtems flora.

Botanical Gazette 1900. Vol. XXIX. Nr.2. I) the development of the pollen grain in Simplocarpus foetidus and Peltandra undulata. - Nerrell, Will. Dayton, A contribution to the life history of Silphium.

\section{Botanische Anstalten, Vereine, Tauschvereine, Exsiccatenwerke, Reisen etc.}

Preussischer Botanischer Verein. V. Sitzung, Königsberg i. Pr., 19. I ärz 1900. Torsitzender Herr Landgerichtsrat Grenda. Dr. Abromeit legte einige benerkenswerte Pflanzen aus der Sammlung des Herm Rektors H e y m in Briesen, Westpr., vor. Die Flora des Kreises Briesen wurde besonders in den westlichen und suidlichen Teilen bereits seitens des Preussischen Botan. Vereins untersteht, wohrei methere anch in pflanzengeographischer Hinsicht wichtige Funde konstatiert worden sind. Bekanntlich wurde in einem unfern von Czystochleb bei Briesen gelegenen sumpfigen See die seltene Aldrovandia vesiculosa im Sommer 1882 durch Caspary entdeckt. Seither ist ein zweiter Funciort diesser interessanten P'flanze in miserem Gebiet nicht konstatiert worden. Herr liektor Hey m hat speziell die nähere I'ngehmeg von Briesen, die durch kleinere Waldungen, Moore, seen und Wiesen atugezeichnet ist, montersucht. Ton seinen P'flanzenfunden wurden u. A. demonstriert: Erymgium plamum, dort schon selten, in Weichselgelände dagegen verbreitet, Lailhyms tuberosus ron einer 


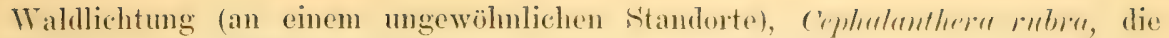
weiterhin im nördlichen Gebiet sehr selten ist, Lysimacha vulyaris fr. Kling-

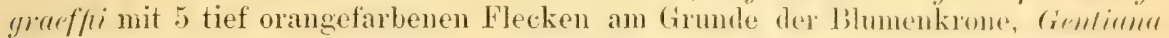
pnenmonanthe in der normalen schmalblättrigen Form nit fein zugespitzten Blumenkronzipfeln vom trockenen Standorte und in der hreitbiatrigen fir. /atifolia solooller mit länglich-eiförmigen his $18 \mathrm{~mm}$ hreiten mittleren sitengelhattern und stumpllichen oder wenig spitzen Blumenkronzipfoln, forner ('uralliorhiza innata unter Juniperus communis angetroffen, was selten beobachtet worden sein dürfte, da Coralliorrhiza sonst nur an feuchten, moosigen Stellen oder auf humosem Boden vorkommt. Sedum reflexum wurde in der bei uns und in Westpreussen wild konstatierten fr.mpestre $L$. an seinem östlichsten Vorkommen im Gebiet von Hrn. Rektor H e y mesammelt. Der Vortragende erwilht ferner, dass wach einer brieflichen Mitteilumg des Herrn K neucker in Karkruhe der aut dem

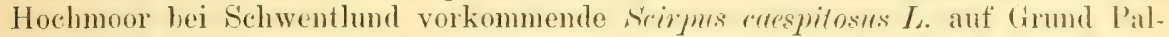
la'scher Untersuchung mit T'richophor'm Austriacum P'alla identisch ist. Bisher' war diese erst vor wenigen Jahren durch Palla aufgestellte Art aus Dentschland nur für Schlesien bekamnt; indessen dürften alle unsere l'unde des alten scrip.us raespitosus $L$. hierher gehören. In pflanzengeographischer Hinsicht reihen sich unsere Fumdorte sehr gut an diejenigen des russischen balticums an. Kar Torlage gelangte ferner die neueste Doppellieferung (8 u.9) der hynopis der mitteleuropäischen Flora von Ascherson u. Graebner, worin ein Teil dex Gramineen sehr eingehend behandelt worden ist. Besonders nind es die formenreichen Arten der Gattungen Agrostis, Calamagrostis, Avena, Aera etc., die hier ausführliche Berücksichtigung gefunden haben. Sehr interessant sind auch die Erörterungen üher die Heimat des Saathafers anf \$. 232 4. 2:3:3, wonach die Koernicke'sche Ammahme, dass der Hafer aus Südosteuropa oder anch wohl aus dem westlichen Asien herstamme, die grösste Wahrschemlichlieit baben dürfte. Herr Apotheker Erich R. Perwo besprach nenere Erscheimmgen aus dem Gehiete der Fachlitteratur. Herr Lehrer $B$ aenge in Wehlan hatte einige benerkens. werte Funde aus der Flora von Wehlau eingesandt, worunter hemerkenswert waren: Orobanche arenaria Borkh., leider ohne Nährpflanze und nur in einem

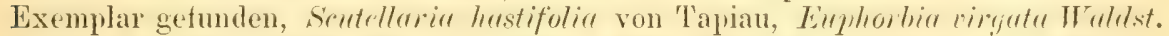
of Kit. vom Alleufer bei Wehlau (Arlventivpflo), Nommen pulla I)C'. als Arlventivpflanze an der Chausseeböschmo anf der strecke von Tapiau nach Eisingen lieobachtet; ferner Allum scorodoprosum und Paparep Rhoress (hier seltene Adventivpflanzen).

Abromeit.

Botanischer Verein für die Provinz Blandenburg. Die Nonatssitzumg am 9. März eröfnete der Vors. Prof. śch hum an $n$ mit der angenehmen Nitteilung, dass der Provinzial-Ausschuss auch in diesem Jahre wieder 500 M. als Beihilfe zu den Kosten für die Drucksachen des Vereins bewilligt habe. Die diesjährige Frühjahrs-Hauptversammlung soll am Sonntag nach Pfingsten in Nenstrelitz stattfinden; dies liegt zwar ansserhalb des Vereinsgebietes, doch sind die hotanischen Bezichumgen der Provinz Brandenburg zu Mecklenburg so imnige, dass eine engere Fühlung zwischen beiden Gebieten eigentlich nur naturgemäss erscheint. IVi: immer wird sich auch diesmal mit der Versammlung eine grössere gemeinschaft. liche Exkursion verbinden, von der sich die Vereinsmitglieder nicht unberleutende Resultate versprechen, da sie gerade in die schönste Jahreszeit fallt. Lndlich teilte I'rof. Schumann mit, dass die Befürchtung, die so hoch interessanten Fenm. im Grunewald könnten gefährdet sein, schon demnächst in Erfüllnug zu gehen drohe, da, wie verlautet, der Forstfiskus beahsichtigt, das Femn zwish hem dem Hundekehlen- und Grunewaldsee zu einer ertragfïhigen Wiese für den Fürster umzugestalten. Das wäre ein unersetzbarer Verlust für die Flora der Mark, der tum so schwerer empfunden werden würde, als schon an einer anderen Stelle des Grunewaldes eine Kette dieser merkwürdigen Hochmoore den Villenanlagen zuliebe in seen umgewandelt worden sind. Der Verein erklïrt sich natiulich damit ainverstanden, dass der Vorstand gegen ein solches Internohmen beim 
Ministerium Verwahrmeg einlegt; da der Kultusninister, wie schon nitgeteilt, der Erhiltung solcher botanischen Denkmäler aus der Vorzeit günstig gesinnt ist, so ist wenigstens Hoffinmog vorhanden, dass eine solche Vorstellung seitens des Vereins nicht auf unfruchtharen Boden fällt. - Als ersten Vortrag spendete Il: Dr. I'ilger eine Reihe ron Eindrieken, welche er als Begleiter des Forschungsreisenden D). Heyer zn den Indianem Brasiliens empfangen hat. Sehr anschanlich und lebendig schilderte er die botanikch so ärmliche Lmgehmug von ('nyabi anl gleichnamigen Flusce in der Provinz Matto Grosso, den spärlichen Baumwuchs aus den Familien der Leguminosen und Apocynacren, den Buschwald mit zwei Meter hohem Grase. Der Cuyahá gehört noch zum Stromgelbet des Para. gualy, erst weiterhin nach Norden, jenseits der Wasserscheide, in dem Oberlauf der Gewässer, die hier sämtlich nach Norden zum Amazonenstrom abfliessen, treten in den charakteristischen Flussthälern, Barancas, dichte Wäder, namentlich prachtvolle Palmen auf. Wieder weiter wird der Boden ein steinharter Lehm, und in der trockenen Jahreszeit stirbt hier die Pflanzenwelt nahezu wänzlich ak. Auch der Tierwelt gedachte der Vortragende, die dem Jäger namentlich in Rehen. Wildschweinen und Straussen reiche Bente zn liefern vermag. Hr. Custos Hennings sprach äher eme Art ron japanischem Forstmerkbuch, welches er der Güte des Hrn. Prot. Shirai verdankt; als auffallendste Beispiele von eigenartigem Wuchs sind darin Exemplare von Pims Thmbergiana zu vermerken, mit eincm sïnlenartigen IVurelhau, zwischen dem ganze Gesellschaften hindurchgehen kömen. Ferner charakterisierte der Tortragende einen Pilz ans dem südlichen Amerika, der iür die Bewohner von Fenerland ein Hauptuahrungsmittel darstellt. - Hr. Reg.-R. v. Tubeut hält die auf dem Berliner Weihnachtsmarkte so zahlreich vertretene und zu erheblich teureren Preisen feilgebotene

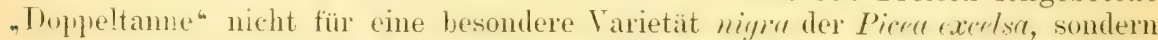
tiir nichts weiter als Gipfelstücke ailterer Bämme, die von den findigen Weih. nathtshaum-Hänllern dem Publikum als eine besondere Raritat aufgehalst werden; Vortragender ist der Meinung, dass der Forstkasse durch den Verkanf dieser in den Bammschlägen sonst als wertlos umherliegenden (iipfel eine nicht merhebliche Einnahme erwachsen könnte. Nun, es soll hier nicht verhehlt werden, dars diese Deutung der J)oppeltanne mit ihren sehr dicht stehenden, säbelförmig nach oben gekrimmten Nadeh bei den Floristen unter den Vereinsmitgliedern Wohl auf einige und nicht ganz mberechtigte Zweifel gestossen sein dürfte. Hr. Prof. sior a ue reprach äber ein grosses Sterben der Kirschbäune am Rhein, dessen Ursache einerseits in einem Pilz, andererseits in der Einwirkung des Frostes gesucht wird. Vortr. glaubt, letzteres annehmen zu können; denn der Girschbaum neigt sehr zum Gummifluss, und daun wird der Frost tödlich, wenn "r die 'Zuwachszone eroreift, so dass die spitze nicht mehr ihre Ernährung finden kamn. Anschliessend daran sprach Hr. Soraner auch über die sogenannten Krebsgeschwïlste hei den (Obsthäumen, die ebenfalls einerseits der Einwirkung eines gewisen l'ilzes zugeschrielen, andererseits als die Folgen bestimmter Frosterscheinungen angesehen werden. - Zum Schluss führte Hr. Prof. Schumann, anchliessend an die letzte vitzmg, mehrere Beispiele von l'flanzen anf, an denen dic Ameisen sich andere (Hrane, als Stanmhöhlungen, zur Wohnmg wihlen, z. B. Blätter, Nebenblätter, sogar Dornen.

W. Lackowitz.

Pflanzengeographische Durchforsehung Badens. Der Torstand des bad. bot. Vereins, H. Prof. Dr. O ltm a n n s in Freiburg i. B., regte in Nr. $171 \mathrm{u.} 172 \mathrm{der}$ Mitteil. des bat. hot. Vereins eine'n schon früher ausgesprochenen Plan zur pflanzengeogr. Durchforschung Badens nach Gradmanns Vorgang an, der schon mehrfach anch anderwaits Tachahmumg gefunden hat. Zunächst sollen nicht mehr als if namentlich anfgeführte species in ihrer Verhreitung studiert werden. Jas ganze Resultat soll dam in Tabellen und Karten übersichtlich dargestellt werden. Anfesestelte Vertranensmanmer haben sich mit den Floristen ihrer Gebiete in Terhimdung zu setzen und das Ergehnis ihrer Arbeit dem fichriftü̈hrer des bad, bot. Vereins, Herrn Prof. Dr. Scheid in Freiburg, abzuliefern. In 
grossen Gebieten des bad. Landes wohnt leider nicmand, der sich für die lolua interessiert, da in Baden gegenüber anderen Lündern der Prozentsatz derer, welche kraft ihrer Vorbildung in der Lage wären, sich mit der Floristik erfolgreich zu beschäftigen, ein verschwindend geringer ist. Wir hoffen und wünschen aber, dass auch in den bis jetzt floristisch weniger hokinnten treilen des bad. Landes noch manche Kräfte sich in den Dienst der schönen Sache stellen möchten.

A. K.

72. Versammlung deutscher Naturforseher und Aerzte. I)icellw fimblet vom 17.-22. Sept. in Aachen statt. Da den allgemeinen Einladungen, die anfancs Juni zur Versendung gelangen werden, bereits ein vorläufiges Programm der Versammlung beigefügt werden soll, so wird geheten, Vortrïge und lemonstrationtu spätestens bis Ende April bei einem der Unterzeichneten ammelden zu wollen.

Es liegt in der Absicht der Geschäftsführung, dem in den Versammlungen zu Frankfurt und Braunschweig hervorgetretenen Wunsche anf lienchrinkun der Zahl der Abteilungen dadurch gerecht zu verden, dass sie versuchen wirl, möglichst viele Abteilungen zu gemeinsamen sitzungen zu vereinigen. Inlem um Unterstützung bei diesen Bestrebungen geheten wird. "risuchen die Interzeichneten ergebenst, Wünsche in betreff gemeinsamer vitzungen einzelner $\Lambda_{\text {h. }}$ teilungen gätigst übermitteln und Beratungsgegenstiunde fïr diese sitzungen hezeichnen zu wollen. Endlich sei jetzt schon mitgeteilt, das's gemaiss einer mit den Herren Vorsitzenden der wissenschaftlichen Hanptgruppen getroffenen Verahredung einstweilen Mittwoch, der 19. Septemher, für gemeinsame Sitzungen jeder der beiden Hanptgruppen freigehalten werden soll. I le für diese Verhandlunge.s in Aussicht genommenen Gegenstamle hofft die leschaftsfiihrung in kurzen hekannt geben zu können. Der Einführende: Prof. Dr. A. W ieler, 'Technische Hochschule; Der Schriftführer: (Wherlehrer Dr. ()nstein, Krakaustr. 30, Aachen.

Internationales bot. Institut in Amazonas. Dr. A. Buscalioni, Asistent am hot. Institut in Rom, ist mit reichen Ergebnissen ans Brasilien zurückgekehrt. Im Auftrage der Regierungen von Parà und Amazonas setzt er sich derzeit mit den Regierungen mo wissenschaftlichen Korporationen Europas in Verbindung zum Zwecke der (tründung eines grossen internationalen Institutis in Amazonas.

(Oesterr'. bot. 'Zeitschr.)

Internationaler botan. Kongress in Paris. Gelegentlich der Wratusstellung findet in Paris in der Zeit vom 1. bis 10. Okt. 1900 ein internationaler bot. Kongress statt. Die "Commission d'organisation" wird von folgenden Herren gebilảet: Prillieux, Präsident; Dutailly, Mussat, Rouy, Vicepräsidenten ; Perrot, Generalsekretar; ( u erin, Lutz, Sekretare; ferner aus 20) Vitgliedern. Die Kommission fordert die Botaniker aller Länder anf, Gegenstande, die auf die 'Tagesordnung gesetzt werden sollen, anzumelden. Bisher wurden folgender Beratungsgegenstände festgesetzt: 1. "Etudes monographiquex"; 2. Espécer. hybrides et métis"; 3. "Unification des mesures micrométrinues"; t. "Influence de la nature du sol et des végétaux qui y croissent sur le developlement des Champignons". Der Anmeldungstermin für Vorträge und Diskusionsthemen endet mit dem 15. September. Auskünfte jeder Art erteilt der Generalsekiretir. Prof. E. Perrot, Ecole superieure de Pharmacie, Paris. - Ein Abdruck der Kongressregehn findet sich 1 . a, im , Journal de Botanique" von I. Morot, 13. 11m. Nr. 10, p. XC-XCII.

(Oesterr. bot. 'Leitschr.)

Arnold. F., Lichenes exsiccati Nr. 1778-1800 ist erschienen. Wie wir erfahren, will der Herausgeber dieses höchst verdienstvolle Werk, welehes er Jahrzehnte hindurch mit grosser. Wühe und unter mancherlei schwierigkeitem und bedentenden Opfern an Zeit und Geld herausgab, vorläufig abschliessen.

Arnold, F., Lichenes Monacenses exsiceati. Lis sind die Nummern l!) t bis 505 erschienen. 
Krieger, W., Fungi saxonici. Fase. XXX. J)ie erschienene Iivef. enthailt $\mathrm{Nr}$. 1451-1500, worin besonder's die Ascomyceten und Hyphomyceten reichlich vertreten sind.

Herbarium Böekeler. Ias Herbarium des verstorbenen Hrn. Ir. Bäckeler ist. zu verkaufen. Dasselhe umfasst (a. 12,000 Arten. Auskunft erteilt die Redaktion der "Botanischen 'Zeitung".

Delectus plantarum exsiceatarum quas anno 1900 permutationi offert hortus hotanicus unives sitatis Jurjevensis.

Der 109 Seiten umfassende Katalog ist durch den bot. Garten in Jurjew (Dorpat) zum Preise von 50 Kop. zu beziehen. Derselbe enthält zahlreiche wertvolle, kriticche Bemerkungen und Liagnosen etc. meist in russischer, aher atch in dentscher und lat. Sprache. Eine Tafel bringt die Abbildung von Myosotic alpestris, anserdem sind einige Abbildungen im Texte beigegeben. Unter den Mitarbeitern ist vor allem Petunnikov aus Moskau zu nennen, welcher grosse Verdienste um die Erforschung der russischen Flora hat. Dic Pflanzen des Katalogs sind in 7 Wertgrupen jeweils alphabetisch aufgeführt. Die Pflanzen der ersten Gruppe sind zu je 3, die der 2. zu 5, die der 3. zu 6, der 4. zu 8, dex 5. zu 10, der 6. zu 15 und die der 7. zu je $20-400$ Einheiten à 1 Kop.= 2,5 Pfg. $=3$ Ctms. bewertet. Wer sich also Herbarexemplare aus der so interessanten russischen F'lora zu erwerben sucht, dem sei der sehr reichhaltige Katalog bestens empfohlen.

Reineck u. Czermak, Plantae Brasiliae meridionalis. Von diesem Exsiccatenwerk sind die 3 arsten Lieferungen erschienen und von dem unterzeichneten Herausgeher zu leziehen. Jede Lieferung enthält 50) Nummern, darunter novae species, und kostet 1t) M. Die Pflanzen sind von Herm Prof. Dr. John Briquet, lienf, hestimmt und gut konserviert. Weitere Lieferungen folgen demnachst. Der Herausgeber ist: Eduard Martin Reineck in Arnstadt, Thüringen, Deutschland.

\section{Personalnachrichten.}

Ernennungan etc.: Dr. Walter Busse hat sich an d. Univ. Berlin für Botanik habilitiert. - Dr. A l fr. Krolopp w. z. a.o. Prof. an d. landw. Schule zu Altenburg ernannt. - Dr. Abromeit, Assistent am bot. Garten in Königsherg, hat sich an der dortigen Lniversitat für Botanik habilitiert. - Geh. Hofrat Dr. Pfeffer in Leipzig und Geh.Rat Prof. Dr. v. Zittel in Nünchen w. zu korrespond. Mitgliedern der Académie des sciences zu Paris ernannt. - N. J. Borsczow w. Subassistent am bot. Garten in Jurjew. - E. A. N. Arber w. Demonstrator d. Palïobotanik an d. Univ. zu Campbridge. - A. B. Fom in, Assistent am bot. Garten in Jurjew, erh. v. Konsul d. kaiserl. geogr. Gesellsch. in St. Petershurg ome silherne Medaille für seine ¿jührige bot. Reisen im östl. Trans. kaukasien u. w. vom Konsul der Univ. Jurjew 1/2 Jahr nach dem Kaukasus abkommandiert, um im bot. Garten zu Tiflis seine bot. Ausbeute zu bearbeiten $\mathrm{u}$. neue Exkursionen nach dem Kaukasus zu unternehmen. - B. B. H ry new ezky nimmt Fomin's Stelle in Jurjew während seiner Abwesenheit ein. - Dr. J. B. Carruthers w. Assistant-Director am bot. Garten in Peradenya. - Dr. Aug. (iinzberger, Profestor am Gymmasium in Krummau, erh. die neue Assistentenstelle am bot. Garten u. Museum der Univ. Wien.

Todesfälle: Nicol. W. (r rign reff, Phytopaläontologe in Charkow, am 16. Juli v. J. - H.Zukal, a.o. Prof. d. Phytop. an d. Hochschule f. Bodenkultur in Wien, am 15. Febr. - James Paget am 30. Dez. in London. - John Ruskin am 20. Januar in Brantwood Coniston. - William $\mathrm{R}$ aw son am 20. Nov. in Süd-Kensington. - Miss F anny E. Langdon am 21. Okt. v. J. in Michigan. - Carl Pollák in Prag am 17. Febr. im 53. Lebensjahre. 


\section{Allgemeine \\ Botanische Zeitschrift \\ für Systematik, Floristik, Pflanzengeographie ete.}

\section{Referierendes Organ}

des bot. Vereins der Provinz Brandenburg, der kgl. bot. Gesellschaft zu Regensburg, des Preuss. bot. Vereins in Königsberg,

und Organ der Botan. Vereinigung in Würzburg und des Berliner bot. Tauschvereins.

Unter Mitwirkung hervorragender Fachmänner herausgegeben

von A. Hneucher, Werderplatz 48 in Karlsruhe.

Verlag von J. J. Reiff in Karlsruhe.

\begin{tabular}{|c|c|c|}
\hline $\begin{array}{l}\text { Ne } 5 . \\
\text { IVI }\end{array}$ & $\begin{array}{l}\text { Erscheint am 15. jeden Mor } \\
\text { Preis: vierteljährl. } 1.50 \mathrm{Mk} \text {. bei freier }\end{array}$ & $\begin{array}{l}\text { 1DOO. } \\
\text { VI. Jahrgang. }\end{array}$ \\
\hline
\end{tabular}

\section{Inhalt}

Originalarbeiten: W.Schmidle, Algologische Notizen (Schluss). - L. C trossNürnberg, Ist Draba 'Thomasii Koch eine gute Art? (Schluss). - Dr. J. MI urr, Phaenologische Plaudereien aus der Innsbrucker Flora - Otto Will, Uebersicht über die bisher in der Umgebung von Guben in der Niederlausitz beobachteten Leber-, 'I'orfund Laubmoose. - A. Kneucker, Bemerkungen zu den "Gramineae exsiccatae".

Bot. Litteratur, Zeitschriften ete.: Dr. H. Poeverlein, Schwarz, August Friedrich, Phanerogamen- und Gefässkryptogamenflora etc. (Ref.). - A. Kneucker, Pax, Dr. Ferdinand, Prantl's Lehrbuch der Botanik (Ref.) - Derselbe, Dalla 'Torre, Dr. C. G. v., u. Harms, Dr. H., Genera Siphonogamarum ad systema Englerianum conscripta (Ref.). - Derselbe, Fonck, Leopold, S. J., Streifzüge durch die biblische Flora (Ref.). - Ders elbe, Hervey, E. Williams, Observations on the colors of flowers(Ref.). W inkler, W., Sudetenflora (Ankündigung). - Inhaltsangahe verschied. bot. Zeitschr.

Bot. Anstalten, Vereine, Tausehvereine, Exsiccatenwerke, Reisen etc.: Preuss. bot. Ver. (Ref.). - Bot. Verein für d. Prov. Brandenburg (Ref.). - Kgl. bot. Gesellschaft zu Regensburg (Ref.). - A. K n e u cker, Flora exsiccata Bavarica (Ref.). Bauer, E., Bryotheca Bohemica.

Personalnachrichten. - Glumaceae exsiceatae (auf dem Umschlag).

\section{Algologische Notizen.}

Von W. Schmidle (Mannheim).

(Schluss.)

16. Nostochopsis Hansgirgi Schmiale n. sp. Die Alge bildet kleine, gelblichbraune, vallertige, feste, a usgebreitete, rundiche Lager an feuchten Felsen. Die Fäden wachsen aufrecht parallel, meist gerade; sie sind wenig oder nicht verzweigt, ann Grunde 1,6 ! dick, cylindrisch mit undeutlichen Scheidewänden, nach oben sich bis auf $2-3 \mu$ verdickend, wobei die Zellen schwach torulös werden mit langelliptischer oder cylindrischer Gestalt mit abgerundeten Enden, mit gelblichgrünem, schwachgefïrbtem Kellinhalt mit hyalinen, später, wenm sie nicht verfliessen, gelblichen, oft etwas rauhen Scheiden; die Zelllänge beträgt 5-6 4 . Grenzzellen sind häufig sowohl intercalar als seitenständig. Die ersteren sind lang- 
clliptish oder meistens beiderseits zugespitzt, $5 \mu$ hreit und ca. $8-10 \mu$ linns, an den knden meist mit vordicktem Knöpfuhen, hyalin. Die seitenständigen sind rund oder halbkroisförmig, ca. 5) fe dick, der Mitte

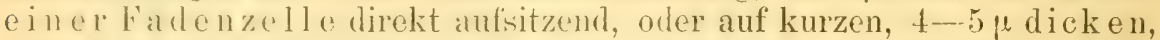

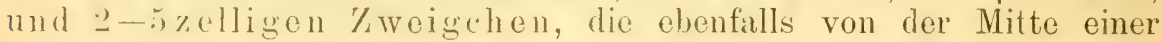
Fatenzelle abguhen, nicht selten sind die Grenzzellen zu zweit am Ende derselben.

18.X.95.

Auf fuchten Folsen zwischen Neral und Matheran bei Bombay;

Von $\Lambda$. lobatus stark verschieden.

1\%. Tolypothrix (IIrssullia) C'eylomica Schmiale n. sl). Die $\lambda$ lge bildet auscelneitete, grime, dünne, sehr weiche und zerbrechliche Leberzïge a uf Holz. Die Fäden sind in denselben parallel aufrecht gerichtet, meist gerade oder wenig gekrimmt, sehr selten verzweigt und dann stets einfach mit kuzen Seitenzweigen, die in spitzem Tinkel abgehen an Grunde einer Grenzzelle, unten 12 $\mu$ dick und sich his auf \& $\mu$ verschmälernd. Die Scheiden sind oft etwas verklebt, sehr zerbrechlich, am Grunde des Lager's leer, gelb oder jung hyalin, anssen sehr rauh, d ünn und nur an der Basis etwas dicker, muscrelichtet. Die 'lrichnme sind stark blaugrün, mit homogenem Inhalt, oben stets cylindrisch, in den älteren Partien dann und wamn schwach torulös; die scheidewände undentlich. Die Zellen sind so lang als breit, oder um die Hälfte kürzer oder länger. Heterocysten sind häutig, gelb, in der Länge und Gestalt sehr variabel.

Ceylon bei Colombo am Mont Lavinia auf altem Holz.

1\%. scytomema maculifomis schmidle n. sp. Die Alge bildet $1-3 \mathrm{~mm}$ grosse, runde, schwarze Flecken auf Baumblattern. Jieselhen bestehen aus vielfach gewundenen und verflochtenen, horizontal kriechenden Fäden; deren Enden aufgebogen sind und die kurze, höchstens $200 \mu$ lange, unverzweigte, meist

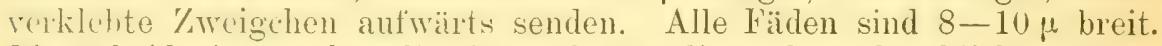
Die scheide ist stark gelb, diinn, fest, anliegend, zerbrechlich, an den Enden der anfsteignden \% weige meist verdickt und schwarzhra 1111 (der Faden hat dort uine Breite bis zu 12 «), das Trichom ist lıalgriun, kïrnig, mit schwer sichtbaren Scheidewänden, cylindrisch, die \%ollen quadratisch oder länger oder kürzer als breit, die HeterocYsten häufig. hyalin, in Länge und Zellform variabel. Die kurzen $\mathrm{Z}$ weige gehen stets doppelt ab.

Mahubeleshwar auf Blättern; 25.X.95.

19). Aurbuenu Mansgivgi Schmialle m. s1). Fäden einzeln. gekrïmmt, in und aul dem schleime einer Nostoc, an den Enden verschmälert, sterile \%ellen 5 / breit, isodiametrisch, oder 2 -3 mal länger (selten bis 1/: kïrzer), fast rechteckig, mit abgerundeten Ecken oder fassförmig, oder kugelig, mit diumer, anliegender, hyaliner Scheide und blaugrünem, homogenem, syïter liörnigem Inhalt. Sporen zu vielen gereiht, mit centrifugaler Entwickelung, von den Heterocysten meist durch eine oder '2 vegetative Zellen getrennt, mit gelbbrauner, fester, glatter Zellhaut, meist fast rechteckig, oder fassförmig, in der Länge sehr variabel (1/2-4 mal länger als breit), zuletzt bis $8,5 \mu$ dick.

An Baumrinde bei Matheran-Bombay; 16.X.95. 
20. Phommilinm Hansgingi Schmialle $\pi$. sp. Die Alge bildet getrocknet feste, spröde, schwarzo oder blan-granschwarze, fencht in Schichten sich ablösende Ueberzüge von der Dicke eine's ('artons. Dieselben bestehen aus dicht aneinandergelagerten, parallelen, meist geraden, langen, 12-16 p breiten Fäden, die durch die verschleinten, mit Chlor-Zink-Jod violett gefärbten, ca. $2 \mu$ dicken, nicht geschichtetrn, zuerst hyalinen, im Alter stark gelbbramen Scheiden verhunden sind.

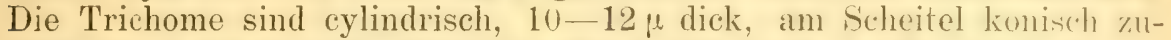
gespitzt, nicht geköpft, blaugrün, die \%ellen kurz, (1-2u lang), rechteckig, mit homogenem oder schwach granuliertem Inhalt; dic Hormogonien sehr kurz, wenigzellig.

In ausgetrockneten Sümpfen bei Elphinstone-Rond und Mahlilakshmi; 13. u. 14.XI.93.

21. Scytomemer Hansgingi Schmirlle \#. sp. Die Alge bildet schwarze Gewebe auf Baumrinde. Die Fäden sind kraus, verhogen, oft winkelig gebogen, kurz, zerbrechlich, wenig rerzweigt mit kurzen, meist einzeln, seltener zu zweit abgehenden Zweigchen, niederliegeind oder aufsteigend, $12-18 \mu$ (meist $16 \mu$ ) hreit, an der spitze unverschmälert, breit abgerundet und oft vorher gebogen Die s'cheiden sind diinn, stark bramngelb, zerhrechlich (an alten Fiiden oft rerdoppelt, eng anliegend. Die Trichome cylindrisch, 10 - 16 is dick, die Tellen rechteckig, äusserst kurz (2-4 le lang, selten bis $6 u$. 8 k), mit blaugrïnem, feinkörnigem Inhalt. Die Endzelle halbkreisförmig oder ein grösseres Kreissegment bildend, heller, gelblich, in keincr oder doch in einer sehr feinen, blassfarbigen sheide steckend. Grenzzellen zerstrent, kurz, abgerundet, so breit wie die Trichome, gelblich.

Auf Baumrinde bei Poona; 20.X.95.

22. Chantransia pulvinatr schmialle r. sp. Bildet ansgebreitete Räschen auf Steinen. Der Thallus ist zweigestaltig. I I untere Teil bildet einen sehr festen, hautartigen. 2010-3110 / dicken [' eberzug. welcher aus eng geflochtenen, reichverzweigten, in verschicdener Richtung wachsenden, stark torulösen, dickhäutigen, 8-16 u dicken, ans runden oder länglich runden Zellen zusammengesetzten Fïden besteht, welche im Quer- und Längsschnitt ein pseudoparenchymatichen (iewele bilden. Aus diesen sprossen zweitens (oder sie gehen in solche ïher) ca. $1 \mathrm{~mm}$ lange, unten 8 ॥ breite, aufwärts sich wenig verschmälermde. an der Basis reich verzweigte, haarlose tüden reichlich hervor, mit cylindrischen, 4-8 mal so langen als breiten Zellen und hyaliner oder unten gelblicher Nembran. [rie Zweige sind meist anliegend. Die Sporen sind vereinzelt auf kurzen seitenïstchen, eirund oder rumi und 10 - $12 \mu$ gross. Der Inhalt aller Zellen ist rötlich violett.

Auf Steinen im Wasserfall von Ponsonby-Spring; 16.X.85.

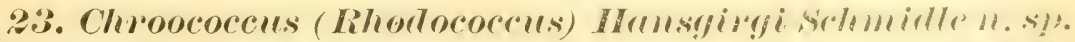

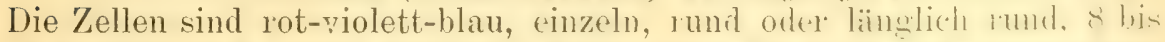
$12 \mu$ gross, mit düner, hyaliner, anliegender \%ellhaut und homerenem Inhalt. In der Zelle entstehen mehrere Tochterzcllen, wehehe dureh das Zerfliessen der Mebmran frei werden.

Auf Schneckenschalen in einem See in der Stadt Poona. 


\section{Ist Draba Thomasii Koch eine gute Art?}

Von L. Gross-Nürnberg.

(Schluss.)

Tnsere kurzen Untersuchungen führten also zu einem in jeder Hinsicht negativen Resultat: wir konnten keine der beiden Arten scharf abgrenzen.

Ergebnisse:

1. Schon Ehrhart nannte seine Art bezeichnend confusa.

2. Auch De Candolle belegt eine hierhergehörige Draba mit diesem Namen.

3. Hoppe verwechselt Diaba confusa Ehrh. mit Diaba confusa DC.

4. Diese Verwechslung geht in die Koch'sche Synopsis iiber.

5. Koch entdeckt den Fehler und stellt seine Dirür Thomasii auf.

6. Die Kochischen Diagnosen lauten 1844 anders als 1838 u. 1846.

7. Die in den Diagnosen gegebenen Merkmale erweisen sich nicht als konstant.

Im Hinblick auf diese Ergebnisse war ich bereits nicht mehr im Zweifel, ob Diuba Thomasii Koch als gute Art gelten kann oder nicht. Da kam mir vor kurzer Zeit das mir fehlende 3. Bändchen (Supplement) der Hausmann'schen Flora in die Hand, in welchem ich folgende Bemerkung las: ") "Diaba incance und Thomasii sind ganz sicher ***) nur 2- oder 1 jährige Exemplare derselben Art und kommen am Schlern meist nebeneinander vor." Die als Ueberschrift dieser kleinen Abhandlung gewïhlte Frage ist demmach von Hausmann schon vor vielen Jahren mit Entschiedenheit verneint worden. v. Hausmann besuchte den Schlern von seiner Vaterstadt Bozen aus zu wiederholten Malen, er sah unsere Pflanze an Ort und Stelle, wie aus einer oben angefïhrten Bemerkung hervorgeht, und war sonach viel eher in der Lage als Kioch, den wahren Sachverhalt zu erkennen. Denu letzterer hatte vermutlich nur getrocknetes Material vor Angen und wir diiffen daher nicht mit ihm rechten, dass el nicht zur richtigen Darstellung der immerhin verwickelten VerhäItnisse gelangen konnte. Da aber der Pietät gegen einen grossen Toten nicht die Kraft innewohnen kamn, einen erkannten Irrtum für immer beizubehalten, dïrfen wir die $\Lambda$ rten Draba incana $L$. und Inabu Thomasii Korh in eine einzige zusammenzichen, deren Diagnose mit möglichster Anlehnung an Koch'sche Ausdruilisweise etwa zu lauten hat:

\section{Draba incana L. erweitert.}

Pflanze ein- bis vielstengelig; ***) Stengel beblaittert, verlängert, einfach oder ästig, Aeste oft schon von Grund an bogig aufsteigend; unfruchtbare Blätterbiischel fehlend oder doch nur in geringer Zahl vorhanden; Blätter ganzrandig oder gezälnt; die wurzelstïndigen rosettig, lanzettlich, die oberen stencelständigen eiförmig bis länglich-lanzettlich; Blätter wie die ganze l'flanze von Sternlaaren mehr oder minder grau; Schötchen lïnger als das Blïtenstielchen, Haumig oder kahl, flach oder schiefgedreht. $\odot \mathrm{u}$. $\bigcirc$. Rauhe, steinige Stellen der Alpen.

Mai(?)-Juli.†) Bth. weiss.

*) pag. 1405. - **) bei Hausmann nicht gesperrt gedruckt.

***) luasst sich also nicht gut in der Sektion Holarge's D('. unterbringen, noch weniger freilich in einer andern Draba-Sektion.

†) Nach Koch Mại-Juni (!). 
Aendert ab:

x. contorta Ehrh. (= Iegitima Lindbl.) mit schiefgedrehten, kahlen Schötchen.

3. confusa Ehrh. (= hebecarpa Koch) mit schiefgedrehten, flaumigen Schötchen.

$\%$ Thomasii Koch (= confusu DC. $)^{*}$ ) mit flachen flaumhaarigen Schötchen.

\section{Phaenologische Plaudereien aus der Innsbrucker Flora.}

Von Dr. Jos. Murr (Trient).

Zu den Haupttugenden eines wackeren Phaenologen gehürt das Heissige Aufzeichnen des jedesmaligen Datums. Leider fehlte mir diese l'ugend wie manche andere seit jeher ziemlich gründlich. Dennoch dürte nachfolgende fichilderung manchem Leser, hesonders den mit den klimatischen Verhälnissen der Alpenländer weniger Vertrauten, von Interesse sein. 'Lu Signatur des Klimas von Innsbruck $(570 \mathrm{~m} \mathrm{s.} \mathrm{m.,} \mathrm{8.} 10$ C. Jahresmittel) gehört im allgem einen ein strenger, bis gegen Anfang April danemder Nachwinter (man vergl. die für die tieferen Lagen allerdings übertreibende Jordtiroler Banermegel: "Pauli Bekehr [25/1], der halbe Winter hin, der halbe her"), ein mbestindiger Frühling mit ofters noch in der 3. und t. Aprilwoche, ja selhst in der 1. Woche des Mai (1886 z. B. am 5. Mai) eintretenden starken Schneefallen, ein niederschlagsreicher Hochsommer und herrliche, klare Herlsstage bei ofter bis in den Dezember hinein schneetweien Gebirgskïmmen. Diese Regehn erleiden nun freilich die engreifendsten und seltsamsten Ablenkungen und zwar einerseits periodische, andrerseits anscheinend ganz willkürliche.

So' fällt der Beginn meiner bot. Sammelthätigkeit in das Ende einer Art Eiszeit mit schneereichen Wintern. Ich machte damals zuerst mit meinem Vater es war nach meiner Erinnerung der 18. August 1877, also ein Datum, an dem in der gegenwärtigen Periode die Gebirgstlora bereits stark im Niedergang begriffen ist - die Partie durch die Kranebitter Klamm auf die Zirler Mähder

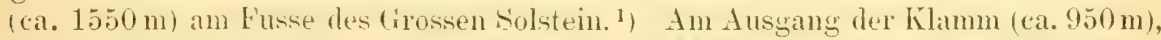
sowie in der bachrunst umittelhar unter den Mähdern lage' tiefe, mehrjührige, firnartige Schnecmassen; dafür aber glichen die freieren Plätze der Buchengestrüppe üher der Klamm und eben-o der tiefere Theil der Mähder einem frischhlühenden, dichten Blumengarten von Orahis maculuta, Gymmalenier romopra und adoratissima, Convallaria maialis, Lilium Martagon, Thalictmum aquilegaifolium u. S. w. Die achtziger und meist auch die neunziger Jahre brachten schneearme Winter nit nachfolgender rascher Vegetationsentwickelnug. Besnchen wir heiläufig 20 Jahre später, etwa Mitte August 1898, die Zirler Mähder. Von alten Schneelagern in der Klamm in lingst jede shur vergangen; aber anch die blumige Wiese der Bergmähder hat sich in magere Hutweide verwandelt, der kaum da und dort ein verspäteter Aster alpims, oder ein aufblühender Busch von Gentiana Stumiana zu einigem Schmucke gereicht. Die Alpenwirtschaft am Schoberwald steht verlassen und zerfallend da. Was hat eine linge heihe von trockenen Wintern gethan!

Und gerade jene schneereichere Periode hatte öfter frähe, schöne Frühlinge, wie sie seitlem kaum mehr wiederkehten. So standen am 28. Februar 1878 am Nagelftuhebruch über Innsbruck bereits Rannnculus bulbosus, Liodim cicu-

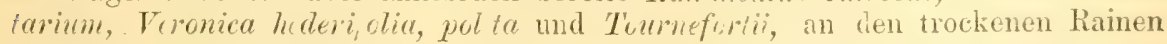
confusa Ehrh.

*) confusa $D C$. muss troz Priosität hinter Thomasii Koch zu1 ü ktreten wegen

') Ich habe diese später von mir oftmals wiederholte Partie eingehend geschillert in dem Aufsatze: Eine Umgehung des Hölenberges bei Innsbruck (Dentsche bot. Monabschr. 1886, S. $150-152,162-171$ ). 
auch Carex humilis in schönster Blüte ${ }^{1}$ ); am 10. April 1879 prangten in der kühlen Mühlaner Ǩlamm allenthalien die rötlichweissen Dolden der Valriani. tripteris und selbst die buschigen Wiesenhänge des anliegenden Arzler-Plateaus (cia. $850 \mathrm{~m}$ ) schmückte sich gleichzeitig mit einer Fülle von üppig entwickeltem, zumteil halbgefülltem Ramunculus montains.

(Schluss folgt.)

\title{
Uebersicht über die bisher in der Umgebung von Guben in der Niederlausitz beobachteten Leber-, Torf- und Laubmoose.
}

\author{
Von Otto Will
}

Seit dem Jahre 1894 heobachtete und sammelte ich Moose in Kreise Guben und in einigen Nachlarget,ieten, wie dem Crossener und Kottbuser Kreise. Den Gubener Kreis habe ich, soweit es die Ferienzeit zuliess, zu verschiedenen Malen durchwandert und zwar nach den verschiedensten Richtungen. Leider verbietet mir einerseits mein schwacher Gesundheitszustand und andererseits mein eigent. liches Berufsstudium (Sprachen und Geschichte), genügende Zeit auf die Untersuchung der gesammelten Funde zu verwenden. Hern Warnsturf (Neuruppin) sowohl, wie Herrn Oberlehrer Limpricht, in dessen Hause ich während meines A identhaltes in Breslau ständig verkehren durfte, hin ich deshalb für fremdliche Bestimmurgen meiner Fumbe, sowie für gütige Ratschläge zu grösstem Danke verpflichtet. Die wichtigeren Arten hat Limpricht alle gesehen. Ausserdem lagern bei mir noch grössere Mengen unhestimmten Naterials, welche ich im nächsten Tahre aufzuarbeiten gedenke.

Was das durchsuchte Gebiet selbst anlangt, so ist darüber nicht viel zu sagen. Es ist zumeist eben, neigt längs der Flussläufe zur sumpfhildung, welche meist in nicht zu grosser Entfermung, oft ganz plötzlich, durch steilaufisteigende (bis $11: 3 \mathrm{~m}$ im hochten Punkte, (ler (iubener "Achnecke") Hügel am weiteren Vordringen gehindert wird. Dies ist der Fall bei der Neisse, welche von Guben aus bis zur südgrenze des Kreises von eine' Hügelkette am linken Cfer begleitet wird; bei der Lubst (rechter Zufluss der Neisse) und der Schlaube (Abfluss der Seen an der N.W.-Grenze des Kreises) finden wir diese Hügel auf der rechten Seite. Meist gehen dieselben dann, wenn man es so nennen darf, in Hochebene iiber. Welche für den bryologen wenig interessant ist, auscer gerade an stellen mit altem Waldbestand, wie zwischen Fünfeichen und Bremsdorf. Den Wald zwischen diesen Orten und Diehlo nenne ich kurz das "Hörnchen", wie es auch im Volkmunde heisst. Ith hahe daselhst schr gute Funde zu verzeichnen gehabt. Diese (iegend, sowie das an Naturschönherten und auch selteneren Hoosen so reiche Schlaubethal habe ich zweimal, das letzte Mal im Sommer 1897 besucht.

In der Nähe der Stadt Guben sind von bryologisch wichtigen Punkten zu nennen: Die Waldungen und Ausstiche hinter Germersdorf, die sog. „Drenziger Schworz"; ferner die diestr mit Bezug aut die Bodenheschaffenheit sehr ähnlichen "Kaltenborner Berge" im S.IV. der Stadt bis Kerkwitz und Atterwasch, und schliesslich die jetzt nicht mehr benützten, früheren Militär-Schiessstände an der Neisce. Letztere zeigen mit ihrer Ahwechslung zwischen trodienem Heideland, Wald nud Sumpf eine der reichsten Floren des ganzen Gebietes. In weiterer Entfernung ron der stailt wire zu nemmen: Der Stadtforst (eine Meile entfernt),

1) Kürzere dureh den Föhn hervorgezauberte, Frühlungsahnungen* mit Blumen-

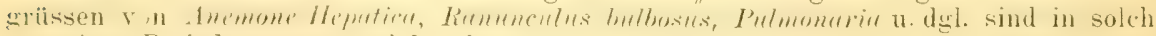
giunstigen Perioden manchmal bereits um den 20. Januar herum zu verzeichnen. Hierher und nicht als Nachblüte vom Herbste ist wohl clie ros.ifarben blühende Potentilla micrantha (Erythrismus infolge des Frostes!) zu bezielien, welche Evers am 11. Februar 1885 bei $1200 \mathrm{~m}$ im Kalkgebirge über Mühlau sammelte. Bekamntlich zeigt sich gerade in dieser (iebirgszone ter Ë̈hn besonders wiksam; ja bis anfangs februar ist die 'I'emperatur hier oben tiberhaupt milder, als in der mehr weniger nebligen 'Thalsohle. 
der Fasanenwald bei Neuzelle (ca. 2 Meilen) und das Gebiet der T'eiche bei Bomsdorf und Schwertzko. Ein Ausflug nach diesen Stellen ist fast reizender als ins Schlaubethal.

Zum Schluss könnte ich noch erwähnen, dass ich im ganzen nur 2 mit Moos bekleidete erratische Blöcke angetroffen habe, einen im Hörnchen, den andern im Schlaubethal.

Von meinen Funden ist Amblystegium rigescens (Limpr.) eine nov. spec., dagegen sind: Octodiceras Julianum, Pogonatum aloides $P$. B. var. minimum (Crome) Limpr., sowie Jungermannia quinquedentata Web. neu für die Mark.

\section{a. Lebermoose.}

1. Riccia giauca (L.). Auf Stoppelfeldern häufig; am Bärbusch; Gubinchen.

2. $T$. crystallina $L$. Auf feuchtem Lehmboden nicht selten.

3. R. fluitans L. forma vulgaris. Zwischen anderen Moosen im Sumpf hinter den Schiessständen.

f. terrestris: In Ausstichen bei Gubinchen; auf Schlamm an Teichufern bei Bomsdorf.

4. Anthoceros punctatıs L. In feuchten Gräben vor dem Bärbusch.

5. A. laeris L. Am Rande von Stoppelfeldern, vor dem Bärbusch.

6. Marchantia polymorpha L. Gemein.

Eine bis $4 \mathrm{~cm}$ lange Form mit fast regelmässiger Verästelung fand ich an Balken unter Wasser bei der Seemühle 1896.

7. Metzgeria furcata (L.) N. v. E. Häufig an Laubbäumen: Bärbusch, Turnplatz, Kaltenborner Berge, Schlaubethal.

(Forts. folgt.)

\section{Bemerkungen zu den ,Gramineae exsiccatae“6*)}

von A. Kneucker.

I. U. II. Lieferung 1900.

Nr. 1. Anthoxunthum orloratum L. Sp. pl. ed. I, p. 28 (1753).

Auf Wiesenboden in der Nähe von Karlsruhe in Baden; sandiges Diluvium. Begleitpflanzen: Alopecurus pratensis L., Bromus ereetus Huds., Festuca pratensis Huds., loa pratensis L., Veronica chamatedrys L., serpyllifolia L. Ca. $117 \mathrm{~m}$ ü. d. M.; 10. Mai 1899。

leg. A. Kneucker.

Nr. 2. Aristidu roenulescens Dest: Fl. atl I, p. 109 (1798).

Auf sehr trockenem, felsigem, eisenhaltigem Kalkbode n bei Malaga in Spanien nahe dem Neere. Begleitpflanzen: Piptatherum multiflorum Beauv., Dactylis glomerata L., Hispanica Reth., Ceratonia siliqua L., Lavandula stoechas L. et multifida.

Ca. $100-150 \mathrm{~m}$ ü. d. M.; April 1890. leg. E. Reverchon.

Nr. 3. Aristida Forshihlei Tsch. Flora 1).506 (18:36); Boiss. Fl. Orient. V, p. 496 (1881).

Auf den Dünen von Beirut in Syrien. Begleitpflanzen: Panicum repens L., Euphorbia Paralias L., peplis L., Thymelaea hirsuta L., Salsola kali

*) Die Revision, bezw. Bestimmung des ausgegebenen Materials und teilweise auch die Zusammenstellung der Litteraturnachweise wurde von Herrn Prof Ed. Hackel in St. Pölten gütigst uibernommen. Ausserdem wirkte noch Herr Schriftsteller W. Lackowitz in Berlin bei Durchsicht der Korrekturabzüge freundlichst mit. Die Schedae sind von dem unterzeichneten Herausgeber selbst zusammengestellt. Da die Anfzählung aller bekannten Synonyme jeder Art zu weit führen würde, hat sich der Herausgeber erlaubt, nur die wichtigsten Synonyme aufzunehmen. In der Anordnung der Genera wurden die Genera Siphonogamarum ad systema Englerianum conseripta * von Dr. v. Dalla Torre und Dr. Harms, wopon erst Fascikel I erschienen ist, zugrunde gelegt. Da der unterzeichnete Herausgeber die Druckkosten der Bemerkungen trägt, kommt die durch die letzteren verursachte Erweiterung des Umfangs der Zeitschrift den Abonnenten gratis zu gut.

A. Kneucker. 
L., Convolvulus secumdus Desr., Ipomaea littoralis L., Echium sericeum Tahl v. hispida Boiss., Statice rorida $\mathrm{S}$. et $\mathrm{Sm}$.

Ca. $30 \mathrm{~m}$ ü.d. M.; 12. Okt. 1899. leg. Ernst Hartmann.

Nr. 4. Stupa gigamter Lay. var. Lregascae Hack: = St. Lagascre K. et Sch. Syst. II, p. $333(1817)=$ St. pubescens Lag. el. p. 3 $(1816)=$ St. gigantea Lay. $f$. pubescens Hack. in "Oesterr. bot. Z." Nr. 4 (1877).

Auf trockenem, schattigem Kalkboden bei Origuela in der Provinz Teruel in Spanien mit Pinus Laricio Poit. und silvestris L. begleitpflanzen: Aera multiculmis Dum., Agrostis olivetorum Gr. et Godr., Brachypodium festucoides Lk., Armeria plantaginea Wlld., Evax Cavanillesii Ry., Arenaria Loscosii Tex.

Ca. $1400 \mathrm{~m}$ ü. d. M.; Juli 1895. leg. E. Reverehon.

Nr. 5. Stupa Reclonshii Trin. in Spreng. neue Entd.II, p. 53 (1821); Uiss. de gram. unifl., p. $183(1824)=S t$. Caragana Irin. in Act. Petrop., p. 74 (1829) = Lasiagrostis Caragana Trin. et Rupr. in Act. Petrop, p. 90 (1842).

Auf trockenem Boden bei dem Dorfe Unal im Kaukasus in Russland in der Landschaft Ossetia; B e gle it p f l anzen: Astragalus Caucasicus Poll., Austriacus L., Caragana grandiflora DC., Andropogon ischaemum L., Linum nodiflorum L., Teucrium polium L.

Ca. $1500 \mathrm{~m}$ ü. d. M.; 26. Juli 1899.

leg. B. Marcowicz. gefallen.

Die Exemplare sind der Fruchlreife nahe, daher die meisten Grannen ab-

Nr. 6. Milimm effusmm L. Sp. pl. ed. I, p. 61 (1753) $=$ M. remale Van Hail. Syn., p. 32 (1821).

Auf diluvialem șandboden in Durlacher Wald (Laubmischwald) bei Karlsruhe in Baden. Begleitpflanzen: Luzula campestris DC. v. multiflora Celak., nemorosa C. A. Meyer, var. rubella Gaud., pilosa Wlld., Carex pallescens L., pilulifera L., brizoides L., silvatica Hude, Anthoxanthum odoratum L., Melica uniflora Retz etc.

Ca. $117 \mathrm{~m}$ ü. d. M.; 17. Mai 1899.

leg. A. Kneucker.

$\mathrm{Nr}^{2}$ 7. Heleochlor alopecuroides Host Gram. I, p. 77 (1801) $=$ Crypsis alopecuroides Schrud. Fl. germ. I, p. 167 (1806).

Auf Löss am Ufer und im Inundationsgebiet der 'Theiss in Ungarn. Begle it p flanzen: Heleochloa schoenoides L. (Host), Panicum grus galli L., Aster l’annonicus Jacq., Cenopodium polyspermum L. etc.

Ca. $80 \mathrm{~m}$ ü. d. M.; Ende September 1899.

leg. Lajos Richter.

Nr. 8. PMlemm Iochmeri Vibel Prim. H. Werthem, p. 125 (1799).

Auf dem sandigen Hochgestade zwisch. Linkenheim und Leopoldshafen in Baden; Diluvium. Begleitptlanzen: Avena pubescens Huds., Havescens L., Arrenatherum elatius $\mathrm{H}$. et K., Brachypodium pinnatum P.B., Briza media L., Koeleria cristata l'er's. ('arex contigua Hoppe, Equisetum hiemale L., Chondrilla juncea L. etc.

Ca. $115 \mathrm{~m}$ ü. d. M.; 8. Juni 1899.

leg. A. Kneucker.

Nr. 9. Phleum arenuium L. Sp. pl. ed. I, p. 60 (1753).

Auf dem sogenannten Mombacher, Sand" bei Mainz a. Rh. Begleitpflanzen: Poa Badensis Hnke., Koeleria glauca DC., Alsine Jacquini Koch, viscosa Schreb., Anthericum liliago L., Alysum arenarium Gmel., Helianthemum fumana Mill., Erysimum virgatum Rth. etc.

Ca. $130 \mathrm{~m}$ ü. d. H.; 11. Juni 1899.

leg. M. Dürer.

Nr. 10. Phleum paniculatum Huds. Fl. angl. ed. I, p. 23, 26 (1762)= P. asperim Jacq. Coll. I, p. 110 (1786); Vill. Fl. Delph. II, p. 61 (1787). 
Auf Brachfeldern des 'Turmbergs bei Durlach in Ballen; M I schelkalk. Begleitpflanzen: Bromus sterilis L., tectorum L., Agropyrum repens Beauv. v. Vaillantianum Schreb., Brachypodium pinnatum P.13., Poa compressa L., Crepis foetida L., Cancalis daucoides L., Fumaria officinalis L., Galium aparine L., Sinapis arvensis L. etc.
Ca. $245 \mathrm{~m}$
ü. d. M.; 14. u.
u. 29. Juni 1899.
leg. A. Kneucker.

Nr. 11. Alopecurus H'rtensis L. Sp. pl. ed. I, p. 60 (1753).

Auf Wiesenboden, Alluvium, zw. (Karlsruhe) Mühlburg und der Appen. mühle in Baden. Begleitpflanzen: Arrhenatherum elatius II. et K., Dactylis glomerata L., Poa trivialis L., Anthoxanthum odoratum L.

Ca. $114 \mathrm{~m}$ ü. d. M.; 7. Juni 1899.

leg. A. Kneucker.

Nr. 12. Mibora cerma Beame. Agr., p. 167 (1812) = Arprostis minima L. Sp.pl. ed. I, p. $63(1753)=$ Chamagrostis minima Borkh. Fl. v. Katzenellenb. $(1796)=$ Mibora minima Desv. Fl. d'Anj., p. 48 (1827).

Auf sehr sandigen Aeckern und teilweise auf Flugsand in der Nähe des Rheinhafengebietes bei Rheinau, unweit Mamneim. Begle itpflanzen: Holo. stemm umbellatum L., Myosotis arenaria Schrad, versicolor Sm., Cerastium semidecandrum L., Teesdalea nudicaulis R. Br., Veronica praecox All., agrestis L., triphyllos L. ete.

Ca. $100 \mathrm{~m}$ ü. d. M.; 11. Mai 1899. leg. A. Kneucker u. H. Zahn.

Nr. 13. Agrostis Reuteri Boiss. Voy. II, p. 645 (1845).

An feuchten, bewachsenen Stellen bei Kerrata in Algier (Afrika), ca. $50 \mathrm{~km}$ vom Meere entfernt; Kalkboden. Begleitpflanzen: Agrostis alba L., vulgaris With., Carex glauca Murr., distans L. etc.

Ca. $800 \mathrm{~m}$ ü. d. M.; Juli 1897.

leg. E. Reverchon.

Nr. 14. Agrostis tmuculatu 1Pul. Fl. it. I, p. 185 (1848).

An trockenen Orten bei Guadalaviar in der Provinz 'Teruel in Spanien, $80-100 \mathrm{~km}$ vom Meere entfernt; Kalkboden. Begleitpflanzen: Agrostis vulgaris With., Anthoxanthum odoratum L., Aera multiculmis Ilum., Thrincia hispida L.

Ca. $1400 \mathrm{~m}$ ü. d. M.; August 1895.

leg. E. Reverchon.

Nr. 15. Calamagrostis littorere Beame. Agrost., p. 15 (1812); fl. fr. V, p. $255(1815)=$ Arundo littorea Schrad. Fl. germ. I, p. $212(1806)=$ Calamagrostis pseudo-phragmites (Hall.f.) Bmy. En. III, p. 211 (1816).

Unter lichtem Gehüsch am sandigen linken Rheinufer bei Maximiliansau in der bayer. Pfalz; Alluvium; teilweise aber auch an freien Stellen zw. dem Pflaster der Uferböschung. Die Spelzen der Exemplare des ersteren standortes sind meist bunt, während die ganze Inflorescenz der Exemplare des letzteren Standortes ein rotbraunes Kolorit hat. Begle it p flanzen: Phalaris armulinacea L., Poa compressa L., palustris L., Festuca arumdinacea Schreb., Equisetum trachyodon A. Br., Sinapis cheiranthus Koch ete.

Ca. $106 \mathrm{~m}$ ü. d. H.; 12. Juli 1899.

leg. A. Kneucker.

\section{Nr. 16. Calamagrostis littorea Berur. f: inter f: typicam. et laxam (Host) Hacliel.}

An steinigen Stellen in der Nähe des Ardon im Kaukasus in der Landschaft Ossetia. Begleitpflanzen: Rubus caesins L., ('henopodium botrys L., Epilobium Dodonaei Vill., Equisetum telmateja Ehrh., Eragrostis minor Host etc.

Ca. $630 \mathrm{~m}$ ü. d. M.; 15. Aug. 1899. leg. Marcowicz. 
Nr. 17. Calamegrostis littorea Beamr. var. Taxa (Host) Gram. IV, p. 25 (1809).

Fenchtes Ufergebüsch des linken Rheinufers bei Maximiliansau in der bayer. Pfalz; sandiges Alluvium. Begleitpflanzen: Phalaris arundinacea L., Poa palustris L., Rubus caesius L., Equisetum trachyodon A. Br., Heleocharis tcicularis R. Br., Veronica anagallis L. ete.

Ca. $106 \mathrm{~m}$ ü. d. M.; 12. Juli 1899.

leg. A. Kneucker.

Durch lockere Rispe, tiefer eingesetzte und längere Grannen ziemlich deutlich, aber nicht spezifisch von der Hauptform verschieden.

Ed. Hackel.

Nr. 18. Calamagrostis lanceolatre Rth. Tent. fl. germ. I, p. 34 $(1788)=$ C. calamagrostis Karsten Fl. Deutschl. 2. Aufl. I, p. 383 (1895). (In „K. Richter, plant. eur.“ 1890 p. 49 sind von dies. Pflanze 13 Synonyme angegeben.)

Sumpfige Uferwaldungen des linken Rheinufers zwisch. Naximiliansau und Wörth in der bayer. Pfalz; sandiges Alluvium. Begleitpflanzen: Heleocharis uniglumis Lk., acicularis $\mathrm{R}$. Br., Carex panicea L., vesicaria L., riparia. Curt., Scirpus maritimus L., Allium acutangulum Schrad. etc.

Ca. $106 \mathrm{~m}$ ï. d. M.; 4. Juni 1899.

leg. A. Kneucker.

\section{Nr. 18a. Calamagrostis lanceolata Rth.}

Im Rönner Gehölz zw. Kiel und Preetz an sumpfigen und trockenen Waldstellen; sandiger Lehm. Begleitpflanzen: Plalaris arundinacea L., Elymus arenarius L., Fagus silvatica L.

Ca. $40 \mathrm{~m}$ ü. d. MI.; 22. Juli 1899.

le‥ E. $0 \mathrm{hl}$.

Nr. 19. Ammophila arenaria LR: H. herb. berol. I, p 105 (1827)= Calamagrostis arenaria Rth. Tent. fl. germ. I, p. $34(1788)=$ Psamma arenaria R. S. Syst. II, p. 845 (1817).

Jenseits der Festung Friedrichsort am Sandstrande des Kieler Hafens häufig. Begleitpflanzen: Ammophila Baltica Lk., Calamagrostis epigeios Rth., Elymus arenarius L.

Ca. $1 \mathrm{~m}$ ü. d. M.; 14. Juli 1899.

leg. E. $0 \mathrm{hl}$,

Nr. 20. Ammophila arenaria LR: $>$ Calamagrostis epigeios Rth. (LT..) f. subarenavia Marss. Fl. v. Neu-Vorp., p. $563(1869)=$ Ammophila Baltica Lk. f. subarenaria Marss. Fl. v. Neuvorp., p. 563 (1869).

Auf dem östlichen und westlichen Sandstrande des Kieler Hafens. Begleitpflanzen: Calamagrostis epigeios Rth., Ammophila arenaria Lk., Elymus arenarius L.

Ca. $1 \mathrm{~m}$ ü. d. Mr.; 14. Juli u. 6. Aug. 1899. leg. E. Ohl.

Nr. 21. Aluevel spica renti P. B. Agr., p. 7 (1812) = Agrostis spicect venti $L$. Sp. pl. ed. I. p. 61 (1753).

Auf sandigen Brachäckern südlich von Karlsruhe. Begleitpflanzen: Agrostis vulgaris With., Hordeum murinum L., Melilotus officinalis Desr., Hypericum perforatum $L$. etc.

Ca. $117 \mathrm{~m}$ ü. d. M.; 18. Juli 1899.

leg. A. Kneucker.

Nr. 22. Aere caryolly yllen L. Sp. pl. ed. I, p. 66 (1753) = Avene. caryophyllea Weber in Wigg. Prim. fl. hols., p. 10 (1780).

Auf dem sandigen Heideboden des Forchheimer Schiessplatzes unweit Karlsruhe in Baden; Diluvium. Begleitpflanzen: Aera praecox L., Myosotis versicolor Sm., Veronica verna L., officinalis L., serpyllifolia L., Cerastium semidecandrum L., Moenchia erecta Fl. d. Wett. etc.

Ca. $116 \mathrm{~m}$ ü. d. M.; 29. Mai 1899.

leg. A. Kneucker. 


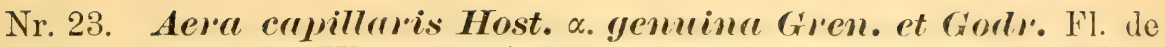
Fr. tom. III, p. $505(1855-56)=$ Avena capillaris $M$. et $K$. in Röhl. D. fl. I, p. 573 (1823) = Aira elegans Willd. in Gaud. Agr. helv. I, p. 130 (1811).

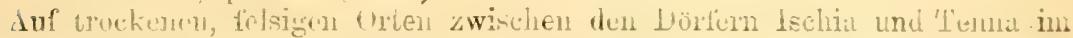
Jal Sugana in Südtirol; Porphyr. Begleitpflanzen: Aera caryophyllea L., Trifolium striatum L., Filago minima Fr., Galium Parisiense L., Pedenontanum All. Ca. $530 \mathrm{~m}$ ü. d. M.; 15. Juni 1899.

leg. Enrico Geimi.

Nr. 24. Aera m.recox L. Sp. pl. ed. I, p. 65 (1753) = Avena praecox Beauv. Agr., p. 89 (1812).

Trockene und sandige Waldränder auf dem linken Lauterufer zw. Kapsweier und der Bienwaldmühle in der bayer. Rheimpalz; Diluvialsand. Begleitpflanzen: Pteridium aquilinum Kuhn, Aera caryophyllea L., Festuca sciuroides Rth., Thesium intermedium Schr. ete.

Ca. $120 \mathrm{~m}$ ü. d. M.; 1. Juni 1899.

leg. A. Kneucker.

Nr. 25. Weingaertmerir canescens Bermh. Fl. Erf., p. 51 (1799)= Corynephorus canescens Beauv. Agr., p. 90 (1812) $=$ Aera canescens L. Sp. pl. ed. I, p. 65 (1753).

Auf den sogenannten, Weissenhurger Linien" zwischen St. Remig und der Bienwaldmühle auf dem rechten Lauterufer im Elsass; Diluvialsand. Begleitpflanzen: Anthericum liliago L., Holcus mollis L., lanatus L., Lychnis viscaria L., Aera caryophyllea L. ete.

Ca. $125 \mathrm{~m}$ ü. d. M.; 5. Juli 1899.

leg. A. Kneucker.

Nr. 26. Deschampsia caespitosa P. B. \% setifolia Bischoff in Koch Syn. 1I, p $914\left(1846^{\circ}\right)=$ A. juncea Vill. H. pl. Dauph I, p. $317(1786)=$ A. setacea Pourr. Act. Toul. III, p. 307 (1783).

Auf sandigem und kiesigem Alluvium am Altrhein bei Leopolds. hafen in Baden. Hegleitpflanzen: Heleocharis miglumis Lk., Carex panicea L., riparia Curt., glauca Murr., flava L., vesicaria L., Equisetum trachyodon A.Br., Hippophaë rhamnoides L.

Ca. $110 \mathrm{~m}$ ü. d. M.; 24. Juni 1899.

leg. A. Kneucker.

Nr. 27. Deschampsia Bottrica Trin. Fund., p. $158(1820)=$ Aira Bottnica. Whlbg. Fl. lapp., p. 36 (1812).

Am Meeresufer bei Landsort in Södermanland in Schweden; Schlamm und Glaciallehm. Begleitpflanzen: Agrostis alba L. $\beta$. maritima (Lam.), Glyceria distans Whlhg., Potamogeton filiformis Pers., Zamichellia pedicellata Fr., Glaux maritima L. ete.

0,3 m ü. d. M.; 16. Aug. 1899. leg. Pastor J. G. Laurell.

Nr. 28. Trisetum oratum Pers. Syn. I, p. 98 (1805) = Bromus ovatus Cav. Jc. VI, p. $67(1801)=$ Avenc ovate Gmel. h. carlsr. (1811).

Auf felsigen und trockenen Stellen bei Origuela in der Provinz Teruel in Spanien ca. $120 \mathrm{~km}$ vom Meere entfernt unter dem Schatten von Quercus ilex L. und Q. toza Bose; Triaskalk. Begleitpflanzen: Festuca ovina L., rubra L., duriuscula L. ete.

Ca. $1600-1700 \mathrm{~m}$ ü. d. M.; Juli 1895 .

leg. E. Reverchon.

Nr. 29. Trisetum Gandinianum Boiss. Voy. II, P. 652 (1845) = Irisetum Cavanillesii Trin. ex p. in act. petr. I, p $63(1830)=$ Avena Cavanillesii Koch Syn. ed. I, p. 713 (1837).

An mehreren Stellen in der Umgegend von sion im Kanton II allis (Schweiz) z. B. bei Fully, Saillon, St. Leonhard, Granges an Wegen und Schuthalden; 
Alluvium von Fluss und Gletscher. Begleitpflanzen: 'Trigonella Mons. peliaca L., Nedicago minima Lam., Viola arenaria DC., Erophila verna E. Mey., Clypeola Gaudini 'Trachsel.

530-560 m ü. d. M.; März u. April 1899. leg. Prof. F. O. Wolf.

Nr. 30. Ventenatu dubia $\boldsymbol{F}$. Schult: Pollichia XX, XXI, p. 273 $(\mathbf{1 8 6 3 )}=$ Avena temis Moench Meth., p. 195 (1794).

Wald- und Feldränder auf der Segnitzer Spitze bei Segnitz in Unterfranken (Bayern); Muschelkalk. Begleitpflanzen: Apera spica venti Beaur. Bromus tectorum L., Potentilla argentea L. ete.

Ca. $260 \mathrm{~m}$ ü. d. M.; 8. Juli 1899. leg. Dr. G. Rostu. Dr. W is licenus. Nr. 31. Avena rersicolor Vill. Prosp. fl. delph., p. $17(1779)=A$. Scheuchzeri All. Fl. ped. II, p 255 (1785).

In den Landschaften Digoria und Ossetia im Kaukasus in Russland. In Ossetia bei der Ueherfahrtsstelle Kion auf Wiesen. Begleitpflanzen: Festuca ovina I., Vaccinim myrtyllus L., Linnaea borealis L., Astrantia helleborifolia Salisb.

Ca. $2700 \mathrm{~m}$ ü. d. M.; 4. Juli 1899.

leg. B. M a r cow icz.

Nr. 32. Avena pratensis L. Sp. pl. ed. I, p. 80 (1753).

In sandigen Kiefernwäldern zwischen Mombach und Budenheim bei Mainz in Hessen. Begle itpflanzen: Onosma arenarium W. et K., Stupa capillata I., Scorzonera purpurea L., Adonis vernalis L., Epipactis ruhiginosa Gaud., Geranium sanguineum L., Pirola chlorantha Sw., Trinia vulgaris DC., Carex supina Whlbg., humilis Leyss.

Ca. $130 \mathrm{~m}$ ü. d. M.; 11. Juni 1899.

leg. M. D ürer.

Nr. 33. Avena mubscens Huds. Fl. angl., p. 42 (1762).

Auf dem sandigen Hochgestade zwischen Linkenheim und Leopoldshafen in Baden; Diluvium. Begleitpflanzen: Phleum Boehmeri Wib, Avena flavescens L., Arrhenatherum elatius M. et K., Brachypodium pinnatum P. B., Briza media L., Koeleria cristata Pers., Carex contigua Hoppe, Equisetum hiemale L., Chondrilla juncea L.

Ca. $115 \mathrm{~m}$ ü. d. M.; 8. Juni 1899.

leg. A. K neucker.

Nr. 34. Beclemanmire erncreformis Host Gram. anstr. III, p. 5 $(1805)=$ Plutaris emeacformis L. Sp. pl. ed. J, p. 55 (175:3).

Auf feuchtem, lehmigem Boden (Alluvium) bei dem Dorfe Mytitschi im Kreise Moskau in Russland. Begleitpflanzen: Alisma plantago L., Alopecurus fulvus Sm., Heleocharis palustris R. Br., Typha latifolia L.

Ca. $140 \mathrm{~m}$ ü. d. M.; 2. Juli 1899. leg. D. Ssyreistschikor.

Nr. 35. Seslerir coemlea Ard. ur. calcaren (Opis) in Bericht. ök. H. v. Boehm., p. $492(1836)=$ S. varia Wettst. in Verh. d. zool. bot. Ges. XXXVIII, p. 557 (1888).

Auf der "Koppe" bei Eschenbach unweit Hersbruck in Nittelfranken (Bayern) auf Dolomit. Begleitpflanzen: Carex ornithopoda Willd., Polypodium vulgare L., Draba aizoides L.

$579 \mathrm{~m}$ ï. d. M.; 29. April 1899.

Nr. 36. Iíoelevir cristutu Pers. Syn. I, p. 97 (1805) \%. genuina Gren.et Godr. Fl. de Fr. III, p. $526(1855-56)=$ Aera cristata L. Sp. pl. ed. I, p. 63 (1753).

Aur̈ dem sandigen Hochgestade zwischen Linkenhein und Leopoldshafen in Baden; Diluvium. Begleitpflanzen: Phleum Boehmeri Wihel, Arena pubescens Huls., flavescens L., Arrhenaterum elatius M. et K.. Brachypodium pinnatum P. B., Briza media L., Carex contigua Hoppe, Equisetum hiemale L.. Chondrilla juncea $L$. etc.

Ca. 115 m ï. d. M.; 8. Juni 1899.

leg. A. Kneucker. 
Nr. 37. Koelerir cristata Per's. v.gracil is (I'ers.) Gren.et Gorl. Fl. de Fr. III, p. 526 (1S55-56).

Auf Heiden am Bisamberge bei Wien; Wiener Sandstein. Begleitpflanzen: Stipa pinnata Lo, Festuca pseudovina Hack, Nedicago lupulina L., Astragalus onobrychis L., Orobrychis viciaefolia Scop., Orobanche gracilis S.m., Inula ensifolia L., Jurinea mollis (L.) Rehb.

$350 \mathrm{~m}$ ü. d. M.; 29. Juni 1899. leg. Dr. Aug, von Hay eck.

Nr. 38. Melica ciliata L. $\propto$. Limnaei Hack. in Halacsy et Braun Nachtr. z. fl. v. Niederösterr., p. $19(1882)=$ M. Nebrodensis Par. Fl. pal I, p. 120 (1845). (Eine kritische Beleuchtung der Nomenklatur dieser Pflanze siehe in Hal. und Braun, Nachtr. 1. c.)

Auf dem sogenannten Gänseberg bei Wenkheim im nördlichen Teil ron Baden; Muschelkalk. Begleitpflanzen: Poa compressa. L., 'Teucrium botrys L., chanaedrys L., Tragopogon major Jacq., Dianthus prolifer L., E1phorbia cyparissias L. ete.

Ca. $230 \mathrm{~m}$ ü. d. M.; 27. Juni 1899.

leg. Arnold.

Nr. 39. Melica mutans L. Sp. pl. ed. I, p. 66 (1753).

In den aus Laubmischwald bestehenden Rheinwäldern des "Kastenwörth" bei Daxlanden in Barlen; Alluvium. Begleitpflanzen: Carex tomentosa l., strigosa Hudk., silvatica Huds., Brachypodium silvaticum R. et Sch., Orchis purpurea Huds. etc.

Ca. $107 \mathrm{~m}$ ü. d. M.; 19. Mai 1899.

leg. A. Kneucker.

Nr. 40. Melica uniflora Reț. Obs. I, p. 10 (1779).

In schattigem Buchenhochwald zwischen Ettlingen und Oberweier in Baden; Buntsandsteinregion. Begleitpflanzen: Carex silvatica Huds, pendula Huds., pallescens L., digitata L., Milium eff̈usum L., Veronica montana L., Sanicula Europaea L. etc.

Ca. $150 \mathrm{~m}$ ü. d. N.; 11. Mai 1899.

leg. A. Kneucker.

Nr. 41. Briwe maxima L. Sp. pl. ed. J, p. 70 (1753).

An grasigen Plätzen bei Abbazia im österr. Küstenland; Sandstein. Begleitpflanzen: Cynosurus echinatus L., Orchis militaris L., ustulata L., Dictamnus fraxinella Pers. ete.

Ca. 20-40 m ü. d. M.; Mai 1899.

leg. Lajos Richter.

Nr. 42. Brian media L. Sp. pl. ed. I, p. 70 (1753).

Auf sandigem Rasenboden des Rheindammes zwischen Maxau und Daxlanden in Baden. Begleitpflanzen: Bromus erectus Huds, tectorum L., Arrhenatherum elatius M. et K., Brachypodium pinnatum Beauv, Spiraea tilipendula L., Achillea millefolium L.

Ca. $109 \mathrm{~m}$ ü. d. II; 10. Juni 1899.

leg. A. Kneucker.

Nr. 43. Cymosurus echimatus L. Sp. pl. ed. I, p. 72 (1753).

Auf grasigen Plätzen bei Abbazia im österr. Küstenland; Kalk - und Sandstein. Begleitpflanzen: Triticum ovatum Gr. et Godr., Briza maxima L., Ophrys aranifera Hul., Orchis picta Lois., Dictammus fraxinella Pers., ('hrysanthemum leucanthemum L. etc.

Ca. 20-40 m ü. d. M.; Mai 1899.

leg. Lajos Richter.

Nr. 4t. sclevochlor dwra P. B. Agr., p. 98 (1812) = Cynosums durus L. Sp. pl. ed. I, p. $72(1753)=$ Poa dwa Scop. Fl. carn. ed. 2 I, p 70 (1772).

An trockenen Wegen bei Kun-Félegyháza in Ungarn; Quarzsand. Begleitpflanzen: Hordeum murinum L., Poa bulbosa L., Lolium perenne L., Plantago lanceolata L, Lepidium draba L., Anthemis Rutheniea M. B. etc.

Ca. $100 \mathrm{~m}$ ü. d. M.; 25. Mai 1899.

leg. Prof. J. W a gner. 
Nr. 45. Poa bulbosa L. Sp. pl. I, p. 70 (1753) var. a. Limn.

An kiesigen Rainen oberhalb Frankfurt a. M. in Hessen-Nassau am rechten Alainufer. Begleitpflanzen: Poa bulbosa L. v. vivipara, Carex praecox Schreh., Muscari racemosum Mill., Saxifraga tridactylites L., Medicago minima Bart., Draba verna L. v. brachycarpa Jord, etc.

Ca. $98 \mathrm{~m}$ ü. d. M.; 10. Mai 1899.

leg. M. Dürer.

Nr. 46. Poa bulbosa L. var.vivipura L. Sp. pl. ed. I, p. 70 (1753) var. B. Linn.

An dem sandigen Hochgestade zw, Rheinau und Seckenheim in Baden; Diluvium. Begleitpflanzen: Carex praecox Schreh., caryophyllea Latour., Bromus tectorum L., Anemone pulsatilla L., Cerastium semidecandrum L., Teesdalea mudicaulis R. Br., Arenaria seruyllifolia L., Saxifraga granulata I. etc.

Ca. $100 \mathrm{~m}$ ü. d. M.; 11. Mai 1899.

leg. A. Kneucker.

Nr. 47. Por prelustris L. Syst. ed. X, p. 874 (1759) $=$ P. serotina Ehrh. Beitr. VI, p. $86(1791)=P$. fertilis Host Gram. III, p. 10 (1805).

Fenchtes Ufergehüsch des linken Rheinufers bei Maximiliansau in d. hayer. Pfalz; sandiges Alluvium. Begleitpflanzen: Calamagrostis litorea DC. v. laxa Host, Phalaris arundinacea L., Rubus caesius L., Equisetum trachyodon A. Br., Heleocharis acicularis R. Br., Veronica anagallis L. ete.

Ca. $106 \mathrm{~m}$ ü. d. M.; 12. Juli 1899. leg. A. Kneucker.

Nr. 48. Festuce ovina L. var. mpicalmina Hack. Monogr. Fest. eur., p. $114(1882)=F$. ovina L.v. alpina Neilr. Fl. v. N.Oester., p. 73 (1859) non Koch $=F$. Halleri Caftisch Fl. v. S.O.Deutschl. et alior. nec All.

Weide der Fürstenalp ob Chur in der Schweiz auf teilweise humosem kalkreichem Lehm auf Bündnerschiefer. Begleitpflanzen: Festuca pumila Chaix, Poa alpina L., Agrostis alba L., Carex capillaris L., Parnassia palustris L., I rifolium badium Schreb., pratense L., Anthyllis vulneraria L., Leucanthemum coronopifolium Vill., Be!lidiastrum Michelii Cass., Campanula pusilla Haenke, Saxifraga aizoides L., Euphrasia Salishurgensis Fek, Plantago alpina L., Polygonum viviparmm I... Salix reticulata L., Selaginella spinulosa A. Br., Lycopodium selago L., Asplenium viride Huds.

Ca. $1900 \mathrm{~m}$ ü. d. M.; 2. Aug. 1899.

leg. Dr. A. Volkart.

Nr. 49. Festura spanticen L. 2 D Dumali Hact: Monogr. Fest. eur., p $167(1882)=$ F. Durandii Claus. in Bill. Adn., p. 163 $(1855)=F$. spadicea Coss. et Dur. Fl. d'Alg. II, p. $298=F$. spadicea L. v. livida Hack. Cat rais., gram. Port, p. 27 (1880).

Unter dem Schatten von Quercus toza Bose, an grasigen Orten auf I'riaskalk hei Tramacastillo in der Sierra de noguero in der spanischen Provinz Teruel. Begleitpflanzen: Dactylis glomerata L., Agrostis olivetorum Gr. et Godr., vulgaris With. Hieracium murornm L.. Leontodon hastilis $L_{\text {.. }}$ Ranunculus flabellatus Desf., Lavandula latifolia Will.

Ca. $1700 \mathrm{~m}$ ü. d. H.; Juni 1896 .

leg. E. Reverchor

Nr. 50. Festuca myumes L. Sp. pl. ed. I, p. 74 (1753) = Iulpia myuros Gmel. Fl. bad I, p. 8 (1805).

Auf sandigen, kiesigen, teilweice mit Kohlenstaub verunreinigten Stellen hein Rheinhaten von Maximiliansau in der hayer. Rheinpfalz. B e gle itp flanzen: Brassica incana Doell, Poa compressa L., Lolium perenne L., Bromus sterilis L., Serofularia canina L., Oenothera biennis L., muricata L. ete.

Ca. $107 \mathrm{~m}$ ü. d. M.; 21. Juni 1899.

leg. A. Kneucker.

Es ist ganz überflüssig, Ehrhart als Autor zu citieren oder F. pseudo-myurus Soy. Will. anzuführen; denn was Linné in der ed. I d. Spec. pl. F. myurus nennt, 
ist sicher die vorligende P'flanze ("floribus scabris"). Erst spiter erhielt er durch Iıöfling die Festuca ciliata, die er mit F. myurus vereiniegte, so dass er in der ed. II sogar in einer Anmerkung zu F. bromoides davon spricht, dass F. myurus ,glumae ciliatae" hat.

Ed. H a c kel.

Nr. 51. Srleropore rigirla Griseb. Spicil. fl. r. II, p. 431 (1844)= Poa rigida L. Am. acad. IV, p. 265 (1759) $=$ Festuca riqida Kth. En. I, p. 392 (1833).

An Mauern, Strassenrändern und beschotterten Stellen bei Abazzia im österr. Küstenlande. Begleit pflanzen: Sisymbrium officinale Scop., Erigeron Canadensis L.

Ca. 10-20 m ü. d. M.; Mai 1899 .

leg. Lajos Richter.

Nr. 52. Bromms albidus M.B. Fl. taur. cauc. 1II. p. 79 (1819) f. intermedia Hackel. (ad B. evectum vergens).

An steinigen Abhängen in den Landschatten Digoria und Ossetia im Kaukasus (Russland). Begle itpflanzen: Silene lacera sims., Anthemis rigescens Wlld, Anchusa Orientalis L., Daucus Bessarabicus DC., Caucalis daucoides L., Gypsophila elegans M. B., Carduus crispus L., Nepeta grandiflora M. B.

Ca. $1500 \mathrm{~m}$ ï. d. MI.; 4. Juli 1899.

leg. B. Marcowicz.

Nr. 53. Lolium rigidum Grand. Agr. I, p. 344 (1811),

In Weinbergen und auf Aeckern in der Umgegend von Sion im Kanton Wallis (Schweiz), steigt bis zur Höhe der oberen Dörfer (ca. $1200 \mathrm{~m}$ ) hinan; Gletscheralluvium. Begleitpflanzen: Lolium perenne L., multiflorum Gaud., Apera spica venti P. B., Adonis aestivalis L., flammea Jacq., autumnalis L., Rapistrum rugosum All., Teslea paniculata Desv., Bunias erucago L., Camelina microcarpa Andrz., Eruca sativa Lam., Brassica campestris DC.

Ca. 500-600 m ü. d. M.; Juni 1899. leg. Prof. F. O. Wolf.

Nr. 54. Agropyron repers P. B. Agr. expl. des plantes p. 13 u. 102 (1812) o. vulyare (Doell.) Fl. d. Grossh. Bad. I, p. 128 $(1857)=$ Iriticum repens L. Sp. pl. ed. I, p. $86(1753)=T r$. arvense Schreb. in Sichweigg. u. Körte Fl. Erlang. I, p. 143 (1804).

Zwischen dem Pflaster der mit Kohlenstaub verunreinigten Böschungen des Rheinhafens bei Maxan in Barlen. Begleitpllanzen: Centaurea solstitialis L., Lepidium perfoliatum L., Diplotaxis tenuifolia DC., Oenothera biennis L., muricata L. etc.

Ca. 107 m-ü. d. I.; 12. Juli 1899.

leg. A. Kneucker.

Nr. 55. Agropyron repens P. B. थ(1). Trallamtinmm (schreb.) in Schweigg u. Költe Fl. Eıl. I, p. 143 (1804).

Auf den Brachfeldern des 'T'urmberges bei Durlach in Baden; Muschelkalk. Begleitpflanzen: Bromus sterilis L., tectorum L., Phleum asperum Jacq., Brachypodium pinnatum Beauv., Poa compressa L., Crepis foetila L., Cau. calis daucoides L., Fumaria officinalis L., Galium aparine L., sinapis arvensis L. etc.

Ca. $245 \mathrm{~m}$ ü. d. M.; 14. u. 29. Juni 1899. leg. A. Kneucker.

Nr. 56. Agrofyron rristrtum P.B. Agr. expl. d. pl., p. 1:3! (1812); Bess. En., p. 41 (1822) = Bromus cristatus L. Sp. pl. ed. I,

p. $78(1753)=$ Triticum cristatum Schreb. Gram. II, p. t. 23 (1772).

In der Nähe von Dobogóhegy bei Ofen in Ungarn an Weinbergsrändern; Tegel. Begleitpflanzen: Koeleria cristata Pers., Dactylis glomerata L., Triticum cylindricum Ces., Achillea Neilreichii Kern, etc.

Ca. $90 \mathrm{~m}$ ü. d. M.; Juli 1899.

leg. L a jos Richter.

Nr. 57. Haymallia rillosa Schu Lin. pl. Transs., p. 807 (1866i)= Secale villosum L. Sp. pl. ed. I, p. 84 (1753) $=$ Triticum villosum M. B. Fl. taur. cauc I, p. 85 (18v8); P. B. Agr, p. 103 (1812). 
In einem Aufbruch von Reken am Nont d'orge nahe bei sion im Kanton Wallis (neu für die Schweiz). Begle it p fla nzen: Saponaria vaccaria L., Achillea nobilis L., setacea W. K., nobilis L. $\times$ setacea IV. K., Silvia verticillata L. etc.

Ca. $600-700 \mathrm{~m}$ ü. d. M., Juli 1899 . leg. Prof. F. O. Wolf.

Nr. 58. Triticmm ovatm G). et God). Fl. d. Fr. III, p. 601 (1855 bis 56) $=$ Aegilops ovata L. Sp. pl. ed. I, p. 1050 (1753).

An trockenen Orten in Olivengäten bei Bordighera in Ligurien (Italien); Sand- und Thonboden. Begle itpflanzen: Brachypodium distachyon R.A., Chlora perfoliata L. etc.

Ca. $5 \mathrm{~m}$ ü. d. M.; Mai 1899.

leg. Clarence Bicknell.

\section{Nr. 58 a. Triticum ovatm G\%. et Godv.}

An Strassenrändern bei Abbazia im österr. Küstenland; Dolomit. Be gleitpflanzen: Briza maxima L., Scleropua rigida Griseb., Capsella bursa pastoris Mnch., Sisymbrium officinarum Scop., Verbascum thapsus L.

Ca. $20 \mathrm{~m}$ ü. d. M.; Mai 1899.

leg. Lajos Richter.

Nr. 59. Triticum cylimaricum Ces. Comp., p. $86=$ Aegilops cylindrica Host Gram. II, p. 5 (1802).

An Weinbergrändern des Petersherges bei Ofen (Budapest) in Ungarn; Dolomit. Begleitpflanzen: Chenopoctum album L., Polygonum aviculare L., Achiliea millefolium L., Inula Britannica L.

Ca. $75 \mathrm{~m}$ ï. d. M.; Juli 1899.

leg. Lajos Richter.

Nr. 60. Elymus arenurius L. Sp. pl. ed. I, p. 83 (1753).

Auf weissem Sand jenseits der Festung Friedrichsort nahe am Kieler Hafen. Begleitpflanzen: Ammophila arenaria Lk. und Baltica Lk.

Ca. 0,5 m ü. d. ML.; 16. Juni $1899 . \quad$ leg. E. Ohl.

Wegen der scharfen, schneidenden Blätter wird das Gras im Plattdeutschen

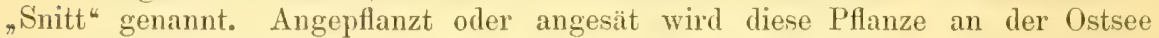
nicht, soviel ich weiss, wohl aber an der Nordsee, wo sie eine wicht,ge Dünenbefestigungspflanze ist. Im Binnenland soll sie auf Flugsand rorkommen; ich habe sie aber dort noch nicht beobachtet.

E. 0 hl.

Nach W. La ck owitz kommt die Pflanze im Binnenlande teilweise massenhaft vor; aber nur zur Bindung des Flugsandes angepflanzt.

A. K.

\section{Botanische Litteratur, Zeitschriften etc.}

Schwarz, August Friedrich, Phanerogamen-und Gefässiptogamen-Flor a der Umgegend von Nürnberg-Erlangen etc. II. oder spezieller Teil. 1. und 2. Heft. Nürnberg 1899.

Im Gegensatze zur Gradmann'schen Flora sucht und findet die Schwarz'sche, die mit ersterer in der äusseren Anlage sowohl, als insbesondere durch die eingehende Beriicksichtigung der pflanzengeographischen Verhaltnisse Vieles gemein hat, ihre Hauptstärke nicht in der Darstellung der Letzteren, sondern in ihrem II. (speziellen) T'eile. Die vortrefflichen Eigenschaften dieses Werkes, über welche in dieser Zeitschrift bereits früher:) berichtet wurde, sind in gleicher Weise auch der neu erschienenen Lieferung eigen, welche auf p. 163-514 die Darstellung der Calycifloren bringt.

Unter den bisher behandelten Familien verdienen besondere Hervorhebung die Rosaceen, namentlich die von Heimich Braun revilierte Gattung Rosa und die auf Grundlage der 'Zimmeter'schen schriften und Bestimmungen erfolgte Bearbeitung der Gattung Potentilla, welch letzterer ich bereits an anderer Stelle die gehouhrende Anerkennmg zollen durfe.**) Bei der Gattung liubus ist nur

*) Appel in Allg. bot. Zeitschr. 1898 p. 28 f.

**) Die bayerischen Arten, Formen und Bastarde der Gattung Potentilla" in Denkschriften der Kigl. hot. tiesellschaft in Regensburg. VII. Bit. (N. F. I. Bd.) p. 147. 
zu bedauern, dass der Verfasser - einem falschen Autoritutsglauben folgend die zum mindesten sehr phantastischen und anfechtharen mul ohendrein nur auf Grund getrockneten Materiales gewonnenen Bestimmungsresultate von Utsch wiedergegeben hat, anstatt - wofür ihm wohl jedermann dankbar gewesen wäre -- seine eigenen, an den naturlichen Fundorten gemachten Beohachtungen der Allgemeinheit zu Besten zu geben.

Von den übrigen ,kritischen "Gattungen, denen ja der Verfasser — wie von jeher, so auch in diesem Werke - sein Hauptaugenmerk zugewendet, seien namentlich noch die trefflichen Bearbeitungen von Prums, Taraxarum und Aster. erwähnt, letztere aufgrumd der von Graebner vorgenommenen Revision und Vergleichung mit dem Material des k. Universitätsherbars in Berlin.

Unter den neuen Formen, welche T'erfasser aufstellt, bilden eine besondere Tategorie die sog. Leinformen: Fumaria officinatis forma linicola; Rophanistrum Lampsana s. livicolum A. Schuarz et Schultheiss; Viola tricolor $\beta$. arvensis forma linicola; Spergula arvensis o. linicola; Anthrmis arvensis forma linicolu, welche samtlich als Charakteristikum einen gestreckten, unverzweigten, bis in die Blütenhühe des Leines sich erhehenden Stengel mit nur wenigen Blüten anfweisen.

Ausserdem ist es dem Verfasser auch gelungen, in der Nürnberger Flora eine Reihe ron nenen Varietaten, Formen und Bastarden nachzuweisen, die teils der Lebersichtlichkeit halher, teils des grossen Interesses, welches die meisten von ihnen auch für weitere Kreise beanspruchen, im Folgenden nebst der Originaldiagnose aufgeführt werden mögeı :

Ancmone nemorosa 8. hirsuta , eine sehr zierliche, kleine Form mit bis zur Basis reichender, abstehender Behaarung des Stengels, breiten Hüllen, die kaum länger sind, als ihr Stiel, breiten, weissen Kelchblättern ";

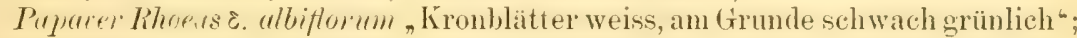

Corylalis sulida $\beta$. multifida , eine äusserst zierliche Form mit feinzerteilten, daher viel zahlreicheren, schmäleren Blattzipfeln";

Erysimum strictum $\alpha_{\text {. typicum }}$ Schoten straff, dem Stengel parallel, manchmal selbst angedrückt";

- - - patns , Schoten samt den Fruchtstielen weit abstehend, wodurch ein ganz anderer Habitus entsteht";

Thlaspi mon'anum a. typicum, Blätter der Stockrosetten spatelig, verkehrteiförmig, langgectielt, zuweilen :elt st rundlich, ganzrandig oder undeutlich gezilunt";

- - ¡. angustifclium, Blätter der Stockrosetten lanzettlich, keilig in den langen Blattstiel verschmälert, deutlich entfernt ausgeschweift gezähnt";

L pidium ccimpcstre $\gamma$ subglabrum, Blätter fast kabl, gr ün, ganzrandig oder undeutlich gezähnt, Stengel viel zarter als beim 'lypus, die Fruchttraube viel weniger dicht";

Polygala vulyaria $\alpha$, rosea ,Blüten rosa, violett bis bläulichviolett, die Flügel kreiter als die Kapsel, spitzlich, wei der Fruchtreife verblassend und alsdann das grünliche Adernetz deutlicher hervortretend";

- - $\beta$. coerulea ,Hlüten intensiv blau, grösser als bei $\alpha$, namentlich die Flügel, welche stumpf oder ganz kurz hespitzt sind, Randnervatur undentlicher als bei $\alpha "$;

Vaccaria parviflora $\alpha$ typica „Kanten des Kelches bis zur Spitze grün und hier schmal häutig berandet;

-. - - B. noblis "Kanten des Kelches an der Spitze purpurn und hier breit weisshäutig berandet";

Stel aria nemorum $\beta$. circreoiles A. Sehwarz ad amicos 1881 , mit glasig

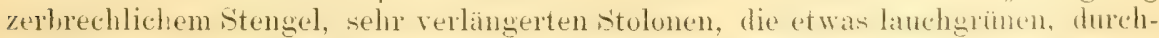
scheinend zarten Blätter Jänglich eiförmig mit herzförmigem Grunde, alle bis an den Beginn des Blütenstandes hinauf sehr lang gestielt, die Rispe, mit nur kleinen Blaittem gestützt, erscheint flatteriger als am 'ylus. I) ge ganze P'flamze, obwohl hoch, ist überaus zart und zerhrechlich, die durchscheinemd dumnen blitter erinnern an Circaea alpina und intermetia"; 


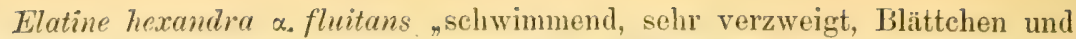
Sitengel grün, Blattsphäre stark entwickelt, die Blättchen meist länger als die Internodien, Blütensphäre wenig auffällig. ('allitvich-ähnlicher Habitus, " in 'Teichen; - - 3. erecta ,Stengel aufrecht, $2-3 \mathrm{~cm}$ hoch, wenig verzweigt, heller, J3attchen weniger entwickelt, viel kürzer als tie Internodien, Montiu-äbnlicher Habitus*, an Teichrïndern;

- - $\gamma$ prostrata, Stengel sehr verzreigt, auf dem Schlamm hinkriechend, Blättchen klein, oft samt den stengeln rotbraun, meist reich und intensiv rosablüthig; ein prachtfarbiges Bild durch die Masse des Vorkommens", auf dem Schlamm abgelassener Weiher;

Geranium Pirrenaicum $>$ pusillum, niedriger und gedrungener als Pyrenaicum, Blätter liüzer gestielt, die Blütensphäre, welche bei G. Pyrenaicum den Gipfel allein eimnimmt, erstreckt sich viel weiter herab, indem sogar grundstan. dige Blütenstiele vorhanden sind Blumenbläter kleiner, hreiter und seichter ausgerandet. intensiv hlau, Kelchblatter breiter, dichter und linger behart als bei G. Pyrenaicum und darin $G$. molle näher stehend. 4. Juli*;

Impatiens Noli tanyere $\beta$. albiflora "Kron- und Kelchblätter weiss mit schwachem gelblichen Ton, nur an der spitze des Spornes hellgelb. Laubblitter etwas kleiner als an der Art, von weniger frischem (trün und beim Trocknen meist rasch braun werdend. Habituell sehr abweichende Form ";

Ononis proeurvens 8. flagelliformis, wie die Parallelform der 0. spinosa" $\gamma$.) flayelliformis A. Luy, $r$ in Celakorsky, I'roulromus der Flora von Böhmen. p. 65\%):

Trifolium mestium $x$ alpestre, Stengel straff aufrecht, frisch wie getrocknet drahtiahnlich starr. Blätchen lanzettlich, spitz, mit am Rande deutlich hervortretender Nervatur. nuten und am hande hehatart, nicht gezähelt. Nebenb]atter schmallanzettlich, längsalerig. der fein pfriemliche Teil lang, straff emporgerichtet. gewimpert. Köpfchen einzehn, kurzahrenförmig, alle deutlich behüllt. Kelche kahl, 10nervig, die fadeutömigen Kelchzahne lïnger, als bei medium und stark behaart, auch die Aehrenspindel stark behaart. Blüten purpurn. Habitus von alpestre, Kelche von medium";

- incamatum $\gamma$. scundens, Die Stengel gestreckt, im oberen Teil um die Kornhalme windend":

Astragalus arenarius $\gamma$. angustifolius, sehr schmalblätterig, die obersten Blätter lineal, mit parallelen Rändern":

- - o. latifolius, die Blättchen breiter, länglich eiförmig, die untersten nahezu kreisrund";

Ervum tetrasprmum $\beta$. tenue , Stengel noch zarter, fast fadenförmig, untere Blätter 1 paarig, 'l'rauben 1blüthig. Hülsen 2 oder 3 samig*;

Ribes grossularia $\beta$. pubscens forma montana, ein straffer, holziger, kleinBhitteriger Strauch, die śteiligen, rotbranen Dornen gewaltig entwickelt";

Saxifragu decipi ns $\beta$. spatulata , unter den typischen 3-5lappigen Rosetten-

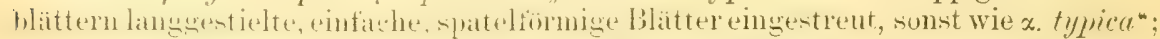
- D. arosifolin slie innersten Lósettenbläter mit hreitem Blattstiel, etwas dicklich, beim Trocknen lange grün und saftig bleibend, perennierende Winterknospen darstellend. Blattstiele und Blätter mit limgen Gliederhaaren. Kronblatter eiförmig, 3mal so lang als der Kelch, sonst wie bei $\alpha$ typica";

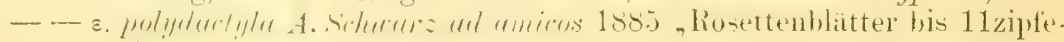

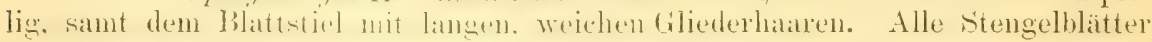
tief 5, dann 3zipfelig, die schmalen Zipfel mit parallelen Seitenrändern. Kronblitter eiförmig mit keiligem Grunde. Eine sehr zierliche form":

Pencedamum palustre ß. simplex ,die ganze Pflanze schlanker und kleiner, Stengel einfach, meist nur mit einer Dolke, oder nur am Gipfel ästig mit 3 Dolden, im unteren Drittel oft rot überlaufen. Hauptdolde 10-15 strahlig; Blätter wenige, die Abschnitte. lineal bis fädlich";

Pastinaca satica $\beta$. montana , die Dolden 6 strahlig, die Stengel schlank,

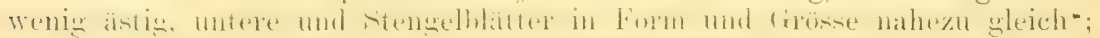


Hedera helix s. acerifotia, ,eine Form, bei der 3 Blattzipfel, namentlich der mittlere, schr verlängert sind, die Buchten zwischen denselhen spitzwinkelig, wodurch eine grosse Aehnlichkeit mit Ahornblättern entsteht";

Sherardia urvensis forma erecta ,mit unverzweigt aufrechten, fast kahlen, nur oben etwas ästigen Stengeln"; teilig“;

Knautia arvensis 8. pinnata ,alle Blätter, auch die grundständigen, fieder-

- arvensis $\times$ silvatica Habitus von sil atica, Stengel mit zweierlei Behaarung, Blätter bis zur Hitte des Stengels wie hei silvatica, dam folgen einige gefiederte. Blättchen der Hülle breiter als bei silvatica";

Anthemis arvensis forma arenosa A. Schwarz 1878 ad amicos ,der Stengel einfach, aufrecht, nur fingerlang oder noch niederer, einköpfig, der Blütenkopf sehr klein. Zwergform dürftiger Sandböden";

Cirsium (oleraceum $>$ acaule) $\gamma$.gigantcum, Formen, welche bis ${ }^{2} / 3$ Mannshöhe erreichen können, der sehr ästige stengel reich mit grossen Blättern hesetzt, die Stengeläste endigen je in einen mittelgrossen Blütenkopf, deren bis zu 12 an einem stock sich finden können, eine grundständige Blattrosette meist nicht vorhanden";

Campanula cervicaria $>$ glomerata? "Grundachse kaum verdickt, oben mit Resten vorjähriger Blattstiele besetzt, Stengel straff-aufrecht, halb meterhoch, kaum kantig-gerieft, Rehaarung wie hei corricariu. Stockblatter lanzettlich, in die lange Sipitze verschmälert, am eifürmigen Grunde plötzlich in den Blattstiel zusammengezogen, Blattrand grob-, fast doppelt-gekerbt, Blattstiel so lang als das Blatt; untere Stengelblätter lanzettlich, gestielt, die obersten sitzend, Behaarung bei allen wie bei cervicaria. Kelchzipfel stumpf";

Vaccinium myrtillus $\gamma$. cordifolium, eine Form mit fast sitzenden Blättern, welche aus herzformigem Grunde loreit-eiförmig his rundlich umd stumpî" sind, wohei jedes Zahnchen mit einer gestielten Drüse versehen ist; die form ist autfallend armblütig".

Dr. Poeverlein.

Pax, Dr. Ferdinand, Puantl's Lehrhuch der Botanik. Verl, von Wilh. Engelmann in Leipzig. 11. Aufl. 1900. 455 S. u. 414 Figuren in Holzschnitt. Preis 4.60 M., geb. $6.10 \mathrm{M}$.

Das wohlbekannte, treffliche Prantl'sche Lehrbuch liegt nun in seiner 11. Auflage vor, welche gegenüber der vorhergehenden um 48 Seiten und $27 \mathrm{Ab}$ bildungen erweitert wurde. Das Werk ist so allgemein bekannt und verbreitet, dass es umötig ist, über seine Anlage und Eimrichtung Ausführungen zu gehen. In der Gewebelehre ist Verfasser num der Schwendener-Haberland t'schen Aufrassung gefolgt. Neu cingeschaltet wurle ene Reihe kurzgefinster biologischer Notizen. 12 phimmakognostische Ahbildungen wurlen ne'll gezeichnet und nehst älteren Figuren des Verlags aufgenommen. Sehr wertvoll für Pharmaceuten ist der Anhang von p. 422-432, welcher eine Uebersicht und kurze Charakteristik der pflanzlichen Drogen der 3. Aufl. des Deutschen Arzneibuches enthält. A. K.

Dalla Torre, Dr. C. G. v., u. Harms, Dr. H., (ienera Siphonogamarum ad systema Englerianum conscripta. Verl, v. Wilh. Engelmann in Leipzig 1900. Fase. I. $4^{0}$ p. 1 -80. Preis 4 II.

Das im Erscheinen begriffene Werk soll eine Uebersicht über die Familien, Gattungen und Sektionen der bis jetzt in Englers natür. Pflanzenfamilien zum Abschluss gekommenen Syphonogamen bringen. Der erste Teil soll die syste.

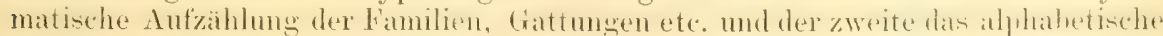
Register enthalten. Besondere Sorgfalt wird den Synonymen der Genera unter Beifügung der vollständigen Litteraturnachweise gewidmet. In abgekürzter Form wird auch die geogr. Verbreitung berührt und die Anzahl der bekannten Arten der einzelnen Genera, die fortlaufend nummeriert sind, angegeben. Der Umfang fles Werkes ist auf 100 Bogen zu 8 Seiten in $4^{0}$ (1U Lief. zu 10 Bogen à $t$ II.) 
und der Gesamtpreis anf etwa 40 II. berechnet: für die Abnehmer, die das Werk nach dem Erscheinen vollständig zu erhalten wünschen, auf $30 \mathrm{M}$. Wie hisher Endlicher's Enchiridion und Durand's Index wird das Buch vor allem für das Ordnen von Herbarien nach dem neuesten Stande der Wissenschaft geradezu unentbehrlich werden und einen Ueberblick geben über die Anordnung u. Reihenfolge der verschiedenen systematischen Gruppen und deren Gliederung. Einen hohen Wert besitzen aber anch die ausfühlichen Litteraturnachweise, welche zu erlangen bisher einen grossen Aufwand von Mühe und Zeit erforderte. A. K.

Fonek, Leopold, S. J., Streifzüge durch die biblische Flora. Herdersche Verlagsbuchhandlung in Freiburg i. B. 1900. $167 \mathrm{~S}$. Preis $4 \mathrm{M}$.

Eine recht verdienstvolle Arbeit liegt hier vor, welche nicht nur zusammenfasst, was in manchfacher zerstreuter Litteratur über die biblische Flora zu finden war; sondern sich auch vielfach auf eigene Studien gründet, zumal der Verfasser in den Jahren 1895 11.96 sich in Palästina aufhielt. Das Werk gliedert sich in 5 Kapitel, deren Ueberschriften lauten: 1. Am Meeresstrand; 2. Auf Berges Höhe; 3. In öder Steppe; 4. Durch Feld und Flur; 5. Bei den Wassern des Todes. Manche in der Bibel genamnten Pflanzen werden wir heute wohl kaum mehr ganz sicher feststellen können, und es lässt sich vielfach nur mutmassen, welches Gewächs der biblische Schriftsteller jeweils mit dem betr. Namen be. zeichnen wollte. In dem Buche erfrenen uns manche śchilderungen von Tegetationsbildern, und zahlreiche Citate, Litteraturnachweise und etymologische Erklärungen dienen zur näheren Erläuterung. Bei seinen Bestimmungen beruft sich der Verfasser vielfach auf Post's Flora von Syrien und Palästina, sowie auf Boissier's klassische Flora orientalis.

A. K.

Hervey, E. Williams, Observations on the culors of flowers. Verl. v. E. Anthony \& Sons in New Bedford, Mass., U. \&. A. 1899. $105 \mathrm{~S}$.

In dieser vorliegenden Broschüre spricht der Verfasser sich hauptsächlich aus über die Bezieh'mgen zwischen dem Insektenbesuch und der Farbe der Blüten. Auf p. 55 kommt er auf die im Kontrast zu der Grundfarbe der Blïten stehenden verschiedenen Punktierungen, Streifen etc. zu sprechen, die gewissermassen den honigsuchenden Insekten als Wegweiser zu den honigführenden Nektarien dienen. Als Beispiele nennt er u. a. Mimulus luteus, Ponlederia cordata, Lespedeza polystachya, Sisyrinchium angustifolium, Solanum dulcamara, Tropaeolum ete. Die Lektüre des recht anregend geschriebenen Werkchens sei besonders den Biologen empfohlen. Die beigcagehenen Abbildungen lassen hingegen zu wünschen übrig.

A. K.

Winkler, W., Sudetenflora (Ankündigung). Binnen kurzem erscheint im Verlage von C. Heinrich in Dresden-N. ein Werk, betitelt "S udetenflora*. Eine Auswahl charakteristischer Gehirgsplanzen. - Nach naturlichen Familien mol unter Berückichtigung des Limë schen Systems bearbeitet von W. Winkler, Hauptleher in shreiherhatu. Nit 1033 nach der Natur aufgenommenen P'flanzenabbildungen auf 52 Farkentafeln. Ein ,Prolog von Herrn Sanitätsrat Dr. Baer in Hirschberg, dem Verfasser gewidmet, geht dem Werke voraus. Preis des fein gebundenen Exemplares 10 M. Besprechung erfolgt erst nach Erscheinen. des Werkes.

Oesterreichische bot. Zeitschrift 1900. Nr. 4. V i e r h a p p e r, F., Arnica doronicum Jacq. und ihre nächsten Verwandten. - Li n sba u er, L. u. K., Teratologische Befunde an Lonicera Tartarica. - Kick, J., Eine neue Sclerotinia. - Polack, J. M., Untersuchungen über die Staminodien der Scrophulariaceen. - hechinger, C., Ueber Lamium Orvala L. u. I. Wettsteinii liech. Scholz, J. B., Chenopodium opulifolium, ficifolium u. album. - Bornmüller, J., Ein neuer, bisher verkannter Bürger der europäischen Flora. - Jenčič, A., Entgegnung. 
Deutsche bot. Monatschrift. 1900. Nr.3. Kuntze, Dr. O., Nomenklaturanfang und Reform internationaler Kongresse. - Baner, Dr. E., Bryologischer Bericht aus dem Erzgebirge. - Fi gert, E., Aira caespitosa $>$ flexuosa n. hybr. - Murr, Dr. J., Zur Kenntnis der Kulturgehölze Südtirols, besonders Trients. - I eimbach, Dr. G., Die Volksnamen uncerer heimischen Orchideen.

Botan. Centralblatt. 1900. Nr. 14. II üller, Karl, Bericht üher die im J. 1899 in Baden gesammelten Lebermoose. - Warnstorf, C., Weitere Beiträge zur Kenntnis der Torfmoose. - Nr. 15. Müller, Karl, Wie in vor. Nr. Warnst orf, C., Wie in vor. Nr. - L udwig, Dr. F., Ueber Variationspolygone und Wahrseheinlichkeitskurven. - Nr. 16. W arnstorf, C., Wie in vor. Nr. Herzog, Th., Einiges über Neckera turgida Jur. und ihre nächsten Verwandten.

Nr. 17. Kükenthal, G., Species generis Uncinia Pers. in America meridionali extratropica sponte nascentes. - Kr a us e, H. L., Floristische Notizen. - D a m mer, Dr. Udo, Ein Vorschlag zur Litteraturfrage.

Zeitschrift far Gewässerkunde. Im Verlage von S. Hirzel in Leipzig erscheint unter vorstehendem Titel eine neue Zeitschrift, herausgegeben von Professor Dr. H. Gravelius in Dresden. Das 1. Heft enthält einen grösseren Aufsatz von Dr. 13. Schorler in Dresden über das Plankton der Elbe bei Dresilen.

Jahresbericht des Preussischen bot. Vereins 1898/99. Der reiche und interessante Inhalt gliedert sich in den Bericht ïher die 37. Jahresversammlung des Vereins am 4. Okt. zu Thorn und in die Berichte über die monatlichen Sitzungen des Preuss. bot. V. im Winter 1898/99. In abgekurzter Form sind diese Berichte auch in der "Allg. bot. Zeitschrift" jeweils erschienen.

\section{Botanische Anstalten, Vereine, Tauschvereine, Exsiccatenwerke, Reisen etc.}

Preussischer Botanischer Verein. VI. Sitzung, Königsberg i. Pr., 23. April 1900. Herr Oberlehrer Gustav Vogel sprach über einige nenere bemerkenswerte Arbeiten auf dem floristischen Gebiet, u. A. über die seltenen durch Geisenheyner beobachteten Farnformen, sowie üler die durch Kražan ausführlich behandelten Formen von Knautia arernsis, von denen die meisten in der Kultur sich als mbeständig erwiesen haben. Der Vortragende machte auf die durch Warnstorf, Ascherson, Grütteru. a. beobachtete Einwanderung des Bidens comnatus aufmerksam, der östlich von der Weichsel hisher noch nicht beobachtet worden ist, und verweist auf die durch Ascherson im Mecklenburger Archiv veröffentlichten Beobachtungen. Auch auf den in Europa weiter vordringenden Bidens frondosus, der von Dr. Graebner bei Lenzen bei Elhing festgestellt worden ist, wurde vom Vortragenden hingewiesen. - Dr. A bromeit demonstrierte hierauf einen durch sehr starke Knospen - bezw. Sprossbildung ausgezeichneten Wipfeltrieb von Picea alba Link. Letztere wird in den Dünenwaldungen und auch sonst viel kultiviert und erträgt unser Kilima sehr gut. Das vorgelegte Exemplar war den Dünenwaldungen von Cranz entnommen. Es ist sehr wahrscheinlich, dass der. Wipfeltrieh im Frühlinge die Nidehn rerloren hatte, worauf sich, wie bei der gewöhnlichen Fichte, eine gesteigerte Proluktion von Knospen und Triehen aus den schlafenden Angen der ichimmelfichte einstellte. Eine derartige reichliche Triebshildumg aus schlafenden Augen wurde bei Piced excelsa bereits von Rober t $\mathrm{Hartig}$ beobachtet und, Ersatztriehsbil. dung" genannt. Die Ersatztriebe entwickeln sich nach voraufgegangener Entnadelung, z, B. durch Raupen besonders bei der gemeinen Fichte sehr oft. J)ic Blätter zeigten bei $P$. alba am Grunde der Ersatztriebe eine ungewöhnliche Breite 
und waren an der Spitze teilweise ausgerandet. Zur Vorlage gelangten ferner Exemplare der bei uns seltenen Gagea spathacea, die von Herrn Rektor Ka l muss in Elbing bei Gross Wesseln gesammelt und nebst anderen bemerkenswerten Pflanzen eingesandt worlen war. Auch wurden einige Exemplare der in der Flora des Kreises Elbing verbreiteten Veronica Jilleni Crantz, ehenfalls von Herrn Rektor Kalmuss gesammelt, vorgezeigt. Zum Schluss wurden mehrere Pflanzen von der Kergueleninsel demonstriert, die von Hern Dr. Vanhöffen gelegentlich der Tiefsee-Expedition der "Valdivia" dort gesammelt worden waren, wie z. B. der "Kerguelenkohl" Pringlea antiscorbutira Ilook., I'ou Cookii Hook, I'. Kérguelensis

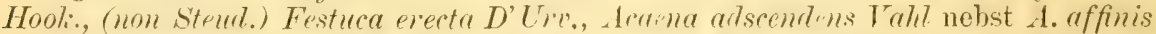
Hook., welche nur eine Form der ersteren vorstellt, ferner Colobunthus Kevouplensis Hook., Ranunculus crassipes Hook., R. trullifolius, Blechnum Penna-Murina Poir. (Lomaria alpina), Azorella Selago ete.

A bromeit.

Botanischer Verein fur die Provinz Brandenburg. In der Monatssitzung am 6. April, welche im bot. Museum (Schöneberg) stattfand, machte der Vors. Prof. Schumann Mitteilung von dem Tode des langjährigen Mitgliedes W. A. Meyn, welcher als geschickter Zeichner die Mehrzahl der lithographischen Tafeln für die Verhandlungen des Vereins gearbeitet hat. Prof. Ascherson widmete dem Verstorbenen einen ehrenden Nachruf. Der Vors. machte dann die ferneren Mitteilungen, dass die Vorstellung seitens des Vorstandes um Erhaltung der Grunewaldfenns an den Minister für landwirtschaftl. Angelegenheiten ahgegangen sei; dass das erste Heft der diesjährigen T'erhandlungen demnächst zur Ausgahe gelangen werde, das auch eine grüssere Arbeit über die Moose der Mark von I.eop. Loeske enthalte, der in nächster 7eit mit Unterstützung des Vereins die Timgegend von Trenenbrietzen inbezug auf die Noosflora eingehend zu untersuchen gedenke. - Hr. Ḱustos Hennings machte die Versammlung wieder mit einigen merkwürdigen japanischen Pilzen bekannt, die auf anderen Gewächsen krophitartige Auswïchse erzeugen, wie C'uroma deformans auf Thujopsis, Pachyma Hoelen auf Pimus-Wurzeln, Uromyces truncicola auf Sophoria. - Den Vortrag des Abends hielt $\mathrm{Hr}$. Apotheker Werth über die Bestäubung der Blüten durch Vögel, auf Grund zahlreicher eigener Beobachtungen in Ostafrika, wo die Stelle der amerikanischen Kolibri durch die Familie der Blumensauger (Nectarinidue) vertreten wird. Unter den Ornithologen ist man noch immer nicht einig, ob diese Vögel dem Blumenhonig nachgehen und infolge dessen für die Bestäubung der Blüten in den Tropen dieselhe Rolle spielen. wie bei uns die Bienen, oder ob sie nur von kleinen Insekten leben, die sie vornehmlich in den Blumen aufsuchen. Dem Vortragenden unterliegt das erstere keinem Zweifel, da nicht allein der lange, gekrümmte, luftdicht verschliesshare Schnabel mit der sehr langen, weit vorstreckharen, mit einer Längsimne versehenen und an der Spitze in zwei Fiden gespaltenen Zunge einen vollkommenen Saugapparat bildet, sondern auch der Bau vieler Blüten, welche ron den Blumensaugern emsig besucht werden, derart beschaffen ist, dass der Zugang zu ihren Honiggefässen den Insekten oft durch Haare und andere Vorrichtungen völlig verschlossen ist. Auch die Gestaltung der Blüten und ihrer inneren Organe lässt darant schliessen, dass sie nicht sowohl für den Anflug von Bienen und Faltern, als vielmehr für den gekrïmmten Vogelschnabel eingerichtet sind. Es lassen sich solcher Nectarinienblumen deutlich rechs Typen unterscheiden. Bei den Blüten der Vyrturen (wie Jambosa, Baringtonia) liegen die Nectarien so tief, dass nur der lange Vogelschnabel sie zu erreichen vermag. Bei Hibiscus würden Insekten gar nicht imstande sein, in die Honighehälter himein zu kriechen, resp. den Honig zu finden. Der Typus der Aloe lässt schon in den verschiedenen Blïtenstadien erkemmen, dass er dem Besuche der Insekten nicht zugänglich ist. Sehr deutlich ist der Unterschied der ornithophilen und Bienenblumen bei den Lippenblumen (wie Kigelia, Salvia, Lobelia ete.), bei denen Blüten mit hervorragender Unterlippe (wie bei salvin protensis) dem Anflug der Insekten Vorwchub leisten, während die nur dem Togelschnabel zugänglichen Formen (wie talvia anten) zuriekge- 
schlagene Unterlippen zeigen. Dem Lippenblumentypus würde sich anch Musa anschliessen lassen. Einen fünften Typus bildet biryflivinu, dem anch wohl Intsin, Vurpu, Poinciana zugerechnet werden künnen; einen sechsten Iorunthus, dem sich die Proteuten anschliessen lassen. Nanche Blüten schliessen anch schon durch die Massen des erzeugten Honigs den Besuch der Insekten aus, wie

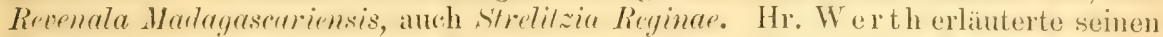
Vortrag durch rasche, aher mit sehr sicherer Hand ausgeführte Zeichnumgen an der Wandtafel. Aus der ausserordentlich interessanten Darstellung ging hervor, dass die ornithophilen Blüten sich nicht allein durch den eigentümlichen Ban ihrer imneren Organe, sondern auch schon durch äussere Gestaltung und liarbe kennzeichnen. Sie sind stets gross und weitröhrig, während die Bienenblumen kurzröhrig und die Falterblumen zwar länger, aber engröhrig erscheinen; sie sind stets brennend scharlachrot oder lenchtend gelb, welche weithin sichtharen Farben durch weiss vertreten werden, wenn die Blüten auch in der Dunkelheit von Nachtfaltern hesucht werden. Alle diese Einrichtungen sind in der 'That erst verstäntlich, wenn der Besuch der kleinen Vögel und ihre emsige Beschäftigumg an und in diesen Blumen mit in Rechnung gezogen wird. - In der daran schliessenden lebhatien Diskussion, in welcher namentlich der Zweifel mancher' Ornithologen an dem Honigsaugen der Vögel erörtert wurle. gab Hr. Werth zu, dass er es durchaus nicht für ausgeschlossen halte, dass die Honigsauger nebenbei auch Insekten verzehren; dass sie indessen die Blumen nicht lediglich der kleinen Insekten wegen, die darin festgekleht sein könnten, besuchen, dafür liefert die von ihnen viel umschwärmte Kigfli, einen Beleg, deren Honigbehälter durch eine Haarvorrichtung gegen das Eindringen von Insekten völlig geschützt sind. Jedenfalls werden spätere Forschungen klarlegen, dass die Nectariniden in den Tropen für die Blütenhestaubung dieselbe Rolle spielen, wie bei uns die Insekten.

W. Lackowitz.

Kgl. bot. Gesellschaft zu Regensburg. Floristisehe Sitzungen. 29. Nov. und 13. Dezember 1899. Herr Lehrer Anton Meyer sprach über neue Beobachtungen an den Sulix-Arten der hiesigen Flora.*) Er legte namentlich kritische Exemplare von st. purpurea und purpurea $x$ riminulis Wimmer vor und bemerkte dazu, dass Wimmer in seinen "Salices Europaeae" Vratislaviae 1866 bei Darstellung der Varietätenreihe von $s$ purpurea auffallenderweise bei einigen Varietäten (erianthu, styliger', furcutu) nichts von der Gestalt der Blätter erwähne, wihrend umgekehrt von den auf der Blattform basierenden Varietaten (gracilis, Lambertiana, strirea) angenommen werde, dass sie die normalen Geschlechtsorgane besässen und die weitere Blattvarietat Helix Koch bei Wimmer überhaupt nicht berücksichtigt ist. Vortragender zeigte nun an einer Reihe von instruktiv gesammelten Exemplaren, dass die Varietät sericen zugleich auch als Varietät eviantha, dass ähnlich styligere sowohl mit typischen Blittern, als anch mit den Blïttern der IIrlir vorkommt. Er kam zu dem Schlusse, dass die von Wimmer aufgestellte Varietiterireihe an dem Fehler krankt, dass siimtliche Varietäten koordiniert erscheinen, während einige, insbesondere die durch Form und Integument der Blütenteile abweichenden, den Blattvarietiten subordiniertwerden müssen. Thatsache ist, dass jede Blüten form, also styligere, triantha und furcatu, mit jeder Blattform, der typischen, der Lumbertiana, gracilis und sericea zu finden ist.

Bei Besprechung der einzelnen Kombinationen des Bastardes S. pur mur $a \times$ riminalis war besonders bemerkenswert, dass die mucrostigmu Wimmer auch mit der Blattform von typischer mbin, ferner ein zu siricea hoch gehöriger Bastard mit ganz kleinen Narben und sehr kurzen Griffeln gefunden wurde; eine weitere

*) Vgl. A. Meyer "lie Weiden des Regensburger Florengebietes" in Denkschriften der Kgl. bot. Gesellschaft in Regensburg. VII. Bd. (N. F. I Bd.) p. $63 \mathrm{ff}$. - Ders., "Einige Salix-Bastarde der Regensburger Flora" in Allg. bot. Zeitschr. 1896 p. $6 \mathrm{ff}$. ibid 1897 p. 203. 
Kombination, welche sich mit keiner der Wimmer'schen Diagnosen deckt, wurde als die S. oliracen Thuillier angesprochen, da die vorgelegten Exemplare genau der Diagnose dieser P'flanze entsprechen. Von besonderem Interesse ist auch die weitere Bemerkung des Vortragenden, dass die Kombination rubra teils mit nur an der Basis zusammenhängenden Filamenten sich findet, wie sie der S. clurrgmifolia Trusch eigen sind, teils mit his nahe an die Staubbeutel verwachsenen, wie es bei S. Forbyana Smith **) der Fall ist. Durch diese sämtlichen Beohachtungen wird die fïr die gesamte Erkenntnis der physiologischen Natur der Bastarde iunserst wichtige Thatsache bestatigt, dass der einzeho Gastard in den Blïttern zwar die Hitte zwischen zwei Stammarten einhalten, aber trotzdem in den Befruchtungsorganen eine deutliche Annäherung zur einen oder zur anderen Stammart zeigen kann. Nähere Ausführungen hierüber wird der Vortragende in dem demnächst erscheinenden VII. Berichte des hiesigen Naturwissenschaftlichen Vereins bringen.

Dr. Poeverlein.

Flora exsiceata Bavariea. Fase. IIL. 1900.

Die vorliegende 3. Lief. enthält die Nr. 151-250 und wurde, wie auch die vorhergehende Lief. von der $\mathrm{Kgl}$. bot. Gesellschaft zu Regensburg unter Mitwirkung des Bot. Vereins Deggendorf u. d. Bot. Vereinigung Würzburg herausgegeben. Zur finanziellen Lnterstützung des höchst verdienstvollen Unternehmens wurde vom bayer. Staatsminister des Inmern 400 M. in Aussicht gestellt, wodurch es ermöglicht werden kann, in Zukunft sämtliche Etiquetten durch Druck herzustellen. Der Preis des III. Fasc. beträgt 20 M., im Abonnement 16 M. Aus dem reichen Inhalte seien z. B. genannt: Erysimm hieracifolium L., Subularia uquatica L., Hi lianthemum polifolium Koch, Eirum oirobus Kittel, Rosa dumetorum Thuill. f. comata Schwertschl. nov. f., micrantha Sm. v. permixta (Déségl.) Gren., yrareolens Gren.et. Godr. v. Iypica Ehrh.f. hispida L. Schulze, Mutriraria discoidea DC.f.pygmare, Doronirum Austriacum. Jacq., Alectorolophus Tollmanni Pärer lein n. sp., Salix purpurea L. $>$ (purpurea L. > repens L.) n. hybr. T', (caprea L. $>$ cinerea L.) v. Reichardtii Kern. ., (capr. × cin.) v. semicinerea A. Mayer $n$. var. $f$. monstrose, (capr. $<$ cin.) v. Gilensis A. Mayer n. var. f. monstrosa 9 , (aurita L. $\times$ cinerea L.) v. auritaformis A. Mayer nov. v. ., Cyperus longus L., Azolla Caroliniana IVlld., Scolopendrium vulgare Sm. monst. irispum II lld. Die beiden ersten Fascikel sind ebenfalls, soweit der Vorrat reicht, noch zum Preise von je $15 \mathrm{M}$. (im Abonnement zu je 12.50 M.) zu beziehen.

A. K.

Bauer, E., Bryotheca Bohemiea. II. Centurie 1899. Die 2. Centurie dieses verdienstvollen Exsiccatenwerkes ist nun erschienen und kann zu dem mässigen Preise von 20 M. von dem Herausgeber Dr. E. Bauer in Smichow bei Prag, Komenskygasse Nr. 961 bezogen werden.

\section{Personalnachrichten.}

Ernennungen etc.: Dr. G. 'Tischler w. 2. Assistent am bot. Institut zu Heidelberg. - Prof. Dr. K. Fr its ch in Wien w. z. a.o. Prof. mit d. Titel u. Charakter eines ordentlichen Professors an d. Universität Prag ernannt. - Prof. Dr. R. v. Wettstein w. vom deutschen naturw.-mediz. Verein "Lotos" in Prag z. Ehrenmitgliede, ron der k. k. Gantenhangesellschaft in Wien zum korrespond. Nitglied ernannt. - Dr. Otto A p pel in Charluttenburg w. v. der Nürnberger Naturfor: schenden Gesellschatt zu ihrem korrespond. Mitgliede ernannt. Fermer wird derselbe vom Kaiserl. Gesundheitsamt zu Berlin vom 1. Juni bis 1. Juli zur Ausstellung nach Paris entsendet (Adresse dort: Paris, 13 rue Pauquet). p. $54 \mathrm{f}$.

**) Vgl. über diese meine Bemerkungen zur „Flora exsiccata Bavarica." Fasc. I. 


\section{Allgemeine}

\section{Botanische Zeitschrift}

für Systematik, Floristik, Pflanzengeographie ete.

\section{Referierendes 0rgan}

des bot. Vereins der Provinz Brandenburg, der $\mathrm{kgl}$. bot. Gesellschaft zu Regenshurg, des Preuss. bot. Vereins in Königsberg,

und Organ der Botan. Vereinigung in Würzburg und des Berliner hot. 'Tauschvereins,

Unter Mitwirkung hervorragender Fachmänner herausgegeben

von A. Héncher, Werderplatz 48 in Karlsruhe.

Verlag von J. J. Heift in Karlsruhe.

Die Herren Mitarbeiter tragen für Form und Inhalt der von ihnen unterzeichnetèn Arbeiten volle Verantwortung.

\begin{tabular}{c|c|c}
\hline li 6. & Erseheint am 15. jeden Monats. - & $\begin{array}{c}1900 . \\
\text { VI. Jahrgang. }\end{array}$ \\
\hline
\end{tabular}

\section{Inhalt}

Originalarbeiten: Eg gers, Bot. Beobachtumgen auf meiner Reise nach dem Orient 1899. - 'T' h. Hellwig, Florenbild der Umgegend von Kontopp im Kreise Grüberg in Schlesien (Forts). - Dr. E. K Blümml, Beiträge zur Flora von Niederösterreich (Schluss). - Dr. J. M urr. Phaenolsgische Plaudereien aus der Innsbrueker Flora (Schluss). - Otto Will, Uebersicht über die hisher in der Umgebung von Guben in der Niederlausitz beobachteten Leber-, 'Torf- und Lambmoose (Forts.). - O t to Kuntzo und 'T'om von Post, Nomenklatorische Revision höherer Pflanzengruppen und über einige 'I'ausend Korrekturen zu Englers Phaenogamen-Register.

Bot. Litteratur, Zeitschriften ete.: Inhaltsangahe verschied. bot. Zeitschr.

Bot. Anstalten, Vereine, Tausehvereine, Exsiceatenwerke, Reisen ete.: Preuss. bot. Ver. (Ref.): - Bot. Verein für d. Prov. Brandenburg (Ref.). - Preisausschreibung. - Raciborski, M., Cryptogamae parasiticae in insula Java lectae exsiccatae. - Bot. Reise nach 'Transkankasien.

Personalnachrichten. - Corrigenda.

\section{Botanische Beobachtungen auf meiner Reise nach dem Orient 1899.}

Von Eggers in Eisleben.

Mit der Schnelligkeit eines heimwärtsziehenden Wandervogels reiste ich Fnde Februar des verflossenen Jahres ïber Münthen, Brennerpass, durch Nord- und Mittelitalien zunächst bis Brindisi. In Deutschland lag die Pflanzenwelt trotz des milden Winters noch im tiefen Schlummer. Auf den Alpen starten die Gefilde und Höhen unter Schnee und Eis, und auch in der lombardischen Tiefebene streckten nur ganz vereinzelt, soweit ich es vom Eisenbahnzuge aus beobachten konnte, die ersten Frühlingsblumen schïchtern ihr Haupt hervor. Je weiter aber nach Süden, desto mehr war auch die Vegetation entwickelt. In Bologna wurden auf den Strassen schon Veilchensträusse verkanft. Vom Wagen aus erblickte ich blühende Aprikosen, Hahnenfuss, Hirtentïschel, Marienblümchen, Ehrenpreis. Die Brombeersträncher bleiben auch hier im Winter belaubt. Hinter Arkona sahe ich in den Gïrten grüne, beblätterte Bäume, wie Myrten, Mandel- und Orangenbäume, Enealypten. 
Auf den Feldern standen eine Buff- oder Saubohmenart und viele unserer Gemüsegewächse (Crucifcren) schon in voller Blüte: weisslich schimmernde Myrten zieren hier, wie in der Lombardei der Maulbeerlaum, die Felder, Anhöhen und Hügel. In der Umgegend von Foggia hatte sich stellenweise schon der Roggen gelagert.

Aber eine ausgedehnte Kältewelle, welche damals Europa durchzog, erstreckte sich auch bis nach Siiditalien. In Brindisi hatte es sogar vor einigen Tagen geschneit, für die dortige Gegend eine seltene Naturerscheinung. Manches zarte Frühlingsblümchen war unter dem eisigen Hauche erstorben. Als ich am 1. Mäiz abends 10 The in Brindisi anlangte, wehte eine harte, kalte Luft. 1)a das Schiff Habsburg (Oestreichischer Lloyd) erst am Abend des andern Tages abfuhr, so benutzte ich die mir am Tage übrigbleihende Zeit zum Botanisieren in der Umgegend von Brindisi. 20 Pflanzenarten waren meine Ausbeute, die ich nachher in aller Ruhe auf dem Schiffe präparierte: Limcric reftexu Isesf.,

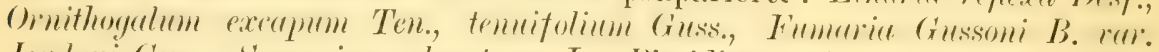

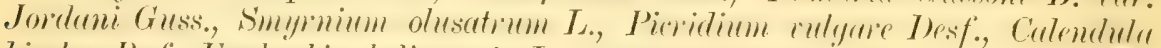

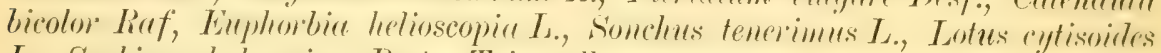

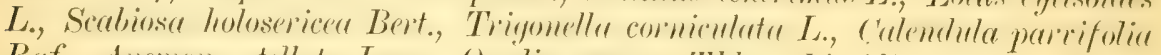

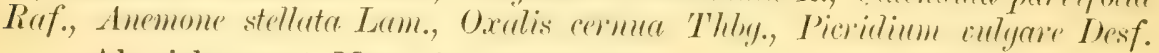

Als ich am 5. März bei Alexandrien den Boden Afrikas betrat, schien ich mich plötzlich in unsern Sommer versetzt. Weizen und Gerste hatten volle Aehren, der Flachs blühte, der Klee wurde iuberall gemäht, und warme Sommerlüfte belebten die Natur. Die Vegetation schien ihren Höhepunkt erreicht zu haben. In dem herrlichen Esbeckiye Garten zu Kairo, in den Anlagen des Museums von Gizeh und in dem daran stossenden zoologischen Garten, früher Haremsgarten, konnte ich nicht genug die Fülle und Bliitenpracht der südländischen Strüucher und Gewächse bewundern. Sonst ist aber das Nilthal kein geeignetes Feld für Botaniker. Jedes Stückchen Land, welches nur irgendwie zu kultivieren ist, wird von den fleissigen und thätigen Fellachen oder Landbewohnern bewässert und zum Ackerbau verwendet. Fruchtland und Wüstenland grenzen sich scharf ab. Aber auch die Wüste bietet nicht viel. Sowohl die libysche wie auch die arabische Wüste sind fast vegetationslos. Auf dem Kies- oder Sandboden hinschreitend, durchzieht man eine monotone, von kleinen Erhebungen kreuz und quer durchschnittene Ebene, in der sich weit und breit nicht die geringste Spur eines organischen Lebens bemerkbar macht, in der kein Baum oder Strauch mit frischem Grün unser Auge labt, sondern wo wir ringsumher unausgesetzt nur die der Wüste eigenen graugelbe Farbe am sonnverbrannten Gestein erblicken. Nur wo in der Wüste Senkungen, Vertiefungen oder Wasserrinnen auftreten, macht sich Pflanzen- und auch Tierleben bemerkbar. Wenn aber im April der heisse, trockene Wüstenwind, der sogenannte Chamsin, der die Luft mit feinem Staub erfüllt, zur Herrschaft gelangt, wird bald jegliche Pflanze in der Wiiste vernichtet. Im Monat März erreicht deshalb die Vegetation im Wüstenlande Egyptens den Höhepunkt, um von nun an rapid abwärts zu sinken. In den Vertiefungen neben den drei grossen Pyramiden von Gizeh fand ich 23 Pflanzenarten, auf dem Totenfelde bei Sakkarah 10 Arten, neben den Chalifen- und Mamlukengräbern 12 Arten und auf dem Wege nach der Mosesquelle, nicht zu verwechseln mit der eigentlichen Mosesquelle 
bei Suez, 20 Arten. Bei den Pyramiden: (ínylusin erunescens Mil., New-

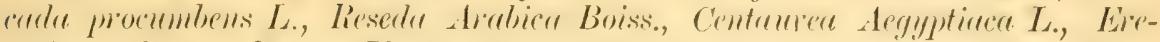

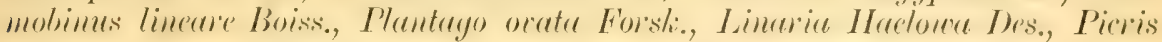

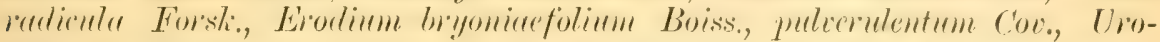

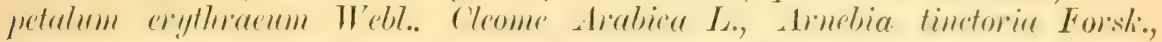

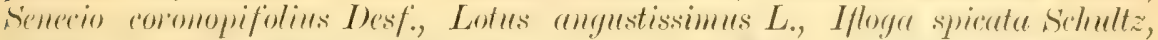

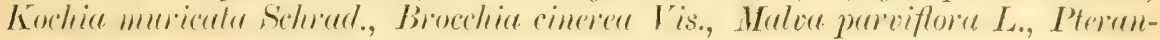
thus echinatus Des., Zygophyllum album L.

Bei den Chalifen- und Mamlukengräbern: Zolitoferiu mulicanlis

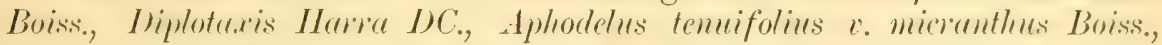
stupu tortilis Itest., Cynodon dertylon Is., IIeliotropium luteum Boiss., Eiru-

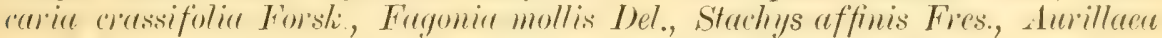
Gacini DC., Sporobolus spicatus Kth., Picridium orientale DC.

Auf dem Wege nach der Mosesquelle: Fayonia mollis Del., Senebieru

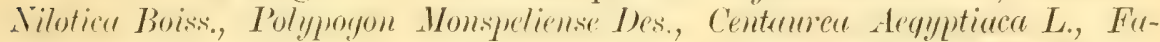

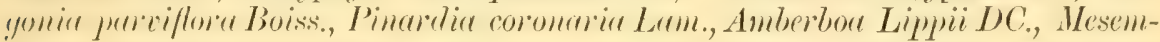

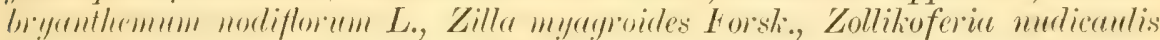

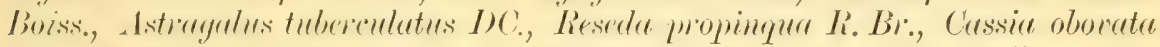

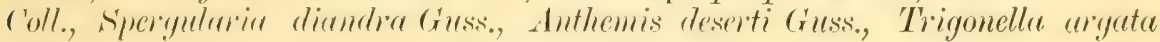
Vis., Cotula anthemoides L., Phelypaea Muteli.

Die Mosesquelle, in Reisehandbïchern wohl ihres Pflanzenreichtums wegen erwähnt, ist von Kairo ans in etwa 'Stunden zu erreichen. Lin Fahrweg führt nicht dahin, sondern ein schwach betretener ?fad zicht sich in der 15-20) $m$ breiten, meist trockenen Wasserrinne bis zur Mosesquelle, der Fremde int somit unbedingt auf Führer angewiesen. Im Winter mag diese Rinne mit Wasser gefüllt sein, zur Zeit meines Besuches war sie trocken und vielfach mit Pflanzen bestanden. Da ich ausser meinem Eseltreiber noch einen kleinen Araberjungen aus einem Orte neben den Chalifengräbern zum Pflanzensuchen mitgenommen hatte, so ist mir wohl kaum eine Pflanzenart entgangen, und wenn ich trotzdem nur 20 Arten auf dem 2 Stunden langen Wege gefunden habe, so ist jene Erwïhnung in den Reisehandbüchern wohl nur vergleichsweise aufzufassen. Denn abseits der Rinne lat man auf dem Wege zur linken Seite die unabsehbare, vollständig vegetationslose Wüste vor Augen, während auf der andern Seite das ebenfalls pflanzenlose Kalksteingebirge Mokattam sich bis zur Mosesquelle erstreckt. Schnelle Eidechsen, langbeinige Kïfer, meine Fangflasche füllend, und eine Heidelerchenart trieben ihr Wesen zwischen den grünenden Pflanzen, sonst war alles ringsumher öd und tot. Der Anblick der Wïste, die Einförmigkeit und das tiefe schweigen, welches auf dem Ganzen ruht, macht einen gewaltigen Eindruck auf jeden, der sich zum ersten Male in solche Welt versetzt sieht.

In der Tmgegend von Port Said, das nach Asien fahrende Schiff liegt einen Tag im Hafen, fand ich 11 Arten; der Ort liegt auf einer diirren, pflanzenlosen Sandscholle, und nur in weiterer Entfernung am Suezkanale gedeihen Pflanzen.

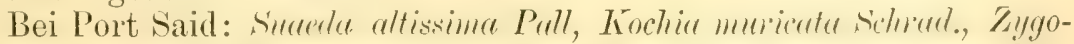

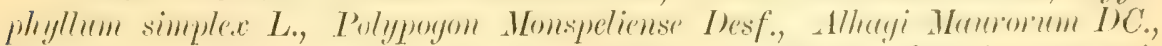

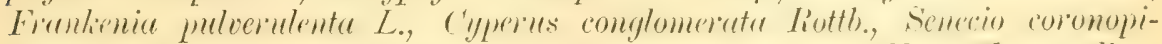

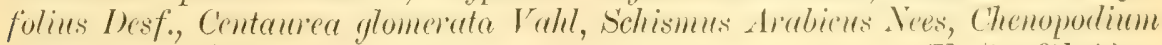
polyspermum $L$.

(Forts, folgt.) 


\title{
Florenbild der Umgegend von Kontopp im Kreise Grünberg in Schlesien.
}

\author{
Von 'Th. Hellwig.
}

(Fortsetzung.)

An Gallen sind zu nemnen für l'otentilla argentea: Diastrophus Mayri Reinh. (Hieron. Nr. (19), für P. Wiemanniana: dieselbe (Vgl. Hieron. Nr. 619), für P. opaca: Xestophanes Potentrỉae (Vill.) Frst. (Vgl. Hieron. Nl。621).

Pimpinella Saxifraga ist mit dem Aecid. von Pucinia Pimpinellae (Strauss) vertreten und mit der Galle (Iieron. Nr. 157) besetzt.

Galium boreale und G. Mollugo haben Puccinia Galii (Pers), i. Mollugo ausserdem Phacidium repandum Fr.

Als Gallen besitzen G. boreale eine Cecidomyia sp. (Hieron. Nr. 429), G. Mollugo Nr. 106 (Hier.) und G. verum Nr. 117, nach oben und unten gerollt (Hieron.).

An Gypsophila fastigiata, Scabiosa Columbaria und Jasione montana ist nicht selten Leptosphaeria ogilviensis (Berk. et Br.), an letztgenannter auch Pleospora vulgaris Niessl.

Lactuca muralis ist mit Puccinia Prenanthes (Pers.) versehen, Senecio viscosus und S. silvatica mit Coleosporium Senecionis (Pers.), Helichrysum arenaria mit Entyloma Aschersonii (Ule) und Erigeron acer mit Gloeosporium Erigeronis.

Hieracium vulgatum und $H$. tridentatum erzeugen Conidien von Erysiphe Cichoracearum (DC.), H. murorum dagegen Leptosphaeria modesta (Desm.).

Gallen bringen hervor: Hierac. Pilnsella (Hieron. Nr. 615), H. Auricula (Vgl. Hieron. Nr. 448), H. umbellatum Aulax Hieracii (Bouchè) Schenk (Hieron. Nr.616), H. vulgatum dasselbe (Hieron. Nr. 617) (auch eine unbeschriebene am Grunde des Stengels), H. boreale ebenfalls (Hier. Nr.613), H. murorum desgleichen (Hier. Nr. 614), H. tridentatum (Vgl. Hieron. Nr. 613); H. vulgatum hat ausserdem Aphis Hieracii Kalt. (Vgl. Hieron. Nr. 329).

Campanula rotundifolia ist mit Synchytrium aureum Schroet. durchsetzt und mit Coleosporium (ampanilae (Pers.) besetzt. Die Gallen von Cecidomyia Trachelii Wachtl (Hieron. Nr.396, a u ch an der Kiapsel) und einer andern Cecidomyide ( $\mathrm{Vgl}$. Hieron. Nr. 395) befinden sich auch daran. C. persicifolia bringt Pleospora herbarum (Pers.) hervor.

Melampyrum pratense und Euphrasia nemorosa werden durch Coleosporium Euphrasiae (Schum.) geziert. Veronica officinalis zeigt Peronospora grisea (Ung.). Linaria vulgaris träigt das so seltene Melanotaenium caulium (Schneid.) (Grïnwald bei Kolzig). Betonica officinalis bietet ebendaselbst die auch seltene Puccinia Betonicae DC. Thymus Serpyllum (T. angustifolius) besitzt eine Galle (Hieron. Nr. 252).

Auf Stümpfen, moderndem Holze u.s.w. wachsen von Myxomyceten: Lycogala Epidendron (L.), Stemonitis fusca Roth, Comatricha nigra Pers., Craterium minuta Lers., Physarum virescens Ditm. und P. leucophaeum Fr. (diese beiden auch auf Moos). Badhamia hyalina (Per's.) und Fuligo septica (L).

An verschiedenen morschen Hölzern kommen vor: Corticium calceum (Pers ) und eine andere Cortic. sp., Stereum purpureum Pers., Gran- 
dinia crustosa (Pers.), Polyporus zonatus (Nees), I'. squamosus (Ifuds.), P. orumalis (Per's.), Ochroporus ignirius (L.), I'hacoporus applinatus (Pers.), Gleophyllum saepiarium (Wulf) an Brückeubohlen, Sphacroholus Carpobolus L., Stictis radiata Pers., Nectria Peziza ('Tode), Ilypoxylon serpens und eine andere Hypoxyl. sp., sowie cine Helminthosporium sp.

An faulenden Stengeln erbeutete ich: Helotium virgultorum (Vahl), H. scutula (Pers.), Ophiobolus porphyrogonus (T'ode) und staganospora dolosa.

Von holzbewohnenden Flechten sah ich in diesem Landschaftsbilde: Usnea barbata (L.) f. glabra, Ramalina polymorpha (Ach.), ('ladonia pyxidata (L.), C. Floerkeana Fr. c. fr, Xanthorina purietina L., Physcia pulverulenta (Schreb.), Gasparrinia murorum (Hoffin.), Bacidia inundata (Fr.) f. lignorum und Biatorina pineti (Schrad.).

IIolz als substrat liebt anch ein Laub- und ein Lebermoos: Neckera pumila Hedw. und Lophocolea heterophylla (Schrad.) N. v. E.

Als Erdhewohner unter den Flechten gehören hierher: l'eltigera rufescens Hoftim., (Tadonia cocifera (L) (C. cormucopioides L.), Biatora granulosa (Ehrh.), B. uliginosa Schrad., B. fuliginea (Ach.), Sphyridium byssoides (L.) P. carnem Flke.; auf Ziegeln ergab sich eine Lithoidea sp.

Von Lebermoosen bemerkte ich: Sarcoscyphus Funckii (W. et M.) N. v. E., Plagiochila asplenioides (L.) N et M., Scapania curta (Mast.) N.v.E., Jungermannia licrenata Lindenb., .J. Starckii (Herb. Funck) N.v.E., J. divaricata (Engl Bot.) N. v. K., Lepidozia reptans (L.) N. v. E., Pellia epiphylla Dillen, Blasia pusilla L. und Aneura latifrous Lindenb.

(Fortsetzung folgt.)

\section{Beiträge zur Flora von Niederösterreich.}

Von Dr. E. K. Blüinml (Wien).

(Schluss.) neuburg.

Lilimm Martayon L. f. album, mh. ${ }^{2}$ ) Mit weisser Blïte. Kloster-

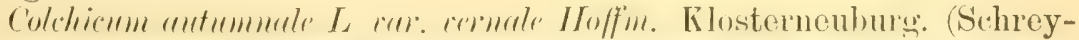
bers sagt in seinen Notizen zum . Jahre 1865) unterm :30. April: ('oldricum rermum erschien heuer (1865) samt den Blättern häufiger als je, ist wohl: coldhicum antumnale ror., die im Herbste nicht mehr zur Blïte kommt.)

Polygynatum latifolinum Inesf. Suchlau bei Klosterneuburg (leg. Huher).

Orchis coriophora $L$. Kritzendorferau.

Orchis militaris L. f. albiflora mh. mit reinweissen Blüten. Haschberg bei Kierling.

Orehis purpurea Inuds. Rohrwald.

Orchis pallens $L$. Siedersgraben bei Klosterneuburg.

Butomus umbellatus L. Donausümpfe bei Klosterneuburg.

Salix daphnoides Vill. Kritzendorferau.

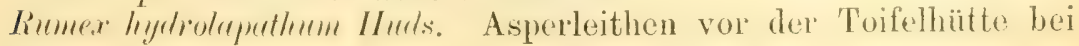
Weidling am Bach.

$\left.{ }^{1}\right)$ Ich benenne diese Form allum, da dieselbe wohl häufig in der Litteratur angegeben wird, mir jedoch keine wissenschaftliche benemnum moch unterkam. auch nicht in Beck's sehr detaillierter Flora von Niederösterreich. Dasselbe gilt auch von den folgenden neuen Farbenformen. 
Sidhisothere rosere (releli. Dei Nusselorf und sanz ohen auf' dem Leopoldsberge (1865).

Amarantus blitum L. Weingärten zu Klosterneuburg.

Alsine verna Bartl. Raxalpe (leg. Fr. Leithe).

Stellaria palustris Eihh. Kierlingthal gegen den Haschberg zu.

Cerastium silvaticum $W$. et $K$. Bei der Windischliüte.

Dionthus superbus L. Haschberg gegen Kierling zu.

Portulace oleracea $L$ Bei Klosterneuburg.

Ramunculus aquatilis L. Bei Klosterneuburg (leg. Kräitz).

Thalictrum flavm L. Sumpfwiesen bei Hadersfeld.

Roripa Austriaca Bess. Bei Klosterneuburg.

Hesperis matronalis L. Raxalpe (leg. Leithe).

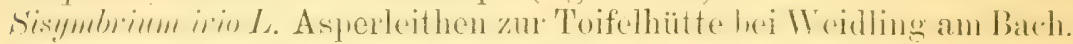

Enysimum Pannonicum Cr. van. dentatum Koch. Rohrwald.

Erysimum repanchm $L$. Wüste Plätze zu Klosternenburg.

Camelina saliou Cr. Weidling,

Bunias emecago L. Hadersfeld.

Helianthemum vulgare Gëintn. Kierling.

Aristolochin clematitis L. Leopoldsberg (1865).

Geranium rotundifolinm L. Leopoldsberg.

Geranium molle L. Bei Klosterneuburg.

Oxulis corniculete L. Stiftsgarten zu Klosterneuburg.

Ruta graveolens L. Verwildert bei Klosterneuburg (leg. Krätz).

Koelventeria paniculata Laem. Ver wildert bei Nussdorf a. d. Donau (leg. Höfer). Nach lieck das erstemal rerwildert, wurde noch nie verwildert angetroffen.

Pimpinella magna L. var. orientalis Gouan. Zwischen Windischhütte und Weidlingbach.

Apium graceolens $L$. Bei Weidlingbach zum Hameau hin.

Laserpitimm I'vethenimm L. Asperleithen ror der 'Toifelhuitte hei Weidlingbach.

Sedum acre L. var. sexamgulare (L.). Bei Hadersfeld. W a bis jetzt für Niederösterreich zweifelhaft.

Saxifraga gramulata L. Eichenhain, Haselgraben.

Rubus plicatus Weihe. Zwischen Kahlenberg und Nussdorf, bei Kierling.

Potentille supina L. Zwischen Weidlingbach und Hainbach.

Rosa canina L. var. syntrichostita Rip. Zwischen Weidling und Weidlingbach.

Rosa rubiginosa L. Bei Klosterneuburg.

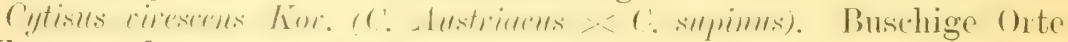

bei Klosterneuburg.

Ononis spinosa L. f. albiflora Neilr. Nussdorf.

Ononis hircina Jac\%. Kahlenberg.

Colutea aborescens L. Bei Klosterneuburg.

Vicia lathyroides $L$. Weidling.

Vicia faba L. Verwildert zu Klosterneuburg (1865).

Ticia monantha Desf. Beim Hameau.

Centaurium inapertum Rafn. (Erythraea pulchella Fries). Gugging.

Gentiana pnemmonanthe L. Haschberg gegen Kierling. 


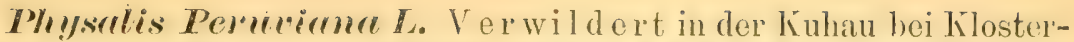
neuburg (2\$. IX 1865 in einem Exemplar vou 4 schuh liohe und 1 Klafter Durchmesser). In Niederösterreich noch nie beobachtet, aus I'eru stammend.

Inture stromonimm I. Bauplatz an der Donau bei lilosterneuburs. Asperugo mocumbens L. Kwischen Leopolds- nnd Kahlenberg.

Anchuse officinalis L f: avoulis lichb. Rothgraben bei Weidling.

Lycopsis arvensis M. a. B. Schüttau bei Klosterneuburg.

Mentha piperita L. Verwildert vor der Toifelhütte bei Weidling am Bach.

Mentha arvensis L. Kritzendotferau.

Stachys amme L.f. rlbre m. Ganz weisse BI üten. Haschberg gegen Weidling,

Lamium album L. Klosterneuburg.

Ajugu puramidulis I.. Am Ilaschherg bei Kierling, im Rohrwald.

Verbascum speciosum Schrad. Bei Hadersfeld.

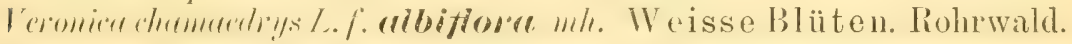

Veronica 'Toumefortii Gm. Schüttau bei Klosterneuburg.

Mclamp!rum pratense L. Kierling.

Orobanche major L. Zwischen Leopolds- und Kahlenberg.

Plantago major L. f. rubra mh. Staubäden und Kronsaum

rot. Kritzendorferau.

Plantago altissima L. Donausiompfe bei Klosterneuburg.

Campame Sibirice L. Hagenthal vor St. Andrä.

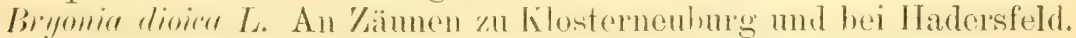

Sambucus racemosa L. Kahlengebire bei Klosterneuburg.

Scabiosa columbaria L. var. agrestis $\mathbf{I}$. et $K$. Donaunfer bei Klosterneuburg.

Inula Germanica L. Bei Weidling und bei Weidlingbach.

Xanthium spinosum L. Schüttau bei Klosterneuburg.

Biclens cemua $L_{\text {. }}$ Kierlingermühlbach.

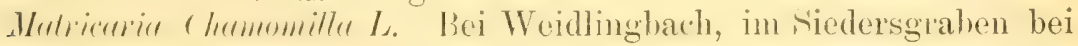
Klosterneuburo.

Clmyernthemmm seyetum I. Unter der Sat bei Klosterneuburg (1865).

Chrysanthemum coronarium L. Verwildert zu Klosterneuburg.

Artemisia pontica L. Leopoldsberg.

Senecio crucifolius L. Kierling zum Haschberg.

Senccio saracenicus L. Kuhau bei Klosterneuburg.

Calendula officinalis $L$. Verwildert in der Kritzendorferau.

Centaniea montana I. Leopoldsberg.

Cichorium intylus L. f. album mh. Schneeweisse Blüten. Am Haschberg gegen Weidling.

Crepis setosø Hall. Muchlau bei Klosterneuburg (leg. L. Huber), Wiesen bei Kierling.

('iepris tectormm L. Twwischen Kahlenberg, Nussdorf und Grinzing (1865).

Hieracium bifurcum M. B. (H. echioides $>$ H. pilosella). Bei Weidlingbach.

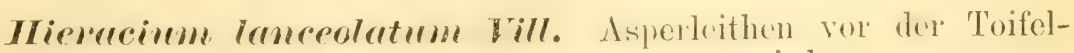
hütte bei Weidlingbach. Neu für Niederösterreich. 
Hieracium Sabaudum $I$ var. silvestre Tansch f. ovatum Mey. Aspèrleithen vor der Toifélhütte bei Weidlingbach.

Hieracium Sabaudum L. var. silvestre T'ausch f. temifolium Host. Leopoldsherg, Asperleithen vor der 'Toifelhiitte bei Weidlinghach.

Tragopogon major $L$. Zwischen Leopolds- und Kahlenberg.

Scorzonera laciniate $L$. Schüttau bei Klosterneuburg.

\title{
Phaenologische Plaudereien aus der Innsbrucker Flora.
}

\author{
Von Dr. Jos. Murr (Trient).
}

(Schluss.)

Aber auch in die zumeist schmeearme Periode der zwei letzten Decennien

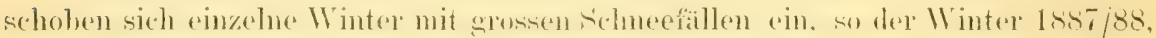
der mit seinen Stanblawinen im Innsbrucker Kalkgebirge und am Haller Salzberge weite strecken alpinen Buchenwaldes niederwarf mol so anch die üppige Krautvegetation mancher chedem heschatteter stellen anf Itacennien, ja vielleicht für immer vernichtete, damn der Winter 1895/96, der sich anch noch in einen ïberaus regnerischen Sommer fortsetzte, welche beiden Umstände eine nie gesehene Hemmung der Gebirgsflora zur Folge hatten.

So sammelte ich am 10. August 1896 im Graben unter der Höttinger Alpe (bei ca. $1300-1350 \mathrm{~m}$ ) Viola sciaphila - neben V.collina die frühblühendste Art der untersten Höttinger Gehänge, die sich in den oben geschilderten gïnstigen Vegetationsperioden hereits Mittr Mar\%, hier when spaitentems Mitte Mai entfilter -

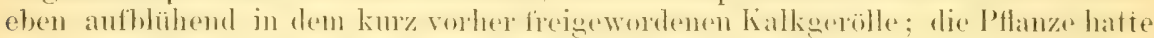
also durch die angehäuften Schneemassen eine Retardation von einem vollen Vierteljahr erfahren. Am 5. September desselben Jahres war Ifieracium subspeciosum Nacy. subsp. comolepin N.I. bei $1850 \mathrm{~m}$ auf exponiertem, lawinenfreiem Standorte unter der Frau Hitt grösstenteils noch nicht aufgeblüht und am 10. Feptember herelissten wir im Lssthale des Haller salzhereses bei $14-1500 \mathrm{~m}$ die gesamte Frühlingsflora, Anemone nemorosa, Primula clatior, Salix glabra, weiter unten Convallaria maialis, Lonicera niqra u.s.w., teils in ihrem Erwachen, teils in schionster Entfaltung.

Viel häufiger als solche Fälle sind im Innthale, wie bereits oben erwähnt, die langen, schönen, sich his genen die Jahreswende erstectienden suatherdste. An einem solchen womnigen Herbstage (20. Nov. 1s8:?) grüksten mich von den Kalkfelsen der Zirler Strasse hart über dem Inn am Fusse der Martinswand dic

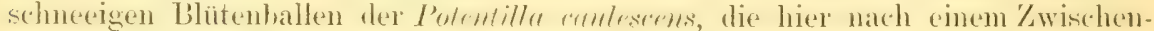
raum von vollen 5 dlonaten zum zweitemmal frühlingslust in sich versuärt hatten, und erst am 5. Dezember nahm ich im Höttinger Berge bei ca. $1200 \mathrm{~m} \mathrm{Ab-}$ schied von den letzten Blütensternen der Saxifraga aizoides. ${ }^{1}$ )

In solchen schönen Spätherbsten, oder vielmehr Vorwintern zeigt sich insbesondere die Flora der Brachäcker an sonnigen Gehängen nochmals in ihrer ganzen Ueppigkeit, so z. B. am 7. Dez. 1878, wo ich in Gesellschaft meines verehrten Lehrers P.Jul. Gremblich über 'Thauer unsere sämtlichen Arten von Acker-Ehrenpreis, Euphorbia Helioscopia, Lithopermum arvense, Sherardia u.s.w. in schönster Blüte beobachtete. ${ }^{2}$ )

$\left.{ }^{1}\right)$ Ein solcher Herbst, wie sie gerne auf einen kithlen Sommer folgen, war auch der von 1875, wo am 16. Okt. P. Gremblich bei $2000 \mathrm{~m}$ am Lavatschjoch bei Hall noch 23 Arten in Bliite fand. Vgl. die ausgezeichnete Schrift Gremblich's: Unsere Alpenwiesen (Programm des k. k. Gymn. Hall 1885) S. 9.

$\left.{ }^{2}\right)$ Noch schönere Aufzeichnungen konnte ich im Spätherbste 1891 in dem freilich viel wärmeren Marburg a. D. $\left(11.2^{\circ}\right.$ C. Jahresmittel) machen. 20. Nov.: Doriycnium herbaceum in Nachblïte; 6. Dez.: Tanacetum vulgare und Solidago am Pyramidenberge; 11. Dez.: Ajuga reptans, Bellis in der Brühl; 14. Dez: Cerastium glomeratum in schöner

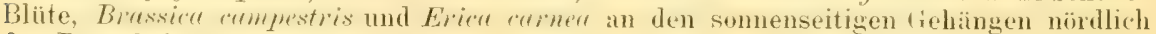
der Drau bei Wildhaus. 
Ja selbst bis Weihnachten und noch - weiter erstreckt sich unter dem Einfluss vorherrschenden Föhnes diese Nachblüte, Im gewiscrmassen dem viachsten Frühlinge die Hände zu reichen.

An einem solchen Weihnachtsabend (1888) pflückte ich blühende Erica am Höttingerbühel und noch mehr als weitere drei Wochen später, am 17. Jan. 1889, war an der sonnigen Klostermauer von Thurnfelel bei Hall noch immer

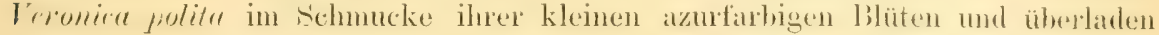
von vollen, eben ausreifenden Kapseln zu beobachten. Dieses Datum bildet freilich die äusserste Grenze der Sachblüte für Nordtirol. Im folgenden 'latge trat strenger Winter ein und als der Frühling wieder erwachte, zeigte es sich, dass ein grosser Teil der Pyramidenpappeh an der leeichsstrisse vor llall das Warme, trockene "Influenza-Wetter jener asten Winterhälfte mit dem 'ívile viler vmheilbarem Siechtum hatte bezahlen müssen.

Damit war aber jene italienisch-milde Dezember- und Januartemperatur noch nicht hinlänglich gerächt. Im folgenden Juni schmeite es immer wieder bis in die Nähe der Thalsohle und am Morgen des 13. Juli 1889 stack das ganze Innthal in tiefer Schneehülle, die sich erst im Laufe einer Woche wieder in das höhere Gebirge zurückzog. Einer fernen Kanonade gleich konnte man in jenem Morgen das Krachen der frechenden fobshaine an den nördlichenteländen des Thales vernehmen und tausende von Lärchen komnte derjenige in jammervoller Weise entwipfelt sehen, welcher durch die unnatürliche Winterlandschaft von Innsbruck die Brennerbahn hinauffuhr.

Noch viel grellere Fälle lange aushaltender Herbstflora liessen sich natürlich aus Südtirol anführen, wo ich z. B. am 2. Dez. 1898 Heteropogon glaber im Grieser Berg bei bozen, damn gleichlabls als Nachblüte einen prichtigen, blumeniibersïeten Rasen von Potentilla. Bolzanensis Vimm. am 5. Januar 1898 bei Fort

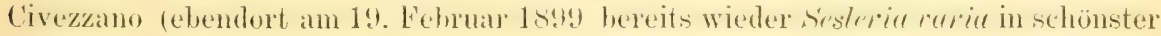
Blüte) beobachtete. Doch hierher driugt ja bereits der Hauch des Mittelmeerbeckens, durch den hier überhaupt schon winterblühende Arten, wie Ruscus aculeatus, dann an Kulturpflanzen Calycanthus praceox und Eriobolrya Saponica

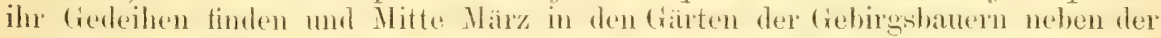
I'firsichblüte bereits starke, hohe stengel von goldig blühendem Kohle unser Auge erfreuen.

Trient am 18. Februar 1900.

\title{
Uebersicht über die bisher in der Umgebung von Guben in der Niederlausitz beobachteten Leber-, Torf- und Laubmoose.
}

Von Otto Will.

\author{
(Fortsetzung.)
}

8. Aneura pinguis (L.) Dmrt. Gemein.

9. Anewra miltifida (L.) Dmrt. An einem nassen Wegrande der Kaltenborner Berge.

10. Blasia musilla $L$. Auf feuchtem Lehmboden häufig.

11. Pellia epiphylla Dillen.' Turnplatz an nassen 'T'ümpeln. Hörnchen an einer Quelle.

12. Frullania dilatala (L.) N.v. E. Häufig. efr. Bürbusch.

13. F. Tamarisci (L.) N. v. E. Gubener Stadtforst am Grunde von Stämmeń; an Wegräudern im Hörnchen.

14. Radule complanata (L.) Durt. Gemein an Baumstämmen.

15. Trichocolea toment.lla (Ehrh.) N. v. E. Auf sumpfigen Wiesen bei der Kieselwitzer Mühle (Schlaubethal!).

16. Blepharozia ciliaris (L.) Dmrt. Gemein, oft Massenvegetation, c. fret. Stadtforst, Hörnchen. Oefter auch als var. ericetorum $N . v . E$. 
17. Pleuroschisma tritobatum (L.) Dmvt. Stadtforst, Bremsdorfer Mühle.

18. Lepidoziu reptans N.v.E. Häufig: Stadtforst, Hörnchen; cfret. Drenziger Schweiz, Schlaubethal bei der Bremsdorfer Mühle.

19. Colyponfer trirhomanis (Dill.) Corde. An lehmigen, fenchten Wegrändern der Drenziger Schweiz.

20. Lophocolea bidentata (L.) N.v.E. Gemein. efret. Bärbusch.

21. L. heterophylla (Schrad.) N.v.E. 'Tumplatz, Stadtforst, bes. am Grumde der Baumstïmme.

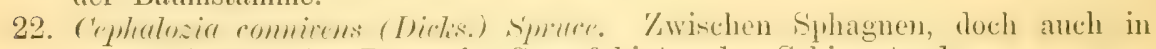
reinen, fruchtenden Rasen im Sumpf hinter den Schiessständen.

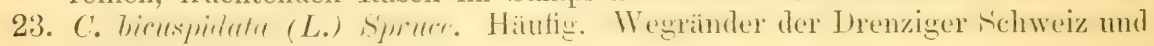
in den Kaltenborner Bergen.

24. C. catemulata (Hïb.) Spr. Neist am Grunde alter Kiefern: Gubinchen, Hörnchen.

25. J. diearicata N.v.E. E Cephalozia byssacea Dmrt. Am Grunde von Kiefern bei Reichenbach.

26. J. Starkii (Hrb. Funcli) N.v. E. = Cephalozia divaricata Durt. Ziemlich häutig: Schiessstände, Lawitz, Hörnchen bei Diehlo.

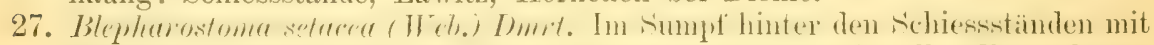

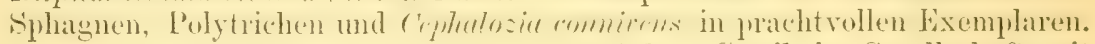

28. Jungermannia quinquedentata Web. Steril in Gesellschaft mit Lophocolea bidentata auf sandigem Boden in den Kaltenborner Bergen legi 2.IV.96. Bisher einziger Standort der Mark.

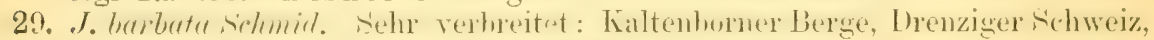
Schlaubethal, am Seeberg bei Atterwasch, Staltforst.

30. J. excisa (Diclis.) Lindb. Auf Heideland hinter den Schiessständen.

31. J. Limprichtii Lindb. An mehreren Stellen, besonders reichlich an Wegrändern in den Schiessständen, trotzdem dieser 'Teil meist mit $\mathrm{L}$ a u b holz bestanden ist!

32. J. bicrenata Schmid. Auf Waldwegen am Seeberg bei Schenkendöbern.

33. J. exentroser Diclis. Auf feuchtem Wialdhoden im Fasanenwald bei Neuzelle.

34. J. Myalina Hook. In der Drenziger Schweiz auf Lehmboden.

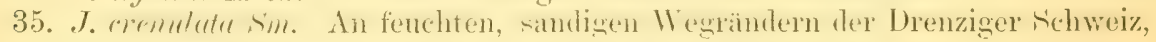
sowie an Ausstichen im Sumpf hinter den Schiessständen.

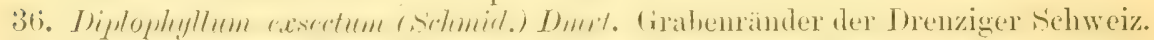

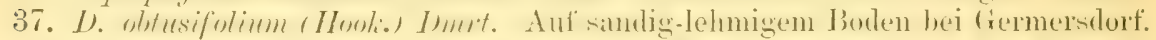

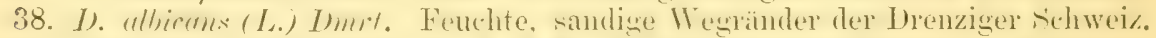

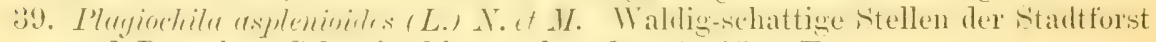
und Drenziger Schweiz, hier auch o. humilis N.v. E.

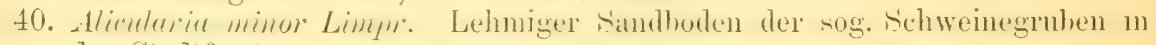
der Stadtforst.

41. A. scalaris (Schrad.) Corda. Wegränder der Drenziger Schweiz, im Kiefern wald vor Sembten und in den Kaltenborner Bergen.

\section{Nomenklatorische Revision höherer Pflanzengruppen} und über einige

\section{Tausend Korrekturen zu Englers Phaenogamen-Register.}

Von Otto Kuntze und 'Tom von Post.

Vergangenes Jahr kündigte Herr Direktor T'om von Post in Upsala ein Wörterbuch der phanerogamen Gattungsnamen an: ich schrieh ihm, dass dieses Unternehmen verfehlt sei, wemn er sich nicht der internationalen und wissenschaftlichen Yomenklatur anschlösse. Daranf relanlasste er nich, die Tomenklatur der Kólasien, ()rinungen, Familien auch noch nach internationalen liegehn zu revidieren und sandte mir ein Verzeir.hnis von ca. 1000 im Engler schen Re- 


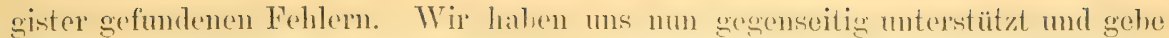
ich im 1. 'T'eil die vorläufigen Resultate meiner Revision. Bei einer Kollation de's lew Index mit. Englers Register zum \%weck, die systematischen Differenzen bei Gattungen zu ermitteln, hatte ich ausserdem noch viele Hundert Fohler gefunden, die im 2. 'Teil zu ergänzen und in Englers Register noch mehr zu be-

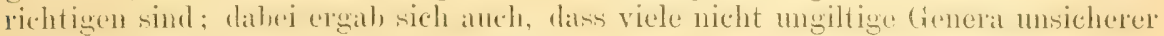
Stellung in Englers Werk völlig fehlen.

San Remo, 1. Juni 1900.

Dr. Otto Kuntze.

Der Pariser Codex von 1867 mit den Lois de la nomenclature botanique enthält für Namen von Divisio (z. B. Ciryptogamae), Subdivisio (z. I3. MLonocolyIrdones), Classis (\%. B. Filices), Subchessis (z. B. Calyciflorae), Cohors (z. B. Daphnales, Geraniales, Piperinae, Primalinae), Subcohors (z. B. Geraniineae) nur für letztere Namen den Wunsch im Artikel 20, sie müchten womöglich mit der gleichen Endung an Finniliemnamen angefingt gebildet werden, also gleiche huffixe erhalten.

Die höchsten Gruppen erhalten dagegen nur Namen, die aus Pluralia simplicia bestehen. Die Cohorten (auch wohl Series oder Ordnungen genannt) wurden von Lindley mit dem Suffix - ales benannt und dem ist Engler in seinen Natürlichen Pflanzenfamilien gefolgt, was nur zu billigen ist. Dagegen verwendet Engler für Subcohorten = Unterreihen das Suffix -ineae, wogegen er - ince für Subtribus, also ganz anders als vor ilm Brongniart, Al. Braun u. a., gebraucht. Gelegentlich ist Engler auch inkonsequent und wendet - inae auch für höhere Gruppen an, z. B. Cycadinae, Coniferinae, Filicinae, Lycopodinae, Guettardinae, I'sychotrinue, Gurdeninae an. Den ersten Namen hat er zwar später in Cycadales korrigiert, aber es kommen sonst genug Verwechslungen dieser Suffixe - inae und - incae vor; ausserden giebt es auch nicht wenige gleichauslautende 'Tribusnamen, wie Borragineae, Alsineae, Cytineac, Commelineae, die zu Verwechslungen führen künnen, so dass es sich empfiehlt, andere Suffixe anzuwenden. Praktisch anwendbar sind nur bisher wenig gebrauchte und Ver-

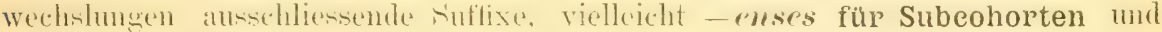
-ance für Subtribus. Artikel 20 möchte also dahin geändert werden: Die Cohorten sind nach einer ihrer Familien mit dem Suffix - ales zu benennen; die Subcohorten mit dem Suffix-enses. Artikel 24: Die Namen der Tribus bilden sich nach einer ihnen angehörigen Gattung mit dem Suffix - eae, die der Subtribus mit -anae.

Der Artikel 21 des Pariser Codex stellt die Endung - aceae an einen Gattungsnamen für Namen von Familien (auch Ordnungen genannt) fest, z. B. Rosaceae, Ramunculucede, und Artikel 22 sub 4 erlaubt auch synonyme Gattungsnamen zu diesem Zweck, z. B. Hippocastamum für Hippocastancae oder vielmehr

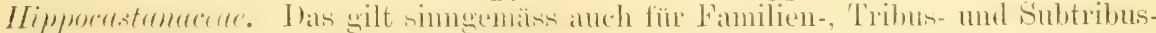
Namen. Wenn also ein Gattungsname geändert wird, darf deshalb weder ein Familienname, noch ein Cohortemname, noch ein Tribus- oder Subtribus-Name im Wortstamm geandert werden. Dadurch wird stabilitit in der Nomenklatur höherer Gruppen gesichert, aber dagegen ist oft genug gesündigt worden.

Die Endung auf - aceae für Familiennamen erleidet-nach Artikel 22 sub 1 für Namen der 3. Declination Ausnahmen, z. B. Salicinece, und sub 2 für zu lange Namen, wie Dipterocarpece, anstatt Dipterocarpaceae; jedoch diese Ausnahmen sind überflüssig, und sub 1 auch verwirrend wegen der sonst anders verwendeten inede-Endung: Sub 1 und 2 des \$ 22 werden daher meist nicht befolgt und sind schon in Artikel 22 sub 2 des Codex emendatus aufgehoben worden. Artikel 22 sub 3 erlaubt einige Ausnahmen von - acere für alte Namen, wie Leguminosae, Compositae, Cruciferae $=$ Cruciatue, Palmae, Graminae ete. Letztere 2 Namen fallen weg, da Palma und Gramen alte Gattungsnamen sind, sodass die korrekte Benennung I'almaceae und Gimminaceae lautet. Nach meiner Revision bleihen bei Lharnoganen mu 3 dusmahmen übrig 
und auch diese können mit dem ganzen Absatz 3 des 22. Codex-Artikel wegfallen, wenn man gemäiss Artikel 65 die Suffix-Endungen - osae, - itae, -atue auch in - aceae ändert, vie es schon manche Autoren, z. B. Lindley, SaintLager mit Cruciacene und Umbellacene gethan haben. Schreibt man also auch

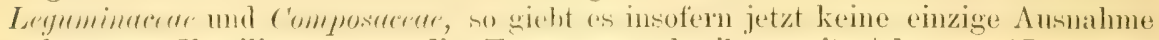
mehr unter Familiennamen; die Franzosen schreiben seit Adanson 1763 stets "Composées", woraus sich consequent Composaceae ergiebt.

Auch die alte klassische Familien-Endung - alae, z. B. Umbellatae, Inun-

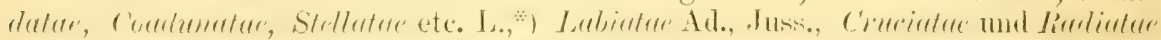

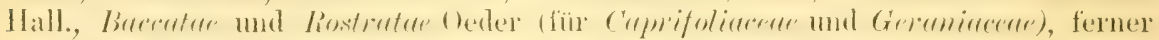
in Papilionatae, Polygonatae, Lythratre, P'asserinatae, Pingriculatae, Tiliatae,

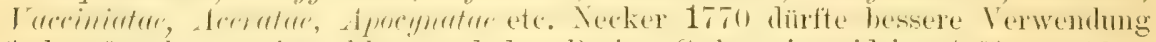

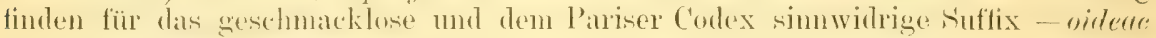
bei Subfamilien-Namen. Dieses Suffix - oideae stimmt schlecht mit dem Sinn des Kornmental zu Artikel 22.2, welcher lateinische Endungen befürwortet; aber Artikel 2:3 selhlägt fïr Sultamilien die Endung - re vor, welche Endung schon für 'Tribusnamen vergeben ist. Um ntm Verwechselungen vorzubeugen, muss wohl ein anderen suftix für Subfamiliemamen genommen werden, aber das darf nicht - oideae, sondern kann nur - atae sein. Das Suffix - oideae ist erst von Ventenat 1799 eingeführt, z. B. Acanthoideae, Cactoideae, Cistoideae, Daphnoideue, Hypericoideae ete. für Familiennamen. Für Subfamiliennamen finden wir dieses an lateinischen Nannen hässliche suffix - vilden in Ascherson's Publikation des Natürlichen Systems von Alexander Braun (1864 Flora der Provinz Brandenbura). Indess sind dort nur wenig subfamilien legrindet worden, während in Engler's N. P'flanzenfamilien fast alle Tribus-Namen aus BHgp. (Jentham und Hooker gen pl.) in sulfimilien mit - videre-Lmonfung erhöht wurden, während die von Bth. u. Hk. meist gut diagnosticierten Sektionen, die Bth. 11. Hk. zu benemen verschmäht latten, neute 'Trihusnamen in Engler's Werk im Ueberfluss erhielten. Diese nenen Tribus sind zwar oft nicht halthar, so dass gar oft nichts ïhrig bleibt, als die massenhaften nenen Engler'schen Namen für diese Gruppen, dabei über 500 mit - videue, die andere Autoren in den meisten Fiallen nach wie vor mit - eue als Tribus werden behandeln müssen.

Nun giebt es ausser Namen mit - atae, von denen z. B. Stellatue bei Ru-

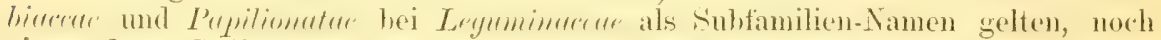
ein anderes Suffix, das manchmal für Subfamilien Anwendung fand: -ideae,

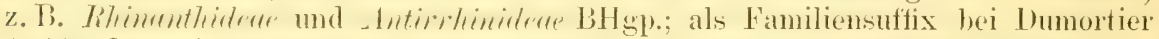

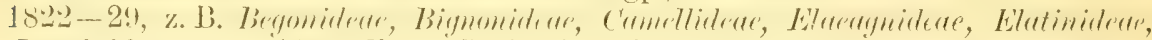
Jasminideae ete. Aber dieses Sulfix ist nicht bloss neueren Datums, hat also keine Priorität vor -atae, sondern ist auch leicht mit gleichlautenden 'TribusNamen zu verwechseln, z. B. Arabideae, Capparideae, Anthemideae, Celtideae,

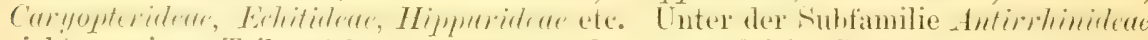
giebt es einen Tribus ITemimerideae, so dass 2 ungleiche Gruppen gleiche Wort-

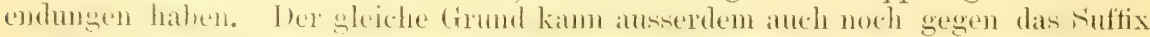

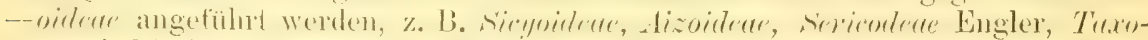

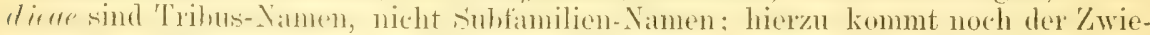
spalt, dass konsequente, also Ordnumg liebende Gelehrte nicht das eine Mal -odece und dass andere Mal - oidede schreiben werden, sondern nach dem Codex emendatus für oideae nur odece anzuwenden hätten. Also viele Gründe sprechen dafür, bei Subfamilien-Namen das Suffix - atce anstatt - oidere anzuwenden. In Artikel 23 ist daher am Schluss anstatt des anderweit schon verwendeten Suffixes - eae zu setzen - atue.

Eine Veränderung der Citation des legalen ersten Begründers einer Familie ist mit der Suffix-Ansetzung oder Suffix-Veränderumg des Namens nicht verbunden, weil dies nach Artikel 65 des Pariser Codex als erlaubte Emendation

C'fr. Ordines naturales am Schluss der 6. Auflage von Linné's Genera plantarum 1764, welches Natürliche System füix unsere Revision in Betracht kommt. 
gilt, so dass die Prioritait nicht verloren geht. \$6.) lanted: "Die Namen für Klassen, Tribus und andere äher der Gattung stehende (iruppen können andere Endungen erhalten, durch welche sie den liegeln und dem fiehrauch angepasst werden."

Dass Pflanzennamen höherer Gruppen auch der Prioritiit unterliegen, ist ausser Kweifel, wenn sie auch bei manchen Autoren fast fïr vogelfrei gelten und in Engler's Natürl. Pflanzenfamilien sogar abweichend von rein wissenschafttichen Werken ohne Autoreitat aufgeführt werden.*) Es kann anch nicht hehauptet werden, dass Familien grösseren Schwankungen systematisch unterliegen, als Genera oder Arten. Meist sind zuerst grosse Gruppen aufgestellt worden, die gelten bleiben, so lange mindestens die Hïlfte davon dabei bleilht; nach uni nach sind einzelne Teile davon abgetrennt worden und dürfen bis zur Hälfto abgetremnt werden, ohme dass die Priorität des legal ersten Begründer's verloren geht, soweit der Gruppeuname überhaupt giltig bleibt. Manchmal sind auch kleine Gruplen zuerst benamnt worden, an denen nach und naich viel sich ankrystallisiert hat, auch dadurch geht die Prioritat des ältesten Namen nicht verloren.

Intolge der Anregung von Prof. Greene (cfr. Rev. gen. pl. III I pag. CCCLXII) hat zuerst Mr. John Hendley Barnhart 1895 im Bulletin of the Torrey Botanical Club XXII S. 1-25 eine Revision der Familien-Nomenklatur vorgenommen; "N hat aber nur eine Revision der Namen mit dem Suffix - aceae vorgenommen, gleichviel ob dieselben für Fimilien oder Sektionen anfgestellt wurden, hat also Artikel 65 des Pariser Codex nicht befolgt, sondern alle Namen, die nicht auf - aceae auslauten, ausgeschlossen. Nun sind aber sowohl von Adanson als von Jussieu die allermeisten Familiennamen nur Pluralia eines Ciattungsnamens, so dass gerade die meisten ältesten Familiennamen von Bamhart vernachläsigt wurden. Dalurch ist er zu ganz falschen Resultaten gelangt. Er ist auch, wie er mir selhst schrieb, von der Unzulänglichkeit seiner "rent-Liste überzengt, aber dieselhe hat mir als Vorarbeit doch gute Dienste geleistet. Die zahlreichen Subfamiliennamen mit acere-Suffix mussten bei der Familien-Revision selbstverständlich ausgeschlossen werden; es sind deren von HBK., DC., Richb., Dumortier, Endlicher, Meisner, Chamisso \& Schlechtendal, Link, I'resl, Lessing, Grisebach viel aufgestellt worden, manchmal mit der Bezeichnumg gemmimer oder reror, um sie von den gleichen Familiennamen zu unterscheiden, manchmal wurde aber auch das Suffix - eae für Familien und - aceae für Subfamilien bevorzugt, und erst durch BHgl. und den Pariser Codex wurde - areae von den Subfimilien vollständig ausgeschlossen.

Ausserlem hat Barnhart, anstatt mit Adanson 1763 anzufangen, mit Jussien 175. angefangen, und die betreffenden Angaben wohl nur aus P'feiffer's Nomenklator entnommen. Aber Pfeiffer hat seine Angaben über „Bernard de Jussieu Ordines naturales in Ludovici XV horto Trianonensi dispositi anno $1759^{\prime \prime}$ doch nur aus Ant. Laur. de Jussieu's Genera plantarum 1789 pag. LXIII-LXX entnommen, wo dieses System von Bernard de Jussieu zum ersten Male veröffentlicht ward. Wenigstens wird es vorher von Niemand citiert, auch von Adanson nicht, der gewissenhaft im 1. Band seiner

*) Das soll in einem Ergänzungswerke "Genera Siphonogamarum" von Dalla 'Torre of Harms nachgeholt werden. Aber das ist fickarbeit und wissenschaftlich verkehrt; denn echte Monographen haben vorher alle Synonyma zu prüfen und dabei gestaltet sich die Systematik oft anders. Nicht aber dïrfen einer unordentlich gefertigten Arbeit, wie Engler's N. Pflanzenfamilien, von fehlenden Synonymen bloss solche, die dem trügerischen Zweck entsprechen, dass nichts geïndert werden darf, hinzugefügt werden. So fehlt $\%$. B. auf der dem 2. Buchbämllerprospekt als Prohe beigefiigten seite hei dem einzigen Familiennamen Commetinacer der ältere siltige Name Ephemere Batsch 1802 , der nach dem Gattungssynonym Ephemerum Moench benamnt ist, so dass also Ephemeracene für Commelinaceae zu gelten hat. Auch ist letzterer Name nicht erst von Reichenbach anfgestellt worden, sondern schon von h. Brown 1810 als Commelineae und Rehb. hat den Namen bloss korrigiert. 
Familles des plantes äher alle frïheren Systeme referierte, Auch citiert Pfeiffer dazu keine Seitenzahlen und bei Pritzel fehlen sowohl der Druckort als dio Seitenzahl, wie er dies doch sonst stets von selbstandig erschienenen Publikationen angiebt. Anch habe ich dieses System von Bernard de Jussieu bisher vergeblich in Bißliotheken gesucht, so dass sich die Pritzel'sche Angabe höchstens auf einen Separatdruck aus A. L. Jussieu gen. pl. 1789 beziehen kann. Also das System von Bernard de Jussien ist in der That erst 1789 publiziert worden. Es ist ein Verzeichnis von Nomina seminuda, würde aber doch, wenn es schon 1759 publiziert worden wäre und man damit anfangen wollte, die Verïnderung von 7 Familiennamen in meiner Revision zur Folge haben.

Barnhart's Vorschlag, dass nur an er k annte Gattungsnamen für Familiennamen dienen sollen, stanmt von Engler, der ihn aber auch nicht konsequent durchiuhrte (cfr. Rev. gen. pl. IIII pag. CCCXVIII)。 Dieses Prinzip ist, wio wir schon sahen, gesetzwidrig und hat in der Engler'schen Nomenklatur schon genug Verwirrung angestiftet. Schliesslich hat Barnart noch das Prinzip anfgestellt, dass Familiennamen, um anerkannt zu werden, lateinisch publiziert sein müssten. Num haben aber Lamarek, A. de Candolle, St. Hilaire, A. L. Jussieu 1804 eine grosse Anzahl Familiennamen zuerst bloss französisch aufgestellt, die sich eingebürgert haben, md die verschwinden müssten, wenn man das Barnhart'sche P'rinzip anerkenmen wollte; z. B, füx Violucés Lam. 1878 käme Jonidia Vent. 1802 = Jonidincede zur Geltung. Und doch hat dieses Prinzip in gewisser Hinsicht eme Berechtigung: man kann nämlich nur solche Namen anerkennen, die an den lateinischen Wortstamm eines Gattumgsnamen das französische acées oder ées angefügt erhielten, wie dies sogar noch in neuester Zeit in Baillon's Histoire des plantes und von Van 'l'iegham (vergl. z. B. Bull. soc, bot. France 189t:1t4) geschehen ist. Man kam aber nicht Wörter wie Camelies St. Hil. $180 t$ (= Cneorme plural) für ('neoraceqe Link 1831, Figniers Lam. 1787 Enc. II: 488 für Artocarpeae DC. 1805 anerkennen, wohl aber z. B. Mirobalanées Juss. 1804 für Combretacene R. Br. 1810. Der Artikel 65 des Pariser Codex bedarf also noch folgenden Zusatzes:

Ein solcher Name, der zuerst in einer anderen internationalen Sprache veröfentlicht ward, gilt nur, wenn er von einem lateinischen Gattungsnamen abgeleitet ist; seine Endung wird latinisiert.

Wir folgen also, un die verschiedenen höheren Gruppen durch Endungen zu kennzeichnen und die grenzenlose Verwirrung und Ungleichheit bei deren Namen zu beheben, folgendem Schema, das der Pariser Codex zum grössten 'Teil gar nicht oder zumteil unrichtig vorgesehen hatte:

Primäre Gruppen bis Subclassis: Pluralia simplicia

Cohors: -ales

Subcohors: -enses

Familia : - acede

Subfamilia: - alae

Tribus: - ene

Subtribus: - anae.

Nun 々ur Revision selbst, soweit sie Verïnderungen betrifft; wir legen die Nomenklatur des Engler'schen System behufs Korrektur zugrunde, nicht, weil dieses System etwa das beste sei - o nein - , sondern weil es das zuletzt ausgearbeitete ist und behandeln die Kryptogamen, welche noch nicht fertig ausgearbeitet sind, hier nur sehr kur. Wegen der Büchercitate sei in dieser vorlïufigen und abgekürzten Nitteilung auf Pfeiffer's Nomenklator verwiesen; die hier angegebenen Jahreszahlen deuten das weitere dort zu Suchente an. Auch die citierte acear-Liste von Barnhart giebt genaue Citate.

Cryptogamae L. 1763 em. exel. Palmae, welche von Adauson zuerst als Familie separiert wurden. Syn. Cryptoc arpeae Fabr, \& Heist. 1763 
Enum. pl. horti Helmst.: 377. Cryptantherae Oeder 1764(-68) Elem. bot. Classis 1. - Die zuerst aufgestellten Klassen sind:

1. Fuci Ad. 1763 enthält nicht bloss Fucacere. sondern auch Landalgen, resp. Süsswasseralgen und der Name Fui hat für Algae Roth 1800 zu gelten. Was Linné 1763 als Algae bezeichnete, enthält noch Lichenes Hepaticae, Lemma, Mrorsilea. Zu den Fuci gehört ein T'eil der Byssi Ad., während der andere 'Teil zu den niederen Pilzen gehört; quasi intermediäir.

2. Fungi Ad.

3. Hepaticae Ad. 1763 (Musci hepalicae Hedw. 1798).

4. Mrusci Ad. 1763 II : 482 ex sect. II. typica (Musci fiondosi Hedw. 1782; Musci foliosi Engler 1889).

5. Filices Ad. em. incl. \$ 1 Muscorum nune Lycopodiacede (Pteridede S. F. Gray 1821; Cormophyta Al. Br. ex Aschs. 1864; Pteridopluyta Cohn 1871, Engler).

Plecenogamae Reb. \& W. 1804 n. corr. (Tegetabilia phaenongma" Willdenow in Rebentiseh Flora Neomarch. pag. VI und "Phaenogamia" Reb. 1. c. XXV.) Syn.: "Nuptice plantarum publicae" L. 1767 Syst. XII. Unverwendbare Bezeichnung, weil aus 3 Wörtern bestehend. Der von Ascherson als linnéisch bezeichnete Name "Ihanerogamae" ist von Linné niemals gebrancht worden. Eimbryonatae Rich. 1808.

Cotylédones DC. $1813=$ Cotyledonede DC. 1818.

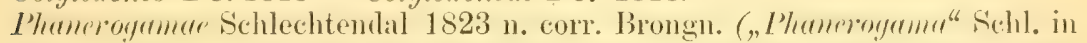
titulo Florae berol,; "Phanerogamen" liuthe 1827; "Ihaneroymme" Batrt. 18:30. Der Name kommt schon 1813 bei I)C. vor ; aber nicht als Klasse, sondern un für eine Sektion der Monocotyledonen).

Anthophyta Al. Br. 1864 ex Aschs. Fl. Brand.

Siphonogamen $=$ Spermaphyten = Embryophyta siphonogama Engler 1889

Nat. P'l. II ${ }^{I}: 1$.

Subdivisiones:

I. Polycotyledones Gaertn. 1788 em. (Gymnuspermar Ldl. 1830 nom auctorum priorum; Archispermae Strassburger 1878).

II. Monocotyledones ()eder 176t Angiosyrimar Lall. 1830 non auctorum 1II. Dicotyledones Gaertner 1788*\} priorum = Meterpermar Strassh.1878. $\mathrm{I}+\mathrm{II}=$ Polycotyledones Fabr. \& Heist. $1763=$ Dicolyledones Juss. 1789.

III mit 3 Klassen:

1. Stamineae Fabr. \& Heist. 1763 1. c. 353 (Incompletae Oeder 1764/8; Apetalae Juss. 1789; Monochlamydeae DC. 1818).

2. Polypetalae Oeder $1764 / 8 \mathrm{em}$. Juss. 1789 incl. Calyricaipar + Cralycicanthemae Oeder = Thalamiflorae + Calyciflorae DC. 1818; Dialypetalae Endl. 1839; Eleutheropetalae Al. Br. \& Doell ex Aschs. 1864; Choripetalae Engler 1889.

Klasse $1+2=$ Archichlamydeae Engler.

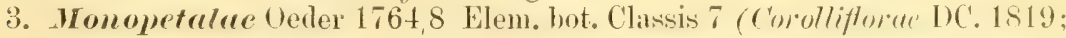
Sympetalae "Synpetalae" Rehb. 1828; Gamopetalae Endl. 1838; Metachlammdeae MacMillan 1892). Monopetalum wurde bei den Patres für. Corolle gebraucht; efr. Lam. dict. IV: 267. Der Name ist daher passend; überhaupt "Un nom est un nom" und darf auch ein unpassender Name nicht verworfen werden.

\section{Familiennamen-Korrekturen:}

Salisburyaceae Link 1831 (Gingloaceae Engler 1897). Auf dem 1. Buch-

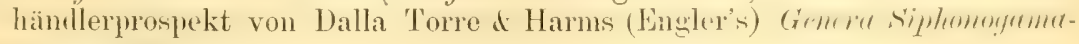

methodica.

*) Gaertner, De fructibus ... I, p. CLXXIX-CLXXXII, Synthesis plantarum 
Inm vom Januar 1899 mit einer Probeseite des 'Textes wird der rechtsgiltige Name Salisburyaceae auch verschwiegen. Dieses Werk ist schon den Probeseiten nach zu urteilen, irreführend und unwissenschaftlich. Selbst die famosen Aprilnomenklaturregeln, die nur für Gattungen und Arten gelten sollten, kömnen das Fehlen solcher legaler älterer Namen nicht entschuldigen.

Thoaceae Ag. 1825 (Ephedracene Dumort. 1827; Gnetacea Ldl. 1834).

Ephemeraceae Batsch 1802 "Ephemera" (Commelineae R. Br. 1810, -aceae Richb.).

Roxburghiaceae Ldl. 1832 (Stemonaceae Fr. \& Sav. 1879).

Narcissaceae Ad. 1763 "Narcissi" (Lencojaceae Batseh 1802; Amaryllidea? St. Hil. 1805, - accue n. corr. Ldll.).

Zingiberacea Ad. 1763 "Zingiberes" (Scitamineue I. 1764; Camae Juss. 1789 ; Marantacea Ldl.) BHgp. führen das incl. Musaceae Juss. "Musae" als Sritamineare auf, dabei die Section Cameae BHgp., welche in Engler's Werk, nebst der Section Maranteae BHgp. leichthin zu besonderen Familien erhoben ward: Jussieu nahm Canneae "Camnae" im weitesten Sinn, wovon Lindley Marantaceae incl. Cama abtrennte.*)

Nuculaceae Lam. \& DC. 1805 (Juglandeae DC. 1813, - aceae Ldi.).

Castaneaceae Ad. 1763 "Castaneae" n. corr. Baill. (Betulideae S. F. Gray 1821,

- aceae Ag. \& Faginae Rehb. 1828, - aceae Drude = Cupuliferae Rich. 1808 ;

Corylaceae Mirb. 1815; Quercineae Juss. 1816).

Artocarpaceae DC. 1805 (Fici Lam. 1805 syn. ex DC.; Curicae Link 1822;

Sycoideae Link 1829; Woreae Endl. 1833, - aceae corr. Ldl.).

Vis cacea Bart. 1802 "Tiscinae" n. corr. Miers (Lorantheae Juss. 1808, - acate Don.).

Osyridaceae Juss. 1804 "Osyrideae" (Santalaceae R. Br. 1810).

Ophiraceae Rehb. 1828 "sOphirea" (Grubbiaceae Endl. 1838).

Cytinaceae Brong. 1824 "Cytineae" n. corr. Lal. (Rafflesiaceae Dumort. 1829). Persicariaceae Ad. 1763 "Persicariae" (Polygonatae Neck. 1770, - aceae Ldl.). Blitaceae Ad. 1763 "Blita" (Atriplices Juss. 1789; Chenopodeae Vent. 1799,

- aceae Dumort.).

Jalapaceae Ad. 1763 "Jalapae" (Nyctayines Juss. 1789, - aceae Ldl.)

Ficodacea Juss. 1789 "Ficoideae" (Aizoidea Spr. 1818; Aizoacene Al. Br. 1864). Alsinuceae Ad. 1763 "Alsines" (Caryophyllei L. 1764, -aceae Rachb.).

Seda ceae Ad. 1763 ,Sedae" n. corr. Neck. (Sempervivae Juss. 1789; Succulentue

Vent. 1779 non L.; Crassulaceae DC. 1801).

Chailletiaceare R. Br. 1818 ,Chailleteae (Dichapetalaceae Engler 1898).

*) In Engler's bot. Jahrbichern 1899 S. 258-350 hat Prof. C. Schumann die Kenntnis der Zingiberaceae durch viele neue Arten selu erweitert, woraus auch resultierte, dass meine Emendation der Sektionen Horaninow's unhaltbar geworden ist. Schumann's erweiterte Genera Alpinia und Amomum sind aber jetzt durch kein einziges reelles Merkmal mehr verschieden, seine Umgrenzung dieser Genera ist wissenschaftlich unhalthar und müssen sie nun unter Alpinic L, vereinigt werden. Bei seiner Polemik versteigt sich Prof. Schumam aber zu der kühnen Sentenz: Man kann ein Gebilde aus Blattscheiden doch niemals einen Caulis nennen " und erlaubt sich deshalb von nicht genügend geschulten Botanikern zu reden. Num, mich trifft das nicht, dem ich habe in Rev. gen. II : 689 von Scheinstengeln fir diese Sache geschrieben und die Definition "Caulis e vaginis involutis... compositus" gegeben, also wie $\mathrm{BHgp}$. diesen Ausdruck Caulis für den Spezialfall elläutert gebraucht. Wemn aber dieser Sentenzler derart auch Bentham und Hooker als nicht geschulte Botaniker hins'ellt, so ist das von ihm, obwohl Professor, nicht ernsthaft und ausserdem in der Sache entstellt. Bei dieser Gelegenheit erlaubt er sich auch 1. c. 264, die von ilm verfassten Aprilnomenklatur-Regeln, die er thatsächlicb meist nicht befolgte, als "Berliner Regeln" zu bezeichnen; da diese Regeln nur von Beamten des Berliner bot. Museums unterzeichnet wurden, ist es - zart gesagt - unrichtig, sie als "Berliner Regeln" zu bezeichnen; dies umsomehr, als sehon vorher auf den Missbrauch und die Unzulässigkeit dieses Ausdruckes hingewiesen ward. 
Tithymalaceae Ad. 1763 "Tithymali" (Tricoccae L. 1764; Euphorbice Juss. 1789 , - acere St. Hil.).

Terebinthaceae Juss. 1789 ex $\$ 1$ u. 2 typ., p. maj. (I'istacias Ad. sed ex parte minore; Anacardicae R. Br. 1818, - aceae Ldl.).

Phytocrenaceae Walker-Arnott 1834 "Phytocreneae" (Barrereaceae Martius 1835; Cardiopterideae Bl. 1847; Icacinaceae Miers 1852).

Millingtoniarea Wight a Arn. 18:34 (Wrllingtoniarene Meisn. 1840; Meliesmeat Endl. 1840; Sabiaceae Bl. 1851).

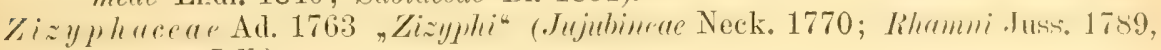
- aceae Ldl.).

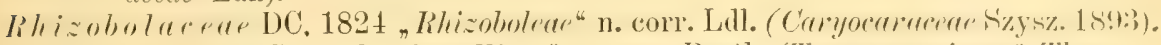

Camelliaceae DC. 1813 "Camellieae" n. corr. Bartl. (Temstroemieae de Theaceae Mirb. Dec. 1813. In DC. prod. I : 529 wird der im gleichen Jahre publizierte Name prioritatis causa vorangestellt; Ternstroemiaceae R. Br. 1818). IIyper icaceae Juss. 1789 „Hyperica" Gen. pl. pag. 254 ex toto, n. corr. Ld1(incl. Guttiferce Juss. 1. e. pag. 255 ex parte).

Samyda ceae Gaertn. 1805 „Samydeae" (Flacourtianae Rich.1815, - aceae Ldi.) Papayaceae Bl. 1823 (Caricacene Dumort. 1828).

Salicariacear Ad. 1763 ,Salicaria" (Lythratae Neck. 1770; -aceae Ldl.). II ens low iaceare Lill. 1834 (Crypteroniea Endl. 1847; Sommeratieae \$ Miq. 1855, - aceae Engler; Blattiaceae Niedenzu 1892).

Granataceae Don 1737 ,Granateae (Punicnceae Horan. 1843).

Napoleonaceae Beauv. 1807 , Napoleoneae" (Belvisiene R. Br. 1811; Lreythidrae Rich. 1824, - aceate Lill.).

Paleluvievaceae Lam. 1796 ,famille des Palétuviers" Enc. IV : 966 (Rhizophoreae R. Br. 1814, - aceate Lell.).

Myrobalanaceae Juss. 1804 "Mirobalanes" (Termmaliaceae. St. Hil. 1805; Combretaceae R. Br. 1810).

Epilobiacea Vent. 1799 "Épilobiana" n. corr. DC. (Onagrarieae St. Hil. 1805; n. corr. Dumort. Non Onayrae Bd., Juss. familia confusa).

Hygrobiaceae Rich, 1808 "Hygrobiae" (Halorageae R. Br. 1814= Halomhagidacene KI. \& Garcke).

ITederaceae L. 1764 (Araliae Juss. 1789, - aceae Vent. 1799).

Umbellaceae Ad. 1763, Uimbellatae" n. corr. Ldll. (Umbelliferae Crantz 1767).

Mo notropaceae Nutt. "Monotropere" (Piroleae Lill. 1821, - aceae Drude).

Vacciniaceae Ad. 1763 "Vaccinia" (Rhododendra of Ericae Juss, 1789, Ericacerte DC).

Ophiospermataceae Vent. 1800 "Ophiospermae" (Ardisiaceae Juss. 1810; Myrsineae R. Br. 1810, - aceae Lill).

Anagallidaceae Ad. 1773 ,Anagallides" (Preciae L. 1764; Lysimachiae Juss. 1789; Primulacere Vent, 1799).

Jasminaceae Ad. 1763 "Jasmina" n. corr. Don (Lilaceae Vent 1799; Oleina" Hfg. \& Link 1806, - aceae Ldl.).

Strychnaceae DC. 1813 „Strychneae" (Spigeliaceae of Loganieae Mart. 1828).

Hydroleacea HBK. 1818 (IIydroplygleae R. Br. 1823, - aceae Ldl.).

Pinguiculaceae Nesk. 17.70 "P'mgniculatue" (Lentibulariene Rich. 1808, -acene Ldl.).

Aparinaceae Ad. 1763 "Aparines" (Stellatae L. 1764; Rubiatae Neck. 1770, - aceue Juss.).

Scabiosaceae Ad. 1763 "Scabiosae" (Dipsaceae Juss. 1789).

Bryoniaceae Ad. 1763 "Bryoniae" (Cucurbitaceae L. 1764).

Goodenoughiaceae R. Br. 1810 ,Goodenoviaceae" n. corr. K. Schum. Goodenieaceae Dumort, 1828).

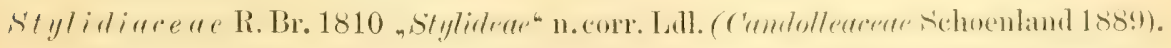

Von den 277 phaenogamen Familien sind in Eugler's N. P'llanzenfamilien 53 , also rund $20 \%$, der fünfte Teil unrichtig, mgenetzlich mol unwissenschaft. 
lich benamnt. Nach ainer schriftlichen Aeusserung Engler's auf die im Manuskript gelesenen betreffenden Einwendungen des Herrn Tom von Post, bindert Engler sich jetzt hei Familimumen überhaupt an keine Regeln mehr; er hat also das verwirente Prinzip, dass ein Familienname nach einem giltigen dattungsnamen zu henennen, resp. zu verändern sei; anfgegeben. Dadurch wurden übrigens nur wenige Familiemnamen berührt. Das Crestamlnis, keine Regel bei Familiennamen mehr zu hefolgen, ist wenigstens ehrlich; hei der Gattungsnomenklatur stellte

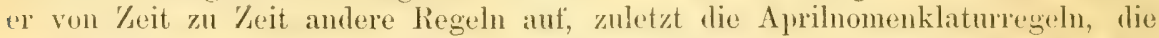
nachweislich meist nicht und nur zum Schein durehgeführt wurlen. ${ }^{*}$ Das Resultat bleiht, dass sowohl seine Familiemonenklatur zu 1/5 und die Nomenklatur fiir 17000 (hattungen etwa \% "1/18 mrichtig ist, wovon anch nach seinen cigenen letzten Regeln der grössere T'eil nicht gelten kanu.

\section{Tribusnamen-Korrekturen.}

Zeeae Rehb. 1828 "Zeaceae" (Mayderte Matthieu 1853).

Sorgea Ad. 1763 "Sorga" (Andropogoneae Presl 1830).

Poeae Ad. 1763 "Poae" (Poaceae R. Br. 1814; Armmlinaceae HBK. 1815; Festuceae Nees 1829).

Triticeae Ad. 1763 "Tritica" (Hordeae Ldl. 1847).

Cryptangieae BHgp. 1883 (Hoppieae Pax 1887, Bisboectelerieae Pax 1897).

Cal ameae HBK. 1815 (Metroxyleae Drude 1889).

Dracontieae Schott 1832 (Lasiene BHgp. 1883).

Pythonieae Endl. 1837 (Amorphophallinae Schott 1860).

Richardieae Schott 1856 (Zantedeschieae Engler 1889).

Arisareare Schott 1832 (Arinae Schott 1856 n. enrr. Areae Engler).

Terotea Rehb. 1828 (Lomandieae Engler 1888).

Cevere Ad. 1763 ,Cepae" (Allicae Kth. 1842).

Lilieae Ad. 1763 "Lilia" ('Tulipeae Koch 1837).

Querceae Dumort. 1829 (Castaneae Prantl 1859).

Bragantiear Rehb. 1837 (Apameae Solereder 1889).

Calligoneae C. A. Neyer 1840 (Atraphaxidede Dammer 1891).

Boerliaaviea Rehb. 1837 (Mirabileae Meisn. 1841).

Hydropeltideae DC. 1821 (Cabombene Hk. f. \& Th. 1855, - oidene Casp. \& Engler 1891).

Nymphacea DC. 1821 ('Tetrasepalae Casp. 1891).

Perseat Miq. 1857 (Cimamonieae Pax 1891).

Hunnemannieae Bernh. 1833 (Platystemoneae Rehb. 1837, Eschscholtzicae Rehb. 1841).

Fotheryillea.e DC. 1830 (Parrotiene Gardner 1849).

Amygdaleae Vent. 1799 (Prmeae BHgp. 1865, - oidene Focke).

Pivea Dumort. 1829 (Pomeae Ldl., Pomaceae § Juss. 1789 sed nomen delendum.

Pomm ist kein Gattungsnamen, wie es $\$ 23$ des Codex verlangt).

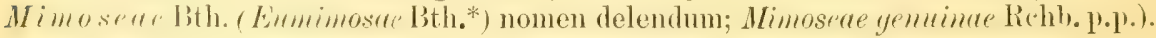

*) Rev. gen. pl. IIIII pag. 28-140 der Einleitung. Die Aprilnomenklaturregeln sind in Engler's Jahrbüchern 1899 Beiblatt Nr.63 durch eine "Erklärung" ergïnzt worden, die vom 11. Juli 1899 datiert, also zu einer 'Leit, als die Hälfte der unters zeichneten Beamten in den Ferien war (!). Eine Erklärung, die also auch ohne deren vorberige kollegiale Beratung verfitst ist. Sie bestätigt nur meine Angaben, dass viele Monographien und von Engler's Nomenklatur alweichende Publikationen anf einen Index librorum prohihitorum bei Anwendung dieser heheinregeln gesetzt, d. h. also sar nicht berïcksichtigt wurden. Vergl. auch Bot. Centralblatt LXXIX : 409 sub 3.

***) Bei Namen höherer Gruppen ist diese Vorsilbe "Eu“ nicht erlaubt, nur bei Subgenera. Ich eitiere aber stets den Autor (in diesem. Falle also Bth.), der für die giltige begrenzung dex Gruppe verantwortlich ist; ebenso wie eventuell bei z. B. Mimoseae genuinae oder verae Rchb. nur Rchb. zu citieren wäre, obwohl genuinat oder verae wegfiele. Nachdem - aceae nur für Familien und-eae nur für Tribus angewendet werden, ist die früher dafür angewendete Unterscheidung L't-, genuina', wroe iiberflïssig geworden. - Gemina, rera, typica, normalis sind bloss noch bei Varietiten- 
Detarieae DC. 1825 p. p. (Cynometreae Bth. 1841 p. p.).

Lagonychieae Rehb. 1842 (Adenanthereae Bth. 18t2).

Brovone ea Rchb. 1827 (Amherstieae Bth. 1840).

Cercidea Brom 1822 "Cerceae" (Bauhinieae Bth. 1840).

Astraguleae Ad. 1763 "Astragali“ (Galegeae Bromn 1822).

Coronillea Ad. 1763 "Coronillae" (Hedysareae DC. 1825).

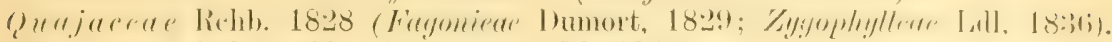

Quasica Rehb. 1828 (Simambeae P'l. 1846).

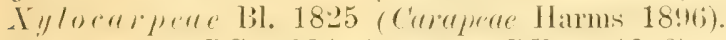

IIyptayeare DC. 1824 (Hivaecre BHgp. 1862).

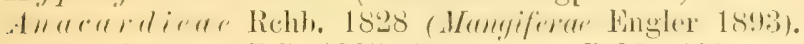

Sumachineae DC. 1825 (Pistaciene Rehb. 1836; Rhoideae Engler 1893).

Evonymeae DC, 1825 (incl. Eucelastreat Loesener = Celastroideae Loesener mit völlig unhaltbarer Differenz und willkürlicher Schaffung von Sub. familiennamen).

Elacodendreae Endl. 1840 (Cassinioideae Loesener 1892, Tribus- und Sub)familiemame zu gleicher 'Leit).

Allophyleae Bl. 1847 ('Thonimieae Bl. 1850 em. Radlk.).

Ceanotheate Rehb. 1828 (Rhammene BHgp. 1862, non priorum).

Malvavisceae P'resl 1831 ,Malvariscaceae \$" (Pavonieae Rehb. 1837, Urenecre Rehb. 1841).

Eriodendreat Rehb. 1837 (incl. Adrmsonieae Rehb. 1837, aber nachher und p.p.). Tridesmeae Rehb. 1837 (Cratoxyleae BHgl\% 1862),

Delimeae DC. 1824 (Tetracereae Gilg 1893).

Gordonieae DC. 182t (Camellieae Rehb. 1827; Therae Baill. 1872).

henennungen anwendbar. Die Praefixe Eu-, Auto-, Typo-, Archi-, Proto- wendet man gern bei den iltesten, resp. typischen Sektionen eines Genus an und ist es all-

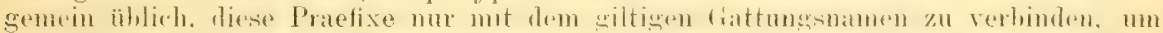

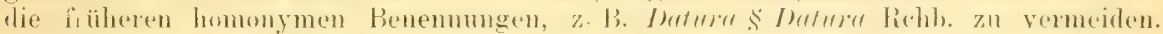
Wenn also ein Gattungsname geändert wird. so wird auch der betreffende Name der typischen Tntergattung geandert. Fingler ging sogitr noch weiter umel indecte, als er Lomandra Lab. 1804 für Xerotes $\mathrm{R}$. Br, 1810 bevorzugte, nicht bloss \& Euxerotes Bth. in \$Eulomandra um, sondern auch \$Schoenoxeros Bth. in \$ Schoenolomandra Engler. Das letztere ist aber gegen den allgemeinen Gebrauch; eine solche Umänderung betrifft sonst stets nur die typische Sektion, immer vorausgesetzt, dass nicht vorher iiltere lattungssynonyme als suhgenusnamen Verwendung fanden, z. B. für die somst

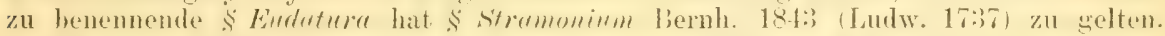
Inas Praefix lin der Subgeneranamen ist erst seit $180 \mathrm{~s}$ durch I) four die typischen Sektionen in Aufnahme gekommen; jetzt giebt es etwa 1500 soleher Subgeneranamen. Ohengenannte substitute fiur lin sind erst nenerdings aufgekommen und notwendig geworden. Vor 1 se wurden typische Soktionen meist nur mit dem Gattumesnamen gleich benannt. Für Familiennamen und 'l'ribusnamen war es ähnlich üblich und wurde später auch $E$ - für 'Tribusnamen verwendet, z.B. Euhedysareae, Euguetturdece; aber bei 'l'ribusnamen ist Eu wenig eingeführt und jetzt durch den Pariser Codex dafür verboten, da Familien und 'Tribus durch aceac und eae unterschieden werden. Ebenso wie plurale Sektionsnamen bei Erhöhung zu Gattungsnamen. nicht zu verwenden sind, ebenso kännen sie in P'rioritätsfällen mit normalen singularen Sektionsnamen in Konkurrenz kommen. Sie sind daher am hesten ganz zu vermeiden. Das erfordert noch einen Zusatz zum Pariser Codex, der sich aus meiner später zu veröftentlichenden Revision der Subgeneranamen ergab. Ich hatte gehoffr, in Rev. gen. ILII den Codex emendatus zum Abschluss gebracht zu haben, aber ich hatte damals noch nicht die Nomenklatur der Familien, 'Tribus und Subgenera vorgenommen und man lernt bloss durch umfassende nomenklatorische Praxis die Lücken des Codex kennen und richtig ausfiilen. Lis ist demnach zu Artikel 53 sub 4 des l'ariser Codex noch himznzufiign: Der Name der typischen Sektion mit Eu-, Auto-, Archi一, Proto-, Typo-beginnend, ist nur mit dem giltigen Gattungsnamen zu verbinden. Sind vor einer solehen benennung schon ïltere diat tungsSynonyme als Sektionsnamen verwendet worden, so haben diese zu gelten. Mit dem Gattungsnamen gleichlautende Sektiousnamen, wie Gerbera Gerbera Less. und plurale Sektionsnamen sind zu verwerfen. 
Symphonieare Choisy 1824 (Moronobeae EndI. 1840).

Prockieae Rchb. 1837 (Abatieae BHgp. 1867).

Casearieae Rehb. 1827 (Samydeae Dumort. 1829).

Salicarieae DC. 1826 (Lythreae Wight d Arn. 1834).

Lagerstroemiear DC. 1826 (Nesaeeae Köhne 1891).

Cercodieae Rehb. 1828 (Halomhageae Petersen 1893).

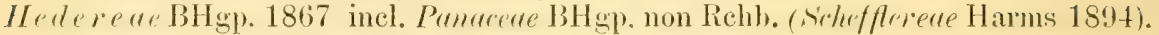

Daboecieae Richb. 1841 (Phyllodoceae Drude 1889).

Dentellariea Rehb. 1828 (Plumbagineae Ldl. 1836).

Chironieae Rehb. 1828 (Gentianeae Dumort. 1829).

Carissea Bartl. 1830 (Arduinerie K. Schum. 1895).

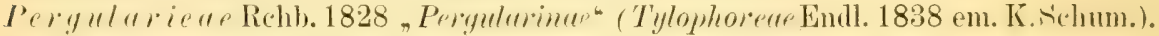
Cynancheae Rchb. 1828 (Asclepiadeae K. Schum. 1895).

Westringieae Bartl. 1830 (Prosteranthereae Bth. 1834, - oideae Briq.).

Pentaraphieae BHgp. 1876 (Gesnereae Fritsch 1893 non priorunı).

II edy otide ae DC. 1830 "Hedyoteae" (Oldenlandieas K. Schum. 1891).

Aparineae Link 1809 (Gulieae Dumort. 1829).

Caprifoliea HBK. 1818 "Caprifoliaceae verae (Lonicereae Rehb. 1828 non

R. Br.; R. Brown's Lonicereae ist Familienmame und wurde für Caprifolia

$\$ 1$ Juss 1789 substituiert. Nur diese 1. Sektion Jussieu's gehört zu Capri-

foliaceae Ad., dessen Name auch nur aus der 1. typischen Sektion gilt).

Whandirobeae Ag. 1825 (Fevilteae BHgp. 1867).

Bidenteae Ad. 1763 "Bidentes" ex 16/17 (Heliantheae DC. 1810).

Cardueae Ad. 1763 „Cardui" (Carduinae Cass. 1818; Cynareae Less. 1830).

Lactuceae Ad. 1763 "Lactucae" (Cichoriaceae Spr. 1818).

Also tso 'Trihusnamen sind in Engler's N. Pflanzenfamilien prioritatis cansa vollstandig durch ältere giltige zu ersetzen. Es sind noch viel mehr, das doprelte etwa melur, wenn man die in diesem Werke oft leicht zn Subfamilien erhobenen 'Tribus amderer Autoren noch revidiert, I)a hierbei systematische Beurteilungen mol Erigterungen inbetracht kommen, so will ich mir das für die spätere ansführlichere Arbeit vorbehalten. Recht leichte nene 'Tribus flndet man \%. B. bei

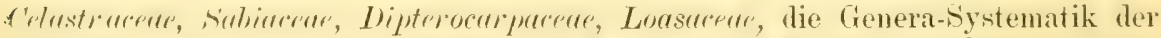

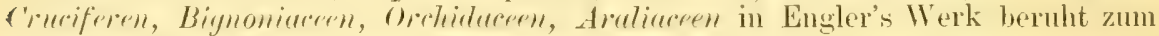

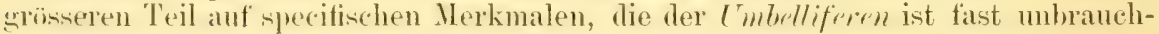
bar, die der Comolvuluceen fictiv (cfr. Rev. gen. IIIII : 207-212). Es ist viel schablonenhafte, von sehr ungleichwertigen Hitarbeitern hesorgte Sulitersystematik darin, die mit dem 'Titel "Natürliche" P'flanzenfamilien gar nicht recht zusammenpasst.

(Forts. folgt.)

\section{Botanische Litteratur, Zeitschriften etc.}

Oesterreichische bot. Zeitschrift 1900. Nr. 5. Linsbauer, I. u. K., Eimige teratologische Befunde an Lonicera 'Tatarica. - Horák, B., 'Zweiter Beitrag zur Flora Montenegro's. - Polak, J. M., Untersuchungen über die Stammodien der Scrophulariaceen. - Blocki, Br., Ein kleiner Beitrag zur Flora von Ostgalizien. - Wettstein, R. v.. Die nordamerikanischen Arten der Gattung Gentiana; Sekt. Endotricha. - Vierhapper, F., Arniea doronicum Jacq. mul ihre nächsten Verwanden. - Litteratur-Uebersicht. - Nachtrag. Bei der Inhaltsangabe der Nr. 2 der "Oesterr. bot. Z." wurde die Angabe folgender Beilagen aus Versehen weggelassen: A ruold, William Nylander U. Massalango (2 kurze Biographien mit Porträts).

Deutsehe bot. Monatsehrift 1900. Nr.4. Höck, Dr. Fr., Allerweltspflanzen in unserer heimischen Phanerogamenflora. - Murr, Dr. J., Beiträge und Bemerkungen zu den Archieracien von Tirol und Voralberg. - Hanemann, Die F'lora des Frankenwaldes, hesonders in ihrem Verhailnis zur Fichtelgebirgstloria. Schmidt, H., Neue Funde aus d. schlesischen Vorgebirge. 
Bot. Centralblatt 1900. Nr. 18, K ïkenthal, (t., Species (ieneris Uncinia Pers. in Americal merilionali extratropica sponte natsentes. - Nr. 19. Derschau, M. v., Die Entwicklung der Peristonzïhne des Lauhmoossporogoniums. - Nr. 20. Derschau, M. v., Wie in vor. Nr. - Nr. 21. Enthailt nur Referate.

Mitteilungen der bayerisehen bot. Gesellsehaft 1900. Nr.15. K' ï $17 \mathrm{le}$, J., Die Adventivflora Bayerms. - M üller, Ed., Phänologische Tieoharhtungen im Haag (()berhayern). - F ischer, Dr. (:., Ueher eine für liayern nene Varietät von Chrysanthemum inodorum I. - W $\mathrm{W} n \mathrm{genm}$ a yer, H., P'ulmonaria montana Lejeune $>$ officinalis L. - W W iss, Dr. J. E., Ueber den gegenwïrtigen Stand der Bekimpfung der Pilzkrankheiten unserer Kulturgewiichse.

Berichte der deutschen bot. Gesellschaft 1900. Heft 3. Heydrich, F., Eine systematische Skizze fossiler Melobesieae. - Vries, Hugo de, Das Spaltungsgesetz der Bastarde. - Lemmermann, E., Beiträge zur Kemutnis der Planktonalgen.- N a t a n s o hn, A lex ander, Ueher Parthenogenesis bei Marilia und ihre Abhängigkeit von der Temperatur. - He in richer, E., Nachtraige zu me iner Studie üher die Regenerationstähigheit der Cystopteris-Arten. - Ule, E. Verschiedenes über den Einfluss der Tiere auf das Pflamzenleben. - Nr. 4. Cza pek, F., Ein Thermostat für Klinostatenversuche (mit 2 'Tafeln V.). Lemmermann. E., Beiträge zur Kenntnis der Planktonalgen. - Sehmidle, WV., Beiträge zur Kenntnis der Planktonalgen, mit 1 Holzschnitt. - Correns, C., (i. Mendels Regel über das Verhalten der Nachkommenschaft der Rasienbastarde. - Burgerstein, A., Ueber das Verhalten der Gymospermenkeimlinge im Lichte und im Dunkeln.

Verhandlungen der k. k. zoologisch-bot. Gesellschaft in Wien 1900. Heft 2 u. 3. Lütkemüller, Dr. J., Dermidiaceen aus der Umgehung des Millstätter Sees in Kämten. - Fritsch, Dr. C., Leher den Formenkreis des Orobus luteus. - Keller, Louis, Zweiter Beitrag zur Flora von Kärnten. - Heft 4. W it a sek, J., Campanula Hostii Baumgarten und (. pseudolanceolata Pantocsek. Strasser, P. Pius, Pilzflora des Sonntagberges (N. Oestr.).

Bulletin de l'association Française de botanique 1900. Nr.27. Brachet, Fl. Excursions botaniques de Briançon aux sources de la ('larée et la Durance.I3 la ne ha $\mathrm{r}$, 'Th., Liste des noms patois de plantes aux environs de Matlezais.Sudre, H., Excursions batalogiques dans les Pyrenées. - Oliviers, H. l'abbé, Exposé systématique et description des Lichens de l'(uest et du Nord-Ouest de la France. - Letacq, A. L., l’abbé, Le Gui de chêne. - Nr. 28-29. Leveillé, H., Les formes de l'Epilobium palustre I. - Brachet, Fl., Excursions botaniques de Briançon aux sources de la Clarée et de la Durance. - Deysson, J. et C'assat, A., Contribution i l'étude des phénomenes de tératologie végétale. Sudre, H., Wie in vor. Nr. - Olivier, H. l'abbé, Wie in vor. Nr. - N. 30. Beleze, Mlle. Mte., Liste de quelques Mousses et Hepatiques de la foret de Rambouillet (Seine et Oise). - Blanchard, Th., Wie in Nr.27. - Olivier, l'abbé, Wie in $\mathrm{Nr} .27$.

Bulletin de l'académie internationale de géographie botanique. 1900. Nr. 125-126. Léveillé, H., Onothéracées japonaises. - Le Gendre, Ch. Vieux arbres. - Simons, Lug., Sur le conditions de végetation du ciui. Claire, Ch., Un coin de la flore des Vosges. -- Léveillé, H., Contributions à la flore de la Mayenne. - Petitmengin, Sur quelques plantes rares et adventives en Lorraine. - Léveillé et Castanet, Les plantes utiles de la Mayenne. -- Capoduro, De la conerescence en botanique et en tératologie végétale. - Monguiljon, E., Catalogue des Jichens du département de lat Sarthe. - Nr. 127. Petitmengin, Sur quelques ()rehidees du platean de Mirzéville. - A cloque, La digestion des Népenthes. - I zo a d, Lu cals tératologique de Vinca ninor. - C a poduro, De la concrescence en botanique et en 
tératologie végétale. - Jamin, V., Ohservations fongiques dans la Sarthe. Claire, Ch., Un coin de la flore des Vosges, - Léveillé, H., Wie in vor. Nr. - Monguillon, E., Wie in vor. Nr.

Botaniska Notiser 1900. Nr. 3. Nilson, N. H., Om de subarktiska Poaarterna vid Lenafloden. - Sernander, R., Om livetets äldre historia. - Juel, O., Om apogamion tos Balanophora jümford med Antennaria alpina. - Gustafsson, J. P., 'T'vit svenska Alopecurus-hybrider. - Kjellman, F. R., En del organogratiska medelanden. - Krok, 'Ih., Förteckning på lärarne i botanik vid Sveriges högskolor, kögre och 5klassiga allm. lärlverk seminarier m. fl. varterm. 1900. - Nordstedt, O., Lokaler för nigra skandinaviska växter i Lunds universitäts herbarium. - Nyman, E., Botaniska exkursioner pi Java.

Botanical Gazette 1900. Vol. XXIX. Nr. 3. smith, (irant, 'The haustoria of the Erysipheae. - Copeland, Edwin Bingham, Studies on the geotropism of stems. - Nr. 4. Smith, R. Wilson, The stucture and development of the sporophylls and sporangia of Isoetes. - Taxter, Roland, Note on the structure and reproduction of Compsopogon. - A rthur, J. C., Cultures of Uredineae. - Nr.5. Davis, Bradley M oole, The fertilization of Albugo can. dida. - Hasselbring, H., Comparative study of the development of Trichurus spiralis and Stysanus stemonitis. - Smith, R. Wilson, Wie in vor. Nr.

Bolletino del R. Orto Botanico di Palermo. Anno III. Fase. I II. 1899. Fanales, Filippo, Contributo alla conescenza della flora delle sciare di Marsala. - Borzi, A., Descrizione ed illustrazione del R. Orto Botanico di Palermo. 'I'erracciano, A., Le piante nuove o rare descritte et illustrate nei "Delectus seminum * e nell' ,Hortus Panormitanus" dall' anno 1856 al $1896 .-$ Derselbe, Osservazioni fenologiche.-- Fasc. III-IV. Riccobono, Vincenzo, Monografia delle specie e varieta dio Agrumi coltivate nel R. Orto Botanico di Palermo.

La Nuova Notarisia. April 1900. p. 49-96. Filippi, Domenico, Contributo alla florula diatomologica della Carizia. - De T'oni, G. B., Ugo Zukal, Cemno necrologico. - Borge, O., Uebersicht der nen erscheinenden Desmidiaceen-Litteratur IX. - Derselbe, Register zu G. Lagerheim's und O. Borge's Uebersicht der nen erscheinenden Desmidiaceen-Litteratur.

\section{Botanische Anstalten, Vereine, Tauschvereine, Exsiccatenwerke, Reisen etc.}

Preussischer Botaniseher Verein. VII. Sitzung, Königsberg i. Pr.,

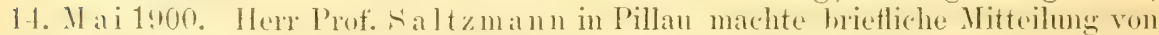

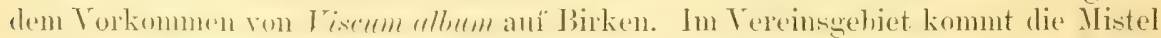
auf den verschiedenartigsten Holzpflanzen vor, jedoch wurde sie auf der Eiche nachweishich noch gar nicht, auf der Kiefer sehr selten, auf der Fichte (Picea (acelser) Eatr nicht und anf Birken rerhaltnismäsige wenig beobachtet. An meisten kommt die Mistel hier auf Populus monilifera, Tilia cordata und Sorbus ancuparia vor. Nachdem ein Anschreiben des Herrn Dr. Klinge aus Petersburg hetreffend sammlung und Zusendung einheimincher orchideen aus der Terwantschaft der Orchis incamata, O. maculatu und 0 . latifolia verlesen worden war, machte Herr Postverwalter a. D. P'hoedovius einige Horistische Mitteilungen. Er legte u. a. eine Kollektion der Gentiana Amarella b. uliginosa Willd. vor, die er vor 20 . Jahren im Kreje l'illkallen hei Willuhnen, (Istpr., gesammelt hatte. Auf einer 'Torfwiese hatte er in Gesellschaft der im Gebiet verbreiteten, erwähnten Form auch 2 Exemplare der östlich von der Weichsel bisher noch nicht beobachteten $G$. compestris gefunden, die er demonstrierte. Immerhin muss letztere Art bei uns sehr selten sein, da sie während der plammässigen Erforschung 
einzelner Kreise noch niemals in Ostprenssen und in Westprensten ïstlich von Ter Weichsel bemerkt worden ist. Ausserdem demonstrierte Herr Phoedovius eine Form der Cardamine pratensis, die sich der fir. acaulis sehr nähert. Herr Lehrer Thielmann referierte über botanische Litteratur. Von dem wissenschaftlichen Lehrer Herr Kar I Bra un erfolgten Mitteilungen üher starke Bämme,

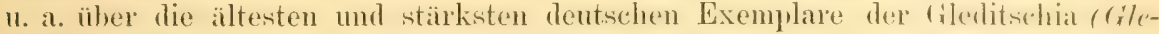
ditschia triacanthos) im Park von Oranienburg, wo er auch einen bemerkenswerten Fill von Verwachsung eines Lindenastes mit einem Eichenstamm heohachtete und eine skizze derselhen vorlegte. Hieranf demonstrierte der Vortrigende

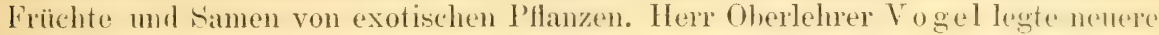
Fachliteratur vor und bespuch einige bemerkenswerte Arbeiten. J)r. A brome it zeigte mehrere Pflanzen vor, die Herr Lehrer Hans Preuss in diesem Jahre an nenen Fundorten in Kreise Pr. Stargard in Westprenssen enteckit hatte. Lis waren darunter Clarlium Mariscus vom Niedatzsee, in dessen Nähe er auch das im Vereinsgebiet seltene Equisetum variegatum Schleich. konstatiert hat, das in Westpreussen westlich der Weichsel an 3 Standorten und an ebenso vielen auch in Westpr. östlich vom genannten Strome und in Ostpreussen bekannt geworden ist. Ferner hatte Herr Preuss anch die seltenen Bastarle Pulsatill patens $>$ vemalis, Senecio vernatis $\times$ vulgaris und Viola arenaria $>$ Riviniana eingesandt, die demonstriert wurden. Schliesslich erfolgte noch die Vorlage interessinter Pflanzen ans anderen Gebieten, die der Tortragende durch ciute des Herrn Rentner Retzdorff in Friedenan erhalten hatte. A bromeit.

Botanischer Verein für die Provinz Brandenburg. Die Monatsitzımg am 11. Mai eröffnete in Abwesenheit des ersten Vorsitzenden der zweite Vors.

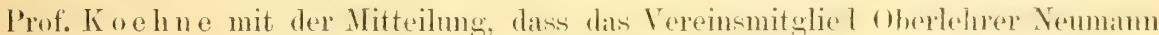
in Neuruppin gestorben sei. Daran schloss sich der Antrag, Hrn. Prof. Klebahn in Hamburg, dessen Verdienste um die Pilzkunde Prof. Sorau er noch besonders hervorlsob, zum korrespondierenden Nitgliede zu ernennen, welcher Antrag einstimmig genehmigt wurde. Nähere Mitteilungen über die am Sonntag nach Pfingsten in Neustrelitz stattfindende Frühjahrshauptversammlung und die am 'Tage zuvor schon stattfindende grössere Lixkirsion schlossen den kurzen geschütlichen Teil der sitzmng. Danach berichtote Dr. Loesener über die Einginge zur V'reinsbibliothek und legte ein Exemplare von drabis alprina $L$. vor, welches durchwachsene Blüten entwickelt hatte, so zwar, dass sich an Stelle des Fruchtknotens eine neue Blüte entwickelt und deren Fruchtknoten wieder zu einem 13lïtenansat\% sich ausgebildet hatte. I) Tortragende sprach die Vermutume aus, dass dergleichen Misshildungen infolge von Apisstichen eintreten kimnten. Prof. Ascherson, welcher danach an Prof. Koehne's Stelle den Vorsitz über-

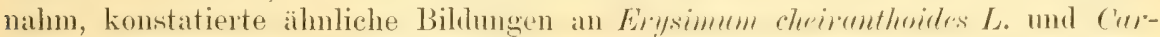
damine pratensis L. Mitglied Lehmann zeigte Blütenzweige eines Ulex curopaeus $L$. vor, die schon zu Anfang des Winter's von einem Strauche im Garten des Joachimthal'schen Gymnasimns abgeschnitten, in Wasser gestellt und so nicht nur zur Blüte, sondern durch künstliche Bestäubung sogar zum Ansatz der Fruchtbildung gebracht worden sind. Prof. Sora uer sprach danach über die Regehnässigkeit in der Stellung der stacheln bei den Rosen, die unterhalh eines Auges am reichlichsten entwickelt sind mol in demselhen Vasse nachlas:on, wie sie sich vom Auge entfernen, Er glaubt konstatieren zu können, dass diese bisher noch wenig oder way nicht beachtete Erscheinung im Kusammenhanges mit dem Verlaufe des Leitgewebes in den Zweigen steht. Dr. Diels gab der Versammlung eine kritische Uebersicht der Einteilung der Farne nach sori, Vervatur, Indusium, wie dieselbe sich seit Linné durch die Arbeiten eines Smith sen., Rohert Brown, Sprengel, Swarts, Presl, Smith jum., Will. Hooker, Mettenius us.s.w. ausgebildet hat und berïhrte dahei anch die neweren Anschanumen von ('hrist, Ascherson und die Prinzipien, nach welchen er selbst in Engler \& Prantl verfahren ist. Der vornehmlich historische Vortrag hot viel des Interessintern. Zum Schluss besprach Geh.-Rat Prof. Engher die Erscheinung, dass in den Früchten 
des Mangobaumes, Mangiferc indica, welche regelmässig nur eine Samenanlage enthalten, mehrere Keimlinge vorkommen, und zeigte ein Blatt von einer im hiesigen hotanischen Garten erzogenen Aracee, Anthurium Gustani Rory, vor, auf dessen $1 \mathrm{~m}$ langem Stiele eine herzfömige Blattspreite sitzt, welche die Grösse eines Schirmes erreicht hat.

W. L a ckowitz:

Preisausschreibung. Die Ahteilung für 'lier- und Pflanzenschutz der Gesellschaft von Fremilen der Naturwissenschaften zu Gera fordert zur Bearbeitung les 'Themas: "Deutsche Jugend iibe Pfanzenschutz!" auf. Die Schriften sollen der Jugend den Wert der Pflanzen im Haushalte der Natur an das Herz legen und sich gegen den Missbrauch wenden, P'flanzen zwecklos zu schïligen oder zu zerstören. I)ie 3 hesten Arbeiten gehen in das mbeschränkte Verlagsrecht der Abteilung über und werden dwch Ehrenurkunden und Preise im Betrage von 100, 60 und to M. ausgezeichnet. Jede Arbeit soll den Umfang eines Druckbogens nicht überschreiten und durch ein Merkwort gekennzeichnet sein. Die genaue Adresse des Verfassers ist in einem verschlossenem Briefumschlage, mit gleichem Merkwort versehen, beizufügen. Redaktionelle Kürzungen und Abänderungen bleiben vorbehalten. Die preisgekrönten Arbeiten sollen als Broschüre in den Schulen zu den denkhar billigsten Preisen verbreitet werden. Einsendungen sind bis zum 1. Juli d.J. an den Vorsitzenden Emil Fischer in Gera (Reuss), La asener Str. 16, zu richten.

(Bot. Centralblatt.)

Raciborski, M., Cryptogamae parasiticae in insula Java lectae exsiccatae.

Fasc. I. Nr. 1-50. Buitenzorg 1899.

Diese fammlung enthält 28 neue von dem Herausgeber aufgestellte Arten und ist die erste Śammlung parasiturer Pilze, welche von ter Insel Java ersehienen ist.

Botanische Reise nach Transkaukasien. Der Assistent des .Jurjewer (Dorpater) hotanischen Gartens, der jetzt in 'T'iflis am bot. Garten arbeitet, hat eine Reise in die tetepen des östl. Transkaukasien unternommen (Bos-dagh), und von dort beabsichtigt er, Hocharmenien zu besuchen und die Gegend um Nachiczawanj botanisch gründlich $\mathrm{zu}$ studieren.

(Bot. Centralblatt).

\section{Personalnachrichten.}

Ernennungen etc.: Walter H. Show w. Assistant-Professor d. Botanik am Pomona-College, Claremont, Col. - Hugo de Vries in Amsterdam w. z. Jitglied kgl. dan. Akat. der Wissenschaften ermannt. - Ceheimrat P'rof. P'feffer in Leipzig und (feheimrat Prof. Sch o endener in Berlin w. z. korrespondievenden Mitgliedern der Académie des sciences in Paris èrnannt. - Geheimrat Dr. A. Engler in Lerlin erh. d. Adlerorden III. Klasse. - Prof. Dr. O. Drude in Dresden w. d. k. russ. Stanislausorden verlieheu. - Herm. Za h $\mathrm{n}$ in Karlsruhe w. v. d. kgl. bot, (res. zu liegenshurg nach Beschluss der sitzmg vom 17. Mai z. korrespond. Mitglied ernannt.

Todesfälle: Eduard Joseph Lowe in Shirenewton Hall bei Chepstow. Ernst Kernstock, Lichenologe. Professor an der Oherrealschule in Klagenfurt, am 14. April im 48. Lebensjahre. - Hjalmar Fred. Chr. Kia erskou, Inspektor am bot. Museum in Kopenhagen, am 28. März d. J. - A ndr. Petterson Wins. $10 \mathrm{w}$ in Göteborg, am 28. Jan. d. J. - Gustav Anders Lindber.g in Stockholm, am 3. Februar d. J.

\section{Corrigenda.}

Nr. 4 p. 66 der "Allg. bot. Zeitschr." wurde unter Nr. 26 bei Lusula confusa C.J. Lindbg. aus Versehen der einzuschaltende Zusatz "Ad L. arcuatam IVhlenby. spectans" weggelassen.

Druck von J. J. Reiff in Karlsruhe. 


\section{Allgemeine \\ Botanische Zeitschrift für Systematik, Floristik, Pflanzengeographie ete.}

\section{Referierendes 0rgan}

des brt. Vereins der Provinz Brandenburg, der kgl. bot. Gesellschaft zu Regensburg, des Preuss. bot. Vereins in Königsberg,

uml Orwan der Botan. Vereinigung in Würzburg und des Berliner bot. Tauschvereins.

Unter Mitwirkung hervorragender Fachmänner herausgegeben

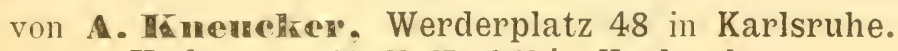

Verlag von $\mathbf{I}$. T. Reifr in Karlsruhe.

Die Herren Mitarbeiter tragen für Form und Inhalt der von ihnen unterzeichneten Arbeiten volle Verantwortung.

\begin{tabular}{|c|c|c|}
\hline 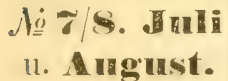 & $\begin{array}{l}\text { Erscheint am 15. jeden Monats. - } \\
\text { Preis: vierteljülrl. } 1.50 \mathrm{Mk} \text {. bei freier Zusendung. }\end{array}$ & $\begin{array}{l}1000 . \\
\text { VI.Jahrgang. }\end{array}$ \\
\hline
\end{tabular}

\section{Inhalt}

Originalarbeiten: A. Petunnikov, Ueber den Wert anatomischer Merkmale zur Unterscheidung der Abies-Arten. - Eggers, Bot. Beobachtungen auf meiner Reise nach dem Orient 1899 (Schluss). - Eduard Martin Reineck, Floristisches vom Strande ron Bahia Blanca (Provinz Buenos Aires, Argent.). - 'Th. Hellwig, Florenlild der Umgegend von Kontopp im Kreise Grünberg in Schlesien (Schluss). - Frhr. von Spiessen, Die Wiesselsheimer Salzwiesen in der Wetterau. - Karl Ortlepp, Abnormität in der Blütenstellung zweier Orchideen. - O t to W ill, Uebersicht über die hisher in der Umgebung von tiuben in der Niederlausitz beobachteten Leber-, 'T'orf- und Laubmoose (Schluss). - Ott o Kuntze und T om vo n P os t, Nomenklatorische Revision höherer Pflanzengruppen und üher einige Tausend Lorrekturen zu Englers Phaenogamen-Register (Forts).

Bot. Litteratur, Zeitschriften ete.: A. Kneucker, Wildeman, E. de et Durand, Th., Plantae Thomnerianae Cogolenses ou énumération des plantes récoltées on 1896 par M. Fr. 'Thonner dans le district des Bangalas (Ref). - D erselbe, Sehinz, Dr. Hans u. Keller, Dr. Robert, Flora der Schweiz (Ref.). - Derselbe, Bubani, P., Flora Pyrenaea per ordines naturales gradatim digesta (Ref.). - D e r s elbe, IVinkler, W., Sudetenflora (Ref.). - Derselbe, Ascherson, P. u. Graebner, P., Synopsis der mittelcuropäischen Elora (Ref.). - D e r s el be, Wiesbaur, J. B., Unsere Misteln und ihre Nïhrpflanzen (Ref.). - Derselbe, Wünsche, Dr. Otto, Die verbreitetsten Pflanzen Deutschlands (Ref.). - D e r s elb e, Ruschhaupt, Dr. G., Bau und Leben der Pflanzen (Ref.). - Os wald We ige l's botanischer Lagerkatalog. Neue Folge. Nr. 95, 96 u 97. Inhaltsangabe verschied, bot. Zeitschriften. - Eingegangene Druckschriften.

Bot. Anstalten, Vereine, Tauschvereine, Exsiceatenwerke, Reisen ete.: Kgl. bot. Gesellschaft zu Regensburg (Ref.). - 8. Jahresversamml. d. dentsch. dendrolog. Gesellsch. - Wetzstein, Exsiccaten aus Ohio. - Gross, L. u. Kneucker, A., Bot. Reise durch Istrien; Dalmatien, Montenegro, die Hercegowina und Bosnien.

Personalnaehrichten. - Zur Nachricht.

\section{Ueber den Wert anatomischer NIerkmale zur Unter- scheidung der Abies-Arten.}

Von A. Petunnikov in Moskau.

Fs wurde von vielen seiten versucht, die anatomischen Merkmale zur L'nterscheidung der höheren Pflanzen in systematischer Hinsicht zu benutzen, nachlem Duval-Jouve dieses Verfahren, welches als histotaktisches ron ihm genamnt wurle, mit viel Erfolu zuvor an den Lipnistum-Arten und nachher mit wenig Glück an dy'm!nmu-Arten in An- 


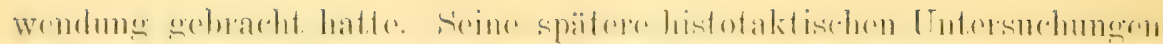

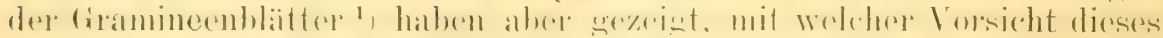

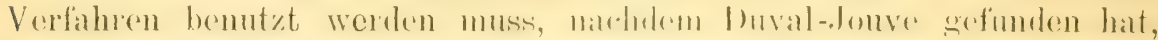

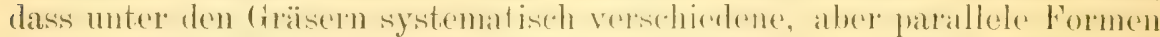
existiem, welehe doch ganz densellnen anatomischen ball zeigen, wio

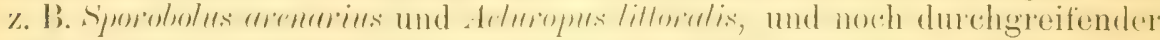
scheint analoger Parallelismus im lian der biatter dreier verschiedener Alyopymam- und Brachypodim-Arten ${ }^{2}$ ) zu existieren.

Die Unzaverlässigkeit dieses Teftahrens hat sich besonders an den

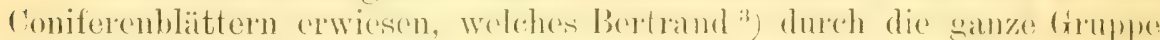
durchfiihren wollte, doch alle cupressineren ansgesclylossen, in derem

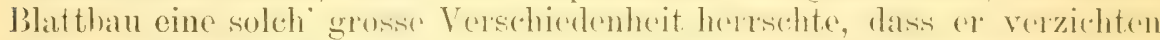

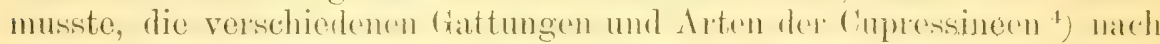
anatomischen Merkmalen za unterseheiden. Aher auch unter den $\Lambda$ hietineen schien die Sache nicht viel besser zu gehen, da Berlrand in mehren Gattungen dieser (truppe kesmen Unterschied im anatomischen Ban zwisthen oinigen systematisch versehiodenen \rten binden konnte.

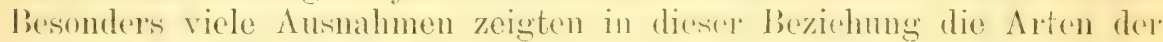
Gattung dbies, welche uns hier ganz spezill interesiolen: nald bertrand sind wenigstens 4 Arten angegeben, die mit 1 anderen, je zu zwei, sanz identisch schant, also anatomisch nicht zu nuterscheiden sind."

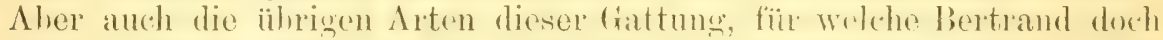

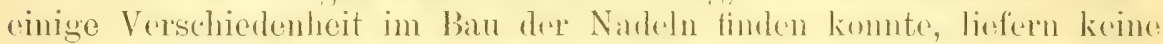

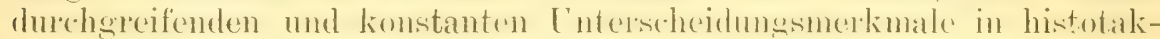
tischer Hinsicht, wie es sich später herausgestellt hat.

In seiner synoptischen 'Tabelle der anatomischen Merkmale muter-

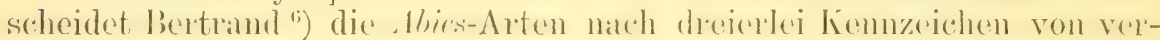
schiedener Wertstufe, und in erster Linie teilt al alle von ihm untersuchten Aitem nath der Lage der Iarzgänge in den Nadeln in z) dirmp-

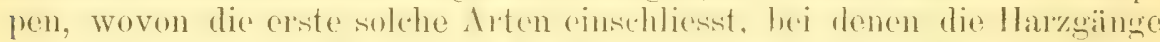

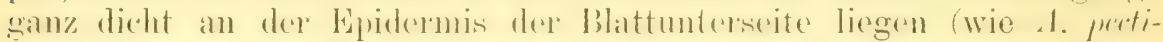
motri); dagegen bei den $\Lambda$ ten dex zweiten liegen dio Harzginge mitten im Blattparenchym (wie A. balsamea, Sibivica ete.).

1) Histotaxie des feuilles de Graminées, Annales des seiences natur. Botanique. VI. Série. 'T'ome ì. 1875. p. 294-371.

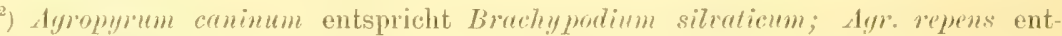
spricht $13 r$. pinnetum; Agr. intermedium entspricht $B r$. plhoenicoides. ${ }_{n}$ Ce parallélisme, bemerkt Duval-Jouve, est si complet et si parfait, que, pour le décrire, il n'y aurait qu'à répéter sur une espèce ce qu'on aurait dit de la correspondante." Ibid, p. 36.5.

$\left.{ }^{3}\right)$ Anatomie comparie des tiges et de fouilles chez les Gnétacées et les Conifères. Ann. des sc. nat. Botanique. V. Série. 'lome XX. 1874. p. 5-153.

$\left.{ }^{4}\right)$ „ll n'y a pas lieu de rechercher les caractères que la structure anatomique des Cupressinées pent fournir pour diflérencier les genres et Jes espèces. En effet, d'un individu à l'autre, dans une même espèce, bien plus, d'un raneau à l'autre sur un même individu, la strueture anatomique varie dans des limites plus étendues que les variations que l'on observe d'un genre à l'autre. Par conséquent, j'ai dû renoncer à caractériser anatomiquement (du moins par des earactères tirés seulement de la strueture de la tige et de la feuille) les genres et les especes des (upressinées. Ibid. p. 138.

$\left.{ }^{5}\right)$ L'Abies Nordmamiana me parait it peine différent de 1'db. pectinata $D C$ : (1. c., 94). Ab. Webbiana Lindl. ne diffère pas anatomiqnement de l'Ab. P'indrouri Sipuch. Ab. religiosa Lindl. - de 1'Ab. brecteata IIooli. et Arnott; Ab. amabilis fiorbes - de l'A b. Froseri Lindl. (loc. cit. p. 95).

Loe. cit. 1. 93. 


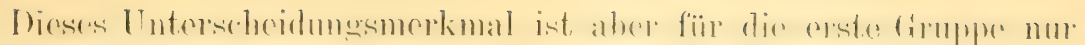

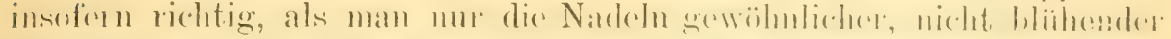

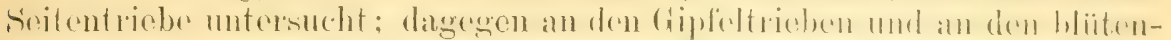

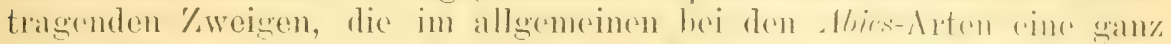

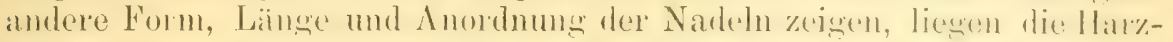

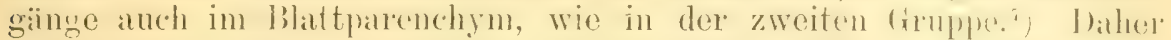
wurde spaiters) dieses Unterscheidungsmerkmal nur füi dio nichth]iihomelen '/weige anwendbal.

Andererseits fand Kochne ${ }^{9}$ ) auch Ausnahmen unter den Arten der' zweiten Gruppe, und zwar bei A. balsomea, homolepis und firma liegen

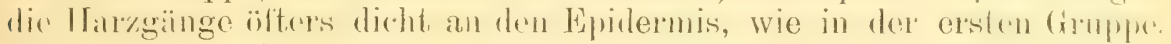
Mir ist es auch gelungen, selbst solch einen Fall an A. Silvirien zu

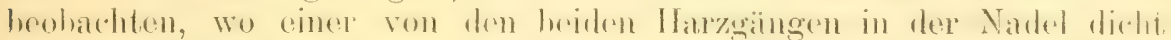

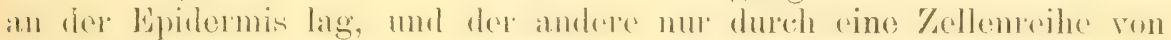
derselben getrenut war.

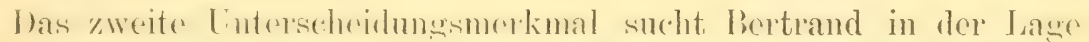

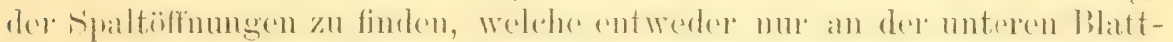
Häche liegen (wie es meistens der Fall ist, bei $\Lambda$. pectimutu etc.), oder anch auf der oberen Blattfliche, wie bei $A$. bulsomer. Indessen müssen

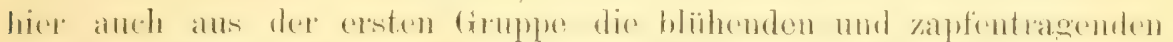

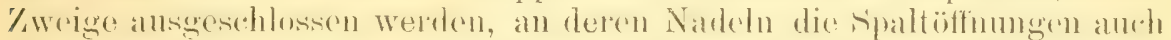

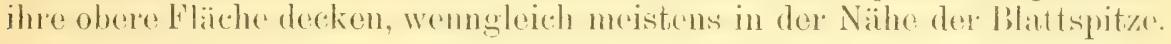

Das dritte und letzte Kenmzeichen, um die Abies-Arten näher unter-

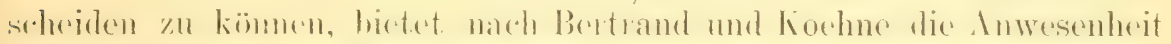

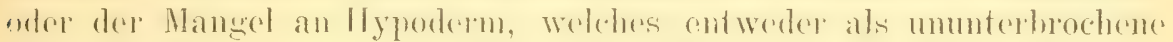

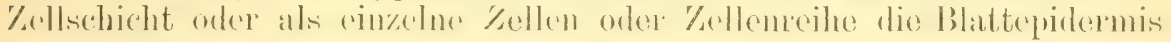
auskleidet.

Nun hat sich herausgestellt, zuvor' an den Nadeln einer 'Tamne,

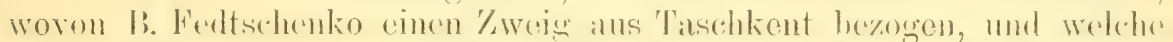
er mit dem Namen A. Semenovi belegt hat, ${ }^{10}$ ) dass an Querschuitten

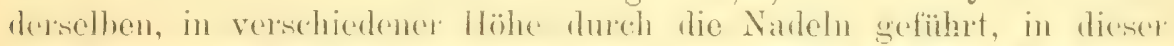

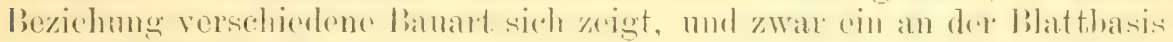

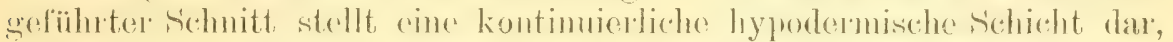

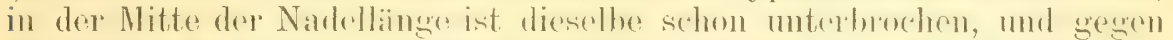

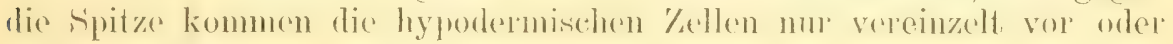
fehlen gänzlich.

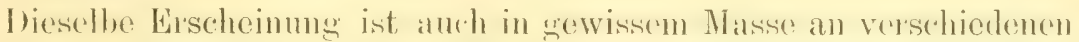

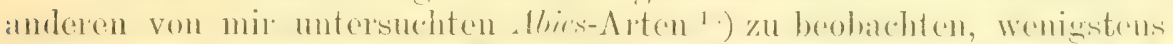

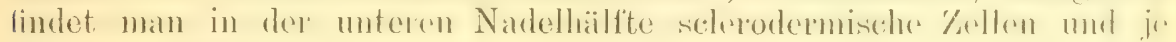

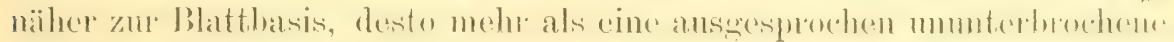
hypodermische \%ellschicht.

${ }^{3}$ Vergl. Hemmpel u. Wilhelm. Die Bäume und Sträncher des Waldes; ïber A. pectimete, p. 88.

$\left.{ }^{8}\right)$ Vergl. Koehne, Deutsche Dendrologie, Stuttgart, 1893. p. 13; auch A scherson u. Grabeber, Symopsis der mitteleuropïischen Hlora, p. 190;

") lace rit. 1. 1:i.

10) B. Fedtschenko, Abies Semenovi mihi, eine nene Tame aus Centralasien, Botanisches Centralblatt, 1898, p. 210. Pinsapo.

11) Abies Sibirica, balsamea, Weilehii, Sachalinsmis, pectinatu, Nordmanniana, 
Besonders durchgreifend ist dieser Unterschied im Baue der Nadelbasis von dem übrigen Blattteile bei $A$. Sibirica, die ich aus verschiedenen Gegenden zu untersuchen Gelegenheit hatte, und die überhaupt eine grosse Mannigfaltigkeit im Baue der Blattnadeln zeigte betreft's des Entwickelungsgrades der hypodermischen Schicht, des Endoderms und der Sclerenchymzellen.

Eine relativ starke Entwickelung des Hypoderms stellt die in Taschkent wachsende Tanne dar, wie es zuerst B. Fedtschenko gezeigt hat, welcher nur aus diesem Grunde irrtümlicher Weise die Taschkent'sche Tanne für eine neve Art hielt und Lt. Semenoni benannte, obgleich er weder den Baum, noch die Zapfen davon gesehen hat. Seitdem wurde diese Tamne in Taschkent von Herm Litwinov mit Zapfen gefunden, auch von dort durch H. Prof. Kusnezoff bezogen und hat sich als echte $A$. Sibirica erwiesen.

Diese Thatsache kamn als gründlicher Beweis dienen, dass die anatomischen Merkmale zur Unterscheidung der Tannen-Arten und wahrscheinlich aller höheren Pflanzen keinen wissenschaftlichen Wert haben können, im Gegensatze zu der Behauptung Koehne's, welcher sich in seiner Dendrologie zu Gunsten dieses Verfahrens äussert.

Moskau den 12./25. März 1900.

\section{Botanische Beobachtungen auf meiner Reise nach dem Orient 1899.*)}

Von Eggers in Eisleben.

Reichhaltiger und auch manigfaltiger ist um diese Jahreszeit die Flora Palästina's. Hier haben die Pflanzen noch nicht von der Dürre gelitten. Bis Mitte April fällt meistens ergiebiger und durchdringender Regen. Bei Jaffa verlässt man das Schiff und betritt den Boden Palïstina's. Ungeheure pflanzenlose Sanddïnen, auf denen zumteil Jaffa liegt, erstrecken sich landeinwärts. Aber das Hinterland, die Ebene Saron, ist sehr fruchtbar und zum Leidwesen der Botaniker fast iiberall beackert; nur an den Tregen, Rainen oder Bächen wird er seine Ausbente finden. In den 4 Tagen, welche ich in .Jafta verweilte, fand ich 40 Pftanzenarten. Citicu pilulifer L., welche Pflanze ich vor Jahren

*) Vur melireren Wochen erhielt ich von Herrn Hans Guyot in Kairo ein Päckchen egyptischer Pflanzen, welche er in den ersten Monaten dieses Jahres sammelte. Ich sandte dieselben zur Bestimmung an Hern Baurat.J. Frey n in Prag-Smichow und die Grïser an Herm Prof. Ed Hackel in St. Pölten und erlaube mir nun, dieselben, soweit sie noch nicht p. 103 dieser Zeitschrift an den betreffenden Stellen genamt sind, hier aufzuführen, da dieselhen zur Vervoliständigung des Florenbildes heitragen. Bei den Pyramiden: Lithospermum callosum Vahl, Reseda Boissieri Müll?, Heliotropium Bovei Boiss?, Malcolmia degyptiaca Spr., Paronychia Arabica L., P. desertorum Boiss., Monsonia nivea Boiss., Juncus bufonius L., Polypogon maritimus Willd., Aristida plumosa L., Sporolobus spicatus Kunth, Stupa tortilis Desf. - Bei Kairo: Panicum colonum L. B. leianthum Boiss. - Wüste bei Helnan: Cleome Arabica L., Caylusea canescens St. Hil. - Purrha Wüste: Fugonia Bruiguieri DC. - Am Nilufer bei Kairo: Irifolium resupinatum L.f. mit kurzen Blütenstielen, Melilotus Indica All., Medicago apiculate Willd., Trigonella laciniata L., Phalaris paradoxa L., Ph. minor Retz., Polypogon Monspeliensis Willd., Agrostis verticillata Vill., Lolium multiflorum Lam. 3. muticum Doell. - An den durch Bewässerungsgräben gebildeten Sümpfen: Cyperus rotundus $L_{\text {., }} C$. esculentus $L$, Heleocharis palustris $R$. Br., Cynodon dactylon Pers.

A. Kneucker. 
auf dem Schlosse zu Mansfeld fand, sah ich wiederholt an Hecken und Einfriedigungen.

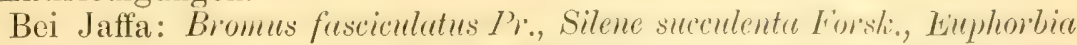
cybirensis Boiss., Anagyris foetida L., Pinardia coronaria Len., Sitatice Tho-

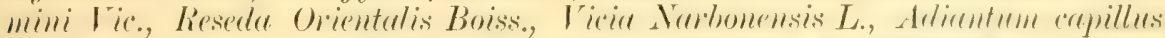

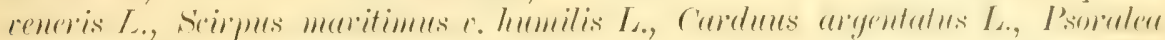

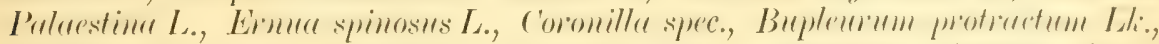

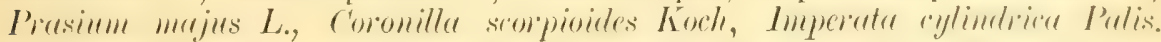

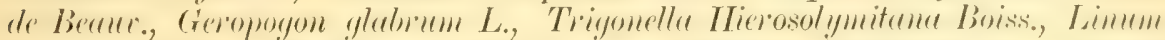

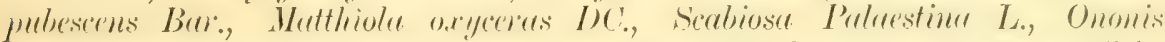

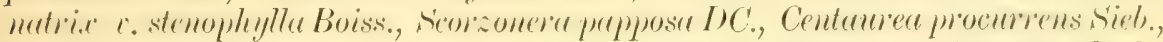

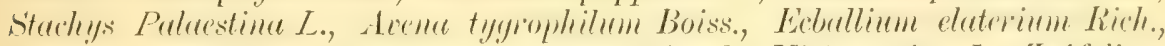

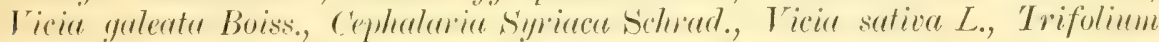

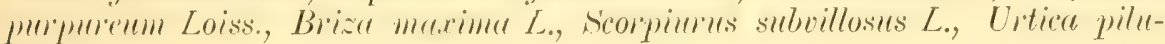
lifere $L$.

Lohnender sind die Exkursionen in der Umgebung Jelusalems. Ist auch das Kralkgebirge Juda, auf welchen Jerusalem liegt, wie alle Gelirge Palästina's vollständig waldlos und wenig fruchtbar, so liegt doch hier der Boden zum grössten Teile unbenutzt, auf dem die wildwachsenden Pflanzen ungestört ihr Dasein fristen können. Der Araber hegnügt sich für seinen Ackerbau mit einem geschützten und dazu giinstig gelegenen Terrain, auf welchem er ohne viele Miihe den Boden hearbeiten kann. An Feuchtigkeit fehlt es um diese Jahreszeit den Pflamzen noch nicht; his Mitte April und zuweilen auch Ende April ist der Niederschlag auf dem Gebirge Juda nicht unbedeutend. Damn aber hört die Regenzeit auf; bis Ende Oktober ist der Himmel fast ununterbrochen wolkenlos. Die llitze wird zuweilen unerträglich. Infolge des Regenmangels verliert die Natur in sommer ihre schönheit und erhält sich nur frisch, wo himreichend IVasser vorhanden ist, um den Boden günstig zu bewässern. Derselbe ist damn nur noch mit dürren liräuterstengeln bedeckt. Die Brumnen und (quellen versiegen nach und nach, und ganze Viehherden sollen dann, da der Araber nicht gewohnt ist, für die heisse Sommerzeit Futter einzuernten, dem Hunger erliegen. Ich fand in der Umgegend von Jerusalem, wie im Kidron- und Himnonthale, auf dem Oelberg, auf dem Wege nach Bethanien und Bethlehem, bei dem Dorfe Lista u.s. w., wïhrend meines 7 tägigen Aufenthaltes 120 Arten, immerhin genug, wenn man bedenkt, dass die Sehenswürdigkeiten Jerusalem's mich zunächst in Anspruch nahmen und ich die F'lora erst in 2. Linie berücksichtigen konnte.

Bei Jerusalem wurden gefunden: Trifolinm clypertum L., stotice

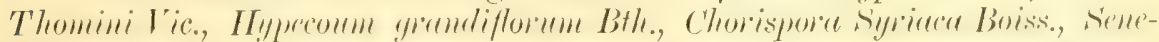
cio vernalis IV.K. . f. humilis, Malcolmia cremulata Roiss., Ononis natrix v.

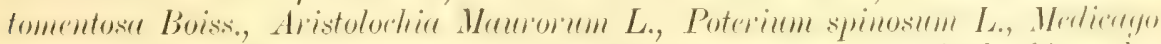

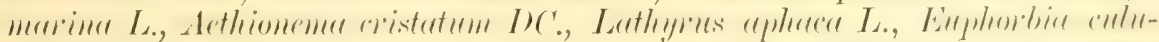

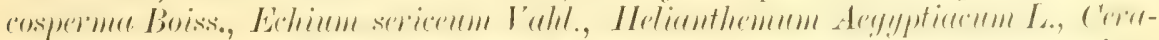

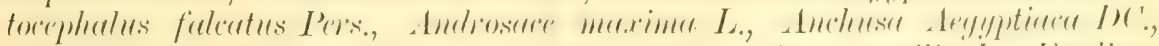

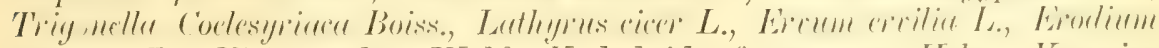
gruinum L., Vicia cordata. Wulf., V. hybrida t. puipurea Hshu., Veronica

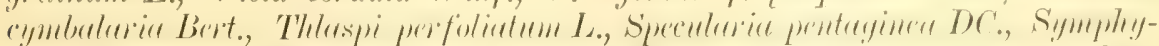

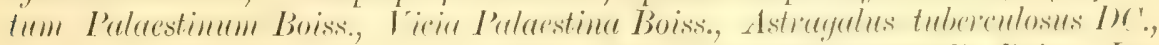

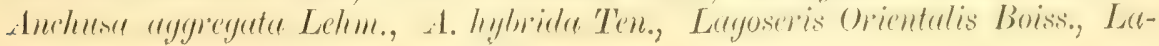




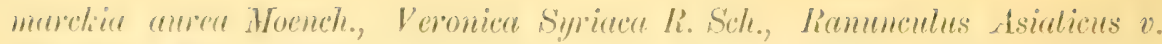

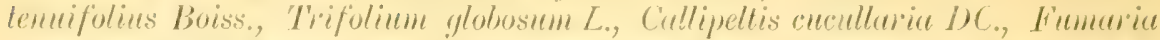

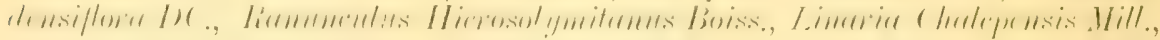
Galium articulatum Boiss., Anargallis coerulea, Adonis dentatu DC., Scorp)i-

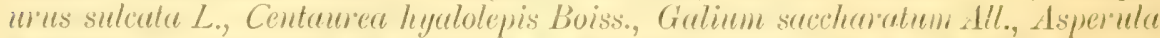

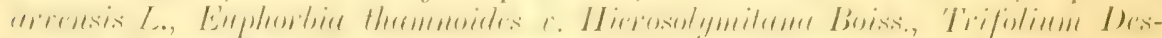

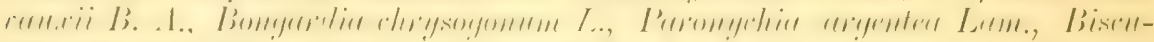

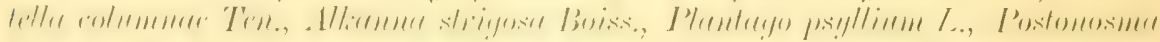
Syjrace Boiss., Astragulus senctus Boiss., Fibigia rostratu Schents., Allinm Hierochuntimum Boiss., Lepirlium draba L. Lammum moschutum v. micrenthem Boiss., bellis silvestris Cyr, Limum. Orientule Buiss, Viciu hybridu f. purpurea L., Lathyms Hierosolymitumes Boiss., liesedu alba L., Trifolium supinum L., Fumaria Arabia Boiss., Onosma frutescens Lam., Stellaria media

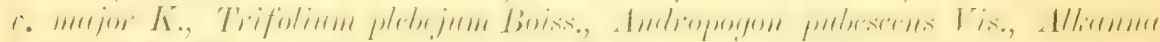

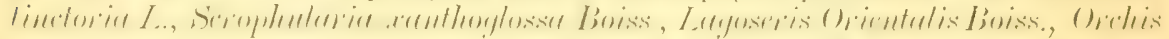

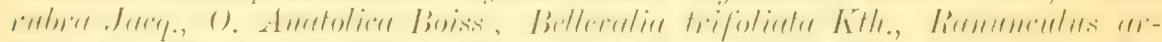

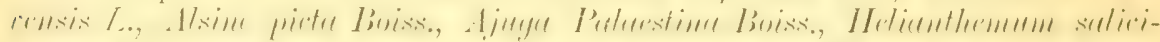

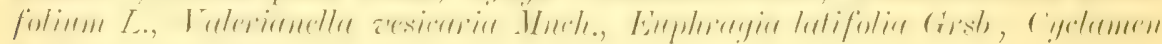
hederacfolium Act., Adonis dentuta I)sf.

Einen ganz anderen Charakter trägt un diese Zeit das untere

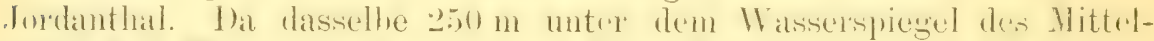

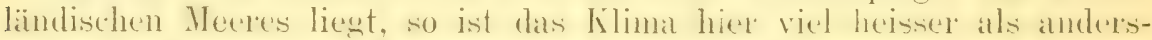
wo in Palästina. Die T'emperatur steer wilnemel meines dufenthaltes

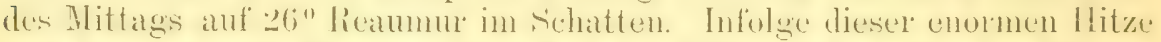
nut der weringen Niedersehläge sind die f'flamzen auf den das Jordanthal cinsehliessemen Höhen sänzlich vereloret. Ilicker Stauh liegt anf

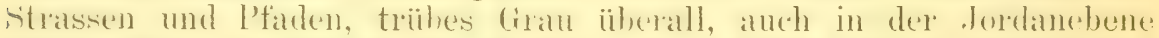
scolbst. Nur wo (guellon zutage treten, oder bäthe und kiinstliche be-

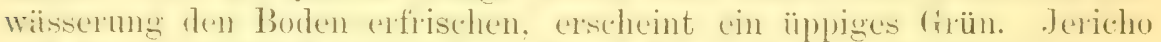
und scine nächste Umeghnng wird von der sehr wasserreichen sultansyuelle, welche eine halbe, itunde vom ()rte am Fusse des Gebirges Juda

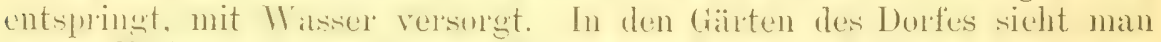

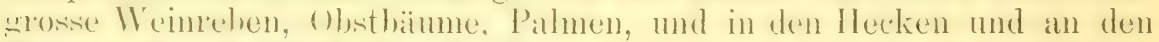

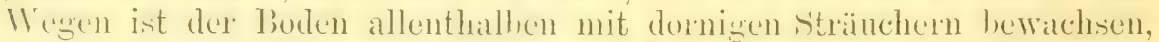

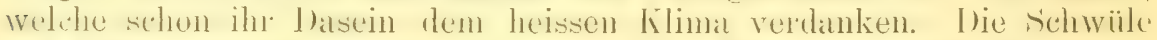
den 'lages, welche mir hei dex geringsten bewegung grosse sehweisstropten entluckte, benahm nil fist jesliche Lust zum P'flanzensuchen, so dass ich nur 12 Pflanzenarten in meine Presse aufnahm.

Bei Jericho fand ich: Boerharevia plumbaginea v.glabrata Boiss., Limmi pubescens liav., Tetranonolobus Pelaestina Boiss., Oiobanche spec., Noto. bersis Syriace Cass., Silybum Marianum Gitn., Bryonia Syriace Boiss., Mandragore officinarum I., Solanum cougulans Forsti., Tunice velutina Guss., Chamaemelum auriculatum Boiss.

Der IVeg von Jericho nach dem Toten Meere führt durch eine

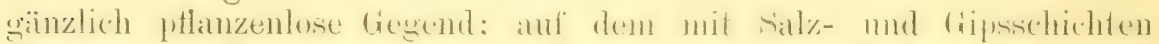

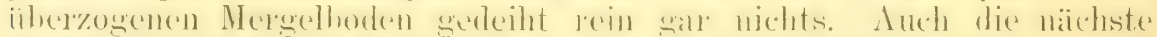

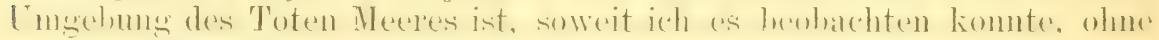

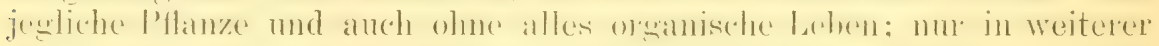
Entfernung fand ich drei Pflanzenarten: P'ulicuric umlulate L., Statice Thomini Vic. und eine mir unbekannte Komposite. 
An den Ufern des Jordans hofite ich eine reiche Ausbente an Pflanzen zu machen: aber als ich am 25. März an der Jordanfurt das

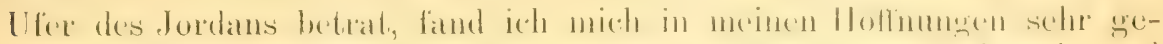

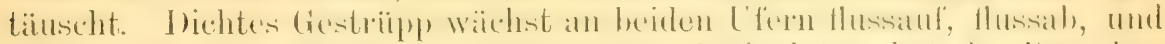

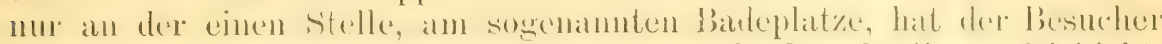

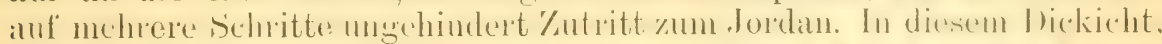

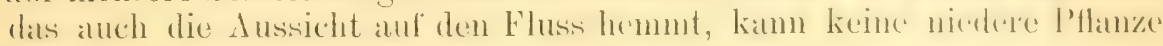
gedeihen.

Am 31. März fuhr ich in Gesellschaft zweier Damen und eines

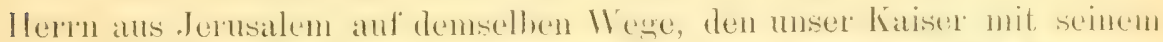
(iefolge auf seiner licise mach derusalem in lleloste lisgs benutzte,

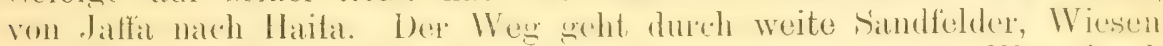

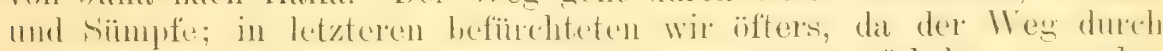

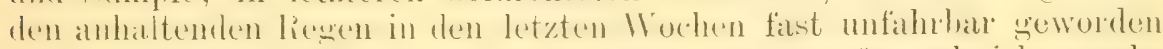
Wall, stecken zu heihen. Auf den Wiesen und simpten sah ich manche

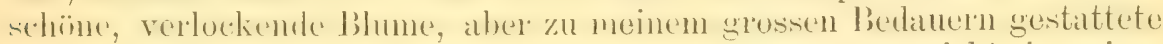
mil der Fuhrmann nicht, vom Wagen zu steigen, um nicht in seiner ciligen Fahrt durch mich aufgehalten zu werden. $\Lambda$ ls wir aber am

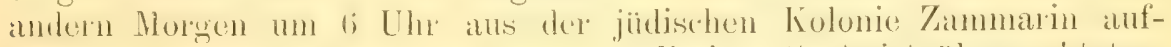

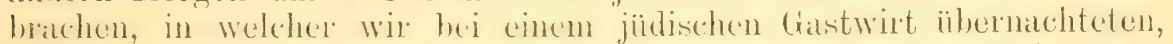

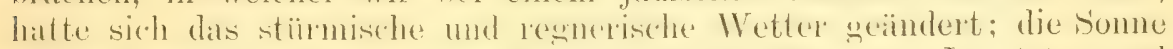

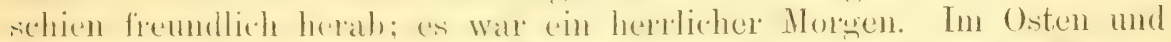

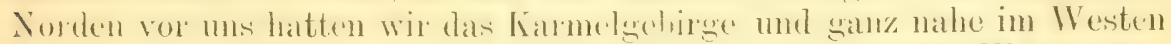

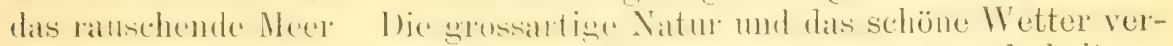

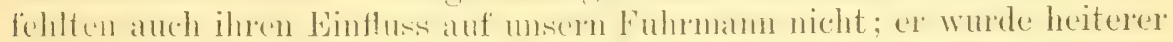

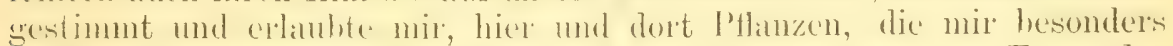
aufitelen, meiner Sammlung einzuverleiben, und als wir am Fusse des liamel anlangten, wo sich der Wry vielfach hergatuf sehlängelte, watl

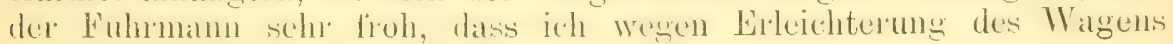
denselben ibberhaupt nicht wieder hestieg.

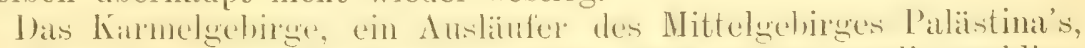
lesteht wie dieses alls hallistein und ist ebenfills vollständig walllos. bas grallgrüne, nicht firischerinte lussehen des gamzen Gehilges ver-

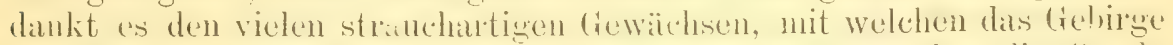
vom Fusse bis zum reheitel hestanden ist: moter diesen hat die staude ("istus rillusus L., die dimbls in schönster libite stand, den hervorragendsten Anteil. Auf dem liamel lind ich abex solche grosse Ausbeute

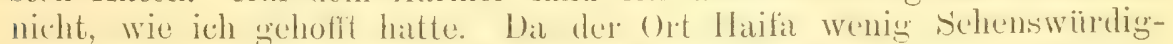
keiten atulzuweisen vermag, so lionnte ich mich während medmes jtägigen

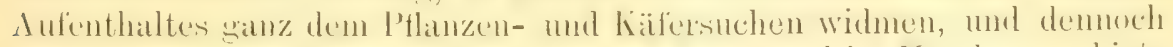

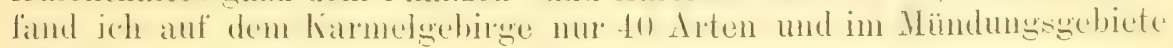
des lison 10 Arten.

Auf dem Karmel sammelte ich: Vmbilicus horizontalis DC., Lun-

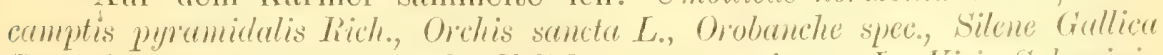
L., Schoenus mueronatus I'arl., Hedichrysum sanguineum L., Vicia Suluminia Boiss., Thymbre spieate I.., Parietarie Juduice IIoffin., Nigella ciliar is DC.,

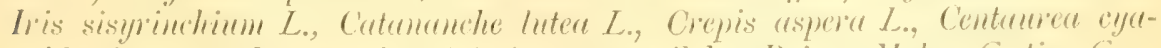

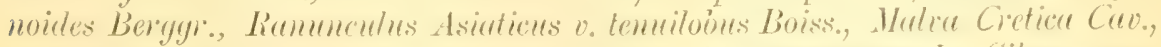
Crupina crupinastrum Moris., Vicie hybrida f. purpureseens L., Silene spec., Salvia triloba L., Astragalus medicaginas Bois., Cistus villosus L., Scleropoa

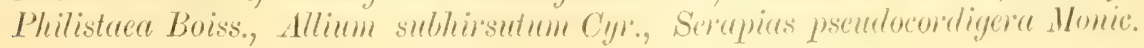




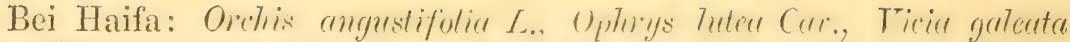

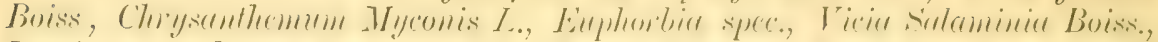

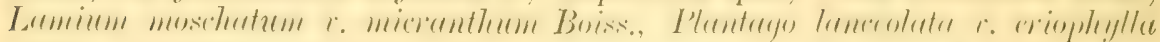
Boiss., Il. stricta Schousb., Lavandula stoechas L., Phannalon Tenosii Pr.,

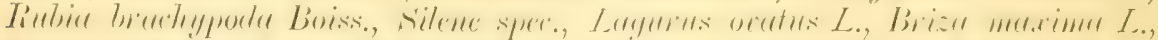

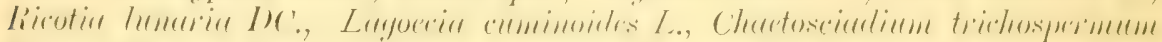

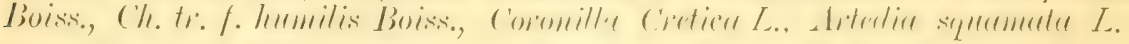

Fast alle I'flanzenarten sind mir von Herrn Hofiat II a usskuecht in IV eimar, der sich bereitwilligst der müherollen Arbeit unterzog, determiniert worden; ihm sage ich an dieser Stelle meinen wärmsten Dank.

\section{Floristisches vom Strande von Bahia Blanca (Provinz Buenos Aires, Argentinien).}

Drei Tage schon hatte mser l)ampfer die hrasilianische Hafenstadt hio Grande verlassen, und dic Geckrankheit hatte unter den woniger befahenen an Bord einige ()pfer auserwälte als geegen Mittas des vierten Tages ein Matrose, nach dem Horizont dentend. zu mir sagte: Dort ist Land! Wirklich! Toch ferne hoh sich ein schmaler branngrelber streifen aus dem Wasser, die argentinische Kïiste, an der unser. wackeres Schiff, um zu laden, station machen sollte. Inmer dentlicler. trat gegen Ahend das Land hervor. Tonnen bezeichneten die Fahrstrasse, da das II asser hier stellenweise sehr flach und für tiefgehende Fahrzeuge gefährlich ist; das Leuchtschift, von dem der Lotse an Bord kam. ward passiert. Mit einbrechender [) ämmerung ging es an drei aluentinischen Kriegsschiffen vorbei, und un 6 Thr rasselte der Anker nicder, da wir wegen eingetretener Ehbe nicht an der Landumgsbrücke anlegen komnten. - Am nächsten Moren zur Flutzeit ving es weiter und um 11) L'hr vormittags ward das sehitf mit starken Tanen und Drahtseilen an den Eisenringen der Brïcke befestigt. Die somne des schönen ()ktobertages lag golden üher Nleer und Land, und eine weite Fernsicht bot sich dar. Die auf mächtigen Trägern ruhende Landungshriicke schob sich vom flachen strande ein stück ins Meer, unalı̈ässig rasselten darauf die Eisenhahmzïge und die Drehkrahnen kreischten, um die hier Ladumg nelmenden Dampfer nit Marren Alesentiniens, meist IIänten und

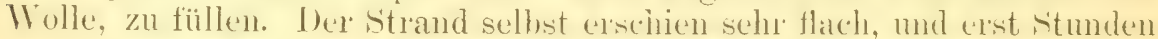
landeinwälts wude die Kïiste von langestreckten Iligelketten mmzogen; in weiter Ferne gewahte ich ein ansehnliches ciebinge. Telere dem Wasser Hogen zahlexehe Mören, ihre Bente erspäherd, pfeileseschwind hin und her, Weithinein ins 11 asser scholen sich languestrectste mit Secgräsern bewalhene Moraststreifen, eine triigerische fohicht und grefähliche stellen, was mancherlei kleinere segler bewiesen, die hies

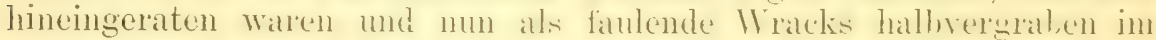
Sande lagen.

Den folgenden somntagmorgen mud einen der nïnsten Vormittage benutzte ich, um an Land zu gehen und etwas zu botanisieren; eine primitive Pressvorrichtung hatte ieh mir in meiner Kahine unter Kofferm und listen zurechtumacht. Ich will versuchen, in den folgenden \%eilen (ine kleine skizze von dem Eindruck zul get)en, den die Kïste, soweit ich sje besuchen lomnte. in floristischur Hinsicht anf mich machte. Wenn 
in meinem Aufsatz manches mangel- und lïckenhatf bleibt, mag mich einesteils die knappe \%eit entschuldigen, die mir fïr Fiskursionen zun

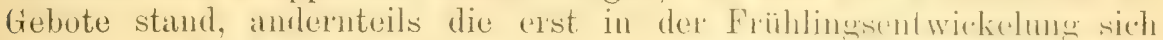
betindende Flora, so dass ich mich bei einheinischen l'flankn faist mur auf Nennung der Fannilien beschnänken musste, dit die meisten Arten eben noch nicht blühten.

In herrlichsten Wetter stieg ich vom theliffe und gine die Dnlengbriicke himunter. Elhbe war eingetreten und ich gewahrte an den monteren Teilen der mächtigen Traghalken eine Nuschelant, die sich dort in will\%en Bänken angesicelelt hatte. Die mit der Angel am Wasser sitzenden Fischäger sagten mir, dass die Muscheltiere tssbar seien und fïhrten mir den Beweis ad oculos vor. Ich heobachtete nun die dewälche, die sich rechts und links an die Böschungen der Bahngeleise drängen, welche. vom Lande bis ans Ende der Bröicke führen. In ganzen P'olstern war

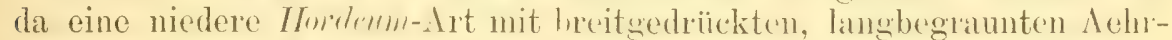

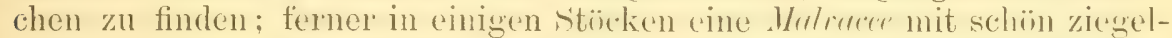
roten Blüten; (ine side-Art, die hier wohl nicht ursprünglich ihren Standort haben mochte, viehmehr durch dic Bahn verschleppt wurde, wie so manche Species der Umgebung.

Ferner fesselten präichtige Exemplare von cirduns Murimurs, der Mariendistel, meine dufmerksamkeit: die schön weiss-geaderten Blaitter mangten im frischesten (ìrün, und die Blïten waren in der Éntwickelung begriffen. - Ich verliess nun die Bahngeleise und ging mehr landein-

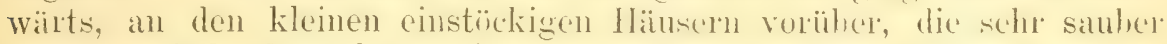
gehalten sind. Vor den meisten deriselben standen auf den Veranden allerlei biihende 'Topfgewiache, wie sehartathgeranien und weisse und rote Levkoien.

Bäume bemerkte ich so gut wie keine im Strandgehiet, doch waren um viele der Iläuschen hohe und statke Témentrir mit hellsoten Blïtenrispen angepflanzt, um welche eifrig die Bienen summten.

Man sagte mir, dass die Flut in frïheren Jahren viel weiter hinein ins Land gegangen sei, und auf den starken fílpetergehalt des Bodens wies nicht allein dic Filera hin, es hatte sich stellenweise anch der Salpeter auf ganzen Flïrhen aligelagert, und in fonnenglanze schien es, als sei die kirde stellenweise bereift. bigentliche Wiesen waren in der ganzen fiegend, die ich durchwanderte, nicht zin finden, das cinzige grössere stüekchen äberblähtes Land fand ich mehr landemwärts. liss war von einer zicrlichen Pflanze, Verbenu dissectu, bedeckt, die ich übrigens anch in brasilien, doch kräftiger entwickedt und nit mehr dunkelvioletten Blumen, angetroffen habe.

Mitten durch's flache Gelände zicht sich die Bahn, welche vom

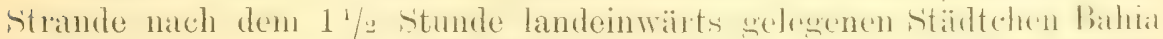

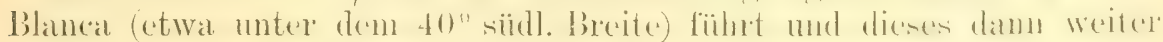
mit der Landes- und l'rovinzhanptstadt lsnenos-dires verhindet. An den Böschungen fand ich recht häufig Sinapis arvensis, Mecticano denticulutu

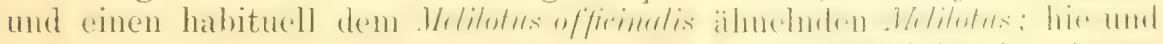

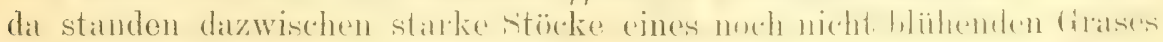

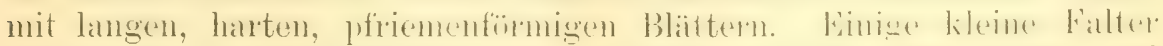

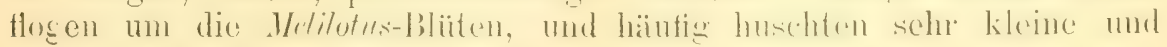
zierliche Eidechsen hin md her. 


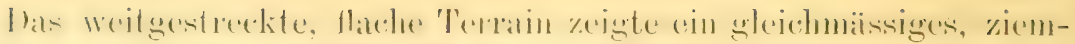

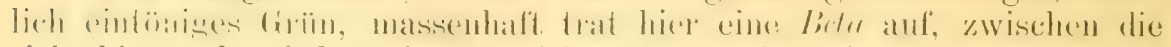
sich hin und wieder ein spärliches Gras oder eine kleine Crncifere drängten. Von alten Bekannten begriisste ich ferner Capsella bursa perstoris, unser "Hirtentäschel", das nicht selten war. Wo die Betu dimmer standen, drä̈gten sieh iiherall kleme salzptlanzen dazwiselen,

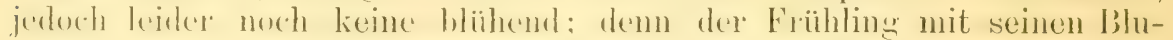
men, der in Brasilion, wonjestens im sïden, im september erwatst. macht sich hier immerhin etwas später bemerkbar.

Ich wanderte cin gut Stiick landeinwïrts, ohne jedoch, ausser cingen blühenden Gräsern, etwas wesentlich. Neues zu finden. Bei

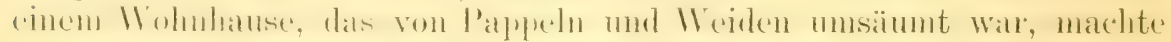

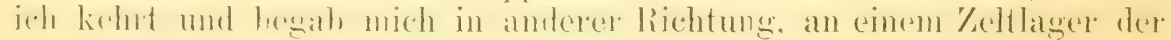

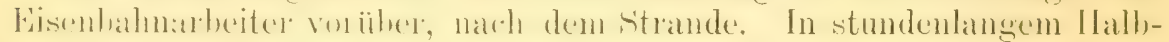
kreise konnte ich ihn überblicken: braun, rötlich und graugrün, das waren die Farben, dic ich sah. Mehr nach den Wasser zu war der Slland sänzlich ohne lewetation: von fonchylien sammelte ich dort cinise kleine Arten, sowie sicheren einer Taschenkrebart, die hier und da zerstrent lagen.

Aus dem Gewir der artarmen Strandflora hoben sich zwei niedere; sparrige llaihsträtchlein empor, die serade in blüte standen und natiis-

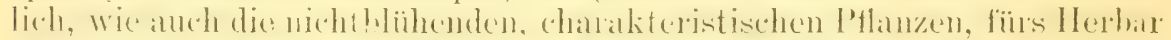

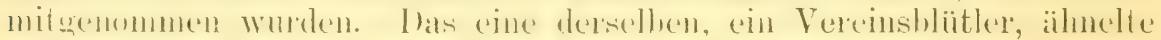

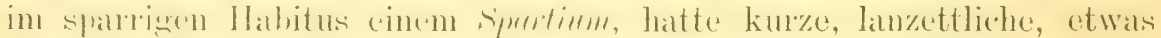

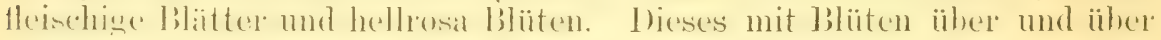

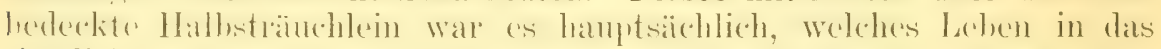

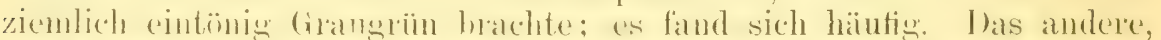

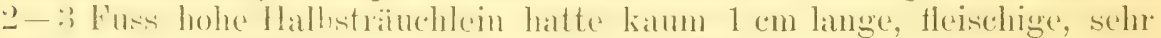
dicht sitzende libittehen, die an den rutentörmigen \%weiglein an sehe

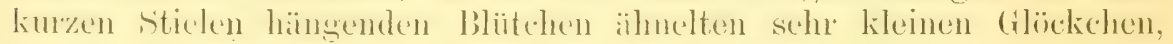
waren weiss und im Grunde schön dunkelblau geadert.

Die ibrigen Strandpflanzen - es waren ausser wenigen Gräisern (i) siten - bildeten entweder kaum fusshohes Gestripp), oder kompakte,

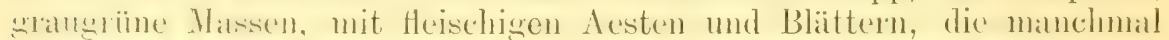

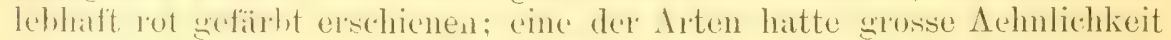
mit einer Saliconia. Jede der Species war sehr häufig vertreten.

Eincn wertvollen Fund machte ich noch in der Nähe des Bahn-

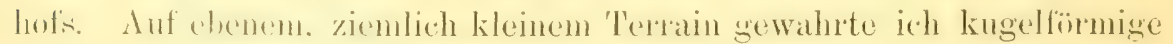
Cacteen, oft von Kopfgrösse und dariber, einzeln oder zu zweit

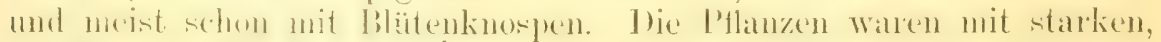

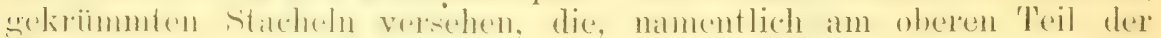
l'flanzen, oft gegen 'Z Zoll lang waren. Neben einem Cactus fand ich eine reizende Oxalis mit hellrosa nickenden Blütchen, leider nu' in einem Exemplare. Von den Cacteen grub ich mir 3 schöne Stïcke aus, um sie mit mil zu nehmen; vielleicht dass es mir gelingt, dieselben in Europa weiter zu kultivieren. Schwerbepackt, in der einen Hand ein mächtiges Pflanzenbiundel, in der anderen die Cacteen, gelangte ich endlich zum Dampfer und bedauerte nur, dass mein Freund und treuer

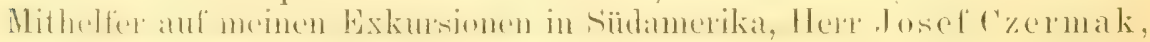
nicht an dieser Fixkursion teilnehmen komnte.-- 
Am zweiten Vormittage darauf botanisierte ich noch eimmal in

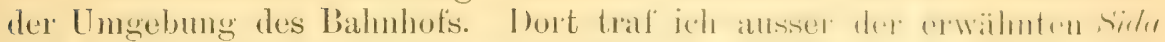

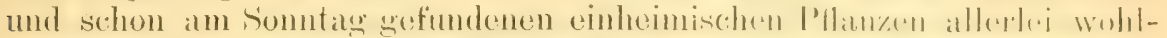

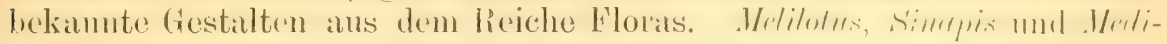
cago habe ich schon erwähnt, ebenso Capselle burse pastoris, das dort äberall zu finden war. Nahe am Bahmgleis land ich ein stattlithes brfr-Exemplar mit weissgeränderten Blättern, „foliis villegatis", wie

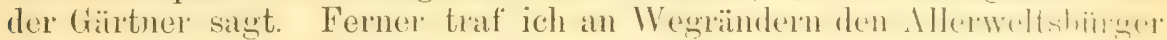

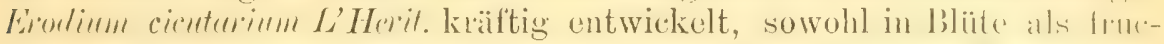

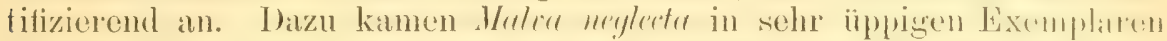

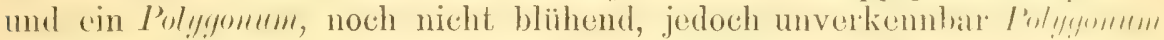

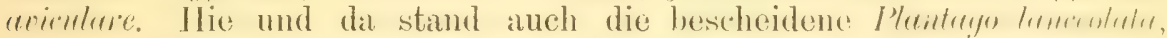
die ich anch in sïdhrasilien häufig antraf. '/um schluss erwähne ich,

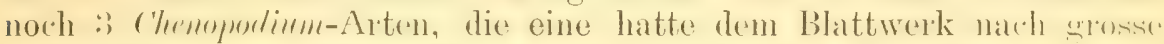

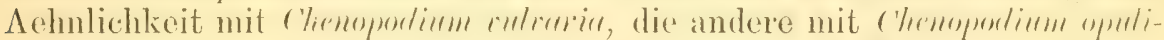
folium, war aber von kïrzerem und gedrängterem Wuchse und kalun

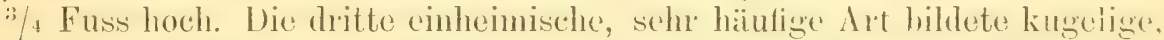
oft iiber 2 Fuss hohe lixemplare von schr gedrängtem Wuchs, die kloinen, rundlichen, dicken Blätter, von denen an gröiseren Exemplaren nuzählige sassen, waren von mattschimmerndem Glanze. Le iden traf ich diese Art, so sehr ich umherspähte, in keinem Exemplare blïhend an.

1)ies in kurzen Zügen meine botanischen Beobachlungen ann Strande von Bahia Blanca. Während ich dies schreibe, sthlagen die Willen

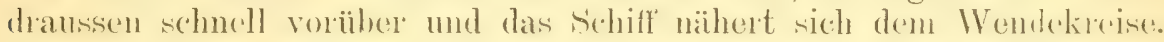
Von den botanischen Auslïgen auf argentinischem Boden blieh dio Himnermng und das kleine Fascikel P'llanzen, die ich dort gesammelt. Und schon frene ich mich wieler darant, nach langer $\Lambda$ hwessenheit vom Vaterlande die bescheidenen kinder der heinischen Elorat wieder zu begriiissen.

An Bord des „Maceió

Eduard Martin Reineck.

auf hoher See, am Freitag 27. Okt. 1899.

\section{Florenbild der Umgegend von Kontopp im Kreise - Grünberg in Schlesien.}

Von 'I'. Hellwig.

(Schluss.)

Aus den Laubmoosen habe ich als gefunden verzeichnet:

Hylocomium squarrosum (L.) Br. et Sch. $\beta$. subpinnatum Lindb., Hypum cordifolium Iledw., straminem I)icks. var. tenue Milde, Hui-

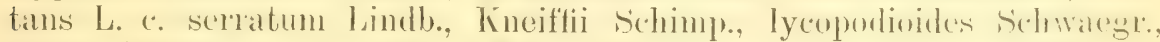

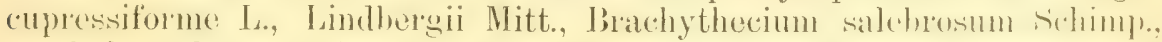
rutahulum Br. et Seh., Plagiothecium silvaticum (1)ieks.) Br. ot reh. h.

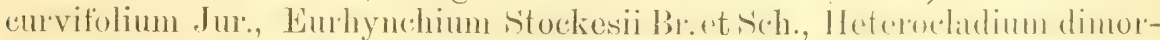

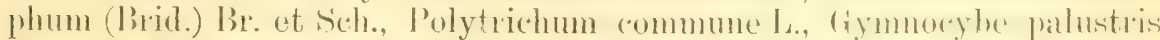
(L.) Frr., Mnium cuspidatum Hedw., hormum (I)ill.) Hedw. IV (herat nutans (S.chel) ) Weh., Grimmia pulvinata (L.) smith, 'Trichodon rylindricus

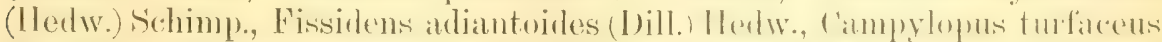

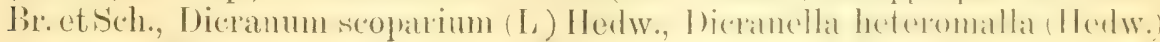

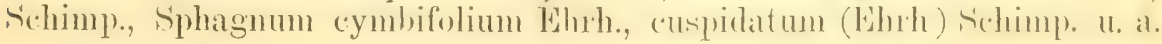


Auf Hylocomium splendens (Dill.) Hedw. fand sich die Flechte Barcidia albescens Ach. Wwwischen Sphagnum wuchs Agaricus (Collybia) (Jusilis Fr., zwischen Moos, Gras und Laub eine Peziza sp., sowie Derminus hypni (Batsch.), Limacium vitellum (Alb. et Schw.) (Hygrophorus hypothejis Lr.), Lactarius subdul(is (Bull.), Marasmius androsaceus (L.), ('oprinarius dichrous (Pers.) (Psilocyle uda?") und liussuliopsis laceata Scop.

Eine Insekten-P'uppe war hevölkert mit Torrubia (Cordyceps) militaris (L.) (Isaria farinosa Fr.), liot mit Isariopsis erythrocephala und Hasen-Losung mit einer Sordaria sp.

Schier endlos ist die Artenzahl der auf dem Erdboden wachsenden Ilymenomyceten: Telephora terrestris Ehrh. und caryophyllea (Schaeff.), Hydnum graveolens Fr., Ochroporus perenuis (L.), Boletus rufus schaeff., rutilans und rubescens Trog., Cantharellus aurantiacus (Wulf.) und cilarius Fr., ('oprinus stercorarius (Bull.) und noch eine C. sp. auf Mist, Hygrophorus sp., Lactarius mitissima (Fr.) und eine andere L. sp., Marasmius alliaceus (Schaeff.) und caryophylleus (Schaeffi.), beide gern auf Graswurzeln und stöcken, P'sathyra sp., P'silocybe pennata (Fr.), mit Vorliebe auf Holzkohle, Psalliota viridula (S'chaeff.) (Stropharia aeruginosa Karst.), Astrosporina lanuginosa (Bull) (Inocybe lan.), Cortinarius albo-violascens (l'ers.) und zweite (. sp., Naucaria furfuracera (Pers.), I'holiota sphatemorpha, A ga ricusepipterygius scop. (Hycena e.), filipes (Bull.), tenacellus Pers. (Collyhia stolonifer Jungh.), striatus Schaeff. und ('Tricholoma) equestris L., als speisepilz hier unter dem volkstünlichen Namen Kasche oder Kottrontle bekannt, der wahrscheinlich slavischer Herkunft ist.

Aus den Gasteromyceten schliessen sich an: Lycoperdon pyriforme Schaeff., gemmatum Batsch. und caclatum Bull., Scleroderma Bovista Fr. und verrucosum (Bull.), Melanogaster ambigua (Vitt.), eine Seltenheit, und Rhizopogon virens Alb. et Schw.

Rhizina inflata (Schaeff.) und Peziza aurantia Miill. kommen als Discomyceten hinzu, Hypomyces aurantius (Pers.), auf Laub und Flechten, als Pyrenomycet.

Es erührigt für die Totalansicht dieses Bildes noch die Angabe der Phanerogamen, die nicht lereits als Nährpflanzen für Pilze genannt sind. Es sind dies: Viola canina L. a. montana (L.) var. ß. lucorum Rehb, silene chlorantha Ehrh und nutans L. var. glabra schk., ('erastium glomeratum Thuill., Radiola millegrana (imel., Hypericum montanum L., Genista tinetoria L.. ()nonis procurens Wallr., Trifolium agrarium Poll.; líubus radula L., I'otentilla argentea L. var. tomentosa Döll, P. Wiemamniana Giünth., P'. argentea Wiemanniana, P'. mixta Nolte, P'. silrestris Neck., I'lmaria filipendula (L.); Sedum sexangulare Wimm. (s. boloniense Loisl.), Scabiosa suaveolens Desf.

Von Compositen: Hieracium pilosella L. trifurcatum Uechtr., H. tridendatum Fr. var. coronopifolia Ki.h., H. trid. f. tenera submonocephala Lechtr, Il. umbellatum L var. Estenophyllum W. Gr. (var. lincarifolium (i. F. W. Mey.), II. umh. s. coronopifolium Rernh, Erigeron Jroebachiensis (). l. Nüll. E. ('anadensis > Druehachiensis, Gnaphalium silvaticum L., Ilelichrysum arenarium 1)( form. anuntiaca, Carhina vulgaris L. und Centaurea Rhenana Boreau.

rodamn: ('himophila mmbellata (L.) Nutt., Monotropa hipopstis L., Gentiana pnemonamthe L., Erythraca ramosissima (Vill.) L'ers. (E. pul- 
chella Fr.), Melampyrum pratense L. var. integerrima Döll., Euphrasia nemorosa (Pers.) E. v. Mart. varr. parviflora, Stachys silvatica und s. palustris L., Thymus serpyllum L. var. pyenotrichus Uechtr., Galeopsis speciosa Mill. (G. versicolor Gurt.), Prunella vulgaris L.; P'olygonum dumetorum L.

Weiter: Alnus incana DC. var. argentata Norrlin forma viridior Call., Anthericum ramosum L., Eriophorum vaginatum L., Luzula campestris (L. z. t.) DC, stark bekleidete Form, Carex disticha IIuds., (: ligerica Gay, C. canescens L., C. Goodenoughii Gay var. ju juncella Th. Fr., C. Good. var. \%. chlorostachya Rehb. (b. chlorocarpa IVimm.), (: frood. var. melaena Wimm., C. acuta (L. z. t) Fr. var. E. sphaerocarpa Lechtr., C. ac. f. brachystachya Uechtr., C. pallescens L.

Ferner an Gräsern: Calamagrostis epigeios Rth. var. glauca lichb., Weingaertneria (Corynephorus) canescens P. B., Aira caryophyllea Wigg., Koeleria cristata Pers. $\beta$. glauca DC., Festuca ovina L., F. duriusculi L sp. plant., F. gigantea Vill.

Als seltenere Juniperus-Formen hat das besprochene Gebiet Juniperus communis L. form. pendula Aschers. und J. intermedia Sichur.

An Gefäss-Kryptogamen sind hier zu nemnen: Botrychium lunaria Sw. und B. matricariaefolium A. Br., Polypodium dryopteris L., Aspidium spinulosum Siw. var. tanacetifolium Sm. (b. dilatatum Hoffm ), Lycopodium inundatum L., L. annotinum L., L. clavatum L. und L. complanatum L. var. anceps Wallr., sowie b. chamaecyparissus A. Br.

\section{Das Ackerbild der Diluvialhügel.}

Eingeschlossen sind die liaine und lïnder, sowie die linderalflora der Dorfstrassen. Bei diesem Bilde sind die Lebensgemeinschaften nicht so eng gefügt.

Hervorstechende Typen sind: Asperula glauca (L.) Bess., Helianthemum chamaecistus Mill., Sanguisorba minor Scop., Bromus erectus Iluds., Alyssum calycinum L. var. reflexum Fiek, Festuca psendomyurus Soyer-Willemet, Anthemis Ruthenica M. B., Nigella arvensis L., Medieago faleata L. var. glandulosit Ǩch., (irsium acaule (L.) All., Agrimonia odorata Mill. und Rosa ruliginosa L. var. pimpinclloides G. F. Mey., sowie var. parvifolia Rau.

Für die grundlegenden Linien bei der Zcichnung dieses Landschaftsgemäldes, in dem dann die kleinen Gewächse ihr I'litzchen bequem finden, dürften sich auch hier wieder die Bäume und Strüucher, weil Strassen und Hecken begrenzend, eignen.

Es wächst an Populus von Flechten: Lecanora sambuci (Pers.), interessante Form (besonders an l'. tremula), Bacidia rubella (Ehrh.), Pragmapora lecanactis Mass. und Opegrapha varia l'ers., an l'. nigral insonderheit Bilimbia cinerea (Schaer).

Von P'izen wählen L'. Italica als Träger: Melampsora populina (Jacq.) und Mycosphaerella populi (Auersw.) (Meptoria populi lesv.); von Gallen sind darauf die von Pemphigus bursarius L. (Hieron. $\mathrm{Nr}$. : 15 ). P. spirotheca Pass, (Hieron. Nr. 346), P. ovata-oblongus liessl. (Hieron. Nr. 347).

Tilia hat nachstehende Flechtenflora: Bacidia rubella (Ehrh.) $\alpha$. luteola (Borr.), B. rosella Pers., Lecidella sabuletorum (rehreh.) Y. enteroleuca (Fr.) f. euphorea (Flk.), Callopisma auriuntiatcum (Lghtf.), L'hlyctis 


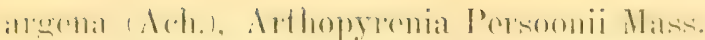
'T'. ulnifolia bringt lasnon erispum Bremi (llieron. Nr. 267) hervor.

Die Galle von Schizoneura lanuginosa (Hart.) (Hieron. Nr.361)

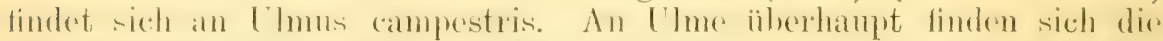

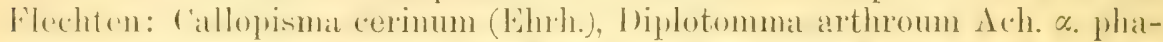
ridia i.leh.), Arthonia populina Mass., leptorhaphis Wienkampii Lahm., an U. var. suberosa (Ehrh.) Strickeria Kochii Khr.

Lothinia trägt P'agmaporal Lecanactis Mass. und ron Pilzen ('uceur-

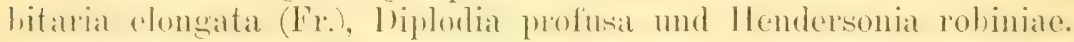

Flechten wurden von l'irus communis mitgenommen: Lecidella

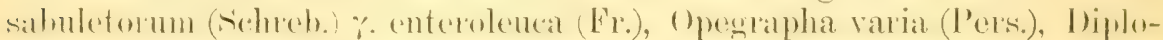
tomma alino-itrum (lloihm.) \%.corticolum (Ich.) f. lencoülis Ach. (letztere an beure blanche).

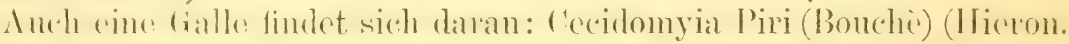

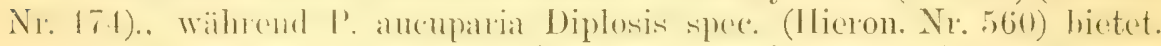

An Evonymus Europaea nistet sich Aphis evonymi Eb. (Hieron. Nr. 315) ein. Reich ist die Gallenwelt an liosa vertreten: An li. tomen-

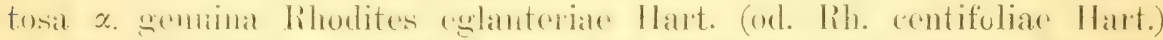

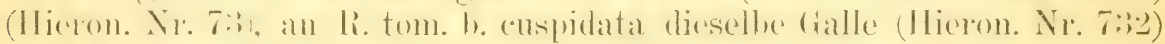
und hih. rosae (L.) Hart. (Hieron. Nr. 733), an R. cinelea letztere Galle

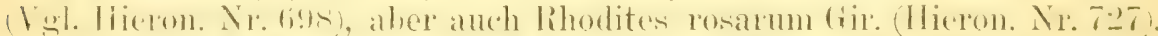

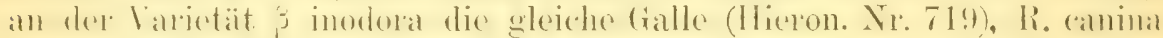
Rh. rosae (L.) Hart. (Hieron Nr.698).

Pilze zeigen Rosa canina: Sphaerotheca pannosa (Wallo.), Rubus

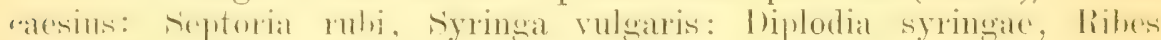
grossularia: Microsphaera grossulariae (Lev.)

Dis Aecker bieten an Capsella bursa pastoris: Cystopus candidus

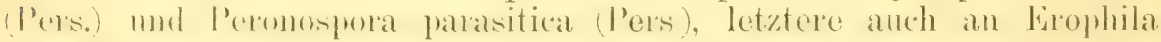
verna; an Cochlearia armoracia: Cystopus cand. (Pers.) und P'hyllosticta anceps.

levelien ergeben sich an risymbriun officinale: Thiplosis ruderalis

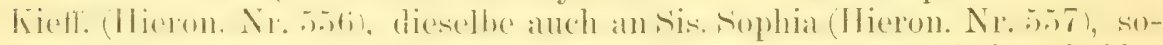

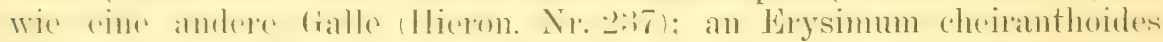

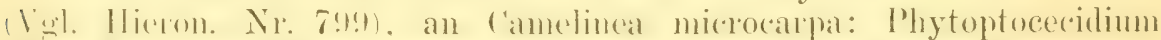

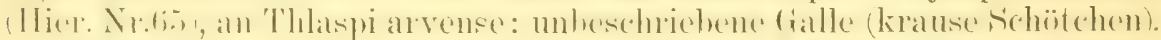

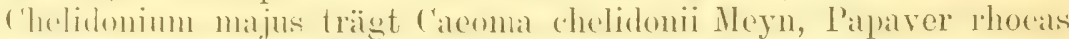

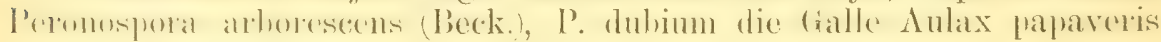
(Perris) G. Mayr (Hieron. $\mathrm{Nr}^{2}$ 618).

fom Ilelianthemum chamaecistus worde eine septoria sp. anfgenommen, an Viola tricolor Ramularia agrestis.

Dianthus anmelia und D). ('arthusianorum sind mit l'leospora dianthi de Not. bestreut, erstere auch mit Phoma herbarum.

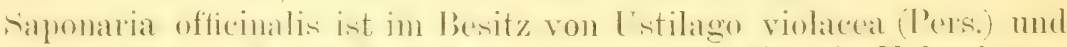

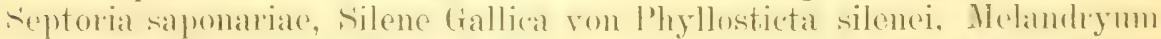

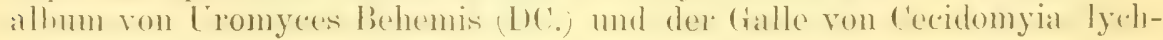
nitis Heyd. (Hieron. Nr.466).

Sperendaria rubra krankt an P'oromospora ohovata (Bom.), Arenaria serpyllifolia an l'oron. arenariate (berk.) nud Holosteum umbellatum an Peron. holostei (Casp.).

Mit hallen wurden gefmulen Stellaria palustris (alanca): l'hytoptocecolium (Hierom. Nr: :-47) mol l'erastimm triviale: Trioma cerastii (II. 


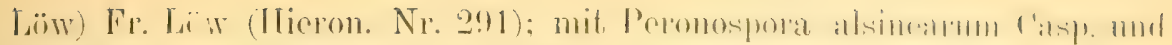
Sorosporium saponaxiae Rud. behaftet Cerast. alvense.

Fiur Geranium molle wurde angemerkt L'eronospora conglomerata

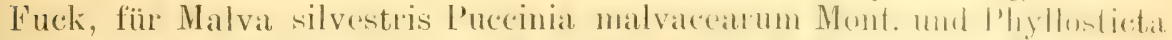
destructiva, für M. neglecta (M. vulgaris) anch I’uce malv. Hont. mad die Galle von Aphis urticaria lialt. (Hieron. N. 33:30).

An Lupinus luteus kommt, vor l'leospora herbarum (l'ors.) an Hreli-

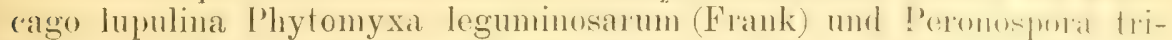
foliorum de Bary, an Medicago falcata Phacodium medicagrinis Lih. (l'sondopeziza Trifolii Biv.-Bern.), an 'Trifolimm filiforme Hronyers striatus suhroet.; an 'Trif. arvense die seltene Galle von 'Tychius polylindatus Germ. (Hieron. Nr. 802).

Von Vicia cracea wurde beigebracht Uromyces fabae (Pers.), von Vic. sativa l'eromospora viciae (Berk.); von Vice angustilola dic diall. zu Cecidomyia viciae Kieff. (Hieron. Nr. 595).

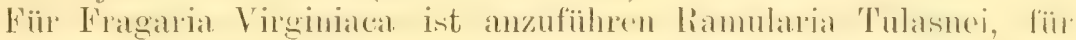

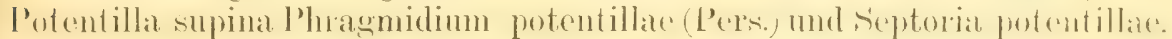

Septoria oenotherae, kommt auf Ocnothera biemnis vor, Sept. seleranthi an folcranthus perennis, Peronospora seleranthi liabenh. an

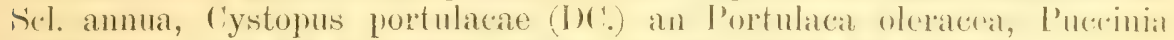
hermintace Ung an llermiaria glahra (in der Nähe bei l'irnige).

An Valerianella dentata wurde l'eromospora valerianellar Fuel.

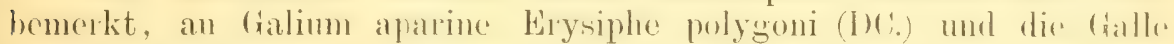
von Cecidophyes galii Nalepa (Hieron. Nr. 103).

Cirsium arvense ist von Cystopus spinulosus De Bary, einer Helo-

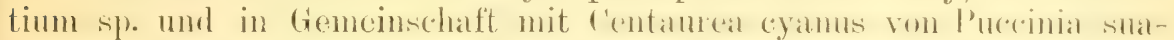

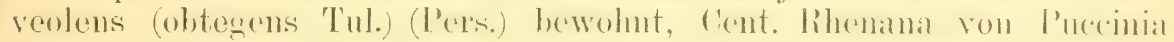
hieracii (Schum.), Artemisia absinthium von Puce tanaceti DC., Art.

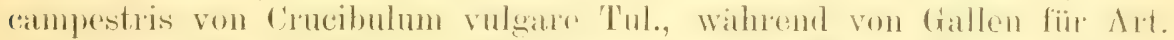
Alsinthim Ne: :37 (Hieron.), liir Art. campestris N1. 11 (Hieron.), ('eci-

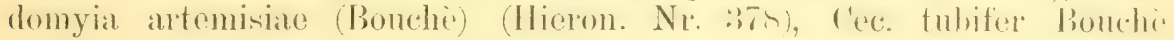
(Hieron. Nr. 379), für Art. vulgaris Nr. 43 (Hieron.) zu buchen sind.

Anthenis Ruthenica steuert l'oronospora leptospenoma l) liary leci, Tanacetum vulgare liamularia tanaceti, Sumerio vulgaris und s. vuluatris - vernalis Bremia lacturate lieg., letzteres anch coleosporium sonecionis (Pers.) und Ramularia senecionis.

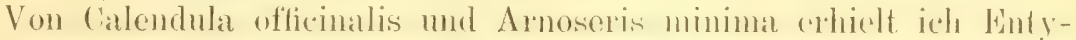

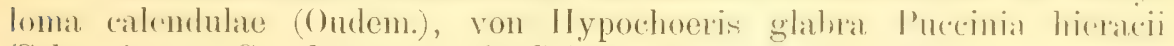
(Schum.), von Sonchus arvensis Coleosporium sonchi (I'ers.).

An Gnaphalium uliginosum sass die Galle von P'emphigus gnaphalii Kalt. (Hieron. Nr.321).

An ('onvolvulus erblickt man Thecaphora hyalina Finderle. mol dir Galle Nr. 79 (Hieron.) mit abnormer Behaarung.

Von Echium vulgare ist die Galle Nr.90 (Hieron) zu nennen und

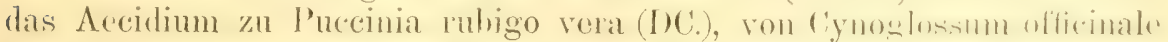
mol Lithospermum arvense l'opomospora myosotidis llo liary. vom ver-

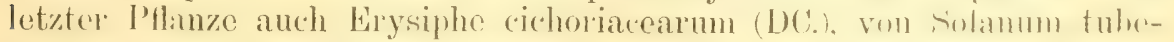
rosum Vermicularia atramenta.

Linaria vulgaris hatte sich die Galle von Gymnetron linariae

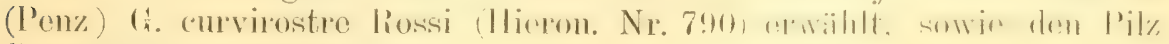
Peronospora linariae Fuck. 
Telhascum thapsiforme zuit iet liamularia varahilis, Veronica hederifolia l'oromospora grisea (Lng.), Alectorolophus majus Coleosporium enphrasiae (Schum.).

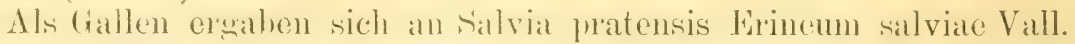
(Hieron. Nr. 2.2!), an (t)echoma hoderaceal recidomyia bursaria Br. (Hieron. Nr. 442) und Aulax glechomae (Hieron. Nr.611).

Ballota nigra, (Xalcopsis terahit. Leomurus cardiaca sind mit Frysiphe galeopsidis (1)(') hesit. Marruhime rulsare mit ()vularia lamii.

Auf l'lantago arenaria gerleiht l'homa polygramma, auf l'olycnemmm arvense cladosporium horlarum, an Imarantus letroflexus mor $\Lambda$. hlitum Cystopus bliti (Biv.-Bern.), auch in Oosporen gefunden.

An Chenoporlimm hyluidum und o : opulifolium wïchst Peronospora effusa cirev, an letzterer Nïhrpflanze, sowie an $\Lambda$ triplex Tatariea Septoria chenopodii, an ('h. vulvaria l'hyllosticta chenopodii, an ch. bonus hemicus Ramularia huni hemici, sowie ein Phoma, an Atriplex angustata Phoma longissima.

An I'olygomum convolvulus crscheint Lstilago anomala .J. Küühn, an Rumex acetosia Lromyeres acetosile fichoet. (Uledo), an Rium. acetosella eine Galle (spiralig gerollte und gedrehte Blätter).

Ton Fuphortial heliosenpia mo fs. peplus heimsen wir ein Melampsora helioscopiac (l'ers.), an Litica mens liamulatia urticae und Septoria urticae.

An Avena sativa erhielt ich Puccinia graminis Pers., an Lolium temulentum Puce. rubigo-vera (D) an recale cereale Lophodermimm

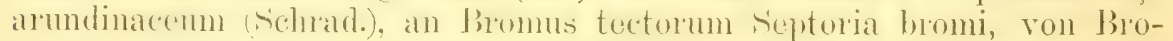
mus mollis eine Galle (Hieron. Nr.61).

Von Ilutpilzen liessen sich blicken: ('halymotta ('anacolus) campanula (L.) mul P'salliota campestris (L.), von Gasteromyceten: Bovista plumbea Pers. und Cyathus olla (Batsch).

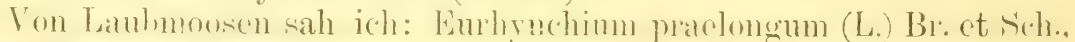
Iottia (Ilymenostommm) trumata (L.) Br. ot tich., Gymnostomum microstomum Hedw. und G. rostellatum (Brid.) Schimp.

Aus den Phanerogamen sind noch zu erwähnen: Ranunculus arven-

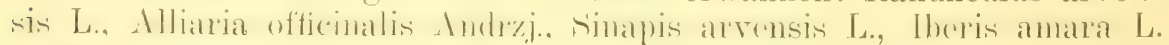

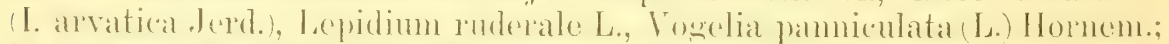

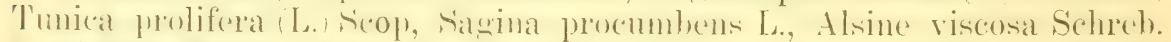

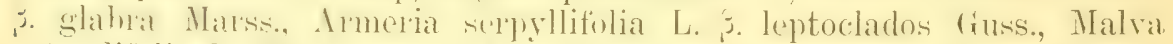

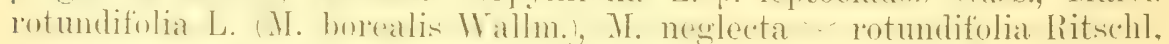
freanim pusilnm L., (r. molle L. f. alliflora, ()xalis strictal L., Anthyllis

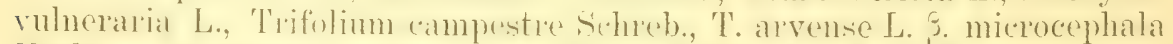

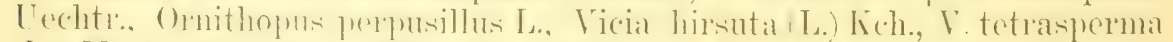

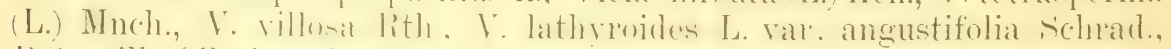

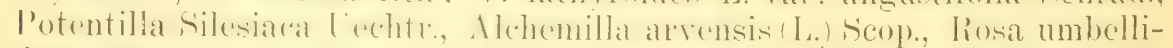
flora riwatz, li. dumotorum 'Thuill. var. uncinella Bess, li. spinossima

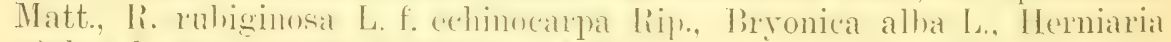

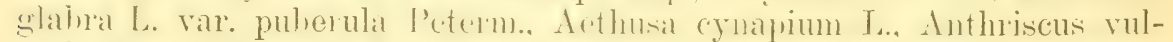

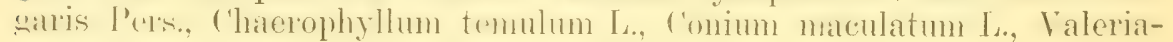
nella olitoria (L.) Poll., Sherardia arvensis L.,

Aus den Compositen: Erigeron Canadensis L., Inula helenium L.

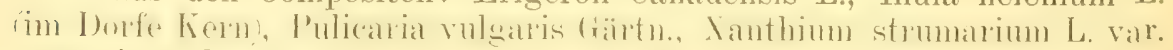
areminm Lasch., (xnaphalimm luten-album L., Anthemis arvensis L., 


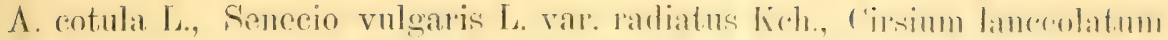

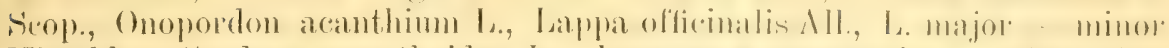

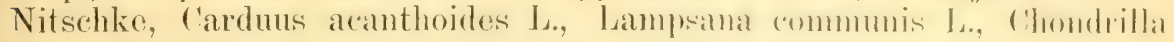
juncea L., Crepis biennis L., C virens Vill.,

Alsdann: Cuscuta epilinum Weihe, Anchusa officinalis L., A. al'vensis (L.) M. B., Myosotis aremaria Schrad. (M. strictil Lik. M. Versicolor (Pers.) Sm., Solanum nigrum L., Hyoscyamus niger L., Jatural stratmonium L., Verbasem phlomoides L., V. nigrum L., Veronica arvencis L.,

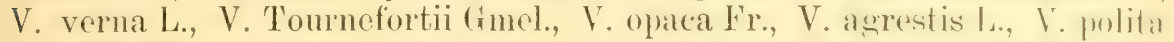
Fr., Mentha arvensis L.f. tenuis, Lamium purpureum L., (ialeopsis latdanum L. (z t.), G. pubescens Bess., Ballota nigrat L., Ácutellatria walcriculata L var. canescens Fiek, Teucrium scordium L.

Ferner: Anagallis arvensis L, Centuneulus minimus L., I'lantago

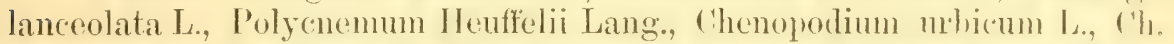
glanc:um L., (h. album L., (h. polyspermum L., $\Lambda$ trip)ex nitens sehk.

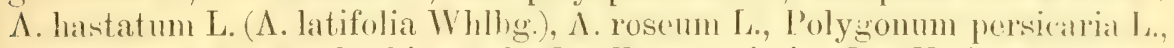
I’. aviculare L., Euphorhia esula L., E. cyparissias L., Urtica dinica l.

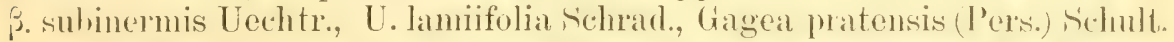

Endlich: Juncus capitatus Weig., Panicum samguinale [., Tetaria viridis (L.) P. J., S. verticillata (L.) R'. B., S. glatuca (L.) I'. B., Phlemu Boehmeri Wrib., Agrostis spica venti L., l'oa ammai L., Bromus secalinus L.., B. tectorum L. P. glahratus Sonder', Equisetum arvense L. valr. ramosal A. Br. und E. litorale Kïhlew. (L. arvense, limosum Lasch).

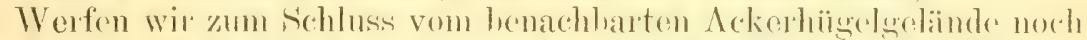

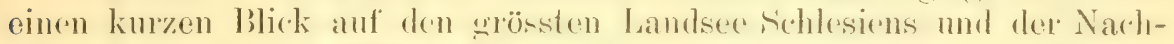
happrovinzen, so gewahren wir am Ufer oder nicht weit rom lleer des

\section{Schlawa-See's}

als Typen: Equisetum hiemale I. $\gamma$. ramigerum $\Lambda$. Br., subforma poly-

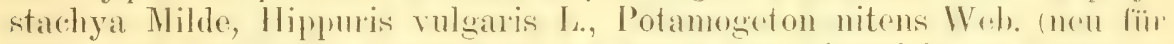
Schlesien), sowic die ('hara stelligera Baner (von Limpuricht fior Schlesion entdeckt) und Ch. aspera (Deth.) Willd.

Ferner sind dort: Botrychium lunaria L, Aira praecox L. und als Pilz auf Equisetum hiemale Libertella Equiseti.

Von $\Lambda$ lgen konnten festexestelt werden: Bolbochacte minor $\Lambda$. Br.,

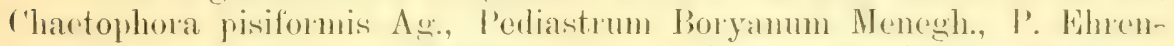
bergii $\Lambda$. Br., Cosmarium Meneghinii Breb., C. phascolus Breb, Fuastrum gemmalum Breb., l'immilaria viridis sm., Naricula cuspidata Kg., N rhyonehocephala Kig., N. amphishaena Bary, Cymbela maculata lig., kineyonema caespitosum lig., Amphora ovalis lig., Cocconeis commmis lleil..

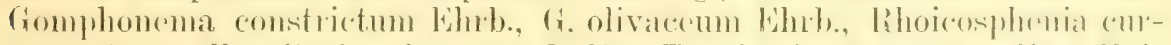
vata Grum., Fragilaria vireseens Ralfs., Taluelluta fenestrata Ke.. Kipi-

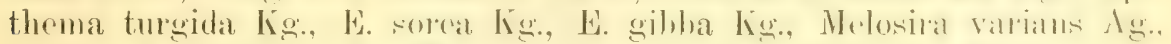

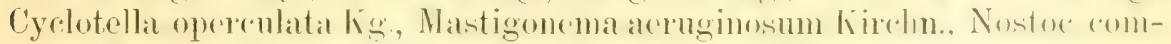

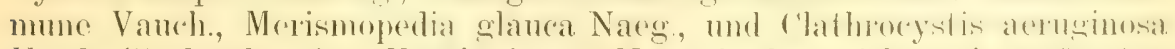

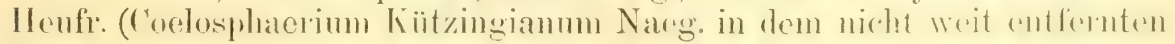
Kölmchen'er See).

Las ist zwar nur din kleiner $\Lambda$ nfing der volls und man kann sagen, die Algenwelt des Sees ist noch so gut wio un-

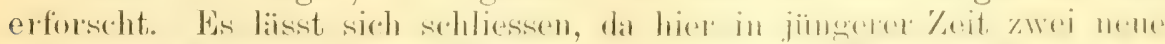

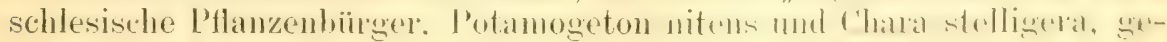


funden wurden, dass anch aus den $\Lambda$ legen eine erhebliche $\Lambda$ nzahl $\Lambda$ rten zu den bekannten schlesischen Arten hirzukommen dïrfte.

Der verstorbene I'rof. Ir. Gehroeter-bieslan hatte gütigst die verzeichmeten Algen bestimmt. (Obwohl dessen wissenschaftliches Arbeiten allerdings in erster Linie der Erforschung der l'ilze zugewendet war, wovon sein klassisches Werk "I)ie l'ilze schlesiens" Zeugnis giebt, so hatte er doch vor, wie or in seinem Briefe vom 11.I.8s bemerkt, die Algenflora des Schlawa-See's möglichst genau festzustellen. Der zu fï̈̈he 'Tod hat ihn daran gehindert.

Schroeter schrieb: „Interessant wäre gewiss eine Zusammenstellung der Algen aus dem Schlawa-See. Ich wïrde eine solche gern unternehmen, wenn Sie mich mit dem nötigen Material versehen wollen. Im Frühjahre komme ich jedenfalls bald eimmal in die Grünberger Gegend, wir könnten dann darüber weiter verhandeln."

Die Mooswelt, Laul)- wie Lebermoose, um den Schlawa-See und die Nachbarseen, ist durch G. Limpricht mit bekamnter Meisterschaft erforscht und geschildert worden (18. Jahreshericht d. schlesischen Gesellschaft für vaterländische (ultur. Breslan, 1871. Seite 106-118).

Alles in Allem kann man nach dem Besprochenen von der Kontopper (kegend wohl hehaupten, sie ist äusserst mannigfaltig und für den Botaniker ein höchst dankbares Gebiet.

Grünberg i. Schl., 2.III.97.

\section{Die Wisselsheimer Salzwiesen in der Wetterau.}

Wenn man den hinter dem Bahnhofe Barl Nauheim gelegenen Höhenzug übersteigt, gelangt man in das 'Thal der Wetter', von der die liegend die Wetterau genannt wird, und sieht vor sich ein Wiesenthal liegen, die Salzwiesen hei Wisselsheim oder das Loewenthal genannt, eine Gegend die sich durch eine grosse $\Lambda$ mzahl charakteristischer talzpflanzen auszeichnet. Bevor man den Höhenzug iihersteigt, kamn man hei der Saline Nauheim noch etliche interessante l'flanzen finden. Ich

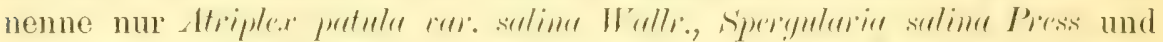
Leprilimm graminifolinm L. Am Johamisberg, ̈̈ber Nauheim gelegen,

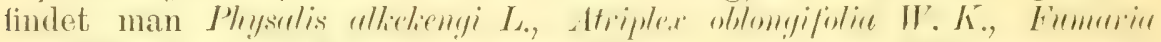

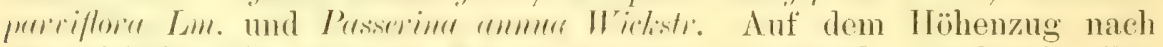
Wisselsheim hin steht in Menge Pulsutilla rulyorris $L$. Doch wir eilen zu den Salzwiesen. Dass der boden da sehr salzhaltig ist, zeigt sich an der ganzen Vegetation, man findet die P'flanz'n bei [oürre mit Salzkrystallen inkrustiert, wer aher zweifelt, mag nur einen 'Tropfen Wasser der vielen Grähen versuchen. Friiher wurde sialz dort gewomnen, es scheint sich aber nicht rentiert zu hahen, da sich von den Gradierwerken nicht eine Spur mehr vorfindet. Mitten dureh das Wiesental schleicht träge die Wetter und scheidet es in 2 ungleiche Trile. Im oheren Teile liegt das Oekonomiegnt Loewenthal, das sein Wasser von den henachbarten Ilöhen bezichen muss, da Brumen nur Salzwasser liefern würden. An den entgegengesetzten Seite zieht sich die Chaussee von Steinfurt nach Wisselsheim hin.

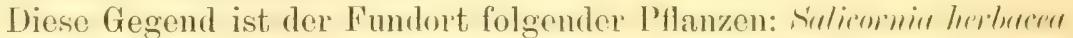

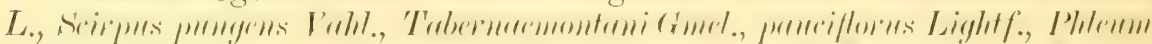

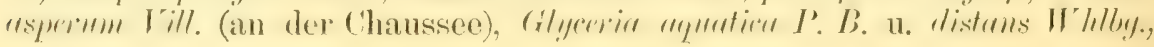


Ilordeum matense Thuds., Pantago manitima Is., Glaux mavitima I., Samolus

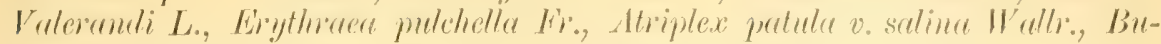
plenum temissimum I. (an der Chaussee), Limm temifolium L. (das.), Juncus bufonius L. v. ranarius P'er. et Lomg., Gerardi Lois., compressus .Jacy., Schenchzeria palustris L. (?), Iriglodin maritina L., litaline hydropiper L. (?), Spergularia salina P'jest, Nigella arvensis (an der Chaussce), Senebiener cononopus I'oir., Lotus tenuifolius libeh., Ihrincia hivte h., Orais incumate L., Kammichellia palustris L. und pedicellata $F$ s.

Winkel, Rheingau.

Freiherr v. Spiessen.

\section{Abnormität in der Blütenstellung zweier Orchideen.}

An einer Lisieve ovata L., welche ich in meinem '/simmer im 'Jopfe

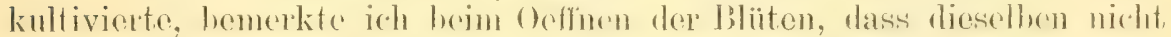
mit der lippe nach unterl gerichlot, sondern so gestellt. Wallen, dass die lippe hei den unteren und oberen libiten anfiväts, hei den mittleren dagegen wagrecht und zwar nach links stand.

Die ganze Pflanze war über der Elde gemessen nur $9 \mathrm{~cm}$ hoch, mol die bibiten befanden sich seht dicht hei einander und waren, ahgerselien von ihrer Stellums, normal. Ans den Wmrolstock dieser P'flanze kam noch ein zweiter 'Trieb, welcher indes nicht blühte.

line wie oben beschriohens anormale stellume hemerkte ich noch

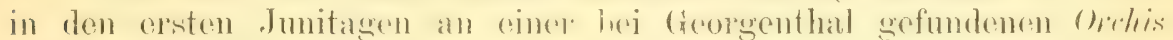

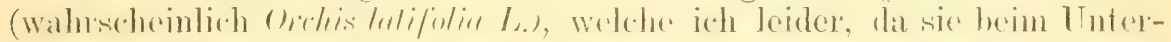
suchen sthon zu welk und kein Knollen daran war, nicht mehr ganz sicher bestimmen konnte. Hier war aber nur eine einzige Blite und zwar eine der obersten mit der Lippe nach aufwärts gerichtet.

Karl Ortlepp, Gotha.

\section{Uebersicht über die bisher in der Umgebung von Guben in der Niederlausitz beobachteten Leber-, Torf- und Laubmoose.}

Von Otto Will. (Schluss.)

\section{B. Torfmoose.}

1. Splurymm cymbifolium (Whrh.) Limpm. Gemein, efret. Hörnchen, Sichiessstände.

2. S. pupillosum Lindb. Im Sumpfe hinter den Sehiessstanden.

3. S. medium Limm. Im Hörnehen und in den Schiessstimden.

1. S. subsecundum (N. no E.) Limpr. Sumptiviesen an der 'T'enfelsskalar.

5. S. contortum (Schultz) Limp). In tiefen Gräben auf Sumpliviesen im Hörnchen. (i. S. compactum I) C: Hörnchen bei Diehlo.

7. S. cuspidatum (Eh\%.) h. et IV. Wiesen zwischen Bomsdorf und Schwertzko.

i. S. squarosum Pers. Am gleichen Standort wie vorige Art.

!. S. acutifolimm (Eh\%.) R. et IV. Häutig.

\section{Laubmoose.}

10. Physcomitrella patens (Hedw.) Schimm. Oderufer bei Fürstenberg.

11. Acaulon muticum (Stheh.) C. Mïll. Auf lehmigen WVegen des Wxerzierplatzes.

12. Phasemm bryoides Diclis. Auf lehnigem Ackerboden, Standort jetzt durch Anlagen des Verschönerungs-Vereins vernichtet. 
13. Ph. cuspridutum Schrob. Häufig, selten ist b. curvisetum (Dictis.).

14. Ph. piliferm S'cheb. Am Oderdamm bei Newzelle.

15. Plemidime allermifolinm Br. o Sch. An Ausstichen bei Gubinchen.

16. P. subulatum (L.) Br. \& Sch. Sandig-fenchter Boden vor dem Bärbusch.

17. P. nitidum (Hedw.) Br. \& Sch. Sehr verbreitet.

18. Hymenostomum microstomum Schimp. Sandig-feuchter Wiesenboden bei Schmachtenhagen vor dem Bärbusch.

19. Dicranoweisia cirrhata (ILedw.) Lindb. Auf dem alten Holzzaune des Bergschlösschens in Guben und an alten Holzscheunen in Horno und Jaenischwalde. Ueberhaupt scheint diese Art auf alten Zïunen, Holzdichern etc. sehr verbreitet zu sein.

20. Dicranella heteromalla (Iedw.) Schimp. Sehr häufig.

21. D. varia (Hedw.) Schimp. Auf Lehmborlen verhrestet.

22, D. cerviculata (Hedu.) Schimp). Sandiger Hoorboden in den Schiessständen und am liande von 'Torfstichen bei Nenzelle.

23. D. Schreberi (Itedw.) Schimp. In sandigen, lehmig-fenchten Ausstichen bei Germersilorf, Kaltenborn und Bomsdorf.

24. Dicramum undulatum Hedw. Häutig.

25. D. palustre (Lap.) B. \& Sch. Nieht zu häufig. efret. Hörnchen.

26. D. spurium Helw. In Kiefernwaldungen verbreitet. efret. im Walde hinter Sembten.

27. D. scoparium (L.) Hedw. Gemein.

28. D. flagellare Hedw. Am Grunde alter Baume bei Treppeln und an der Seemiihle steril. efret. Stadtforst.

29. D. montanum IIedw. Seltener; an Bäumen im Stadtfort.

30. Leucobrym glaucum $(L$. Schimp. Gemein. efret. seltener: Stadtforst, Atterwasch, Kaltenborner Berge.

31. Fissidens adimtoides (Dill.) Medw. Auf Wiesen bei Jaenischwalde in Menge, auch efret. Steril am kleinen 'l'reppelsee.

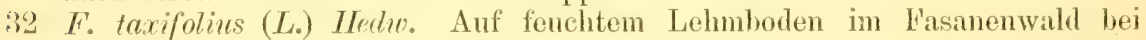
Neuzelle.

3. F. osmundioides (Sw.) Hedu. Drenziger Schweiz.

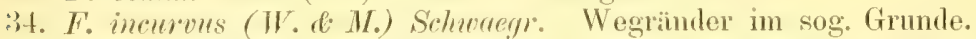

35. F. bryoides Iedw. Lehmig-sandiger Boden im Barbusch und an den schweinegruben im Stadtforst.

31. Octodiceras Juliannm (Savi) Brid. Unter der Eisenbahnbrücke bei den Schiessstinden an Steinen im Neissebette legi 15. Juli 1895, wieder gesammelt 13. Juli 96. Jetzt ist der Standort infolge von Arbeiten an der Brücke durch Steinmassen verschiittet.

37. Pottie lanceolute (Dicles.) C. Miull. An Wegrändern in Lawitz.

38. T. mimulula (Schuregy).) Fiirm. An Ausstichen bei Gubinchen.

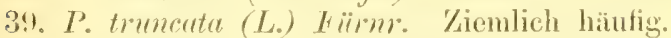

40. P. intermedic (Tume) Fïm. Verbreitet.

41. Didymodon rubellus (lioth) Br. o Sch. Kerstrent.

12. Ceratodon purpmeras (L.) Brid. Gemein.

4:. Ditrichum tortile (Schrad.) Hampe. Auf feuchtem Sandboden in der Nähe des Schiesshanses in Neuzelle.

44. Barbula fallax Meatw. Häulig.

45. B. unguiculata (Dill.) Hedw. Sehr verhreitet auf Lehmboden.

46. Tortula muralis (L.) Timm. Gemein.

47. T. ruralis (L.) Ehrh. Gemein. Vorwerk Cöhne, Nenzelle cfrct.

4.. T. subulate (L.) Hedu. Häufig.

49. T. montana (Nees) Limalb. 'Ziegeldächer in Germerstorf.

51). T. pulvinata (Jur.) Limm. Zerstreut an Laubbämen.

51. Racomitrinm canescens (Dill.) Brid. Gemein. Auch b. ericoides (Dirks.) nicht selten. 
52. Schistidinm apocarpum (L.) Br. ent. Häutig: alter Kirchhof auf Sandstein, am alten Holzzan des Bergschlösschens; auf Steinen bei Diehlo, Schwertzko ete.

53. Grimmia pulvinata (L.) Smith. Genein.

54. Hedwigia ciliate (Dill.) Hedw. Auf Steinen im Hömchen, Schlaubethal und der 'Teufelsskala.

55. Ulota Bruchii Hormseh. An Waldbäumen bei Atterwasch.

56. U. crispa (11edw.) Brid. Gubener Stadtforst an Lichen; Schlaubethal an Buchen.

57. U. crispulu Bruch. Am gleichen Standort wio vorige Art im Stadtorst. U. Ludwigii wird von Rabh. in Flor. lus. bei Guben angegeben; ich konnte sie noch nicht auffinden.

5.. Orthotrichum diaphenum Sched. Auf alten Holzzïumen in Germerselorf.

59. O. patens Bruch. An Weiden bei Kaltenborm.

(i). O. pumilum Su. Häutig an Weilen, Pappeln ete.

(i1. O. fallax Schimp. = O. Schimperi Ilammar. Kiemlich häulig.

(i). O. anomalum. IIedue. An Feldsteinen bei Deulowitz.

(ii). O. Lyellii Hook. o' T'ul. An Lichen des Staultorstes.

(i). O. leiocarpum Br. d Sth. An Bäumen bei Atterwasch.

(i.). O. speciosum N. v. Li. Zienulich häutig.

(iti. O. affine Schrad. Sehr verbreitet.

67. O. fastigiatum Br. var. (qp)endiculatum (Schimp.) Limpr. An Akazien des alten Kirchhof's in Guben.

is. O. obtusifolium Schrorl. An Buchen hinter Sembten.

(i!). Encalyptu vulyaris Iledwo. Kerstrent auf alten Mauern: Lawitz, Neuzelle ete.

70. Plysscomitrim pysiforme (L.) Brid. Auf 'Torfboden häufig. Ein kleines Riischen fand ich am Manerwerk des Bahnübergangs vor Kaltenborn!

71. Hunaria hygrometrice (L.) Hedwo. Gemein.

72. Leptobryum piriforme (L.) Schimp. In Mauerritzen: Eisenbahnbrücke vor Gubinchen, Feldbricken bei Kaltenborn, in den Oderwiesen bei Nenzelle etc.

73. Nebera albicans (Ithlbg.) Schimp. Kerstreut, doch nur steril.

74. W. nutuns (Schreb.) Hedue. Häufig; in Waldungen manchmal Massenvegetation.

75. II. sphagnicola Br.eur. 'Zwischen Sphagnen und Hypnen im Hoor hinter den Schiessstinden legi 1896.

7t. W. crude (Scheb.) Schimp. Schattige Erdabhänge am wendischen Kirehlof in Guben und im Fasanenwald bei Neuzelle.

77. Bryum inclinatum (Sw.) Bland. Auf Moorboden in den Schiessständen.

7s. B. pendulum (Hoinsch.) Schimp. Am Gemïner einer Lriicke in den Oderwiesen bei Neuzelle.

79. B. pscudotriquetrum (Hedw.) Schwaegr. Sumpfige Torfwiesen bei Nenzelle.

80. B. capillare L. Verbreitet. Barbusch, Neisseufer vor den Schiessstanden.

81. B. argentenm L. Hiufig.

82. B. cuespititium L. Nicht selten. Germersdorf, Lawitz.

8:3. B. intermedium ( $W$. d M.) Brid. An alten Mattern in Kaltenborn.

it. B. bimm Schreb. Ausstiche vor Bomsilorf.

55. Rhodobryum roseum (Dill.) Scheb. Verbreitet: Germersdorf, Dietrich, Kaltenborner Berge etc. Im Kiefernwäldchen hinter Reichenbach cfret.

sti. Mnium stellare Helw. Abhänge im Kiefernwald hinter Bonsdorf, Schlaubethal: an Abhängen des WVeges von der Bremsdorfer zur Kieselwitzer Mühle.

87. M. serratum (Schrad.) Bril. Erdlehnen des Kerkwitzer Kirchweges.

Ss. M. hormum (Dill.) ILedu. Nicht selten. Hier und da auch efiet. (Turnplatz, Drenziger Schweiz, Kaltenborn.)

s!). H. undulatum (Dill.) Hedw. Gemein. efret. Kaltenborn, Bremselorfer Mühle.

90. U. affine Bland. 'I'urmplatz; 'Lorfwiesen bei Schwertzko. An beiden Urten cfret.

91. M. cuspidatum Hedw. cfret. Bomselorf, Reichenbach, Fasanenwald bei Neuzelle. 
!'.. M. punctanm I. Zerstrent. cfret. Schlaubethal, zwischen Bremsilorfer und Kieselwitzer Miihle, Sumpliwiesen an der Lisenbahn bei Kaltenborn.

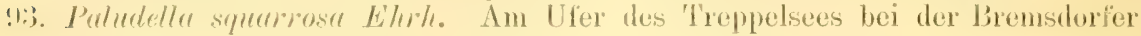
Mïhle, steril, logi 1897.

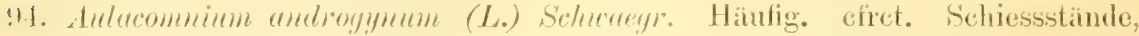
Barbuseh und besonders üppig an Wegrändern in Schlaubethal.

15. Aul. pulustre (L.) Schugr. Auf Wiesen, fenchtem Waldhoden gemein. Im Hörnchen efret. var. polycephale (Dill.) Sehimp. Schiessstände.

!hi. Bartramia pomiformis (L.) Ilede. Sandige Wegränder mol Abhänge: Drenziger Schweiz, Kaltenborn, Bomsilorí, Lawitz, schlaubethal.

17. Philonotis fontuner (L.) Brid. In Sümpten hinter den Schiessstinden in r) Exemplaren, steril auf Wiesen im Schlaubethal.

15. Cutharinaed undulala (L.) W. ot M. Gemein.

!!9. C. tenella Röhl. Selten an WVegrändern: Lawitz, Germersdorf.

100. Porgonatum umigernm (L.) Schimp. In Ausstichen der Drenziger Schweiz.

101. P. aloides (Dill.) P. Benn. An Erdlehnen der sog. Schwenegruben im Stadtforst und in den Kaltenborner Bergen.

var. minimum Lim \%. An feuchten, lehmigen Abhängen des Kerkwitzer Kirchweges oberhalb Kaltenborn legi Ostern 1898 und Juni 99.

10.2. P. namm (Dill.) I'. Beomv. Auf Heideland: Schiessstände, Deulowitz.

10:3. Polytrichum commune L. Staltforst, Hörnchen.

104. P. jumiperimum Willd. Desgleichen und am Secberg.

10天. P. piliferum Schreb. Schiessstande, 'T'entelsskala, Bomsdorf.

10ti. P. fomosum IIedw. Hörnchen, Schlaubethal, Stadtforst.

107. Georgia pellucida (L.) Rabh. An Wegrändern bei der bremsdorfer Mühle reich fruchtend. 'Iumplatz in Guben.

10s. Diphysermm foliosum (L.) Hohr. In einer Fuche im Kiefernwald der Drenziger Schweiz.

10!1. Buxbanmin aphylla L. Häufig anf Waldboden: Drenziger Schweiz, Stadtforst, Schlaubethal, Kaltenbomer Berge.

110. B. indusiata Brid. Zumteil mit voriger an denselben Standorten. An einem Graben im Kiefermwalde vor Gubinchen.

111. Fontinulis antipypetica (Dill.) L. Im Neissebett und in besond. prïehtigen, grossen Exemplaren im langen See bei Bomsdorf.

112. F. gracilis Lim7b. An Steinen im Neissebett unter der Eisenbahnbrücke bei den Schiessständen. Es ist dies derselbe Standort wie für Octodiceras; aber, wie bei dieser Art schon bemerkt, jetat vernichtet.

11:. Leslien polycarpa Ehr\%. An Bäumen ziemlich häufig.

111. Anomodon viticulosus ITook. of Tayl. An alten baumstämmen: Bärbusch, Dietrich, Staltforst (Hohle Berge).

115. Thuidium tumariscimum (IIedw.) Br. S SeT. Bärbusch, Kaltenborn, Atterwasch. Im schattig-feuchten Gebüsch am linken Schlaubeufer nahe der Kieselwitzer Mühle e. fi.

116. Th. delicatulum (L.) $13 \%$ o \& Se\%. Seltener als vorige Art. Waldlehnen am Schlatubenfor unweit der Bremsdorfer Mühle c. fr.

117. Th. recognitum (L.) Limlb. Anf Waldboden im Schlaubethal am kl. 'Treppelsee, und am Grunde alter Eichen des Stadtforst bei den Schweinegruben c。 fr.

11s. T\%。 abietimu (L.) B\%, d Seh. Häufig, doch stets nur steril.

11!). Neckere complanate (L.) Hïboner. An Buchen längs der Schlaube nahe der Bremsilorfer Mühle.

120. Lencodon sciuroides (L.) Schuraer). Häufig, doch immer steril.

121. Antitrichia curtipendula (L.) Brid. Steril an alten Eichen des Gubener Stadtforstes; reich fruchtend an Buchen bei der Bremsdorfer Nühle.

12.). Climacium dendroides (Dill.) W. \& M. Gubinchen, Vorwerk Cöhne, Dietrich, Bremsdorfer Mühle c. fr.

1:2: Pylaisin polyanthe (Schreb.) Schimp). An Weiden vor den Schiessständen und vor Kaltenborn; an Eirlen im Bärbusch. 
124. Homalia trichomanoides (Schreb.) Br. of Se\%. Verbreitet. Auf feuchtem Wurzelwerk bei Schmachtenhagen und am Grumle vou Bäumen im Ilönchen c. fr.

125. Isolhecium myurum (Poll.) Brid. An Laubbäumen steril im Bäbusch.

1206. Homelothecinm sericenm (L.) Br. \& Sch. Barbusch; Dietrich c. fir.

127. Camptothecinm lutescens (Huts.) Br.eur. Gubener berge, Atterwaseh, Fasamenwald bei Nenzelle, überall cfret.

1.3. Eurhynehimm striatum (Schreb.) Br. \& Sch, Verbreitet; Bärbusch, Kaltenborner berge und bei Atterwaseh c. fr.

139. L. strigosum (Hoffm.) Schimp. Auf Waldboden zwischen Deulowitz und Kerkwitz.

130. L. protongum (L.) Br. o Sch. Auf schattigem Boden des alten Kirchhofis in Guben.

131. Li. Stoliesii (Tw\%.) Br. Sch. Auf Wurchn und Steinen an einer Quelle nahe am Bahmhof Neuzelle.

1:30. Rhynchostegium rusciforme Br. \& Sch. An Steinen im Dorchebache bei Schwertzko.

133. Brachythecium albicans (Neck.) Br. of Sch. Buderose, Gubinchen.

134. B. salebrosum (ILoffm.) Schimp). Häufig.

135. B. velutinum (Dill.) Br. \& Sch. Nicht selten.

136. B. rutubulum (L.) Br. of Sch. Gemein. Sehr formenreich!

137. B. sericeum W Wrnst. Auf Laubholzstümpfen des Bärbusches legi 27.III.1896.

138. B. rivulure (Bruch.) Br. \& Sch. Im Sumpf am Bahnübergang bei Kaltenborn.

139. B. populenm (ILedw.) Br. \& Sch. An Sandsteindenkmälem des alten Kinchhof's in Guben, unter Gesträuch auf blosser Erde im sog. Grunde und an Wurzeln auf dem 'Turnplatze.

140. Plargiothecinm silcaticum (L.) Br. \& Sch. Bärbusch, Hörnchen, Schiessstände.

141. P. Roeseanum (Hampe) Br. \& Sch. Am Grunde alter Laubholzstimme auf dem 'I'urmplatze und an Wegrïndern im Fasanenwald bei Neuzelle; auch im Schlaubethal verbreitet.

142. 1'. denticulatum (Dill.) Br. o Sch. Erdlehnen der Drenziger Schweiz und des linken Schaubeufers nahe der Bremsdorfer Mühle.

143. P. Silesiacum (Sel.) Br. \& Sch. Auf faulem Holz in Kiefernwald bei Sembten.

144. Amblystegium riparium (L.) Br. \& Sch. An Stemen in einem Bach vor dem Bärbusch, auf altem Holz in der Lubst und Neisse.

145. A. serpens (L.) Br. o Sch. Reich fruchtend an Steinen im Bett der Dorche bei Schwertzko.

146. A. rigescens Limpr. nov. spec. An dem Gemäuer einer Feldbrücke vor Kaltenborn detexi 4.VII.1896. Dieselbe Art an Samdsteindenkmälern des alten Kirchhofs in Guben legi 12.VIII.96.

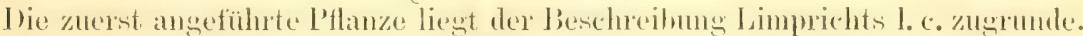

147. Hypmum chrysophyllum Brid. Steril in sandigen Ausstichen hinter Bresinchen.

148. H. stellatum Schreb. Steril im Bärbusch.

149. II. polygamm (Bi. \& Sch.) Wils. In Ausstichen bei Kaltenborn.

150. II. gigantem Schimp. In Gräben auf Wiesen bei Jaenischwalde nahe der Haltestelle der Eisenbahn.

151. H. purum L. Nicht selten. An schattigen Waldlehnen in der Nähe des sog. Lauschützer Hammers e. fr.

15:. 11. cuspidatum L. Häutig.

153. H. Scheberi IV Ylld. Häufig. Grund, Kaltenborn, Hörnchen, Vw. Cöhne e. fr.

154. II. stramineum Dichs. Steril im Moor hinter den Schiessstanden, an einer Quelle bei den Kaltenborner Bergen.

155. II. palustre IIuds. Gubinchen, Schlagsdorfer Brïcke.

156. II. crista-castiensis L. Spärlich zwischen Birkenwurzeln im Hörnehen bei Diehlo rechts vom Wege nach Bremsdorf. Steril.

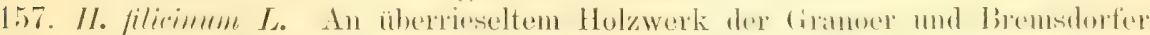
Mühle, hier auch efret. Auf Steinen im Dorchebach bei Schwertzko.

158. H. uncinatum Hedu. Sumpf hinter den Schiessständen cfret. 
159. II. fluiluns L. Wiesen bei Gubinchen und Grano.

160. II. exammatum Giamb. Henchte Wiesen in der Nähe des Haltepunktes Jaenischwalde, steril.

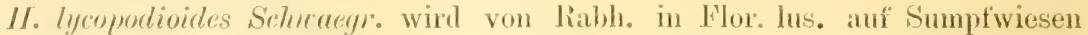
bei Guben angegeben, doch habe ich es noch nicht beobachtet.

161. II. seorpioides L. In Gräben längs der Eisenbahn bei Jaenischwalde.

162. II. Kneiffii Schimp. Im Sumpte hinter den Schiessständen.

163. II. vemicosmm Lindl). Steril in Wasserlöchern bei Schmachtenhagen und anf Wiesen bei Jaenischwalde.

164. II. cupressiforme L. Gemein.

var: filiforme $\mathrm{Br}$ \& Sch. An Bämmen: Stadtforst, Hörnchen. Steril.

165. Mylocomium splendens (Heder) Br. o Sch. Grund, Hörnchen, Schlaubethal, Bremstorfer Mïhle e. fr.

166. II. triquetmm (L.) Ji. o Sch. Schattige Stellen in Grunde und bei der Bremsilorfer Mühle c. fr.

167. II. squurrosum (L.) Br. of Sch. An den gleichen Standorten wie vorige Art e. fir.

\section{Nomenklatorische Revision höherer Pflanzengruppen} und iiber einige

\section{Tausend Korrekturen zu Englers Phaenogamen-Register.}

Von Otto Kuntze und 'T'om vou Post.

(E'ortsetzung.)

'I'ribusnamen früerer Autoren, die in-oideueverändert oder o hne eue-Suffix a f g estelltwurden.

(Die vielen 'Tribusnamen, die bei Engler zu Subfumilien erhoben und dabei in - videae umgeändert wurden, soweit sie darmer noch 'Tribusnamen erhielten, sind hier weggelassen.)

IIydrophylleae (Ilydrophylloideae Aschs. (inerke).

Tha las siene BHgp. 1883 (Thalessioidea Aschs. \& Guerke 1859).

Pistiene HLK. 1815 "Pistincenes“ n. corr. Richb. $18+1$ (Pistividene Engler 1889).

Le mue ue (Lemmoilene Engler).

II olffiea (ITolffioideae Engler).

Herierieace (Irererioidene Engler).

ophiopoy o neae (Ophiopogonoideae Engler).

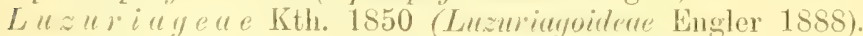

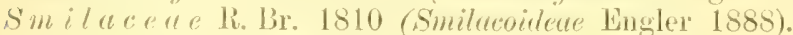

Ay avea sal. 1806 (Aguroidene Iax 1888).

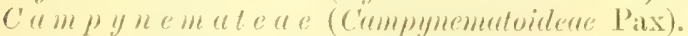

Crocea e Bkr. 1876 (Crocoilene P'ax 1888).

Ul mea Ag. 1825 em. BHgl). (Ulmointene Engler 1889)

Celtide a e Gaud. 1826 (C'eltidnidene Engler 1889).

Conoceph al e a e 'l'récul 1847 (Conocephatoidene Engler 1589).

Ca nn a b e a e Bl. 1825 "Camabinae" (Cammaboiltea Engler 1889).

Wyst o pet a lea e Eichler 1868 (Mystropetaloidene Eingler 1889).

Ductyla th the a e BHgp. 1880 (Dactylanthoidene Engler 1889).

Sur co phytoae Endl. 1836 (Sareophylhoideae Engler 1889).

If ollugineare Spr. 1818 (Holluginoideae Pax 1889).

Nel "mb n ne a e DC. 1821 (Netumbonoideae Casp. a Engler 1891).

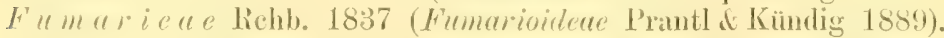

II y p e co e a e l)umort. 1827 (Ilypecoidecte Prantld Kündig 1889), 
Cleo meae DC. 1824 (Cleomoidene Pax 1891).

Dipterygieare (Dipterygiouleae L'ax).

Royd sieare (lioydsioideae Pax).

Calyptrothecea (Calyptrothecoideae Pax d Gilg).

Emblingieare (Emblingioideae Pax).

Francoea e Richb. 1841 (Frane(o)oideae Engler 1890).

Pterostem o ne a e (P'terostemonoideae Engler).

Es se allonica e DC. 1830 (Escallonioidene Engler 1890).

Ribesie ae BHgp. 1865 (Ribesioidea Engler 1891).

$B$ a ue rea e DC. 1830 (Baneroideae Engler 1891).

Neuradea DC. 1825 (Neuradoideae Focke 1888).

A ugeeae (Auyeoideae Engler).

Peganea (Peganoideae Engler).

$N$ it $r$ a riea $e$ Meisn. (Nitrarioideae Engler).

T'etradiclidea e (T'etraclidoideae Engler).

B a la nit ea e Baill. em. (Balanitoideae Engler).

Poranthereac Rehb. 1841 (P'orentheroidene Pax 1890).

Ricinocarpea Muell. arg. $186+$ (Ricinocarpoideae Pax 1890).

Tripteryge ae (Tripterygoideae Loesener).

Groue pie a e Baill. 1876 (Gonepioideae Loesener 1892).

Sta phyle ea e (Staphyleoidece Pax).

T'a pis ciea e (T'upiscioidene Pax).

Lophopy xideac (Lophopyxidoidea Engler).

Cardiopteridea (Cardiopterygoidea Englex).

Vitea e lichb. 1837 (Vitoideae Gilg 1896).

Le e e a L Lal. 1847 (Leeoidene Gile 1896).

L'udodes mi ea e (Éndodesmioideae Engler).

C'alophyllea choisy 1824 (Calophylloidea Engler 1893).

Bl at tie a e (Blattioideae Niedenzu).

Crypteronie a e (Crypteronioideae Nielenu).

Foetidieare (Fotidioideae Niedenzu).

Planchonie a e (Planchonioideae Niedenzu).

Na p o l e o ne a e (Napoleonoideae Niedenzu).

Le cy thide ae (Lecythidoideae Niedenzu).

An is ophyllece BHgp. 1867 (Anisopluylloideae Schimper 1892).

Gar rye a e Baill. 1879 (Garryoideae Hams 1897).

Nys sea baill. 1879 (Nyssoideae Hams 1897).

Davidieare (Davidioideae Harms).

Ala nyicae Miq. 1855 (Alangioidene Harms 1897).

IL a sea e DC. 1834 (Matsoidene Pax 1889).

Aegicerateat DC. 1834 "degicereae" (Aegiceratoideae Pax).

bud l le e a e (Buddleioidene Soleveder).

Cordiea Rehb. 1837 (Cordioideae Guerke 1893).

Ehretieat Don 1837 (Ehretioidene Guerke 1893).

Heliotropieat Ag. 1825 (Heliotropioidene Guerke 1893).

St ilbeate BHgp. 1876 (Stilboideae Briq. 1894).

Caryopteridea Schaner 1847 (Caryopleridoidea Briq. 1894).

Symphoreme a Me Misn. 1840 (Symphoremoidene Briq. 1894).

Avicenuie ae Meisn. 1810 (Avicemioidene Briq. 1894).

Cat op herieare (Catopherioidea Briq.).

Nelsoni e a e Nees 1832 (Nelsonioideae Lindau 1895).

Mendonciear (Mendoncioideae Lindau).

Th " ub ergie a Dumort. 1829 (Thumbergioidene Lindau 1895).

Cyp th ie a e Rchb. 1841 (Cyphioidea Schoenland 1889).

Lobeliea S. F. Gray 1821 (Lobelioidene Schoenland 1889).

Goodeniea Dumort. 1829 (Goodmioidede Schoenland 1889).

Brunouiea non 1834 (Brunonioidene Schoenland 1889). 
Also 81 'l'ribus wurlen nicht mit dem Suffix - eae benannt, wie es sonst in Engler's N. P'lanzenfamilien stattínd, und zwal anch bei solchen, wo 'Tribus = Subtamilie ist, z. B. Dictyolomene = Dichyolomoidece, Flindersieae $=$ Fthuder-

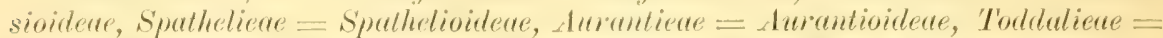

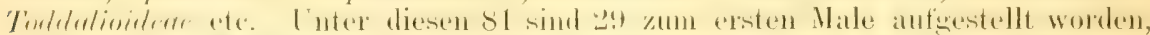

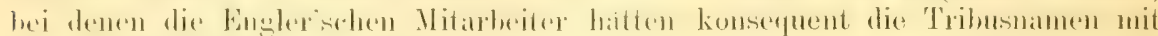
-ene neben dem + imaginären Subfamiliennamen auf - oideue bilden sollen. In den restierenden 52 liallen sind aber die 'l'ribusnamen frïherer Autoren verschwiegen worden und dafür Namen mit - oidene gesetzt worden, zu denen dam in Dalla 'T'orre a Harms' Supplement- Werk die neuen Namenbildner als 1. Autoren für Auftellung dieser Gruppen zu citieren sind, da sie in Engler's Werk zum Schein so hingestellt sind. "A kind of piracy" kam man das mit amerikinischen Botanikern nemnen. In wissensehaftlich botanischen Werken sollte jeder Autor, wie das jetzt viele exacte Botaniker thun, falls ein nener Name gebildet wird, stets sem verantwortiches Autorcitat dazu setzen, sonst entsteht leicht Verwirumg. Nomenklatorische Eigenmichtigkeit ist es, 52 schon von anderen Autoren aufgestellte und benannte Gruppen neu zu benennen, resp. Immataufen, und die früheren Namen und deren Autoren totzuschweigen, wie es Engler veranlasst und getham hat.

Die enzehen Engler'schen Mitarbeiter sind lierbei wohl zum teil durch die Dispositionen des Direktors entschuldigt, Dispositionen, die wohl für ein populäres Werk genügen, wo man die Citationen der Synonyme und Publikations-

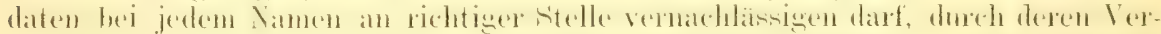

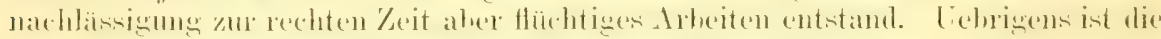

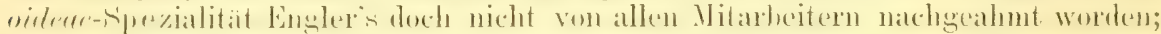

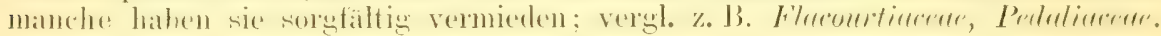

\title{
Wissenschaftliche Korrekturen und Ergänzungen zum Gesamt-Register II --IV von Engler's Natürlichen Pflanzenfamilien.:)
}

\author{
Ausgearbeitet von \\ Tom you ost. \\ Direktor der Samen-Kontrol-Anstalt zu Upsala.
}

In mnserer Zeit, wo nach Professor E. Köhne**) jährlich über 7000 botanische Schriften erschemen, kömnen nur noch solehe vom systematischen Stand-

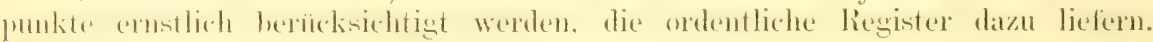

*) Diese Dissertation, ehe sie hier zum Abdruck gelangte, ist mit Ausnahmo einiger Nachträge zur Aufnahme in die Botanischen Jahrbiucher Herm Prof. A. Engler angeboten worden. Her. Lngler hat aber nur einen alphabetischen Extrakt für Aus. lassungen und Verbesserungen seines Registers in einem neuen Nachtrag zu seinen "Natiirl. Pflamzenfamilien" publizieren lassen wollen. Das wäre nur Flickarbeit geworden. Ausserdem sollte etwa die Hälfte meiner Arbeit mit Berichtigungen von Englers mol seincr Vitarbeiter Fehler mol seines unhaltharen Nomenklaturstandpunktes didurch unterdricht werden. Für meine biblographische Arbeit, die der Herstellung

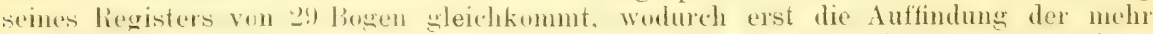
als lowo fehler möglich ward, erlaulte sich llere bingler die keineswegs provocierte Bemerkung, dass für einen solchen Extract, der dann kaum einen Bogen füllen dürfte, diese drbeit mit also hörhstens 1 bogen Honorar ,ordentlich honoriert" werde. Auf diesen Vorschlag bin ich nicht eingegangen. Ich hatte übrigens nur 75-100 Freiexemplare anstatt Honorar beansprucht

Da ich ausserdem für Engler's Werk nur ein pflichtschuldiges ordentliches Register für Phaenogamen und zugleich Kíryptogamen für nötig halte, so erlaube ich keinerlei Nachdruck, insbesondere nicht den Druck eines alphabetischen Extraktes aus dieser meiner P'ublikation.

'T'om vou l'ost.

**) 'The Botanicai Gazette 1894 S. 250. ${ }_{n} \Lambda$ defense of the Botanische Jahresbericht." 
Die Register in Engler's botanischen Jahrbuichern weichen von denen anderer botanischer Zeitschriften dadurch unvorteilhatt ab, dass die Bandregister, also mit Ausnahme sehr seltener Spezialregister, keine alphabetisch geordneten Gattungsnamen enthalten. Dadureh werden solehe P'ublikationen, welche doch meist

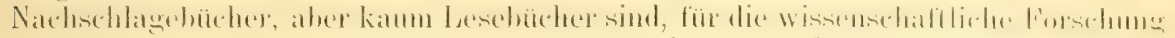
stark entwertet, dem das Suchen nach den einzelnen Namen der Novititien mol systematischen oder nomenklatorischen Veränderungen wirl durch diese Defekte so zeitraubend, dass es meist ganz unterbleiben mus. Das ist ein so grosser Uebelstand, dass Dr. Otto Kuntze nicht mit Unrecht im Bulletin de liherbier Boissier 189+ Seite 467 einen besonderen Zusatz zu Artikel 73 des Corlex emendatus, der auf den internationalen Lois de la nomenclature botanique, L'aris 1867 basiert, gegen schlechte oder fehlende legister vorschlug.

Während Baillon zu jedem der 13 Bände seiner Histoire des plantes, ebenso wie Bentham of Hooker zu jedem Bande ihrer Genera plantarum ein genames Spezialregister mit Synonymen und Autorcitationen gab, musste man bei den Natïrlichen Pflanzenfamilien über 11 Jahre warten, ehe ein mit diesen unentbehrlichen Details versehenes Gesamtregister zu band II-IV erschien, das die 2t 'Teilregister, welche der Synonyme und Autorcitationen entbehren, ersetzen sollte. lch hatte während der vieljährigen Publikationsilauer für die 2t, resp. mit dem im Jahre 1897 erschienenen Nachtragband 25 Teilbinde der Phaenogamen die Register mit den fehlenden Details erginzt und fand num nach Erscheinen des jetzt erschienenen Gesamtregisters, dass darin über 1000 Defekte und unichtige Angaben sich finden.

Dieses Gesamtregister weist eigentumliche Fehler auf und scheint übrigens von einem bibliographischen Fachmann, aber kaum von einem erfahrenen Botaniker") angefertigt und von Engler selbst erst nach vollentetem Druck revidiert zu sein. Da Engler's eminentes und teueres Werk indess kaum oder nicht bald ein noch so nötiges gemeinsames Register für Phaenogamen und Kryptogamen erhalten dürfte, so seien die wichtigsten Ergänzungen und Korrekturen zum Nutzen der zahlreichen Freunde dieses Werkes hier mitgeteilt. Sie lassen sich wie folgt gruppieren.

\section{Corrigenda systematischer Verwechselung'en.}

Actinotinus Oliv. ist keine (Caprifoliac.) sondern eine Mystifikation. Adinandra Jack ist Theacee, nicht Elaeocarpacee.

Amorenxia Moc. \& Sessé ist Cochlospermacee, nicht Bixacee. Aphylleia Champ. ist Trinviduce, nicht Hydrocharitacee.

dracococcus Brong. ist Bromeliace, nicht Liliacee.

Arthrostachys Desv. ist Graminee nicht Cyperacee.

Aubletia Gaertn. Someratiacee, nicht Blattiacee.

Azerediu Allem. Cochlospermacee, nicht Bixacee.

Basisporia Ndz. Sommeratiacee, nicht Blattiacee.

Biatherium Desv. ist Graminee, nicht Cyperacee.

Blancou Blume, die Sapindacee, ist mit der Palme Blancou Blume konfus zusammen registriert.

Blatli Ad. Sonneratiace, nicht Blattiacee.

Bromia HBK. Fouqueriacee, nicht Tamaricace.

Canella Murr. Canellacee, nicht Winteranace.

Champereia Griff. Opiliace, nicht Santalace.

Chiratia Montr. Somneratiacee, nicht Blattiacee.

Cinnamodendron Endl. Canellacee, nicht IV'interanace.

Cimamosma Baill. Canellacee, nicht Winteranace.

Cochlospermum Kth. Cochlospermacee, nicht Bixacee.

Coclocaryon Warb. Mirristicacee, nicht dionacee.

*) Allenfalls von einem Lokalfloristen, der mit exotischen Gattungen und Funilien nicht vertraut ist. Der Verfasser des Engler'schen Registers ist Prof. Dr. C. G. de Dalla 'I'orxe nach Engler's brieflicher Mitteilung. 


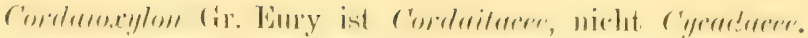

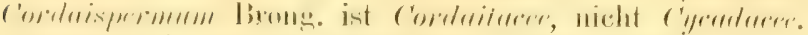

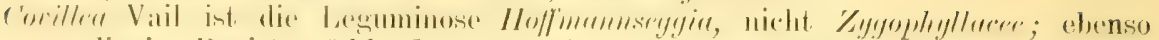
die im liegister fehlende Laver Ort. (non Cav.). Beide sind an richtiger Stelle, also unter IIo/fmanneggio zu eitieren vergessen worden.

Cryosophila B1. ist Palme, nicht Riaputeacee.

Cinpteroniu BI. Sonneratiace, nicht Blattiacee.

Clenoholhon Oliv. nicht Olacacee, nach N. 204 wohl Linacee.

Cyanastrum Oliv. nicht Commelinacee, wohl Pontederiacee.

Cyclocarpus Goepp. ist Cordatuce, nicht Cycadaces.

Dasystachys Oerst. Palme, keme Rubiacee.

Dialyenthera Warb. ist Myristicace, nicht Anonace.

Dialyearya Mast. Tiliacee, nicht Bombacacee.

Digomphlia Bth. ist Bignonacee, nicht Acanthacee.

Diperium Desv. Graminee, nicht Cyperacee.

Diplasanthum Desv. Giominee, nicht Cipperacee.

Diplotesta Gr. Eury ist Cordailace, nicht Cycadacee.

Diporamlia DC. Cochlospermacee, nicht Bixacee.

Dolerophyllum Sap. ist IDolerophyllacee, nicht Cordaitacee.

Duabanga Ham. Somneratiacee, nicht Blatliacee.

Elachanthera F. V. M. Liliacee, nicht Iridace.

Eremorhlou bïse ist Graminee, nicht Cyperacee.

Eublatli Nalz. Somneratiacee, nicht Blatliacee.

Eucrypteronia Ndz. Somneratiacee, nicht Blatlacee.

Eufouqueria Ndz. Houpuieriacee, nicht Tamaricacee.

Eumaximilianeu Warb. Cochlospermace, nicht Bixuece.

Fingenthe Ch. ¿ Schl. Cochlospermace, nicht Bixacee.

Evodianthus Oerst. Cyclanthace, nicht I'alme.

Floyeria Neck. Gentianacee, nicht bricacee.

Gomorlega li. \& P'. ist Gomortegacee, nicht Laurace.

Gonianthes B1. Burmaniacee, nicht Orchidacee.

Gramerium Desv. ist Graminee, nicht Cyperacee.

Ginmucrenthere Warb. Myristicacee, nicht Anonacee.

Hensloria Wall. Sommeratiacee, nicht Blattiacee.

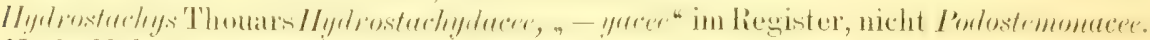

kéenle Hol. Gomortergace, nicht Lauracee.

Irredilier Coss. \& Dur. Graminee, nicht Lilincee.

Lancisic Lam. IV 5:276 und Gaert. IV $5: 280$ sind in letzterem Falle durch

einen Lapsus von 0 . Hoffmann verwechselt.

Leiogyme K. Schum. "Fritsch" ist Bignoniacee, nicht Gesneracee.

Lutaie Brong. Cyclanthace, nicht Palme.

Hacrodendion T'aub. Mnienacee, nicht Cunoniacee.

Macrosanonia Cogn. ist Cucurbitace, nicht Composite.

Malulucban Blanco Opiliatere, nicht Samlalacee.

Mreventia Vog. Lerfuminose, nicht Dipterocarpace.

Massonie 'Thbg. Liliace, nicht Oichidacee.

Mandoutchia Warb. Mypisticacee, nicht Anonacee.

Maximilianea Mart. d Schr. Cochlospermaces, nicht Bixacee.

Microsplenimm Hk. f. Rubiacee, nicht Caprifoliacee.

Mimurie DC. Composite, nicht Grominee.

Moriche Drude ist L'alme, nicht Bromeliacee.

Muchlbergella lieer ist Campanulace, nicht Composite.

Nagcin Gaertn. ist als Myjichere und Taxacee registriert, aber gar keine Myricacee.

Nowodicorstiya P'resl Graminee, nicht Liliacee.

Ophininella Desv. Graminee, nicht Cyperacee.

Opiliastrmu Baill. Opiliacee, nicht Sumalacee.

Osteocapum H.. V. M. Chenopodiace, nicht Saxifragacee. 
Osteophleam Tirrb. Myristicacee, nicht Anonacee. Oxycaryum Nees Cyperacee, nicht Graminee.

Payapata Sonn. Sommeratiacee, nicht Blattiacee.

Parophiorhiza Clarke Loganiacee, nicht Rubiacee.

Paspalanthium Desv. ist Graminee, nicht Cyperacee.

Pectinaria Bth. die Orchidacee II 5:216 ist mit P'ectinaria Bth. N. 40 Gramine konfundiert.

Pentaphragmene sind Campamulaceae, nicht Curcurbitaceae.

Pentapyxis Hk. ist Caprifoliacee, nicht Rubiacee.

Pistites Hos. \& v. d. M. Cycadacee, nicht Aracee.

Pittiera Cogn. ist Cucurbitacee, nicht Composite.

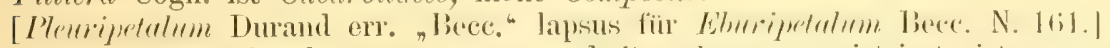

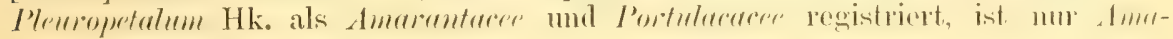
iantacee.

Phryganocydia Mart. Bignoniace, nicht Gesneracee.

Prionanche Nees und Prionanthium Desv. sind Gramineen, nicht Cyperacenn.

Podoon Baill. Anacardiacee, nicht Phytolaccacee.

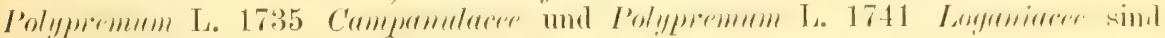
konfus zusammen registriert.

Pycnanthus Warb. Myristicacee, nicht Anonacee.

Pyy.hosa Endl. Myristicacee, nicht Anonacee.

Quilamum Blanco Somneratiacee, nicht-Blattiacee.

Ravenea Hild. \& Bché. Palme, nicht Lemnacee.

Rhodoclada Bkr. nur Theacee, nicht Linacee.

Rhynchotheca R.\& P. Geraniacee, nicht Gesneracee.

Rodschiedia Miq. Polygalacee, nicht Legmminose.

Rosenbachia Reg. ist nur Labiate, nicht Verbenacee.

Samaropsis Goepp. ist Cordaitacee, nicht Cycaldeee.

Scialostigma Ndz. Somneratiacee, nicht Blattiacee.

Scyphocephalium Warb. Myristicacee, nicht Anonacee.

Sebophora Neck. Myristicacee, nicht Anonacee.

Sendelia Goepp. ist Rubiacee, nicht Myrsinaree.

Sibbaldia L. ist Rosacee, nicht Loasacee.

Sicyocodon Feer ist Campamulacee, nicht Composite.

Slackia Griff. giebt es dreierlei:

1 Gesneracee, 1 Palme und 1 Lardizabalace;

im Engler'schen Register sind sie unglïcklich vereint.

Sphaerosepalum Bkr. Cochlospermace, nicht Bixaree.

Stachyanthemm KI. ist Cyrillacee, nicht Celastracee.

Standia Warb. Myristicacee, nicht Anonacee.

Tetracentron Oliv. ist Magnoliacee, nicht Trochodendiacee.

Themeda Forsk. ist Gramince, nicht Cyperacee.

Thespesocarpus l'ierre ist Ebenacee, nicht Sapotacee.

Thollonia Baill. ist Icacinacee, nicht Rosacee.

Tombea Br. \& Gr. Sommeratiacee, nicht Blattiacee.

Trapa L. ist Onagracee, nicht mehr IIydrocaryacee.

Trematocarpus ist Campamulacee, nicht Composite.

Urceolaria Mol. Gesneracee N. 300 ist mit der limbiacee Urceolaria Coth. Eonfus zusammen registriert.

Taseyanthus Cogn. ist Cucurbitace, nicht Composite.

II arburgia Engl. Canellacee, nicht IVinteranacee.

Winterana L. p. p. Canellacee, nicht Winteranace.

Wittelsbachio Mart. ¿ Schr. Cochlospermace, nicht Bixacee.")

*) In diesem Teil, der grösstenteils im Manuskript vor dem ersten 'T'eil fertig war, ist Engler'sche, noch nicht die reformierte Familion-Nomenklatur anguwendet. 
Es sind dies zumteil offenhare Verwechselungen der Familien, zum kleinen

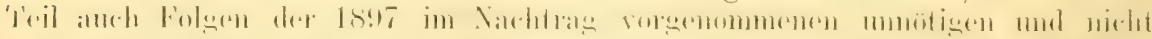

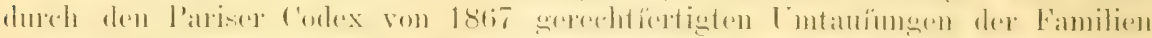

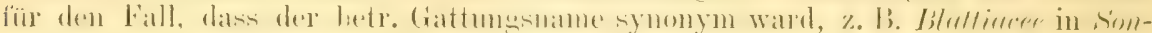
neratiacce, Winteranacer in Canellacere. Dass diese und andere Veränderungen

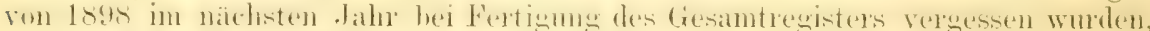

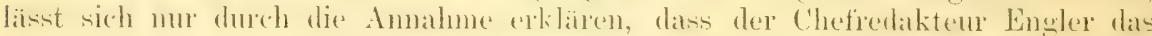
liegister erst etwas mehr angesehen hat, als es im Druck fertic war und dass

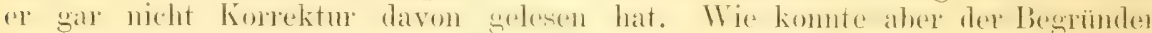

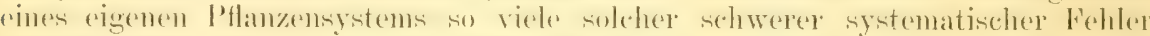
stehen lassen, ohne sie im liegisternatehtrag zu berichtigen!?!

\section{Namenfehler bei Familien und anderen höheren Gruppen.}

Als gültige Familiennamen sind im Register anfgeführt, aber synonym sind nach dem letzten 'J'ext:
Ampelidacere, sind $=$ Vilaceae.
Apiaceae, sind $=$ Umbelliferere.
Blattiaceace, sind = Someratiuceae.
Comitere, sind $=$ Pinaceae.
Hydroceryacene, sind = Onurgraceue.
Roxburdhaceae, sind $=$ Siemmaceae.
Ternstioemiacene, sind $=$ Theacene.
Thelygonacere, sind $=$ Cynocrambaceae.
Wintranacere, sind = Canellacear.

Bei manchen Alternativnamen, 2. B. Camellearcae (Stylintiacene), ist der 2. Name richtig als synonym registriert. Betreff Coniferae ist zu bemerken, dass sie 1897 in Engler's Nachtrag als Klasse behandelt und in Pinaceae, Ginglioarear, Taxurate geteilt wurden, aber der Name Coniferae ist im Register nicht mit den 'Typen für Klassen. gedruckt. Die übrigen Fehler beruhen anf dem

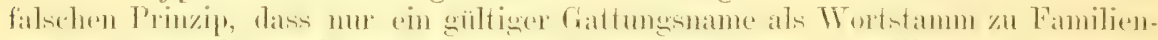
namen verwendbar sei, wodurch eine Menge geläufiger Familiennamen umötig von Engler verändert wurden; ummötig, denn nach Artikel 22, Absatz 4 des Pariser Codex kömmen s y n on y me Gattumgsnamen auch dazu dienen. Von solchen Namen hat Engler sogar 15 gegen sein neues Prinzip unverändert gelassen: Aquifoliacene, Balsaminaceae, Cactaceae, (Cactus gilt bei Engler nicht), Caprifoliacene, Caryoplugllaceae, Cucmbitacene, Ebenaceae (Ebemus L. gehört nicht in diese Familie), Graminate (richtiger Graminaceae geschrieben, denn Gramen ist

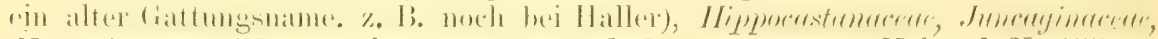
Nyetarinaceae (Nyctayo ist synonym und Nyctarinia von Heimerl N. 155 zu Boerhania eingezogen worden), Lentibulariacere, Palmae (richtiger Palmaceae,

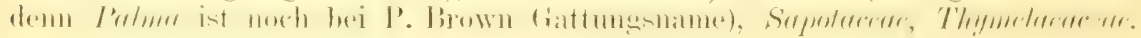

Folgende 7 Familiennamen haben üherhaupt keinen Gattungsnamen zur

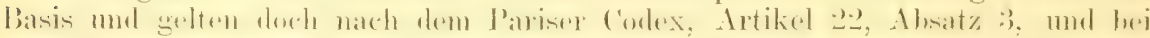
Engler gegen sein Prinzi): Chlaenctere (es giebt keinen Gattungsnamen Chtuen, ebenso wenig wie ein Genus IIydrocrrya für die früher bei Lngler gültigen IIydrocaryaceac), Compositue, Cruciferae = Crnciatue, Umbelliferae = Umbellatue,

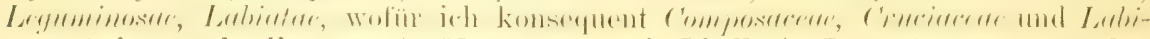
acene (wie manche l'ranzosen), Umbollacede (wie Lindley), Legminucere anwenden werde.

Bei Subfamilien, resp. Tribus sind Tristeginede nach dem ungiltigen Namen Tristegis und Ulmarieae nach Utmaria, wofür Engler 1897 Filipendula annahm, gegen sein Prinzip stehen geblieben. Anch die Namen höherer Gruppen, wie

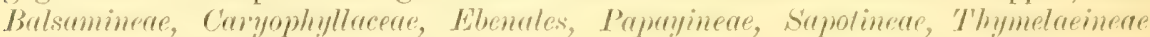
lassen die Durchführung dieses Prinzipes vermissen. Es lïsst sich eben nicht

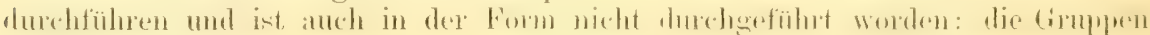


werden durch - ales (Reihen oder Ordnungen), -ineae (Unterreihen oder Unterordnungen), -aceae (Eamilien), - oideae (Unterfamilien), -eae ('Tribus), - imae (Subtribus gekenmzeichnet; aber z. B. Cupress-inear, Abirt-inear, Scandir-inere sind 'Tribus und keine Unterordnungen und hatten Cupress-eae, Abiet-eae, Scandiceae lauten müssen, wie Engler sonst anch richtig Rumicene, Viticene (von Vitex), Mirabileae, Parideae etc. schrieb. Ausserdem ward in Band II, S. 2 -inene auch für Klassen gebraucht.

Bei den Familiensektionen sind im Register gültige und ungïltige Namen gleich gedruckt, sodass man bei konkurrierenden Namen nicht ersieht, welcher Name gilt, z. B. Luzuriagoidene = Enargeoideae.

Ansserdem fehlen in Engler's Werk bei Familienmamen genane Citate jedes Autors und der ersten Aufstellumg, wie man solche Citate in Limolley's Natural System, Endlicher's Genera Plantarum und Bentham \& Hooker's Genera plantarum findet.

\section{Citatfehler bei Gattungsnamen.}

1. Zunächst einige grobe Verwechselungen von Antorcitationen:

Abroma Jaeq., nicht L. f., der den Namen in Ambroma änderte.

Amasonia L. f., nicht L.

Amplicome Royle, nicht Lall.

Aristotelia L'Hér., nicht DC.

Arthraevua O. Ktze., nicht Sehinz.

Berylias Sonn., nicht Lam. = Capmepiria Gm., nicht L.

Brucen Miller, nicht Mueller.

Binganthus Gim., nicht Steller.

Campelia L. C. Rich., nicht L.

Campulosus Desv., nicht Desf.

Chamaeorchis Rich., nicht L.

Corycium Sw., nicht P'fitz.

Crassina Scepin, nicht Scop.

Deerimgin R. IBr. (1810), nicht P. Br. (1756) und bei Guidonie P. Br. (1756), nicht R. Br. (1810) mmgekehrt. Fine Verwehsolung dieser hänfig citierten Autoren P. Br. und R. Br. ist recht irreführend; im Spezialfall wird die Prioritit von Deringia (Deringa) Ad. 1763, welcher Name bei vielen Antoren jetzt gilt, dadurch verdunkelt. Noch unklarer ist das Citat ohne Vornamen bei Diplopogon. Brown, Leptaspis Brown, Microchloa Brown und andere Gramineen.

Diuris Sm., nicht Sw.

Dysophylla Blume, nicht Lour.

Ecestripliyllum P. Bro, nicht Rich.

[Fradinia "Mill." IV 5:391 ist ein Lapsus für IIeleniestrmm und ganz zu streichen.]

Gayg Gaudin, nicht ,Drude", der dafür Gaud. schrieb; aber Gaudin sollte nicht abgekürzt werden, da Gaud. für Gaudichaud gilt.

Gomphocarpes R. Br, nicht L.

Grophephorum Desv., nicht Pers.

Habenaria "L." giebt es nicht; N. 98-99 ist unter Mabenaria W. zu stellen.

Heteracia F. M. und H'. v. M., soll Fiseher \& Meyer $=$ F. d M. heissen.

Iydrilla Rich., nicht L.

Ismene Salisb., nicht Bkr.

Knantia L., nicht Coulter.

Leiog!yne K. Schum., nicht Fritseh.

Lencosalpa Elliott, nicht Engler.

Limmobium Rich., nicht L.

Nanophylum Less, nicht L. 
Paromia Cavo, nicht $L_{0}$; letateres Citat verdunkelt die Prioritat von Malache 'Trew und Jassa Ad.

Poligmeria K. Brr., nieht L.

l'seudoconnums liadlk., nicht L.

Iferongyon L. Muell., ans der ungenïgenden (itation , F. M." entstanden, muss Fischer \& Meyer heissen.

Puschlinia Adams, nicht Adanson.

Khamnicastrum nur L.; Ludw. dafür giebt es nicht; dass es in der Nachtragsrevision dazu citiert wird, ist nur ein Zeichen der Flüchtigkeit dieser Namensverwerfungen.

Richardia Ad., nicht Bartl.

Richardsomia Kth., nicht L., ist = Richamia L.; das falsehe Citat verdunkelt den Sachverhalt, was anch durch das Fehlen von Richardia Kth. II 3 : 136 im Register geschieht.

Rottboellia L. f., nicht L.

Sciadoseris Kunze (G. Kze.), nicht Kuntze (O. Ktze.).

Schistemthe G. Kze., nicht O. Ktze.

Seraphyta Hisch. \& Meyer, nicht bloss Fisch.

Simsia R. Br. = Stivlimgia Endl., die Autoreitate sind vertanscht.

Sphenoderme Jack, nicht Jaequin.

Strobilorhechis Kl., nicht Lk.

Stylisma Raf., nicht Hochst.

Styplelie Sm., nicht Sol.

Suringarin Pierre, nicht L.

Thamatophorns 'Lipp. corr. Walp., nicht Rdlk.

Trilobmm Saporta, nicht Scopoli.

Triodom IOC., nicht Cham. \& Schl.

Tros Haworth, nicht Harvey.

IVormia liottb., nicht Blume.

Zeyher(i), Spr., nicht W.

2. Unzulässige, alleinige Citation vorlinnéischer Autoren. Im Nachtragband, hezw. noch bei der Registration hätten vom Engler'schen 1753-Standpunkt nachstehende Autorcitate durch die von Limné oder späteren, den betref-

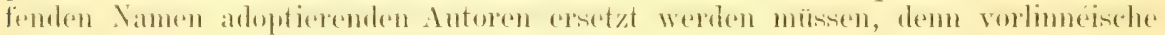
Antoreitate sind sowohl gesetzlich, als nach den Aprilnomenklaturregeln verjäht. Acyntha Commelyn, Alums 'Tourn., Ancthm 'Tourn., Anguria Plumier, Argyrodewlogs Commelyn, Betula 'Tourn., Bryanthus Steller, Butomus 'Tourn., Canam/a Rumpf, Camabis Tonirn., Carpinus 'Tourn., Casuarina Rumpf, Cistus 'Tourt., Codianm limmpt, Corylus Toum., Cynomorinm Micheli, Damasonimm 'Lourn., Diospypos Dalechamps, Galium 'Tourn., Gentiana 'Lourn., Gingko Kaempfer, Guazmma l'lumier, Hysterophome Vaillant, Jacanatia Maregraf, Laratas Pumier, Lencodendron Hermam, Malvariscus Dill., Menispermum Tourn., Mitella 'Toum., Narthecinm Moehring, Oddentandia Plumier, Onagra 'Tourn., Parietaria T'ourn., Partheniastrum Nissole, I'eireshia Plumier, Pentagonocurpus Micheli, Petiveria Plumier, Pinguirula 'Toum., Pins 'Toum., Pisonia 'Tourn., Polygonatum 'Tourn., Pongelion Llieede (das ex Adanson zu gelten hat), Porophyllum Vaillant, Potomogeton 'Tourn., Plevocephalus Vaillant, Qumoclit 'Tourn., Rivinn Plumier, Rondeletia Plumier, Sherordice Dill., Sparganophorus Vaillant, Spermacoce Dill., Tetragonocarpus Commelyn, Timonims Rumpf, Tribulus 'Tourn., Utmaria 'Toum., Vallisneria Micheli, Teratrum 'Tourn., Vitis 'Tourn.

Es sind solche vorlimnéische, (olme Ergänzung des giltigen Autors) alleinstehende Antorcitate mindestens noch dremal soviel bei Synonymen zu finden, von denen Engler sogar - allerdings ohne Datum - Hivnahe Thevet aus dem Tahre 1558 und Therde Piso aus dem Jahre 1648 unter Pradosia aufuahm.

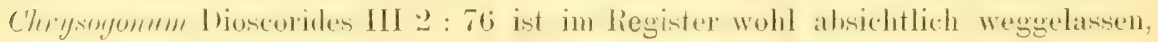


aber die anderen vorlinnéischen Autoren haben elonsowenig lierechtigung selbst nach Englersehen liegeln. Nan muss also folgern: Fintwedre das Korrekturbedürfnis nach den eigenen Regehn ist hei Englex - 0 (glateh Null), orler hotanische (ieschichte = Censul :3. Vom 1753-Standpunlit scheiht man Pirmella L., nicht Brunella, was derart auch unkorrigiert blieb.

3. Verspätete Autorcitate. Z. B. Arisarm Targ.-Toz. 1810 ist schon von Indwig 1737 und Adanson 1763 anfgenommen, eine Verjïlnumg liegt in keiner. Weise vor, aher Engler citierte Targ.-Toz. und korrigierte sich nicht. I)erartige Fehler liegen wenigstens :300 vor, aber sie nach dem 1753-Standjunkt antzuzähleı, Iohnt nicht, da dies für Genera ein unwissenschaftlicher und inbezug auf Namenveränderumgen äusserst schädlicher Standpunkt ist. Nur der Anfang mit 1737. ist wissenschaftlich und zugleich ökonomisch.

4. Verfehlungen gegen $\$ 50$ des Pariser Codex, wonach das Citat eines Autors, welcher zuerst einen Manuskriptnamen eines anderen Autors publizierte, nicht fehlen darf, während in folgenden Fälen nur das keine Publikationsquelle lietende Citat des Manuskriptautor's in Engler's Register steht. Dypsis 'Thouars "Noronha", elenso hei Nisu, Paropsia, Philomerla und Zymmm steht Noronha anstatt Thonars. Duennia und Plutonia Hask, "Noronha". Platymetra Salish. "Noronha". Pliloga BHgp. "Noronha", ex Thouars n. n. Die etwa 100 Gattungsnamen, welche Noronha 1790 publizierte, sind nur Nomina nuda (Nur 4 / fimyia 1785 ist von ihm mit Diagnose publiziert). Der Rest ist aus nicht publiziertem Manuskript und Abhildungen von einigen Autoren von $1806-185$ s recognowiert worklen; auch das nicht ohne Ungleichheiten, z. I3. Scobir Noronha, die in P'feiffer's Nomenklator noch fehlt, wird in Engler's Werk zur Lyllureer Lagerstroemia, im Kew Index mit ? zur Verbenacee Premna gesetzt. Auf alle Fälle ist der Aufkliirung liefernde spätere Autor an 1. S'telle zu citieren und Noronha nicht allein. Noch verworrener ist diese Sache bei Commerson $=$ Commercon, dessen Manuskriptnamen von 1786-1887 von wenigstens 11 verschiedenen Antoren publiziert sind, und bei Solander, resp. Banks d Solander, dessen Manuskriptnamen von $1786-1878$ ron mindestens ! verschiedenen Autoren publiziert sind. Es sind dies: Fermelin Lam. 1786 "Comm.", ebenso Fortidiu, Gustromia, Spronin Lam. "Comm." Antirrhoed Juss. 1789 „Comm.", ebenso Bongainvillea, Cordy-

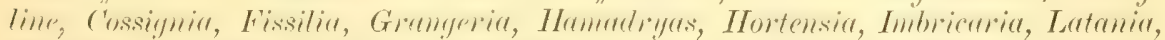

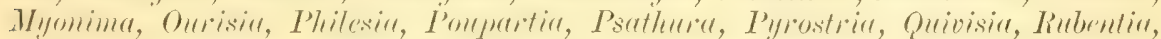
Serissa, Zucca, Juss. „Comm.“ 1789.

\section{Ardinghelia Ad. Juss. 1824 "Comm.", ebenso Tepris.*)}

*) Vepris A. Juss. 1825 "Comm. ${ }^{*}$ wird unrichtig bei Engler III 4 : 178 citiert. als Comm. emend. ${ }^{\star}$ A. Juss; Endlicher und BHqu. schrieben dafür Comm. ex A. Juss. In Engler'schen Register sind dann sogar zweierlei C'itate aus Engler's falschem Citat geworden: Vepris A. Juss. und Vepris Comm.! Ebenso konfus ist die Engler'sche Systematik dieser (iruppe: el trennt abweichend von BHgp. Trewis von Tuddalid durch Stamina doppelt soviel als Petala: Stamina ebensoviel als Petala; das ist schon im Widerspruch mit den Abbildungen l. c. Seite 176, wo Vepris glomerate Fig. Y, die unrichtig als dimer bezeichnet ist, 3 Petala und 4 Stamina hat, wälirend Fig. ' $Z$, die unrichtig als trimer bezeichnet ist, 3 Petala und 5 Stamina zeigt. Eine andere Konfusion findet sich unter angeblicher Vepris lanceolata; es wird 1. c. unter Fig. II eine mach A. Jussien kopierte Blite mit \& gleichlangen Stamina abgelildet: dats bezieht sich aber auf den Jussieu'schen Typus Toddalia paniculata Lam. = Tepris paniculata Engler, während Tepris lanceolate Don = Boscia Thbg. = Asaphes DC. = Vepris Harv. non Jusś. intermediär ist, dafür 4 lange und 4 abortierte kurze Stamina hat, welch letztere von Thunbers sogar ganz ähersehen wurden. Ausserdem schrieb Engler irrig Vepris tanceolata Juss. anstatt $V$. lanceolata Don. Noch eine Flüchtigkeit zeigt Engler 1. e. 178 in dieser Gruppe, indem er Toddalia Juss. mit dem Datum April 1789, C'rantzia Schreb., welchen Namen er willk ürlich verwirft, mit fuli 17x? aitiert: umgekehrt sind diese wichtigen Data richtig.. Das ist ziemlich viel Engler sche Flïchtigkeit, Konfusion und gewaltsame Systematik auf recht wenig Raum.

Otto Kuntze. 
Calraria Gaertn. f. 1805 "Comm." Colophonia, Masqueria und Mariqnia Kth. 182t „Comm." Jussinia DC. 1828 "Comm." Sublimia-Arten "Comm." ex Mart. 1836. Sophisteques, Roelana und Tenelia Endl. 1840 „Comm." Thomina Pl. 1887 "Comm." In allen diesen Faillen ist es fehlerhaft, Comm. allein zu citieren. In anderen Fällen fehlt dagegen Comm. bei Engler, z. B. Lodoicea "Comm.", welche zuerst von Saint-Hilaire 1805, dann auch von Labillardiere 1807 publiziert ward. Ausserdem gebraucht Engler auch Comm. für Commelyn oder verwechselt beide hei Argyrodendros Engler, syn. ex Adanson und bei Tetragonocarpus \$ Rehb.

Ayeleia Pl. „Sol.“; Cratites Miers 1878 ,Sol. “; Macrydinm Forst. 1786 ,Sol.“; Danais Vent. "Sol."; Diplanthera R. Br. "Banks d Sol."; Hepetis Swartz, Sol."; Myopyrum Forst. "Banks d Nolo."; Myzodemlrum Forst. „Banks d Sol."; Nertera Gaertı. „Banks d Sol.“; Philydrum Gaertn. „Banks"; Pimelia Gaertn. „Banks d Sol.“; Pollichia Aiton ,Sol."; Pomax Gaertn. ,Sol."; Thamnea Brong. „Sol."

Aehnlich darf anch bei folgenden Manuskriptautoren deren Citat nicht allein stehen: Agastuche Gronov. ,Clayt."; Butesia BHgp. "Spruce"; Caleanthes R. d S. "Seidel" (nicht Seidl); Monopterix Bth. "Spruce*; Pinillosia DC. "Ossa"; Plectrocarpa Bth. "Gill."; Sympieza R. \& S. "Lichtenst." u. s.w.

5. Komische Autorcitate. Agoseris Raf. \& Greene; Athamantha Linné, Parl. \& Vis,; Cordyloblaste Moritzi, Benth. \& Hk.; Doratoxylon Thouars \& Hk. f.; Hariola Adans. d O. Ktze.; Libanotis Crantz, Grenier of Godron; Pladera Sol. d Roxb.; Pleurandra Labill., Benth. d Hk.; saimala Hamilton, Aschers. d Gürcke; Siebera Rehb., Benth. ¿ Hk.; Tirgilia L'Her. d Smith; Taenidia Torr. \& Gray, Drude.*)

Diese komischen Autorcitate kam man nur in Engler's Register finden; sie sind eine Verquickung von verstorbenen und lebenden Autoren oder von Autoren, die nie gemeinschaftlich etwas publizierten. Diese Citate entstammen der Sorglosigkeit und fehlender einheitlicher Behandlung der Citate von Subgenera, Emendationen, wobei das Zeichen ș oder () oder die Angabe em. wegtiele; ferner sind Fälle dabei, wobei das ex des Textes mit $\mathfrak{d}$ im Register falsch übertragen wurde.

Während das d-Zeichen im Register sehr häufig zu finden ist, fehlt es bei folgenden Citaten, die wesentlich von den Kompositen und Orchideen herstammen. In kleinen Druckereien mögen ja soviel d-Zeichen, das bei Autorcitationen international stets das lateinische und framzösche ,et", das deutsche nund", das englische , and" ersetzen soll, nicht vorhanden sein, aber bei einem Werk, wie Engler's N. Pflanzenfamilien, das in Leipzig gedruckt wird, ist diese Entschuldigung nicht erlaubt, sondern sein Fehlen, resp. das Weglassen von \& nur eine komische Spezialitit der betreffenden Nitarbeiter: R. P. anstatt R. \& P. oder Ruiz \& Pavon bei Amyulna, Gongora, Itumboldtia, Rodriquezia und a. Orchideen; bei den Kompositen Chatunthera, Solicu u. s. w. Bei der Orchidee Sobralia jedoch richtig, ebenso bei Citaten anderer Mitarbeiter. Kuhl., Hass. anstatt Breda, Kuhl \& van Hasselt oder kürzer B. K. ‘ H. hei Cionisacens, Hiproglossum, Polychilos, Psychechitus, Pteroceras, Colontostyles, Sestochilus u. a. Orchideen. Bei Orehipedum ist

*) Anstatt Taenidia § Torr. \& Gray em. Drude. Die Subgenera sind im Englerschen Register ohne nähere Bezeichnung, s. B. das sonst dafür übliche Zeichen §, aufgenommen worden, wie bei Endlicher, BHgp., Baillon und das schliesst gewissermassen, wenigstens in letzteren Werken, die nomenklatorische Konkurrenzfähigkeit der Namen von Genera und Subgenera in sich ein. In Veränderungsfällen zwischen Genera und Subgenera wird aber im Engler'schen Register sehr ungleich citiert, z. B. S'ynsepalum A. DC. muss \$ A. DC. em. Baill. heissen; bei Hesperidopsis DC., O. Ktze muss § DC. em. O. Ktze. gesetzt werden. Tetracerutium [DC.] O. lítze. in N. 176 oder L'ncarina (Baill.) Stapf = Baill., Stapf im Register sind unrichtige Manieren für § DC. em. O. Ktze. bezw. \$ Baill. em. Stapf. Bei Tetrastigma Pl., welche Gruppe andere Autoren zu T'itis stellen, fehlt vorher $\$$ Miq. Das darf nicht fehlen in solchen Fällen, sonst wird die historisch-systematische Entwickelung und Priorität verdunkelt. 
erst im Register das \& hinzugefügt und auch der Name korrigiert worden.*) Da hinter Kuhl ein Abkiirzungspunkt meist gesetzt ward, aber nicht stehen darf und Breda, welcher die Diagnosen besorgte, stets im Citat fehlt, so muss man wohl annehmen, dass der Engler'sche Orchideen-Monograph das Werk dieser 3 Autoren niemals angesehen hat, ähnlich wie er 'Thonars' Orchideen-IVerk grïndlich vernachlissigte. Knowl. \& Weste. wird von Pfitzer anch olme d gebrancht.

P. E. = Poepp. Endl. anstatt P. siten und Orchideen, z. B. Paranephelins, Prionolepis, Comparettia, Itiudenium. Letztere erhielten aber im Register das \&-Zeichen.

T. G. anstatt T. \& G. oder 'Torrey \& Gray nur bei Kompositen, aber häutig, z. B. Apargidium, Brachyacta, Centauridium, Cosmella u. s. w.

H. A. anstatt Hk. \& Arn. bei der Komposite Anisopappus.

Coss. \& D. R. anstatt Cosson \& Durieu oder Coss. \& Dur. bei den Umbelliferen Ammodaucus, Selinopsis.

H. B. Kth., resp. H. B. Kunth bei der Umbellifere Ottoa HBK. (oder H. B. ( K.) macht den Eindruck, als ob H. B. ein abgekürzter Vorname von Kunth sei; ähnlich bei B. \& K. Sch., welche komische Abkürung Prof. K. Schumam 1897 im Nachtrag S. 301-302 anstatt Bureau d K. Schumann oder Bur. d K. Schum. bei Nematopogon, Pachyptera, Hanburyophyton, Pseudopaegma und 24 Arten eingeführt hat. Bur. kann man annehmen, aber B. lässt auf noch einen anderen Schumann schliessen und ist also rätselhaft.**)

Mehr Lapsus-Fehler sind wohl folgende Fälle in Engler'schen Register: Aus Labillardiere wurde bei Mitrosacme "Labille", aus Lagasea bei Microplemr "Lagaica", aus Cavanilles bei Mitraria "Cavan.", aus Banks \& Sol bei Misodendron "Benth. \& Sol.", aus Muell. arg. bei Oligyogynap scheinbar 2 Autoren: "Huell., Arg.", aus Baillon bei Rolliniu muserse "Beull." aus Hallier, der ja nur wenig bei zweifelvollen Comolvulaceen und Cypripedium-Sektionen citiert wird und mit Haller = Hall. verwechselt werden kömte, steht Hall., resp. bei S'̈gmapetalum bloss "Hall"; dieser Name sollte nicht abgekürzt werden. Auf Seite 84 des Engler'schen Registers wird Rumpf $=$ Rumphius mehrmals als Rumph, also irrig ohne Abkürzungspunkt citiert. Cautlea Boyle ist Royle. H. K. = Hk. bei Argyrophyton. Mck. = Neck. bei Amordica

Raphionacme-Apoxanthera Hochst. Solch einen Namen hat Hochstetter nie gegeben; das Wortungetium beruht nur auf einer Flüchtigkeit von Prof. K. Schumann. Raphionacme ist ein Name von Harvey, den Schumann in Raphiacme unzulässiger Weise kürzte, wozu er dam obiges monströse Wort in () setzte und in einer Fussnote die Sache klarstellte, die aber der Registrator nicht verstanden hat. Die Autoren müssen eben zu ihren Arbeiten die Register selbst fertigen (am besten auf kleinen Zetteh von bestimmter Grösse, damit sie alphabetisch zu ordnen sind, und von mehreren Arbeiten verschiedener Antoren in ein einziges Register vereinigt werden können); dann können Fehler und solcher Nonsens nicht leicht registriert, sondern leicht gefunden und korrigiert werden.

*) Im Text steht Orchiped Kuhl Hass., was im Register wahrscheinlich wegen Orchipeda K. Schum. in Orchipedum Kuhl \& Hass. geändert wurde. Damit ist wenigstens das Prinzip von Korrekturen im Register anerkannt, wie das von DC. (cfr. z. B. Nectouxia 1821 Syst. II : 737, 745), Saccardo Syll. fung. X mit Fusnoten, u. A. sehon angewendet wurde. Mustergiltig könnte auch für Engler Linné's fienera plantarum 17:37 sein, worin kurz vor dem Register alle Korrekturen aufgeführt wurden und dann im Register mit einem $\uparrow$ darauf hingewiesen ward.

**) Auch andere Autoren thun in Abkürzungen eigener Citate des Guten oft zu viel und werden dann bei isolierten Citationen für Andere zu Rïtseln. Besonders Anfänger sinnen meist schon bei der ersten Publikation auf möglichste Küirzung ihres Autorcitats. Doch sollten Autorcitate erst stark abgekiirzt werden, nachdem sie vielhundertfach vorkommen. Eine Nützlichkeit und Berechtigung für solche starke Abkürzungen liegt bloss bei bei Autorcitaten vor, die mehr als $1000 \mathrm{Mal}$ vorkommen. 
Etwas komisch ist anch die Ungleichheit mancher Antoreitate in Engler"s Register, \%. B. P. B. (Solenumlin), Beans. (()plismrmus), P. Beauv. (Spermadom), Pal. Beauv. (Omplahlariu), P'al. de Beanv. (S'timularir), [Pal. oder Palis. = Palisot gehrauchte P'feiffer u. a. früher auch dafür allein, cfr. Trirhospermum] gilt für ein und dasselbe Citat, wofür man am besten, da P. B. und Pal. zu Verwechselungen führen, nur Beauv, gebraucht. Ferner z. B. Du P'etit Thouas (1'rr-

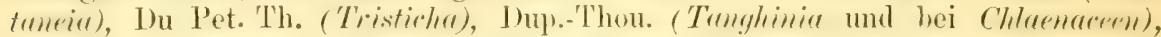

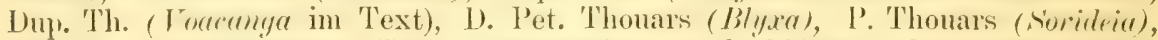
Thouars (Spirospermmm), Thou. (Alismorchis u. a. Orchideen): also s verschiedene Abkürzungen, wofür man am besten nur Thouars verwendet.

\section{Namenändernde und alphabetstörende Schreibfehler.}

Abmuon Engler = Abumon Ad.

Albolboa Engler = Abolboda H. \& B.

Allacanthus Engler = Allaeanthus 'Ihw.

Anthrima K. Schum. = Anthrme Lour. N. 332, im Register nicht korrigiert.

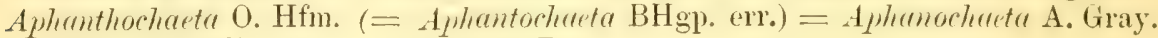
Arthrotaxis Endl. err. = Athrotaxis Don.

Bapusia Engler = Bopusia Presl.

Barreira Engler = Barreria Scop.

Bottinaed rectius Bottionea Colla sind als zwei geltende Gattungsnamen registriert. Brossaca Engler = Brossaea L.

Buckia Engler \& Petersen = Buehia Giseke.

Calyptocarpus Less. (nicht Calyptrocarpus).

Caramotis Engler = Camarotis Ldl.

Careyna Engler = Careyana Pfitzer.

Carpocace Engler = Carpacoce Sond.

Catacoryne Krasser = Catocoryne Hk. f.

Chelusia O. Hfm. = Cheliusia Sch. bip.

Chorisandra $\mathrm{Pax}=$ Chorizandra $\mathrm{R}$. Br.

Chiliocaly $x$ Pax $=$ Chilocaly $x \mathrm{KI}$.

Cinctosandra Walp., Drude = Cinetosandra Kl.

Cloradenia ist zu streichen, weil Lapsus für Chloradenia.

Coleostephus IV 5:389 Lapsus für Coleosanthus.

Cosmespermastrum Engler $=$ Comespermastrum Steetz.

Coptosapelta Korth. = Coptospelta K. Schum.

Coryla Engler $=$ Cordyla Blume.

Criociris Engler = Crociris Schur.

Crucifera Engler ist die Palme Cucifera Del., wofür unrichtig Dill. steht.

Culmeiroa Engler = Colmeiroa Reut.

Cusambium Engler \& Rdlk. = Cussambium Rumpf, Lam.

Cystostemon, nicht Cystistemon.

Dupresmenilia Briq. = Dupremesnitia F. v. M.

Deronetia $0 . \mathrm{Hfm} .=$ Deronetia Boiss. \& Bal.

Desmocelis Krasser = Desmoscelis Naud.

Geyera Engler = Geijera Schott.

Heeppiella Engler = Heppiella Rgl.

Hormium Engler $=$ Horminm L., Bth., Noench.

Hornaida Engler $=$ Homaida Ad.

Hovellia Schoenland $=$ Howellia A, Gray.

Hymantophyllum Engler = Himantophylham Spr.

Hyperstelis $\mathrm{Pax}=$ Hypertelis E. Mey.

Ischnocarpus Engler = Ichnocarpus R. Br.

*) Es handelt sich hier nicht um Auslautverïnderung, denn เov = Veilchen ist ein besonderes Wort griechischen Ursprungs. Im Register unter I, J ist ausserdem $11 \mathrm{Mal}$ der Intellektfehler zu finden, dass dieses Ion mit J steht. 
Isodendron*) Engler = Isodendrion A. Gray.

Lejica Engler = Lepia Hill.

Melissea Wettst. = Mellisia Hk. f.

Mikroschienkia Wettst. = Microschwentia Bth.

Monochetum Engler $=$ Monochaetum Naudin.

Notaphoebe Gris., Pax = Nothaphoebe BI.*)

Nothothlaspi Prantl $=$ Notothlaspi Hk. f.*)

Pallisya Engler = Palissya EndI, non Baill,

Paryella Engler = Parryella Gray.

Pentamera K. Schum. (4 Mal) = Pentamua Bl.

Pentastemon "Mitch. ${ }^{*}=$ Pentstemon Mitch.**)

Perieilema Engler = Pereilema Presl.

Philomela Engler $=$ Plilomeda Noronha.

Pleurispora Engler = Pleuricospora Gray.

Pomereulla Hackel $=$ Pommereulla L.f.

Prionanche Engler = Prionachne Nees.

Psilotrophe Engler \& Hoffm. = Psilostrophe DC.

Quiaio Engler = Quinio Schl.

Raphiacme K. Schum. = Raphionacme Harv.

Reboucha Engler = Reboudia Coss. \& Dur.

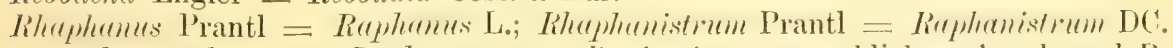

wurde geändert, aber Raphanora'pus, Rophunistrormpus blieben ohne h nach R.

Rhaphispermm Bth. wurde in Raphispermum in Engler's Werk geaindert, umgekehrt aber Raphistemma Wall. in Rhaphistemma.

Rhorlochlamys Schamer (non Bkr.) wurde in Rodorhlamis geandert und steht num vom Backerschen Rhodochlamys sehr entfent.

Ripogonum Forst. wurde in Rhipogonum geändert.

Ropalocarpus Bojer, nicht auch Rhopalocarpus,

Rynchospor'a Vahl, nicht Rhynchospora.

Rynchostylis Bl., nicht Rhynchostylis.

Rigtidophyllum Mart., nicht Rlyytidoplygllum; Rytirlo wird ausser Rlyy-- auch sonst manchmal Rutido, Rhutido und Rhitido geschrieben.****)

Rhodea Endl., Engler = Rohdea Roth.

[Ruelingia R. Br. ist korrigiert aus Rulingia.]

[Rulingia Ehrh, ist verdorben aus Ruelingia.]

Salpingaria Engler = Talpinaria Karst.

Schellhammera Engler = Schelhammera R. Br.

Schiechia Pax = Schickia Meisn.

Schima Engler = Sehima Forsk.

Sclerophyran Engler, Hieron. = Seleropyron Arn.

Scoliopsis Engler = Scoliopus 'Torr.

Serrapylis Engler = Serrastylis Rolfe.

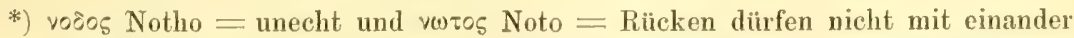
verwechselt werden.

**) Solche Korrekturen mögen berechtigt sein, es darf aber der alphabetisch entfernte, sehr übliche, ursprüngliche Name Pentstemon nicht fehlen.

***) $R a, R e, R i, R o, R u, R u: R y: R i=R h a, R h e, R h i, R h o, R h u, R h y: R h u: R h i$ wird hei den Autoren so verschieden behandelt, dass es eine Qual für Nomenklaturisten ward. Otto Kuntze schlug in $\$ 73$ des Codex emendatus vor, dieses h vor $\mathrm{R}$ in Wörtern griechischen Ursprunges künftig wegzulassen mit einigen Ausmahmen, die ein Kongress feststellen möchte. Da wir gezwungen sind, schon in unserer nächsten Arbeit hierzu Stellung zu nehmen, so werden wir die Ausnahmen nur für die klassischen oder altringebürgerten Namen Rhammus, Rheum, Rhodo-, Rhus, nebst Derivaten, sowie die im Innern eines einfachen Wortes stehenden $r h$, bezw. $r h, z$. B. in Myrrhis, - arrhena bloss heibehalten, die ührigen $/$ nach $/$ griechischen Ursprunges aber einfach und konsequent bei gültigen Namen weglassen. Auch bei cirrus fiel das $h$ im alten Latein schon aus, sodass die Schreibweisen cirrhosus, cirhosus, cirosus zu vermeiden sind. 
Sicyodon Engler $=$ Sicyocodon Feer in Engl. Jahrb. XII : 613 .

Sylvianthus K. Schum. = Silvianthus Hk. f.

Tafalia Engler = Tafalla R. \& P. (besser: Tafallaea).

Tecophyllum Wittm. = Thecophyllum André (fide Mez).

Thynanthus und Tynnanthus K. Schum. = Tynanthus Miers.

Tridemis Engler =- Tridesmis Lour.

Triostema K. Schum. IV 4:82=Triosteum L.

Trochetia Engler =: Troschelia KI.

Tananthes Engler = Vauanthes Haw.

Yaundea Gilg N. $190=$ Jaundea Gilg III 3 : 188.

Da ein besonderer Registrator nicht genannt ist, muss man wohl oder übel den Chefredakteur Engler für diese Fehler im Register verantwortlich machen.*) Bei den meisten Abbildungen, die wirklich vorzïglich und wissenschaftlich vollendet sind, und die Nanche sogar für das Branchbsrste des Werkes halten (Kuntze Rev. gen. IIIII : 232) - wenigstens unter Lmst.unden - ist der fremde Zeichner auch nicht genannt. In beiden Fillen ger ins: Fngler die Erute: Ruhm, resp. - Beileid. Die Zeichnungen von J. Pohl sin: stisserordentlich gut und zuverlässiger als der Engler'sche 'lext.

Es ist nicht ausgeschlossen, dass einige der obigen falschen Schreibweisen nur Druckfehler sind, deren man auch noch bei folgenden korrigierten Namen beim Vergleich finden wird, die dam meist in alphabetischer Ordnung nicht stören:

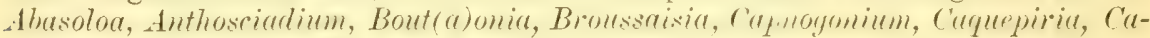
thestrenm, Coleotrype, Comostemum, Cotulimu, Civitishantisia, Dissoluena, Evtrlu,

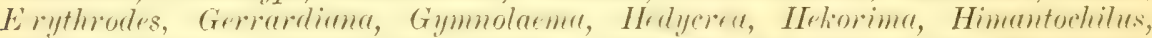
Himantostemma, Hypogynimm, Lenzial, Lettsomia, Macrarharmium, Harimima,

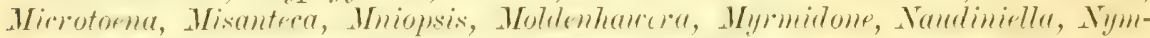

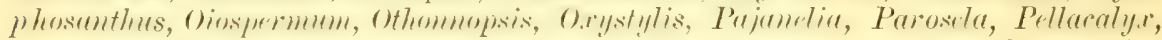
Picralima, Pleuranthesis, Prionanthium, Prionopsis, Quetettia, Ramatuela,

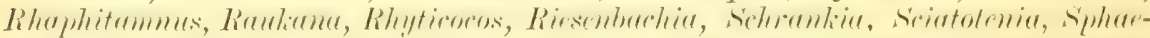

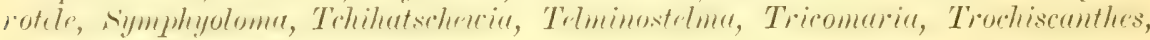
Uralepsis, Venegasia.

\section{Veränderungen der Auslautsilben.}

Achaeta Fourn., nicht - e; Achroanthes Raf, nicht auch - us; Aeranthes Ldl., nicht - us; Adenostemum Pers., nicht auch -on; Agathaea Cass., nicht -ea; Ampelodesma Beauv., nicht - os; Andradea Allem., nicht - aca; Angostyles Bth., nicht - is; Anigozanthos Lab., nicht - us; Anisotoma Fenzl, nicht - $e$; Aphyllocladus Wedd., nicht -os; Argyranthus Neck., nicht - es; Asemmantha Hk. f., nicht -e; Asteranthos Desf,, nicht - us; Asteridea Ldl., nicht - ia; Astertinon Hfg. \& Link, nicht - um; Athalmum Neck., nicht - us; Axanthes Bl., nicht -ea; Baskervilla Ldl., nicht - ea; Benjamina Vell., nicht -ia; Bernieria Baill., nicht - $a$; Bisboeckelera O. Ktze., nicht - ia; Blechrodea Bl., nicht - ia; Bosqueia Baill., nicht -iea; Branciona Sal., nicht - ia; Brewstera Roem., nicht -ia; Bunburia Harv., nicht - ya; Burmeistera Karst., nicht -ia; Byrsanthes Presl, nicht - us; Candidea Ten., nicht - ia; Cardiopteris Wall., nicht - yx; Chamaecistus Oed., nicht-um; Chamaespartium Ad., nicht -tum; Chapelievia Rich., nicht - ra; Christiana DC., nicht - ia; Chuquiraga Juss., nicht - ua;

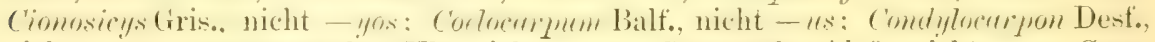
nicht - us; Coprosmanthus K1., nicht - os; Coresantha Alef., nicht - e; Coryanthus Nutt., nicht - es; Craspidospermu Boj., nicht - a; Cremocarpon Boiv.,

*) Zumal er auch noch behauptet, wie er mir schrieb, dass er das Register vorher durchgesehen habe.

'T'om von Post.

Diese leichtfertige Revision seines Registers beweist anch nur Engler's erschreckliche Lnordentlichkeit und Flïchtigkeit, die nur noch von seiner Willkür übertroffen wird, wofiir ich in Rev. gen. ïberreiche Beweise lieferte. Ob dies Eigenschaften füir einen botanischen Srstemverbesserer sind, darf wohl bezweifelt werden. Otto Kuntze. 
nicht - us; Crocosmia P1., nicht - a ; Crossostylis Forst., nicht - es; Cryptantha Lehm., nicht - e; Cyrtochilum HBK., nicht auch - us; Dactylophyllium Schott, nicht - um; Dicuelospermum Clarke, nicht - a; Dicrastylis Drumm., nicht - es; Dictyostega Miers, nicht -ia; Diomycha Naud., nicht - in; Diploryatha N. E. Brown, nicht - us;*) Dracontomelon Bl., nicht - um: IMryopwalon A. Gray, nicht - um; Emmenosperma F. v. M., nicht - um; Encholivium Mart., nicht - on; Epilasia Bge., nicht - is; Flüchigera O. Ktze., nicht - ia; Gamelythrum Nees, nicht - tron; Ginoria Jacq., nicht - ra; Glossopetalon A. Gray, nicht - um; Gonyanthes Bl., nicht - e; Gymmophyton Clos, nicht - um; Hermatoxylum L. 1753, nicht - on; Haenselera Boiss., nicht - ia; Haplophyton DU., nicht - um; Heslonu Lour., nicht - $\rho$; Hemistylus Bth., nicht - is; Heterodendrum Desf., nicht - on; Heterosperma Cav. = -um W.; Hicorius Raf., nicht - as; Hieronima All., nicht - ia; Hippophae L., nicht - es; Hololachna Ehrb., nicht - e; Homonoia Lour., nicht - oya; Hydrocleys Rich., nicht - eis; Hymenocarpos Savi, nicht - us; Julostylis Thw. nicht - es; Lathriogyna E. \& Z., nicht -e; Lecostomon (rectius - stemon DC., prod. II Register), nicht - ion; Lefeburea Rich., nicht - ia; Leptonema .Juss., nicht - ea; Leptorhynchos Less., nicht - us; Leucocarpum Rich., nicht - on; Limatorlis Bl., nicht - es; Lophopterys: Juss., nicht - is; Vreceaya R. Br., nicht - rya; Marrochordion De Vriese, nicht - ium; Mitracarpum Zuce., nicht - us; Musineon Raf., nicht - enm; Nanouluytom Less., nicht - nm; Ochrorarpos Thouars, nicht - us; Octomeria Don, nicht — is ; Oliganthes Cass., nicht - os; Omphalophlhalmum Karst., nicht - us; Onrostemum Juss., nicht - on; Oporanthus Herb., nicht-es; Oreobambos K. Schum., nicht - us; Oroxylon Vent., nicht - um; Osyridicarpos DC., nicht - us; Oubamguia Baill., nicht - gia; Oxydemdrum DC., nicht - on; Pacouria Aull., nicht - ea; Parlatorea Rodr., nicht - ia; Petalostylis R. Br., nicht - es; Petrea L., nicht - aea; Phaeoptilum Radlk., nicht -on; Pierrea Hance, nicht-a; Pigafettia Bece. ,Bl.", nicht - a ; Podopterus Hb.d Bpl., nicht - is ; Poitea Vent., nicht - aea; Prionotes R. Br., nicht - us; Prossephaleium Korth., nicht - lium: Fteleocarpa Oliver, nicht - us; Pterygosturhyum Nees, nicht - imm; Qualea Aubl., nicht -ia; Quapoya Aubl., nicht -ia; Ragala Pierre, nicht - e; Renarda Rgl., nicht -ia; Rhaphiostylis Pl., nicht - os; Rhazya Dene., nicht - ia; Rhodotypos S. \& Z., nicht - "rs; Ricimocarpos Desf., nicht - us; Roptrostemon BI, "Ropho", nicht - um; Schauera Nees, Ldl., nicht - ia; śrheelea Karst., nicht - ia; Schizopetalon Sims, nicht - um; Schoenobiblus Mart., nicht - os; Schoenorhm Lab. nicht -on; Schoenoprasum Don, nicht - ium; Schweiggera Mey., nicht -ia; Scoria Raf., nicht - ias; Serenoa Hk.f., nicht - ara; Seringia Gay, nicht -ea; Sestochylos Kuhl \& Hass., nicht -us; siphonanthus L., nicht auch —a; Speirantha Bkr., nicht - e; Sphenodesme Jack, nicht - a; Sporadanthus F. v. M., nicht - a; Stylocoryna Cav., nicht - e; Tetractomia Hk., nicht - ma; Tinaea Garcia, nicht Tinea; Toxanthes Turez., nicht - us; Trigymaen Schl., nicht - ria: Trimea Salisb., nicht - in; Tristegis Nees, nicht - ia; Trophinnthus ścheidw. nicht -es; Tuberostylis Steetz, nicht - es; Tella L., nicht Vellea; Zeyheria Mart., nicht $-r a$; Zosima Hoffm., nicht Zozimia.

Nach dem Pariser Codex und Codex emendatus gelten solche Verinderungen als orthographische Licenz $=$ differente Sichreibweise (erlaubte Korrekturen), bedingen also keine andere Autorcitation. Da aber Engler gegen den vorherrschenden Gebranch derartige Differenzen für zweierlei Namen gelten lässt, so muss man ihn nach semen Prinzipien als Autor zu den in seinem Register derart veränderten Namen citieren. Damn hätte jedoch Engler z. B. auch Frlücligerin Rushy neben Flärhigera (). Ktze, Ermochlor Buese nehen Errmochlor Wats. etc., gelten lassen und damn häte er diese geänderten Schreibweisen in den Auslaut. silben, die seine Mitarbeiter angewendet hatten, konsequenterweise nach seinen Regeln im Register korrigieren müssen.

*) K. Schumann hat gegen die Aprilnomenklatur-Regeln den Namen absichtlich verändert, weil im Griechischen diese Endung nicht vorkommt; aber bei lateinischer Namengebung von Pflanzen darf Jeder das halten, wie er will. 
Trem Eugler aber trotzdem, wie vorstehende Beispiele zeigen, solche Korrekturen als erlaubt hält und sein Prinzip nicht durchführt, so darf er auch nicht die gesetzlich zuliissige orthographische Licenz, die überhaupt von Otto Kuntze nur und zwar ökonomisch geregelt wurde, verwerfen. Dann sind aher noch einc Anzahl Genera anders zu benennen, z. B. Salpingu DC. 1828 und Salpingia Reim. 18:3:3 können nicht neben cinander bestehen, letzteres wird Cralpriniar Britton, ein Name, der in Engler's Werk tehlt. Esmopolda und Lismaruldid gelten hei Engler für 2 verschiedene Genera.") Von Molinuen "Comm." Juss. 1789 und Molinie Schrank 1788, beide nach Wolina benamnt, ersterer Name in korrekter Form, kann selbst nach der 9. Engler'schen AprihomenklaturRegel nur einer gelten, so dass Molinaea Juss. zu Gelonim Gaertn. wird. Es ist das em ïhnlicher Fall wie Hopree und Hopren, die Engler selbst nicht neben einander bestehen lässt, efi. dessen Registernachtrag, Seite 461. Doch solche und andere Korrekturen, sowie weitere Erginzmgen fehlender Nomenklatur in Engler's Werk, deren ich am S'chluss noch einige andente, sollen in einer späteren Arbeit, mit der ich jetzt schon beschäftigt bin, gegeben werden.

In diesem 5. Abschnitt sind übrigens relativ wenig Widersprüche zwischen Text und Register; die meisten Auslautverimderungen sind schon in 'Text vorhanden und zumteil von Engler's Mitabeitern veranlasst, zunteil ans neneren Arbeiten nachgeschrieben worder. Neme die sogenamenten Monographen von Engler's Werk wirkliche Quellenstudien gemaldht hitten, wimblen sie die richtige Schreibweise dieser Namen gefunden hahen. Diese 15: oft spaiteren Autoren nachgeschriehenen Namen-Vexanderungen beweisen unwillkürlich, dass meist nu kompiliert wurde.

(Fortsetzung folgt.)

\section{Botanische Litteratur, Zeitschriften ete.}

Wildeman, E. de et Durand, Th., Plantale Thonnerianac Congrolenses ou ćnumciration des plantes récoltées en 1896 par M. Fr. Thonner dans le district des Bangalas. Avec une introduction de M. Fr. Thonner 23 plantes et une carte. Bruxelles 1900. Société Belge de Librairie Oscar Schepens \& Cie, editeurs, 16 rue Treurenberg. $\mathrm{XX}$ u. 49 pp. Pr. 8 Fres.

Das vorzüglich aungestattete Terk enthalt die wisscnschaftliche Bearbeitung der botanischen Ausheute einer Forschungsreise, welche Monsieur Fr. Thonner 1896 an den oberen Congo und in das Bassin von Mongala unternahm. Verfasser schildert eingangs des Werkes in recht anregender Weise den Terlauf seiner Reise. Suite $1-49$ wird die ganze botanische Ansbeute in systematischer. Reihenfolge aufgeführt. Darunter sind 50 Pflanzen für den Congo und unter diesen 23 Arten und 4 Varietäten überhaupt neu. Auf den 23 Tafeln sind die nenen Arten mit allen nötigen Detailzeichungen ganz vorzüglich zur Darstellung gebracht. Die neuen von Wildeman und Durand beschriebenen Pflanzen sind: Dioscorea Thomeri, Urera Thomneri, Monodora Thomeri, Salacia Congolensis, Impatiens Thomeri, Scaphopetahm Thomeri, Onratea laxiflora, Dicranolepis Thomeri, Tabernaemontane Thomeri, Solanum symphyostemon, Harveya Thonneri, Sesamm Mombanzense, S. Thomeri, Thunbergia Thonneri, Asteracantha Lindaviana, Bertiera Thonneri, Geophila renaris, Uragoga Thonneriana, Panicum brizanthum Hochst. v, polystachyum, I'. diagonale Nees $v$. hirsutum, Phytolacea Abyssinica Hoff. v. macrophypla, Mussaenda stenocarpa Hiern v. latifolia. Von anderen Spezialisten wurden beschrieben und neu aufgestellt: Gruyonia intermedia Comianx, Dinophora Thomeri Cogniaux, Loranthes Thomeri Engler, Listrostachys Thomeriana Kracnalin, Pycnocoma Thomeri Pax.

A. K.

*) Weitere solche Fälle in Kuntze Rev. gen. III 2, S, 130-131 der Einleitung. 
Schinz, Dr. Hans und Keller, Dr. Robert, Flora der Schweiz. Verl. v. Albert Raustein in Zürich 1900. $628 \mathrm{~S}$. Preis $6 \mathrm{MI}$.

Am Eingang der Vorrede wird in erster Linie als Zweck des vorliegenden Buches angegehen, ,den Schülern der mittleren und höhcre!n Lechanstalten die Kenntnis der schweizerischen Gefïsspflanzen zu vermitteh." Wrem wir diese neue Schweizer Flora nur als Schulbuch besprechen wollen, so müissen wir bekennen, dass die beiden Verfasser ein recht brauchbares Werk geliefert haben, welches auch durch die in text zerstrenten Abhildungen den sichiolern zweckdienlichen Anfischluss gieht. Die Anordnumg der l'flanzen erfolgte nath dem naturrlichen system von Engler"s und P'rantl's "Natürlichen l'flanzenfamilien". Den einzelnen Eamilien sind schlüssel vorangestellt, durch wellehe der Vame der Genera gefunden wird, wïhrend Difterenzialdiagnosen das Anftinden der Arten ermöglichen. Die Diagnosen der Arten sind ziemlich ausführlich gehalten und die synonyme und Auturennamen weggelassen: hingegen wurle am sichlusise ein "Verzeichnis der in Gremli's Exkursionsflora der Sichweiz (8. Autlage) vorkonmenden Synonyme" beigegehen, nebst einem liegister der lateinischen und einem Register der dentschen l'flanzemumen. (1) die Weglassung der Antomamen im 'Texte, die allerdings im Register der lateinischen l'flanzennamen beigefïgt sind, sich allgemein einbürgern wird, ist sehr zweifelhaft. Bei einer einheitlichen Nomenklatur kömte man allerdings die Autornamon als einen muötigen ballatst cutbehen; so lange uns aher eme solche fehlt, kamm man auf die dutornamen nicht wohl verzichten. Dem Zweck des Buches gemäss wurde unter den vielen Varietiiten und Formen eine Auswahl getroffen. und ebenso wurlen die Bastarde an Schlusse fler Genera mur namentlich angefühnt. Freilich hätten die zahlreichen Floristen und Fremele der Sichweizer Flora es sehr begruisst, wenn die beiden mit der Schweizer Pflanzenwelt so vorzüglich vertranten Verfisser die Varietäten,

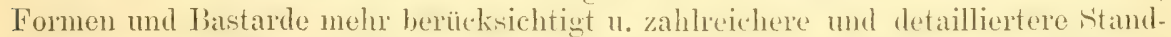
orte angegehen hitten. Sollten sich die beiden Herren entschliessen, neben diesem recht guten schulhuche auch eine ausführliche, alle hekannten P'lanzen, Formen und Bastarde der Śchweiz herieksichtigende Flora auszuarbeiten, so würden sie sich den Dank aller Floristen erwerben.

A. K.

Bubani, P., Flora I'yrenaea per ordines naturales gradatim digesta. Opus posthumum editum curante O. Penzig, in Athenaeo Genuensi Botanices Professore. Verl. v. Ulrico Hoepli, Milano. 1900. 2. Bd. 618 S. Preis $20 \mathrm{~L}$.

Wie schon p.130 des Jahrgangs 1898 dieser Zeitschrift hervorgehoben wurde, ist das Bubani'sche Wrek ein wahres Kleinod für den Floristen, der die Vegetation der Pyrenäen studieren will. Ungemein gross ist die Zahl der Standortsangaben, aus denen sich der Botaniker zum Zwecke einer Reise in die Pyrenäten die nötigen Notizen mathen kimn. Die genanen mol allsührlichen I)iagnosen

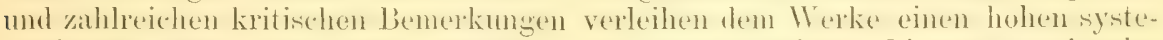
matischen Wert, und ebenso wertvoll sind die zahlreichen Litteraturnachweise des gewissenhaften und geleheten Verfiksters, der in der Anwendung des l'riorititsprinzips freilich zu weit geht und atuch dic Autoren vor Limme zur liceltung lningt.

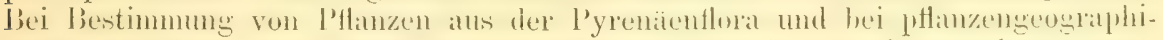

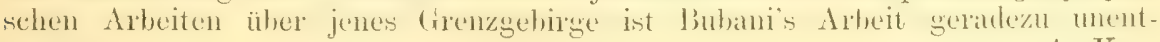
behrlich.

A. K.

Winkler, W., Sudetenfloral. Eine Anzahl charakteristischer riehirguntluzen. Mit 52 Tafeln. Verl. v. C. Heinrich in Dresden N. 1900. 190 Seiten. P'reis 10 M.

Ein prächtiges Buch, das so recht geeignet ist, allen Naturfremden, be-

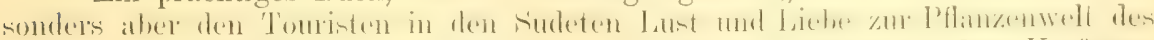
Gebirges zu erwecken. An Eingang des Buches bringt Dr. Bater dem Verfisser der Sudetenflora eine poetische Widmung, dann folgt: I. Vorwort als Einleitung 1. 5-11, II. Aus d. Leben d. Pflanze p. $12-14$, III. P'flanzensysteme 1. $15-25$, IV. Eine botanische Wanderung vom Thal bis auf das Hochgebirge p. 26-32,

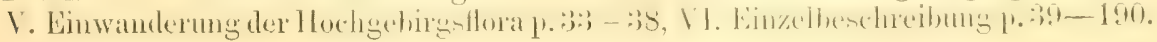


In frischer und lebendiger Sprache schildert der Herausgeber nur 103 besonders schöne und auffallende Pflanzenarten des genannten Gebietes, die auf 52 Farbentafeln abgebildet werden und berücksichtigt in seinen Beschreibungen ausser der Diagnose alles, was sich über die betr. PHlanze sagen lässt: Biologie, Pflanzengeographie, Sagen und Aberglauben, welche sich daran knüpfen, einschlägige Poesie etc. etc. Die Gewächse werden gewissermassen als vernünftige Lebewesen betrachtet und in bilderreicher Sprache vor Augen geführt. Man sieht sich beim Lesen dieser herrlichen Schilderungen in das Halbdunkel des Hochwaldes versetzt, hört das geheimnisvolle Rauschen der Wipfel, fühlt den kühlen Hauch, der über die alpinen Höhen des Gebirgszuges weht und klettert im Geiste dem Verfasser nach, wemn er an fast unzugänglichen Stellen die seltene, hochnordische saxifraga matis sich herabholt. Man merkt es ihm an, dass er seine heimatlichen Höhen schon unzählige Mal mit lieben Freunden durchwanderte, dass er jedes Plätzchen genau kennt, wo seine Lieblinge wohnen; denn wer in solcher Weise schreibt, muss auch alles selbst erlebt und gesehen haben. Allen Touristen und Gebirgswanderern sei das schöne und populäre Werk bestens empfohlen; aber auch für den Floristen wird es schon seiner poetischen Sprache halber eine angenehme Lektüre sein.

A. K.

Ascherson, P. u. Graebner, P., Synopsis der mitteleuropäischen Flora. 10. u. 11 Lief. Verl. v. W. Engelmann in Leipzig. 1900. 8". II. Bd. p. 305-464. Preis jeder Lief. 2 M

Jie beiden Lieferungen enthalten in vorzüglicher Bearbeitung einen weiteren Teil der Gramineen und zwar folgende Genera: Inuthonia, Gandinia, Echinaria, Sissteria, Cortaderia, Ampelodesmos, Arundo, Donar, Molinia, Diplachne, Melica, Korleria, Arellinia, Eragrostis, schismus, Dactylis, Aelwopms, scherochloa, Poa, Briza, Catabrosa, Glyceria, Festuca.

A. K.

Wiesbaur, J. B., Unsere Misteln und ihre Nährpflanzen. Duppau in Böhmen 1899. Selbstverlag des Verfassers. Eine Tafel mit 9 Figuren. $25 \mathrm{~S}$.

Der in der botanischen Litteratur rühmlichst bekamte Verfasser legt in dieser Schrift u. a. die Ergebnisse seiner Untersuchungen darüber nieder, wo und auf welchen Baumen Misteln wachsen. Obgleich an 50 Baumarten Misteln bekannt sein sollen, hat Wiesbaur trotz eifrigster 16 jährigen Beobachtungen in Böhmen nur an 5 Baumarten Misteln gesehen. Die Frage, giebt es verschiedene Mistelarten?* beantwortet er aufgrumd seiner Untersuchungen damit, dass er neben Tiscum album L. noch die auf Pinns Austriaca Hiss wachsende Vistel als Tiscum Alustriucum Wiesh. aufstellt und diagnostiziert, ausserdem werden noch einige Formen unterschieden. Im Uebrigen sei auf die interessante Arbeit selbst verwiesen.

A. K.

Wünsche, Dr. 0tto, Die verbreitetsten Pflanzen Deutschlands. Ein Uebungsbuch für den wissenschaftlichen Unterricht. Verl. v. B. G. 'Teubner in Leipzig und Berlin. 1900. $282 \mathrm{~S}$. Preis $2 \mathrm{M}$.

Das vorliegende Büchlein ist vor allem als Schulbuch zum Bestimmen der in Deutschland am häufigsten vorkommenden fewächse zu benützen und wird wohl auch von den vorgeschritteneren Schülern mit Erfolg auf Exkursionen gehraucht werden können. Die Anordnung der Pflanzen erfolgte nach dem syllabus der Vorlesmgen üher spezielle mol medizinisch-pharmacentische Botanik von Adolf Engler 1892. Mls Anhang ist beigegeben eine "Uehersicht einiger nach den Blüten nur schwierig zu hestimmender P'flanzen" und eine Tabelle zum Bestimmen der Holzgewächse nach dem Laube.

A. K.

Ruschhaupt, Dr. G., Bau und Leben der Pflanzen. Kurzer Leitfaden zur Einführung in die Anatomie, Physiol gie und Biologie der Pflanzen. Verl. v. F. Richters Buchharidlung in Helmstedt. 2. Aufl. 1900. $51 \mathrm{~S}$. Preis $1.60 \mathrm{M}$.

Jer vorliegende Leitfalen eignet sich gut beim Unterricht in Mittelschulen, Feminarien, Landwirtschaftsschulen etc. 24 Abbildungen im Texte dienen zur 
Erklärung. Der Verfasser legt nicht mit Lnrecht Wert darauf, das Büchlein mit Papier durchschiessen und die den Text erlüternden Figuren nachzeichnen zı lassen. Das Werkchen kanı für obengenamute Anstalten gut empfohlen werden.

Oswald Weigel's botanischer Lagerkatalog. Neue Folge. Nr. 95, 96 u. 97. Vorliegender Katalog ist bei der Firma Oswald Weigel in Leipzig, Königstrasse: I erschienen und enthält 1. a. die hinterkssenen Bibliotheken der verstorbenen Herren O. Böckeler in Varel, Dr.J.Lange in Kopenhagen und 1)r. K. B.J. Eorssell in Karlstadt in Schweden.

0esterreichische bot. Zeitschrift 1900. Nr. 6. Wettsteill, R. v., Die nordamerikanischen Arten der Gattung Gentiana; Sekt. Endotricha. - Frey n, .J., Nachträge zur Flora von Istrien. - Linsbauer, L. u. K., Einige teratologische Befunde an Lonicera Tatarica. - Vierhapper, F., Arnica doronicum Jacq. und ihre näheren Verwandten. - Horak, B., Zweiter Beitrag zur Flora Montenegro's. - Podpera, J., Beitrag zur Flora von Böhmen. - LitteraturUebersicht. - Nr. 7. Celakovsky, L.J., Die Vermehrung der Sporangien von Gingko biloba. - Ott, L., Beiträge zur Härte vegetabilischer Zellmembranen. - Degen, A. v., Bemerkungon ïber einige orientalische Pflanzenarten. Velenovsky, J., Eine interessante Missbilulung in den Blüten des Ramunculus acris. - Schlechter, R., Acriopsis Reinw. und ihre Stellung zu den Podochilinae. - Palla, E., Die Unterschiedsmerkmale zwischen Anemone trifolia u. a. nemorosa. - Freyn, J., Nachträge zur Flora v. Istrien. - V ierhapper, F., Wie in vor. Nr. - Lehm ann, G., Verzeichnis von Hutpilzen aus der Umgebung von Liebwerda und Friedland.

Deutsehe bot. Monatsehrift 1900. Nr. 5 u. 6. Murr, Dr. J., Zur Kienntnis der Kulturgehölze Südtirols, besonders 'Trients. - Hasse, W., Bestimmungstabellen für die Rosen von Meisen u. Ungegend. - sthmidt, H., Nene Funde aus dem schlesischen Vorgebirge. - Becker, IV., Bemerkmugen zu den Violae exsiccatae. - Zsch acke, H., Beiträge zur Flora Anhaltina. VIII. - Lei mbach, Dr. (i., Die Volksnamen unserer heimischen Orchilcen. V. - Usteri, Beiträge zur Monographie der Gattung Berberis. - Suksdorf, Washingtonische Pflanzen. - Traunste iner, Dr. J., Carduus personata $>$ platylepis Saut.

Bot. Centralblatt 1900. Nr. 22. Hansgirg, Dr. Anton, Zur Pyllobiologie der Gattung Ficus L., Coffea L. md Kibara Endl. -- Neger, F. IV., Weiteres über Phyllactinia. - Nr. 22/23. Bokorny, Dr. 'Th., Einiges über die Proteinstoffe der Samen. - Nr. 25. Enthält nur Referate.

Berichte der deutsehen bot. Gesellsehaft 1900. Heft 5. Butkeritsch, IVl. Ueher das Vorkommen proteolytscher Enzyme in gekeimten timen und ihre Wirkung (vorläutige Nitteilung). - Nestler, A., Die hatreizende Wirkung der Primula obconica Hance und Primula sinensis Lindl. (mit 2 Tafeln). Schütt, F., Zur Porenfrage bei Diatomeen. - Stein brinck, C., Zur' I'erminologie der Volumanderungen pflanzlicher Gewehe und organischer fubstamzen bei wechselndem Flüssigkeitsgehalt. - Naw aschin, S., Ueber die leefruchtungsvorgünge bei einigen Dikotyledonen (vorläutige Nitteilung) (mit 1 'Tatel).

Zeitschrift d. bot. Abteilung des naturwissenseh. Vereins d. Prov. Posen. VII. Jahrgang. I. Heft. 1900. Niller, Zur Flolat dex I'mgenend von 'Tirsehtiegel im Kreise Heseritz. - Hempel u. Holztuss, Kur Flora des liegierungsbezirks Bromberg. - Szulczewski, Posener l'flanzensagen und P'flamzenaberglauben. - Śpribille, Linige Aufzeichnungen aus dem Š̈uden der L'rovinz. 
Missouri Botanical Garden. Eleventh annual report. 1900. Schenk, If ermann D., A dinease of 'Taxodium distichum known as peckiness, also a similar disease of Libocedrus decurrens known as pin-rot. - Rose, J. N., Agave expatriata and other Agaves flowering in the Washington Botanical Crarden in 1898. - Norton, J. B. J., A revision of the American species of Euphorbia of the section 'Tithymalus occurring north of Mexico. - Smith, Jared G., Revision of the sprecies of Lophotocarpus of the Lnited states; and description of a new species of Sagittaria.

Bulletin de l'assoeiation Française de botanique 1900. Nr.31. ()f ne r,J., Sutes sur la Flore printamiere de loisans. - Beleze. Mlle. Mte., Liste de quelyues Mousses et Hépatiques de la forit de Ramboullet (seme-et -Oise). Bla nchald, 'Th., Liste de noms patuis de plantes aux environs de Maillezais (Tendee). - sudre, H.. Excursions hatologiques dans les Pyrénées. - Olivier, l'ahbe, Exprosé systematigue et description des Lichens de l'Onest et du Tord. Ouest de la France.

Verhandlungen der k. k. zoologisch-bot. Gesellschaft in Wien. 1900. Heft 5. Matouschek, L'rof. F'ranz, Bryologisch floristische Mitteilungen aus Oesterreich-Ungarn, der Schweiz und Bayern.

Bulletin de l'académie internationale de géographie botanique. 1900. Nr. 128. Reinier, Alf., Téritable nom de la plus remarquable Fougère provençale. - Ca p oduro, Mar., De la partition des Fougères. - Monguill q n, E., Catalogue des Lichens du département de la Sarthe. - Guignon, l'abbé, Correspondance: A propos du Gui.

Eingegangene Drueksehriften. Fonck. Leop. S. J., Streifzïge durch die bibl. Flora. Herdersche Verlagsbuchhandlung in Freiburg i. B. 1900. - Halácsy, Ed. v., Conspectus Florae Graecae. Vol. I. Fase. I. Verl. v. Wilh. Engelmann in Leipzig 1900. - Bericht der bayer, bot. Gesellschaft 1900. Bd. VII, 1. Abteilg. - Jaap, O t to , Beiträge zur Noustlora der Lmeegend v. Hamburg (sep. aus den Verhandl. des naturw. Ver. in Hamburg 1899, 3. Folge VII). - Lindberg, Harald, Bidrag till kiannedumen om de till sphagnum cuspidatum-gruppen (Sep. aus Acta societ. pro fauna et flora Fennica. "1899. T. XVIII. Nr. 3), - Derselbe, Om Pohlia pulchella (Hedw.), l'. carnea L. weh năgra med dem sammanblandade former sep. aus Acta societ, pro fauna et Hora Fennica, T. XVI. Nr. 2, 1899). - W W es baur, J. B., Unsere Misteln und ihre Nährpflanzen. Duppau in Böhmen. 1899. Selbstverlag d. Verfassers. - Grecescu, Prof. Ur., Plantele indigene din Romaniea. Bucuresti 1900. - Dersel b e, Plantes de la Macédonie appartenant au vilayet de Monastir. Bucarest 1899. - Derselbe, Plantele indigene din Româniea. Bucuresti 1899. - Alberg, Albert, Frost Flowers on the Windows. Fraternal Printing Co. Chicago 1899. - Mïller, F., Otto Böckeler, Nachruf (Sep. aus d. ,Bericht d. deutsch. bot Ges.“ 1899. Bd. XVII. Generalversammlungsh. (I). - Kuntze, Dr. Otto, Nomenklaturanfang u. Reform internationaler Kongresse (Sep. aus d. Weutsch. bot. Monatschr. 1900). - Pax, Dr. Fer inand, Prantl's Lehrbuch der Botanik. Verl. v. Wilh. Engelmann in Leipzig. 11. Aufl. 1900. - Grecescu, Ir., Conspectul Florei Romaniei. Tipografiea Ureptatea in Bukarest. 1898. -

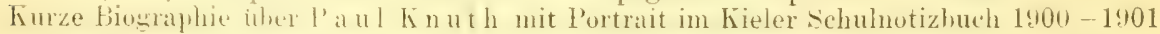
p. 36-37. - Gerassim off, J. J., Ueber die Lage und die Funktion des Zellkerns. Moskau 1900. - Müller, Karl, Zusammenstellung der Lebermoose aus d. Reichslande Elsass-Lothringen (Sep. aus d. "Bot. Centralblatt". Bd. LXXXI. 1899). - Derselbe,

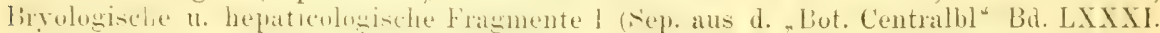
1900. Nr. 6). -- Fritsch, Dr. K., Schulflora für die österreichischen Sudeten u. Alpenländer (mit Ausschluss des Küstenlandes). Verl. von Gerold's Sohn in Wien. 1900. Keller, Lou is, Zweiter Beitrag z. Flora v. Kärnten (Sep. aus d. VerhandI. d. k. k. zool.

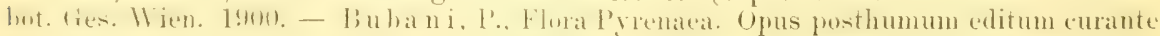
O. Penzig. Verl. v. Ulrich Hoepli in Mailand. 2. Band. 1900.-- Buchenau, Dr. Fr.,

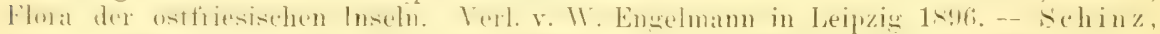
Dr. H. u. Keller. Dr. R., Flora der Schweiz. Vell. v. Alb. Raustein in Zürich 1900.S'ehube, 'Ih., Ergebnisse der Durchforschung der schlesischen Phanerogamen- und (iefiisskryptogamen-Flora im J. 1899 (Separatabdruck). - Derselbe, Ergebnisse der phanologischen Beobachtungen in Schlesien im J.1899 (Separatabdruck). - Ascher- 
son, P. u. Graebner, P., Synopsis der mitteleuropaiischen Flora. Verl. v. W. Engelmann in Leipzig. 10. u. 11. Lief. 1900, - Wirtgen, F., Beiträge zur Flora der Rheinprovinz (Sep. aus d. „Verhandl. d. naturw. Ver. der preuss. Rheinlande, Westfalen 11 . des Regierungshezirks Osnabrück") 1899. - Focke. W. (). Treber die Keimptlanzen der Stein- und Kernobstgewächse (Sep. aus, Ahhandl. d. Nat. Ver. liremen. 1!g00). Bad. XVI. Heft 3. - K ik kenthal, G., Species generis Uncinia Pers. in America meridionali extratropica sponte nascentes (Sep. aus "Bot. Centralhlatt" Nr. 17/19.1900). - Freyn,

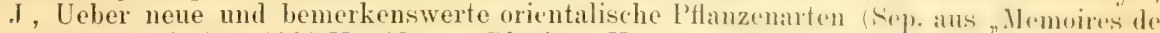
l'Herbier Boissier. 1900 Nr. 13). - Christ, Herm., Les Fongeres des Alpes maritimes. Aus Émile Burnat, „Materiaux pour servir a l'histoire de la flore des alpes maritimes. Verl. v. Georg \& Co. in Genf, Basel u. Lyon. 1900. - Schin z, Dr. H. u. Kell er, I r. R., Flora der Schweiz. Verl. v. A. Raustein in Zürich 1990. - W iinsche, I'r. Otto, Die verbreitetsten Pflanzen Deutschlands, Verl. v. B. (\%. 'Teubner in Leipzig 11. Perlin 1!n(k).

De Wildeman u. Durand, 'Th., Plantae Thonnerianne Congolenses. Verl. v. Oskar Schepens \& Cie. in Brüssel 1900. - Winkler, W., Sudetenflora. Verl. v. C. Heinricl, in Dresden 1900. - Ruschliapt, Ir. G., Bau und Leben der Pflanzen. Verl. v. F. Richters Buchhandlung in Helmstedt 1900.

J a hresbericht des preuss. bot. Ver. 1898/1900. - Separatabdruck aus Zeitschrift für Gewässerkunde 1900, Heft 1. - O esterre i c h. bot. Zeitschr. 1900 , Nr: $2-7$.

Deutsche bot. Monatschrift 1900, Nr. 3-6. - Botanical Gazette XXIX. 1900, Nr. 3-5. - Verhandlung. d. k. k. zool. bot. Gesellsch. 1900, Nr. 2-5. - L a Nuora Notarisia 1900, p. 49-96. - Bolletino del R. orto botanico di Parlermo 1899, Fase. I-IV. - Bulletin de l'académie internationale de geogr. bot. 1900, Nr. 125-128. - Bull et in de l'association Française de botanique 1898, Nr. 1-6 u. 1900, Nr. 28 - 31. Le monde des plantes 1900, Nr. 6. - Berichte der deutschen bot. Gesellsch. 1899. Generalversamml.-Heft II, Registerheft und 1900 Heft 2 - 5. - Berichte der bayer. bot. Gesellschaft. Bd. VII. 1. Abt. 1900. - Z eits chrift d. bot. Abt. d. naturw. Ver. der Prov. Posen VI. Jahrg. 3. Heft. 1900, VII. Jahrg. 1. Heft 2. Juli 1900. - Mitteil ungen des bad. bot. Vereins. Nr. 171 und 172. - Botaniska Notiser 1900, Nr.2-3. - B ota nical Magazine 1900. Nr. 155-159. - III. D e l e c t us plantarum exsiccatarum quas anno 1900 permut. off. hortus bot. universitatis Jurjevensis. - Z e i ts e h r i f t für angewandte Mikroskopie. V. Bd. Heft 9 u. 12; VI. Bd. Heft 1. - 'I' he Botan ical Ex ch ange Club of the British Isles. Rep. for 1898. - Mitteilungen d. bayer. bot. Gesellsch. 1900. Nr. 15. - L e Botaniste. 7. Serie. 26. Mat 1900. - Botanischer Lagerkatalog Nr.95 u. 96 Oswald Weigel in Leipzig 1900. - Missouri botanical Garden Eleventh annual report.

\section{Botanische Anstalten, Vereine, Tauschvereine, Exsiccatenwerke, Reisen etc.}

Kgl. bot. Gesellsehaft zu Regensburg. Floristische Sitzungen : 13. und 27. Dezember 1899. Dr. Poeverlein besprach im Anschluss an Wettstein's Monographie und unter Zugrundelegung einer selhstgefertinten liestimmungstabelle die bayerischen Arten der Gattung Ehuhrosiu, wobei ein reiches. durel! die Liebenswürdigkeit des Herm I'rof. Ir. R, von Wettstein anthentisch bestimmtes Herbarmaterial vorzügliche Dienste that.*)

Das dileiche warr der F'all bezüglich der vom Referenten weiters hesprochenen Gattung Alectorolophus, aus der bis jetzt für Bayern folgende Arten nachgewiesen sind:

Alectorolophus alectorolophus Sterneck: Im grössten Teile Bayerns unter den Namen "Glaffen," "Kloft," "Feldgeld" u. A. verbreitet.

1. patulus stemerl: Jenseits der Thettachhrïcke momittelhar hei obrestdorf (det. Vollmann 17. Juli 1899).

A. major Reichenbach: Ziemlich verbeitet; scheint jedoch im Innem des Bayerischen Waldes zu fehlen.

*) Der Raummangel gestattet leider nicht, über die oben erwähnten Besprechungen der Gattungen Euphrasia, Potamogeton, Hieracium und Rosa an dieser Stelle ausführlichere Referate zu bringen. Dieselben mögen daher ciner anderweitigen be. handlung vorbehalten bleiben. 
A. serotinus G. Beck: Hierher gehören die meisten von Prantl zu "Rhimanthus amyustifolius Guelin" gezogenen standorte. Nach Schwarz im nördlichen Frankenjurat um Huggendorf und Pottenstein, anch um Velden und Plech überall hiutig, austerdem am Kauerlacher Weiher auf Keuper (leg. Schultheiss). Nenerdings auch von host hei Schweinfurt und Veitshöchheim, von Kraenzle im Dachanermoor und von Poeverlein im Gomnersdorfer Moor (T'ertiair) und am Lausbuckel bei Regensburg aufgefunden.

A. lanceolatus Sternech: In den bayerischen Alpen ziemlich verbreitet, zuweilen auch in den Vorbergen, z. B. zwischen Eurasburg und Wolfratshausen (leg. Schwarz).

A. Vollmanni Poeverlein nov. spec. (Eine zwischen A. lanceolatus und A. anyustifolius die Mitte haltende Form, bei der die Scheidung zwischen Atstivales und Autumnales noch nicht vollzogen ist; habituell dem A. angustifolius rar. subalpimus sternech ähnlich, jedoch nicht damit identisch): Hölitobel bei Gerstruben im Algän auf Kalk, 1050-1100m (det. Vollmann 24. Juli 1899).*)

A. angustifolius Heynhold: Bei Sterneck nur für die Alpen und die Garchingerheide angegeben, wurde diese Art neuerdings von Schwarz bei Augsburg, ansserdem in Menge im Riedenburger Jura um Altmannstein und Tettenagger, sowie bei Holenstein und Anersberg, von Schultheiss bei Grossaffalterbach, von Rost im Edelmannswald und bei Randersacker gefunden.

A. major Reichenbach $\times$ minor Wimmer et Grabowsky: Nach Sterneck von Haussknecht auf den Bodenseewiesen bei Lindau gefunden, jedenfalls aber mit den Eltern weiter verbreitet.

A. minor Wimmer et Grabowsky: Die verbreitetste Art.

1. stemophyllus Stemerl: Von Schwarz bei Mandlau nahe Pottenstein für Bayern neu entdeckt, neuerdings im Juli 1899 von Vollmann anch an mehreren Standorten bei ()herstdorf (Trettachanlagen und Zwingsteg) anfgefunden.

10. Januar 1900. Herr Lehrer Anton Mayer sprach an der Hand der Bearbeitung in Ascherson-Graebner's Synopsis über die um Regensburg vorkommenden Arten der Gattung Potamogeton.*)

24. Januar 1900. Herr Prof. Linder sprach über die um Regensburg einheimischen Arten der Gattungen:

Pulmonuriu ( $P$. officimulis, bereits in Kerner's Monographie für Regensburg angegeben; I'. olsarur, weitaus die häufigste Art; $P$. tuberosu, sehr selten).

Fumaria ( $F$. officinalis, verbreitet; $F$. Taillantii, selten; $F$. capreolata, q.sp. im städt. Alleegarten zu Regensburg).

Corydatis (C. cava, nicht selten, besonders auf Jura; C. intermedia, selten, z. B. Falkensteiner Park, Fronberger Park bei Schwandorf, Höllbachthal bei Wiesent, im Jura bei Laaber).

14. Februar 1900. Herr Justizrat Vogel besprach an der Hand des von ihm zusammengestellten Bestimmungsschlüssels, sowie eines reichen, namentlich von ilm und Herrn Prof. Vollmamn gesammelten Herbarmaterials die um Regenshurg vorkonmenden Hirva ium-Arten aus der Sektion Archlli r ac i "m.**)

28. Februar 1900. Herr Prof. Seywald erörterte die bayerischen Arten und Formen der Gattung Knauti ", wobei er in erster Linie die Krasan sche Abhandlung „Untersuchungen über die Variabilitä der steirischen Formen der Kunntia silvatica-arvensis." in Mitt. des Naturw. Vereins f. Steiermark. Jahrg. 1898. p. $65 \mathrm{ff}$. zugrunde legte. Tortr. führte aus, dass als typische Form $K$. arvensis zu gelten habe, die - wie sich durch fortgesetzte Kulturversuche gezeigt habe - bei Versetzung in Waldborlen wesentlich abünlere und zwar in der Richtung gegen $K$.

*) Von Vollmann gesammelte Originalexemplare dieser auffallenden Form wurden in Fase. III der "Flora exsiccata Bavarica" sub Nr. 205 ausgegeben.

**) Vgl. hiezu "Allg, bot. Zeitschrift 1899 p. 181 f. 
silvatica, indem die fiederspaltigen, grangrünen Bläter in ungeteilte, gesiigte oder gekerbte, grasgrüne Blatter mit verlängerten Blattstielen ( h. I'unnonicre

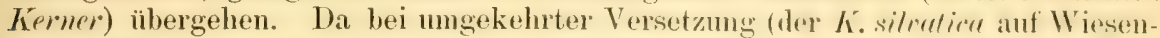
boden) keine oder nur unbedentende Variation stattfinde, so habe nach Krašan $K$. arvensis als Stammform zu gelten. K. Penmmica werde dann hei ihrer weiteren Höhenwanderung zur $K$, dipsacifolia, die Wettstein als präalpine Form der $K$. silratica Duby erklärt, während er die noch höher hinaufgehende car. "ruminatu Opiz als die Normalform der $K$. silvatica bezeichnet. Da mit der zunehmenden Höhenlage die Blätter sich ständig verlïngem und verschmälern, so scheine die alpine $K$. Tongifolia das anders Extrem der von den Thalwiesen aufwairts gewanderten $K$. arensis darzustellen. Dass zwischen $K$. arrensis, Pannonica, dipsacifolia, silvatica, typica und longifalia wieder zahlreiche Zwischenformen exi. stieren, sei bei einer so variablen Gattung, wie Krnautia nicht zu verwundern. Wenn Krašan l.c. als charakteristisch für die silvaticue die Blattfurbung, für die Arvenses den Standort bezeichne, so sei diese Auswahl der charakteristischen Merkmale keine ganz glückliche, da sie nicht als korrelativ zu betrachten seien. Vielmehr dürften die Innovationsverhältnisse weit verlässigere Unterscheidungsmerkmale darbieten, bedürften jedoch noch weiterer Beobachtung.

14. und 28. März 1900 . Herr Prof. Petzi besprach die um Regensburg vorkommenden Arten, Formen und Bastarde der Gattung $R o s a$, wohei er einen von ihm verfassten Bestimmungsschlüssel derselben verteilte.

Generalversammlung: 17. M a i 1900. In Stellvertretung des Vorsitzenden gedachte der Sekretär, Herr prakt. Arzt Dr. Fürnrohr, des am 22. Fehruar 1900 erfolgten Ablebens des Direktors, Herm Kreisregierungs- und Medizinalrates Dr. Hofmann, und hob in warmen Worten dessen Verdienste um die Gesellschaft hervor. Sodann erstattete derselbe Bericht über die Thätigkeit der (iesellschaft im abgelaufenen Jahre: Von den 30 ordentlichen Mitgliedern verstarben im Jahre 1899 die Herren Bildhauer Geyer und Privatier Anton Schmid. - Die Exkursionen und wissenschaftlichen Sitzungen wurden in der bisherigen Weise fortgesetzt. - Von Publikationen erschien der III. Fascikel der Flora exsiccata Bavarica, diesmal zum ersten Male in einer Stärke von 100 Nummern. Das Herbarium erfuhr insbesondere Zuwachs durch eine Schenkung von Pflanzen aus der Nümberger Flora durch Herm K. Stabsveterinär A. F. Schwarz, durch Amold's Lichenes exsiccati, sowie durch die kiuflich erworbenen Carices exsiccatae, herausgegeben von A. Knencker. - In gleicher Weise hatte sich auch die Bibliothek eines stattlichen Zuwachses, teils durch schenkungen, teils im Tauschwege zu erfreuen.

Auf Vorschlag des Dr. Poeverlein wurde der Entwurf der nenen Statuten einstimmig angenommen.

Bei der darauf folgenden Vorstandswahl wurden gewählt:

zum Vorsitzenden Dr. Fürnrohr;

zum Sehriftführer Dr. Vollmann;

zum Rechnungsführer Dr. Herrich-Schaeffer;

zum Bibliothekar Prof. Lindner;

zu Konservatoren: Dr. Familler, Anton Mayer, Petzi, Dr, Porverlein, Vogl.

Zu Mitgliedern der Redaktions-Kommission wurden gewählt: Irr. F'ürnrohr, Dr. Vollmann, Prof. Lindner; zu Mitgliedern der Kommission für die Herausgabe der Flora exsiceata Bavarica: Dr. Poeverlein als V'orsitzender, Dr. F'ürnrohr, Mayer, Petzi, Dr. Vollmann.

Zu korrespondierenden Mitgliedern der Gesellschaft wurden einstimmig ernannt: Kreisspitalarzt Dr. Joseph Schatz in Geisingen (Baden), Reallehrer Hermann Zahn in Karlsruhe (Baden).

Dr. Poeverlein. 
8. Jahresversammlung der deutschen dendrologisehen Gesellschaft. Dieselhe findet vom 5.-7. August d. J. in der Orangerie und dem Camelienhaus

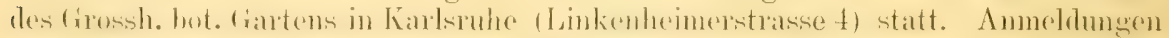

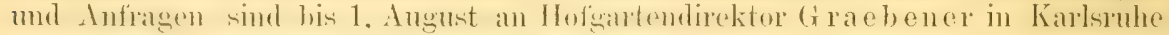
zu richten. Vortrïge sind zugesagt von: Graf Fritz von Schwerin, Ham m (Karlsruhe), Drude (Dresten), Pfitzer (Deidelberg), v. St. Paul-Illaire (Fischbach), Beissner (Poppelstorf). Weiteres siehe Programm.

The Botanical Exehange Club of the British Isles. Report für 1898.

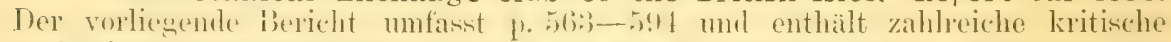
und wissenschaftliche bemerknngen. liei den Vutersuchungen und bestimmungen hahen eine lieihe bekamer botaniker mitgewirkt, z. H. W. Moyle logers (Rubi),

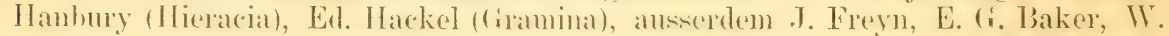
H. Beery, Arthur Bennett, J. Britten, (4. ('. I)ruce. E. F. Linton u. E. S. Marshall.

Wetzstein, Exsiceaten aus 0hio. A. Wetzstein, Prof. of the German Language Stt. M ary's, ()hio, U.s. A., offeriert nordamerikanische Ex siccaten zum Kauf und bittet um Aufträge.

Gross, L. u. Kneucker, A., Botanische Reise durch Istrien, Dalmatien, Montenegro, die Hereegowina und Bosnien. Der Untermeichncte healsichtight mit Herrn Gymmasialoberlehrer L. G ross aus Nürnberg im Monat August obengenannte Länder zu besuchen. Herr Gross wird schon vor Mitte Juli in Istrien

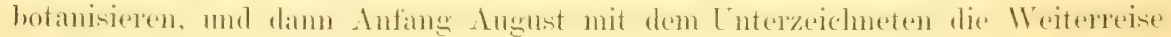
von Fiume aus antreten.

A. K.

\section{Personalnachrichten.}

Ernennungen etc. Dr. Kolkwitz, Privatdozent an der Univertitait, hat sich anch an der $\mathrm{Kgl}$. Jandw. Hochschule zu Berlin für Botanik habilitiert. -

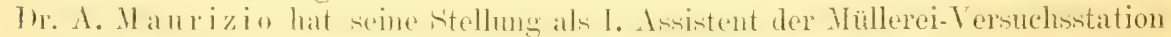

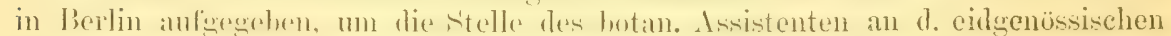
landwirtsch. Versuchsstation in Zürich zu übernehmen. - Prof. L. Nicotra, von der Universituit Sassari, kommt an die Universitit Messina. - Dr. G. B. de 'T'oni kommt an die Universitit von Camerino. - Dr. Domenico Saccardo w. Assi-

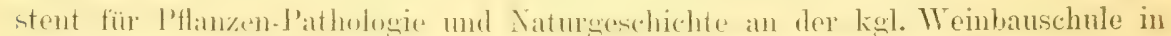

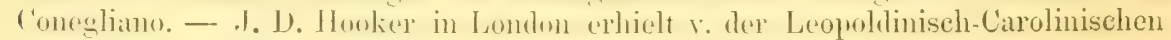
Akademie die Cothenius-Mrdaille.

Todesfälle: Dr. S. Ge orghieff, Professor der Botanik in Sofia, Bulgarien, am 22. Mai in Görberslorf. - Dr. Georges Clautrian, Assistent am bot. Institut der Universitut Brüssel, am 23. Mai in Davos.

\section{Tur Nachericht.}

Die nächste Nummer der "Allg, bot. Zeitschrift" erscheint Mitte September. Vom 28. Juli bis 3. September befinde ich mich mit Herrn Gymmasialoberlehrer L. Gross ans Nürnberg anf einer bot. Reise in Dalmatien, Montenegro, der Hercegowina und Bosnien.

Die während meiner Abwesenheit in Karlsruhe eintreffenden Briefe etc. können erst nach meiner Rückkehr beantwortet werien.

Die Herren, welche im Laufe dieses Sommers Material für die „Glumaceae exsiccatae" (einschliesslich der "Carices exsiccatae") gesammelt haben, werden gebeten, dasselbe nun spätestens im Laufe des September an meine Adresse zu senden.

Karlsruhe in Baden. A. Kneucker, Werlerplatz 48 . 


\section{Allgemeine \\ Botanische Zeitschrift \\ für Systematik, Floristik, Pflanzengeographie ete.}

\section{Referierendes Organ}

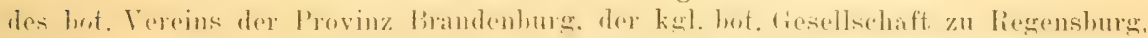
des Preuss. bot. Vereins in Königsberg,

und Organ der Botan. Vereinigung in Wüirzburg und des Berliner bot. 'lauschvereins.

Unter Mitwirkung hervorragender Fachmänner herausgegeben

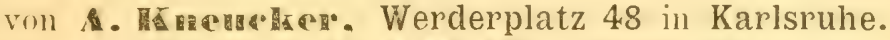

Verlag von .J. J. TE

Die Herren Mitarbeiter tragen fïr Form und Inhalt del von ihnen unterzeichneten Arbeiten volfe Verantwortung.

\begin{tabular}{|c|c|c|}
\hline Jis. & $\begin{array}{l}\text { Erseheint am } 15 . \text { jeden Monats. - } \\
\text { Preis: vierteljihrl. } 1.50 \text { Nik. hei freier Zusendung. }\end{array}$ & $\begin{array}{l}\text { 190\%. } \\
\text { VI.Jahrgang }\end{array}$ \\
\hline
\end{tabular}

Inhalt

Originalarbeiten: Pfarrer J. G. La urell, Ueber einige Carcx-Hybriden aus Schweden. - Frhr. v. Spiessen, Das Suiskenbruch bei Dülmen in Westfalen. - I. Gross, Anemone trifolia [. forma biflora. - Dr. Franz Bloŕski, Zur Chronik der preasischen lifora. - - ottohuntza mo Tom von Post, Nomenklatoriarbe Revision höherer Pflanzengruppen und über cinige 'T'ausend Korrekturen zu Englers Phaenogamen-Register (Schluss).

Bot. Litteratur, Zeitschriften ete. I a to uschek, Gauchery, Paul, Untersuchungen über den "Nanismus" der Pflanzen (Ref). - Inhaltsangabe verschied. bot. Zeitschriften.

Bot. Anstalten, Vereine, Tausehvereine, Exsiceatenwerke, Reisen ete.: Schulz, Paul, T'auschvermittlung für Herbarpflanzen. - A 11 escher, A., u. Sch nabl, J. N., lumgi bavarici exsicrati. - Vestergleu. 'Tyeho, Nicomyetes rariores selecti. - liriegar, W., Fungi saxonici. - Cusick, Wm. C., The Eastern Oregonand Western Idaho-Flora. - Collins, Fr. Sh., Holden, J. and Setchall, W. L.,

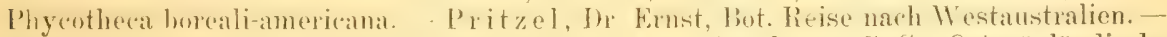
Sinten is, P., Bot. Reise nach 'I'ranskaspien ete. - A m dru p, C. G., Ostgrönländische lixpedition. - I'anlsen, Ove, C'entrabisiatische Reise. - Schmidt, Tohs., Reise

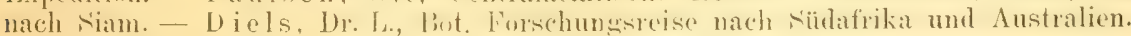

Personalnachrichten. - Zur Nachricht. - Anfrage.

\section{Ueber einige Carex-Hybriden aus Schweden.}

\section{Von Pfarrer J. G. La urell.}

Innerhalb der Gattung Carex kannte man in Schweden bis zum Jahre 1881 nicht mehr als nur eine Hybride; diese war Carex fulva $\times$ (lura, von I)r. A. Mmpuist in shohweden entaleckt. Nun kömen wil in

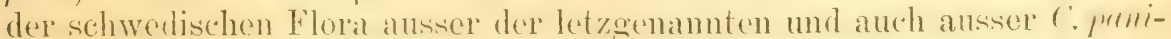
culata $\times$ vemota (Boeminghanseniana IV eihe), elongata $>$ loliacea, canescens $\times$ Norvegica, c. $\times$ loliacea, $c .>$ dioica (microstachya Ehrh.) noch 15 oder 16 andere Kreuzungen aufzählen.

Dazu können wir auch num C. rostrate (amprellacea) $\times$ filiformis und

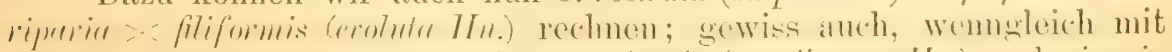

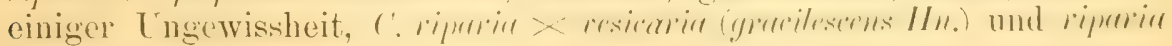
$\times$ rostrota. 
Betreffs $C$. rostrata $>$ filiformis sei auf $\mathrm{Nr} .11$ der "Allgemeinen Botanischen \%eitschrift" 1899 von $\Lambda$. Knencker, Karlsuhe, verwiesen. Die folgenden :3 Hybride wollen wir num hier einer genauen Betrachtung unterziehen.

Zunächst Criparia $\times$ filiformis.*) Diese Hybride ist eine von den in Fhweden zuerst gekannten. In Deutschland wurde sie jedoch (a. 25) Jahre vorher, namentlich i. J. 1857 in der Flora von Schlesien von Fr. Wimmer heohachtet. Gleichwohl war sie jedoch in Schweden schon 1818 in den Verhandlungen der Königlichen wissenschaftlichen Akademie unter dem Namen C. evoluta bekannt.

Dass diese Carexart nur eine Hybride zwischen $C$. riparia und $C$. filiformis ist, diurfte durch folgende Diagnose der Pflanze des Standortes von der Gemcinde Torö (Herrhamra) in Södermanland hervorgehen. Das Rhizom des ährentragenden Halmes hat schwächere Ausläufer als die Blatttriebe, die zuweilen lis zu mehr als $1 / 3 \mathrm{~m}$ lange, kräftigere Ausläufer mit vielen Scheiden hesitzen. It al m einzeln oder unten mit mehreren oder wenigeren sterilen Blattrieben beisammen, deren Blätter zuweilen $1,10 \mathrm{~m}$ in der Länge messen. Der Halm ist meist nicht $1 \mathrm{~m}$ hoch, unter den Aehrchen rauh und scharf 3 kantio, nur hisweilen stumb-kantig und wenig rauh. Sicheiden, hesonders untere ziemlich netzaderig, hell und pupurot. Bläter meist flach und dazu gekielt, das olierste lisweilen sämtliche Achrchen üherragend. Von den Tragb]ättern überragt in der Regel nur das unterste den Halm.

Weibliche Aehrchen meist 2. Von 24 Stöcken hatten 17 nur 2 weilliche Aehrohen und erinnesten somit an (i. filiformis. Unterstes zuweilen bis zu 7 cm lang, nach unten verschmälert und ausserdem zuweilen fast 2 cm lang gestielt. Männliche Aehrehen meist 3. Von 24 Pflanzen hatten 12 je 3 männl. Achren und erinnerten somit an C. ripariu; nur 1 stock hatte $t$ männliche Aehrchen. Oherstes männl. Aehrchen hisweilen $T$ cm lang mod meist sehr chmal. Schlä uche lraun oder graugrïn, nath unten konisch verechmälert, nach ohen allmählich in den Schnahel äbersehend. Deckschupen der weiblichen Aehrchen meist

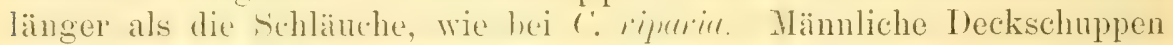
dunkel purpurrot lis schwarzhraun, länglich lanzettlich, mit der grössten Breite in der Nitte.

Es versteht sich von selbst, dass die fruchttragenden Teile der Hyhride riemlich selwwach ausgelildet erscheinen; sogar im August waren noch keine fertilen und reifen Schläuche zu finden.

Was das Vorkommen der Hybride in Schweden betrifft, ist sie

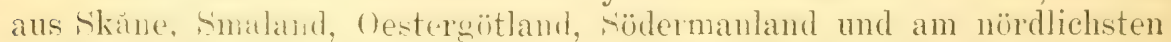
ans Testmanland und Cphland hekannt. Aus södermanland kemnt man sie auch; nicht aber so lange aus Södertöm. Hier in diesem Landesteil kommt die Hybride ca $18 \mathrm{~m}$ ii. d. M. in einem alten Teiche am Moore bei Herrhamra in der naturschönen Gemeinde Torö nur in Begleitung eines Elterutcils, der r'. filiformis vor. Dies lïsst sich daraus erklären, dass die ganze Gegend vor ca 100 Jahren ein See war, der

*) Ditse interessante Hybride wurde von mir anfangs Juni 1900 in Gesellschaft meines Freundes Dürer aus Frankfurta. M. und des Herrn Dr. v. Palézieux auch anf einer sumpigen Waldwiese bei Obertshausen unweit Offenbach a. Main in der hessischen Provinz Starkenburg in grosser Henge mit den beiden Eltern zusammen entdeckt und für die Carices exsiccatae präpariert. A. K. 


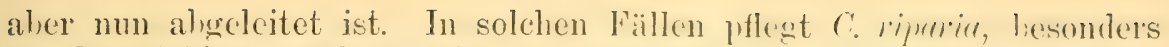
wo das Gebiet angebant wird, zu verschwinden. ['msomede ist ahere

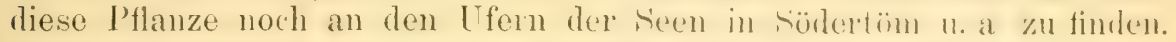

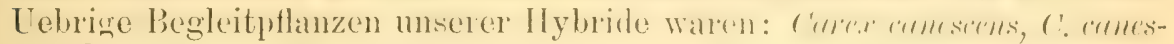
cens $\beta$. subloliacea Laest, Comann palustre, Equisetum palustre, Galium palustre, Spiraea ulmaria f. denudate Hayne u a. Diese Kreuzung kommt wohl noch an viclen Onten vor und mag wohl in versehiedenen 'Teilen unseres Landes übersehen worden sein.

(Schluss folgt.)

\section{Das Süskenbruch bei Dülmen in Westfalen.}

Vie oft denke ich noch daran zurïck, dass ich, ein junger Hensch, mit meinen Geschwistern oder anch allein zum Sïskenhruch wanderte, um dort seltene P'flanzen dinzusammeln. Ich fiand dort fiast bei jedem Besuche etwas Nemes und Interessantes, und erimere mich noch u. A., dass bei einer Exkursion 1s7! von den Herren Medizinalasiateor Dr. Wilms, Dr. Kronenterg, Apotheker Leiss etc. ans Minster unter meiner

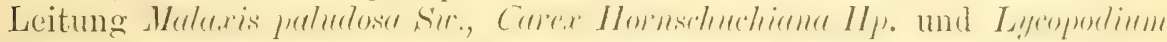
Selergo $L$. als neu, und mir hisher entgangen. anfefunden wurden. Teitdem mein Vater gestorben und mein Bruder von Diilmen fortgegingen, war ich nicht mehr dort. Nanches mag sirh scitdem veründert hahen, manche l'flanze mag verschwunden sein, seitdem das Gelände, was früher zumteil gemeinschaftliche Weide für die Lïdinghauser Strasse zu Dülmen war, unter die Interessenten verteilt ist, aber ich zweifle kaum, dass sich trotzdem noch sehr viel Interessinter mol seltenes vorfinden wird. Der Weg fühte bei rolon Winkelmann vorbei, wo in dem 'Teiche sich ausser verschiedenen, nicht seltenen I'otrmmugeton-Arten, Istmm minor L., trisulca L., gibba L., polyprhiza $L$. im trauten Verein fanden, ïber Bulshergs Heide, auf der, ansser wenigen I'mlsutillu bulyntis Hill.,

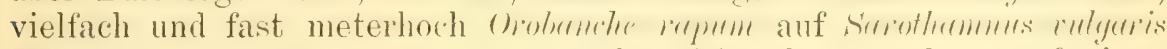
Wimm., das „Braam" genannt, wuchs. Man konmt dann auf einen breiten Sandweg, der das Siirkenlunch in 2z verschiedene 'T'eile scheidet. Doch bevor man hinliommt, selangt man noch anf eine kleine, zumteil sumpfige Heide; in Grahen dort rechts vom Wege finden sich schon

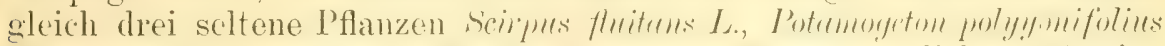

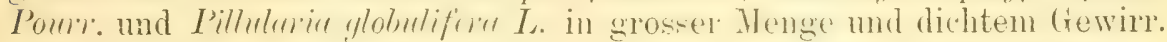
Auf der Heide selbst steht Erica tetralix L., Gentiana meumonanthe L., Pinguicula vulgaris L. und in Menge Lycopodium imundatum L., nebst clucutum $I$. Wir hetreten nun das eigentliche sïskenhruch. liechts des Weges ist ein smmptiges J'errain, durchzogen von Grähen und mit Wassertämpeh. Der Untergrund ist liaseneisenstuin, der hindert, dass die stagnierenden Gewïsser in die Tiofe abziehen kömen, und wird anch der Lisenstein entfernt, er bildet sich immer wieder mach, so lange nicht die Gegend entwässert wird; und das hält dort sehr schwer, da die Gegend völlig eben ist und keinen Alfluss bildet. II de dieses Terrain, die frühere, oben erwähnte Kuhweide, durchstühern will, ist genütigt. sofern er es nicht vorzicht, sich seiner Fusshekleidung zu rntledigen, von Bülte zu Bïlte zu springen, wemn el nicht sein hichuhwerk voll Wasser haben will. (ieföhrlich ist die sache hö̈hstene fïr dic Brelkleidung, nicht für den Menschen sellst. da die Gewässere Grähen und Tïmpel so seicht sind, dass kaum ein kleines hind ertrinken kïnnte. 
An der Ostscite bildet die Fisenbahn ron Wanne nach Münstere, die auf hohem Damm sich hindurch zicht, etwa die Gronze; in Sïden und Norden liegrn kleine hiefernwälder vor, anch teilweise ant sumptigem Boden; im Westen ist olienbenamiter, lreiter Weg, der zur lintenkoi

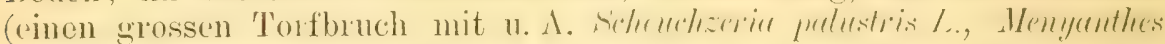
trifoliata L., Aspidium cristatum Siu., Calla palustris L. etc.) führt, die Grenze. Auf diesem sumpfterain, in dem der Kilsit\% in Mlenge nistet, finden sich eine grosse Zahl soltener Sumpf- und Moorpflanzen, welche hier nachfolgend sollen bentunt werden. Thuldetrum flormm L. (nehen

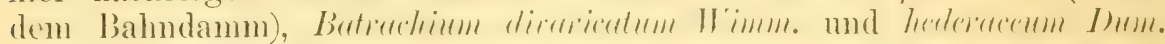

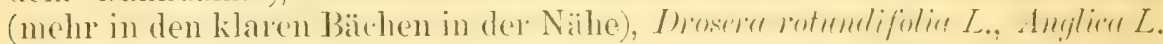

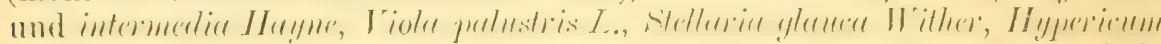
clodes L., Myriophigllum verticillatum L., spicatum L., alterniflorum DC.,

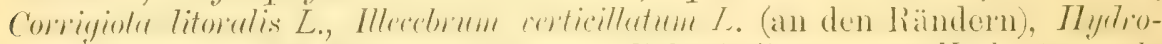

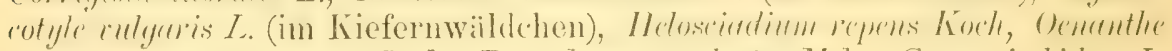
fistulosa L. und aquatica Lmk, Pencedanem palustic Meh., Corcopsis bidens L.

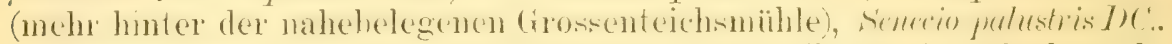
Limosella aquatica L., Pedicularis palustris L. und silicatica L., Mentha pulegium L., Utricularia minor L., IIottonia palustris L., Littorella lacustris L., Potamogeton polygonifolins 'Iour., Orchis morio L. (auf mehr trockenen Stellen), auch weissblühend, Lipactis palustris L., Liparis Loeselii Rich., Malaxis paluelosa Su., Narthecium ossifragum I., Juncus supinus Mch. mit var. uliginosus Rith., filiformis L., tenageja kinh., Cyperus fuscus L., Rhynchospora albe Vahl und fusca R. et Schult., Clendium marisens R. Br., Carex Hornschuchiana Hpe., Osmunde regalis L., Lycopodium selago L. Ausser diesen und dem schon erwähnten imendatum $L$. findet man noch der Gegend hinter Hausdiilmen (in desinen spurlos verschwondenen liurg 1535. Johann ron Leyden und die ührigen Wiedertäufer gefangen gehalten) am Vogelsberg Lycopodium annotimum. $L$.

Der zweite Teil des Süskenbruchs, jenseits des breiten Weges, besteht aus grössten 'Teils lehmigem Boden, anf dem sich freilich stellenweise siimpfe finden, auch Wassergräiben und 'Tümpel, der aher anch leidlich gute Wriesen bietet und deshalh zum grossen t'eil von seinem

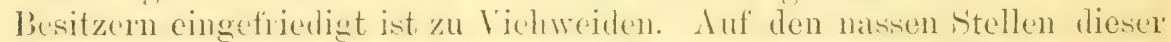
Tïmpe tinden sich eine grosse Anzahl der vorbenannten l'flanzen, aber

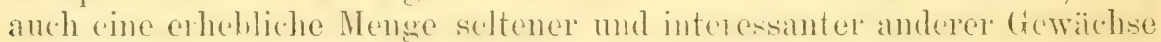
trifft man dort an. Man findet daselbst I'amussice pulustris L., Polygale depressu Wendr., Genista Anglica L., Comaram palustre L. mit Peplis portula L. und Isnadia palustris L., sowie Helosciadium inndatum im Graben der zweiten Wiese (Isuardia pulustris findet sich in der Nähe noch bei

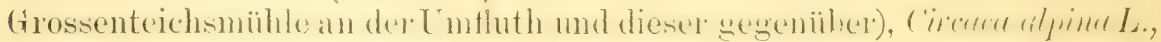
Carm carvi L, (in der ganzen Gegend sonst selten), Therincia hirta Leys, Phyteuma nigrum Schm. (nicht orbiculare, wie in den Floren steht und wofür ich die P'llanze selbst früher gehalten), Faccinium oxycoccos $L$. und Andromedr polyfolia $L$. (nur an der äussersten Grenze nach der

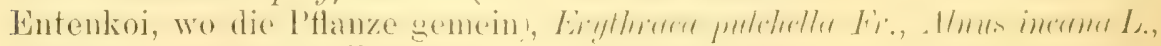
Alisma ramunculoides $L$. (unctuns $L$. findet sich auf dem Spelderbockfelde und beim Drügenputt). Lemma arhiza fand ich früher dort mehrfach und mehrere Jahre nacheinander in einem Tümpel, später war der 'T'ümpel zugeworfen, und deshall, ist die l'flanze fort. Givernthes antum-

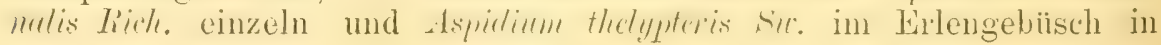




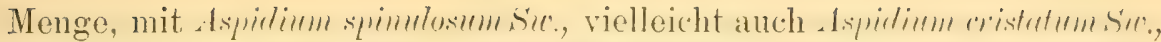
das diesseits bereits 1868 in der benachharten Entenkoi grefunden wurle.

Wer sich nun erguicken will, findet fiir alle billigen Bediirfnisse des Leilies anf Grossenteichsmühle gesorert, und wemn ep noch weiter botanisieren will, so trifft er dort Hippuris vulgaris, Carex arenaria und Arundo arenaria L., Potamogeton densus L., Rudbechia lacinirete L. und manches Andere, von nicht häufigen Pflanzen, und auch Asplenium trichomanes $L$. (der einzige Standort weit und breit).

Winkel, Rheingau, Februar 1895.

Frhr, v. Spiessen.

\section{Anemone trifolia $L$. forma biflora.}

Die Bozen-Meraner Bahn passiert zwischen Bozen und sigmundskron ein dichtes Laubgehöl, in welchem man während der Fahrt eine scharf ahgeurenzte Kolonje von Anemone trifolien $L$. erblicken kann. Am 20. April d. J. stattete ich dieser Kolonie cinen Besuch ab, wohei ich innerhalb weniger Hinuten zwei Exemplare der genannten l'flanze mit je zwei Blüten sammelte. Ich hätte noch mehr solcher Pflanzen gesucht und wohi auch gefunden, wem ich damals schon gewust hïtte. dass diese äusserst selten sind, ja bisher vicleicht üherhanpt noch nieht heohachtet wurden. In keincm der zahlreichen Florenwerke wenigstens, die mir zur Verfügung stehen, geschieht 2 blütiger Exemplare Erwähnum, ja einige Autoren betonen andrücklich, dass solche nicht vorkommen. So schreibt z. B I'ospichal in seiner. püchtigen Flora des issterreichischen Küstenlandes (Band II, p. 76): "Blüte stets einzeln."

Das grössere der beiden von mir gesammelten 2 blütigen trifoliaExemplare misst vom Girunde his zum Gipfel des itengels $21 \mathrm{~cm}$ (mit dem gröseren der heiden Bliitenstiele $28 \mathrm{~cm}$ ), das kleinere aher $18 \mathrm{~cm}$ (resp. $2: 3(\mathrm{~cm})$. Die beiden gipfelständigen Blütenstiele sind von 5) (!) dreizähligen IIüllblättr'n gestiitzt, der eine ist nicht nur dünner, sondern auch um ca. $2 \mathrm{~cm}$ kürzer als der andere und trägt eine Blüte, die in allen Teilen etwa halh so gross ist, als die am lüngeren Bliitenstiel. Die Blumenblïtter der kleineren Bliite sind anf der lïickseite, hesonders längs der Dittellinie, hlasslila, sonst weiss, wie die Blumenl, ätter der grösseren. Drei der fünf erwähnten Itüilbläter haben normale Grösse, zwei davon, welche offenbar der kleineren Blüte zugeordnet werden müssen, sind auffallend klein; ihre Blattahschnitte messen nur 1-2, in der Länge, während die Blattahschnitte der :' grösseren I Liillblätter $4,5-6 \mathrm{~cm}$ lang sind. Ein ähnlicher Unterschied lässt sich beziiglich der Breite feststellen.

Nürnberg, Mitte Mai 1900.

L. Gross.

\section{Zur Chronik der preussischen Flora.}

Von Dr. Franz Bloński (Spiczyńce, Ukraina).

Die ungünstige Wirkung der menschlichen Kultur auf die Urflora eines Landes ist eine allbekannte Sache. Jede Lokalflora kamn dazu überzengende Beispiele liefern. Aus der preussischen Flora will ich nur das Verschwinden der Clematis recta $L$. in Westprenssen erwähnen, welche Pflanze in ưsprünglich wildem Zustande in der ersten Hiilfte des 19. Jahrhunderts bei Thorn (der einzige Standort derselben in ganz Preussen) von Nowicki in Nenge entdeckt, 1848 noch 
spärlich heobachtet mol nemerdings an dientem (irte vergehens gesucht wurde. 1)a die enenamnte l'flanze in Königreiche Polen ziemlich häutig an der Weichsel rorkummt, rermutet Dr. J. Abromeit (Hlora von Preussen, Kö̈nigsberg 1898-1900), dass sie wieder nach Westpreussen einwandern könnte.

Eis wïre üherflüsig, zn heweisen, dass ähnliche Vernichtumgen von Pflanzen durch hintur am leichtesten an den firenzen ihrer Verbeitungshezirke stattinden. Für Clematis recta $L$. war Thorn ein nördlichster Fundort in Mitteleuropa, da sie nördlicher (z. 13. hei Königsherg) nur als dartenflüchtling beobachtet wurde.

Nun will ich die deutschen Botaniker auf einen ihnen unbekannt gebliebenen, weit nach Norden vorgeschobenen Posten von Myricaria Germanica Desv. in Westpreussen aufmerksam machen. Die Quelle, aus welcher ich meine Nachricht schönfe, war seiner Zeit den dentschen Taturforschern nicht unzuganglich, da sie in lateinischer Sprache verfasst und ihr 2. T'eil (Auctuarium etc. genannt) in Danzig erschienen ist. Der Titel dieses Werkes, dessen Verfasser der Jesuit (rabriel haaczriski int, lantet: Historia naturalis curiosa regni Poloniae, magni ducatus Lithnaniae, annexarumque provinciarum in tractatus $\mathrm{XX}$ divisa: ex scriptoribus probatis, servata primigenia eorum phrasi in locis plurinis, ex M. S.S. rariis. testihus oculatis, relationibus tide dignis, experimentis, desumpta. sando.

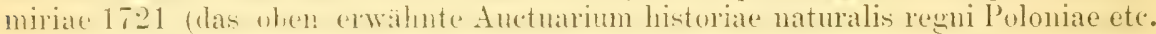
ron demselben Verfasser erschien in Danzig 1746).

Doch "habent sua fata libelli." Beide Werke wurden in kurzer Zeit selbst von Polen vernachlässigt und vergessen, anch vielfach bis zur nenesten Zeit einer zu scharfen Kritik unterworfen.*)

Nur etliche polnische Naturforscher, und zwar Hieronymus Łabencki [richtiger Eabęcki*)], Gustav Belke**) und Verfasser dieser Zeilen***) haben Rzaczyriski zum beilentendery Verdienste angerechnet, dass er zerstreute und jetzt fast inzugänglich gewordene phisographische Angaben über alle chemaligen polnischen Liander in se'ner Historia naturalis curiosa und dem dazu gehörigen Anctuarimm zusiummengetaset und mit seinen eigenen beobachtungen und hrieflichen oder münlichen lielationen der anderen Naturforscher bereichert hat.

In oben besprochener Historia naturalis sagt lizączynski (S. 203-204) über das Torkommen seines .Tamariseus officinis, Myrica Plinio, Tamarix silvestris Dioscoridi, Tabernaemontano etc., nobis (d. h. bei Polen) Tamaryszek" folgendes: ,nascitur uberrime in Roxolania, penes fluvios Dniestr, Bystrzyca, Stryj, San, Strwiaz etc., in Montano Tractu ultra Cracoviam ad Sola, Dunajec, Skawina, $\mathrm{Skawinka,} \mathrm{Raba,} \mathrm{Rabka,} \mathrm{Orawka} \mathrm{amnes:} \mathrm{prope} \mathrm{Vistulam} \mathrm{villamque} \mathrm{Maty-}$ aszów ete., Pulatinutus Sundomiriensis: ad radices montium Beszkid excelsior crassiorque quam alibi caeditur: in Prussia prope Torunium crescit."

(Schluss folgt.)

*) Ich führe nur die Beurteilung beider trefflicher Kenner der polnischen Flora: Prof. Dr. J. Rostafiński's und J. A. Knapp's an. „Die Arbeit von G. Rzączyński - sagt der Verfasser des Florae Polonicae Prodromus - hat in botanischer Hinsicht kaum etwas Bemerkenswertes." Das Knapp'sche Urteil ist noch schärfer: "Der Jesuit Gabriel Rzaczyński suchte alte Finsternis zu verbreiten, doch gelang ihm dies bei seinen Leb-

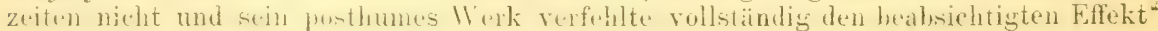
(Die Pflanzen Galiziens und der Bukowina, Wien 1872, S. XII).

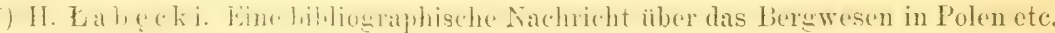
(in Biblioteka Warszawska 1841. 4. Band, S. 103-104).

**) G. Curier. Geschichte der Naturwissenschaften (ins Polnische übersetzt von G. Belke und A. Kromer). Wilno 1853-55.

***) Dr. Franz Błoúski. Ueber den Iürchenschwamm in Polen in Wszechswiat -

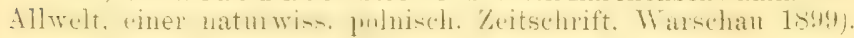




\title{
Nomenklatorische Revision höherer Pflanzengruppen
}

\author{
und über einige
}

\section{Tausend Korrekturen zu Englers Phaenogamen-Register.}

Von Otto Kuntze und Tom von Post.

(Schluss.)

\section{Nicht registrierte Gattungsnamen.}

Tollstandig fehlen in ciesantregister folgende Namen, lie in 'Text der Natürlichen Pflamzenfamilien an den dazu citierten Stellen existieren:

Abapus Ad. II 5 : 108. - Aberia Hochst. III 6 a : 44. - Abietites Carruth. II 1: 8t. - Abolboda H.\& B. II 4:20. - Abumon Ad. JI 5:53. - Acacallis Ldl. II 6:166. - Acoridium Nees N. 105. - Acoropsis Conw. II 3:118. - Adrastcea DC. III $6: 116,117$ - Amygdalopsis Ldl. III 3 : 53. - Anacharis Rich. II 1 : 251.Analyrium Mey. III 8:236. - Anatherum Beauv. II $2: 28$ - Androstephium Torr. II 5 : 58. - Antoschmidtia Steud. II $2: 64$ - Aphlebia Presl p.p. II $1: 27 .-$ Apocynophyllum Ung. IV 2 : 119. - Arausiaca Bl. II 3 : 55. - Argyropsis Roem. Il 5: 107. - Armoracia Fl. Wett. III 2 : 184. - Aroites Kovats II $3: 110$. Aronites Heer II $3: 110$. - Artocarpidium Ung. III 1:83. - Artocarpoides Sap. III 1: 83. - Asclepiodora A. Gray IV 2:239. - Athrodactylis Forst. II $1: 206$. Atylosia IV.d A. III 3 : 373. - Audibertiella Briq. IV 3 a:287. - Azaola Blanco IV 1: 133. - Balania Van Tiegh. N. 150. - Bambusium Ung. II 2 : 96. -- Banksia Forst. N.260. - Bathatherum Nees II 2:26. - Beccariodendron Warb. N. 161. -

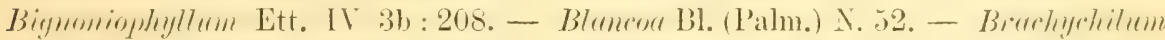
R. Br. II 6 : 20. - Brassaia Endl. III 8:36. - Burckella Pierre N. 272. Cacara Thouars III $3: 383$. - Callirhoë Nutt. III 6:40. - Calocedrus S. Kurz N. 25. - Calophanes Don IV 3b:302. - Calvelia Moq. III 1a:80. - Calycosia A. Gray IV 4 : 113. - Camarotis Ldl. II 6 : 218. - Camnophyllites Brong. II 6 : 32.Capellenia 'Tijsm. \& B. III 4:90. - Cardiopteris Schimp. II $1: 27$, III $5: 257$. Carpentaria Rece. II 3:73. - Caryophyllaster Rumpf N.332. - Cephalophorus Lem. III 6a:182. -- Cephaloschoenus Nees II 2 : 116. - Celastrinanthium Conw. III 5:185. - Chamaccatiaria Port. III 3:16. - Chloropsis Herb. II $5: 118$. Choretis Herb. Il 5: 110. - Choriceras Baill. III 5:32. - Chrosperma Raf. II 5:23. - Chymocarpus Don III 4:26. - Cinchonidium Ung. IV 4: 14. Cissites Heer III 5:438. - Clav Kth. II $5: 31$ - Colmeinoa Reut. III 5 : 18. -

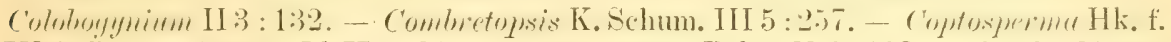
IV 4:74.-Cordyla Bl.II 6:219. - Corydandra Rehb. 11 6:132. - Cotylelobiopsis Heim III 6:268. - Courantia Lem. III 2 a:34. - Cratericarpinm Spach III $7: 212$. Cratopleura Web. N.157. - Cremosciadium Boiss. III 8:234. - Criosanthes Raf. II $6: 82 .-$ Crocivis Schur II $5: 142$. - Cryptocaula Hanst. IV 3 b : 180. Culmites Ung. II 1:186. - Cumingia Don II 5:122. - Cupanites Schimp. III 5:298. - Cupunoides Bowerb. III 5:298. - Cupirana Miers IV 2: 132.Cupressinoxylon Goepl. II 1 : 116. - Cyclopteris Brong. p. p. II 1:27. - Cyrthanthemum Oerst. IV $3 \mathrm{~b}: 158$. - Daemonorops B1. II $3: 52$. - Damatris Cass. IT 5:309. - Dasystemon DC. III 2a : 37. - Debesia O. Ktze. N. 73. - Dendrophita Bl. IV 3 b :230. - Deringa Ad. III 8:271. - Dieranostigma Hk. f. \&'Th. III 2:139, - Dicrypte Ldl. II 6:187. - Diplocalyx Presl IV 3 b: 162 - Disocuctus Ld1. III 6a:183. - Dolichites Ung. III 3:98. - Doliostrobus Sap. \& Mar. II 1:89. - Dombeyopsis Heer III 6:74. - Dufourea HBK. IV 3 a : 17. - Du-

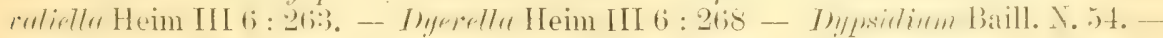
Ecbolium L. N. 309. - Eehitonim Ung. IV 2:119. - Eigeria Pl. II 1:251. Elissarhena Miers III 2:88. - Enantioblastes Goepp. o Ber. IV 1:15. - Endrachimm Juss. IV 3 a : 23. - Eremitis Doell H 2:89. - Eriobroma Pierre

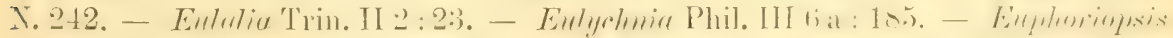

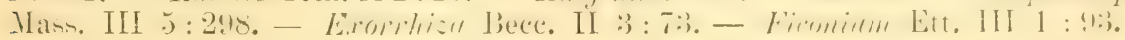
Folliculites Zenk. N. 39. - Fussia Schur If 2 : 54. - Gasteranthopsis Oerst. 
IV 3 b : 159. - Geijein Schott III 4: 112, 119. - Gelonium Thouars III 5 : 342.-.Gelbrea B1. III 7:83. - Gigantabies Nees II 1:86. - Globocarpus Caruel III 8: 204. - Glumosia Herb. II 5 : 151. - Gongrothammus Steetz IV $5: 126$. Gonzalea Pers. IV 4:61. - Goyazia Taub. N. 300. - Grevellina Baill. N. 208. Groentandia Gray II 1 : 209. - Guindilia Gill. III 5 : 310. - Gulubia Bece. II $3: 73 .-$ Gymmema KI. (Hippocr.) III $5: 228$. - Gypostachys Pers. II $3: 113$. Hagaea Vent. III $1 \mathrm{~b}:$ 87. - Hamamelidanthium Conw. \& Hamamelites Lesq. III 2 : 129. - Haynaldia Kanitz IV $5: 67$ - Heliconiopsis Miq. II 6 : 10. Heliotropites Ett. IV $3: 80$. - Hemigyrosa Bl. III $5: 315,319,320,345,346$. Hemingsocarpum O. Ktze. N. 336. - Heppiella Rgl. IV 3 b: 176. - Herzogia K. Schum. III 4 : 121. - Heterostylus Hk. II 1 : 225. - Heterotaxis Lill. II 6:187. Hexatheniu Sond. II 1:213. - Heyderia C. Koch II 1: 96, - Heydia Dennst. N. 142. - Himantop7yllum Spr. II 5:105. - Holocarye Durand IV 4: 133. Holosetum Steud. II 2 : 35. - Hopeoides Heim III 6:266. - Hyalis Sal. II 5 : 15t.Ifydrotrophus Clarke II 1 : 253. - Hymenolepis IV $5: 270$. - Jacquinotia Hombr. IV 1:73. - Jardinia Sch. bip. IV 5:124. - Ibidium Salisb. H 6:113. - Iboga Baill. IV 2: 189. - Ichnocarpus R. Br. IV 2:162, 178. - Idria Kellog III 6 : 298.Illicites F. v. M. III $2: 19$. - Ividinm Heer II $5: 146$. - Isias De Not. II $6 ; 89 .-$ Kinginda 0. Ktze. N. 161. - Kitchingia Bkr. III 2a:35. - Krugella Pierre N. 275. - Ktenosachne Stend. II 2:53. - Lahaya R.d S. III 1b:87. - Langscorffia Raddi N. 56. - Larochea Pers. III 2a:36. - Larrea Ort. III 4:354. Latania hort. II 3: 35. - Leguminosites Lesq. III 3:98. - Leichhardtia Sheph. II 1 : 94. - Lepidoc(1\%yopsis Stur II 3 : 90. - Lepidorhachis Wendl. \& Dr. II 3 : 69.Lepilatna Drum. II 1 : 213. - Leptophoenix Becc. II 3 : 75. - Linhia Pers. II $2: 50$. - Londesia F. \& M. III 1a:70. - Lueddemannia Rehb. II 6:165. Macrostachya Bth. II 5 : 51. - Macrostemium Horan. II 6:20. - Malanthos Stapf 1. 266. - Melastomites Geyl. III 7 : 142. - Micranthes Haw. III 2 a : 56. Micrenthes Haw., 'lausch III 2 a : 56. - Millingtonia Roxb. III 5 : 371. - Mimosites Lesq. III 3:98. - Mitriostigma Hochst. IV 4:75. - Mollia WV. III 1 b:87. Monadem Salisb. II 5: 24. - Moriera Boiss. III 2: 165. - Mucunites Heer III $3: 98$. - Nyptillocactus K. Schum. III 6a : 178. - Nacaretia R. \& P. IV 3a:50. - Nectris Pursh III 5: 137. - Nemostylis Herb. II 5: 148. - Neritinium Ung. IV 2 : 119. - Nenropteris Brong. p. p. II 1:27. - Nicolia Ung. II $3: 91$ - Nivaria Noench II $5: 105$. - Nothaparlytes Bl. III $5: 219 .-$ Nuno Bth. II 5 : 151. - Octorton Schum. \& Thon. IV 4:144. - Odontocarpa Neck. IV 4: 177. - Orchiontes Trew N. 101. - Oreopolus Sehl. IV 4 : 30. - Oricic Pierre N.208. - Pachuderma Bl. IV 2 : 11. - Palaeolobium Ung. II $2: 10$. Palmarella Gray IV 5: 69. - Papiria 'Thbg. II 5: 108. - Parilizm Gärtn. IV 2: 15. - Paryella Gray III $3: 263,264$ - Payeria Baill. (Meliac.!) IV $4: 31 .-$ Periballia Trin. II 2:54. - Pereilema Presl II 2:44, 48. - Peripteryginn Hask. III 5:257. - Petrocarei Tausch III 8:206. - Petrophiloides Bowerb. III 1 : 156 . Pharetrella Salisb. II 5 : 122. - Pharim Herb. II 5: 58. - Phaseolites Ung. III 3:98. - Phitetaeria Liebm. IHI 6:298. — Phryssocarpus Miers IV $2: 148$. Phyllocereus Miq. N.258. - Phyllotaenium André II 3: 140. - Phytoxys Spr. IV 3 a :290. - Piclieringia Nutt. IV 1:94. - Picria Lour. IV 3 b: 80. - Pierrea Heim III 5:268. - Pityrosperma S. \& Z. II 2: 59. - Platycladus Spach II 1:95. Pleurachne Schrad.II 2:111. - Plinia L. III $7: 81$. - Poissonella Pierre N. 276. Polia Lour. II 16 : 87. - Polyplethia Van Tiegh. N. 150. - Polypremum Ad. IV 4:177. - Pomatothece F. v. MI. III 1b:42. - Pritchardia Ung. N. 50. Pritchardioxylon Drude N. 50. - Prummopitys Phil. N. 21. - Pseudosantalum Rumpf III 8:32. - Psendoresicaria Rupr. II 2:166. - Psilosiphon Welw. II 5 : 156. - Psilostachys Hochst. III 1b:111. - Psittacoglossum Ll. \& Lex. II $6: 187$. Pterospermites Heer III 6:74. - Pycnophyllum Brong. II 1:27. - Raffenaldia Godr. III 2 : 182. - Rapistrella Pomel III ' $:$ 181. - Reimuardtia Spr. IV 3 a : 17.Rhizomites Geyl. III $3: 98$. - Richardia Kth. II 3:136. - Richea Thonars N.261.Robinsia M. \& G. III 3 : 383. - Roumea Rich. III 6a :"44. - Rubiacites Web. IV 4:14. - Saccopetalum Benn. III 2:29. - Sarcosiphon Bl. II 6:48. - Sar- 
cynpia Baill. III 7 : 88. - Scabrita L. IV $2: 15$. - Schedonume Beauv. II 2 : $75 .-$ Schizonotus Raf. N. 186. - Schizotheca Ehrb. II 1:254. - Schoenlandia Cornu N. 70. - Schoenleinia Kl. N. 102. - Schubertia. Mirb. II 1:91. - Scituminophyton Mass. II 6:32. - Scytanthemum Kl. IV 1:56. - Scytopetalum Pierre N.244. Selima Forsk. II 2 : 26. - Sequoiopsis Sap. II 1 : 92. - Sericura Hask. Il 2 : 38. Serpicula L. f. II 1:250. - Serpicula Pursh. II 1 : 251. - Sisymbrella Spach III 2 : 184. - Sphaerosacme Wall. III 4:297. - Sphallerocarpus Bess. III 8: 151. Stereosterma BI. IV 2:11. - Styloconus Baill. N. 79. - Styracina Schenk IV 1 : 175. Symepiluena Baill. IV 3b: 179. — Symisoon Baill. IV 4: 74, 155. - Taeniocarpum Desv. III $3: 383$. - Taeniosapium IIüll. arg. III 5:95. - 'Talpinaria Karst. II 6:139. - Temocydia Mart. IV 3 b : 219. - Tetralepis Steud, II 2:117. - Tetrapilus Lour. IV 2 : 11. - Tetrapoma Turcz. III 2 : 184. - Theophrasta L. IV $1: 89$. Tonguea Endl. III 2 : 170. - Treubia Pierre III 5 : 257. - Triandea Karst. II 1 : 25 Tricholobos 'Turez. III $2: 170$. - Trichospermum Lour. en. K. Schum. N. 234.

Trigella Salisb. II $5: 122$. - Tristylinm Turcz. III 6:189. - Tubith Tausch III 8: 205. - Tylosepalum Kurz III 5 : 84. - Typhaeolopium Ung. II 1 : 186. - Typhopsis Bth. II 5 : 51. - Udora Nutt II 1 : 250, 251. - Urceocharis Mast. N. 78. Urceolaria MLol. N. 300. - Uropedi(l)um Lal., Pfitz. II 5 : 84; N.98. - Taginariu Pers. II 2 : 111. - Tauanthes Haw, III 2 a : 37. - Teronicites Heer IV 3 b : 48. Williamsonia Carruth. N. 15. - Borraginites Heer IV 3 a : 80. - Caloplyyscu Krasser (Calophysa DC.) III $7: 133,138,139,140$ (ward später unterdrückt!). - Carpolithes Gerv. III 5 : 171. - Collania Herb. II 5 : 120. - Hydastylus Sal. II 5 : 151.Podocarpus Lab. N. 21 (nicht L'Hér.). - Ertela Ad. III 4: 168.

Die im Nachtrag des Gesamt-Registers Seite 457- 562 erfolgten Berichtigungen sind extra nachzutragen.

\section{Falsche Angaben über Gültigkeit der Gattungsnamen.}

a. Als synonym registriert, aber gültig sind im Text unter Berücksichtigung der Veränderungen im N achtrag von 1897.

Acanthostachys!!, Acrotome, Actinophora!, Actinostigma, Adenogymum!, Adesmia!, Agatites, Alania, (Alloplectus), Almeidea, Alonsoa, Alsodeia!, Alysicarpus!, Alyxia!, Ammi, Anisotes Nees, Ameslea Wall.!, Anplectrum!, Antirhoea, Apera, Apluda, Asterolasia, Athamanta L., Balansaephytum, Bartlingia F. Aluell.!, Blephlaridachne, Bonplandia Cav., Brevoortia, Bronmea Jacq.!, Calamovilfa, Calyptrochilus, Cancrinia, Canella!, Cantharospermum, Carajaea, Centotheca, Centrosema!, Chaetopappa (Choritaenia), Clianthus!, Cloëzia, Coelococeus!!, Conradiu Nutt.!, Corymbarchis!, Corynella!, Corynostylis!, Cyathochaete, Cynorchis!, Dacalepis Wight \& Arn., (Deplanchea), Derris!, Diclidocarpus!, Dipteryx, Diptychocarpus! Dulacia!, Earina, Echinospermum!, Elytraria!, Eremochloa Büse, Eucommia, Foumiera, Fusams, Garuleum, Gestroa, Gethyum, Gilibertia R.\& P., Glancidim, Globifera!, Glossogmne, Glossostema, Gnephosis, Gomphocalyx, Goniolimon, Gonospermm, Gratuizhia, Guaduella, Gustavia!; Gymnoschoenus!, Holigama!, Holosteun, Horsfieldia Willd.!, Hydranthelium, Hymenocarpus!, Hymenocharis!, Isabelia, Ixophoms!!, Kalopanax, Keteleeria!!, Korthalsella!! Lathrogyna, Lecanosperma!!, Linociera Sw.!, (Lophotocarpus), Macrolobium!, Metopium, Moronobea, Myrianthus, Myroxylon L. fil.!, Nanodea Banks, Navia, Nephlropetalum, Notothlaspi (ausserdem irrig Nothothlaspi), Otiveriana, Orleanesia, Omocarpum!, Ormithochilus!!, Ottoa, Oxylobium!, (Paranomus), Par(r)yella, Parthenocissus, Pectinaria Haw., Petalostemon!, Ribes, Petroselinum, (Phaylopsis), Pholidostuchys, Piper, Placea, Planera Gmel., Polunisia,

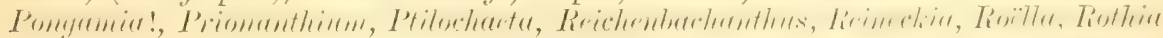
Schreb. ist IV : 391 anerkamnt. (Rothia Pers. wirt Westonia), Sabadilla!, Santivia, Saunurus, Schrebera Roxb.!, Selloa HBK., Sempervivum L., Senecio, Sesamothamms, Seseli, Siolmatra, Stelestylis, Stimpsonia, Tetravia Beaur,!, Themeda!, Tiarella, Thrixspermum Lour.! N.234, (Trichospermum em. K. Schum.), Trichosandra, Twrnera, Warscewiczella, Washimgtonia Wendl.!!, Virola!!, Wistaria!, Xylosma Forst.!, Marlierea, Meconella!, Shepherdia:, Speroularia!, Sirartzia! 
Autorcitate sind hier weggelassen worden, wenn keine Verwechselung möglich ist. Die Namen in () sind schon im Nachtrag zum Gesamtregister berichtigt.

b. Alsgeltend mit Sperdruckregistriert, abersynonym sind nach Engler's letzten Veränderungen.

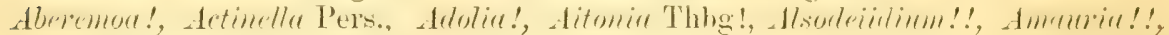
Amburana!, Andreoshia Rehb.!, Androchilus, Antherma, Anthistivia!, Antitragus, Antochortus, Apalatoa!, Arduina!, Argyrorchis!!, Astrace Kl., Augusta!, Aulacodiscus!, Aydendron!, Barlaea!!, Batschia Vahl!, Bechium (Berendtia Gray), Biolettia!!, Blatt!!, Boldu, Bonateria!, Bradburya Raf. Burchardia R. Br.!, Byronia!!, Calesium!, Callistachys Vent.!, Calyptrion!, Camerinheira, Cantuffa!,

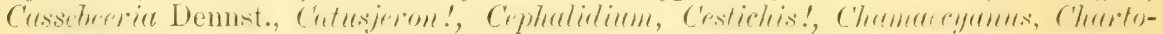

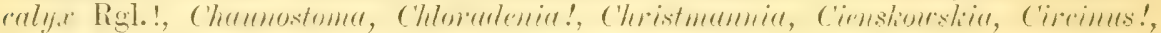

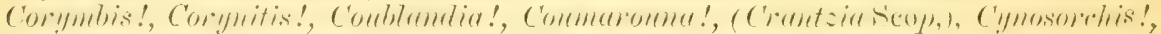

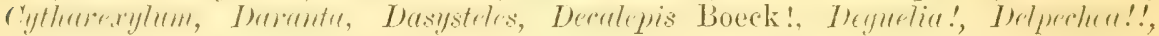
Dialycarpa!!, Dichelocalyx, Digomplia!, Diphaca!, (Diplanthere Banks it Sol.), Diplectria!, Distoeclea!!, Donia Don!, Douarrea!!, Duxana, Echinothammes!!, Elymanthus!, Enaryea!, Endolithodes n. n. [E Symisoon], Eremochloë Wats!, Fabricia Scop.!, Fadoyia!, Fistularia!, Galedupa!, Gastroglottis!!, Glycideras!!, Grundelia, Gynopogon!, Gymmopsis, Halochloris, Halodule!, Hastingsia, Hazardia!!, Heleastrum, Helenium L. 1735 „O. Ktze.", nicht IV 5: 262, Helicophyllum Schott!, Henricia Cass., Hepetis!, Hermesias!, Heterocroton, Hexastemon Kl., welches IV 1: 64 als Subgems eingezogen ward. Holopleura!!, (Hoppia Nees Reg. N. 461), Hormogyme!!, Horsfieldia Bl.! N. 166., Hymenopappus IV 5: 391 sub Rothia angedeutet, Hypocistis!, Japotabita!, Japaramdiba! Jenlinsia, Intsia!, Ipmum!!, Ischnosiphon!, Kampmannia, Krannhia!, Kuhnistera!, Lappula!, Laxmamma R. Br.!, Ledenbergia!, Lepidopirania A. Rich., Lepargyraea!, Libanotis, Liriosma!, Lophanthus, (Lophiocarpnes Miq.), Lupsia!, Macranthera!, Marantochloa!!, Macrorthynchus, Marilanidium!, Massungea, Maximitianea Mart. \&

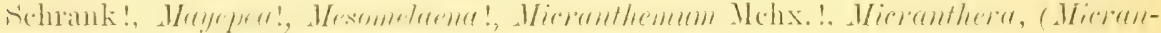
thus Wendl.), Microsplenium!!, Mikroschuentia Wettst. = Microschentia Bth.!!, Miltus, Hoendoe, Moghania!, Montanoa!, Montolivaea!!, Moquilea!!, Mountnorrisia!, (Müllerothammus), Myroxylon Forst.!, Nathusia!, Naucleopsis, Neobaionia! 1. 338, Nerviplacentaria, Newastlia, (Nentonia Baill.), (Nivenia R. Br.), Nyetaginia!! N. 155., Ochagnvia!!, Oenoe, Oligogynium!!, Ophiocaulon!!, Opoidia,

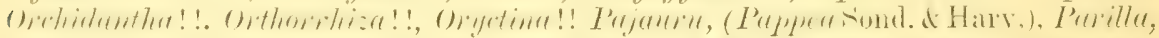

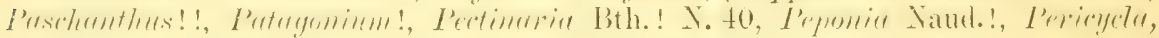
Picrium, Rosenbachia, Platycoryne!!, Platymerime, Platystigma Bth.!, Pleio-

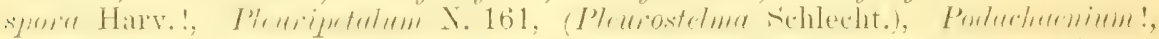
Polychisma, Polyschistis!!, Prantleia!, Prionachne, Psidiastrum, Pteroj. fulum!!, Pusatha!, Quinaria!, Qumio, Raphistemma, Reinia*), Rielchela, Rhacoma L.!,

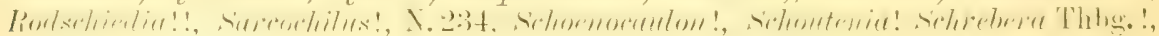
Seridia, Sophronia, Soranthus, Stachyanthemum, Sychnosepahm!!, Symandro-

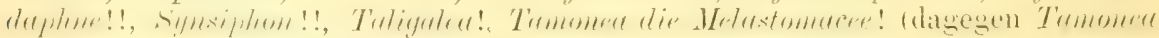
die Terbenaceal bleibt geltend) Taonabo!. Tetradenia Nees 1831 wird wegen

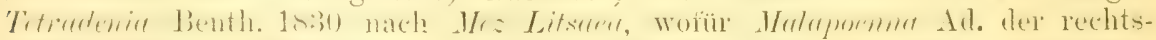

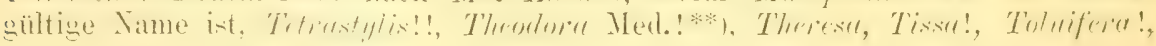

*) Aus dem 'Text geht hier, wie in manchen anderen Fällen, nicht klar hervor, dass Reinia ein Symonym ist; es ist eben Pflicht der Autoren, die Register ihrer Arbeiten selbst zu besorgen, wobei sie meist noch manche Unklarheit finden und im Register berichtigen können. Bei Stachyanthemum z. B., das nach dem 'Text = Cyrilla

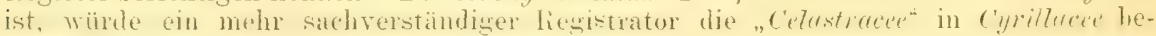
richtigt haben.

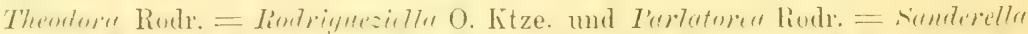
(). Litze sind in Engler's Nachträgen stellt, aher kinfus. indem die 2 cieners ron Rodrignez 7 der tinen. dagegen deren 2 Synonyme von Kuntze zur anderen Gattung gesetzt sind; das kann unmöglich richtig sein! 
Tounatea!, Trenatocarpus Zahlbr.!!, (Trianthera Wettst.), Trichospermum BI.!, Tubiflora!, Tylodendron, Clmaria!, Vitellaria!!, Vitiphoenix, Togelia Lan.!, Vouacapona!, Touapa!, Wehuitschia!, Winterana!, Tanthochymus, Zycona!! Gaslondia, Riddellia.

Es sind noch mehr solcher Fialle, da die Genera excludenda, die ofter am Schluss der Familien mul im Nachtrage anfgeföhrt sind, hahl suiltig, hald als ungültig registriert sind. Die mit ! und !! versehenen Namen betreffen Verïn. derungen, die erst 1897 im Nachtragband vorgenommen sind, und zwar gilt ! für

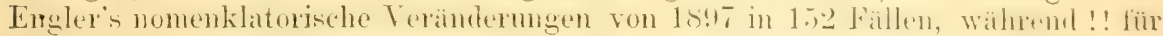
seine systematischen Veränderungen von 1897 in 39 Fällen gilt. Also schon aus Liste 7a of b ergeben sich 191 Fälle der Genera-Korrekturen von 1897, die 1898 beim Registerantertigen orler 1899 beim Registerrevidieren ühersehen sind; dem im Registernachtrag sind hierzu nur die 17 Fälle in ( ) berichtigt worden.

Diese massenhafte Vergesslichkeit innerhalb 1 bis 2 Jahren seiner eigenen

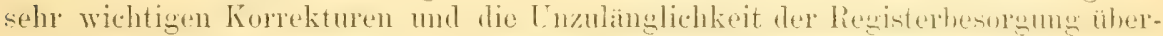
haupt ist für ein übrigens so wissenschaftliches und grossartiges Werk, wie die Pflanzenfamilien, zu bedauern; mindestens jedoch fehlt es an der zur Sache umbedingt nötigen Ordnung. Die anderen Listen, insbesondere die erste, bestaitigen dasselbe Factum.

Man darf nicht etwa behaupten, dass die Fehler, so wie sie im 'Text von Band II-IV stehen, als solche registriert seien, weil die Corrigenda des Nachtragbandes in diesen Fällen damn allermeist im Register fehlen; anch müsste man dann registrierte Fehler (z. B. mit ,err.") als solche kenntlich machen.

In Engler's Werk findet sich eine zweimalige Umänderung der Nomenklatur: erstens manchmal in den Nachträgen der 24 einzelnen Abteilungsbände und am Schlusse mancher grösseren Familien, meist nach den internationalen Gesetzen; zweitens*) in Nachtragband vom Jahre 1897 Seite 1-330 angeblich nach Privatregeln des Berliner botanischen Huseum, die thatsächlich aber in den meisten Fälen weder befolgt wurden, noch befolgbar sind. Dies hat Otto Kuntze in Revision gen. IIII Seite 58-134 der Einleitung gründlich bewiesen, wemn auch leider in allzu schroffer Form - doch durch die Umstände entschuldbar. Diese Beweise gegen die historisch sogenannten - zum Unterschied von früheren

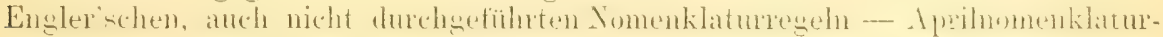
regeln sind meiner Ansicht nach nicht widerlegt worden, dagegen von kompetenten Kritikeru als meisterhaft bezeichnet worlen.**)

Von den nomenklatorischen Veränderungen in den PHanzenfamilien des Jahres 1897 beruhen die meisten auf der zweiten Aprilnomenklaturregel und das wurde also meist im Register übersehen oder nicht vom Registrator respektiert. Diese zweite Regel beruht wiederum auf einer soust bisher mit Recht, anch von

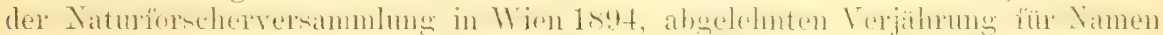
vou "nicht allgemeinem Gebrauch"; aber der "allgemeine Usus" ist für die meisten Namen undefinierbar, also nicht für eine Regel verwendbar. Vgl. auch Botan. Centralblatt LXXIX (1899) S. 409-410. Der nachträgliche Kommentar zur 2. Aprilnomenklaturregel, die Erk!ärung vom 11. Juli 1899 im Beiblatt Nr.63 zu Engler's Botanischen Jahrbücher ändert an der Kuntze'schen Beweisführung sehr wenig, zumal Kuntze ihm statistischen Beweis anbot, dass auch in diesem Falle seine 2. Regel meist nicht befolgt wurde. Die Zumutung Engler's an die übrige botanische Welt ausserhalb seines Museums, dass man gewisse Werke und künftige Nonographien, soweit ihre Nomenklatur von der Engler'schen abweiche, ausschliesse, also auf einen "Index librorum prohibitorum" setzen

*) Manchmal findet sich sogar dreierlei Nomenklatur für dasselbe Objekt; z. B. Sitria Allem. III 2 : 123 im Jahre 1891 = Neositvia Pax im 1897-Nachtrag Seite $174=$ Mezilanrus O. lítze. im Register-Nachtrag 1899 Seite 462!

**) Hiern im Journal of botany 1898 S. 494 schreibt z. B. 'This (Engler's Code of April 1897) is critized in a masterly way. The rule 2 is valiantly resisted by Dr. Kuntze, who, besides arraying powerful arguments of his own against it, quotes adverse opinions of several botanists from widely distributed centres of thought. 
könnte - und das ist die Quintessenz dieser seiner "Erklärung" zur 2. Aprilnomenklaturregel - ist wohl ein allzu grosser und naiver Anspruch Engler's.

Ganz unzulänglich und ein bedenklicher Missbrauch der Unterschreiber der Aprihomenklaturegeln int es, dass diese Regehn trotz mehrfacher Einsprïche

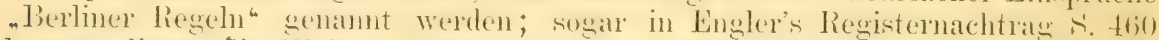

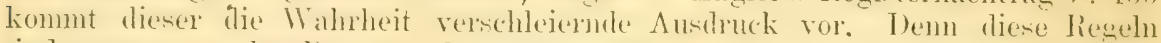
sind auscer von den beanten des berliner Musenus von kemem anderen berliner Botaniker motersehrieben worken. Solche Behauptungen, dass der vier jenter Name nach den Berliner Regeln ungültig oder gültig sei, sind eben nu leere Behauptungen, denen die internationale Yomenklatur nicht ohne Wreiteres folgen dürfte.

Die Besitzer von Engler's N. Pflanzenfamlien mögen gehofft haben, dass die Konfusion, welche Engler in der Nomenklatur dieses seines Werkes durch öfteren Prinzipienwechsel während der Publikation angerichtet hat, durch das Generahegister geklairt werde; aber die 1!1 Fehler, die gegen seine letzte Nomenklatur-Veränderung unkorrigiert stehen blieben, beweisen nicht bloss, dass das Register zur Erkenntnis reiner letzten Nomenklatur ganz mutaglich ist, sondern anch, dass Englex dienen Teil der. Wiscmschaft gar nicht heherseht. Die Nomenklatur ist ein besonderer Zweig der Wissenschaft geworden, denen sich Nichtspezialisten bescheiden zu fügen haben, sonst richten sie eben nur Verwirrung an, und bei Engler ist die Nomenklatur-Terwirrung jetzt so gross, dass er sich selbst nicht mehx darin zurechtfinden und herausfinden kann.

\section{Zu berichtigende und wichtige zu ergänzende Zahlencitate für Genera; alphabetische Satzfehler.}

Acicalyptus III $7: 85$ (nicht 83). Alepyrum II (nicht III). Asteriscodes N. 323. Bahel IV 3 b : 296 zu streichen. Bothriochloa N. 40 (nicht 90). Brasenia N. 157. Bucephaton III 1 : 73 (nicht 72). Camillugenia N. 99 (nicht 199). Campamula N.319. Cyclopterygiam 174 (nicht 173). Dermophyllites + IV 4 : 15. Diplngon 389 (nicht 189). Disisocactus III 6a: 183. Gevardanthus + N.254. Heteranthera II 4 (nicht II 5). Hexastemon + IV 1:64. Horsfieldia BI. N. 166. Jenkinsia 256 (nicht 254). Ixophorus II 2 (nicht II 3). Lechlera 152 (nicht 154). Lindera III 2 (nicht 3). Meconella 138 (nicht 438). Mizopetalum III 5 : 346 (nicht II 4 ete.). Nenga II (nicht III). Neobaronia N.338. Neopringlia + N. 336

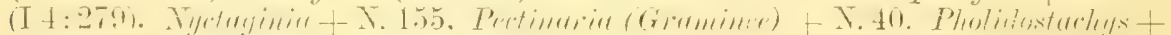
N. 52. Phragmopedilum 98 (nicht 198). Pleuripetalum "Bece." + N. 161. Prionophyllum N.68. Psilostrophe + IV 5: 253. Rinorea 252 (nicht 152). Riothia

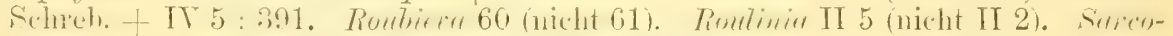
chitus + N.234. Sencletia + IV 4:15. Themistoclesia IV 1: 56 (nicht 64). Thrixspermum Lour. + N. 234. Trichocalyx Koern. II (nicht III).

*) Gute Register enthalten weniger als 1\%o (1 auf 1000) Fehler, z. B. das Petermann'-

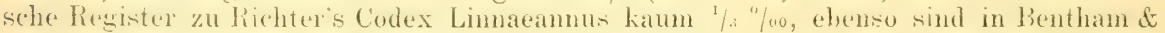
Hooker gen. pl., Endlicher gen. pl., Baillon's Hist. d. pl. nach jahrelangem Gebrauch kaum soviel finden. Bei anderen als Registerarbeiten darf man bis $2 \%=20 \%$ Fehler gestatten. In Zeitschriften, die periodisch pünktlich erscheinen, wird man oft noch mehr Fehler entschuldbar finden; denn diese Korrektur wird vom Autor manchmal gar nicht gelesen oder es wird ihm nicht genügend Zeit dazu oder nicht doppelte Korrektur zu lesen erlaubt.

Register aber müssen möglichst fehlerfrei sein, sonst verfehlen sie ihren Zweck. In Engler's legister aber kommen auf etwa 40000 Namen 2600 Fehler ungefähr, die dem Registrator zur Last fallen, jetzt schon, obwohl verschiedene Namengruppen noch nicht näher geprüft sind. Das ergiebt $\frac{2600}{40600}=65 \%$ mindestens; aber selbst wenn es nur 1/3 soviel Fehler wären, dari man getrost sagen, dass Engler's Register unter den schlechtesten botanischen Registern des vergangenen Jahrhunderts wohl den ersten Platz einnimmt. Dass der verschwiegene Prof. Dalla Torre mur einen 'Teil der Fehler verschuldete, ist ausser Zweifel. Für das Lehrige und auch für das Ganze ist Direktor Engler verantwortlich. 
Diese Zahlenfehler sind relativ so sparsam, dass der Registrator alles Lob verdient, đlenn ganz fehlerfreie Register giebt es überhaupt nicht.*)

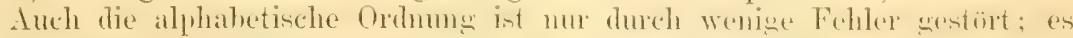

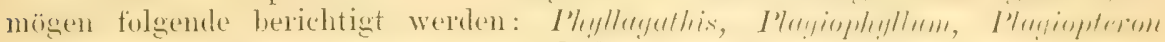
Raphiostyles, Rhodocastim, Royena, Ribus, Sarcotoechia, Siphaeroma.

\section{Fehler bei Sektionsnamen.}

Von den etwa 2100 pluralen Subgenera-, Sektions- und Subsektions-Namen, die in Engler's Werk vorkommen, ist kaum der 4. 'Teil registriert und der Ausschluss dieser ungefäh 1550 Namen ist in keiner. Weise berechtigt. Entweder man schliesst alle plurale solche Namen aus und erkennt damit an, dass-sie neben singularen Sektionsnamen keine Berechtigung haben, oder man muss alle

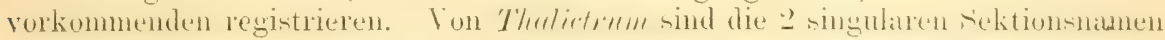
registriert, von den pluralen z. B. Macrocarpa, Platycarpa griechischer Ahatammung, dagegen fehlen die lateinischen semst gleich in Rang stehenden Pluralnamen $\leqq F l e x u o s a, ~ S S p a r s i f l o r a$ u.s.w., atch Dioica, das man lateinisch aber Dioeca zu schreiben hat. Bei $T a m a v i x$ sind z. B. Obdiplandrae und Obdiplostemones registriert, die höher im Rang stehenden, ungeschickten Niedenzu'schen Subgenusnamen Sessiles, Amplexicuules aber nicht. Bei

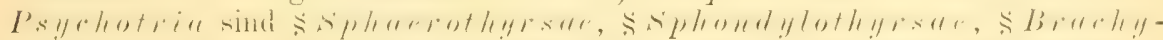

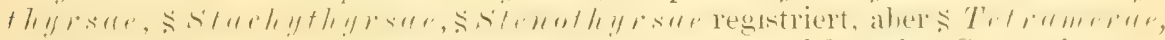
SCephalothyrsae, $\$$ Inumatae, $\$$ Capitatae nicht; eine Separation von lateinischen und griechischen Sektionsnamen ist also weder vorgenommen worden, noch hatte sie irgendwelche Berechtigung. Der plurale Gruppenname von Basilicum, also Basilica ist registriert unter Ocimum, aber SIIantia und Gratissima nicht. Lachnaiocarpae Briq. ist registriert, Leiocurpat und viele andere Briquet'sche plurale Sektionsnamen sind ausgelassen. Der Re-

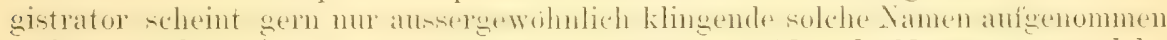
zu haben, oder bei Hieracium. Dendrobium, Bolbophyllum u. s. w. solche

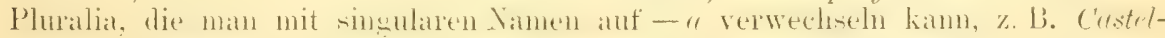
lanina, Auriculina, Eriophara, Batarnoidea, Beceariana u. s. w. Ton den 64 gleichwertigen Sektionsuamen von Mesembrinthemm sind nur 12 registriert, auch die 2 höheren (Subgenera-) Namen Epapulosa und Papulosa fehlen; das ergiebt für diesen Fall allein 5/6 Manco, sodass 1550 fehlende solche Namen in Engler's Register als Ninimalberechnung gelten darf. In einem Register, wo selbst Vermacular-Namen registriert sind, durfte dies nicht vorkommen.

Erörtern wir jerloch einmal im Algemeinen, welche plurale SubgeneraNamen zu verwerfen und durch normale Namen in Singular zu ersetzen sind. Die hoheren stektionen, wie subgenera n. . w. Wekommen sellistintige singulare Namen; dagegen Americanae, Asiaticae, latifoliae u. s. w. sind keine selbständigen

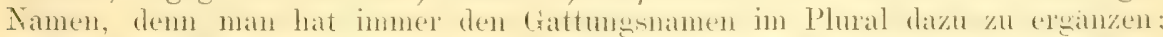

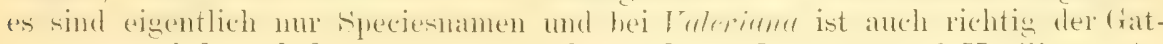
tungsname jedesmal davor gesetzt worden, z. 13 . $\$ V$. montanae, $\$ Y$. ciliatce etc.

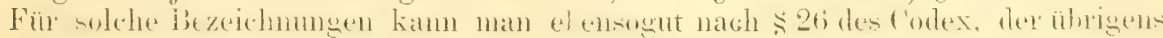
nicht von P'luralia spricht, Nummern oder laufende Buchstaben zur Gruppenbezeichnung oder nichtlateinische Namen wählen, welche nicht konkurrenzfähig mit wirklichen Namen sind. Diese kann man in Registern und Wörterbüchern ganz weglassen. Wo aber Konkurrenz bei Subgenera- und Sektionsnamen eintritt, müssen sie durch normale singulare Namen ersetzt werden; da haben sie keinerlei

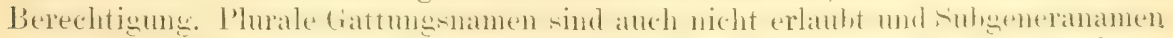
künnen mit Gattungsnamen in Prioritätsfällen konkurrieren; sie konkurrieren auch unter sich, sodass schon aus Prioritätsriicksichten für anerkennbare Sektions-

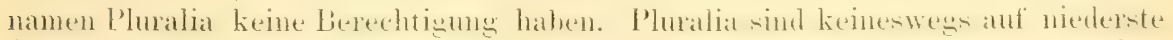
Sektionsnamen beschränkt geblieben, vergl. ausser citierten Tamarix, Mesembri-

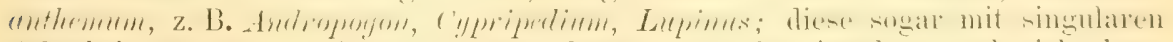
fubselionsnamen. Keineswegn aber düren normale singulare und nicht livilkurenzfähige plurale Namen durcheinander gestellt werden. 
In welch verwirrender Weise die Nomenklatur der Sektionen ausserdem oft behandelt wird, sei nur an einem Beispiel gezeigt. Drude führt in Engler's N. P'flanzennamen merer Piruln 4 Sektionen anf, von denen er 2 unter dem nenen überflussigen, umberechtigten Namen Eupirola Drude zusammenfasste. $\$ 1$ ist

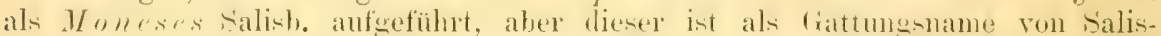
bury in S. H. Gray's Werk 1821 aufgestellt; für eine Sektion hat Endlicher zu-

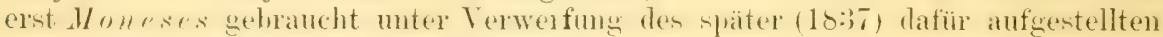
Koch'schen Namens Parnassiodes.

Bei $\$ 2$ citiert Drude zu Actinocyclus, Klotzsch", der diesen Namen 1857 aber für eine Gattung anwendete; man muss daher. $\$$ Actinocyclus Drude schreiben. Als Gattung führen Garcke u. a. diese Gruppe unter den älteren Namen Ramischia Opiz 1852 auf, aber schon 1821 hatte S. F. Gray - wie man auch, wenn man das seltene Werk von Gray nicht hat, in Pfeiffer's Nomen-

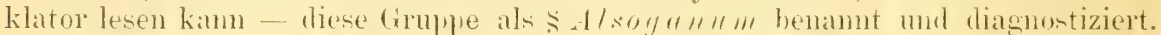

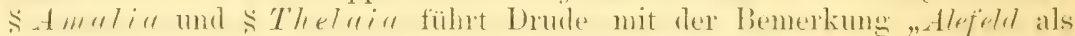
Gattung" auf, sodass man Drude als Autor für diese Sektionen citieren könnte; aber vor Drude wurden sie schon von BHgp. als Sektionsnamen behandelt. Ausserdem hat Amelia Alef. 1856 in Erxlebenia Opiz 1852 einen älteren Namen und für Thelaia hatte S. F. Gray auch schon 1821 einen älteren Namen mit tadelloser Diagnose der Sektion: $O$ rpheus aufgestellt.

Resultat: $3 / 4$ der Drude'schen Nomenklatur sub Pirole absolut unrichtig und zum geltendbleibenden Sektionsnamen Moneses ein unrichtiges Autorcitat. Ausserdem fehlt bei Drude der Name der S Scotophylla Nutt., die auf die merkwïrdige Pirola aphylla L. basiert und welche noch am ehesten generisch abzutrennen ist, da sie sich der Saprophyten-Unterfamilie Mo notrop ea nähert. Es ist bemerkenswert, dass je willkurlicher ein Autor wie Drude mit der Nomenklatur ums suringt, dass el sich (Vergl. auch Kuntze's Rev. gen. III 2:322--323).

Im Engler'schen Werke sind aber von den mehr als 4600 singularen Sektionsuamen nach bisherigen Erfahrungen der Revision etwa $\mathrm{J} / 4$ unrichtig und die Hälfte mit falschen gegen $\$ 51$ des Corlex verstossenden Autorcitaten versehen, sodass mindestens 3300 Fehler bei solchen Namen vorkommen. Bei der

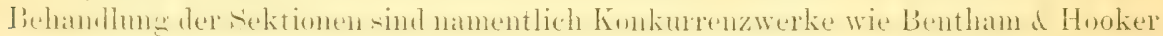
g. pl. und Baillon's histoire des plantes, worin zahlreiche Sektionen zum ersten Hale aufgestellt wurden, tapfer benützt worden, ohne dass diese Quellen als Autoritäten der Sektionsmamen citiert wurden!

Wir werden den ausführlichen, ohen nur exemplifizierten Beweis hierfür in einem besonderen Werke nächstes Jahr erbringen. Es ist richtig, dass manche andere Autoren ehenso unrecht verfuhren in solchen Fïllen wie Drude und Engler, und es zumteil noch thun, aher der Pariser Codex soll eben diesen faulen Zuständen ein Ende machen. Für Einschaltung dieses Kapitels bin ich Herrn Dr. Otto Kuntze verbunden; ich selbst hatte die Subgenera- und Sektionsnamen im Register nicht kontrolliert.

\section{Defecte betreffs Artencitation.}

Es fehlen meist Hinweise auî zerteilte Genera, die durch Citation der synonym gewordenen 'leile oder deren Arten nötig sind. Z. B. von Eryth ophlocum lat Harms zwei neue Genera im Nachtrag 191-192 abgetrennt, ohne dass unter Erythrophloem dies im Register angegeben ist. Von Panax III 8 : 58 wurde Nothopanax abgetrennt und die fiederblattrigen Arten wurden zu Polyscias ge-

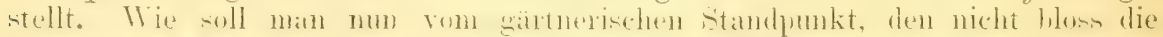

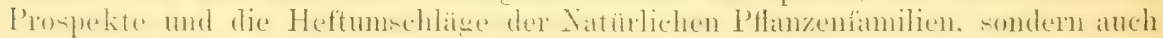
die Jotive 21 den Aprilnomenklaturregeln besonders betonen, finden, dass die unter dem Namen Panax aborenm Forst. und Panax sambucifolium. Sieh. kultivierten Pflanzen, die auch so im Kew Index gelten, jetzt Nothopanax arboreum Seem. 1. c. III 8: 48 und Polyscias sambucifolius Harms 1. c. 45 heissen? Es ist doch lästig, 34 Seiten auf Aialiaceen-Arten deshalb durchzusuchen. 
Im Register sind ausserdem trotz der für die Praktiker warm empfohlenen specieshearteitungen in den Natürlichen l'flanzenfimilien doch in dem Register mur die wenigen Artemnamen der ahgebildeten species anfgentmmen worlen, wo dass dieses Register für diese Praxis ziemlich geringwertig ist.

Im Register sind manchmal die Seitenzahlen für giltige Namen und für Synonyme deselben Namens in getrennten Zeilen gegehen worden: haintiger aber sind Arten betreffende Synonyme, die also damn zu anderen Genera gehören, mit dem giltigen Gatumgsnamen anf einer Zcile kmnfus registriert worden: ich kïmnte davon eine lange Liste mitteilen; es sind mindestens 200 Fälle. Wenn beide auf eine Zeile zu stehen kommen, müssen die Citations-Zahlen für giltige Arten desselben Namens durch anderen Druck oder Sternchen hervorgehoben werden. Für ein solches Werk ist es aber wohl besser, die im 'lext vorkommenden ansgeschiedenen Arten desselben Gattungsnamens auf einer besonderen Zeile als synonym zu registrieren, wobei dann der synonyme 'Teil, weil die Arten oft zu mehreren Genera gehören, kein Autorcitat zum Gattungsnamen zu führen hätte. Sollen also im noch ausstehenden und erneuerten Register nicht alle Arten mit Namen registriert werden, so müssten wenigstens die Seitenzahlen der vorkommenden Synonyme auf besonderen Zeilen registriert werden, wemn dadurch auch mindestens 1000 Zeilen mehr im Register entstehen, die jetzt eben fehlen.

Die Wissenschaft muss fordern, dass ihr ordentliche Register geliefert werden; die Gesamt-Register der Pflanzenfamilien II-IV können auf Zuverlässigkeit und genügende Ordnung keinen Anspruch machen. Das Anfertigen von

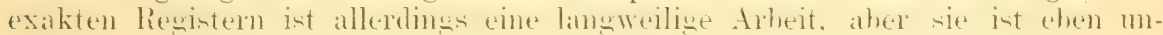
entbehnlich für die Wissenschaft und darf nicht untergeordneten Kräften überlassen werden. Diese langwierige Arbeit lässt ausserdem oft dem Autor manche seiner Fehler finden, was einem fremden Registrator selten moggich ist, und sie

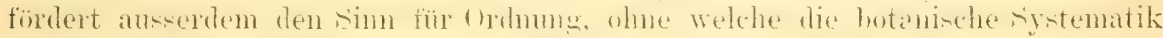
und internationale Harmonie nicht existieren kann.

Ordnung soll für die "Natürlichen Pflanzenfamilien" betreff's der massenhaft fehlende Citate und Synonyme noch durch ein Nachtragwerk: „Genera Siphonogamarum" durch De Dalla Torre und Harms herheigeführt werden. In der 'That halten die Pflanzenfamilien diesbezüglich nicht recht einen Vergleich mit Bentham \& Hooker's "Genera plantarum" und Baillon's "Histoire des plantes" ans, worin sich mehr als 50 Mal soviel Citate von Publikationsdaten jeder Gattung, ihrer Arten, Abbildungen und Synonyme finden. Aber indem Bentham, Hooker,

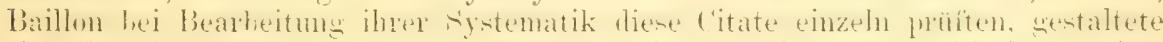
sich ihre Systematik oft anders, während hier an ein derart fehlerhaft aufgebautes Ganze das gerügte Fehlende, die Citate und Synonyme, nur so weit ausgesucht werden, dass das Falsche nicht geändert werde. Das scheint mir wissenschaftlich verkehrt und gar nicht richtig zu sein.

Es ist besser, erst ordentliche Register*) zu liefern und damit das bisher Geleistete fü̈ sich und andere verdaulich zu machen, überhaupt weniger und ordentlich wissenschaftlich zu prorluzieren, als auf ebenso unortentlicher wie will-

*) Namentlich für Engler's Botanische Jahrbicher in nun 27 Bänden, die sonst systematisch nur zum kleinsten Teil verwendbar sind. Es wird jetzt eine Liste der netuen Arten aus 25 Bänden publiziert, aber mit dieser blendenden Liste wird das Bedürfnis der notwendigen Registration nur wenig erfüllt. Um das nur an 1 Beispiel zu zeigen, sei auf Hallier's Convolvulaceen-Arbeiten XVI : $453-591$ und XVIII : $81-160$ hingewifsen. Ton diesen 268 Seiten werden also nur die 47 neuen Arten registriert; auf die Begründung der neuen Genera (zumteil ohne neuen drten) wird in diesem Register keine Rücksicht genommen; das IVichtigste der Arbeit werden die \pm 500 Speciesidentifikationen Hallier's bleiben, gerade weil die Gattungsbegriffe in dieser Familic so sehr verschieden bei den Autoren sind. Wenm num Jemand über Convolvulaceen srlocitet, muss er wegen jeder Art womüglich 100 Seiten durchblättorn, weil die Arten

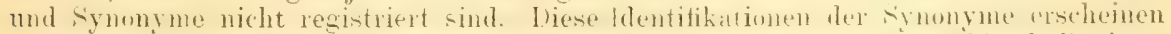

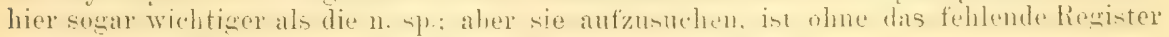
wegen des erforderlichen Zeitverlustes fast unmöglich. 
Jiblicher Jiasis klamkhatt weiter zu arheiten mol - fügen wir noch nachträglich, aber nachdrücklich hinzu -... durch bedenkliche Nassenproduktion zu blenden.

Ordnumg in der botanischen Nomenklatur und internationale botanische Harmonie kamn nur durch strenge Befolgung der simngemäss emendierten internationalen Regeln des Pariser Codex von 1867 mit dem ökonomischen und wissenschaftlichen Anfang für Genera mit 1737 gedeihen.*)

Bis jetzt sind mehr Differenzen zwischen 'l'ext und Register des Engler' -

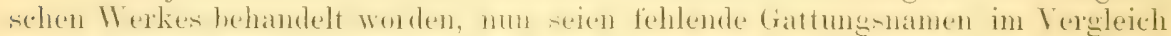
mit anderen Werken hervorgehoben.

\section{Fehlende Namen von Gattungen unsicherer Stellung aus dem Kew Index.}

Es hätten wenigstens solche ergïnzt werden sollen, welche dort als fettgedruckte Gattungsnamen gelten oder noch unsicherer Stellung, bezw. non satis nota sind. Es befinden sich auch darunter nenere bis 1885 publizierte, aber in Bentham \& Hooker gen. pl. zumteil übersehene, wohl giltige Gattungen. Noch manche vernachlässigte Gattung von I,oureiro, Aublet etc. hätte von Monographen im British Nluseum of nat. hist., resp. in Paris, in Leyden etc. durch Revision der Originalexemplare aufgeklärt werden sollen. Selbst im Willdenow'schen Herbar im Berliner botanischen Nusemm lif gen noch unaufgeklärte Genera, und wie z. B. P'ntarhaphis HBK. = Strombodurus W. erst vor Kurzem Aufklärung fand, so ist das auch noch von Dicaryum W., Epactium W. zu erwarten. Klotzsch's Gattungsnamen aus Schomburgk's Fauna und Flora von Britisch Guiana sind wohl unbeschrieten, also Nomina nuda, ebenso die von Bowdich, aber sonst sind viele folgender Genera non satis nota bloss vernachlässigt. Bentham \& Hooker haben ja meist nur anf Kew-Material basiert; Baillon hat ziemlich viel solcher Gattungen aufgeklärt, aber die anderen kontinentalen Botaniker sollten doch das ihnen bequemer Erreichbare aufarbeiten.

Acophorum Gaud., Amaraboya Linden, Amorphocalyx Kl., Amgadenia Miers, Angiopetalum Reinw., Autelaed Gaertn., Apaputu Gris., Aptotheu Miers, Areynospermem Turcz., Arisacontis Schott, Asciadium Gris., Aspidandra Hask., Atelandra Bello, Aulacorhymelus Nees, Axiu Lour., Bulangue Gaertn., Banjolea Bowd., Bargenontia Gaud., Barreria L., Baryxylum Lour., Beloanthera IJask., Bembix Lour., Bouzetia Montr., Bmea Gaut., Bruxanetia Demst. Calycotropis Turez., Campylus Lour., Catonia P. Br., Catostemma Bth., Cattleyopsis Lem., Celsa Vell., Cerberiopsis Vieill., Cerium Lour., Cervia Rodr., Chamaeyeron Schrenk, Chambeyroniu Vieill., Chibaca Bertol., Chomanthus Phil., Cleobula Vell.. Coelopyrum Jack, Coptocheile Hfg., Corallospartium Armst., Courimari Aubl., Covilhamia Korth., Curinila R. «S., Cycnia Griff., Cylindria Lour., Cystostemon Balf. 1., Dadia Tell., Daniela Mello, Dendrosma Panch. \& Sebert, Diastatea Scheidw., Dicaryum W. ex R. « S., Didymeles Thouars, Distomanthera 'Turez., Dodecadia Lour., Dolichosiphora Phil., Donzellia Ten., Doyerea Bello ,Grosoudy", Drebeetia Zoll. 1857 non 1816, Dugezia Montr., Endocellion 'l'urez., Endoponon Raf,, Endosteira 'Turcz., Entrecastearxia Montr., Epactinm W., Epatitis Raf., Ephobopodon Nees \& Meyen, Eriolithis Gaertn., Enucaria Cerv. 1870, Eteriscius Desv., Euphroniu Vell., Eurystyles Wawra, Evacidim Pomel, Gabila Baill., Guuropsis Presl, Geruma Forsk., Giganthemm Welw. Gigliolia Rodrig., Gomianthes A. Rich., Gonocitrus S. Kurz, Halmolobos Tausch, Haskertia Neisn., Haynea Schum. \& Thom., Hemitria Raf., Heterapithmos 'Turez., Holoplenn Rgl. ¿ Schmalh, non Casp., Hygea Kl., Hygen Hanst., Hypnoplyglanthus Rgl., Koenniclea Kl., Kumlienia Greene, Kunzmamiu Kl., Lachnostachys Hk.**), Lasianthemm Kl., Lencymmaea Presl, Lepidocroton Kl.,

*) Cfr. Otto Kiuntze: "Die Vorteile von 1737 als Nomenklatur-Anfang." Gärtnerisches Centralblatt 1899 Nr. 2 und in der Allgem. Bot. Zeitschr. 1899 Nr. 4.

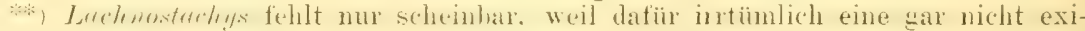
stierende Lachnocephalus Hk. steht; Lachocephalus Lepidotus 'l'urcz. gehört aber nicht zu dieser Gattung, sondern ist nach Bentham etc. = Mallophora globifera Fndl. 
Lepidospora F. v. M., Lorentzia Hieron.*), Loureire Meisn., Mracrochatium Steud. (efi. Durand it Schinz Consp. H1. afr.), Whanothrix Miers, Mussoiu Bece., Helanococce Bl., Meristostylis K1. (Gentianac.), Nicrolobius Presl, Moschop(s) is Phil., Munbye Pomel, Nephrostigma Griff., Octas Jack, Denosciadium Pomel, Perengenipe Baill., Peltostegia Turcz., Petrusia Baill., Phyllopidium Raf., Physopodium Desv., Piptosaceos T'urez., Plegerina Arruda it Koster, Potamobryon Liebm., Pterotum Lour.. Quaqua N. E. Brown, Reynoutria Houtt., Gm., Ribeirea Arruda, Riqueiria R. \& P., Roebelia Engel (non Engelm., non Engler), Ropourea Aubl., Rumplia L.. Sarcocodon N. E. Brown, Schieckea H. Karst., Schtschurowshia Rol. \& Schmalh., Selenocera Zipp., Semenovia Rgl. \& Herd., Serresia Montr., Sherboumia Don (= Amaralia Welw.), Singana Aubl, Solenospermem Zoll., Sonzaya Marchand, Spilocerpus Lem., Stelechospermum Bl., Stenocalyx Turcz., Stenostachys Turez, Tachibote Aublo, Tampor Aubl., Tapirocarpus Sagot, Tayotum Blanco, Thamnoseris Phil., Thorwaldsenia Liebm., Thuraria Mol., Thysanus Lour., Temnadenia Miers, Tephea

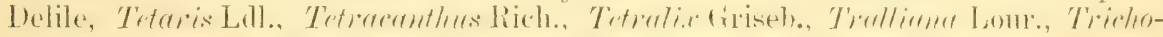

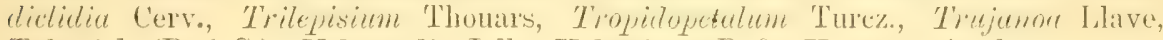
Tula Ad. (R. \& S.), Valcarcelia Ludl., Valentience Raf., Votomita Aubl.

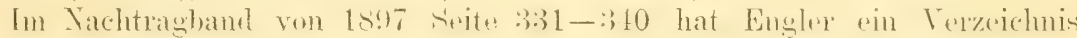
von Phaenogamen-Gattungen unsicherer Stellung hesorgt. Wie fürchterlich lücken-

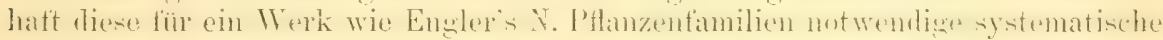
Arbeit ist, beweist vorstehende Liste. Es ist das Aufklären **) solcher Gattungen

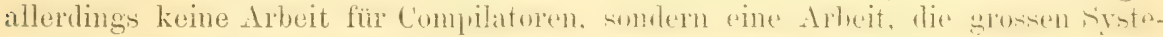
matikern als Prüfstein dient.

\section{Fehlende Namen aus Otto Kuntze's Revisio und Hiern's Werk.}

In Rev. gen. IIIII Seite 101-125 der Einleitung sind solche legal reformierte

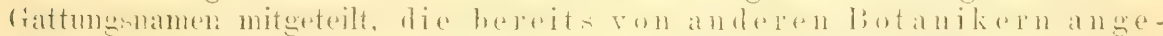

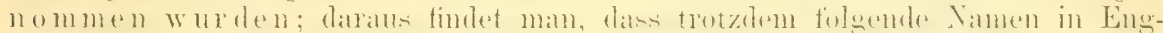
ler's Werk fehlen; es sind ausserdem noch einige Namen aus Kuntze's Register ergänzt. [Kuntze's Rev. I-II ist in Engler's Werk ausgiebig - wenn auch oft verkehrt - benutzt.

Anidrum Neck. (Umbellif.), Arduina Ad. (Umbellif.), Benthamantha Alef., Bichea Stokes, Calappa Rumpf, Cammarum Hill, Capnorea Raf., Cicendiopsis O.Ktze., Cuminum L. 1735 non 1742 (Umbellif.), Denisia Neck. [corr. ex - rea, eia], Dolicholus Med., Dondia Ad., Fritschiantha O. Ktze., Furera Ad., Galpinsia Britton,

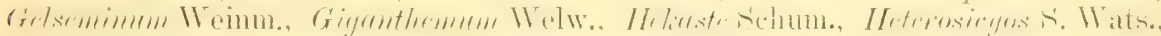

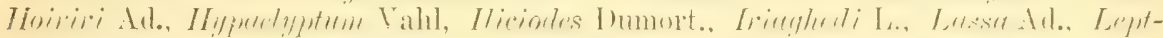
orchis 'Thouars, Levchea Hall., Lilaeopsis Greene (Umbellif. = Hallomïllera O. Ktze.,

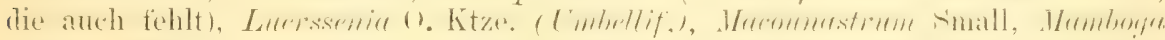
Blaneo, Marsea Ad., Myrrodes Fabr. (Umbellif.), Mystacime Rai., Nymamia Lindb., Oxytria Raf., Perfoliata Burm., (Umbellif.), Phryminm Loefl., Piaropus Raf., Pneumaria Hill, Pogomesia Raf., Psendechinopepon Cogn., Ptyxostome Vahl, Pyxidaria Hall., Quamasia Raf., Richtera Rehb., Seemamia Hk., Sherbournia Don, Spermacaceodes O. Ktze., Solulus Rumpf, Tephrothammus Sweet, Wagnera Ad., Ziggia P. Br.

*) Lorentzia Hieron. dürfte im Berliner botanischen Musem vorhanden sein und ist wohl nur ubersehen worden, weil das dem Huseum gehörige Herbarium Hieronymus in dessen Piivatverschluss gehalten wird, anstatt ordnungsgemäss ins Hauptherbarium inseriert zu werden. Fremde Botaniker mögen nicht, wie ich es gethan, nach Berlin gehen, um seltene Argentiner Pflanzen zu studieren, denn das wird ihmen dort fast unmöglich gemacht. Wie Larentzia Hieron. beweist, bleiben diese selbst Engler verborgen. Otto Kuntze.

**) Bei Aufklärung dieser 163 Genera neglecta darf selbstverständlich nicht das unwissenschaftliche und willkürliche, selbst in den fimosen Aprihomenklatur-Regeln Engler's verleugnete, von ihm früher befürwortete Prinzip der ,ordnungsgemaissen Diagnose", wonach Nomina seminuda = Nomina nuda seien, angewendet werden, cfi. Botan. Centralblatt LXXIX 1899: 407. Diese Berliner Botaniker haben es bekanntlich fertig gebracht, den Namen mancher sehr gut beschriebenen und abgebildeten neuen Gattung von P. Browne 1756 als angebliches nomen nudum zu verwerfen!! 
Diesen lassen sich noch mehreve von Hiern im Catalogue of Welwitsch's African Plants I-III erneuerte Namen hinzufügen, welche zumteil aus dem Engler'schen unhaltharen Standpunkte 175:3 für Genera-Anfang resultieren, z. B.

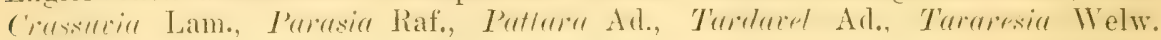
Einige sind schon oben erwähnt. Hinzu kommen noch von Hiern's nova genera: Epinetram, Campilochiton, Chalarocarpus, Justeniu, Pentacarpea.

J)iese offenhar absichtliche, aher mwissenschaftliche Vernachlässigung der Tomenklatur sehr vieler und zumteil recht namhafter botanischer schriftsteller, die noch dazn in gewissenhafter Ansführung der Prioritä nach internationalen oder konventionalen Regeln handeln, seitons Engler, der angeblich nach privaten Regreh seine Nomenklatur ron Zeit zu Zetit willkürlich ämlerte, macht sich auffallend erst in den letztialnrigen Heiten seiner "Natürlichen Pflanzenfamilien", namentlich bei den ['mbelliferen und hei den hiryptogamen traurig bemerkhan.

Um das in mancher Hinsicht hervorragende Werk Engler's, das gerade, weil es in der /e it der grössten nomenklatorischen Reformation pulliziert wurde, in Nitleidenschaft gezogen ward, möglichst wieder gesund zu machen, müsste an Schluss ein so wie so nötiges Gesamtregister für Phanerogamen und Kryptogamen gegehen werden, in denen die manenhaften tehler des Teihegisters für Band II-IV zu vermeiden wären; eines 'T'eilregisters, in dem Engler selbst nicht imstande war, in hunderten ven Fibllen anzugeten, welcher (rattungsname eigentlich silt oder nicht. Wie soll sich da ein Dritter in diesem Wirmis zurechtfinden?

Nanche Autoren liefern Korrekturen im Register als Fussnoten. Da aber die Korrekturen hier zu zahlreich sind, so müsste vor dem Gesamtregister erst ein Terzeichnis aller korrigierten Gattungsnamen geliefert worden, wobei die gesetzmässige internationale Nomenklatur anzuwenden wäre.

Robert Brown zog*) seinen berihmenten, jetzt in der Originalausgabe äusserst

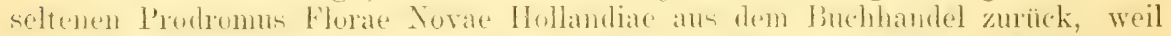

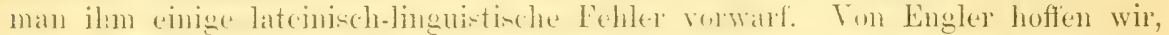
dass er sein Register zu Band II-IV durch ein korrektes Gesamtregister für Band I-IV ersetzen und damit den Käufern des Werkes ein brauchbares Register zu entsprechend billigerem Preise liefern werde; denn das kaum brauchbare Register kostete 18, bezw. 21 \% Mark.

\section{Statistik.}

Zum Schluss gebe ich eine zahlengemässe Uebersicht, wozu ich bemerke, dass noch mehr solcher Corrigenda zu finden sein werden, da ich einerseits eine Anzahl dubiöser Fälle hier ausser Acht liess, andererseits die Arbeit nicht zu dem Zwecke gemacht wurde, die Fehler herauszusuchen, sondern nur, um die Teilregister zu ergänzen.

Falsche Angaben von Familiemamen und über Familienzugehörigkeit laut 1. Liste

$\begin{array}{cc}\text { Citierte und } & \text { Nur } \\ \text { berechnete } & \text { angedeutetc } \\ \text { Fille } & \text { Faille }\end{array}$
angedeutetcolle
Fialle

Register-Fehler betreff Familiennamen laut $\$ 2$.

Autorcitatfehler bei Gattungsnamen laut $\$ 3$ : Terwechselungen 57 ; vorlinnéische alleinstehende Citate 58 (Synonyme +180 ); verspätete Autorcitate \pm 300 ; allemstehende Citate von Manuskriptautoren 68; komische Autorcitate 30

Alphabetstörende, namenändernde Schreibfehler laut 4. Liste w\%-Fehler laut Fussnote zu $\$ 4$.

Kleinere Namenfehler

Teränderungen von Auslautsilben laut こ̌. Liste

Nicht registrierte Gattung-namene lant di. Liste 
Falsche Angaben über Gattungsgültigkeit im Widerspruch zu dem 'Text laut Liste 7 a uud 7 b

Citatzahlen-Korrekturen und alphabetische Satzfehler

$\begin{array}{cc}\begin{array}{c}\text { Citierte und } \\ \text { berechnete } \\ \text { Fille }\end{array} & \begin{array}{c}\text { Nur } \\ \text { angedeuteto } \\ \text { Finlle }\end{array} \\ \text { Uebertrag } 844 & 480\end{array}$

Citierte und Nur
berechnete angedenteto

Fehler bei Sektionsnamen laut $\$ 9$

1. im Register fehlende Namen berechnet

365

51

2. unrichtig Autorcitate nach \$51 des Pariser Codex

1550

3. falsche Selitionsnamen . . .

Konfuse Registrationen von ausgeschlossenen Teilen unter

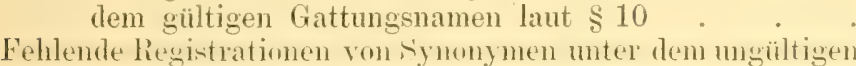

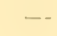

2200

1100

Fehlende gültige trattungsnamen, liezw. von Genera incertate sedis aus dem Kew Index

Fehlende gültige Gattungsnamen anderer Autoren . . 59

\section{- $\quad 200$}

$-\quad 1000$

\section{3}

zusammen 8173 Fehler: $\overline{3193+4980}$

Hierzu die von Otto Kuntze im 1. Abschnitt unserer Dissertationen mitgeteilten Fehler:

Unrichtige Familiennamen bei Phaenogamen . . 53

Unrichtige Gattungsnamen im ganzen System etwa . 940

Unrichtige 'Tribusnameñ bei Phaenogamen . . 68

Falsche Suffixe bei T'ribusnamen

\section{+ obige Fehler 8173}

1. Serien Fehler in Engler's N. Pflanzenfamilien und Register -9315

Berichtigungen (Zahlen in [] für den Sonderdruck):

Seite 114 [4] Zeile 20 [41] von oben lies: Tiolacées Vent. 1799

, $114[4], 21[42],$, , Ionidicecre

" $114[5], 2[34]$, unten " Cryptogamia" nom corr. Bernh., anstatt em. excl. Palmae ... (welche von Limné als incertae sedis den Kryptogamen nachgesetzt wurden).

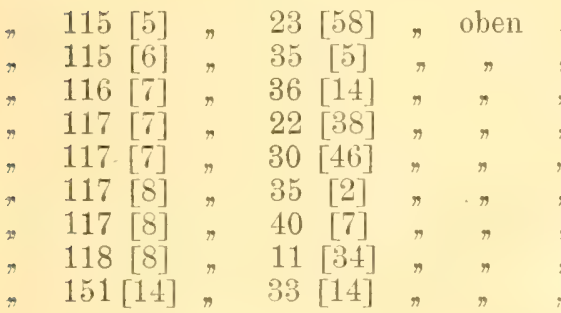

"Phanerogamia"

$\mathrm{I}+\mathrm{III}=$ Polycotyledones

Sede

Don 1826

Onargrae Ad.

Nutt. 1818

Ad. 1763

fuir +1700

Oliv. ist weder Caprifoliacee noch Celastivacee

" $151[14], 45$ [26] " " ergänze: Blepharipappus Greene ist nur Composite, nicht auch Graminee.

" $154[17], 3[31]$, unten lies: Caryopluyllineae.

Im Dictionnaire inédit des Herbarium De Candolle findet sich eine Bemerkumg von A. IC'. worauf mich der Custos Herr h. Buser anfinerk-am machte,

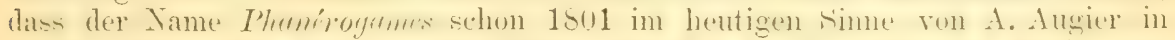

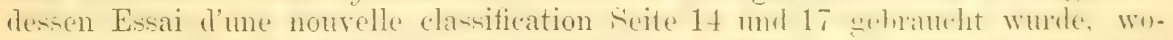

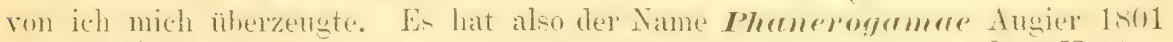
die Priorität ron 3 Jahren über Phaenogamae Reb. \& II. 1S04. Otto Kuntze. 


\section{Botanische Litteratur, Zeitschriften etc.}

Gauchery, Paul, L'ntesuchungen äher den „Xanismus der I'flinzen (Amnales des Sciences naturales 1899 . VIII. Bot. 'T. 9 . pag. 61 u. ff.).

Verfasser hefasst sich in seiner vorliegenden morphologisch-anatomischen Arbeit mit dem konstitutionellen Nanismus (Zwergwuchs). Während dieser auf uns noch unbekannte, aber sicher bereits im Samen gegebene Gründe zuriickzuführen ist, ist es andererseits bekannt, dass alpine und nordische Z Zwergpflanzen durch Einwirkungen äusserer Art, z. B. das kalte Klima, den Boden, entstanden sind. Ton diesen natürlichen zwei Arten des Zwergwuchses ist schliesslich der künstliche zu unterscheiden, den wir dem Gärtner verdanken und der auf die Atrophie einiger oder vieler Organe zurückzuführen ist. Wir wollen die beiden ersten Arten des Nanismus vergleichend betrachten. Bei alpinen Z wergp fla nzen sind die unterirdischen Teile stärker entwickelt als die oberirdischen, die Nittelstämme sind stärker verzweigt als bei der Normalform, die Internodien zahlreich, aber kurz; die Blätter sind zahlreich vorhanden, so beschaffen wie bei der Normalform, nur natürlich kleiner und dicker. Infolgerlessen sind numerisch mehr Spaltöffnumgen vorhanden. Die Bekleidung mit Haaren ist eine reichlichere. Die Zahl der Blüten ist eine bedentende, die letzteren zumeist nicht kleiner als bei der Normalpflanze entwickelt. Der anatomische Bau der Zwergformen gleicht im allgemeinen dem der Normalpflanzen, nur sind die Schntzgewebe stärker und namentlich das Pallisadengewebe reichlicher entwickelt. - Ganz anders verhalten sich Pflanzen, die konstitutionellen Nanismus zeigen. Verfasser macht namentlich auf folgende Punkte aufmerksam: Die Gesamtheit der oberirdischen Teile ist weniger reduziert als die unterirdischen T'eile, die Internodien sind verküuzt und in geringerer Zahl vorhanden, der Stengel nicht sehr verzweigt. Blatt und Blüte sind reduziert, z. B. sind die Blitter rund eingeschnitten. So findet sich häufig an den Zwergen nur die Form der Primordialblätter, wenn die entsprechenden Riesenformen mehrere Blattformen aufweisen. Die Blütenstände sind wenig verzweigt. Während aber die Samen und die Blüten der Zwergform absolut genommen kleiner sind, als die des Riesen, so ist doch die Grösse der Blüte im Verhältnis zur Gesamtgrösse der Zwer'form sehr auffallend. Einige sehr interessante Ergebnisse zeigt die an atomische Untersuchung der Zwerge: Die Zahl der Gefässbundel ist eine geringere, die Gefässe zeigen stets eine grosse Rerluktion. Das Rindengewebe ist am Stengel mächtiger (auch in absoluter Hinsicht) entwickelt, als bei den grossen Pflanzen. Die Endodermis ist gut ausgeprägt, das MIark ist in relativer Hinsicht mehr entwickelt als bei den Riesenformen. Dafür aber ist das Rindencollenchym, der Pericykel, das secundäre Holzparenchym sehr schwach entwickelt. Jie Wurzehn zeigen ganz analoge Abweichmgen wie die Stengel. Alle secundären Bildungen, z. B. Periderm, secundäres $\mathrm{Holz}$ sind nie so mächtig entwickelt wie bei den Riesenpflanzen. Nan sieht daraus, dass der primäre Bau durch Sklerose der Elemente fixiert wird. Der Bau der ausgewachsenen Zwergptlanzen entspricht einem geringeren Stadium der Riesenpflanzen "mit gleichsam sklerosierten Geweben." Die Zwergpflanzen stellen daher keine "Miniaturen " der Art vor; sie besitzen ihre besonderen Eigenschaften und sind gegenüber den Riesenformen nicht proportional reduziert.

M a to uschek (Ung. Hradisch).

Desterreichische bot. Zeitschrift 1900. Nr. 8. Schiftuer, IV., Kritische Bemerkungen über Jungermannia collaris. - Celakovsky, L. 'J., Die Ver' mehrung der Sporangien von Gingko biloba. - Nagnus, P., Eine Bemerkung

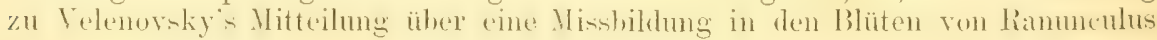
acris. - Schlechter, R., Acriopsis und ihre Stellung zu den Podochilinae. W ettstein, R. v., Die nordamerikanischen Arten der Gattung Gentiana, Sekt, Endotricha. - Bubák, F., Zweiter Beitrag zur Pilzflora von Tirol. - Lit. teratur-Uebersicht. 
Deutsche bot. Monatsehpift. 1900. Mr. 7. Suksdorl, Makhingtonimelu

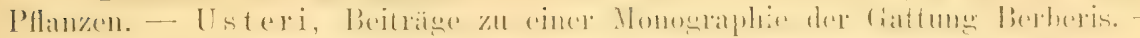

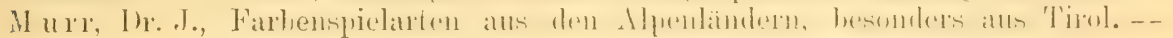
Jacobasch, E., Neuere Beobachtungen über Lanosa nivalis, den Schneepilz. -

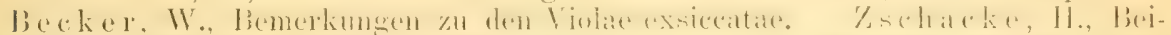

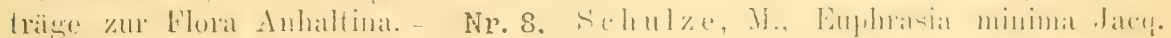
in Thüringen. - M urr, Dr. J., Wie in vor. Nr. - Geisenheyner, Is., $A b$ norme Orchileenblüten. - Goverts, W. J., Mykologische Beiträge zur H'lora des Harzes. - Blümml, Dr., heferat über Pöverlein: Die baycrischen Arten, Formen und Bastarde der Gattung Potentilla. - Becker, W, Wie in vor. Nr.

Botan. Centralblatt. 1900. Nr.26. Nïller, Karl, Vorlüufige Bemerkung zu einer Monographie der europäischen Scapania-Arten. - Nr. 27. Lö vinson, Os kar, Ueber Keimungs- und Wachstumsversuche an Erbsen in Lösmgen von

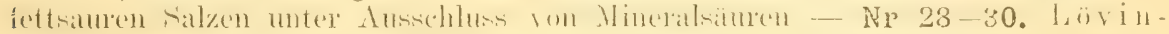
son, Oskar, Wie in Nr.27. - Nr. 31. Lövinson, Oskar, Wie in Nr. 27. Kosaroff, Dr. P., Die Wirkung der Kohlensäme auf den Wassertransport der Pflanzen, -- Nr. 32. Küster, Ernst, Bemerkungen über die Anatomie der

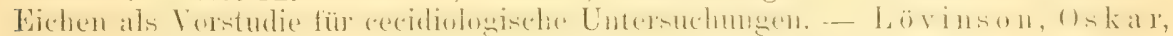
Wie in Nr.27. - Nr.33/34. Lövinson, Oskar, Wie in Nr.27. - I3rand,

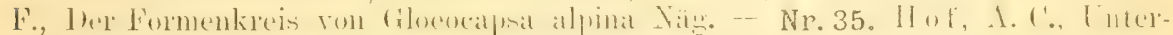
suchungen über die Topik der Alkiliverteilung in ptlanzlichen Gewehen. Brand, F., Wie in Nr.33/34. - Fischer-Benzon, R.v., Zur Geschichte des Kürbis.

Verhandlungen del k. k. zoologisch-bot. Gesellschaft in Wien. 1900. Heft 6. Ma touschek, Prof. Franz, Die 2 ïltesten bryologisehen Exsicentenwerke aus Böhmen. - IV a gn e r, Rud., Zur Anisophyllie einiger Staphyleaceen. Strasser, P. Pius, Pilzflora des Somntagsherges (Nied. Oest.). - Wagner, Rud., Zur Morphologie von Dioscovea auriculata Poeplp. - Brunnthaler, Jus., Plankton-Studien, - Heft 7. Straser, P. Pius, Wie in vor. Nr. - Matouschek, Prof. Franz, Dr Alois Poech"s, Musci botanici". - Brunntlialer, Jos., Plankton-Studien.

Beriehte der deutsehen bot. Gesellsehaft 1900. Heft 6. 'I' 4 chermak, H., Ueber künstliche Kreuzung von l'isum sativum. - Foslie, M., Die Systematik

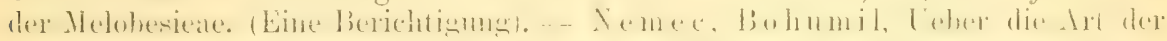

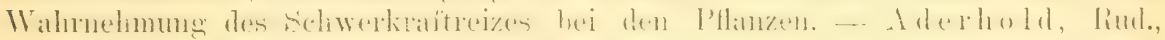
Mrycosphaerella cerasella n. sp., die Perithecienform von Cercospora cerasella Sace. und ihre Entwickelung. - U le, E., Verschiedene Beobachtungen vom Gebiet der

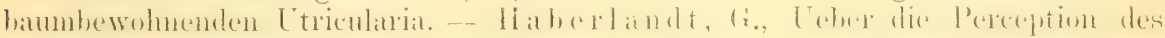
geotropischen Reizes. - L e m m erma n n, E., Beitrag zur Kenntris der P'lankton-

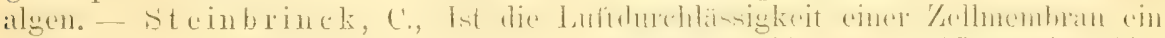
Hindernis für ihre Schrumpfelung? - L'rianischnikow, D., Ueber den Einfluss der 'T'mperatur auf die Energie des Eiweisszerfalles. - Z ' a leski, W., Zur Aetherwirkung auf die Stoffumwandlung in den Ptianzen.

Helios, 17. Band. 1900. Usteri, A., '/usammenstellung der Forschungen über die Reizerscheinumgen an den Filamenten von Berberis.

Botanieal Gazette 1900. Vol. XXIX. Nr.6. Smith, Ralf E., liotrytis and Scherotinia: Their relation to certain plant diseases and to each other. - Con rad, Abram H., A contribution to the history of Quercus. - Hill, H. J., Floria of the white lake region, Michigan, an its ecologieal relations, - Vol. XXX. Nr. 1. Johnson, Dunean S., On the endosperm and embryo of Peperomia pellucida. Cardot, J., and Thériot, J., New or mrecorded mosses of North America. Wiegand, Karl M., The development of the embryo-sace in some monocotyledonous plants. - Nr. 2. Timberlake, H. G., The development and function 
of the cell plate in higher plants. - Moore, George Thomas, Contributions from the cryptogamic laboratory of Harward University: New or little known unicellular algae. I. Clorocystis Cohnii.

Bulletin de l'académie internationale de géographie botanique. 1900. N. 129-130. Spalikowsk, Dr. Ed., Encore le Gui. - Capoduro, Mar., De

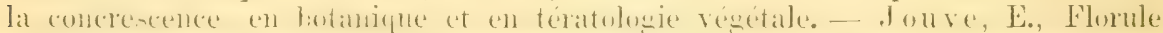
de Montmurat (Cantal). - P'etitmengin, M., Sur l'adoptation aux sols calcaires des plantes silicoles. - Castanet, Les plantes utiles de la Mayeme. II onguillon, E., Catalogue des Lichens du département de la Sarthe.

Bulletin de l'association Française de botaniq e 1900. Nr. 32-33. Gagne pa in, Sur une nouvel hybride artificiel: Oenothera suaveolens $>$ biennis. Sudre, H., Excursions batologiques dans les Pyrenées. - Beleze, Mlle, Mte.,

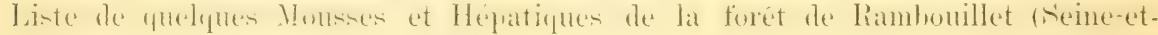
Oise). - Olivier, l'abbé, Exposé systématique et description des Lichens de l'Ouest et du Nord-Onest de la France.

Bulletin de l'assneiation Pyrénéenne. Dixième année 1899 1900. Yutes critiques sur le plantes distribuées: Anemone coronaria L. var. primigenia A. Gubler, Lychnis coronadia Desv. v. albida, Rubus castaneicola Sudre. R. lacertosus Sudre, R. subrotundus Sudre, Calendula ceratosperma Viv., stellata Cav., parviflora liaf., sublanata Rchb., Cirsium oviforme Gandg., Hieracium pallidifrons Sudre, H. Sudreanum Arvo-T., Digitalis purpurascens Roth. und Polygonum salicifolium Delile non Broussonet.

La Nuova Notarisia. Juli 1900. 1.97-144. Forti, A., Pugillo di Diatomate del laghette di Lasés nel 'Trentino. - Borge, O., Register zu G. Lagerstein's und O. Borge's Uebersicht der neu erscheinenden DesmidiaceenLitteratur.

\section{Botanische Anstalten, Vereine, Tauschvereine, Exsiccatenwerke, Reisen etc.}

Schulz, Paul, Tausehvermittlung für Herbarpflanzen. Ien Teilnehmern und Interessenten zur Nachricht, dass auf mehrseitiges Ersuchen nummehr auch P'flanzen auf Strohpapier, Makulatur etc. zum Tausch zugelassen werden. Der diesjährige Katalog wird anch Characeen, Algen und Pilze berücksichtigen, Die Ammeldungen für denselben erbitte ich in Kürze.

Paul F. F. Schulz,

Berlin NO., Virchow Str. 9.

Allescher, A., u. Schnabl, J. N., Fungi Bavarici exsiceati. Münchenl. Die 7. Centurie dieser verdienten Sammlung ist 1900 zur Ausgabe gelangt.

Vesterglen. Tycho, Micromyeetes pariores selecti, quus aljurantihus Prof. Dr. Fr. Bubák, Direktore Jos. Em. Kabat, Prof. Dr. G. Lagerheim, Prof. Dr. P. Magnus, P. Sydow, arjectis fungis a beato C. J. Johanson relictis distribuit. Fase. XI, Nr. 251-275. Fase. XII, Nr. 27t-300. Upsala. April 1900.

Krieger, W., Fungi saxonici. Königstein a.d. Elbe. Fascikel XXXI dieses hekamiten Werkes ist 1900 erschienen.

Cusiek, Wm. C, The Eastern Oregon- and Western Idaho-Flora. Elitio 1899. 144 Nummern. 49 Mark.

Collins, Fr. Sh., Holden, J. and Setchell, W. L., Phycotheca borealiamericana. Erschienen Fasc. XIV. 21.25 Mark.

Pritzel, Dr. Ernst, Botan. Reise nach Westaustralien. [)r. Linst l'rit\%el (Kgl. bot. II Zeit die Kolonie Westaustralien bis nördl. zum Murchison zu bereisen, um dort 
botan. Sammlungen anzulegen. Dieselben werlen, nit vollstänligen liestimmungen versehen, den eventuellen Abonnenten zum l'reise von 40 Mark für die Centurie angeboten. Die Verteilung der Sammlungen hat das $\mathrm{Kgl}$. botan. Museum zn

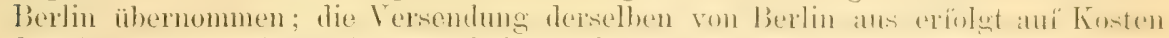
der Abnehmer. Anmeldungen sind möglichst bald an obige Adresse zu richten. Die Ordnung aller Angelegenheiten hezüglich der Simmlung wahrend der Ahwesenheit Pritzel's ist Herm Dr. Robert Pilger im Kgl. bot. Nuseum zu Berlin übertragen worden.

(Oesterr. bot. Zeitschrift.)

Sintenis, P., Botan. Reise nach Transkaspien ete. L'. 'inten is hat Ende Februar l. J. die angekündigte Reise nach 'Transkaspien angetreten und

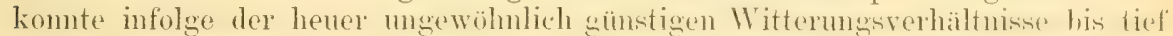
in den Juni um Askabad sammeln. Das Ergebnis ist vorzüglich. Seit Ende Juni ist Sintenis im persischen Grenzgebirge südwestlich von Askabad in einem schwäbischen Dörfchen, wo er in einer höchst ergiebigen HochgebirgsGegend sammelt, so dass die Abnehmer seiner l'flanzen hochbefiedigt sein werden, trotzdem die Austehnmg der Reise bis Chorassan wegen unzurehehender Mittel unmöglich ist.

Amdrup, C. G., Ostgrönländische Expedition. Als Botanikex nehmen Teil: N. Hartz und C. Kruuse. Amdrup wird das Schiff verlassen und die unbekannte Küste von 690-670 $22^{\prime} \mathrm{n}$. Br, geographisch untersuchen, währenddem der wissenschaftl. Stab unter Leitung von Hartz Scoresby Sund und die nöräl. davon gelegenen Fjords untersuchen wird.

Paulsen, Ove, Centralasiatische Reise. Ove Pausen ist von seiner centralasiatischen Reise nach Kopenhagen zurückgekehrt.

Schmidt, Johs., Reise nach Siam. Johs. Schmidt ist von seiner Reice nach Siam nach Kopenhagen zurückgekehrt.

Diels, Dr. L., Botan. Forsehungsreise nach Südafrika und Australien. Dr. L. Diels hat am 3. August eine auf 2 Jahre berechnete Forschungsreise nach Südafrika und Australien angetreten.

(Bot. Centralblatt.)

\section{Personalnachrichten.}

Ernennungen etc: Prof. Dr. Oskar Loew in Washington erhielt den kaiserl. Japan. Verdienstorden. - Dr. Lorenz Hiltner w. kaiserl. Regierungsrat und Mitglied des Gesundheitsamtes zu Berlin. - Dr. Aladar Richter,

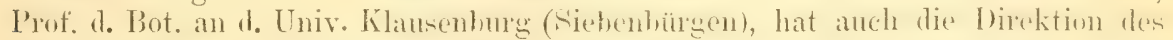
bot. Instituts und Gartens daselbst übernommen. - Prof. Dr. J. Behrens in Karlsruhe w. Vorstand der neu begründeten Weinbau-Versuchs-Anstalt in Weinsberg in Württemberg. - Dr. Julia W. Snow w. Instruktor d. Bot. am Rockford College, Illinois. - Mr. Frederick 0. Grower w. Prof. d. Bot. am Oberlin College. - Dr. Rodney H. True w. Lecturer d. Bot. an d. Harward University während der Abwesenheit des Dr. George L. Goodale im nächsten Jahre. John Bretland Farmer w. z. Mitglied der kgl. Gesellsch. d. Wissenschaften in London erwählt. - Dr. L. Kolderup Rosenvinge w. Dozent d. Bot. an der polytechn. Lehranstalt in Kopenhagen. - C. Ostenfeld w. Inspektor des bot. Nuseums in Kopenhagen. - F. Borgesen w. Bibliothekar d. bot. Gartens in Kopenhagen. - Frau Olga Fedtschenko erh. v. russ. Kriegsministerium die silberne Medaille "Für Feldzüge in Centralasien 1853-1895". - R. H. Y app w. Assistant-Curator an dem Herbarium der Universität Cambridge. - Stu art W eller w. Instruktor der Palaeontologie an der Universitat Chicago. - Hofirat Prof. Dr. J. W iesner erh. das Kommandeurkreuz des schwedischen Nordstern- 
Ortens. - Dr. Adolf Ciesla r erh. d. russ. St. Ammen-Orden. - Prof. Dr. R. v. TV ettstoin w. z. wirkl. Mitglied d. kaiserl. Akademie d. Wissenschaften in Wien crnamnt. - Prof. Dr. K. Fritsch in Graz erh. von der "Académie internationale de géographie hotanique in Le Mans die "Médaille scientifique internationale." Prof. J. H of fm a nn (/Wwittau) w. z. Prof. an der Staatsrealschule in Elbogen und Dr. J. M. Polak (Prag) \%. Prof. an d. Staatsrealschule in Plan ernannt.

Todesfälle: Prof. Dr. Ed. Formínek in Brünn (Mähren), verlienter Forscher der Orientflora und geschïtzter Mitarbeiter unseres Blattes. - Miss Nary H. Kingsley in Simonstown, Sürlafrika. - Kjedahl, Direktor der chem. Abteilung des Carlsberg-Laboratoriums in Kopenhagen, verunglückte am 18. Juli, ca, $50 \mathrm{~J}$. alt, beim Baden.- Hjalmar Ki a er'sou, Systematiker, 18. März, 64 J. alt. - Mr. Charles E. Smith, bedentender Botaniker, in Philadelphia. - Dr. Paul Marès in Mustapha hei Algier am 24. Mai d. J. - Ernest Roze in Caton am 25. Mai d. J.

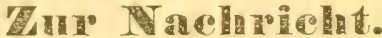

Alle Nitarbeiter der Glumaceac exsiccatae (Gramineae, Juncaceae, Cyperaceae und Carices exsiccatae) werden höflichst ersucht, die gesammelten Pflanzen im Laufe des Monats September mit den nötigen Notizen über Be-

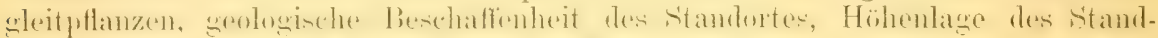

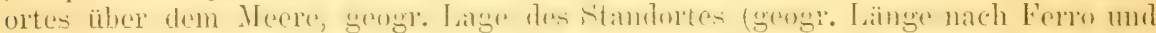
geogr. Breite) und Datum und etwaigen anderen Bemerkungen versehen, an die

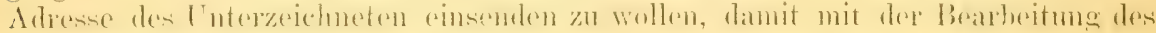
Materials sofort begomnen werden kamn und die Ausgabe der Lieferungen keine Verzögerung erleidet.
Karlsrulue in Baden.
A. Knencker, Werlerplatz 48.

\section{量}

Am Nachmittag des 6. Juni d. J. (ca. 2 Uhr) wurde in der Rheinprovinz eine in Packpapier verpackte grössere Partie lebender Gräser zur Post gegeben, ım nach ihrer Ankunft der Präparation für ein Exsiccatenwerk unterzogen zu

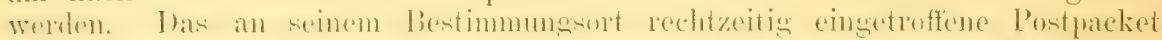
wollte von dem derensiten am 8. Jumi vormittags zwischen 10 und 11 ohr in

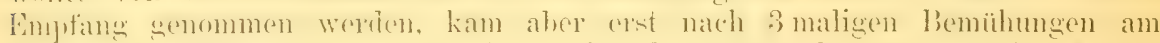
9. Juni nachmittags $1 / 21$ Uhr in Besitz desselben, also statt nach 44 erst nach 70 Stumden. Die Pflanzen waren nun grösstenteils verdorben und können jetzt, obgleich sie sofort präpariert wurden, nur zum ganz geringen 'Teil für das

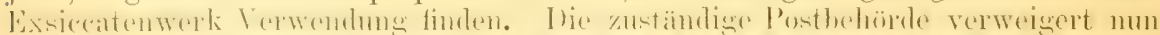

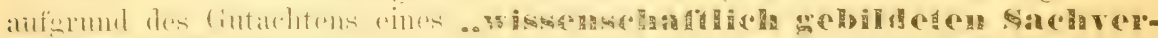

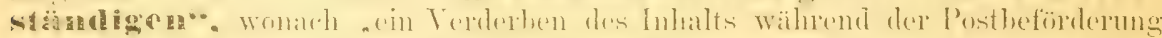

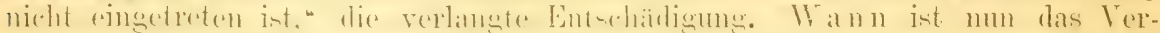
derben eingetreten, wenn die P'flanzen in tadellosem Zustande frisch gesammelt

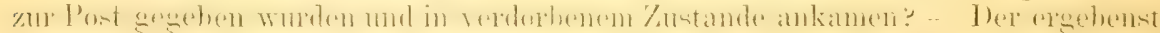
Unterzeichnete erlaubt sich daher, an alle Botaniker, die anf dem Gebiet der

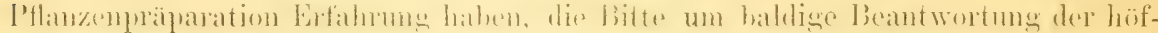
lichen Anfrage zu jichten, ob lebende Pflanzen, die statt 44 Stundeu 70 Stunden in einem Packet eingeschlossen waren, sich zur Präparation für ein wissenschaftliches Exsiceatenwerk noch gleichgut eignen und ob das Gelb- u. Schwarzwerden der Blätter des angekommenen Pflanzenrnaterials, welches nachweisbar frisch gesammelt und trocken verpackt zur l'ost gegeben wurde, auf das um 26 Stunden längere Verbleiben desselben in einem geschlossenen Packet zurickznfïhren ist.

K a r lsin lie.

A. Kneucker, Werderplatz 48.

Druck von J.J. Re iff in Kitrlsruhe. 


\title{
Allgemeine
}

\section{Botanische Zeitschrift}

für Systematik, Floristik, Pflanzengeographie ete.

\section{Referierendes 0rgan}

des but. Tereins der Provinz Brandenburg, der kgl. hot. Gescllschaft zu Kegenslurg, des Preuss. bot. Vereins in Königsberg,

tund Organ der Botan. Vereinigung in Würzburg und des Berliner bot. 'lauschvereins. Unter Mitwirkung hervorragender Fachmänner herausgegeben

von A. Mnesedrer, Werderplatz 48 in Karlspuhe.

Verlag von . T. TReif in Karlspuhe.

Die Herren Mitarbeiter tragen für Form und Inhalt der von ihnen unterzeichneten Arbeiten volle Verantwortung.

\begin{tabular}{|c|c|c|}
\hline $\begin{array}{l}\text { Ni } 10 . \\
\text { Oktober. }\end{array}$ & $\begin{array}{l}\text { Erscheint am } 15 . \text { jeden Monats. - } \\
\text { Preis: vierteljährl. } 1.50 \mathrm{Mk} \text {. bei freier Zuseudung. }\end{array}$ & $\begin{array}{l}\text { 1900. } \\
\text { VI.Jahrgang. }\end{array}$ \\
\hline
\end{tabular}

\section{Inhalt}

Originalarbeiten: Pfarrer J. G. La urell, Ueber einige Carex-Hybriden aus Schweden (Schluss). - E. Palla, Die Gattungen der mitteleuropäischen Scirpoideen. Dr. J. M ur r, Ein Nachwort zu meiner Abhandlung ,Ueber einige kritische ChenopodiumFormen." - Dr. Franz Bloński, Zur Chronik der preussischen Flora (Schluss). Ot to IV ill, Uebersicht über die bisher in der Umgebung von (xuben in der Niederlausitz beobachteten Leber-, Torf- und Laubmoose (Nachtrag).

Bot. Litteratur, Zeitschriften ete. : L. Ge is e n h e y n e r, Jaennicke, Friedr., Studien über die Gattung Platanus L. (Ref.). - A. Kneucker, Lackowitz, W., Flora von Berlin und der Provinz Brandenburg (Ref.). - Derselbe, Blïcher, H., Praktische Pflanzenkunde (Ref). - Inhaltsangabe verschied. bot. Zeitschriften. - Eingegangene Druckschriften.

Bot. Anstalten, Vereine, Tauschvereine, Exsiceatenwerke, Reisen etc.: Die Königl. botan. Gesellschaft zu Regensburg. - B o cker, W., Violae exsiccatae. Palla, Dr. Ed., Reise nach Java. - Bornmüller, J., Botan. Forschungsreise nach den canadischen Inseln.

Personalnachrichten. - Mitteilung.

\section{Ueber einige Carex-Hybriden aus Schweden.}

\author{
Von Pfarrer J. G. La urell
}

(Schluss.)

Es folgt nun C. riparia $\times$ vesicaria (C. riparia * gracilescens $H n$.). Diese Pflanze habe ich in "Enumeratio plantarum Suecia" etc., p. 18, 1897 von Hermann Hamberg in Stockholm, als eine nunmehr ausgemachte Hybride sowohl aus Schweden, als auch aus Dänemark angeführt gesehen, ebenfalls in der Flora von Deutschland, p. 661, 1898 von Dr. Aug. Garcke. Somit dürfte nun auch unsere schwedische C. riparia* gracilescens $H n$. ein Bastard zwischen \%. riparia und C. vesicaria sein. Dann aber muss gesagt werden, dass der Typus der $C$. riparia bei allen deutschen und schwedischen Exemplaren, die ich unter dem Namen $C$. viparia * gracilescens $H n$. gesehen habe, so deutlich zum Ausdruck gelangt, dass die Pflanze nur wenig an $C$. vesicaria erinnert.

Nach Exemplaren von Södertöm anbei folgende Diagnose: R hizom mit wenigen schmalen Augläufern. $\mathrm{Halm}$ nicht so dick als bei $C$. riparia. 
Blatter schmialer als bei (. ripuriu, am Rande sehr rauh, in ihrer ganzen Länge gekielt. Tragbläter auch schmäler als bei c. rifuriu und dazn kiurzer: oberstes jedoch sïntliche Aehrchen überragend. Weibliche Aehrehen 1 bis zu 5, meist 2-4 cm lang, bisweilen jedoch länger, bis an $8 \mathrm{~cm}$ und sngar manchmal noch viel ]änger gesticlt, besondws das unterste. Il äm liche Achrchen 1-t, meist

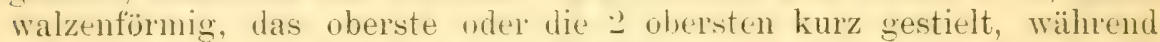
hei (. ripnrin die mänml. Aehren iiberhaupt nicht gestielt sind, wohl

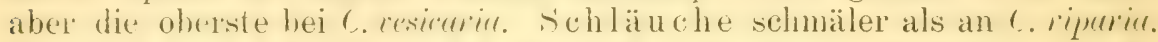
Ebenso ist ilı rehnabel tieler gespalten, wie bei C. iesicuria. Deckschuppen der weiblichen A ehrohen von der Länge der Schläuche oder meist länger und lanzettlich. Deckschuppen der männlichen A ehrchen mehr oder meniger pfriemenspitziy und sehr lang.

Dies alles ungeachtet, erscheint die Hybride habituell als eine ziemlich schmalblättrige C. riparia.

Das Vorkommen betreffend, kenne ich mit Ausnahme von der Gemeinde Kungsăra in Vestmanland keine anderen standorte in Schweden als die von mil 18!: nahe Styrehorg und 18:t6 am Nordwestufer des Sees Styran (Botan. Notistr, p. 104, 185s; in der Gemeinde Sorunda und ron Dr. . Bereglen (stockholnu) an sühufer des sees bei Djursnäs in der Gemeinde (j)mo gefundenten, alle in rödermanland, ersterer ca. $25 \mathrm{~m}$ ü. d M., letzterer aber nur ca. 3 oder $4 \mathrm{~m}$ ü. d. $\mathrm{M}$. An allen Orten wachsen auch die Eltern nebst anderen I'tanzen als Begleiter.

Natürlich dürfte auch diese Kreuzung hin und wieder in Lande ïbersehen worden sein.

Endlich folgt C.riparia < rostrata. Die Kreuzung wurde bereits 1889 ron Figert in der "Deutschen but. Monatschrife" p. I85 als Corex Berlimanminma beschieben und in bot. 'Tauschvereinen unter seinen bot. Freunden verbreitet.

Die Hylnide wude 1-99 am Ser Ellwiken in der Gemeinde Ösmo (a. dim ï. d. I. von Ir. J. Berggren, Lehrer an der Nemen Elementarschule in Stockholm, gefunden.

Zur Charakterisierung derselben, so gut es sich nach einem mir zugänglichen Exemplar niachen lasst, diene Folgendes: Inas lihizom mit mehreren oder wenigen Ausläufern. Der $\mathrm{H}$ alm fast $1 \mathrm{~m}$ hoch und ziemlich cick. nach miten stumpt rundlich mol fest, dazu glatt wie bei C. instrotr, nach ohen 3 kantig und rauh. blatter an Grunde gefaltet, geren die Ilitte und spitze flach und ziemlich scharf, leicht gekielt, nach mten shit, ron der Mitte gegen die spitze an dem liande und áuf den Nerven rauh und ca. 7 bis $8 \mathrm{~mm}$ breit. Tragblätter wie bei den Eltern hreit, unterstes 6,5 mm lreit, simtliche Aehrchen überragend. Weibliche A ehrohen 2 , oberntes fast $1 \mathrm{~cm}$, unterstes fast 2 cm lang gestielt, mehr cylindlisch und sthmälex als das der C. ripuriu. Mämnliche tehrehen 4, häutich, hesonders das oberste etwas walzentörmig (bis linealisch) und gestielt, wie dies nitmals bei c. ripura, in der hegel

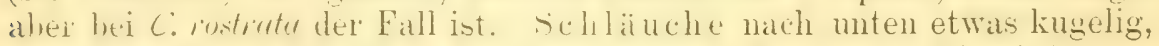
dahte ziemlich pötzlich in den etwats langen, -2 spitzigen schmabel übergehend, hangrian. Deckschuplen der weibhchen Aehrchen fatst ovallanzettlich. Mämliche Deckschuppen an den händern der spitze weisshäutig, übrigens breit und stumpf mit kurzer Spitze u. s. w. 
Die Farbe der l’flanze ist intensiv blaugriü. Der glatte Halm, der stiel des obersten, männlichen Aehrchens und vor allen Dingen die blaugrüne Farbe und noch mehr die breiten Tragblätter und die nach unten etwas kugeligen schläuche erimern sehr an C: rostratu.

Für Schweden ist somit nun auch diese seltene Hylride C. riparir $>$ rostrata aufgefunden worden.

Aspö, Strengnäs im August 1900.

\section{Die Gattungen der mitteleuropäischen Scirpoideen.}

Von E. Palla.

Ton dem Herm Herausgeber der "Allgemeinen Botanischen Zeitschrift" ist der Wunsch ausgesprochen worden, ich möge für die Leser seiner Zeitschrift eine kurze Uebersicht über die Gattungen geben, in welche die seinerzeit unter siripus beschriebenen und lange fortgeführten Cyperacen der deutschen Flora heutzutage gestellt werden. Ich komme diesem Wunsche hiemit nach, will mich aber im Nachfolgenden nicht auf die ehemaligen sicirmes-Arten Deutschlands allein beschränken, sondern auch die übrigen Scirpoideen der Flora Deutschlands, Oesterreichs und der Schweiz in Betracht ziehen.

Bei der weitgehenden Reduktion der Blütenverhältnisse bei den Cypereceen ist es verständlich, warum Linné und seine Nachfolger die wahren Verwandtschaftsverhältnisse innerhall, dieser Familie vielfach falsch auffassten und eine Anzahl rein künstlicher Gattungen schufen; Scivpus und S'hoemus im Linnéschen L'mfunge sind zwei gute Beispiele dafür, wie man seinerzeit die Cypercern-Arten zusammenstellte. Die in neuerer Zeit vor allem von $R$ adlk of er inaugurierte, anatomische Methode" hat endlich auch bei den (yperceen angefangen, allmählich Wandel zu schaffen, und es hat sich herausgestellt, dass in vielen Fällen der innere Bau allein uns Anhaltspunkte giebt für die richtige Errkenntnis der Phylogenie der Arten dieser Familie. So beweist uns, um nur ein paar Beispiele zu nemmen, das Studium des anatomischen Baues des Stengels bei Scirpus premenlus R. S. und pauciflurus Lightf. auf das unwiderleglichste, dass beide Arten, trotzdem ihnen die sonst charakteristische Verdickung der Griffelbasis fuhl, echte Ileleocheris-Arten sind, wofür ja im ïbrigen auch der ganze äussere Habitus spricht; und ehenso folgt aus dem anatomischen Bau des stengels des siripus cuespitosus L. die zwingende Folgerung, dass diese fyperucce der allernächste Verwandte des Livophm alpinum L. ist. Die phylogenetischen Konsequenzen, die man aus dem Studium der ('yperceen-Anatomie ziehen muss, bringen allerdings für manche Krejse das Unangenehme, dass einige der neven Gattungen ihren äusweren morphologischen Verhältnissen nach von anderen kaun oder gar nicht auseinanderzuhalten sind. Allein die Systematik kamn sich, sofern wie wissenschaftlich hleiben soll, nicht un die praktischen Bedïrfnisse der Gïrtner und solcher "Systematiker" kümmern, die bloss sammeln und möglichst bequem bestimmen wollen; sie muss vielmehr, wenn sie hestimmte phylogenctische Be-ziehungen zwischen verschiedenen Arten aufgedeckt hat, dies gegebenenfalls auch äusserlich durch Handhahumg der Nomenklatur lekmden. Hentzutage, da Zoologie und Botanik ganz auf dem standpunkte der Abstammungslehre stehen, besagt eine Ciattung viehmehr als zu Linné's 
Zeiten, sie ist der Ausdruck des rellen verwandtschaftlichen Zusammenhanges, den wir beziiglich einer Anzahl von Arten erkannt haben gegeniiber anderen Arten deselhen Familie; und deshalb ist es eine viel wichtigere Sache der Systematik, natiirliche Gattungen zu schaffen, als beispielsweise ül,er P'rioritätsansprüche nachzugrübeln; ob damn eine Gattung morphologisch oder nur anatomisch definierbar ist, bleibt fur die wissenschaftliche Botanik gleichgiltig.

Im Nachfolgenden will ich aufgrund der bereits rorliegenden Arbeiten, die sich unter Berïcksichtigung des inneren Baues mit dex Phylogenie der (yperueen beschäftigt haben, ${ }^{1}$ ) sowie aufgrund einiger von mir noch nicht veriffentlichten Beobachtungen zumächst eine Uebersicht über die Gattungen geben, in welche den neneren Beobachtungen zufolge die mitteleuropäischen Scirmideen gestellt werden müssen; daran soll sich ein Bestimmungsschlïssel für die rerschiedenen Gattungen anschliessen; den Shchluss möge ein doppeltes Synonymen-Verzeichnis bilden, in welchem die älteren Namen den jetzt geltenden gleichgesetzt sind und umgekehrt.

\section{Lebersicht der mitteleuropäischen (yperuern-(iattungen.}

Die angeführten morphologischen Nerkmale passen auf einzelne Gattungen ganz oder zumteil nur insoweit, als deren mitteleuropäische Vertreter in Betracht kommen, und kïmnen daher nicht iiberall als allgemeine Gattungs-Charaktere angesehen werden. Andererseits sind die anatomischen Verhältnisse nur da ausfühlicher geschildert, wo es unbedingt notwendig ist, sonst nur flüchtig gestreift oder unberücksichtigt gelassen. Es sollen ehen hier die Gattungen hauptsächlich so vorgeführt werden, wie sie sich in unseren einheimischen Arten verhalten. Eine eingehende allgemeine Begründung der Gattungen, die zumteil nur auf anatomischer Grundlage möglich ist, würde hier zu weit führen.

\section{Cyperaceen.}

\section{Scirpoideen.}

1. Chlorocypereen Rikli, a. a. O.

Die grünen Zellen des Blattes und des Stengels in einer einzigen Schicht unmittelbar an die Gefässbündel-Schutzscheiden angrenzend, um die kreisrunden kleinen Gefässbündel herum einen geschlossenen, um die ovalen oder elliptischen grösseren Bündel einen an der Grenze zwischen Holz- und Siebteil unterbrochenen oder nur einseitig am Siebteil angedeuteten Kranz bildend ${ }^{2}$ ); innerhalb der Gefässbündel selbst, von der Schutzscheide umgeben, ein anderer einschichtiger Kranz chlorophyllhaltiger Zellen, in seiner vollständigen oder unvollständigen Aus-

1) Es sind dies: E. Palla, Zur Kenntnis der Gattung "Scirpus" (Botan. Jahrb. f. Syst., X, 1888, S. 293). - M. Rikli, Beiträge zur vergleichenden Anatomie der Cyperaceen mit besonderer Berücksichtigung der inneren Parenchymscheide (Jahrb. f. wiss. Botanik, XXVII, 1895, S. 485). - E. Psll a, Zur Systematik der Gattung Eriophorum (Botan. Zeitung, 1896, S. 141). - Ders., Einige Bemerkungen über Trichophorum atrichum und caespitosum (Ber. d Deutsch. Botan. Ges., XV, 1897, S. 467).

$\left.{ }^{2}\right)$ Die anatomischen Verhältnisse sind hier immer so geschildert, wie sie sich am Querschnitt des Stengels, bezw. Blattes darstellen. 
bildung meist genau mit dem äusseren übereinstimmend. Eine farblose Parenchymscheide um die Gefässbündel herum nicht vorhanden. Perigonborsten fehlen.

a. Griffel nicht vom Fruchtknoten abgesetzt, olne deutliche Grenze in ihn iibergehend.

1. Chlorocypems Rikli. Tragblätter der Blüten (die Deckblätter oder Spelzen der Aehrchen) streng zweizeilig angeordnet, an der Spitze nicht oder kaum grannenartig. Staubgefïsse in der Regel 3. Inflorescenz gewöhnlich eine deutliche Spirre.

Einheimische Arten: Chl. longus (L.) Palla. ${ }^{1}$ ) - Chl. bredius (Dsf.) Palla. - Chl. glomeratus (L.) Palla. - Chl. glaber (L.) Palla. - Chl. serotimus (Rottb.) Palla. - Chl. pennonicus (Jacq.) Rikli. - Chl. flecescens (L.) Rikli.

2. Galile Parl. Tragblätter zweizeilig, mit kurzer Granne. Staubgefïsse :3. Aehrchen dicht kopfförmig zusammengehäuft.

Unterscheidet sich von Chlorocyperes hauptsächlich durch die xerophile Ausbildung des Stengel- und Blattgewebes; besonders die Epidermis- und Wassergewebszellen sind sehr derb gebaut.

Einzige Art: G. meronata (L.) Parl.

3. Dichostylis Beauv. Tragblätter zweizeilig oder - bei unserer Art - mehrzeilig, an der Spitze mit deutlicher, häufig zurückgekrümmter Granne. Neist nur 1 Staubgefäss. Aehrchen dicht kopfförmig zusammengehäuft.

Einheimische Art: D. Micheliana (L.) Nees.

b. Griffel am Grunde verdickt und vom Fruchtknoten deutlich abgesetzt (ähnlich wio bei den meisten Heleocharis-Arten).

4. Fimbristylis Vahl. Tragblätter mehrzeilig.

Einheimische Arten: F. dichotoma (L.) Vahl. - F. ammu (All.) R. S.

Ausser den 4 einheimischen gehören zu den Chlorocypereen nach Rikli's Untersuchungen noch folgende aussereuropäische Gattungen: Lipocarpha R. Br., Hemicarpha Nees, Ascolepis Nees, Kyllingia Rottb. ${ }^{2}$ )

Die Gattungen der Chlorocypercen können, nachdem bisher nur ein geringer Teil der aussereuropäischen scirpoideen daraufhin untersucht worden ist, ob sie zu den Chlorocyperee'n oder Encypereen gehören, vorläufig nur als provisorische Gattungen angesehen werden; die Klärung' der Verwandtschaftsverhältnisse ist erst künftigen Untersuchungen vorbehalten. Sellsst Fimbristylis, scheinbar durch den Griffelbau eine der bestbegründeten Gattungen, ist in gewissen Arten von Dichostylis ausser eben durch den Griffel nicht zu trennen, und es scheinen sich diese beiden Gattungen so zu einander zu verhalten, wie die Ueberzahl der Heleocharis-Arten zu H. paciflore und parvula. (Forts. folgt.)

$\left.{ }^{2}\right)$ Chlorocyperus longus wird von Rikli (a. a. O.) zu Eucyperus gestellt. Rikli muss bei seinen Untersuchungen eine ganz andere Art vorgelegen sein; demn Exemplare von Baden in Niederösterreich, die ich untersuchte, zeigten den für Chlorocypereen charakteristischen Bau.

2) Rikli rechnet hieher auch seine neue Gattung Chlorocharis, unter der er eine Anzahl Heleocharis-Arten abtrennt, allein mit Unrecht, da das (mehrschichtige) Assimilationsgewebe der betreffenden IIeleochuris-Arten wohl nicht homolog ist dem äusseren Assimilationskranz der Chlorocypereen.. 


\section{Ein Nachwort zu meiner Abhandlung ,Ueber einige kritische Chenopodium-Formen." ${ }^{1}$ )}

Von Dr. J. Murr (Trient).

Die in der Oesterr. bot. Zeitschrift, Jahrg. 1900, S. 49 ff. und $93 \mathrm{ff}$. veröftentlichten, studien über Chenopodium opulifolium Schiorler, C. ficifolium Sm. und album $L$., vom Oberlandesgerichtssekretär Jos. B. Scholz" veranlassen mich zu einer Reihe von Bemerkungen über die von mir seinerzeit aufgestellten und behandelten Formen.

1. Chenopodium striatum (Kraš. movar. C. albi) Mur. Indem Herl Sch. zum Schlusse seiner Arbeit auf meine ausführliche Beschreibung in der Deutschen bot. Monatschr. "Bezng nimmt" (in einfachere Sprache ïbertragen: nicht Bezug nimmt), bemerkt er, dass er sich schon an einer anderen Stelle ${ }^{2}$ ) über den negativen Wert dieser Art (von der er mir unmittelbar vor seiner Publikation, nämlich am 26. Okt. 1896, Proben zur Begutachtung übersandt und meinerseits eine kleine Probe aus Tirol erhalten hatte) geäussert habe. Herr Sch. hat in diesem ersten Punkte kürzlich von sehr beachtenswerter Seite, nämlich von Prof. Dr. Vollmann in der Sitzung der $\mathrm{kgl}$. bot. Gesellschaft zu hegensburg rom 8. Nov. $1899^{3}$ ) Succurs erfahren.

Prof. Tollmiann griundet sein negatives Lrteil auf ron mir ausgegebene Originalexemplare und bespricht, stets auf meine Beschreibung wirklich "Bezug nehmend", einzeln die von mir dem Ch. striatum als charakteristisch beigelegten Merkmale. Ich stimme Hrn. Dr. Tr. vollkommen bei, dass die violette Streifung des Stengels, die sich, wenn auch kaum so lebhaft, auch bei mehreren verwandten Formen findet, wenig zu bedeuten hat und dass daher der. Name strutum von Kr rašan gerade nicht sehr glïcklich gewählt wurde; mehr Gewicht lege ich schon auf die feine aber grelle rote Umrandung der Blätter — dieselbe mag immerhin, wie Dr. V. vermutet, als ein besonders durch Herbstfrost beförderter Erythrismus erklärt werden - die ich mich in dieser Schärfe nur bei $C h$. striatum und den Kreuzungen desselben mit andern Formen beohachtet zu haben erinnere; vollends aber halte ich die (bei fast parallelen Seitenrändern!) oft ganz enorm stumpfen untersten und unteren Blätter und deren öfters auftretende eigentümliche plötzliche aber sehr kurze nnd stumpfe Lappung ${ }^{4}$ ) im untersten Drittel oder Viertel (wie selbe ähnlich, aber nicht in derselben Weise nur noch bei $C h$. ficifolium vorkommt) fiur eine specifische Eigentümlichkeit des Ch. striatum, die ich bei den von mir revidierten Chenoportimm-Materialien sonst nirgends auch nur in annähernder Weise heobachtet hatte.

Uebrigens glaube ich, dass der Streit, ob Art oder blosse Race, ziemlich unfruchtbar ist. Was heute noch Race ist, kann sich in so entwicklungsfähigen Gattungen, wie Chenopodium, in nicht allzulanger Zeit als Art konsolidieren. Für die Gegenwart möchte ich umsoweniger halsstarrig an meiner frither ausgesprochenen Ansicht festhalten, als ich im heurigen Juni an der Kaserne Alle Laste bei Trient eine noch

1) Deutsche bot. Nonatschrift $1896, \therefore .32 \mathrm{ff}$.

2) Mitteilungen des Copernikus-Vereins für Kunst und Wissenschaft in Thorn 1896, Heft XI, S. 79 f. Diese Tereinsberichte sind mir natiurlich nicht zugänglich.

s) S. den Bericht darüber in der Allg. bot. Zeitschr. 1900 S. 48.

*) Man vergleiche Fig. 2 und 4 der von mir a. a. O. abgebildeten Blattreihe von Ch. striatum. 
sehr junge Gruppe vou sonst völlig typischem Chemopudium striutum beobachtete, das statt des sonst fiir unsere Pflanze so charakteristischen dunkeln Blattgrimes und der spiegelnden Blattoberseite eine gleichmässige graue Bestäubung auf beiden Blattseiten nach der Art des gewöhnlichen $C h$. clbum, wem auch nicht so intensiv, anfwies. Iass $C h$. striatum, in dem ich sofort bei seinem ersten Auftreten am Bahmhofe in Immshruck (1\&86) eine selhständige, mit unseren sonstigen mä̈hligen Variationen des $C h$. album nicht zu vergleichende Form erkannte, küntig weit mehr gekannt und gewürdigt sein wird, steht mir ausser $/$ weifel und ich freme mich, hiezu die erste wirksame Anregung gegeben zu haben. ${ }^{1}$ )

2. Die Bewertung meiner hybriden $\mathrm{Z}$ wischenformen von $C h$. album L. und $C h$. striatum ( $\left.K r a s_{\text {. }}\right) m h$. richtet sich natürlich nach der Bewertung der letzteren Form. Möge es sich num auch um blosse Racen-Bastarde handeln, mir war es bei den zahlreichen Kreuzungen, wie ich sie besonders um Linz und Trient sammelte und wie sie mir Herr Rohlena von Prag neben typischem $C h$. strutum vorlegte, ${ }^{2}$, stets leicht möglich, den Einfluss des Ch. striutum nicht nur im Blattgriun, der roten Tmrandung und eigentümlich ausgeloissenen Zähnung der Blatter, sondern sueziell durch die auch hei den sonst dem $C \%$. album näher stehenden, also I spitzblättrigen Formen am Grunde der Zweige stets vorhandenen äusserst stumpfen, fast leierförmigen Seitenblättchen zu erkennen.

3. Ch. striatum (Kră̌s) Murr x ficifolium Sm.a.a. O. S. 36 u. Taf. II Fig. $2 \mathrm{a}-\mathrm{c}$ von Linz hahe ich hereits in meinem "Nachtrage zur Flora von Ober- und Nieder-Oesterreich" (Allg. bot. Keitschr. 1898 Nr. 6 S.97) als Form von Ch. striatum (foliis protractis) eingezogen. ${ }^{3}$ )

4. Ch. album L. x ficifolium Sm. a. a. O. Taf. II 4 a-c. Auch diese Form wurde mir in genau gleicher Ausbildung von Hrn. Rohlena aus Prag vorgelegt; doch waren auch seine Stiicke noch nicht fiuchtend, also für einen sicheren Nachweis des hybriden Ursprunges nicht ausreichend. Ich verweise bezüglich dieser Form auf meine Abbildungen (bes. b u. c.), in denen sich der eclatante ficifolium-Typus mit einigem album-axtigem, dem $c \%$. ficifolium sonst fremden Randa ufputz wiederspiegelt, und bemerke dazu, dass ich sehr ähnliche Blattformen z. B. auch vor der Artillerie-Kaserne in Linz rorfand, denen ich aber wegen ihres zahlreicheren Auftretens und der mehr starren,

1) Selbst in Garcke's Ill. Flora v. Deutschland 18. Aufl. S. 515 taucht bereits bei Ch. album die var. c) striatum Kraš. auf.

") Unmittelbar vor Korrektur dieses Artikels erhielt ich auch von Hrn. E. Iss ler in Kolmar (Elsass) eine schöne $F$ rischsendung dortiger (henopodien zur Ansicht und Revision, darunter einen grossen Teil der von mir behandelten formen, nämlich das typische Ch. striatum ( $K r a s ̌)$, ferner die Reihe Chr. album - striatum, sodann Ch. opulifolium Schrad. f. obtusatum Beck und mucronatum Beck, sowie schliesslich Exemplare, die meiner var. betulifolium entsprechen. Letztere var. ist aber nach meiner heutigen Einsicht nicht sehr glücklich aufgestellt; denn sie deckt sich zumteil (Tiffel I '? $e^{\prime}$ ) mit der var. mucronatum Beck, zumteil (Tafel I $2 \mathrm{c}$, dazu die in Rede stehenden Issler'schen Ex., wahrscheinlich auch a) gehört sie entschieden der Reihe ( $h$. opulfolinm.album an.

$\left.{ }^{3}\right)$ Als Ersatz dafür fand ich letzthin bei S. Martino in Trient in einem Ex. die

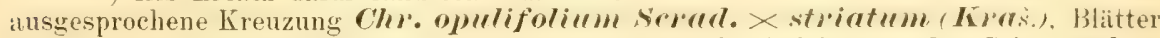
bis auf die obersten lanzettlichen sehr stumpf, dreickig, an den Seitenrändern buchtig grezähnt, dabei aber beiderseits, hesonders oberhalb. lebhaft is rï mit rötlicher Berandung, Stengel sehr lebhaft gestreift. 
ullum-artigen Konsistenz der Blätter (wie auch schärferer Zähmung, anderen Blatterius u. s. w.) einen hybriden Ursprung von Anfang an nicht beileste. sch. bezieht (S.95) sowohl ,b) die von Mur erwähnten

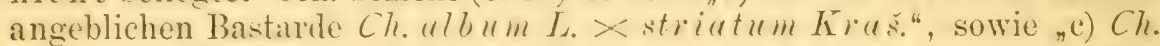
"lbum>ficifolium (vergl. die Tafeln II 1, 3, 4 zu dessen (d. h. meiner) Arbeit", also zwei Formengruppen, die in Form. Konsistenz und Blattsrün kaum eine entfernte Aehnlichkeit mit einander besitzen, die ersteren mit fast stets ziemlich breiten, im Lmrisse + deltoidischen mehr weniger lebhaft grïnen, oft rotherandeten Blättern, die letzteren mit matten, etwas weisslich bestäubten schmalen + parallelrandigen Blïttern rom Zuschnitte des Ch. ficifolium als Verwandte zu Ch. album var. hastatum Klinggraeff.

Da, wie gesagt, die von mir für $C h$. album $\times$ ficifolium angesehenen Exemplare kein ahschliessendes Urteil gestatteten - ich bezeichnete deshalb die Hybride a. a. O. \$. 36 selbst ansdrücklich als „mutmasslich" - so gebe ich nicht nur hinsichtlich der oben erwähnten im Blattzuschnitt an $C h$. ficifolium erinnernden Form von Ch. allum, sondern auch hinsichtlich der ron mir als $C h$. album $\times$ ficifolium vermuteten Pflanzen von Innsbruck und Prag (nicht aber hinsichtlich meiner Zwischenformen Ch. allum — striatum) die Möglichkeit zu, dass dieselben zu der erwähnten Form des Ch. ulbum in nïherer oder fernerer Beziehung stehen könnte.

5. Ch. opulifolium Schrad. $\times$ ficifolium Sm. Obwohl auch bei dieser Form eine Untersuchung des Samens nicht möglich war. könnte $i$ herale hier am wenigsten ron meiner Deutung als Hybriden abgehen. In den auf Tafel II 5 abgebildeten sechs von einem Aste genommenen Blättern zeigt sich der Reihe nach so schön zuerst die ficifolium-artige parallelrandige Verengung des opulifoliumBlattes und sodam der röllige Uebergang zum breitlappiyren ficifolium-Typus, dass die entsprechende Kombination ummöglich anschaulicher und glaublicher konstruiert werden könnte. Ich verweise also nochmals alle, welche sich einigen Simn für habituelle Erkenntnis von Hybriden beimessen zu kömen glauben, auf meine Tafel

Merkwïrdig bleibt hiehei die Ausdrucksweise im Scholz'schen Artikel. Es huisst dort (S. 9t): Weit daron entfernt... an der Richtigkeit der Deutung Murr's Zweifel zu hegen“ u. s. W. Im nächsten Satze ist aber bereits so ziemlich das Gegenteil davon zu lesın: "Die letzteren (d. h. die von Murr Tafel II Fig. 5 abgebildeten in Rede stelenden Blattformen) wiurde man sehr wohl gewissen Gruppen ron Ch. opulifolium einreihen können, wenigstens kenne ich derartige Formen (warum nicht gleich Nïheres über dieselben:) von Standorten, wo Ch. ficifolium in weiten L'mkreisen nicht nachgewiesen ist.

6. Ch.opulifolium Schrad. (var.betulifolium mh.) $<$ album L. Die Cnterscheidung der Lebergangs- (resp. Grenz-) und Hybridformen zwischen Ch. opulifolimm und ullum ist jedenfalls ausserordentlich schwierig; doch springt hei dem von mir Tafel I 2 d abgebildeten Blatte vom Stiftingthale bei Graz (leg. Preissmann 18\&0) aus dem opulifolium-Typus mit aufgesetztem Mittelstücke von Ch. album (wodurch das Blatt geradezu unproportioniert erscheint) das hreuzungsprodukt sofort in die Augen. 
Auch hier (S. 94) verfihirt Her. Sch. in der bekannten Weise. Demn auch auf diese Form bezieht sich die bereits angeführte Bemerkung „Weit entfernt" u. s. w., sowie weiter unten eine nochmalige Versicherung: "Da ich auch die von Murr erwähnte fünfte Hylride [Tafel I Fig. 2d] nicht gesehen habe (übrigens dürfte auch Herr sch. seine Abbildungen von Chenopodium-Blattformen $\mathrm{zu}$ dem Zwecke veröffentlicht haben, damit man sehe und sich ein Urteil bilde), enthalte ich mich jeder vorgefassten Meinung. Im nächsten Satze folgt wieder mit kluger Umschreibung das gerade Gegenteil: „Häufig werden gewisse Varietäten ron $\mathrm{Ch}$. album, besonders eine von mir mit pseudopulifolium bezeichnete... mit Ch. opulifolium verwechselt. Dieser Form wïrde das Blatt des mutmasslichen Bastardes am besten entsprechen."

Ich schliesse, indem ich die Beurteilung dieser Art wissenschaftlicher (?) Behandlung den geneigten Lesern anheimstelle.

Trient, am 19. Sept. 1900.

\section{Zur Chronik der preussischen Flora.}

Von Dr. Franz Błoński (Spiczyńce, Ukraina).

(Schluss.)

Die von Rzączyński aufgeführten galizischen Fundorte des Myricaria Germanica Desv. wurden in neuesten Zeiten fast alle bestätigt und zwar (ich führe nur die zum Weichselsystem gehörigen an):

1. Ufer der Osława, zum Śan-System gehörend (Dr. Wołoszczak in Berichte d. physiogr. Komm. zu Krakau XXIX, S. 65);

2. Nebenflüsse der Sola (J. Krupa ebenda. XIII, S. 178);

3. Ufer des Dunajec in den Centralkarpaten (Uechtritz in Oest.hot. Woch. VII, S. 342); Dunajec in Pieninen (Herbich in Flora XVII, S. 573; Ascherson in Verhandl. d. Brandb. Ver. VII, S. 126; Gustawicz in Denkschr. d. 'TatraGesell. VI, S. 21; Berdau in Flora der Tatra, Pieninen und WestBeskiden, S. 209); Dunajec bei Nowy Targ (Neumarkt) (Ilse in Verhandl. d. Brandb. Ver. X, S. 4); Dunajec im Sandecen Kreise (J. Krupa in Ber.d. phys. Komm. zu Krakau XVI, S. 213 ; Dr. Eu. Wołoszezak, ebenda, XXX, S. 65); Dunajec bei Tarnów (Zaręczny bei Raciborski, ebenda, XVIII);

4. Ufer der Skawin a (W. Besser, Primitiae fl. Galiciae etc., I, S. 282);

5. Ufer der Skawinka bei Bierzanow im ehem. Bochnia'schen Kreise (jetzigem Kr. Wieliczka) nach Herbich's Manuskripten;

6. Ufer der Raba (Uechtritz in Oent. hot. Woch. VH, S. 342; Ascherson in Verh. d. Brandb. Ver. VII, S. 121); Raba bei Dobezyce im Kr. Wieliczka (J. Krupa in Ber. d. phys. Komm. zu Krakau, XVI, S. 213).

Es bleihen nur die Flüsse Rahka und Grawka von galizischen standorten der Myricaria Germanica Disu. in neueren floristischen Arbeiten unerwihnt, was sich teils durch mangelhafte Erforschumg dieser Gegenden, teils durch das Ter-

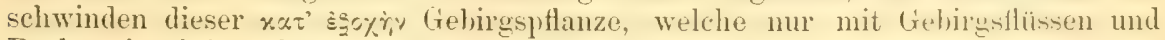
Bächen in tiefere Gegenden herabsteigt, erklärt.

Das noch nördlicher gelegene Dorf Matyaszow am linken Weichselufer, wo nach Rzączynski diese interessante P'flanze wachsen sollte, liegt schon im Königreiche Polen und zwar in dem südlichen Teile des Kreises Sandumir, der

*) Verfasser der Flora Cracoviensis (1859), der Flora der Tatra, Pieninen und. West-Beskiden (1890), und vieler anderer botanischer Arbeiten. 
in hotanischer Hinsicht his jetzt ein terra incognita war. Aus diesem Grunde kamn man nichts Nïheres über diesen ror fast zwei. Tahrhmolerten entreckten Standort der deutechen Mrricarie sagen. Trenn die Pflanze znfailligerweise dort an einer von der Borlenkultur verschonten Lokalitä wuchs, so könnte sie auch hente noch dort vorhanden sein. l)ies ist umso wahrscheinlicher, da Prof. F. Berdau*) sie mehr nach Torden hin auf dem rechten Weichselufer im Kreise Pulawy (jetzt Towa-Alexandria) des frour. Lublin, zwischen Kaliszany und Piotrowin, welchen Fundort C'asimir Lapezyiski in Warsch. physiogr. Denkschr. VII (1887) veröffentlichte, beobachtet hat.

Lapczyíski a. a. O. vermutet, dass die Myricavia-Samen durch den Weichselstrom von den Karpaten nach dem Gouv. Lublin gelangen konnten. Für uns aher ist die sache emfacher. Da anscer den oben anfegefïhten Vebenflüssen der Weichsel (Sola, Skawina, Skawinka, Raha, Dnnajec) noch die Lfer der Skawa [Dorf l'alczowice und Einmündung der skatwa in die Weichsel*)] mit ihrem Vehenfluse skawica**, und die [ter der Wistoka [Stälte Dęhica ***) und Kolaczycet)], nach haacyyiski anch selbst die san-L ler als bestandige standorte für germanische Myrikarie nachgewiesen sind, ham man auf eine minder entfernte Verschleppungsquelle denken. Lud wirklich: da Rzączyoski's Fundert Matyaszow an dem linken Weichselufer fast gegenüher der Mündung der Wisloka liegt, an der bekanntlich diese Myricaria gut gedeiht, so bilden die Wisłoka-Ufern einen mutmasslichen Ausgangspunkt för wahrseheinlich wienlerholte Einschleppung hei Matyaszow, von wo die Pflanze dann durch den Weichselstrom nach dem Gouv. Lublin leicht gelangen konnte.

Zur Rzączyíski's Zeit waren vermutlich noch andere an der Weichsel, wenigstens in der simdomir shen Wojewodschaft grelesene Fumborte der Myr-icaria bekannt, wenn der Verfasser der Historia naturalis curiosa nach dem Worte "Hatyaszów" ein "etc." beifügt. Diese Standorte konnten sich nicht nur zum Gouv. Sandomir, sondern noch weiter nach Norden, z. B. bis Kaliszany oder Piotrowin, wo sie Berdau entdeckte, oder wohl noch weiter bis 'Thorn selbst erstrecken. Da aber das Holz der Pflanze zur Anfertigung von Bechern, Trinkkammen, Fiisschen rorzïglich für Jilzkranke nach Rzaczrniski verwendet wurle, so könnte auf diese Weise die Ausrottung des Gewächses an minder günstigen Lokalitäten leicht stattgefunden haben.

Ton einem dieser an der Weichsel gelegenen Standorte konnte unter gïnstigen Cmstimulen die Pflanze nach Thorn, wenn nicht unmitelhar, so durch einige in den Weichsehiederungen liegende Etappen, ron denen aher jetat keine Spur selhot in der Litteratur zu finden ist, einwandern. Bei Thorn wurde sie von einem, von haaczynski nicht nïher genannten beohachtert†) gefunden und vermutlich in einem verkannten Werke, oder in einem schon lange vernichteten Januskript, wovon Razaczynski später einen anonymen Auszng anfertigte, erwähnt, oder vielleicht direkt mündich von dem Entlecker selbst dem Jestriten Rzączyniski mitgeteilt.

Infolgedessen blieb fast zwei Jahrhunderte dieser neue Bürger der preussischen Flora unbekannt; denn G. A. Helwing (1726), J. Chr. Wulff (1765), E. G. Graff (1809), C. G. Hagen (1818 und 1819), C. G. Lorek (1826-30-37), E. J. Klinggräff'(18to-titi), C. Yatze. E. Mever und L. Elkan (1848-50), H. N. Klinggräff (1880), J. II. Scholz (1896). P. Ascherson mol P'. Grähner (1899), J. Abromeit $(1898-1900)$ u. a. führen diese Pflanze in ihren Werken nicht an.

Es wäre nicht überflüssig, zu erwähnen, dass auch in den Schriften der naturforschenden tesellschaft in Lanzig und in den sichriften der physikalisch.

*) Berdau, Flora Cracoviensis, S. 149.

**) Besser, Primitiae florae Galiciae I, S. 282.

***) Weiss in Verh. d. r.ool. bot. Gesell. in Wien XV (1865), S. 459.

†) Knapp, in Ber. d. phys. Komm. zu Krakau III (1869), S. 1 P4.

iT) Es war keiner der älteren preussischen Botaniker, da sie in ihren Werken die Myrikarie nicht anführen und zwar J. Wigand (1590), J. Loesel (1654), J. Loesel Sohn (1703) und G. A. Helwing (1712). 
ökonomischen Gesellschaft in Köniosherg, in welchen reiche Beiträge zur 'Thorn' schen Flora veroffentlicht wurden, keine spur aber die dentsche Mrrikirie hei Thorn zu finden ist. Dies beweist, dass in der zweiten Hälfte des 19. Jahrlumderts sie von Niemanden bei Thorn und in Westpremsen äherhaupt hemerkt wurde, weil sie an diesem Fundorte schon lange vorher, vielleicht noch in 18. Jahrhundert vernichtet worden ist.

Aehnlich wie mit dem 'Thorn'schen Standorte der Myricaria ging es auch in Polen mit dem anderen von Rzączyński verzeichneten bei Matyaszów im Kreise Santomir, da er seit 1721 his zu meiner jetzigen I'ublikation mbekannt geblieben ist.

Łapczyński a. a. O. hält Berdau für den ersten Entdecker dieser Pflanze im Königreiche Polen und bestreitet ihr Büretrecht in der polnischen Flora, da für diese angehlich ,karpatische" I'flanze die klimatischen Verhälnisse des Königreichs Polen zu unginstio seien. Für uns aher erscheint dies Bürgerrecht in ganz anderem Lichte, indem wir aussel dem nenesten standorte Berdaus (im Gouv. Lublin) noch den hei Matyaszow im Kreise Sandonir und den ehemaligen Standort bei Thorn kennen und nachdem diese schöne Pflanze anch bei Firakau*) am linken Weichselufer sehr nahe der russischen Grenze und bei Jaworzno**) im Kreise Chrzanów in Galizien unweit von Szczakowa, also auch dicht an der Grenze des Königreichs Polen entdeckt worden ist.

Der letzte Standort, weil zu dem Przemsza-System gehörig, welches seinen Anfang nicht in den Karpaten, sondern in dem südlichen Teile des Königreichs Polen nimmt, beweist, dass sich diese Pflanze nicht nur durch diebirgstlüsse und Bäche, sondern auch durch den Wind (z. B. von den Sola-Ufern), zu welchem Zwecke ihre samen mit einem Flugapparate rersehen sind, und rielleicht anch durch Eisenbahnverkehr auf neue Strecken verbreiten kann.

Aus obigen Auseinandersetzungen folgt, 'dass 1. Myricaria Germanica Desv. vor etwa zwei Jahrhumderten hei Thorn wenigstens (ans Süu-Polen) verschleppt. wenn nicht einheimisch heobachtet, später aher vollstandig vernichtet wurde; 2. dass sie jetzt längs der Weichsel nicht nur in Galizien, sondern auch im Königreiche Polen wächst, und 3. dass sie im Künigreiche Polen nicht ganz ungünstige klimatisehe und Boden-Terhältuisse zu ihrer Fortpflamzmg und Verbreitung besitzt.

\title{
Uebersicht über die bisher in der Umgebung von Guben in der Niederlausitz beobachteten Leber-, Torf- und Laubmoose.
}

\author{
Von Otto Will.
}

\section{Nachtrag.}

Durch die Freundlichkeit des Herm Oherlehrel Warnstorf, welcher einen grossen Teil meiner früher gesammelten Moose bestimmte, ist es mir möglich, noch folgende Arten meiner Uebersicht hinzuzufügen.

\section{A. Lebermoose.}

42. Scapania nfmorosa $(L)$ N. r. E. Auf feuchten Wiesen nahe der Eisenbahn bei Jaenischwalde.

\section{B. Torfmoose.}

168. Sphagmum rufescens $(B r$ germ.) var. immersum $W$. Sumpfige Gräben im Hörnchen vor Fünfeichen.

169. S. tres Amgstr. var. subquurrosum H. Auf Torfwiesen zwischen Bomsdorf und Schwertzko.

*) Kulczyński bei Raciborski (in Ber, d. phys. Komm. zu Krakau XVIII (1883).

**) Unverricht in Fiek Flora von Schlesien, S. 158. 
var. imbricatum W. Wiesen bei Schwertzko.

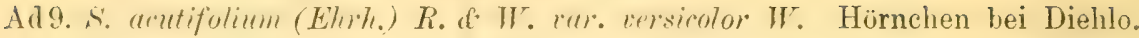

\section{Laubmoose.}

170. Cymodontimm torquescens Limm. Auf nassem Sandboden zwischen Bremsdorfer und Kieselwitzer Mühle gesammelt 6.VIII.1897.

171. Il ebera amnotina (Hedw.) Schuaegr. Auf nassen, lehmigen Wogen im Hörnchen hinter Fünfeichen,

172. Bryum pallens Sw. In Ausstichen bei der Kieselwitzer Mühle.

Ad98. Catharinea undulata $W . \&$ M. var. glabra W. nov. var. Auf Sandboden an einer Quelle bei Deulowitz 6.X.1898 gesammelt.

173. Amb? ystegium rarium Lindb. var. oligorhizon? An nassen Balken der Mühle in Grano.

17. A. currifes Gïmb.? Am Grunde von Baumstämmen am Granoer Fliess im sog. Lauschützer Hammer.

175. Hypnum intermedium Lindb. Sumpfige Wiesen bei Jaenischwalde.

176. H. polycarpon (non Hoffmann) Bland. Wiesen bei Gubinchen.

177. H. cordifulium Hodu. In tiofen Wiesengräben nahe der Eisenbahn-Haltestelle in Jaenischwalde.

\section{Botanische Litteratur, Zeitschriften etc.}

Jaennieke, Friedr., Studien über die Gattung Platanus L. 1892-1897. Halle 1899 aus den Abbandluugen der Kaiserl.-Leop.-Carol, deutschen Akademie der Naturforscher in Komm. bei W. Engelmann in Leipzig. Preis 8 M. 226 Seiten Fol.

Jedem, der sich mit beschreibender Botanik befasst, dürfte bekannt sein, wie ansserordentlich schwer die bei uns vorkommenden Platanenarten von einander zu unterscheiden sind und wie schwer eine richtige Bestimmung derselben fällt. Der Grund liegt nicht so sehr in dem durch Jahrhunderte lange Kultur in den verschiedensten Klimaten erlangten Formenreichtum der Arten, als viel. mehr in der sehr grossen Variabilitit der Individuen betreffs Ausbildung von Blattformen. Dazu kommt noch, dass bei den verschiedenen Schriftstellem in Bezug auf Arten, Unterarten und Formen die schroffsten Widersprüche bestehen. Denn kaum sieht es wohl eine andere liaumgattung, beziglich derer in Theorie und Praxis, in Gelehrten-, wie in Züchter- und Gïrtnerkreisen solche Konfusion, solche oft durchaus wilersprechende Meinungsverschiedenheit herrscht.

Der Verf. oben genannten Werkes hat sich seit Jahren eingehend mit den Platanen beschäftigt, alle einschligglichen Texhälnisse an der lebenden Pflanze und umfangreichem Herbarmaterial studiert und sich bemüht, bessere Charaktere zur schäferen Unterscheidung und Trennung der Arten und Formen zu finden. Wenn num auch nach seiner Ileinung, die erzielten Resultate nicht ganz in Verhiltnis zu der aufgewendeten Summe von Zeit und Arbeitskraft stehen", so muss doch unbedingt ausgesprochen werden, dass die sehr fleissige Arheit in vieler beziehtung Klarheit schaft't und dass sie in Zukunft dem Dendro. logen unentbehrlich sein wird.

Tach einem historischen Ueherhlick üher die Entwickelung der Systematik der Gattung kommt der Terf. zu einer Darlegung der auffallenden Widersprüche in derelben. In zwei umfangreichen Kapiteln werden damn alle die Nerkmale einer eingehenden kritischen Untersuchung unterzogen, die zur Unterscheidung der Arten benutzt worden sind, event. herangezogen werden könnten, z. B. an Blüte und Frucht, Rinde u. s. w. Am eingehendisten werden die aus der Belaubung hergenommenten Merkmale hesprochen, die Nervatur der Bläter, Zahl, Form und Grössenverhältnis der Lappen, die Gestaltung des Blittgrundes u. s. w. und damn hes. auf die grosse Vielfältigkeit der Form eingegangen. Dabei ergiebt sich die bis jetzt noch von keinem Autor ausgesprochene und selbst in genaueren Be- 
schreibungen kamm entfernt gestreifte Thatsache, dass alle unsere Platamen zwei Modifikationen der Belauhumg zeigen, gezälnte Jblatformen und ganzra nd ige und dass mit der Veränderumg der liezahnung sich eine Liungenverinderung des Blattstiels einstellt.

Den Hauptteil des Werkes hildet die sehr eingehende liesprechung und Beschreibung der sechs Arten mit 11 Varietäten, die der Verf. annimmt. Sie wirl durch uber 400 Blattzeichmugen auf 10 Tafeln, darmer nur wenige Re. produktionen, miterstutat. Gegen $40 \mathrm{Abb}$ anf 'Tafel $\mathrm{X}$ sind den drei pacificehen Arten (Pl. racemosa Nutt, Pl. mexicana Moricand, und $P$. Wrightii I Watson), die ja in Europa sehr wenig hekannt sind, gewidmet, die a anderen Tateln bringen Blattformen der drei Konfuionserzenger (Pl. orifntalis und arcilentalis L. und acerifolia Willd.) und tragen sehr wesentlich zum Verstandnis des Textess bei. Tach des Verfassers überzeugenden Darlegungen kommt bei uns I\%. acrifolius an allermeisten vor und nur in verschwindend wenigen Exemplaren findet. sich $P l$. orientalis angeptlanzt.

Ein Schlusskapitel gieht eine Uehersicht über die geologisehe Entwickelung der Platanenarten, wie sie sich nach des Verf. Annahme aus der tertiären Pl. aceroides Goepp. abgezweigt haben könnten.

L. Geisenheyner.

Lackowitz, W., Flora von Berlin und der Provinz Brandenburg. Verlag von friedberg \& Mode in Berlin. $297 \mathrm{~S} .12$. Auflage 1900.

Das rorliegende Büchlein ist ein Taschenbuch für den Floristén und als solches ganz vorzüglich bearheitet, da es anch Schüler und Anfinger zum Bestimmen der Pflanzen mit Nutzen gebrauchen können, zumal ein kleiner Abriss die Kemntnis der morphologischen Grundbegriffe vermittelt. Alsiann folgen Tabellen zur Bestimmung der Familien. Für den in der Pflanzenkenntnis vorgeschrittenen Floristen enthält es meist im Kleindruck auch die Formen und Bastarde vielgestaltiger Genera nebst kurzen Diagnosen. Dass eine Lokalf'lora 12 Auflagen erlebt, ist wohl eine grosse Seltenheit und der beste Beweis für die Beliebtheit des Lackowitz'schen Taschenbuches.

A. K.

Blacher, H., Praktische Pflanzenkunde. Verlag von Otto Paul in Leipzig. 107 S. Preis gebunden 50 g.

Auch dieses Büchlein gehört, wie das p. 198 des vor. Jahrgangs besprochene der "Niniaturbibliothek" an und bringt im Dreifarbendruck eine Anzahl der wichtigsten wildwachsenden und kultivierten Pflanzen unter besonderer Berücksichtigung der Giftpflanzen. Das Büchlein eignet sich besonders zur Volksbelehrung auf Spaziergängen etc. Der Preis ist ein ausserordentlich niederer.

Deutsche bot. Monatsehrift. 1900. Nr.9. Murr, Dr. J., Zur Kenntnis der Kulturgehölze Südtirols, besonders Trients II, - Suksdorf, W., Washing. tonische Pflanzen. - Goverts, W. J., Mykologische Beiträge zur Flora des Harzes. - Jacobasch, E., Ueber die Ursache der vermehrten Anzahl von Laubblättern in einem Quirl. - Blümml, Dr., Referat über Pöverlein: Die bayrischen Arten, Formen und Bastarde der Gattung Potentilla. - M urr, Dr. J., Beiträge und Bemerkungen zu den Archieracien von Tirol und Vorarlberg. Leimbach, G., Die Volksnamen unserer heimischen Orchideen. - Nr. 10. Steig er, Dr. Fr., Beobachtungen über Formationsfolge im Kaiserstuhl. - H ö ck, Dr. Fr., Allerweltspflanzen. - Murr. Dr. J., Wie in vor. Nr. - Suksdorf, Wilh. M., Wie in vor. Nr. - Leimbac h, Dr. G., Wie in vor. Nr.

Berichte der deutschen bot. Gesellschaft 1900. Heft 7. W inkler, Hans, Ueber den Einfluss äusserer Faktoren auf die Teilung der Eier von Cystosina barbata. - Lemmermann, E., Beiträge zur Kenntnis der Planktonalgen. Heydrich, F., Weiterer Ausbau des Corallineensystems. - Ule, E., Ueber weitere noue und interessante Bromeliaceen. - Nestler, A., Zur Kenntnis der hautreizenden Wirkung der Primula obconica Hance. - Gai dukov, N., Ueber das Chrysochrom. 
Jahresbericht des preussischen bot. Vereins 1899/1900 enthïlt ausser dem von Dr. Ahromeit erstatteten ausführlichen und interesianten Bericht über die 38. Jahresversammlung des Vereins in renshurg noch die Berichte über die monatl. Nitzungen des hetr. Vereins in Winter 1899/1900, welche anch in der ,Allg. bot. Zeitschrift" jeweils erschienen.

Oesterreichisehe bot. Zeitsehrift 1900. Nr.9. It ettstein, R. v., Der internat. bot. Kongress in Paris und die Regelung der hotan. Nomenklatur. D e gen, A. v., Bornmillera Iieckii n. sp. - Peter, A., Ceber hochzusammengesetzte Stäkekürner im Endosperm von Weizen, Roggen und Gerste. - Bubák, Fr., [eher nelle und bekannte ansserenopaische Pilze. - Freyn, J., Weitere Beitrage zur Flora ron Steiermark. - Celakovsky. L., Die Vermehrumg der Sporangien ron Gingko biloba. - Litteratur- Lebersicht. - N. 10. Palla, E.. Kéminis der P'ilobolus-Arten. - Freyn, Wie in vor. Nr. - Wettstein, R. v. Euphrasia Cheesemani 11, sp. - Hayek, A. v., Leber eine biologisch bemerkenswerte Eigenschaft alpiner Compositen.

Botan. Centralblatt. 1900. Nr.36. Brand, F., Der Formenkreis von Glueocapsa alpina Xïg. - Garjeanne, A. J. M., Ueher ein monströses Köpfchen von Bellis lerennis. - Nr.37. Gillain, Gust, Beiträge zur Anatomie der Palmen- und Pandanaceenwurzeln. - Nr.38. Gillain, Gust., Wie in vor. Nr. - Nr.39. Gillain, Gust., Wie in vor. Nr.

Berichte der sehweizerischen botanischen Gesellsehaft 1900. Heft X. Fischer, Ed., Entwicklungsenschichtliche Lntersuchumgen ïher Rostpilze (Fortsetzmang). - Rickli, Lr. II.. Nitteilungen aus dem bot. Museum des eidgenöss. Polytechnikums in Zürich: Dic schweizerichen Dorycnien. - Schellen berg, H. C., Graubundens retreidevarietaten mit besonderer Ruicksicht anf ihre horizontale Verbreitung.

Botaniska Notiser 1900. Nr. 4. Krok. Th. (). B. N., Srensk hotanisk literatur 1s99. - Nordstedt, O.. Samblems flora. - Bohling, K., Eth exempel pă ömsesidig vikawiering mellan en fjäll-och en kustform.

Botanical Gazette 1900. Vol. XXX. Nr. 3. Lawson, Anstruther, A., Crigin of the cones of the multijular spindle in Gladiolus. - T imberlake, H. G., The derelopment and function of the cell plate in higher plants. limbach, A., Physiological ohervations on some peremial herbs. - Nelson, Aven, Contribution from the rocky mountain herbarium I.

Bulletin de l'aeadémie internationale de géographie botanique. 1900. Nr. 131 u. 132. Léveillé, H., Oenotheraceae japonenses. - Carreiro, T.S.

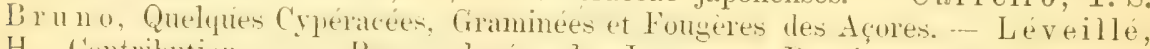
H., Contrihutions anx Renonculacéte du Japon. - Renier, Alf., Botanique rmale: m petit coin de la l'rovence, - Renandet, Georges, Les principes chimiquen des plants de la Flore de France. - Olivier, l'abbe, Quelques Lichens

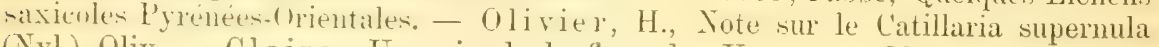
(Nyl.) Oliv. - Claire, Un coin de la flore des Vosges. - Monguillon, E., Catalogue des Lichens du département de la Sarthe.

Eingegangene Drueksehriften. Dall a 'Torre, Dr. C'. (x. et Harms. Dr. H., fenera Siphonogamarum. Fase. II. Verl. v. IV. Engelmann in Leipzig 1900. A scher-

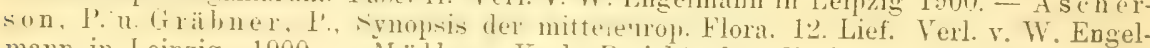
mann in Leipzig. 1900. - II iller, Karl, Bericht iber die im Jahre 1899 in Baden

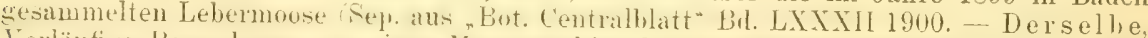

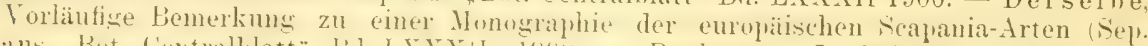

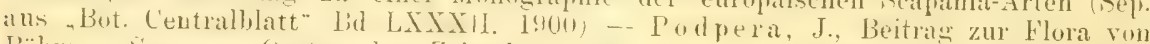

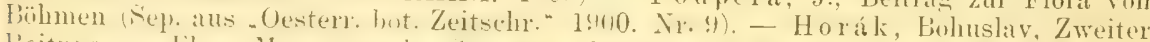

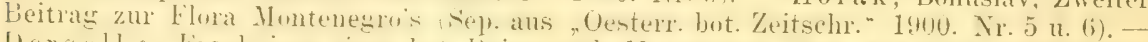

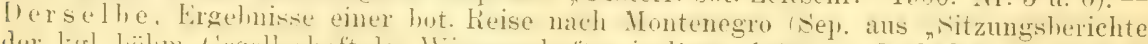

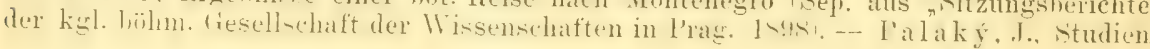


über die Verbreitung der Moose (Sep. aus den "Sitzungsberichten der kgl. böhm. Gesellschaft der Wissenschaften in Prag. 1900). - A bromeit, Dr. J., Die Pflanzenwelt Masurens (Sep. aus d. Werke: Masuren, Samland und das Pregelthal v. Dr. All). Zweck. Verl. v. Hobbing \& Büchle in Stuttgart 1900). - 'T'release, William, Some twentieth century problems (Reprinted from Science, N. S. Vol. XII., Nr. 269. Pages 48-67. July 13., 1900). - Blü cher, H., Praktische Pflanzenkunde mit 100 farbigen Abbildungen. Verl. v. Otto Paul in Leipzig 1900. - Hallier, Dr. Hans, Das proliferierende persönliche und das sachliche, konservative f'nioritätspriozip in der bot. Nonenklatur (Sep. aus „Dr. H. Hallier, Ueber Kautschuklianen und andere Apocyneen nebst Bemerkungen über Hevea und einem Versuch zur Lösung der Nomenklaturfıage. Jahrbuch der Hamburgischen wissensch. Anstalten. XVII. 1899. 3. Beiheft: Arbeiten des bot. Museums). - Kuntze, Dr. Otto, Exposé sur les congrès pour la nomenclature. botanique et six propositions pour le congrès de Parisien 1900. - Lackowitz, W., Flora von Berlin und der Provinz Brandenburg. Verl. v. Friedberg \& Mode in Berlin 1900. - Frey n, J., Nachträge zur Flora v. Istrien (Sep. aus d. „Oesterr. bot. Zeitschr, “. 1900 Nr. 6 u. 7). - Petunnikov, A., Ueber den Wert anatomischer Merkmale zur Unterscheidung der Abies-Arten (Sep. aus d. ,Allg. bot. Zeitschrift" $1900 \mathrm{Nr}$. 7/8). Geisenheyner, L., Abnorme Orchideenbliiten (Sep. aus „Deutsche bot. Monatschr." 1900 Nr. 8). - Stuckert, Theodoro, El Vinalillo una nuova planta arbórea de la. familla de las leguminosas perteneciente á la flora Argentina (Sep. aus nanales des Museo National de Buenos Aires." 'Tom. VII. p. 73-79. 1900).

Oesterr. bot. Zeitschrift 1900, Nr. S-10. - De uts che bot. Monatschrift 1900 , Nr. 7-10. - Berichte der deutschen bot: Gesellschaft 1900. Heft 6 u. 7. - Verhandlungen d. k. k. zool. bot. Gesellsch. 1900. Nr. 6 u. 7. - Helios. 17. Bd. 1900. Botanical Gazette XXIX. 189\%. Nr. 6 und XXX. 1900. Nr. 1-3. - Bulletin de l'académie internationale de géogr. bot. 1900, Nr. 129-132. - Bulletin de l'association Française de botanique 1900, Nr. 32-36. - B u ll et in de l'association Pyrenéenne. Dixième année 1899/1900. - La Nuova Notaris i a 1900. p. 97-144. Societatum Litterae. XIII. Jahrgang. 1899. -.. The Botanical Magazine 1900, Nr. 160 u. 161. - Le mond e de plantes 1900, Nr. 7 u. 8. - Rousset, Jules (36 rue Serpente Paris), Catalogue mensuel de livres d'occasion. - Zeitschrift für angewandte Mikroskopie. VI. Bd. Heft 5 u. 6. - Botaniska Notiser $1900 \mathrm{Nr}$ 4. Berichte der schweizerischen bot. Gesellschaft. 1900. Heft X. - Jahresbericht des preuss. bot. Vereins 1899/1900.

\section{Botanische Anstalten, Vereine, Tauschvereine, Exsiccatenwerke, Reisen etc.}

Die Königliche botanische Gesellschaft zu Regensburg heabsichtigt, in der schon seit mehreren Jahren in ihrem Selbstverlage erscheinenden Flora exsiceata Bavarica ummehr anch die Zelikryptogamen zur Ausgabe zu hingen.

Es soll zunächst in Jahre 1901 mit der Herausgabe der Bryophyten begonnen werden, denen sich damn je nach Möglichkeit und Bedart die übrigen Zellkryptogamen anschliessen sollen.

Die Stärke der Auflage ist vorläutig auf s0 Exemplare festgesetzt, welche in durchgängig gleich grossen Enveloppes aus starkem braunem Papier mit gedruckter Etikette in fortlaufender Nummerierung geliefert werden. Je 4 oder 5 Dekaden werden alsdamn in einem $\mathrm{Pappkarton}$ vereinigt, sodass sich die Sammlung bequem unterbringen lassen wird.

Die einzelnen Faszikel können unabhängig von den im Exsiccatenwerke zur Ausgabe gelangenden Phanerogamenfiastikeln entweder kibulich das einzelne Exemplar einschliesslich Ausstattung zu 15 lieichsptemigen oder im fansche hezogen werden, bei weleh letzterem die f'flanzen in 6 ll ertklatson eingenchatzt werden und für je 2 Einheiten eine Dekate als Aequivalent gecgeten werken sull. 
Dieshezägliche Anfragen heliebe man schon jetzt an den Leiter der Kryptogamenahteilumg, Herrn Lr, phil. J gna $\mathrm{z}$ amiller in Karthans-Prüll hei Regensburg, zu richten.

Becker, W., Violae exsiceatae. 1. Lief. 1900. W. Becker in Wettelroda hei Sangershausen in der Prov. Sachsen versandte neulich die 1. Lief. seiner "Violae exsiccatae*. Die Ausstattung des Werkes ist eine tadellose. Die Pflanzen liegen in starken Bögen weissen Papieres, und letztere sind wieder in Pappdeckelmappen eingeschlossen. Die schedae sind durch Druck hergestellt und enthalten ausser kritischen bemerkungen meist noch Angaben über legleitpflanzen, geologische Lnterlage ete. Im Kaufe kostet die vorliegende Lieferung hloss 8 Mark. Thesonders wertrolle Formen sind: Tiolle Vulteria All., alba $>$ odorata, scanica, purpurea Stev., nummularifolia All., strieta Horn., canira $>$ stagnina, 7 amerikanische Veilchen etc. etc. Im Interesse der Sache wäre zu wünschen, dass recht viele Botaniker aus allen Erdteilen sich an diesem wertvollen Exciccatenwerke beteiligen möchten.

Palla, Dr. Ed, Reise naeh Java. Jr. Ed. Palla, Privatdozent der Botanik an der Universitit Graz, hat am 3. Oktober eine von der $\mathrm{k}$. Akademie der Wissenschaften in Wien subventionierte Reise nach Java angetreten. Er wird sich hesonders mit der Erforschung der javanischen Phykomyceten. und Cyperaceen. Flora beschäftigen.

Bornmuller, J., Botan. Forschungsreise nach den eanadischen Inseln. Born müller ist von s. Forschungsreise zurückgekehrt. (Oest. bot. Zeitschr.)

\section{Personalnachrichten.}

Ernennungen etc.: Prof. J. W. Toume y w. Assistant-Professor der Forstwissenschaft an der neuen Forstschule der Yale.University in Arizona. - Dr. Vogel in Hamburg w. Bakteriolog der landwirtsch. Versuchsstation in Posen. Direktor Dr. R. Thie le in Visselhövede erh. v. der deutschen Landwirtschaftsgesellschaft ein Stipendium zur Ausbildung in der Bakteriologie und siedelt am 1. Oktober nach Halle a. S. über. - C. A. Moore w. Prof. der Biologie am South Carolina College, Columbia, S. C. - Prof. Dr. K. Mikosch w. für d. Schuljahr $1900 / 1901$ z. Rektor der techn. Hochschule in Brünn gewählt. - Prof. Dr. Fr. Krašan in Graz w. anlässlich seines Uebertritts in den Ruhestand der Titel ,Schulrat* verliehen. - Dr. L. Kolderup Rosenvinge w. z. Dozenten der Botanik am Polytechnikum in Kopenhagen ernannt. - Bei der Personalnachricht betr. Dr. Aladar Richter in Nr. 9 p. 195 soll es statt Klausenburg (Sieben. bürgen) heissen: Kolozsvár (Ungarn).

Todesfalle: Hofgärtner C. Ts chernikl in Innsbruck. - Prof. Dr. Ahles in Stuttgart. - Henrick Gustav Lübeck in Karlskrona am 2. Sept. 1900. Johannes Emanuel Planander in Linköping am 3. Sept. 1900. - Erik Ol of Aug. Nyman in München am 29. Juli 1900. - Emmerich Rathay, Professor und Direktor der önologisch-pomolog. Gesellschaft in Klosterneuburg bei Wien, 9. Sept. d. J. - Günther v. Bün a u, Reichsgerichtsrat, am 17. Sept. $1900 \mathrm{im}$ Alter von 55 Jahren.

\section{Plitteilung.}

Mit der Versendung der Novembernummer (Nr. 11) werden die noch ausstehenden Abonnementsbeträge für die „Allg. botan. Zeitschrift" unter Nach. nahme erhoben. 


\section{Allgemeine \\ Botanische Zeitschrift}

für Systematik, Floristik, Pflanzengeographie ete.

Referierendes Organ

des bot. Vereins der Provinz Brandenburg, der kgl. bot. (iesellschaft zu Regenshurg, des Preuss. bot. Vereins in Königsberg,

und Organ der Botan. Vereinigung in Würzburg und des Berliner hot. Tauschvereins.

Unter Mitwirkung hervorragender Fachmänner herausgegeben

von . Herrerer, Werderplatz 48 in Karlsruhe.

Verlag von \$. J. Heifr in Karlsruhe.

Die Herren Mitarbeiter tragen für Form und Inhalt der von ihnen unterzeichneten Arbeiten volle Verantwortung.

\begin{tabular}{|c|c|c|}
\hline $\begin{array}{l}\text { No } 11 . \\
\text { Novenaber. }\end{array}$ & $\begin{array}{l}\text { Erscheint am } 15 . \text { jeden Monats. - } \\
\text { Preis: vierteljährl. } 1.50 \mathrm{Mk} \text {. bei freier Zusendung. }\end{array}$ & $\begin{array}{l}\text { 1DOO. } \\
\text { VI.Jahrgang. }\end{array}$ \\
\hline
\end{tabular}

\section{Inhalt}

Originalarbeiten: E. Palla, Die Gattungen der mittelemopäischen Scirpoideen (Forts.). - L. Gross und A. Kneucker, Unsere Reise nach Istrien, Dalmatien, Montenegro. der Hercegovina und Bosnien im Juli und August 1900. - B. Marcowicz, Lappa Palladini sp. n. - A. Kneucker, Brmerkungen zu den Cyperaceae (exclus. Carices) et Juncaceae exsiccatae.

Bot. Litteratur, Zeitschriften ete.: A. Kneucker, Haláesy, Dr. E. v., Conspectus florae Graecae (Ref.). - Derselbe, Dalla Torre, Dr C. G. v, u. Harms, Dr. H., Genera Siphonogamarum ad systema Englerianum conscriptà (Ref.). - Derselbe, Uyptogamae Japonicas iconibus illustratae (Ref.). - Derselbe, Phanerog imae et Pteridophytae Japonicae iconibus illustratate (Ref.). - Inhaltsangahe versehied. hotan. Zeitschriften. - Eingegangene Druckschriften.

Bot. Anstalten, Vereine, Tauschvereine, Exsiceatenwerke, Reisen etc.: Botan. Verein der Provinz Brandenburg (Ref). - Botan. Tauschverein in Arnstadt. - Siegfried \& Sickenberger, Herbarien. - Jaczewski, Komarov, Tranzschel, Fungi Rossiae exsiccati. - Botaniker, tropendiensttaugliche.

Personalnachrichten.

\section{Die Gattungen der mitteleuropäischen Scirpoideen.}

Von E. Palla.

(H'ortsetzung.)

\section{Eucypereen Rikli, a. a. 0.}

Die grimen Blatt- und Stengelzellen zu einem meist einschichtigen, einheitlichen oder durch subepidermale Bastbelege der Gufüssbindel zerklïfteten Assimilationsgewebe angeordnet, niemals ummittelbar an die Schutzscheicie angrenzend, sondern von ihr durch rine farblose Parenchymscheide ${ }^{1}$ ) getrennt (beziehungsweise durch Bastgewebe, wemn

1) An Stelle dieser Parenchymscheide tritt bei den Chlorocypereen der ïussere Assimilationskranz auf! Zweifelsohne repräsentieren die bei den Eucyp!ren hezuiglich des Assimilationssystems bestehenden Verhältnisse den älteren anatomischen Typus, und wir haben die den Chlorocypereen zukommende Ausbildung von ihnen abzuleiten (unter Annahme eines Funktionswechsels der inbetracht kommenden (iewebe). Rücksichtlich der Entstehung des imueren Assimilationskranzes kamn wohl kein Zweife] darüber obwalten, dass derselbe aus den an die Schutzscheide anstossenden Leptom- 
ein solches als Bastrippe dem Gefïsshündel anliegt und die Parenchymscheide teilweise unterhicht). - Perigonborsten vorhanden oder fehlend.

a. 'Tragblätter der Blüten zweizeilig angeordnet.

5. Eucyperus Rikli. Perigonborsten fehlen.

Einieimische Art: Euc. fuscus (L.) Rikli.

b. Aehrchen mit mehrzeilig angeordneten 'Tragblättern.

(i. Srivpurs L. Aehrchen mehrere bis vicle; die Inflorescenz dentlich endständig, von mehreren laubhlattähnlichen Hochblättern grestützt. l'erigonhorsten vorhanden, fo (seltener weniger). Das grüne Gewebe des stengels durch his an die peripher gelagerten Gefässhündel sich anschliessende, subepidermale Bastrippen in zahlreiche, selbständige 'Teile zertrennt. Markgewebe mit einem einzigen centralen Luftgang oder ganz frei ron grösseren Luftgängen.

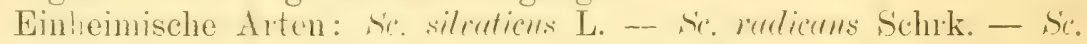
maritimus L. (dieser wohl kaum hieher gehörig).

7. Eriophoum L. Aehrchen 1-viele. Perigonborsten stets vorhanden, zahlıeich, nach dur Bliitczeit stark sich verlängernd. Im anatomischen Bau des stengels im Prinzip mit shor ühereinstimmend (hei $E_{\text {: }}$ grache setzen sich die sulepidermalen Bastbündel nicht oder nur zumteil an die Gefässbündel an).

Einheimische Arten: E. latifolium Hoppe. - E. gracile Koch.E. angustifolium Roth. - E. Scheuchzeri Hoppe. - E. vaginatum L.

8. Hotoschocmus Link. Achrohen zahlreich, zu kugeligen Blütenständen zusammengedränt; Gesantinflorescenz scheinhar seitenständig. Perjgnborsten fehlen. Im anatomischen Ban des stengels an Scirms sich anschliessend, aber dadurch ahweichend, dass die subepidermalen Bastripren sich nicht oder mit sehr verjüngten Enden an die Gefïssbündel ansetzen.

Einheimische Arten: H. vulgaris Link. - H. australis (L.) Fritsch. H. romams (L) Fritsch.

9. Wrehophomm, Pers. Ein einziges endstiandiges Aehrchen.

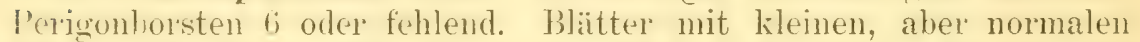
Blattspieiten (wenigstens das oberste Blatt). Gefäsbündel des stengels wenige, in einem einzigen Kreis. Subepidermale Bastrippen in der Anzahl der wröseren Gefässbïndel, sich an sie anlegend. Markgewebe ganz oder mit centralem Luftgang

parenchym-, bezw. Hadromparenchymzellen der Eucypereen hervorgegangen ist. Bezüglich der phylogenetischen dusbildung des äusseren As-imilationskranzes aber muss man vorläufig zwei Möglichkeiten zulassen: entweder sind alle Assimilationszellen der Eucypereen zu dem farblosen Wassergewebe der Chlorocypereen geworden, während gleichzeitis die farhluse l'arenchrmscheide sieh zum Assimilationsgewelse umgewandelt hat. uder es entepricht der änsere Assimilationskanz den umnittelhal an die Parenchymscheide ansrenzenden Assimilationszellen der Encyperen, während die übrigen im Laufe der phylogenetischen Entwicklung zu Wassergewebszellen geworden sind und die Parenchymscheide verschwunden ist. Welche von den beiden Möglichkeiten als thatsächlich gegeben zu betrachteu sein wird, müssen erst künftige Untersuchungen erweisen; wewi-se bei einigen Fimbistylis-Arten verkommende Verhälnisse, anf die Rikli hinweist (a. a. O., S. 532), sprechen vorderhand mehr für die zweite Ansicht. 
Arten: Tr. anstriacum Palla (= Tr. coespitosum [L.] Hartm. z. Th. $\left.)^{1}\right)$ Tr. germanicum Palla (= Tr. cuespitosum [L.] Hartm. z. Th. '). - Tr. apimum (L.) Pers. - Tr. atrichum Patla (= Scirpus alpimus Schleich.).

10. Blysmms Panz. Aehrehen in eine zweizeilige Aehre zusammengestellt. Perigonborsten vorhanden (6) oder fehlend.

Arten: Bl. compressus (L.) Panz. - Bl, rufus (Huds.) Link.

11. Schoenoplectus Palla. Aehrchen mehrere bis zahlreich (ausnahmsweise nur 1). Inflorescenz, wenigstens in der Jugend, scheinbar seitenständig. Perigonborsten bei den meisten Arten vorhanden. Subepidermale Bastrippen des Stengel zahlreich, isoliert. Mark von zahlreichen „rossen Luftgängen durchzogen Gefïssbïndel zahlreich am ganzen Querschnitte des Markes verteilt.

Einheimische Arten: Sch. Iarustris (L.) Palla. - Sil. Tubernuemontuni (Gm.) Palla. - Sh. cerimatus (Sm.) P'alla. -- Sh. Kullumsiii (Aschers., Abrom., Grähn.) Palla. -. Sich. triqueter (L.) Palla. - Sch. pungens (Vahl) Palla. - Sch. littorulis (Schrad.) Palla. - Sch. mucronatus (L.) Palla. Sch. supinus (L.) Palla.

12. Isolepis R. Br. Inflorescenz scheinbar seitenständig, aus 1 oder mehreren zu einem Köpfchen vereinigten Aehrchen sebildet, oder ein einziges endständiges Aehrchen. l'erizonborst.n fehlen. Blätter mit normaler oder rudimentärer Spreite. Anatomischer Bau wie bei Shenoplectus, aber vereinfacht: Gefässbündel wenige, in einem einzigen Kreise, Luftgänge so viele als Gefässbündel.

Einheimische Arten: I. setacea (L.) R. Br. - I. fluitans (L.) R. Br.

13. Heleocharis R. Br. Ein einziges endständiges Aehrchen. Perigonborsten meist vorhanden. Griffel, mit Ausnahme von H. pauciflora und parmla, von Fruchtknoten dentlich abgesetzt. Blattspreiten vollständig fehlend oder kaum angedeutet. Anatomisch ausgezeichnet durch die zahlreichen kleinen isolierten Bastrippen des Stengels, welche entwicklungsgeschichtlich aus je einer Epidermiszelle hervorgehen und an Querschnitte mehr oder minder die F'orm eines Fünfeckes zeigen. Sonst anatomisch mit loolepis, in emigen grossen tropischen Arten mit Schoenoplectus übereinstimmend.

Einheimische Arten: II. pulustris (L.) R. Br. - II. unighumis (Link) Schult. - H. multicaulis A. Dietr. - H. pauciflora (Lightf.) Link. H. ovata (Roth) R. Br. - H. acicularis (Is) R. Br. - H. carniolica Koch. - H. parmula (R. S.) Palla. - H. Lereschii Shuttlew.

Ausser den 9 in unserem Gebiete vertretenen Eucupcren-Gattungen kommt in Europa nur noch 1 vor: Fruiremu Rottb. (= $F^{\prime}$. pulbescens (Poir.) Kth., in Portugal, Spanien und auf Corsica)

Ueber die gegenseitigen Verwandtschaftsverhältnisse der LuchperechGattungen lässt sich, nachdem die aussereuropäischen ścirpoidren in ihrem anatomischen Bau noch sehr wenig erforscht sind, vorderhand nicht viel diskutieren; doch lïsst sich jetzt schon erkemmen, das einerseits

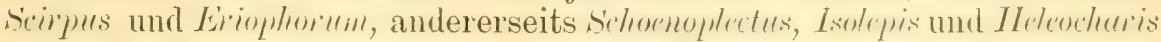
natürliche Verwandtschaftsgruppen bilden.

$\left.{ }^{1}\right)$ Ueber die Zerlegung des $T r$. caespitosum in $T r$, austriacum und germanicum vgl. man meine Abhandlung "Einige Bemerkungen über. Trichophorum atrichum und caespitosum “ (Ber. d. Deutsch. bot. Ges., XV, 1897, S. 468). Eine Besprechung der beiden Arten wird übrigens auch in dieser Zeitsehrift in den bemerkungen zu den "Lrperaceae et Juncaceae exsiccatac" demnächst erfolgen. - (Siehe p. 222 u. 223 dies. Heftes.) 


\section{Cerricuideren.}

Die Unterschiede zwischen den beiden Unterfamilien der Cyperaceen, den Scirpoidtern und C'uicoideen, beruhen auf dem bei beiden verschiedenen Bau der Aehrchen; näheres darüber findet man bei $\mathrm{Pax}$, Beiträge zur Morphologie und systematik der Cyperaceen (Botan. Jahrb. f. Systematik, VII, 1886, S. 287). Ich führe hier der Vollständigkeit halber auch die in unserer Flora vertretenen Gattungen der Curicoideen dem Namen nach an.

\section{Rhynchosporeen.}

14. Cladium Schrad.

15. Rhynehospore Vahl.

16. Schoenus L.

\section{Caviceen.}

17. Elyma Schrad.

18. Kobresia W.

19. Uncinia Pers. (bei uns Uncinia microglochin Spr. = Carex microglochin Whlbg.).

20. Carex L.

\section{Bestimmungsschlüssel.}

Demsclben sind anch die anatomischen Charaktere zugrundegelegt, weil sie rasch zum Ziele führen: die Benützung nur äusserer Merkmale wïrde den Schlüssel sehr kompliciert gestalten. Im übrigen ist der Bestimmungsschlïssel nur zumteil auf (rattungscharaktereu aufgebaut und nur insoweit giltig, als eine einheimische Art auf ilre Gattungszuständigkeit geprüft wird. Mrrkmale nicht strengen Gegensuitzes stehen in Cursiv-Schrift.

Die grünen Blatt- und stengelzellen in einer einzigen Schicht unmittelbar an die (x́füssbundel-schutzscheiden angrenzend; imnerhall, der (iefïssbindel selbst, von der Schutzscheide umgeben, ein zweiter einsehichtiger Kranz chlorophyllhaltiger Zellen. Gefüssbündel ohne farblose Parenchymscheide. Peingonhorsten stets fihlend. Chlorocy pereen.

Die grünen Blatt- und Stengelzellen von den GefässbündelSchutzscheiden durch eine firblowe Parenchymscheide getrennt, zu einem mohrschichtigen, einheitlichen oder durch die Gefïssbïndel mit ihren oleren bastbelegen zerteilten Assimilationsgewebe angrordnet; immerhall, der (ivfïsshündel keine grünen Zellen (wenigstens keine typischen Assimilationszellen). P'erigonlunsten corburden oder fehlend. . . . Eucypereen. . . . . . . 5

Tragblätter stets zweizeilig

Trazhlitter stets mehreilig

Epidermiszellen des Stengels wenig höher oder niedriger als hreit, in den liadialwänden nicht aulfallend dick. Choveryperus.

Epidermiszellen des Stengels zum grossen T'eile noch einmal so hoch als breit, auch in den Radialwänden dick. . Galilea. 
Griffel am Grunde verdickt und vom Fruchtknoten deutlich abgesetzt. Aehrchen in emer einfachen, doldenähnlichen oder zusammengesetzten Spirre. Wenigstens die Blattscheiden behaart. Fimbrel istylis.
Griffel ohne deutliche Grenze in den Fruchtknoten übergehend. Aehrchen in einem Scheinköpfchen. Pflanze kahl. Dirhostylis.

$5\left\{\begin{array}{l}\text { Tragblätter stets mehrzeilig. Perigonborsten vorhanden oder } \\ \text { fehlend. }\end{array}\right.$ fehlend.

Tragblätter stets zweizeilig. Perigonbursten fchlen. Lucyperus.

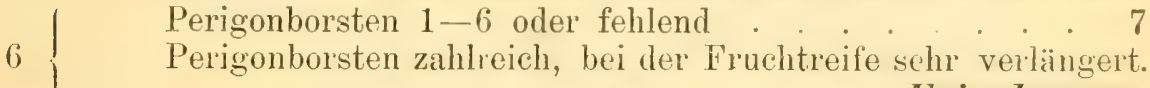

Eviopllom!lm.

Aehrchen in einer zweizeiligen Aehre angeordnet. $\left.{ }^{1}\right)$ Blysmus.

Aehrchen stets nur 1, zur Blütezeit dentlich endständig. 9

8 Aehrchen 2-viele; bisweilen nur 1, dann aber wenigstens bis zur Bliitezeit scheinbar seitenständig, indem das das Aehrchen stützende Hochblatt scheinbar den Halm fortsetzt . . . . 11

Blattspreiten (wenigstens des obersten Laubblattes) stets wohl entwickelt. Griffel nicht vom Finchtknoten abgesetzt . . . 10

Blattspreiten fehlend, höchstens schwach angedeutet. Griffel

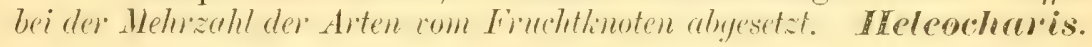

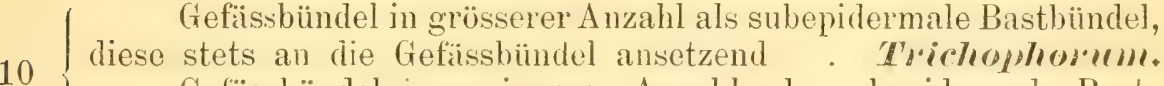
Gefässbiindel in geringerer Anzahl als subepidermale Bastbïndel, diese durchwegs isoliert . . . . . . Isolepis.

Blütenstand wenigstens vor der Blütezeit scheinbar seitenständig (zur und nach der Blütezeit nucht selten dentlich endständig erscheinend). Subepidermale Bastliundel durchgehends isoliert oder hï̈chstens (bei Iloluschoemus-Arten) mit ganz verjingtem Lnde an

die Gefïssbiundel ansetzend . . . . . . . . . . . 12

Blütenstand stets dentlich endständis ª , von mehreren laubblattähnlichen Hochblättern gestützt. Subepidermale Bastbündel an die Gefïssbündel sich anlegend . . . . . Scirlmes.

( Markgewebe mit mehreren bis zahlreichen Luftgïngen . 13

Markgewebe mit einem einzigen centralen Luftgang oder ganz. Aehrchen in kugeligen Scheinköpfchen. Perigonborsten fehlen

Gefässbündel am ganzen Querschnitte des Holoschoemus. oder wenigstens in zwei Krreisen, wovon der innere auch unvollständig sein kann. P'erigonbursten corkenden, b, mer bei sih. supinus in der Regel fehlend . . . . . . . Schoemoplectus.

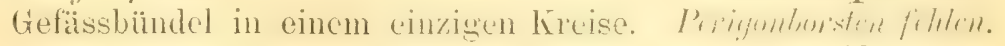
Isolepis.

') Bei Blysmus rufus und Scirpus maritimus besteht der Bllitenstand bisweilen aus einem einzigen Aehrehen und ist dann scheinbar seitenstindig.

$\stackrel{2}{)}$ Bei Blysmus rufus und Scirpus maritimus besteht der Blïtenstand biswcilen in einem einzigen Aehrchen, und dies ist damn scheinbar seitenständig. 


\title{
Unsere Reise nach Istrien, Dalmatien, Montenegro, der Hercegovina und Bosnien im Juli und August 1900.
}

\author{
Von L. Gross und A. Kneucker.
}

Im Verlaufe des Frühjahrs 1900 verabredeten wir auf brieflichem Wege, im Nonat August des namlichen Jahres eine gemeinschaftliche botanische Sammelreise nach oben genannten Ländern zu unternehmen. Ausser Phanerogamen sollten Gefiisskrytogamen und nehenhei anch Woose, Algen und Pilze als sammeloljekte ins Auge gefasst werden. Ein mitgeführter hodak (phot. Apparat) diente zur Tllustration der Reise. Da der eine von uns $\left.\left(G_{*}\right)^{*}\right)$ in der nicht gerade unangenehmen lage war, schon von Mitte Juli an frei über seine Zeit verfügen zu kömen, ging dieser nach Istrien voraus und sammelte dort zunächst mit Hrn. Gugler aus Nördlingen, später allein an verschiedenen Orten. Am 2. August aber trafen wir in Fiume zusammen, um von hier aus den gemeinsamen 'Teil der Reise zu beginnen, über die wir nun berichten wollen.

Wenn wir uns dabei nicht, wie sonst wohl zu geschehen pflegt, auf die hosee 1 iedergahe eines systematiseh geordneten Verzeichnisies der gefundenen Pflanzen hechränken, sondern wenn wir mseren simmelstuft nach Exkursionen zusammenstellen, so geschicht dies hampt-ichlich in der Absicht, gelegentlich anch bemerkungen üher I and und Lente, I'nterkunftsverhälnisse, Beförderungsmöglichkeiten und minches Andere einfliesien lassen zu kömnen, was hesonders für Floristen, welche ebenfalls die betr. Länder besuchen wollen, von Interesse sein dürfte. Wir glauben zudem, dass eine Schilderung der einzelnen 'Touren auch insofern einen Vorteil gewähren kamn, als mitunter ler Leser die Freude des Sammlers bis zu einem gewissen Grade mitempfinden darf, manchmal eine Menge auserlesener Species auf engem Raum beisammen zu sehen. Am besten diurfte ührigens so anch die bei uns vielfach verheitete Ansicht hekimpte werden, als ob's "da unten" in den Sommermonaten nur Steine und Sonne gäbe und Hammelbraten. Gerade im Hinblick auf diese nur kurz angedeuteten Vorzüge

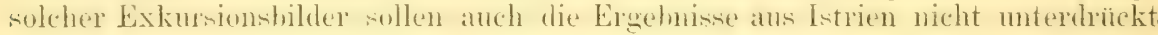
werden, wiewohl Niemand erwarten wird, dass ein Land mit so trefflichen Lokalfloren, wie sie Istrien von Freyn, Marchesetti, Pospichal u. A. gewidmet wurden, den flühtig durchreisenden Fremdling viel mehr, entedecen" lissen sollte, als etwa einige neue Standorte.

\section{A. Küstenland, insbes. Istrien.}

\section{Im Karst von Divača und S. Canzian.}

Wer sich zum ersten Male dem Karste nähert, um einige Zeit daselbst zu verweilen, dem schwellt wohl in der Regel nicht frohes Erwarten die Brust; wem er gar den Wog dahin quer durch's Herz der Alpen gewählt, hat er zweifachen Gruml, sich hanger Giefühle nicht zuschamen und der antsübende" botaniker vollends, der des nämlichen Wreges gezogen kommt, mag fast etwas wie Rene

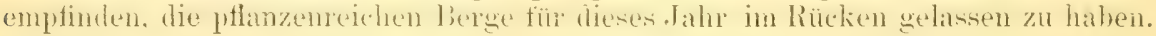
Bei mir $(G$.) selbst mögen mündliche Schilderumgen des vielverrufenen Karstes

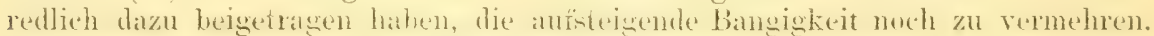
Wie gross war daher die freudige Ueberraschung, als ich am 17. Juli mit Freund Gugler von Villach ïber Laibach kommend von den Fenstern des Eisenbahnwagens aus, wie schon unterwegs, so auch noch um S. Peter, also bereits mitten im Karst, eine fast üppige Vegetation erblicken durfte, die sich anch gegen Divača hin nicht völig in Steine auflöste.

Divača, das wir zu einer späten Stunde des Nachmittags bei grosser Hitze

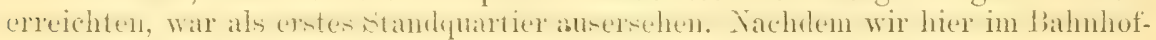
hotel ein leidlich gutes Zimmer mit 2 Betten - andere waren nicht zu haben -

*) Die hie und da vorkommenden Buchstaben (G.) und (K.) sind die Aufangsbuchstaben der Namen Gros: bezw. Knencker. 
mit Beschlag belegt und uns gestirkt hatten, unternahmen wir noch am $\Lambda$ hend einen Vorstoss gegen ein hauptsichlich aus jungen Eichen und Hoplenbuchen gebildetes Gehölz dicht neben dem nach Triest führenden Schienenstrang in der Nöhe des Bahnhofs. Neben den Schienen wuchs Autirhinum orontium L., spärlich Polycremum majus A. Braun, ferner der im süaden unvermeidliche Bromus squarrosus $L$., zwar schon verblüht, aber in einzehen Exemplaren zur Priiparation noch geeignet; auch Ianthium spinosum L. fehlte hier nicht. Die Grenze zwischen Bahnkörper und Gehölz bildet eine lose aufgeschichtete riteimmauer, neben welcher anf der Gehölzseite ein recht verwahrloster und deshalb mit allerlei P'fanzen bewachsener Weg hinzieht. An dieser stelle machten wir gute Bente. Zunïchst fesselte uns Centaurea rupestris L. var. armata Koch mit Uebergängen zur subinemis Koch, welch' letztere Pospichal*) als die typische Pflanze betrachtet. Wir latten hier Gelegenheit, die grosse Variationsfihigkeit des prïchtigen Gewiichses, anch in der Blattbildung, zu bewundern. Es scheint fist, als ob der liang einer V arietit für armuta einerseits und subinermis andererseits zu hoch gegriffen sei. In dieser Ansicht bestarkt mich eine Aellsserung Marchesettis:**) Del resto vi si trovano tutte le forme di passaggio, talvolta sulla medesima pianta.***) Dicht neben der Centanea stand Liphorbia Viequensis All., Hypericum perforatum $L$. rar. stenoplugllum Wimm. de Grab., Rutu divariratu Ten. und einen blauen Farbenton beimischend Érynginm anchystinum L., eine der schönsten L'mbelliferen „im Distelgewand"; mehr im Gehüsch wuchs Dorycninm suffruticosum hisch, nahe der Hauer aber hatten sich Geranium purpureum Vill. und die überaus stattliche Campanula pyramidalis L. ein Plätzchen erobert. Ebenda wuchs auch Teucrium montanum $L$. und zwar sowohl in der typischen, als auch in einer schönen Schattenform mit übex '2 dm hohen s'tengehn, deren Blätter den Grund des nächsthöheren blattpaares in der Regel nicht erreichen. Kanm nahmen wir uns noch Zeit zum Einsammeln eines Thymus, den ich trotz der verspäteten Blütezeit für longicaulis Presl halten muss, und einer Cuscuta epithymum L., die ant Golium purpureum L. und Euphorbia ryparissies L. zugleich schmarotzte; denn gar verfüherisch lockte aus der nahen debiischlichtung eine überaus stattliche Umbelifere, die gerade in gelber Blätenuracht prangte: Ferulayo gulbunifere Koch. Neben ihr standen, an Höhe wetteifernd, Bupleurum gramineum Vill. und Seseli elatum DC., nicht weit entfernt Cnidium apioides spreny., Allium

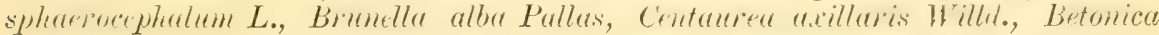
officinalis L. in der Form serotina Host, Veronica anstriaca L. var. bipinnatifida Koch fruct., Campanula bononiensis L., Euphorbia dulcis L. fruct., Linum tenuifolium L., Anthericum ramosum L., Dictammus albus L. fruct., Thesium montanum Ehrh. Melica ciliata L. und Brachyportium pimatum Beane. Nur spairlich finden wir die seltene Innla ensifolie L. und die an Filigranarbeit erimemde Genista silicstris Soop. a) typica P'ospichal; doch sind deren Hülnen nicht kahl, †) sondern gegen die spitze zu und besonders an der Banchnaht behaart. Auch der Keleh der von uns gesammelten Pflanze ist behaart, am st:irksten am liande der Kelchzithne ma auf der inneren Seite derselben.†) In voller Bläte steht dsperesh cynanchica L. rar. scubria Freyn, bei welcher der, Stengel unterwirts von kurzen Papillen körnig rauh “ ist, †††) ein Zusatz, der von Pospichal übersehen worden zu sein

*) Pospichal, Ed., Flora des österreichischen Küstenlandes, Leipzig und Wien, 1897-99, Bd. 11, p. 930.

**) Marchesetti, Dr. Carlo, Flora di Trieste e de suoi dintorni. Trieste 1896-97, p. 322 .

***) Im Original nicht gesperrt gedruckt!

†) cfr. Pospichal, 1. c. II, p. 346: ${ }_{n}$ Hülsen ... kahl ${ }^{\star}$ und Marchesetti, 1. c. p. 109: "legumi romboidali glabrescenti*; dagegen Schlechtendal-Hallier, Flora von Dentschland, 5. Autt., 23. Bd., p. 89: "Die Hülsen sind ... behart."

计) Nach Pospichal soll der Kelch kahl sein. Vergl. dagegen Fritsch, Exkursionsflora für Oesterreich (1897), p. 315!

††) Freyn, J., Flora von Süd-Istrien p. 348, in Verh. der zool.-bot. Gesellschaft in Wien, XXVIL. Band. 
scheint. Allenthalben wächst Triticum intermedium IIost. Ein Cruduus, der viele Aehnlichkeit mit unserm mutans hat, die Köpfchen aber aufrecht trägt, gehört wohl zum Carduns litoratis Borbás. Die Hüllschuppen der zwei Pflanzen, welche ich mitnahm, zeigen freilich eine bedenkliche Neigung zur Einschnürung, so dass der Vertacht, einen nutans vor sich zu haben, nicht ganz abzuweisen ist.

Wir verliessen nicht gern den ergiehigen standort; doch wollten wir noch einem benachharten Hügel einen kurzen Besuch abstatten. Wir erreichten ihn freilich nicht mehr, da die schnell eintretende Nacht fast plötzlich unserer Sammelthätigkeit ein Ende bereitete. Als Schlusseffekt hatten wir noch einen entzückenden Anblick: eine Gruppe von Aurvopryan Gryllus L. in voller Blüte. Teh selber fand ausserdem als letzte Pflanze für diesen Tag ein ziemlich schwächliches Exenplar der Koferia en-sptentens A. grandifora Aschers. of Gratuer.

Wer kann glauben, dass wir am 17. Juli unsern Nachttrunk mit betrübten Mienen genommen hätten?

(Forts, folgt.)

\section{Lappa Palladini sp. n.}

Diese neue Klette war his jetzt noch von niemand unterschieden worden, ohgleich sie im Gouvernement Tereck auf einem tausende von Morgen zählenden Areal vorkommt und oft auf grossen Strecken vorherrscht. Es erkliirt sich dieses daraus, dass alle Botaniker, die den Kaukasus besuchen, zur alpinen Zone emporstreben, wo die hübschen Alpenblumen einen herrlichen Teppich bildon, um dort kaukasische Rarititen zu sammeln. Wer aher, wie ich, infolge seines Wohnortes an eine tiefere Zone des Kaukasus gebannt ist und infolge seines Berufes im Unkraut umherkriechen muss, der hat auch manchmal einen Blick für eigentümliche Furmen der kaukasischen Vorberge. Wie der erste Erforscher der Flora des Kiukasus Marschall von Bieberstein*) (1808), so anch der letzte, Hr. Lijsky (1899)**,, kemnen für den ganzen Kaukasus nur drei Kletten: Lap)pa major Gä̈rtr., L. mimir DC. und L. tomentosa Lam. Meine Exkusionen haben aber ergeten, dass in Ossetien, 'T'schetschnya und Imeretien (Radtscha) lieine von diesen Lappa-Arten sich betindet, sondern hier wächst eine ganz eigene Art, die ich Lappur I'allatimi henaunte. Sie unterscheidet sich von den oben citierten 3 Arten auf den ersten Blick: von $L$. major und $L$. minor durch ihre doldentraubige Infloresens, schwache Spinnwehgkeit und ganz eigene originelle samen, von $L$. tommtosa durch geringere Spinmwebighsit, durch ihren Hahitus ebenfalls durch die sehr runzeligen, gerippten siamen und die gefarbten löffelartigen, inneren schuppen des Köpfchens. Auch der Habitus dieser Lappa ist ganz eigentünlich; demn erstens ist sie sehr hoch und erreicht oft eine Höhe bis zu $4 \mathrm{~m}$, zweitems bilden ilne Aeste einen subcorymbus, d. h. auch die unteren Zweige erreichen heinahe den Gipfel der P'flanze; die oberen und mittleren bilden eine Doldentrablue und liegen ofter in der Richtung des Meridians, so dass die Pflanze dann gleichsam als Kompass dienen könnte.

Diese Klette wird ausführlich in den „Arbeiten des Jurjew'schen botanischen Gartens" Lief. III unter Beigabe der Abbildung***) beschrieben; hier aber gehe ich vorlaulig eine liurze Diagnose: Capitulis subcorymbosis longe pedunculatis. Involucris parm arachnoideis vel glaberrimis, squamis externis subulatis et uncinatis, medis coloratis lineare subulatis vel subulato uncinatis, internis linearis coloratis (purpureis) apice dilatatis in mucronulo recto attenuatis. Acheniis oblongis apice truncatis costatis $t-6$ jugis primariis multo elevatis et secumdariis parvis saepe olsoletis vel prominulis, interea jugis transverse rtugosis, jugis et rugis griseis, foveis nigris. Caule striato ramoso ramis breve villosis subcorymbosis. P'anta 1-10 pedalis hahitat praesertim in pratis silvestris, ruderatis, hortis al margines silvarum in montihus et promontoriis. Floret Julio-Augusto.

B. Marcowicz.

*) Flora Taurico Caucasica Marschall von Bieberstein-Charkow.

**) Flora des Kaukasus. W. J. Lipsky. Petersburg.

***) Marcowicz hat mir eine Photographie dieses mächtigen (iewächses eingesandt. 


\section{Bemerkungen zu den Cyperaceae (exclus. Carices) et Juncaceae exsiccatae*)}

von $\mathrm{A}$. Kneucker.

IX. Lieferung 1901.

Nr. 31. Chlorocyperus laevigatus I'alla $=$ Cignerus lateigatus $L$. Mant.II, p.179 (1771) $=$ C. mucronatus Rottb. Pr. p. $17(1773)=$ Pycreus moronatus Nees Linn. IX, p. 283 (1834) $=$ P. laevigatus Nees in Lin. X, p. 130 (1835-36).

In Salzlaken und in brackigem Wasser am Meeresstrand bei Beirut in Syrien, namentlich an der Mündung des Beirutflusses, Nahr Beirut (Magoras). Die Standorte werden oft vom Meerwasser überspült; Alluvium. Begleitp flanzen: Chlorocyperus distachyus (All.) Palla; oft aber auch allein und bestandbildend.

Meeresstrand; Dezember 1899 und August 1900.

leg. Ernst Hartmann.

Nr. 32. Chlorocyluevus serotimus Prella $=$ Cyperus serutims Rottb. Descr. et icon., p. $31(1776)=$ C. Monti L. f. Suppl.,p. 102 $(1781)=$ Pycreus Monti P. B. in Rchb. Fl. Germ. p. 72 (1830).

An feuchten Stellen bei der Mündung des Flusses Nervia bei Bordighera in Ligurien (Oberitalien). Begleitpflanzen: Chlorocyperus flavescens (L.) Rikli, Juncus lampocarpus Ehrh., scirpus maritimus L., Typha angustifolia L., Mentha aquatica L., Lycopus Europaeus L., Lythrum salicaria L.

Nahe dem Meere, 25. Sept. 1900. leg. Clarence Bicknell\&L. Pollini.

Nr. 33. Dichostylis Michelinur Nees in Gen. pl. fl. germ. IX, tab. 7, f. $1-8(1836)=$ Scirpus Michelianus L. Sp. pl. ed. I, p. 52 $(1753)=$ Jsolepis Micheliana R. S. Syst. II, p. $114(1817)=$ Cyperus Michelianus Sadl. Fl. pest., p. 31 (1825).

Auf sandigem Alluvium an Ufer des Flusses Dniepr bei Kiew (SüdRussland). Begleitpflanzen: Eragrostis pilosa P. B., Heleochloa schoenoides Host, Heleochlea alopecuroides Boiss, Cyperus fuscus L., Juncus bufonins I., L'olygonum lapathifolium L., Rumex Ucrainicus Fisch., Cardamine parviflora L., Limosella aquatica L., Gnaphalium uliginosum L.

88 m ü. đ. M.; 14. Sept. 1900.

leg. N. Zinger.

\section{Nr. 33 a. Dichostylis Micheliana Nees.}

Auf feuchtem, schlammigem, kalkhaltigem Alluvium des rechten Ufers der Rjeka, unterhalb der Brücke bei dem gleichnamigen Dorte lijeka im Gebiete des nahegelegenen Skutarisees in Montenegro in grosser Menge. Begleitpflanzen: Fimbristylis dichotoma Vahl., Chlorocyperus longus (L.) Palla, Heleochloa alopecuroides Host, schoenoides (L.) Host, Gratiola officinalis I., Mentha pulegium L., Crlinus lotoides L., Amamia verticillata I., Oldenlandire Cropensis 'Thaub. fl. (ap). (Letztere nach Bormmüller's briefl. Mitteilung neu fül' Europa.)

Ca. $10 \mathrm{~m}$ ü. d. M.; 15. Aug, 1900.

leg. L. Gross u. A. Kneucker.

*) Die Revision, bezw. Bestimmung des ausgegebenen Materials und die Zusammenstellung der Litteraturnachweise wurde hei den Cyperaceen von H. Dr. E. Palla in Graz (jetzt auf Java) und bei den Juncaceen von Hr. Prof. Dr. Fr. Buchenau in Bremen gütigst übernommen. Auch wirkte noch Hr. Sehriftsteller W. Lackowitz bei Durchsicht der Korrekturabzüge freundlichst mit. - Der Lieferung II wird ausserdem ein Separatahdruck der soehen in der, All@. Bot. Zeitschrift" erscheinenden Pallaschen Arbeit „Die Gattungen der mittelemopiaischen sirirpoideen beigelegt.

A. Kneucker. 
Nr.3t. Fimbristylis dichotoma Vahl in Enum. pl. II, p. 287 $(1806)=$ Scirpus dichotomus L. Sp. pl. ed. I, p. 50 (1753).

Auf feuchtem, schlammigem, kalkhaltigem Alluvium des rechten Ufers der Rjeka, unterhalh der Brücke hei dem gleichnamigen Dorfe Rjeka im Gebiete des nahegelegenen Skutarisees in Montenegro. (Standort derselbe wie bei $\mathrm{Nr} .33$ a; Begleitpflanzen dieselben.)

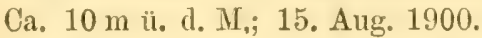
leg. L. Gross u. A. Kneucker.

Nr. 35. Scirpus maritimus L. in Sp. 11. ed. I, p. 50 (1753).

In einem Teiche bei Sion im Kanton Wallis in der Schweiz. Begleitp f'l a n z e 1s: Ranunculus scleratus L., Rionii Lagg., Ceratophyllum submersum L., S'ciruus silvaticus L., Schoenoplectus lacustris (L.) Palla, 'Tabermaemontani (Gmel.) Palla, Carex pseudocyperus L.. acutiformis Ehrh.

$520 \mathrm{~m}$ ü. d. M.; 25. Juni 1900.

leg. Prof. F. O. W olf.

Nr. 36. Scirpus maritimus L. f. compacta (Hoffm.) D. Fl. II, p. $25(1804)=$ S. compactus Hoffim. D. Fl. II, p. 25 (1804).

Sumpfwiesen bei Lichtenhof hei Nürnberg in Bayern; s andgemischter Moorboden. Begleitpflanzen: Glyceria distans Whlbg., Carex vulpina L., Poa pratensis L.

310 m ï. d. M.; 3. Juli 1900.

leg. J. S. Ka ulfuss.

Nr. 37. Trichophormm Austrincum Palla in Ber. d. deutsch. bot. Ges. XV, p. 468 (1897) = Scirpus caespitosus L. Sp.pl. ed. I. p. 48 (1753), p. p. = Trichophorum caespitosum Hartm. Hdb., p. $256^{\circ}(1820)$ p. p.

In dichten, von Sphagnum durchsetzten Rasen auf dem Hochmoor bei Schwentlund üstl. rom s'eehate Crantz im Kreise Tischhatusen bei Königsherg in Ostpreussen. Begleitpllanzen: Eriophorum vaginatum L., Rhynchospora alba Vahl, Vaecinimm oxicoceos L., Andromeda polifolia L., Rulus chamaemorus L., Urosera rotundifolia L., Linpetrum nigrum L., Calluna vulgaris Salish., Scheuchzeria palustris L., Sphagna ete.

$4 \mathrm{~m}$ ü. d. M.; Aug. 1899 u. 6. Juni 1900.

leg. Dr. A bromeit.

Wie ich in meiner Abhandlung "Einige Bemerkungen über Trichophorum atrichum und caespitosum" (a. a. ().) anseinandergesetzt hahe, ist 'l'richophormm caespitosum in zwei Arten zu zerlegen, die sich vor allem durch den anatomischen Ban ihres Blütenstengels unterscheiden. Die anatomischen Differenzen dieser beiden Arten, die ich Tr. Austriacmu mol Germanicum benamnt habe, sind hei Betrachtung des Stengelquerschnittes der Hauptsache nach die tolgenden. Bei Tr. Germanicum findet sich in dem grünen Gewehe zwischen je zwei Gefissbündeln je eine farblose, runde oder elliptische Stelle vor, welche von einigen grossen abgestorbenen '/sellen gehildet wrol, ferner Excretionszellen mit an Herbarmateriale, besonders alterem, lethalt rotbram oder braungetärbten Inhalte; im Leptom der grösseren Grefisshündel hahen die viehröhren durchschnittlich $6-6^{2}{ }_{2} \mu$ im Querschnitte und treten den um die Hailfe und darunter kleineren Geleitmol Cambiformzellen gegeniibes sehr auffillig hervor; die Atemhöhle der spalt. öfinumen ist ziemlich klein (meist nur halh so hoch als die Höhe der schliesszellen). Bei Tr. Austriacum kommen in dem Assimilationsgewebe weder farblose Lellgrumen noch Excretionzellen vor; die Siehröhen der grösseren Gefïssbündel haben durchschnittlich einen Durchmesser von nur $3-31_{2} \mu$; die Atemhöhlen sind gross (meist $1-2 \mathrm{mal}$ so hoch als die Schliesszellen). Ein weiteres, allerdings nicht sanz durchgreifendes Merkmal besteht in dem Bau der Perigonborsten, welehe hei 'lr. Alustriacum glatt sind, bei Tr. Germanicum dagegen im ohersten Tiertel mit l'apillen besetzt elscheinen; doch ist von mir ausnahmsweise 
auch bei Tr. Austriacum Papillenbildung beobachtet worden. A eusserli ch lassen sich Herbarexemplare der beiden Arten von emander leicht durch die oberste Blattscheide unterscheiden; bei 'T' Austriacum ist die s'cheide anf' der' der spreite gegenüberliegenden Seite mässig ansgeschweift, und ihr schmaler häutiger Rand. der dem Stengel ziemlich dicht anliegt, ist geblichweiss oder hellbram, hüchstens schwach rötlichbran gefäht; bei Tr. Germanicum hingegen ist die sicheide viel tiefer ausgeschnitten, und der bedentend breitere Hautrand, welcher nur locker den Stengel umfasst oler sich geradezu blasig von ihm ahhebt, erscheint gewöhnlich lebhaft rostrot gefürbt.

Ir. Germanicum kommt nach meinen bisherigen Untersuchungen vor allem in der norddeutschen 'liefebene vor, sowie in Harz und Schwar\%wald; westlich von Deutschland geht es in das französische Tiefland uiber und findet sich üherdies noch in schottland und schweden vor. Die übrigen für 'Tr. caespitosum bekamnten emopäischen, asiatischen und nordamerikanischen standorte beziehén sich, soviel ich bisher gesehen, durchweg anf' 'l'r. Austriacum. Wie die hier ansgegehene l'flanze beweist, greift T'r. Austriacum von Russland her bis nach Ostpreussen über. Nach meinen Untersuchungen ist auch das bei Kiel vorkommende Trichophorum 'Tr. Anstriacum. Andererseits gehören die von mir eingesehenen P'fanzen aus Pommern (z. B. Greifswald) zu Tr. Germanicum. Es wäre eine dankbare Aufgabe, die Verbreitung der beiden Arten im deutschen Küstengehiete klarzulegen. Auch in Baden konmen heide Arten vor, 'Tr. Austriacum allerdings wohl nur im Süden (bei Ueberlingen am Bodensee!).

P.

Nr. 38. Trichophormm Germanicum Palia in Ber. d. deutsch. bot. Ges. XV, p. 468 (1897) = Scirpus caespitosus L. Sp. pl. ed. I, p. 48 (1753) p. p. = Trichophorum caespitosum Hartm. Hdb. p. $256(1820)$ p. p.

Auf den Mooren der Hornisgrinde im nördlichen 'Teil des badischen Schwarzwaldes; Granit. Begleitpflanzen: Eriophorum vaginatum L., Genista pilosa L. ete.

1100-1166 m ü. d. M.; 14. Juni 1900.

leg. A. Kneucker.

Nr. 3\%. Isolepis setacer IR. Br. in P'rodr. I, p. 78 $(1810)=$ Scirpus setaceus L. Sp. pl. ed. I, p. 49 (1753).

An sumpfigen und quellenreichen Stellen auf verwittertem Granit bei Bermersbach auf der linken Seite der Murg im nördl. 'Teil des badischen Schwarzwaldes. Begle it p fil nzen: Juncus acutillorus Ehrh., lampocarpus Ehrh., bufonius L., Sagina procumbens L., Lotus uliginosus Schk., Galium palustre L.

Ca. 500-500 m ü. d. M.; 25. Aug. 1899.

leg. A. Kneucker.

Nr. 40. Isolepis srovii schult. in Mant. II, p. 63(1824)= Ścipus śuvii Seb.u. Maur. Fl. rom. prodr., p. 22 (1818).

An feuchten Stellen in Val Seborino bei Bordighera in Ligurien (Italien). Begle it pfl a nzen: Equisetum maximmm Lam., Holoschoenus vulgaris Lk. Carex distans L., Mentha aquatica L., Cirsium Monspessulanum All.

Ca. $200 \mathrm{~m}$ ü. d. M,; 9. Juni 1900.

leg. Clarence Bicknell.

Von Isolepis setacea R. Br. vor allem durch die Frucht verschieden: bei I. setacea ist das Nüsschen undeutlich dreiseitig oder nur zweiseitig, dahei lamgsrippig und durch die stark in die lireite verzogenen, aber niedrigen Epilermiszellen der Fruchtschale mehr oder minder ansgesprochen quergestreit, hei I. Savii deutlich dreiseitig, ohne Längsrippen und wegen der (von oben betrachtet) isodiametrischen und mit einer Papille versehenen Epidermiszellen dicht fein punktiert.

Ed. Palla. 
Nr. 41. IIeleocharis ovatu R. Br. in I'rodr. I, p. 80 (1810) - Scirpus ovatus Roth in T'entam. fl. germ. II, p. 562 (1789) et in Catalecta bot. II, p. 5 (1800).

Auf dem Grunde eines abgelassenen Teiches bei Wundschuh, südl. von Graz in Steiemark. Begleitpflanzen: Riccia fluitans L., Carex cyperoides L., Bidens cernua L.

Ca. 340 m ü. d. M.; Juni 1900.

leg. Dr. Ed. Palla.

Nr. 12. ILeleocharis Camiolica Toch in Syn. ed. II, p. $853(1845)=$ Scirpus Carniolicus Simk. En. fl. transs., p. 541 (18E6).

An sumpfigen Waldstellen hei Hohenegg nächst Cilli in Steiermark; Kalk. Begleityflanzen: Poa trivialis L., Carex remota L., echinata Murr, leporina L., Juncus bufonius L., articulatus L., Ramunculus flammula L., Galium palustre L., Myosotis palustris (L.).

$320 \mathrm{~m}$ ï. d. M.; 24. Juli 1900.

leg. Dr. A. v. Hayek.

Nr. 43. Schoemus ferruginems L. Sp. pl. ed. I, p. 43 (1753) = Streblidia fermuinea Lk. H. Ber. I, p. 276 (1827) $=$ Chatospora fermginea Rchb. Fl. exc., p. 74 (1830). "

Auf Noorwiesen bei Moosbrumn in Niederösterreich; Alluvium. Begleitpflanzen: Schoenus nigricans L., Carex Davalliana Sm., stricta Good., Primula farinosa $L$.

$160 \mathrm{~m}$ ü. đ. M.; 13. Mai 1900.

leg. Dr. A. v. Hayek.

Nr. 44. Schoemes mignicans I. in Sp. pl. ed. I, p. $43(175: 3)=$ Chaetospora nigricans Kunth in Enum. pl., p. 323 (1837).

Bei Ardon im Kanton Wallis in der Schweiz, auf sumpfigen Wiesen in der Rhoneebene; Alluvium. Begleitpflanzen: Blysmus compressus Panz., Schoenoplectus lacustris Palla, Carex dioica L., paniculata L., echinata Murr., stricta Good., flava L., Menyanthes trifoliata L., Potentilla tormentilla L. etc.

$460 \mathrm{~m}$ ü. d. M.; 20. Mai 1900.

leg. Prof. F, O. Wolf.

Nr. 44 a. Schoenus nigricans $\mathcal{L}$.

Auf Sumpfwiesen nördlich vorr Waghäusel in der badischen Rheinebene; Alluvium. Begleitpflanzen: Carex flava L., distans L., lepidocarpa T'sch., Hornschuchima Hyle., Lavalliana Sm., teretiuscula Good., (Hrchis laxifiora Lam., Sturmia Loeselii Rchb., Galium boreale L., IVirtgeni F. Schultz etc.

Ca. $100 \mathrm{~m}$ ü. d. M.; Mitte Juni 1898 u. 1899.

leg. A. Kneucker.

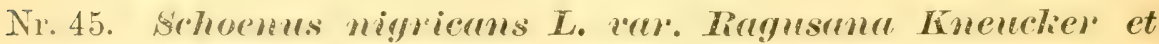
Prilla nov. var.

An vollständig trockenen Stellen auf Kalkfolsen im südl. Dalmatien links des Weges, der von der Strasse zwischen Ragusa und Gravosa nach der Halhinsel Lapad ahzweigt. Legleitpflanzen: Myrtus communis L., Lagurus ovatus L., Seleropoa rigida Griseb. etc.

Ca. $20 \mathrm{~m}$ ü. d. M.; 7. u. 9. Aug. 1900.

leg. A. Kneucker.

Die sehr robuste und derbe Pflanze fällt durch ihre Höhe (besonders grosse Exemplare 2-3 mal so hoch als der gewöhnl. Schoenus nigricans), im lebenden Zustande durch das sehr dunkle Grün der Halme und den äusserst trockenen Standort sofort auf. Herr Dr. Palla in Graz, dem ich einige Halme cinsandte, schrieh mir am 4. Sept 1900 üher die vorliegende Pflanze: ,Der von Ihmen gesammelte Schoenus gehört moweifelhaft zur Terwandtschaft des sehoenus 
nigricans, zeigt aber gewisse Abweichungen; ich werde nächstes Jahr eine genane Untersuchung (auch anatomisch) vornehmen, da ich es nicht für ansgeschlossen halte, dass er gegenüber dem mitteleuropäischen Sch. nigricans eine selbstinndige mediterrane Art darstellt."

A. K.

Nr. 46. Juncus bufomius L. Spec. plant. ed. I, p. 32S (1753); v. Fr. Buchenau, Monogr. Juncac., p. 174 (1890).

Auf quelligen und feuchten Stellen, besonders an Wegrändern und auf Waldboden bei Bermershach im nördlichen Schwarzwald; Granit. begleitp flanzen: Carex leporina L., Isolepis setacea R. Jir., Juncus lampocarpus Ehrh., Sagina procumbens L. etc.

Ca. 500-550 m ü. d. M.; 27. Aug. 1899.

leg. A. Kneucker.

Nr. 47. Juncus trifidus L. Spec. plant. I, p. $326(1753)=J$. tr. var. $\alpha$. vaginatus Neilr. Fl. v. Niederösterr. I, p. 149 (1859); v. Fr. Buchenau, Monogr. Juncac., p. 182 (1890). forma parva miflore.

Trockene Bergweiden über dem Schwarzsee bei Zermatt in Kanton Wallis (Schweiz); Glimmersehiefer. Begleitpflanzen: Juncus Jacquini L., Avena subspicata Clairv., Anemone baldensis L., Halleri All., sulfurea L., Campanula cenisia L., Saussurea alpina DC., Callianthemum rutaefolium (.. A. M., Astragalus Leontinus Wulf, australis l'eterm., alpinus L., Oxytropis Halleri Bumge, sordida Gaud., neglecta (xay, Lapponical Gay, Hieracium glatiale Reyn., piliferum Hoppe et glandulifermon Hoppe, Potentilla aurea L., frigida Vill., multifida L., minima Hall. fil. ete.

$2589 \mathrm{~m}$ ü. d. M.; August 1899.

leg. Prof. F. O. W olf.

Nr. 45. Juncus squerrosus L. Spec plant. ed. I, p. 327 (1753); ed. II, p. 465 (1762); v. Fr. Buchenau, Monogr. Juncac., p. $184(1890)=$ J. Sprengelii Willd. Prodr. Hor. berol., Nr. 394 (1787).

Auf sandigem Diluvium des Exerzierplatzes von Weissenburg i. Elsass. Begleitpflanzen: Drosera rotundifolia L., intermedia Hayne, Lycoporlim inmdatum L., Carex echinata Murr, Oederi Ehrh. ete.

Ca. $123 \mathrm{~m}$ ü. d. M.; 6. Juli 1899.

leg. A. Knencker.

Nr. 49. Juncus Befticus Willd. in Berlin. Magaz. III, p. 298 (1809); v. Fr. Buchenau, Monogr. Juncac., p. $214(1890)=J$. helodes Link Enum. pl. horti berol. I, p. $305(1821)=J$. Balticus Willd. var. a. Europaeus Engelmann, Transact. St. Louis Acad. II, p. 441 et $680(1866,1868)$

Auf dünnem Torf des kieselsandigen Meeresstrandes bei Adö in russisch Fimland. Begleitpflanzen: Agrostis alba L., Heleocharis mighmis Lk., Carex salina Whllog. ssp. cuspidata Whilbg. f. Ostrobottnica Almc., Juncus Gerardi Loisl., filifornis L., Empetrum nigrum L.

Meeresufer; 22. Aug. 1900.

leg: K. W. F outell.

Nr. 50. Auncus Balticus $x$ filiformis FH. Burbenan Honogr. Juncac., p. $216(1890)=J$. imendatus S. Diejer in Kröger's Tidsskrift II, p. 181 (1838).

In tamenbewachsenen T'orfsumpten des kiesels a ndige n Meeresstramides

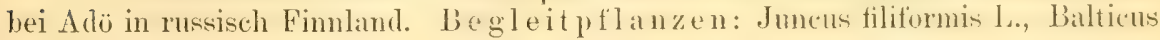


Willd., Gerandi Loisl., Carex vulgaris Fr. v. juncella Fr., Carex salina Whlbg. ssp. cuspidata Whlbg. f. Ostrobottnica Almq., Empetrum nigrum L., Vaccinium uliginosum L.

Meeresufer; 14. Aug. 1900. leg. K. W. Foutell.

Nr. 51. Jumcus filiformis I. Spec. plant. ed. I, p. 326 (1753); v. Fr. Buchenau, Monogr. Juncac., p. 224 (1890).

Auf Moorboden in der Nähe des Hohloh-Sees bei Kaltenbronn im nördl. badischen Schwarzwald; Unterlage Buntsandstein. Begleitpflanzen: Carex canescens L., leporina L., echinata Mur., Juncus squarosus L., effusus L., Andromeda polifolia L., Empetrum nigrum L., Vaccinium oxycoces L., uliginosum $L_{\text {., }}$ vitis idaea $\mathrm{L}$. ete.

900-990 m ü. d. M.; 25. Aug. 1899 und 29. Juli 1900.

leg. A. Kneucker.

Nr. 52. Juncus wortus A. Koor\%er Flora silesiara I, p. 562 (1787); v. Fr. Buchenau, Monogr. Juncac., p. $363(1890)=J$. melananthus L. Rehb. Flora Germ. excurs. I, p. $96(1830)=J$. septangulus W. L. Peterm. in Flora I, p. 361 (1844)

Bei Kiew in Südrussland in Menge am Ufer des Dniepr anf Wiesen, welche im Frühjahr ühershwemmt sind. Be gr le it pflatuen: Heckmamia cruciformis Host., Allium acutangulum Schrad., Iris Sibirica L. ete.

$88 \mathrm{~m}$ ü. d. M.; 1. u. 22. Juli 1900.

leg. N. Zinger.

Nr. 53. funcus anceps J. ae Laharpe Monogr., p. 126 (18.5); v. Fr. Buchenau, Monogr. Juncac., p.:375(1890), v(r). atricupillus Fr. Buchenau in Ber. d dentsch. bot. Ges. I, p. $487-493$ $(1883)=J$.atricapillus S. Diejer in Kröger's Tidsskrift II, p. 182 $(1838)=J$. alpims vel fusco-ater aut. div.

Dïnenthal Hall-Ohms-Glopp auf der ostfriesischen Insel Juist; Sand. Begl eit pflanzen: Hippophaës rhammoides L., Salix repens L., Juncus lampocarpus Ehrh., Epipactis palustris Crntz., P'arnassia palustris L., Linum catharticum L., Sagina nodosa Fenzl.

5-8 m ü. d. M.; 5. Aug. u. Sept. 1900.

leg. Fr. Buchenau und Otto Lange.

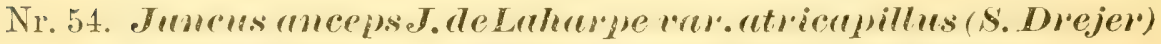 Buchenare > lampocavprs Eh\%. (Buchenau).}

An feuchten Stellen in den Dünenthälern der ostfriesischen Insel Baltrum nördlich vom Osterloog; Dünensand. Begleitpflanzen: die Stammarten, Agrostis alba L., Carex arenaria L., Phleum arenarium L., Jasione montana L., Thrincia hirta Rth.

Ca. 5 m ü. d. M.; Aug. 1900.

leg. Fr. Buchenau.

Rhizom weniger ausgebildet, Wuchs niedriger, weniger straff als bei J. anceps, Blütenstand weit lockerer. Blüten oft fehlschligend; Perigonblatter oft stumpf. Frucht meist sehr viel stumpfer als bei reinem J. lampocarpus.

Fr. Bu eh enau.

Nr. 55. Lasula Forsteri DC. Synops. plant. in Flora gallica descr., p. 150 (1806); v. Fr. Buchenau, Monogr.Juncac., p. $78(1890)=$ Jumcus Forsteri J. E. Smith, Flora brit. III, P. 1395 (1804) $=$ Luzula decolor Barker, Webb et Berthelot Phytogr. des Isles canar. in Histoire naturelle des îles canar. III, p. 350 (1840). 
Auf Muschelkalk zwischen Untergrombach und Bruchsal in Baden, in einem dichten Bestande junger Föhren. Begle itp flanzen: Luzula pilosa Willd., campestris DC. v. multiflora Celak., pilosa $>$ Forsteri (Buchenau), Asperula odorata I. ete.

Ca. $250 \mathrm{~m}$ ü. đ. M.; 31. Mai 1899.

leg. A. Kneucker.

Die beigelegten mit Papiersehlingen versehenen Halme entstammen Exemplaren, welche im Stadtgarten zu Karlspuhe kultiviert und im IIai 1898 gesammelt wurden.

A. K.

Nr. 56. Lusula pilosa Willd. Enum. plant. hort. reg. Berol., p. 39t (1809).; v. Fr. Buchenau, Monogr. Juncac, p. $83(1890)=$ Juncus pilosus var $\alpha$. Limé Spec. plant. ed. I, p. $329(175 \%)=J u n c$. vernalis J.J. Reichard Flora Moeno-Francof. 11. p. $182(1778)=$ Luzula vernalis DC. Flore franç. III, p. 160 (1805).

Unter Laubmischwald auf Hügeln zwischen Grötzingen und Weingarten in Baden; M uschelkalk. Begleitpflanzen: Carex umbrosa Host, montana L., Luzula silvatica Gaud., nemorona E. Meyer, campestris DC. r. multiflora Celak., Pulmonaria tuberosa Schrk. ete.

Ca. 230-250 m ü. d. M.; 21. April 1899.

leg. A. Kneucker.

Nr. 57. Lusula silvatica Grud. Agrostologia helvetica II, p. 240 (1811); v. Fr.Buchenau, Monogr.Juncac., p.91 (1890)=Juncus

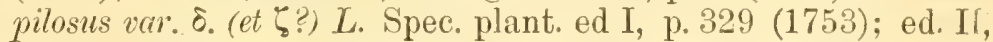
p $468(1762)=J$. silvaticus Huds. Flora anglica ed. I, p. 151 $(1762)=J$. maximus J.J. Reichard, Flora Moeno-Francofurtana II, p. $182(1778)=J$. montanus var. $\alpha$. Lam. Encycl. méth. bot. III, p. $273(1789)=J$. latifolius Wulf. Plant. rar. Carinth. in N. J. Jacq. Collect III, p. $59(1789)=$ Luzula maxima DC. Fl. franç. III, p. 169 (1805).

Auf $\mathrm{MI}$ uschelkalk unter Laubmischwald auf Hügeln zwisch. Grötzingen und Weingarten in Baden. Begleitptlanzen: Luzula pilosa Wlld., nemorosa E. Meyer, campestris DC. v. multiflora Celak., Carex montana L., umbrosa Hst., Veronica latifolia Scop., Pulmonaria tuberosa Schrk, etc.

Ca. 230-250 m ü. d. M.; 21. April u. 9. Juni 1898. leg. A. Kneucker.

Nr. 58. Lusula mmprea Masson teste H. Fr. Link (1825); v. Fr. Buchenau, Monogi. Juncac., p. $88(1890)=$ Juncus purpureus Leop. v. Buch. Canar. Inseln in Abh. Berl. Akad., p. 362 $(1816-17)=$ Luz. elegans Lowe Nov. fl. Mader., p. 532 (1338)

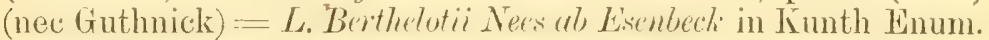
plant. III, p. 298 (18 18 ).

Calçada do Gato bei Coimbra in Portugal; San dboden. B e gle it p flanzen: Festuca sciuroides Roth, Aera caryophyllea L. v. multiculmis (Dum.) ete.

$30-130 \mathrm{~m}$ ü. đ. M.; April und Mai 1900.

leg. M. Ferreira.

Nr. 59. Lasula murans J. Dural-Jome in Bull. soc. bot. France X,

p.77 (1863); v. Fr.Buchenau, Monogr. Juncac., p. $119(1890)=$

Luz. pediformis A. P. DC. Flore franç. III. p. 162 (1805).

Auf $\mathrm{Kalkboden}$ in Fichtenwaildern und Grasplätzen in der Sierra de la Sagra in der Provinz Granada in Spanien, bei der kleinen Stadt Poblo. B egleitpflanzen: Festuca rubra L., scoparia Kern. \& Hckl., Arbutus uva ursi L., Genista Aragonensis, Alyssum spinosum L.

1900 m ü. d. M.; Juni 1900.

leg. E. RGverchon. 
Nr.60. Lusula campestris DC. vr. vulgaris Gaud. $f$. collina G. IF. W. Meyer Fl. hann. exc., p. 582 (184.9).

Am Rande der Jungfernheide bei Berlin, auf den Böschungen des Nordkanals; A lluvialsand. Begleitpflanzen: Holosteum umbellatum L., Erophila verna (L.) E. Mey., Potentilla incana Anch., Tabernaemontani Aschs., Spergula vernalis Willd., Veronica verna L. ete.

Ca. $120 \mathrm{~m}$ ü. d. M.; Mitte April 1899 u. 1900. leg. W. Lackowitz.

Die Pflanze stellt eine niedrige Form dar, die G. F. W. Neyer in d. Flora hannov. als var. collina bezeichnete.

Fr. Buchenatl.

Der Sanmler der vorliegenden Pflanze, Herr W. Lackowitz, teilt noch mit, dass dic spirre dieser Form nur 2-3köptig sei und dass der mittlere Kopf sitzend und die seitlichen verhälnismaissig lang gestielt seien. Jach seinen Beobachtungen ist die $f$. collina die zuerst erscheinende Frühlingsform, die sehr bald verschwindet und der höheren Hauptform mit 4 und mehreren Köpfen Platz macht; von dieser kommen übrigens anch Zwergexemplare vor, immer bilden aber auch dann die Köpfe einen Büschel.

A. $\bar{K}$

\section{Korrektur.}

Durch meinen Freund Kükenthal wurde die Entdeckung gemacht, dass Nr. 63 in Lief. III der "Carices exsiceatae" bei seinem Exemplar aus Elyna spicata Sideral. hesteht. Es scheint diese Pflanze mit C. nardina zusammen vorzukommen, und da zwergfömige Individuen der Élyna mit C. nardina habituelle Achnlichkeit bexitzen, heim Linsammeh teilweise verwechselt worden zu sein. Ich will versuchen, $C$. nardina $F r$. vera nochmals zu erhalten und werde dann den sich meldenden Abonnenten, welche statt $C$. nardina ganz oder teilweise E. spicala erhalten haben, z. Z. die richtige Pflanze übermitteln.

\section{Botanische Litteratur, Zeitschriften etc.}

Haláesy, Dr. E. v., Conspectus florae (iraecite. Verl. v. Wilh. Engehmann in Leeipzig. 1900. Vol. I. Fase. II. p. 225 -576. Preis 8 M.

Nun liegt auch der 2. Teil des I. Bandes vor. Einrichtung u. Erscheinungsweise dieses höchst zeitgemässen und zum Studium der griechischen Flora unenthehrlichen Wretes wurden hereits in Mr. 4 p. tis dieses Jahrgangs hesprochen. Dieser II. Teil enthält: den Schluss der Alsinaceae, die Linaceae, Maliaceae, Tiliaceae, Hypericaceae, Aceraceac, Hippocastanaceae, Ampelidaceae, Geraniaceae, Oxalinacene, Zygophyllaceae, Rutaceae, Coriariacene, Celastracene, Rhamnaceae,

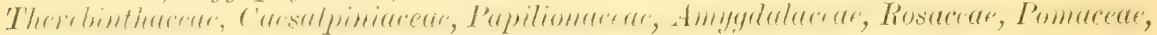
Granataceae, Myrtaceae, Cucurbitaceae, Datiscaceae, Onagraceae, Halorhagaceae, Callitrichaceat, Lythraceae, Tamaricaceae, Portulaceae, Paronychiaceae, Scleranthaceae, Crassulaceae.

A. K.

Dalla Torre, Dr. C. G. v., u. Harms, Dr. H.., fienera siphonngamarum ad systema Englerianum conscripta. Verl. v. Will. Engelmann in Leipzig 1900. Fasc. I. $4^{0}$ p. 81-160. Preis 3 M.

Auf die 1. Lief. ist zieml. rasch die 2. gefolgt. Ueber die Einrichtung und Bedentung des Werkes wurde in Nr. 5 p. 95 u. 96 das Nötige mitgeteilt. Vorliegende Lief. lringt: den Schluss der Iridacene, die Musaceae, Zingiberaceae, Cammacar, Marantaceae, Orchidaceae, Casuarinaceae, Saururaceae, Piperacae, Cloranthaceae, Lacistemacear, Salicacene, Myricaceae, Balanopsidaceae, Leitneriaceae, Juglandaceae, Betulaceae, Fagaceae, Ulmaceae, Moraceae, Urticaceae, Proteaceae, Loranthacere, Myzndendiacene, Santalaceae, Grubbiaceae, Opiliacede, Olaceae, Aristolochiaccae, Rafflesiaceae, Hychoracear, Polygonaceae, Chenopodiaceae, Ama- 


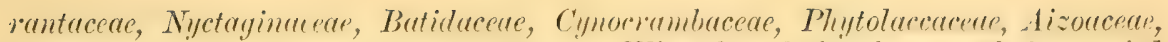
Portulacacede, Baselacere, C'aryophyllacere. Wie schon früher hervorgehohen, wird das Buch als Index zur wissenschaftl. Anordnmeg dex Herbarien unenthehrlich werden. Auch der Unterzeichnete hat der Anordnung der Genera in seinen "Glumaceae Exsiccatae das IVerk v. Dilla Torre u. Harms zugrumde gelegt.

A. K.

Cryptogamae Japonicae iconibus illustratae. Tokio. Lief. 1-8. 1899 bis 1900. Die bis jetzt vorliegenden Lieferungen enthalten die Abbihlungen und Beschreibungen von folgenden Arten: Climarium. Japonicum Limolh., Stirta pulmonacea Ach., Pleurotus ostreatus J., Usnea longissima Ach., Getidium corneum

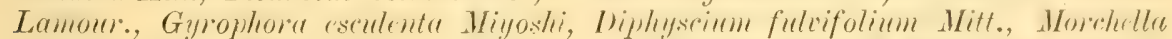

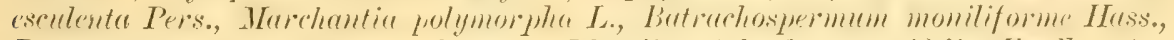

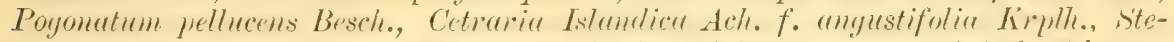

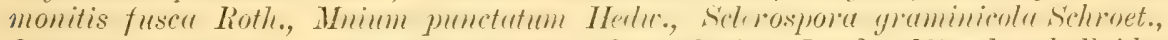

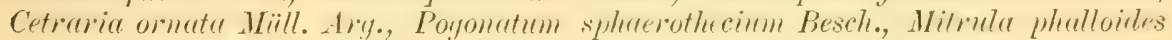
Chev., Rhizopogon mbescens Tul., Conocephalum conicum Necker, Pogonatum otamense Besch., Siicta Miyoshiana Mïll. Arg., Digenea simplex Ag., Lactarius

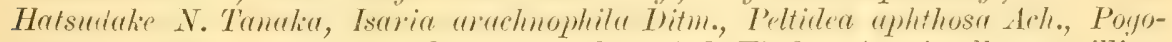
natum alpinum Brid., Ithyphallus munlosus Ed. Fisch., Asterionella gracillima

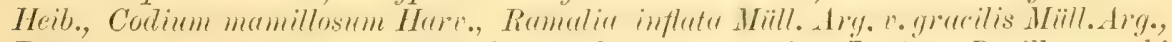
Pogonatum grandifolium Mill., Scytosiphon lomentarius J. Ag., Bacillus typhi

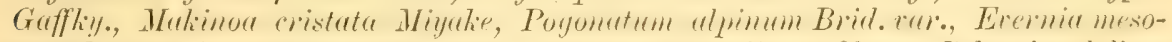

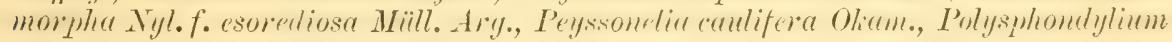
violaceum Bref., Ilirneola polytricha Fries.

Phanerogamae et Pteridophytae Japonicae iconibus illustratae. 'Tokyo. 1900. Lief. 8. Die vorlieg. Lief. enthält die Diagnosen und Abbildungen von: Davallia temifotia Su., D. t. var. Chinensis Moore, Aldrovanda versuulosa L.,

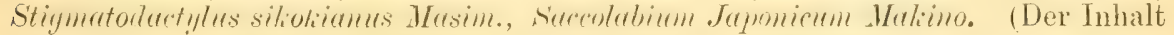
der Lief. $1-7$ ist in $\mathrm{Nr}, 4$ p. 70 der Zeitschrift angegeben.)

Oesterreichische bot. Zeitschrift 1900. Nr.11. Fritsch, K., Teher den Wert der Rankenhildung für die Systematik der Vicieen, inshesondere der Gattung Lathyrus. - Palla, E., Zur Kenntnis der Philobolus-Arten. - Freyn, J., Weitere Beiträge zur Flora v. Steiermark. -. Velenovsky. J., Die Achselknospen der Hainbuche. - Sarnthein, S, Graf, Ein Beitrag zur Pilztlora von Tirol. - Litteratur-Uebersicht.

Botan. Centralblatt. 1900. Nr.40. Hering, Ludw., Zur Anatomie der monopodialen Orchideen. - Nr.41. Kroemer, Karl, Ueber das angebliche Vorkommen von violetten Chromatophoren. - Hering, Ludw., Wie in vor. Nr. - Nr. 42. Hild ebrand, Friedr., Ueber Bastardierungsexperimente zw. einigen Hepatica-Arten. - Hering, Lud., Wie in vor. Nr. - Nr. 43. Correns, Dr. C., Ueber Levkojenbastarde. - Hering, Ludw., Wie in Nr. 41. - Nr, 44. Hering, Ludw., Wie in Nr.41. - Garje anne, A.J. M., Weiterer Beitrag zur Kenntnis monströser Bellis-Köpfchen.

Deutsche bot. Monatschrift. 1900. Nr.11. Rottenbach, H., Zur Flora von Ratzes in Sültirol. - Z schacke, H., Bryologische Spaziergänge in der Umgebung von Mitweida in Sachsen. - Neigen, Dr. Fr., Beobachtungen über Formationsfolge im Kaiserstuhl. - . Iurr, Dr. J., Beiträge zur Flora von lirol und Vorarlberg. - Leimbach, Dr. G., Die Volksnamen unsereu heimischen Orchideen. - Dr. Blïmml, Referat über Pöverlein: Die bayrischen Arten, Formen und Bastarde der Gattung Potentilla. - Kuntze, Dr. O., Bericht über bot. Vorträge auf der Versammlung deutsch. Naturforscher und Aerzte in Aachen. 
Botaniska Notiser 1900. Nr. 5. Lindroth, J. J., Om Aecilium trientalis 'Tranzsch. - Freidenfeld, T., Studier öfver örtartade växter rötter. Erikson, J., Om Sorbus seandica (L.) Fr. $>$ Aucuparia L. - Nilsson, N. H., Nägra ammärkningar heträffunde bladstructuren hos Carex-arterna. - Derselbe, Om några Carex-former.

Bullettin de l'association Française de botanique. 1900. Nr. 34-36. Brachet, Excursion hotaniques de Briançon aux sources de la Clarée et de la Durance. - Carbonel, J., Florule de la commune de Saint-Hippolyte. Blanchard, Th., Liste de noms patois de plantes aux environs de Maillezais (Vendée). - Sudre, H., Excursions batologiques dans les Pyrénées. - Olivier, l'abbé, Exposé systematique et description des Lichens de louest et du NordOuest de la France.

Botanical Gazette 1900. Vol. XXX. Nr. 4. Harper, R. A., Cell and nuclear division in Fuligo varians. - Land, W. J. G., Double fertilizationi is Compositae. - Hefferan, Mary, A new chromogenic Micrococcus.

Eingegangene Drueksehriften. Ir onfeld, Dr. II., Studien äber die Verbreitungsmittel der Pflanzen. I. Teil: Windfruichtlcr. Verl. v. W. Engelmann in Leipzig 1900, - Christ, H., Die Farnkräuter der Schweiz in "Beiträge zur Kryptoganienflora der Schweiz". Bd. I. Heft 2. Verl. v. K. J. WVyss in Bern. 1900. - Hal ácsy, E. de, Conspectus florae Graecae. Vol. I. Fasc. II. Verl. v. W. Engelmann in Leipzig. 1900. - Buchenau, Dr. F., Vortrag über die deutschen Pflanzennamen in d. Schule und im Leben. Gehalten am 27. Sept. 1899 in Lremen (Bericht). -. Schuler, B., Dante's göttliche Komödie in Wort und Bild den Deutschen gervidmet. Selbstverlag des Herausgebers. München 1900. - Geheeb, A., Révision des mousses récoltées en Brésil dans la province de San Paulo par M. Juan J. Puiggari pendent les années 1877-1882. (In "Revue bryologique. 1900. Nr. 5). - Krause, Ernst H. L., Fluristische Notizen. VII-XII (Sep. aus ,Bot. Centralblatt" 1899 u. 1900.

Deutsche bot. Nonatschrift 1900. Nr. 11. - Botanical Gazette XXX Nr. 4. - Bulletin de l'association Française. $1898 \mathrm{Nr} .7-12$ u. $1900 \mathrm{Nr} .34-36$. botanical Magazine $1900 \mathrm{Nr}$. 162. - Zeitschrift für angewandte Mikroskopie. VI. Bd. Ni. 7. - Cryptogamae Japonicae iconibus illustratae. Tōkyō. 1900. Nr. 8. - Phanerogama et Pteridophytae Japonicae iconibus illustratae 1900. Nr. 8. - I. Tauschliste des bot. Tauschvereins in Arnstadt. 1900. - Botaniska Notiser 19:0. Nr. 5. - Mitteilungen des bad. bot. Vereins. 1900 Nr. 173 u. 174.

\section{Botanische Anstalten, Vereine, Tauschvereine, Exsiccatenwerke, Reisen etc.}

Botanischer Verein der Provinz Brandenburg. Vor der Eröfnumg der

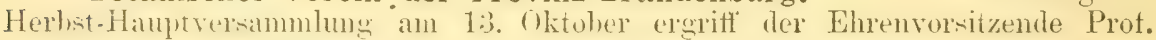
Ascherson das Wort, um den von seiner grossen Reise glücklich zurückgekehrten zweiten Vorsitzenden Prof. Volkens, dem zu Ehren der Vorstandstisch in frischen Blumenschmuck pramgte, im Namen des Vereins in Berlin williommen zu heissen. Nach einigen Worten des herzlichsten Dankes eröftnete dann Prof. Volkens in Abwesenheit des ersten Vorsitzenden die Versammlung mit Begrüssmug einiger von fernher gekommenen Mitglieder, der sich ledider auch die Mitteilung von dem Tode des Prof. Dr. Frank anschliessen musste. - Darauf erstattete Dr. Gilg den Jahresbericht des Vorstandes, dem wir entnehmen, dass der Verein im Laufe des Jahres vom 1. Okt. 1899 bis dahin 1900, nachdem 16 Mitsheder neu hinzngetreten und 13 teils gestorben, teils ansgeschieden sind, von 269 anf 272 Vitglieder gestiegen ist. Der laufende Band der Verhandlungen wird allex. Wahrocheinlichkeit nach bis Anfang. Dezember al)geschlossen sein; in demselben ist der Kryptogamenflora der Mark besondere Aufmerksamkeit gewidmet worden. - In Abwesenheit des Kassenführers W. Retzdorf erstattete 


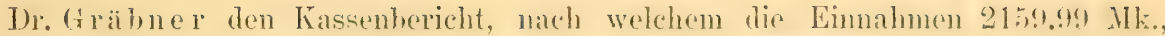
die Ausgaben 1945.52 Mrk, betrugen, also ein Ueberschuss von 214.47 Mk. zu verzeichnen ist, so dass das Gesamtvermögen des Vereins \%. \%. nahezn 7oo() Mlk. betrïgt. Der Vors. ergriff die Gelegenheit, um darauf aufmerkinm zu nachen, dass der Kassenführer, welcher jetzt sein Amt zehn Jahre verwaltet, die Geschäfte übernommen hat, als es um die finanzielle Seite des Vereins sehr misslich stand, mol dass es mur seiner limsicht mol seiner vorsichtigen lirwaltumg zu danken sei, dass die Verhältnisse des Vereins sich so wesentlich gehoben haben. - Der Bibliothekar Dr. Loesener legte die neuesten litterarischen Eingange vor. - Dr. Lindau epstattete Bericht über den gegenwartigen rtand der Arbeiten der Kryptogamenkommission. Er hedauterte, dass der in Angriff genommene Moushand noch nicht hat erscheinen kimmen, da berichte aus einigen Gegenden noch immer fehlen und Hr. Warustorf den Druck nicht dher hesinmen lasien will, als his das Manuskript völlig abgeschlossen vorliegt. - Eine längere Debatte entspann sich über die von dem Herrn Oberpräsidenten der Provinz angeregte Frage, ob der Verein die Herausgabe eines forstbotanischen Merkbuches, wie es Prof. Conwentz für die Provinz Westpreussen hergestellt, nun für die Prov. Brandenburg übernehmen wolle. An der Debatte beteiligten sich die Herren Dr. Potonié, Prof́. Beyer, P'rot. Ascherson, Prof. Winckelmamm, Dr. Lindau, Dr. T'ost, welch' letzterer als Verlagsbuchhändler Auskunft über die etwaigen Kosten erteilte. Bezüglich des erwähnten Merkbuches von Conwentz lag die Angelegenheit insoferm günstig. als das Vinisterium 1000 Lxemplare à 40 Pfg. entnahm, mit welchen 400 Mk. ungefäh die Druckkosten gedeckt wurden; ausserdem hatte Prof. Conwentz die Clichés geliefert. Selbst wenn sich dieser gïnstige Fall hier wiederholen sollte, würde der Verein immerhin die Vorarbeiten zu bestreiten haben, denn es müssen viele Aufforderungen verschickt werden, die einlaufenden Berichte müssen von Mitgliedern, die sich dafür besonders interessieren sollten, auf ihre Richtigkeit hin gepruitt werden, was wieder mannigfache kleinere und grössere Austlüge eriordert u. s. w. Aus ঐlem Gange der Debatte glaubt der Vorsitzende jedloch heransgefunden zu haben, dass die Anwesenden im grossen und ganzen mit Herausgabe eines solchen Herkbuches einverstanden sind, und er bittet, die Erwägung über die Kostendeckung einstweilen dem Vorstande zu ähelasmen, der damn seinerzeit weitere Vorlagen machen wird. - Es folgten nun die Wahlen, als deren Resultat sich folgendes ergab: Vorsitzende die Herren Prof. Volkens, Prof. Schumann, Prof. Koehne; Schriftührer die Hurren Dr. Gilg, Dr. We is se, Dr. Loesener; Kassenführer Hr. W. Retzdorff; Ausschussmitglieder die Herren Hennings, Dr. Gräbner, Prof. Beyer, Prof. Engler, Prof. Sehwendener, Prof. Wittmack. Die botanischen Nitteilungen eröffnete Prof. Ascherson mit der Vorlage

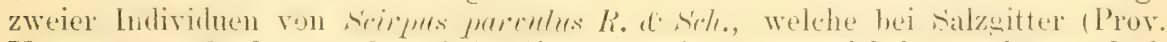
Hannover) gefunden worden sind; die Expl, sind zwar richtig bestimmt, doch hest Prof. Ascherson leise '/weifel an dem findort so tief im Binnenlande. Ferner zeigt er eine neue Spielart von Lathyrus silvester L. vor., die der Entdecker Scholz capillaceus genannt hat, und die in der That so eigentümlich aussieht, dass sie kaum für ein Lathyrus gehalten werden kann. - Dr. Potonié

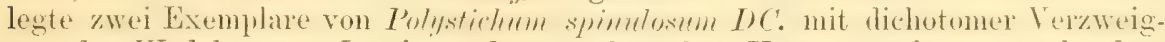
ung des Wedels vor. In einem daran geknüpften Vortrage wies er nach, dass diese Bildung hei farnen in in̈uheren Erdperioden Regel geweren ist und dass die vorliegenden Exemplare ein Beweis dafür sind, wie heute noch ab und zu derartige Beispiele von dichotomer Rückbildung vorkommen. - Dr. Gräbner

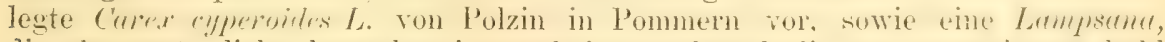
die oherwäts dicht drüsenharrig epscheint, wihrend die drt somst immer kilhl ist, wozu Hr. R. Schulz bemerkt, dass er dieselbe Form auch bei Tegel beobachtet habe und zwar mit Uebergüngen in die kahle Form. Ferner teilte Hr. Dr. Gräbner mit, dass sich im neuen botanischen Garten in Dahlem eine Menge von Adventivpflanzen angefunden haben, wie Solanum rostratum Dun., das nie im alten botanischen Garten gewesen, Cyperus vegetus Willd., Linaria multi- 
cunlis u. a.; dass cin l'olyymmm, welches bis jetzt niemand kennt, ganze Gelände äherzogen hat. - Dr. IT eisse machte Mitteilung von einer Doppelblüte an C'ephalunthera y'andiflora (scop.) Bab., welche er bei Lohme ant Rügen beobachtet hat, ein Vorkommen, das unter den his jetzt hekannten 32 Fiallen von Doppelhlüte!n (meist an exotischen und Gewaichshauspflanzen, nur 8 an wilden) wohl noch nicht gesehen worden ist. - Prof. W in ckelmann (Stettin) legte Erythren mulchellu (sw.) Fr. in den verschiedensten Formen, sowie eine I'opulus tre'mula L. mit elliptischen Blättern vor. - Zum Schluss schilderte Prof. Volkens in grossen Zügen die von ihm zurückgelegte Reise, die ihn über Suez, Aden, Ceylon, Singapore nach Neuguinea, den Carolinen und Marianen, zurück über Japan und China führte, und von der er reiche hotanische schätze mitgehracht hat. Durch eingestreute Details wusste Hr. Volkens seinen Vortrag ausserordentlich zu beleben, so dass man sehr gespannt auf die mehr ins Einzehne gehende Fortsetzung sein kamn, die er für die Folge in Aussicht stellte. Während des Vortrags circulierten unter den Zuhorern ganze ferien prachtvoller grosser Photographien, unter denen die Ansichten von singapore sich hesonders auszeichneten.

W. La ck ow itz.

Botan. Tausehverein in Arnstadt. Die 4 Seiten umfassende gedruckte Liste enthält: 1. Pflanzen aus Ungarn, 2. aus Australien, 3. aus Deutschland und 4. aus dem kaliforn. Florengebiet. Die Pflanzen unter 1 u. 3 werden im Kaufe mit 10-15) М. und die unter 2 u. 4 mit 25-30 M. pro Centurie berechnet. Der Leiter des Vereins ist Prof. und Direktor Dr. G. Leimbach in Arnstadt in 'Thüringen.

Siegfried \& Sickenberger, Herbarien. Für đlas hot. Museum der Univ. Zürich ist das megtithr 100000 Nummern zïhlende Herbar des bekamnten Potentillenkenners s'iegfried in Bülach erworben worden. Die P'otentillensammlung des H. siegfried ist hierin nicht inbegriffen, indessen hat sich die Behörle das Torkaufrecht gewahrt. Hierzu ist für dasselhe Institut hinzugekommen die Erwerbung des Herbars des in Cairo verstorbenen Dr. Sickenberger.

(Bot. Centralblat.)

Jaczewski, Komarov, Tranzsehel, Fungi Rossiae exsiceati. Fase. VI. Nr. 251-300 und Fasc. VII. Nr. 301-350 sind erschienen und bringen interessante Beiträge zur Kenntnis der Pilzflora des russ. Reiches.

(Aus ,Bot. Centralblatt.")

Botaniker, tropendiensttaugliche, jromovierte und dem deutschen Reich angehörige, welche Veigung haben, für einige. Tahre in den Kolonialdienst zu treten, wollen sich unter Angabe ihres Entwicklungsganges und ihrer Arbeitsrichtungen bei A. Englèr, Direktor des Kgl, bot. Gartens Berlin, melden.

(Bot. Centralblatt)

\section{Personalnachrichten.}

Ernennungen etc: Prof. Dr. Oskar Loew hat einen zweiten Ruf als Prof. der Agriculturchemie an der Univ. Tokiō erhalten und die Berufung angenommen. - Dr. P. Beveridge Kennedy w. Prof, d. But. an d. Univ. in Jewarla. -- Dr. E. B. Copeland W. Assistant-Prof. d. Bot. an d. Lniv. von WestTirginia. - Dr. II. Racibors ki w. Z. Protessor an d. landw. Hochschule in Dublan

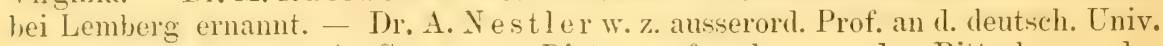
in Prag ernannt. - A. Grunow, Diatomeenforscher, w. das Ritterkreuz des Franz Jos. Ordens verliehen.

Todesfälle: Dr. Alb, Bernh. Frank, kaiserl. Geh. Regierungsrat und Torst. der biol, Abteil. im kaiserl. Gesundheitsant zu Berlin, am 27. Sept. im A. von 62 Jahren. - Prof. T. Mik in Wien am 13. Oktober.

Druck von J.J. Reiff in Karlsruhe. 


\section{Allgemeine}

\section{Botanische Zeitschrift}

für Systematik, Floristik, Pflanzengeographie ete.

\section{Referierendes Organ}

des bot. Vereins der Provinz Brandenburg, der kgl. bot. Gesellschaft zu Regensburg, des Preuss. bot. Vereins in Königsberg,

und Organ der Botan. Vereinigung in Würzburg und des Berliner bot. Tauschvereins.

Unter Mitwirkung hervorragender Fachmänner herausgegeben

von . Mónewer, Werderplatz 48 in Karlsruhe.

Verlag von J. J. Reiff in Karlsruhe.

Die Herren Mitarbeiter tragen für Form und Inhalt der von ihnen unterzeichneten Arbeiten volle Verantwortung.

\begin{tabular}{c|c|c}
\hline $\begin{array}{c}\text { № 12. } \\
\text { Dezember. }\end{array}$ & Preis: vierteljährl. 1.50 Mk. bei freier Zusendung. & $\begin{array}{c}\text { 1900. } \\
\text { VI.Jahrgang. }\end{array}$ \\
\hline
\end{tabular}

\section{Inhalt}

Originalarbeiten: IV. Schmidle, Algologische Notizen. - (r. K ïkenthal, Carex Canariensis Kükenthal nov. spec. - L. Gross und A. K neucker, Unsere Reise nach Istrien, Dalmatien, Montenegro, der Hercegovina und Bosnien im Juli und August 1900 (Forts.). - M. Golds chmidt, Die Flora des Rhöngebirges. I. - A. K n e ucker, Bemerkungen zu den „Gramineae exsiccatae".

Bot. Litteratur, Zeitschriften ete.: L. Gross, Lang. Dr. G., Von Rom nach Sardes (Ref.). - A. Kneucker, Christ, H., Die Farnkriuter der Sihweiz (Ref.). Derselbe, Ascherson, P., u. Gräbner, P., Synopsis der mitteleurop. Flora (Ref.). Derselbe, Kronfeld, Dr. M., Studien über die Verbreitungsmittel der Pflanzen (Ref.). . Derselbe, Kull, Albert, u. Lutz, Dr.K. G. Bilder aus der heimatlichen Vogelwelt (Ref.). - Inhaltsangabe verschied. botan. Zeitschriften.

Bot. Anstalten, Vereine, Tauschvereine, Exsiceatenwerke, Reisen etc.: Botan. Verein der Provinz Brandenburg (Ref). - Preussischer bot. Verein. - Thiir. Bot. Tauschverein - Berl. bot. Tauschverein. - Schultz, Paul F. F., Tauschvermittlung für Herbarpflanzen. - Herbarium normale, ('enturie XL. - Ba en itz, Dr. C., Herbarium Europaeum, Prospekt 1901. - Association Pyrénénne, Liste génerale des doubles, 1900/1901. - Keverchon, Elisée, Catalogue de 1900. - Haglund, Arvid u. Källström, Joh., Katalog getrockneter Pflanzen aus Skandinavien. Ortlepp, Karl, Gesuch um Uebersendung von Labiatensamen.

Personalnachrichten. - Mitteilung. - Zur Nachricht (anf d. Umschlag).

\section{Algologisehe Notizen.}

W. Schmidle-Mannheim.

$\mathrm{XV}$.

1. Camptylonema schmidle in Hedwigia 190(0, p. 181 ist falsch gebildet und muss Campylonema heisen (von xóktùos krumm,

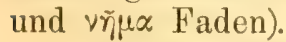

2. Statt Mastigochadus Angelliforme Schmidle 1. c. p. 175 ist $\mathrm{zu}$ schreiben $\mathrm{M}$. flagelliformis.

\section{Crucigenia Morren.}

Seit der zusammenfassenden Arbeit von Wille über Süsswasseralgen $\left.{ }^{1}\right)$ wird von den meisten Autoren der alte Gattungsnamen Circi-

-) Wille in Engler \& Prantel: Pflanzenfamilien I, 2 p. 58.

Allg. Bot. Zeitschrift 12. 
genia Morren statt Stanroyenia Ittzq. wieder gebraucht. Es ist deshalb angezeigt, die von mir unter dem alten Gattungsnamen Stururogeniu unterschiedenen Arten ${ }^{2}$ ) nochmals hier anzuführen. Ich unterscheide:

I. Zellen ohne Stacheln und Fortsätze: Eustaurogenia: 1) Crucigenia rectangularis (A. Br.), 2) Crucig. cruciata Wolle, 3) Crucig. Lauterbornei (schell.), 4) Crucig. emaryinater (W.d.G. West), 5) Crucig. quadrata Norren, 6) Crucig. fenestrata (Schdle.).

II. Zellen mit Stacheln oder Fortsätzen: Tetrachastrum: y) Crucig.

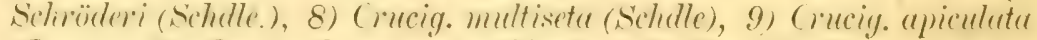
(Lem.), 10) Crucig. heteracenta (Nordst.) O.K., 11) Crucig. alpina $\left(\right.$ Schdle.). $\left.{ }^{3}\right)$

4. Centratractus Lemermamm in den Berichten d. D. bot. Gesellsch. 1900, p. 274 ist richtig ('ntritiactus zu nemen und ebenso die Art Centr. belonophorus statt belonophora Lem.

5. Heneda purmuren Raciborstii in Parasitische Algen und Pilze Javas, I Teil 1900, p. 1 ist offenbar eine Art von Phylloplax Schmille, welche noch keine Hakensporangien getrieben hat. IVenn dieselbe von $P h$. cumdelabrum schmidle verschieden ist, so ist ihr Name Ph. purpureum (Rac.).

6. Microsporr amoena f. Mrassior Wille in New Forms of green algae in Journ. of the New Engl. Botanical, vol. I, 1899 ist identisch mit conferea (Microspora) amoena car. crassion Hansyirg Prodromus II, 189.2, p. 22.2 (erschienen 1893), und dieses mit Hicrospora amoenu car. crusse schmidle in Ber. d. Naturf. Gesellsch. in Freiburg i. B. Bd. VII, 1893, p. 75, tab. I fig. 1, und dieses wieder mit Microspore de Toniana Latertheim in Chlorophyceen aus Abessinien und Cordofan N. Notarisia 1893, p. 137. Es ist höcht wahrscheinlich, dass alle diese Formen zu conferce liuciborstii Gutuinsliy in Flora Glon. Galicyi 1892, p. 7 , tab. III fig. 1 gehören, obwohl Gutwinsky l. c. seine Art ausdrücklich zu Confire stellt und daneben noch Microspor unterscheidet; W. West citiert C. Raciluralii ebenfalls in Notes on Scotsh Freshw. Alg. Journ. Bat. 1893, p. 92 tab. 333, fig. 4.

Lässt man der unsicheren Identitiat wegen r.. Riuciborstiii Crutu. bei Seite, so kamn ich richt entscheiden, welche der 3 Benennungen vom Jahre 1893 Priorität hat.

\%. Radiofilum apieulatum W. d. G. West: New Am. Algae in Journ. of Bot. 1895, p. 52 und Bohlin: die Algen der I. Regnell'schen Expedition in Bih. sv. let. Akad Handl. Bd. 23, p. 10, tal. I fig. 6-8 gehört zu liadiofilum conjunction Shmidle. Ich hahe in meinem Nateriale von Virnheim neben der ron mir gezeichneten Form in Flora 1894 tab. VII fig. 4 u. 5 seituem Zellen gesehen, die vollständig mit der Test'schen Form identisch waren und zumteil an demselben Faden wie die typische Form sich befanden.

2) Schmidle in Ber. d. D. bot. Gesellsch. Mai 1900, p. 156 u. ff.

$\left.{ }^{3}\right)$ Nenerdings hat Chodat 2 weitere hierher gehörende Arten beschrieben in Bull. Herb. Poiss. Juni 1900 und zwar: Staurogenia triangularis Chodat = Crucig. triungnlaris ((hod.), vielleicht eine Form von Crucig. quudratu Lurren (vergl. Alorren in Anm. Sc. nat. 1830 , tab. XX fig. $15 \mathrm{Nr} .3$ ) und Crucig. appendiculata (Chod.) = Staurogenia appendiculata (Chod.) $=$ Hofmannia appendiculata Chodat 1. c. pag. 9 fig. 10. 
9. Pilinia strgmalis G. S. West in Journ. of Bot. 1899, p. 12 , tal. 394 Fig. 6-9) ist zweifellos eine Gomgrosiru = (t. stronalis (G. S. West) und gehört, wie G.S. West selbst angiel,t, in die Nähe von Gr. trentepoh liojsis nob. Noch näher steht aber G. De Baryana, so dass eine Identität nicht ausgeschlossen ist.

\section{Carex Canariensis Kükenthal nov. spec.}

Rhizomate caespitoso; culmo $75-130 \mathrm{~cm}$ alto stricto firmo triquetro apice scahro faciehus concaviusculis ad basin vaginis fuscis dissolutis ciscumdato foliato; foliis culmo brevioribus $4 \mathrm{~mm}$ latis planis subrigidis; spica $6-15 \mathrm{~cm}$ longa interrupta basi + paniculata apice nutante; spiculis $10-17$ apice o basi $q$, superioribus simplicibus approximatis ovatis, mediis et inferioribus remotis compositis interdum brevipedunculatis; hracteis setaceis; squamis lanceolato-ovatis ferrugineis marginibus hyalinis, nervo dorsali viridi in aristam scabram excurrente; utriculis squamas superantibus suberectis rarius demum squarrosis $31 / 2 \mathrm{~mm}$ longis membranaceis ovatis vel oblongo-ovatis pallidis utrinque plurinervosis glabris basi spongiosa attenuatis marginatis in rostrum sublongum latiusculum antice fissum ore profunde bidentatum marginibus subalatum setulosumque subabrupte abeuntibus, dentibus rostri paullum divergentibus; achaenio ovato utriculum fere explente; stigmatibus 2.

Synon.: Carex paniculata Perraudière MS. non L.!

Carex paniculata var? Bourgeau MS. in Bourgeau Plant. canariens. $\mathrm{Nr} .1024$.

Habitat: Canarische Inseln: Auf Hierro, Fuente de Savinosa (G. Bourgeau leg. 18.VI.1845) und auf Teneriffa, in lauretis (Perraudière leg. 20.11I.1855), in lauretis pr. Taganana (Bornmüller leg. 5.VI.1900).

Diese die Multiflorae mit den Paniculatae verbindende Art fand ich im Herhar des Kaiserlich russischen botanischen Gartens in St. Petersburg, welches sich durch die Güte des Direktors, des Herrn Geheimrats Professor Dr. Fischer von Waldheim, seit 2 Jahren zur Revision in meinen Händen befindet. J. Bornmüller, welcher dieselbe Art im Juni dieses Jahres wahrscheinlich am gleichen Standort wie Perraudière (1855) fand, wundert sich (in litter.) darüber, dass eine schon habituell von Carex panimulutu so auffallend abweichende Art so lange unter falschem Namen in den Herbarien gelegen haben sollte. Dennoch ist es Thatsache, dass sie bisher nicht erkannt worden ist.

Bei genauer Untersuchung ergeben sich so viele Differenzen mit $C$. paniculata, dass C. Canariensis ummöglich auch nur als Varietait bei dieser belassen werden kanm. Ein Blick auf die Form und Struktur des Schlauches zeigt, dass sie vielmehr in die Nähe von Curex ulma Bailey oder von Carex leiarhyncha C. A. Meyer gestellt werden muss, mit denen sie die membranöse Textur des Utriculus, dessen an der nicht höckerigen Aussenseite deutlich hervortretende Nervatur und breiten Schmabel gemeinsam hat. Auch die bleichgrüne Färbung der viel weniger ramösen Inflorescenz verweist auf die Multiflorae, da sämtliche Paniculatae dunkle Rispen hesitzen. Ausserdem sind die spitzer auslaufenden Deckschuppen am Rande nicht silberhäutig, die of Blüten sind wenig sichtbar, und die Schläuche verschmälern sich in ihre Basis. Habituell einer langährigen ('. rulpina $L$. car. nemorosa (Kebent.) nicht unähnlich findet $C$. Canariensis ihre natüliche Anknüptung bei den oben genannten nordamerikanischen, bezw. ostasiatischen Arten aus der Gruple IIultiflorae, ohme jedoch mit irgendeiner von diesen zusammenzufallen.

Nach brieflichen Witteilungen Bormmüller's tritt sie in 2 Formen auf, einer mit fast einfacher und einer andern mit zusammengesetzter Inflorescenz.

Grub a. F., den 26. November 1900.

G. Kükenthal. 


\section{Unsere Reise nach Istrien, Dalmatien, Montenegro, der Hercegovina und Bosnien im Juli und August 1900.}

Von L. Gross und A. Kneucker.

(Fortsetzung.)

Die Sonne stand bereits nicht mehr im Horizont, als wir am nächsten Tormittag auf der guten Landstrasse gegen Unter-Leseče zuschritten, um von da das grottenberühmte Karstdorf St. Canzian zu erreichen.

Neben der Strasse selbst wuchs nicht viel, was unsere Aufmerksamkeit fesseln konnte: Diplotaxis moralis $D C$., Alopecurus agrestis $L$, in einigen sehr verspäteten Exemplaren, Euphurbia falcata $L$. und Liapistrum rugosum $A l l$. , in einer fast aukgetrockneten Pfütze Juncus compressus Jacq. Am Rancle sehr kleiner Dolinen,*) welche beiderseits die Strasse flankieren, sammelten wir Plantago madia L. mit gezïhnelten Blättern, ferner die Form sphuerostachya IFimm. de Grab. von Plantago lanceolata L., mit besonderer Liebe aber Plantago carinata Schrad. forma Wulfeni scheb. und forma longebracteata Koch; letztere war selten.

Gleich hinter Leseče zweigt links von der Strasse ein Fussweg ab, Hankeweg genamnt, der uns lald auf mageren kurzrasigen Karstborlen brachte. Auf ihm längere Rast zu halten nötigte uns eine zwar unscheinhare, aber doch sehr hegehrenswerte Pygmäenflora. Ein vorüberkommendes Bauernmädchen blieb voller Terwunderung stehen, als es hier zwei auf den Knien rutschende erwachsene Menschen erblickte, die mit grossen Messern eifrig kleine Pflanzen ausstachen, um sie im abgenommenen Hute sorgfältig zu verwahren. Es hätte sich vielleicht noch mehr gewundert, wenn wir ihm häten sagen können, dass die kleinen Dinger die hochtrabenden Namen führen: Medicugo prostrata. Jacq. var. glandulifere Cr.ban und Bupleurum aristatum Bartl. d. Wendl. ial. mims Facelini. Wir sanmelten von letzterer Pflanze die beiden Formen nana Koch und fustigiata Krasun, doch hat es damit, wie auch Herr Gugler brieflich mitteilt, insofern einen Haken, als sich eine ununterbrochene Uebergangsreihe von der einen zur andern beobachten lässt. Mit dem Kochischen Tamen nana wären wohl alle diese Hungerformen hinreichend gekennzeichnet.

Auch bezüglich der Medicayo mostrata machten wir (Gugler und Gross) heim Bestimmen eine Erfahrung, die nicht verschwiegen werden soll. Der eine entdeckte nämlich, trotz genauer Untersuchung, an seinen Pflänzchen keine Drüsen, der andere sah Drüsenhaare an Stengeln, BIätern, Früchten. An letzteren düren ja wohl auch bei der typischen Pflanze Drüsenhaare vorkommen (= M. Itclincla Kit., $\left.{ }^{*}\right)$ nicht aber an Blätern und Stengelteilen. Der Zwiespalt der Ansichten klärte sich so auf, dass hei genügend starker (etwa 30 facher) Vergrösserung wirklich an allen Pflänzchen Drüsen zu sehen sind. Die Vermutung, dass auch Andere die ausserst feinen Drüsen wegen zu schwacher Vergrösserting ihrer Lupe nicht wahrnehmen konnten, und dass dadurch die ylandulifro in den ihr nicht gebührenden Ruf der Seltenheit***) gelangte, müsste erst noch durch weitere Untersuchungen gestützt werden.

Von Medicago lupulina L. und minima L. f. pubescens Webb., sowie von Trifulium ayrarium Pollich rar. campestris Schich., die an der nämlichen Stelle wuchsen, nahmen wir wenige Belegstücke mit, um damn ein etwas beschlemigtes IIarschtempo einzuschlagen. Nur Carx muricata L. und stachys Germanica I. veranlassten gelegentlich noch kleinere Unterbrechungen.

Bald aber wurle es wieder interessanter, dort nämlich, wo der Hankeweg den wildesten Teil der Krarstlandschaft zwischen Leseče und St. Canzian durchr.lmejot. T'nzahlige Kalkblöcke bedecken oberhalb und unterhalb des steinigen

*) Dolinen sind trichterförmige Bodenvertiefungen, die dem Karste eigentümlich sind. Ueber ihre Entstehung sind die Geologen noch immer nicht einig.

**) cfr. Beck von Mannagetta, Flora von Nicderösterreich, Wien 1890 u. 93, p. 839.

*** *) Pospichal z. B. giebt 1. c. II, p. 356 für die drüsigeVarietät nur e in e n Standort an! 
Pfades die Halde, so dass den kühmen Pflänzchen, die sich in tem l'elsemmeer anzusiedeln wagten, nur schwer beizukommen ist. Vergeblich neckten uns dem auch Sesteria elongata Host, Melica ciliata. L., Rosa mierantha S'm., Marrubinm condidissimm I., Allium spharorephalum L. Da erblickten wir fast gleichzeitig, hier frei auf Felsen, dort im Schutze eines Wachholderbusches, zwei neue ungemein anziehende Gestalten: Dianthus Tergestims Relhb. und Digitalis larvi-

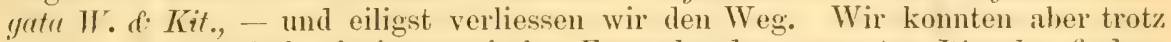
eifrigen Suchens kein einziges typisches Exemplar des genannten Thouthus finden; denn unsere Pflanzen besitzen unterhalb der Blüte statt 4 höchstens 3 Deckschuppenpaare, ausserdem sind die Platten der bleichroten Kronblätter vorn sehr deutlich gekerbt-gezähnt, während sie nach Pospichal*) ganzrandig oder schwach gekerbt, nach Fritsch**) fast ganzrandig sein sollten. Wer über die Wandelbarkeit dieser Pflanze noch mehr erfahren will, möge die diesbezüglichen Bemerkungen Pospichals (a. a. O.) nachlesen.

Unsere Digitalis lacvigata fügt sich mit ihrer rotbraunen Blütenunter. lippe ebenfalls nicht ganz den Angaben einiger Autoren, $\left.{ }^{* * *}\right)$ nach denen diese Unterlippe weisse Grundfarbe haben müsste.

Dianthus und Digitalis zeigten sich übrigens immer häufiger, je mehr wir uns einem Gebüsch näherten, das sich bis zu der nicht mehr weit entfernten Stefanienwarte hinzieht. Kurz bevor wir dasselbe erreichten, sammelten wir in kleinen schüsselförmigen Vertiefungen, die jedes anderen Pflanzenwuchses entbehrten, Euphrasia Illyrica Wettst.; $\dagger$ ) im Gebüsch selbst aber erwartete uns so ziemlich dieselbe Gesellschaft wie tags zuvor bei Divača, darunter auch wieder Scabiosa gramuntia L. var. leiorephala Hoppe; dazu traten noch Rhus cotimus $L$. fruct., Campamula rapmenloides L. f. umbrosa Op., Cyclamen Europaenm L., Thatictram minus L. f. virens II alli, Aethionema saxatile R. Br., Athamanta Matthioli Wulf. mit oft 8 und mehr Hüllchenblättern. ††)

Wer wird aber nicht auf einige Zeit Botanik und seltene Pflanzen ver. gessen, wenn er jetzt mit uns in Gedanken die schon erwähnte Stefanienwarte betritt? Welch' ein Aublick! Gegenüber fast auf gleicher Höhe mit dem Beschauer in eigenartig malerischer Lage Kirche und Dorf St. Canzian, fast senkrecht unter uns, $160 \mathrm{~m}$ tief, die brausende Reka, die sich in einer Doppeldoline wild schäumend über zwei Felsenahsätze stürzt, bevor sie im geheimnisvollen Dunkel verschwindet. Ringsum, dem gewaltigen Bilde einen grossartigen Rahmen gebend, senkrechte Kalkwände! Wäre in St. Canzian nichts weiter zu sehen als dieses Schauspiel, wahrlich, es wäre noch immer lohnend genug, den kaum einstündigen Spaziergang von Divača hierher zu machen. Und doch, wie klein ist dieses grosse Naturwunder im Vergleich zu jenem, das unten auf uns wartet! „Ein gelindes Granen, schreibt Prof. Kleiber, $+\dagger+$ ) beschleicht auch den Nutigen und unwillkürlich dachte ich an Dante's Eintritt zum Inferno - - - Auch uns erschien es wie ein Hinabsteigen zur Hölle und, sei hinzugesetzt, erleichtert atmeten wir auf, als sich nach stundenlanger unterirdischer Wanderung wieder der Himmel erschloss.

Erst im schattigen Hofe des nahen, empfehlenswerten Gasthauses in Matavm fanden wir Nuse, unsere Grottenfunde genauer zu besichtigen. Ausser ca. 10 Höhlenkïfern aus der Familie der Carabiden, die wir bei Kerzenschein erbeutet, hatten wir auch mehrere Pflanzen mitgebracht, die der Erwähumng wert erscheinen: Corydalis ochrolenca Sturm, Saxifraga petraea L., Allium saxalile $M$. Bicb., Geranium nodosum L., Emphrasin Illyrica II 'ettst. (Schattenform), Hieracium

*) Pospichal, 1. c. I. p. 454.

**) Fritsch, l. c., p. 198.

***) cfr. Koch, Synopsis der Deutschen und Schweizer Flora, Leipzig 1846, p. 614 und Fritsch, 1. c., p. 501 .

†) Herr Prof. Dr. Ritter v. Wettstein in Wien hatte die Guite, saimtliche auf unserer Reise gesammelten Euphrasien zu bestimmen.

†) Fritsch schreibt 1. c., p. 415: „B. der Hüllchen 2-3."

$\dagger+$ †leiber, M., Abseits der Touristen-Strasse. Reisebilder aus Dalmatien. Minchen 1899. 
Illyrimm Froies sep. foliciense Nacg. et Pet.,*) Thioncium bupleuroinles Gmel. II) scabriceps a) gemuimm 1) nonmale Naeg. et Pet. und Acer Monspessulamm L. var. Liburmira Pax. Dieser Ahorn wuchs mit Acer campestre L. Iinks neben dem Fusssteige, auf dem die Grotte gewöhnlich verlassen wird; spitzwinklige Blattbuchten, sowie der mehr oder minder gezïlnte Rand der Blattlappen verleihen ihm ein auffäliges Aussehen. Der verdiente Kemer der orientalischen Flora, Herr J. Born müller in Berka (Thüringen), dem wir diese Bestimmung verdanken,**) beobachtete die Pflanze laut briefl. Mitteilung bereits im Jahre 1886 nächst 'Triest, während sie den einheimischen Floristen bisher entgangen zu sein scheint, da sie weder von Marchesetti, noch von Pospichal***) erwähnt wird.

Durch das Einsammeln der wenigen soeben aufgezählten Pflanzen hatten wir unserem wackeren Führer, der bei seinem Vortrag sicher noch nie unaufmerksamere Zuhörer gehaht, leider grossen Kummer bereitet. Diese Thatsacheoder war's vielleicht nur der halsbrecherische Weg? - hatte uns daran gehindert, mehr Arten mitzumehmen. Doch notierte ich alsbald nach dem Verlassen der Grotte noch einige der Zurückgebliebenen: Prenanthes purpurea L., Digitalis ambigna Murr., Salvia glutinosa L., Lumaria redivira L. Bezügich der letzteren bemerkt Marchesetti (a. a. O. p. 36): La $L$, rediriva $L$. non venne trovata ancora entro il nostro distretto, sebbene cresca a poca distanza a Prevald, Adelsberg, sul M. Nanos, ecc." Da C'anzian noch zu Marchesetti’s Gebiet gehört, kann er künftig Lunaria redirira $L$. zu den Seinen zählen.

Ein mehrtagiges Standquartier im oben erwähnten (xasthaus wäre warm zu empfehlen, hesonders wem sich die aufmerksame Wirtin zur Führumg einiger Conserven nach Pott'schem System entschliessen könte. Uns selber nötigte die Verabredung des Hernn Gugler mit seinem definitiven Reisegenossen zur Rückkehr nach Divaca, wo wir nur ungern eine zweite Nacht verbrachten; denn die zahlreichen Gütcrzüge, welche hier durchlaufen, lassen erquickenden Schlaf kaum zu. Wir begrüssten deshalh mit Frenden den Anbruch des nächsten Tages und die Stunde der Abfahrt nach 'Triest.

(Forts. folgt.)

\section{Die Flora des Rhöngebirges. I.}

Von M. Goldschmidt-Geisa.

\section{Einleitung.}

Es kann an dieser Stelle nicht meine Aufgabe sein, eine allgemeine Schilderung des Rhöngehirges zu geben. Für seine Erschliessung wirkte in dcr 2. Hälfte des 19. Jahrhunderts in warmem, vou tiefhegrundeter Liebe zengendem Eifer der verstorbene Lehrer B. Spiess in Meiningen durch sein eingehendes, mehr geographisch gehaltenes Werkchen "Die Rhön", sodam aber in unablässiger Thätigkeit der verstorbene Geheime Medizinalrat Dr.Joseph Schneider zu Fulda und dessen Sohn, Herr Dr. Justus Schneider, der unermüdliche Präsident des seit 1876 hestehenden Rhönklubs. In den arheitsvollen Jahren seiner Prisidentschaft ist es ihm gelungen, nicht nur seinen Verein zu ununterbrochener Erschliessung unseres Gehirges durch Bauten, Wegeanlagen, Wegebezeichnungen u. S. w. anzuspornen, sondern auch durch seinen "Führer durch die Rhön", der in der 5. Auflage vorliegt, fowie durch eine Anzahl geologischer und historischer

*) Der bekannte Hieracien-Forscher Herr H. Zahn in Karlsruhe übernahm in dankenswerter Weise die Bearbeitung unseres Hieracien-Materials und konnte u. a. 2 neue $\mathrm{Z}$ wischenformen (aus Bosnien) konstatieren, deren Beschreibung an spätere. Stelle elfolgen wird.

**) Wie auch viele andere Bestimmungen schwieriger Species!

**2) Dir Shreibweise monspesulanum bei Pospichal, Flora 1, p. 52 darf wohl als Druckfehler angemerkt werden? Dass die Blätter des A. Monspessulamum "völligr $\mathrm{kahl}^{*}$ sind, stimmt nicht in allen Fällen. Oft firden sich unterseits auf den Hauptnerven, hesonders in der Nähe des Blattstiels Hatre, so auch an der von uns gesammelten Pflanze. 
Arbeiten und Vorträge die Freunde einer frischen, frohen, freien Wanderung für dieses lange verkannte, reizende Glied des dentschen Mittelgebirges zu erwärmen. Alljährlich wird die Zahl der Naturfremde, die dieses Gebiet durchziehen, eine grössere, und wer erst nur eimmal Körper und (iemüt in schönen Sommer- und Herbsttagen in diesem, seinem Aufhan nach so formenreichen Gebirge gestairkt, den zieht es immer wieder dahin zurück, in seine lieblichen Längs- und stillen Querthäler, auf seine langgedehnten Hochmatten, wo die Brust freier atmet, auf seine kegelförmig aufragenden Gipfel, von denen jeder ein neues, reizvolles Nah. und Fernbild dem Auge hietet, zu seinen gigantischen, oft grotesken Felsmassen, in seine freundlichen städtchen, zu seinen genïgsamen, ehrlichen, schlichten Bewohnern. Hier ist noch Raum für alle jene vom Getriche des Berufes und unserer hastenden Zeit abgespannten Menschen, welche, in freier Natur Erholung suchend, aus mancherlei Gründen dem Gewïhle moderner Reiseziele ausweichen wollen. Nicht die Mode hat den Ruhm von der Schönheit der Rhön ausposaunt; ihr unberührter Liebreiz wird erkannt, sobald man sich ihr. naht, und wer ihr Wesen erst erkannt hat, der liebt sie, kehrt wieder und findet sie noch schöner.

Wer zu seiner Wanderung durch die Rhön sich über sie selbst, ihre Bewohner, sowie ihre Geschichte u.s.w. unterrichten will, den verweise ich auf die beiden vorgenannten Werkchen, sowie auf den "Rhönspiegel" des Rhönsängers Pfarrer Hoehl; auch das Büchlein des Lehrers Arno Fuchis quer durch die Rhön" giebt manch schönen Fingerzeig zur Orientierung. An Sprezialkarten empfehle ich - ausser den im Schneider'schen Rhönführer gegebenen - ans eigener Erfahrung die in Wischmanier gehaltene Thein'sche (Würzhurg) und die in farbigen Höhenstufen ausgeführte Hossfell'sche (Eisenach, H. Kahle).

Der Geschichte der Erschliessung dieses Gebirges entsprechend, ist die Erforschung seiner reichen botanischen schäze erst in den letzten $3-3^{4}$ Jahrzehnten in Aufschwumg gekommen. Eine einheitliche Bearbeitung hat nur clas Reich der Laubmoose durch einen der besten Kenner der Rhön, ihren immertrenen Verehrer, den scharfsichtigen Bryologen Herm A d albert G ehe eb erfahren, dem die glückliche Gabe gewährt ist, seine unermüdliche Forscherthatigkeit mit rlem Lichte künstlerischer Auffassung zu erhellen. Seine Arbeiten über Rhönmoose sind in der "Flora" $1870,71,70,76,84$ und in der "Allgemeinen botanischen Zeitschrift" 1898 erschienen. Das Śchmuckstück semer hierhergehörigen Arbeiten, gleichsam ein hohes Lied auf die Milsehurg, die Perle ler Rhön, nebst einer Beleuchtung ihres überaus reichen schatzes an Laubmoosen, wird die Jubiläumsschrift des linonklubs im Jahre 1901 bringen. Mit der Aufnahme der Gefässkryptogamen- und Phanerogamenfloral waren in dem genannten Zeitraume besonders der vorgenamnte Herr A. (teheeb, Apotheker in Geisa (jetzt in Freiburg i. B.) und der verstorbene emsige Lichenologe Apotheker Dannenberg in Fulda anhaltend beschïtigt. Ein vortrefflicher Kenner besonders der Flora des südichsten Gebietsteiles ist Herr Bezirkstierarzt Vill (jetzt in Bamberg), von dem mir leider keine Aufzeichnumgen vorliegen. Von Meiningen aus hat Herr Prof. Rottenbach (jetzt in Perlin) mehrere Jahrzehnte lang namentlich den östlichen Teil des Gebirges durchforscht. Andere Floristen, die sich um den Pflanzenschmuck unseres Gebietes verdient gemacht hiben, sind, soweit sie ihre Ergebnisse veröflentlicht haben, aus der folgenden Aufführung der einschlägigen Litteratur zu ersehen. Ehe ich jodoch diese Aufzählung gehe, müchte jch an dieser Stelle Herm A. Geheeb herzlichst danken für die uneigennützige Art, in der er mir seine handschriftlichen Anfzeichnumgen aus nahezu $t$ Jahrzelunten zur Verfïgung stellte, sowie für die freundliche Weise, in der er viele Jahre hindurch meine Bemühungen um die Anfinahme der Rhönflora mit Rat und That unterstïtzt hat. Ausserdem verweise ich noch auf das unter Nr. 3, 6 und 8 des Litteraturverzeichnisses Gesagte. Die mir bekante ilteste Veröflentlichung über Rhönpflanzen geschah im Jahre 1870 durch den genamnten Apotheker Dannenberg.

(Forts. folgt,) 


\title{
Bemerkungen $\mathrm{zu}$ den ,Gramineae exsiccatae $\left.{ }^{6 * *}\right)$
}

\author{
von $\mathrm{A}$. Kneucker.
}

III. น. IV. Lieferung 190 .

Nr.61. Erianthus strictus Bluff of Fingerh. Fl. Germ. ed 2 I, p. 105 (1836) = Andropogon strictus Host Gram. Austr. I, p. 2 t. $2(1801)=$ Saccharum strictum Spreng. Plant. Pugill. II, p. 16 $(1815)=$ S. adpressum Kit. in Spreng. Syst. I, p. 282 (1825) = Erianthus Hostii Griseb. Spicil. flor. rumel. II, p. 548 (1844).

Slavonien bei Kamenitz am Wege nach Ireg um das Adamski'sche Haus auf stemigem Lehmboden. Begleitpflanzen: Andropogon ischaemon L., Panicun glaucum L., viride L. v. Weinmannii R. u. Sch., Cynodon dactylon L., Arrhenatherum elatius M.\& K., Calamagrostis epigeios Rth.

$90 \mathrm{~m}$ ü. d. M.; 23. Aug. 1900.

leg. Dr. v. D e gen.

Nr. 62. Andropogon distachyus L. Sp. pl. ed. I, p. 1046 (1753) = Pollinia distachyos Spreng. Syst. I, p. 288 (1825).

Auf Wiesen bei Bordighera in Ligurien (Italien). Begleitpflanzen: Andropogon ischaemon L., Spiranthes autumnalis Rich.

Nähe des Meeres; September 1900. leg. Clarence Bicknell.

Nr. 63. Andropogon Halepensis Brot. Fl. Lusit. I, p. 89 (1804) var. gemuina Hacliel Suites DC. Prodr. VI, p. 502 (1889)= Holcus Halepensis L. Sp. pl. ed. I, p. 1047 (1753) = Sorghum Halepense Pers. Syn. I, p. 101 (1805) = Andropagon Halepensis Brot. 1. typicus Aschers. u. Gräbn. Syn. II, p. 47 (1898).

In Weinbergen bei Riva am Gardasee in Südtirol; Kalk boden.

Ca. 80-150 m ü. d. M.; Juli $1900 . \quad$ leg. Pietro Porta.

Nr. 64. Andropogon hirtus I. Spec. pl. ed. I, p. 1046 (1753) var. genuina Hackel in DC. Mon. Phan. VI, p. $619(1889)=A$. h. 1. typicus Aschers. u. Gräb. Syn. II. p. 53 (1898).

Auf trockenen Hügeln bei Bordighera in Ligurien (Italien); S a nd boden. Begleitpflanzen: Lotus edulis L., Calluna vulgaris Salisb., Dorycnium suffruticosum Vill., Lavandula stoechas L.

$50 \mathrm{~m}$ ü. d. M.; 25. April 1900.

leg. Clarence Bicknell u. Luigi Pollini.

Nr. 65. Andropogon hivtus L. f: inter ra. genuinam Hack. et var. pubescentem (Vis.) Hackel.

Zwischen Ragusa und San Giacomo in Dalmatien auf felsigen und berasten Stellen in grossen Büschen links des Weges; Kalk. Begleitpflanzen: Lagurus ovatus L., Asplenum ceterach L., Punica granatum L., Myrtus communis L. ete.

Ca. 40-60 m ü. d. M.; 6. Aug. 1900. leg. L. Gross u. A. Kneucker.

*) Die Revision, bezw. Bestimmung des ausgegebenen Materials und teilweise auch die Zusammenstellung der Litteraturnachweise wurde von Herrn Prof. Ed. Hackel in St. Pölten gütigst übernommen. Ausserdem wirkte noch Herr Schriftsteller W. $\mathrm{Lack}$ ow it z in Berlin bei Durchsicht der Korrekturabzige freundlichst mit. Die Schedae sind von dem unterzeichneten Herausgeber selhst zusammengestellt. Da die Aufzählung a ller bekannten synonyme jeder Art zu weit fülıren würde, hat sich der Herausgeber erlaubt, nur die wichtigsten Synonyme aufzunehmen. In der Anordnung der Genera wurden die , Genera Ciphonogamarum ad systema Englerianum conscripta ${ }^{\star}$ von Dr. v. Dalla Torre und Dr. Harms, wovon erst Fascikel I u. II erschienen sind, zugrunde gelegt. Da der unterzeichnete Herausgeber die Druckkosten der Bemerkungen zum grossen 'Teil trägt, kommt die durch die letzteren verursachte Erweiterung des Umfangs der Zeitschrift den Abonnenten gratis zu gut. - Den Mitarbeitern und Abonnenteu der Glumaceae exsiceatae diene zur Nachricht, dass nach einer Mitteilung aus NewSil. W Iles in Ausstralien in den nächsten Tagen aus Sydney eine Kiste mit ca. 25 it $1,1,:, 11: 31$ für die Ersiccaten abgehen wird.

A. Kn e u cker. 
Nr. 66. Andropogon hirtus L. var. mbescens (Vis.) Flora XII, 1. Erg. Bl., p. $3(1829)=$ A. giguntens Ten. Fl. Nap. V, p. 285 $(1835-36)=$ A. hivtus L. subv. pubescens Hackel in Monogr. Androp.

Trockene und unkultivierte l'latze um Beirut in Syrien; Küstensand und mioz ïner Grobkalk. Begleitpflanzen auf Sand: Verbascum Tripolitanum Boiss., Aristida coerulescens Desf., Scilla maritima L. etc, anf ('robkalk: Andropogon ischaemon L., Scilla maritima L. etc.
50-100 m ü. d. M.; Dezember 1899.
leg. Ernst Hartmann.

Nr. 67. Adropogon contortus L. Spec. pl. ed. I, p. 1045 (175:3) と(1. glaber Hacliel in Mart. u. Eichl. Fl. Bras. II 3, p. 268 (1878 bis 83) sube. Allionii Hacliel in DC. Mon. Phan VI, p. 587 $(1889)=$ A. contortus All. Fl. Pedem., p. 260 t. 91 fig 4 $(1785)=$ A. Allionii Lam. \& DC. Fl. Fr. III, p. 97 (1805) $=$ Heteropogon Allionii $R$. \& Sch. Syst. veg II, p. 835 (1817).

Bei Riva in Südtirol auf Felsen am Monte Brione; Sandboden. August 1900. leg. Pietro Porta.

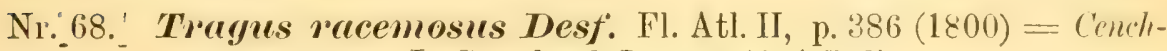
ms racemosus L. Sp. pl. ed. I, p. 1049 (1753).

Trockene, sonnverbrannte Heiden des Hügels von Valeria bei Sion im Kanton Wallis (Schweiz); Quarzit. Begleitpflanzen: Andropogon ischaomon L., Kentrophyllum lanatum DC., Achillea setacea W. K., Allium sphaerocephalum L., Eragrostis pilosa P. B., Ephedra Helvetica C. A. Meyer etc. Ca. $625 \mathrm{~m}$ ü. d. M.; September 1900.

leg. Prof. F. O. Wolf.

Nr. 69. Panicum sanguinale L. Sp. pl. ed. I, p. 57 (1753) "ar. vulgaris Döll in Rhein. Fl. p. 126 (1843) = Digitariu sanguinalis Scop. Fl. Carn. ed. 2 I, p. 52 (1772).

Auf Kartoffeläckern in der Nähe des Schwimmschulweges bei Karlsruhe in Baden als gemeines Unkraut; Diluvialsaud. Begle itpflanzen: Panicum viride L., glaueum L., Spergula arvensis L., Polygonum persicaria L., Galinsoga parviflora Cav.

Ca. 120 m ü. d. M.; 13. Sept. 1900.

leg. A. Kneucker.

Nr. 70. Panicum sangminale L. var. ciliaris (Retwius):' (Obs. IV, p. $16(1786)=$ Digitaria ciliaris Koel. Deser. Gr. p. $27(1802)=$ P. s. ß. ciliare Döll Fl. d. Grossh. Bad. I, p. 229 (1857).

Auf sandigem und kieshaltigem Boden bei dem Rheinhafen von Naximiliansau in der bayr. Rheinpfalz. Begleitpflanzen: Poa conpressa L., Eragrostis minor Host, Panicum lineare Krocker, viride L., Scrofularia canina $\mathrm{I}_{\text {s. etc, }}$ Ca. $107 \mathrm{~m}$ ü. d. M.; 5. September $1900 . \quad$ leg. A. Kn eucker.

Die Anwendung des Autornamens Retzius hinter eiliare ist nicht ganz einwandfrei; denn Retzius beschrieb aus Java und Indien stammende Exemplare und erwähnt nichts von dem Vorkommen in Europa. In Java und Indien finden sich aber noch andere gewimperte Formen, so dass nicht sicher steht, was Retzius gemeint hat. Am besten wäre zu setzen: var. ciliaris Aut, germ.

Hackel.

Nr. 70a. Panicum sanguinale L. var. ciliuris (Retw.):

Auf Aeckern beim Gardasee in Südtirol unweit Riva; Kalkboden.

Ca. $75-150 \mathrm{~m}$ ü. d. M.; September 1900 . leg. Pietro Porta.

Die Exemplare dieses Standortes liegen mit denen von Nr. 70 auf einem Blatt und sind durch Papierschlingen kenntlich gemacht.

A. K. 
Nr. 71. Panicum sangminale L. f. inter subvar. Aegyptiacam (Retiv) Obs. III, p. 8 (1779-91) et var. vulgarem Döll (Hackel).

Zwischen der spärlichen Vegetation des K üstensandes rechts vom Ausflusse des Nahr Beirut (Marocas) in Syrien. Begleitpflanzen: Salsola kali L., Alhagi Maurorun DC., Lippia nodiflora L., Inula viscosa L., Euphorbia paralias L., peplis L., Cynodon dactylon L., Rottboellia compressa L. f. fasciculata Hackel, Panicum colonum L.

Meeresstrand; August 1900.

leg. Ernst Hartmann.

Im Briefe vom 26. November 1900 teilt $H$. Prof. Hackel einiges über den Wert etc. der hier unter Nr. 69-71 ausgegebenen Formen mit. Ich erlaube mir, die diesbezüglichen Bemerkungen hier inhaltlich wiederzugeben: Bei Nr. 70 und 70 a ist die Var, eiliaris zumteil noch nicht vollständig ausgeprïgt; denn hei dieser finden sich an der 3. Hüllspelze gegen den Rand zweierlei Haare, nämlich kurze, anliegende, feine Wollhare und längere, steife, abstehende Wimpern. Nun kommen aber alle möglichen Zwischenformen (Fehlen der steifen Wimpern an den sitzenden Aehrchen, Fehlen derselben im unteren Teil der ganzen Aehre ete.) vor, so dass eine Begrenzung dieser Varietät ganz unmöglich ist; auch die Exemplare von Nr. 70 und 70 a sind fast sämtlich solche Zwischenformen. Bei var. vulgaris ist die 3. Hüllspelze auch nicht ganz kahl, sondern hat stets zwischen Seitennerv und Rand feine, anliegende Wollhärchen, weshalb solche Fxemplare von manchen dann gleich zu ciliaris gestellt werden. Ganz $\mathrm{kahl}$ ist die 3. Spelze nur bei var. Aegyptiaca, zu der das Material von Nr. 71 nur teilweise zu rechnen ist, weil bei dieser doch auch, wenn auch nicht überall, solche Haare vorhanden sind.

A. $\mathrm{K}$.

Nr. 72. Panicum lineare Kroctier Fl. Siles. p. 98 (1787) non L.= P. glabrum Gaud. Agrost. Helv. I, p. $22(1811)==$ Digitaria glabra R. u. Sch. Syst. II, p. 471 (1817).

Auf sandigem und kieshaltigem Boden bei dem Rheinhafen von Maximiliansau in der bayr. Rheinpfalz. Begleitpflanzen; Poa compressa L., Eragrostis minor Host, Panicum sanguinale L., viride L., Serofularia canina L. ete.

Ca. $107 \mathrm{~m}$ ü. d. M.; 5. Sept. 1900. leg. A. Kneucker.

Nr. 73. Punicum Numidiamem Lram. Encycl. IV, p. 749 (1797)= P. muticum Lk. Hort. Berol. I, p. $206(1827)=P$. leiogomum Sieb. Egypt. exs.

An und in Wassergriben lïngs der Felder und Wege bei Beirut in Syrien; Alluvium. Begleitpflanzen: Erianthus Ravennae L., Arundo donax L., Rottboellia compressa L., Panicum glaucum L., Rubus discolor Boiss.

Wenige m. ü. d. M.; Dezember $1899 . \quad$ leg. Ernst Hartmann.

Nr. 74. Panicum crus galli L. Sp. pl. ed. I, p. 56 (1753) var. breviseta Döll Fl. Bad. I, p. 232 (1857).

Auf Kartoffeliackern am Turmberg bei Durlach in Baden; Muschelkalk. B e gle it p f lanzen: Pamicum viride L., Triticum repens L., Sonchus oleracens L. ete. Ca. $200 \mathrm{~m}$ ü. d. M.; 8. Sept. 1900.

Nr. 75. Punicum cuss galli L. val. Iongisetr Trin. Spec. Gram. tab. 162 (1829); Döll Fl. Bad. I, p. 232 (1857).

Sandiges und hie und da überschwemmtes Ufer der Nord-Ostseite des St. Marys Reservoir bei St. Marys in Ohio (U.S. A.). Begleitpflanzen: Eragrostis hypmoides B. S. P., Panicum crus galli L., Schoenoplectus lacustris (L.) Palla, ('yperus erythorhyzos Mühl., Lophotocarpus calycinus J.G. Sm., Sagittaria arifolia Nutt., P'olygonum Pemnsylvanicum L., Bidens cernua L., Ilysanthes gratioloides Benth.

Ca. $250 \mathrm{~m}$ ü. d. M.; 17. Sept. 1900.

log. Prof. A. Wetzstein.

Das Material von Nr, 75 wurde als Panicum Walteri Pursh eingesandt. A. K. 


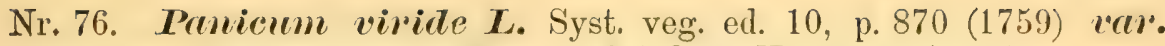
Weimmanni (R. $\boldsymbol{~}$. Sch.) Syst. II. p. 490 (1817).

Am Bahnkörper bei Karlsruhe und auf Kulturland bei Durlach in Baden; Sand - und Lehmboden. Begleitpflanzen: Eragrostis minor Host, Panicum sanguinale L., crus galli L., Polygonum persicaria L. ete.

Ca. 117-120 m ü. d. M.; 7. u. 8. Sept. 1900. leg. A. Kneucker.

Nr. 77. Panicam glancum L. Spec. pl. ed. I, p. 56 (1753) = Setaria glanca P. B. Agrost. p. 51 (1812).

Kartoffelfelder bei Karlstuhe in Barlen, in der Nähe des S'chwimmschulweges; Diluvialsand. Begleitpflanzen: Panicum virile I., sangumale L., Spergula arvensis L., Polygonum persicaria L., Galinsoga parviflora Lav.

Ca. $120 \mathrm{~m}$ ü. d. M.; 13. Sept. 1900.

leg. A. Kneucker.

Nr. 78. Phalaris Camariensis L. Sp. pl. ed. I, p. 54 (1753).

Auf Schuttstellen um Karlsruhe in Baden; Sandboden. Begleitpflanzen: Agrostis vulgaris With., Bromus arvensis L., mollis L., Hordeum murinum L. etc.

Ca. 118 m ü. d. M.; 12. Juli 1900.

leg. A. Kneucker.

Nr. 79. Mierochlö̈ custrulis R. d Sch. Syst. veg. II, p. 514 (1817).

In lichtem Gebüsche von Quercus pubescens Willd., sessiliflora Salisb., Castanea sativa Mill., Ostrya carpinifolia Scop., Colutea arborescens L. und Berberis vulgaris L., östl. von der Kampiller Kirche, nächst Bozen in süultirol auf der Porphyr-Ansehwemmung des von Kampenn herabkommenden Hurbaches. Begleitpflanzen: Carex digitata L., humilis Leyss., Erica carnea L., Chamaebuxus alpestris Spach ete.

Ca. $280-300 \mathrm{~m}$ ü. d. M.; 10 April 1900.

leg. L. Gross.

Nr. 80. Hierochlö̈ alpina R. d Sch. Syst. II, p. 514 (1817). = Aera alpina Liljebl. Flor. ed. II, p. 41 (1798).

An trockenen Stellen auf dem Gebirge Sliäro (Sliravarre) in Kvanangen im Amte Tromsö in Norwegen; Glimmerschiefer. Begleitpflanzen: Betula nana L., Empetrum nigrum L., Dryas octopetala L., Vaccinium myrtillus L., vitis idaea L. etc.

$700-900 \mathrm{~m}$ ü. d. M.; 4. Aug. 1900.

leg. Andr. Notó.

Nr. 81. Stupa cristella C. Syst. Nat. ed, 12. III, p. 229 (1768) = Aristella bromoides Bertol. Fl. It. I, p. 690 (1833).

In Oelgärten und auf trockenen Hügehn bei Bordighera in Lignien (Italien); Conglomerat - und Sandboden. Begleitpflanzen: Psoralea hituminosa L., Oryzopsis miliacea Aschs. u. Schweinf., Pallenis spinosa Cass., Galactites tomentosa Mnch. etc.

$10 \mathrm{~m}$ ü. d. M.; 21. Juni 1900.

leg. Luigi Pollini.

Nr. 82. Stupa pennate L. Sp. pl. ed. I, p. 78 (1753) ssp. Foammis Celak. in Oest. bot. Z. XXXIV, p. 318 (1884); Vgl. Cel. Oest. bot. Z. XXXIII, p. 313 u. 349 (1883) fं. inter ssp. Jormmem. et ssp. Tivsum Stev. Bull.

Im Comitat Pest in Ungarn am "Hármashatárhegy" bei Ofen; Kalk. Begleitpflanzen: St. villifolia Simk., Jurinea mollis Rchb., Hieracium Bauhini Schult., Phleum phleoides ('T.), Briza media L., Achillea Néiheichii Kern., Pamonicum Scheele, 'I'rifolium montanum L., Linum Austriacum L., Gialium verum L. etc.

Ca, $400 \mathrm{~m}$ ü. d. M.; 6. Juni 1900. leg. Dr. A. v. Degen u. C.v. Flatt.

Nr. 83. Stupa pemuatu L. ssp. pulchervima (C. Koch) Linnaea XXI, p. 440 (1848) v'. hirsuta (Velen.) Oesterr. bot. Z. XXXIV, p. 820 (188t) t: vilifolia Simk. in „Természettud. 
Kožl. Pótfuz", p. $46(1895)=$ St. dessy)lıylla Borb. in „Term. I. Pótf." p. 50, non Czern. Consp. pl. Chark, p. 75 (1859) sol. nom.; Lindem. suppl. III ad Flor. Elisabethgrad p. $45(1875)=$ St. penmata L. B. mediterranea Aschs. of Gräbn. prol. mulchervima (C. Koch) rar. hirsuta (T'elen.) Aschs. \& Gräbn. Syn. II, p. 107 (1898).

Im Komitat Pest in Ungarn bei Ofen am Berge "Hármashatarhegy" au! Kalk. Begleitpflanzen: Genau dieselben wie bei $\mathrm{Nr}, 82$.

Ca. $400 \mathrm{~m}$ ü. d. II.; 6. Juni 1900 . leg. Dr. A v. Degen u. C. v. Flatt.

Nr. ¿4. Stupa pennata L. ssp). Gallica Celal: in Oesterr. hot. Z. XXXIII, p. 315 (1883).

Sonnverbrannte Hügel im mittleren Wallis (Schweiz) am Monte d'Orge; Urkalk. Begleitpflanzen: Koeleria Valesiaca Gaud., Festuca Valesiaca Schl., Stupa capillata L., Poa concinna Gaud., Carex nitida Host, Iris lutescens Lam., Hyssopus officinalis L., Galium rigidum Vill., Trigonella Monspeliaca L., Medicago minima Bart., Astragalus onobrychis L., Euphorbia Gerardiana Jacr., Anemone montana Hoppe ete.

500-800 m ü. d. M.; Mai 1900.

leg. Prof. F. O. Wolf.

Nr. 85. Stupa juncea L. Spec. pl. ed. I, p. 78 (1753).

Aus trockenen Hügeln oberhalb Bordighera in Ligurien (Italien); Conglomerat. Begleitpflanzen: Rosmarinus officinalis L., Fumana Spachii Gren. Godr., Goris Monspeliensis L., Pinus Halepensis Mill., Globularia alypum L., Jumiperus oxycedrus L.

Ca. $50 \mathrm{~m}$ ü, d M.; 7. Mai 1900 .

leg. Clarence Bicknell.

Nr. 86. Oryinglsis milincer Aschs ". Schweinf: Mém. Inst. Ég. II, p. 169 ([Sep. 1887] 1889); Vgl. Bnth. \& Hook. Gen. pl. III, p. $1142(1883)=$ Agrostis miliacea L. Sp. pl. ed. I, p. $61(1753)=$ Piptatherum multiflorum P. B. Agrostogr. p. 173 (1812).

In Oelgarten hei Bordighera in Ligurien (Italien) auf trockenem siandboden. Begleitpflanzen: Pallenis spinosa Cass., Koniga maritima R. Br., 'Tunica saxifraga Scop., Psoralea bituminosa L.

$20 \mathrm{~m}$ ü. d. M.; 21. Juni 1900.

leg. Clarence Bicknell.

Nr. 87. Oryzopsis virescens Becl: Fl. v. N.-Oest. p. $51(1890)=$ Milium paradoxum Scop. Fl. Carn. ed. II, p. 58 (1772) = Piptatherum paradoxum Koch Syn. ed. I, p. 786 (1837) $=$ P. virescens Boiss. Fl. Or. V, p. 507 (1884).

Im Spitalwald bei Bruck a. Leitha in Ungarn; Kalk. Begleitpflanzen: Dactylis glomerata L., Bhammus tinctoria W. K., Evonymus verrucosa Scop.s, Viola mirabilis L.

Ca. $250 \mathrm{~m}$ ü. d. M.; 10. Juni 1900.

leg. Dr. A. v. Hayek.

Nr. 88. Oryappis coerulescens Huclel in Denkschr. Ak. Wiss. Wien, p. $75(1885)=$ Milium coerulescens Desf. Fl. Atl. p. 66 t. $12(1798)=$ Piptatherum coenulescens P. B. Agrostogr. p. 173 (1812).

Auf Jurakalkfelsen oberhalb Itenton in den Seealpen (Frankreich). Begle itpflanzen: Andropogon hirtus L., Biscutella laevigata L., Cneorum tricoccum L., Euphorbia dendroides L., Lavatera maritima Gouan, Lactuca perennis L. Ca. 200-300 m î. d. M.; Mai 1900.

leg. Clarence Bicknell u. Luigi Pollini. 


\section{Botanische Litteratur, Zeitschriften etc.}

Lang, Dr. G., Von Rom nach Sardes. Verlag von .. F'. Steinkopf in Stuttgart. 2. Aufl. 1900. Preis kart. 3 M., fein geb. 3.80 M.

Ein präichtiges Büchlein, das anch dem Botaniker, inshes. dem reisenden, warm emptohlen werden kamn, und deshalh an dieser titelle, wiewohl es von Botanik nicht eben viel enthäl, a 1 su a hmsweise angezeigt werlen soll. Der Verfasser schildert in anzichender sjuache Eindrücke und Erfahrungen, die er auf einer 8monatlichen Studiemreise durch Italien, Sicilien, Griechenland und Kleinasien gewann. Da wir uns wegen Raummangel leider versagen müssen, auf den reichen Inhalt hier niher einzugehen, seien wenigstens einige Kapitelüberschriften angeführt: Weihnachten in hom. Papstmesse. Neapel. Besteigung des Vesuv. Sicilien. Ostern in Athen. Ithaka. Troja. Konstantinopel. Muhanmedanischer Gottesdienst. Wer sich durch diese reizen lässt, das Büchlein näher zu prüfen, wird die aufgewendete Zeit nicht bereuen.

L. Gross.

Christ, H., Die Farnkräuter der Rchweiz. Druck u. Verlagg von K. J. Wyss in Bern.

1900. $189 \mathrm{~S}$.

Der auf floristischem Gebiete. so hochverdiente Verfasser, welcher vor 3 Jahren seine "Farnkräuter der Erte" der (leffentlichkeit übergah, hat num anf "Initiative der schweiz. bot. Cresellschaft und auf Kosten der Eidgenossenschaft" in den Beiträgen zur Kryptogamenfora der Schweiz die Farnkräutes bearbeitet, welche als 2. Heft des I. Bandes vorliegen und auch einzeln käutlich sind. Den 38 s. grosse allgemeine 'Teil der Arbeit gliedert sich in verschiedente Absitze mit folgenden Ueberschriften: Das benïtzte Naterial, Litteratur, taxinomische Linheiten, Variation, Varietät und standort, Subspecies in geograph. Beziehung, Hybridation und hybridogene Species, Auswahl und Einfluss der standorte, Anpassungen, Laubdauer, Entwickelungsgrade der Fortpflanzungsorgane, Einflus der Gesteinsart, Grade der V'erbreitung, Gesellschatten, Höhengrenzen, Florengebiete und Verlreitungsareale, Endemismus, Vergleichmg mit der europ. (iesamtflora und Physiognomisches. Jem speciellen 'Teil ist ein S'chlüssel zur biestimmung der Genera und Species vorangestellt. Der specielle Teil heschreibt die vorkommenden Arten, Formen, bastarde etc. auts genaueste und nimmt auch Rücksicht auf die Formen der angrenzenden Länder. Besonder's wertvoll sind die vielen Texthilder, welche vor allem kritische oder neue Formen zur Darstellung bringen. Die Arbeit, welche anf die Verloreitung der einzelnen Arten und Formen eingeht und grenaue standortsangaben enthäl, gehört zu den besten auf diesem Gebiete und wird sicher von den zahlreichen l'teridophytenfreunden mit grosser Freude begrüsst werden. Herr Dr. Christ hatte die Freundlichkeit, sein schönes Werk allen Mitarbeitern der "Pteridophyta exsiccata", welche von Herrn F. Wirtgen in Bonn a. Rh. 'Niebuhrstrasse $\mathrm{Nr} .27$ a herausgegeben werden, gratis zur Verfügung zu stellen. A. K.

Ascherson, P., u. Gräbner, P., Synopsis der mitteleurop. Flora. 12. Liefg. Verlag

\section{v. W. Engelmann in Leipzig. 1900. p. 465-544. Preis $2 \mathrm{M}$.}

Die vorliegende Lieferung enthït die Bearbeitung eines grossen 'Teils der vielgestaltigen Gattung Festura, in welche Ascherson auch die fruhere rilyorid distans IVhlbg. und Terwandte aufnimmt. Aus dem ITmfing, wolehen z. B. Cirmus Festuce eimnimmt, geht für die, welche das Ascherson'sche Werk nicht besitzen, zur Genüge hervor, in welch eingehender Weise die mittelemop. Aynopis sich ihrer Aufgabe entledigt. Selhstverstandlich ist vielfich Bezug genommen auf das hervorragendste Werk in dieser Hinsicht, auf lie Monographie des gelehrten Gramineenforschers Ed. Hackel über die curopiischen Fratur-Arten. A. K. 
Kronfeld, Dr. M., Stuảien über die Verbreitungsmittel der Pflanzen. I. Teil; Windfrüchtler. Verlag v. W. Engelmann in Leipzig. 1900. p. 1-42. Preis 2 M.

Verfasser bespricht in semer Arbeit: Windfrüchtler, Schüttelfrïchtler, Flugfrüchtler, die Kompositen und 'Typhat. Die Arheit ist recht ansprechend geschrieben. Doch würde es zu weit gehen, dieselbe eingehend zu hesprechen. Es seien daher alle Interessenten auf die Broschüre selbst verwiesen. A. K.

Kull, Albert, u. Lutz, Dr. K. G., Bilder aus der heimatlichen Vogelwelt. Herausgegeben von der Kasseler Hafer-Kakao-Fabrik Hausen \& Co. in Kassel.

Da früher schon Erscheinungen auf zool. Gebiet an dieser Stelle kurz erwähnt wurden, so sei hier auch auf ein durch die Kasseler Hafer-Kakao-Fabrik veranlasstes Unternehmen aufmerksam gemacht, welches im hohen Grade der Volkshelehrung und Unterstützung des naturgeschichtl. Unterrichts zu dienen berufen ist. Statt wertloser Reklamebilder legt genamite Firma ihren Haferkakao-Paketen Täfelchen mit kolorierten Bildern aus der heimatlichen Vogelwelt bei, die von Herm Tiermaler A.Kull vorzïglich ausgeführt sind. Im ganzen sind es 120 Täfelchen (in 10 Serien à 12 Stück) und 10 Hefte Text à 24 Seiten. Die Auflage beträgt 240000 . Wie die Firma mitteilt, will sie von demselben Künstler zu demselben Zwecke bimmen Jahresfrist ein ähnliches Werkchen "Hausens Terrarium und Aquarium" herstellen lassen.

A. K.

Botan. Centralblatt. 1900. Nr. 45. Hering. Ludw., Zur Anatomie der monopodialen Orchideen. - Nr. 46. Busse, Walter, Zur Kenntnis der Leitgewebe im Fruchtknoten der Orchideen. - T aliew, Dr. W., Ueber die russischen myrmecophylen Pflanzen. - Nr. 47. Cador, Ludw., Anatomische Untersuchung der Mateblätter unter Berucksichtigung ihres Gehalts an Thein. - Nr. 48. Fedtschenko, B o ris, Kleinere Mitteilungen über einige Hedysarm-Arten. Cador, Ludw., Wie in vor. Nr.

Berichte der deutsehen bot. Gesellschaft 1900. Heft 8. Foslie, M., Bemerkungen zu F. Heydrichs Arbeit "Die Lithothamien von Helgoland. - Möbius, M., Das Anthophaein, der braune Blütenfarbstoff. - W i eler, A. u. Har t leb, R., Leber Einwirkung der Salzsäure auf die Assimilation der Pflanzen. - Butkewitseh, Wrl., Ueber das Vorkommen proteolytischer Enzyme in gekeimten Samen und ihre Wirkung. - Kohl, F. G., Dimorphismus der Plasmaverbindungen. - Hildebrand, Friedrich, Teher Haemanthus tigrinus, besonders dessen Lebensweise. - Steinbrinck, C., Ueber die Grenzen des Schrmmpelns.

Mitteilungen des bad. bot. Vereins. 1900. Nr.173 u. 174. Herzog, Theodor, Standorte ans dem Hlorengebiet Freiburg. - Leutz, Schistostega osmundacea. - Neuberger, Teue Pflanzen und neue Standorte aus dem Freiburger Florengebiet. - Liehl, Neue Funde in der Kiesgrube an der Baslerstrasse. - Knetsch u. Lettau, Neue Standorte.

Zeitsehrift der bot. Abteilung des naturwiss. Vereins der Prov. Posen. VII. Jahrg. 2 Heft 1900. Spribille, l'rof., Einige Aufzeichnungen aus dem Süden der Provinz. - Torka, Anthericum liliago. - Gatzemeyer, Einige phaenologische Angahen über die Lmgegend ron Tremassen a. d. Jahr 1900. Pfuhl, Dr., Die Wirkung des diejührigen heissen und trockenen Sommers aut die Pflanzenwelt. - Derselbe, Der Weimbau in der Provinz Posen.

Verhandlungen der k. k. zoologiseh-bot. Gesellschaft in Wien. 1900. Heft 8. I a gnus, P., Bornmüller, J., Iter Syriacum.

Berichte der bayerisehen botan. Gesellseraft. München 1900. VII. Bd. II. Abteilg. Arnold, Dr. F., Zur Lichenenflora von München.

Botanical Gazette 1900. Vol. XXX. Nr. 5. Livingston, Burton, Elw., On the nature of the stimulus which causes the change of form in polymorphic green algae.-- Millan, Conway Mac; Observations on Lessonia. - Beadle, C. D., Studier in Crataegus, 
Bulletin de l'académie internationale de géographie botanique. 1900. Nr. 133. Gray, Ch.u. Hue, l'abbé, Lichens récoltés à Coonoor (Ghattes). Nilgiris (Indes-Orientales). - Reynier, Alf., Botaniene rurale; m petit coin de la Provence. - Guffroy, Cl., Un nouveau classeur pour herbier. - Monguillon, E., Catalogue des Lichens du département de la Sarthe. - Féret, A., Les Plantes des terrains salés.

\section{Botanische Anstalten, Vereine, Tauschvereine, Exsiccatenwerke, Reisen etc.}

Botanischer Verein der Provinz Brandenburg. Der Vorsitzende Prof. Volkens eröffnete die Sitzung am 9. November mit der Nachricht von dem Tode eines Mitgliedes, Rentner Kirchner, und machte dauach einige ganz interne Vereinsmitteilungen, die sich vorlüufig noch der Oeffentlichkeit entziehen. Dann übergehend 7.11 den botanischen Mitteilungen warf Geheimrat Wittmack die Frage auf: ob die in England und Irland vorgekommene 'Thatsache, dass Elodea Candensis imstande sei, Wasserläufe zu stauen und oberhalb zur Versumpfung zu zwingen, auch bei uns beobachtet worden sei, besonder's in nenerer Zeit? und ob die Pflanze im September und Oktober oder erst im nächsten Frühahr absterbe? Dr. M arsson hat gefunden, dass die Vermehrung der Flodea nicht von der Jahreszeit, sondern lediglich von dem Kalkgehalt des Wassers abhängig sei; verschwinde dieser, so müsse auch sie absterben; habe sie in geschlossenen Gewissern den Kalkgehalt absorbiert, so sei auch ihre Lebensfähigkeit zu Ende, sie gehe damn in Fäulnis über und verursache ein massenhaftes Fischsterhen, Prof. Be y er konstatiert, dass die Pflanze im Kalksee bei Rüdersdorf in fabelhafter Ueppigkeit vorkomme, von Versumpfung aber keine Spur vorhanden sei. Dr. M a rsson findet die Ursache dieser Erscheinung darin, dass in diesem Gewiisser jedenfalls auch viele sauerstofthildende Pflanzen vorhanden sein werden. Die Lebenskraft überdaure den Winter; denn er habe Elodea im T'iergarten unter dem Eise frisch gefunden. Dr. Hoffm a n n macht laranf aufmerksam, dass der von der Pflanze absorbierte Kalk doch nicht verschwinde; denn wenn die Pflanze schliesslich verfault, so bleibt er doch. Prof. Ascherson findet, dass in der Debatte zwei verschiedene Dinge vermischt würden, einmal die Verpestung des Wassers und damn die entstehende Versumpfung. Hier handle es sich jedoch nur um die letztere Frage, und da könne er ans seiner Erfahrung nur bestätigen, dass bei uns ein Beispiel dieser Art nicht bekannt geworden sei. Uebrigens würde sich für die Pflanze die richtigere Schreibart Helodea empfehlen. Prof. Volkens erimnert an die Thatsache, dass die Pflanze seinerzeit auch bei uns hier und da in gewaltigen Massen aufgetreten sei, so dass z. B. der Spandauer Schifffahrtskanal von Zeit zu Zeit hat ausgerïumt werden müssen, ebenso der Seddinsee hei Köpenick, wo schliesslich die Fischerei ummöglich geworden war. Dann aber haben sich die Massen von Jahr zu Jahr vermindert, so dass die Pflanze jetzt wohl nirgend mehr ein Hinderuis bildet. Da die Pflanze an der Luft weiss wird, so enthält sie ohme Frage viel Kalk oder doch Kalksalze - Nachdem noch von mehreren Seiten bestaitigt worden, dass Elonteu üherali im Rückgange begriffen sei und im Herbst nicht absterbe, sondern den Winter überdanre, macht Geheimrat Wittmack die fernere Hitteilumg, dass Jassus sexnotutus, eine Zwergeikade, in diesem Jahre am Getreide sehr schädigend aufgetreten sei. Während das Tier nach Prof. Franks Untersuchungen jührlich zwei tienerationen durchmacht, scheint dieses Jahr deren drei gebracht zu haben, und die Landwirte sind hier und da rein in Verzweiflung gewesen. Der einzige Rat wäre der, die Bestellung des Ackers nicht zu Anfang September, sondern später vorzmehmen; aber auch das sei nicht so leicht durchzuführen, weil die Gefihr nahe liege, dass sich die Getreideptlanzen dann nicht genügend bestocken. 
Professor Ascherson machte die belustigende Mitteilung, dass seine Zweifel an den Fundort des Scripus pormlus in Hamover (s. Okt.-Ber. S. 231) sich hereits bestaitigt hïtten; denn nachdem der angebliche Finder, ein Seminarist, auch noch Schocmus migricuns entleckt und zwar an einer Stelle, wo ihn sonst niemand gesehen, schliesslich sogar ('ypens thermalis auch dort irgendwo gefunden haben wollte, kam der Schwindel zu Tage. - Ferner macht Professor Ascherson darauf aufmerksam, dass der ungewönlich schöne Herbst auch die Blütezeit vieler Gewaichse verlängert hahe, im neuen botanischen Garten zu Dahlem noch eine Menge blühender Alpenpflanzen zu finden seien, und dass es daher wohl anzuraten wäre, über derartige ahmorme Blütezeiten Buch zu führen.Danach sprach Dr. Newes aufgrund der Versuche von Lucien Daniel über das Pfropten von Pflanzen aus verschiedenen Familien, dessen Gelingen man früher angenommen, damn verworfen, in nenerer Zeit jedoch immer wieder behauptet hat und, wemn Daniels Versuche richtig sind, ausser /weifel stehen würden. Daniel hat das Terfahren des Absäugens angewendet, die beiden P'lanzen auf ihrer. Wurzel helassen, dann gepfropft, die Stämme also kreuzweise vereinigt und auf diese Weise gelungene Resultate erzielt mit Somnenblume und Melone, Kohlarten und Tomate, Aster und Phlox, Ahorn und Flieder u.s.w. Diese Pfropfungen sollen eine dauerhafte V'erwachsung ergeben haben. Eine zweite Frage ist die: ob das Pfropfreis Einfluss auf seine Lnterlage hat und umgekehrt. Auch diese Frage ist heantwortet, indem ein zwejjiluriger Helimthus auf ammus gepfropft wurde, das Pfropfreis die Blaitter des ammus annahm und dieser in Verholzung ïherging und längere Lebensdauer erhielt. Da Daniels Veröffentlichungen in den Comptes Rendus seit sechs Jahret. regehmässig wiederkehren, so dürfte die Sache wohl nicht so ohme weiteres von der Hand zu weisen sein, und es wäre wünschenswert, wenn seine Versuche auch von deutscher Seite nachgeahmt würden. Prof. Ascherson wollen diese Versuche nicht viel beweisen: demn wenn jede Pflanze ihre Wurzel hehält, dam ernährt sie sich doch selbst, während der physiologische Begriff der Pfropfung doch der ist, dass eine Pflanze von der andern ernährt wird. Ihm sind diese Versuche also wenig Vertrauen erweckend. Prof. Volkens möchte wissen, was Daniel über die Neubildung von Geweben zu sagen weiss? Da der Vortragende konstatieren muss, dass Daniel von anatomischen Veränderungen keinerlei Mitteilung macht, so scheint Prof. Volkens auch der anatomische Begriff des Pfropfens nicht erfüllt: dass nämlich die Leitungshahn der Lnterlagempflanze mit dem Reis in Verbindung tritt. Wemn jede Pflanze aber auf ihrer Wurzel belassen wird, damn behält auch jede ihre eigene Leitungshahn; es kamn also eine eigentliche Verwachsung nicht eintreten, sondern nur eine Wundkorkbildung und infolge dessen Verklehung. Geheimrat Wittmack hat zwar Erfahrungen über die Pfropfung von Pflanzen aus verschiedenen Familien nicht gemacht, solche von Pflanzen aus denselben Familien gelingen aber sehr schön, wie z. B. Syringen auf Liguster, damit sie klein hleihen, auch mit Birnen auf Quitten hat man gute Resultate erzielt. Immerhin wären Versuche von anderer Seite recht wünschenswert. - Zum Schluss sprach Dr. Loesener über die den Celastraceen zunächst verwandte kleine Familie der Hipnornoterern, die nur zwei Gattungen aufweist, Hippocraten mit reichverzweigten und sulacer mit büscheligen Blütenständen, tropische und subtropische Pflanzen in der alten und nenen Welt.

W. Lackowitz.

Preussischer Botaniseher Verein. Die 39. Jabres̀versammlung des Tereins fand am 9. Oktober d. J. in Elling statt, wo derselbe am 11. Juli 1862 durch Robert Caspary und einige andere Mämer begründet worden ist. An Stelle des am Erscheinen verhinderten Torsitzenden erstattete der Schriftführeı des Vereins, Dr. A bromeit einen kurzen Jahresbericht, worin er auf die vom Verein geleisteten Arbeiten und Aufgaben hinwies und das Vereinsleben schilderte. (iemäss dem anf der 38. Tahresversammlung gefassten Beschluss wurden die Kreise 'lilsit und Ragnit, sowie Teile der Kreise Rosenberg und Marienwerder (Westpr.) seitens der. Fendhoten des Vereins floristiseh untersucht. Ein- 
zelne Mitglieder des Tereins botanisierten gelegentlich in versehiedenen Teilen des Gebiets und teilten ihre Beohathtungen nebst Belegen mit. Herr Professor Dr. Winkelmann in Stettin hatte wahrend der Ferien Reisen im südlichen Ostprenssen, angestellt und seine Beobachtungen, sowie eine Anzahl der dort gesammelten Pflanzen, worunter Galium satatile neu für das (iebiet ist, dem Vereine gütigst zur Verfügung gestellt. Nachdem der Vortragende über die Vereinssammlungen berichtet hatte, erfolgten die Witteilungen der Sendboten über ihre Beobachtungen. Der erste Sendbote des Vereins, Herr Lehrer H a n s Preuss war durch eine militärische Uebung am Erscheinen aut der Versammlung leider verhindert. Derselbe hatte einen kurzen Ueberblick über seine Forschungen in den Kreisen 'Tilsit, Ragnit und Pr. Stargard eingesandt. Seme hemerkenswertesten Funde wurden den Versammelten durch den Vortsagenden vorgelegt. Herr Preuss konstatierte als Adventivpflanzen im Kreise Tilsit neu: Romumeulus streni, sofoia pratensis und an neuen Fundorten Potentillo Norvegiea b) Ruthernier Willd., sowie $P$. intermedia $L$. bei Wallenthal. Er sammelte ferner eine grosse Anzahl von Formen von Ramunculus cassubicus, Silene Tartarica, Stellaria Frieseama, Er.iophorum alpinum an mehreven Stellen, und eine Seltenheit für den Kreis Tilsit: Geranium silvaticum. Trifolium spadicenm war an mehreren Fundorten von ihm bemerkt worden, desgleichen Campamula cerriraria, Empetrum nigr.um, Salix: lirida, Polygonatum rerticillatum, Orchis militaris b) syrciosa, seltener Rosa mollis (2 Stellen), R. glanca b) complicuta Chr.; sehr selten waren: (iymnarlenia conopea, Platanthere vividis Lindl., Coralliormiza imata, 'Poa Chaixi b) laxa und nur in den Schluchten des Schlossberges (litauisch ,pilkálnis" genannt) bei Curmenculken $I$ unar $i$ r lirica, die dort namentlich die quelligen Stellen umrahmt und in Gesellschaft von Lappa nemorosa Lï̈rnicle u. Onocled struthiopteris üppig gedeiht. Bellis peremnis, dort schon ausserhalb ihres Verbreitumgsgebietes, wurde in sehr geringer Zahl anf einer Wiese am Wilkefluss an= scheinend urwüchsig konstatiert. Jie sehr seltenen Umbelliferen Cenolophium Fischeri und Conioselinum Tataricum wurden wiederholt gefunden und eine Anzahl von Exemplaren in Elbing ausgegeben. Ausser Hieracium hyperboreum Fr. (teste Peter), das im vergangenen Jahre von Preuss im Kreise Ragnit an einer Slelle gefunden wurde, hat derselbe auch in diesen Jahre eine Anzahl bemerkenswerter Hieracien in jenem Gebiet gesammelt, die später determiniert werden sollen. Von Asplenium trichomanes, Carex Hornschuchiana und C. fulva Good. wurde nur je 1 Fundort festgestellt. Im Kreise Tilsit ist $O$ enotherabiennisb) parviflora A.Gray die vor. herrschende Form und kommt dort anf Sandlindereien viel vor. Im westpr. Kreise Pr. Stargard beobachtete Herr Preuss neu Pulsatill patens x rernalis, Chenopodium album. b) microphyllum. Coss. et Germ., Diosera angliea $\times$ rotundifolia, Geranium silvaticum b) parviflorum Knaf u. m. A. - Herr OberlandesgerichtseSekretar Scholz untersuchte wïhrend des vergangenen Sommers einige T'eile der westpreussischen Kreise Marienwerder und Rosenberw. Er konstatierte im Kreise Marienwerder Alisma arcuatum. Michalet und Trifolium arense b) mirocephalum $n$. Uechtr. auf sandigem Boden. Im Kr. Rosenherg im Kämmereiorst bei Freystadt in einer Kiefernschonung eine änserst feinbliittrige Form des Lathurus silvester, deren Blättchen vielfach die Breite von $1 \mathrm{~mm}$ noch nicht erreichen und bis $6 \mathrm{~cm}$ lang sind. Diese Form scheint noch nicht beobachtet zu sein. Herr scholz monnte sie $I$. silester b) line ur ifolius, bezw. capillacrns. Ascherson hat sie unter letzterem Namen bereits veröffentlicht (vergl. Nr. 1 I S. 231 dieser Zeitschrift). Es bleibt jedoch noch durch weitere Beobachtung festzustellen, ob diese Form unter verimderten Bedingungen konstant bleibt. der pseudoplatums war dort als Unterholz spontan, obgleich einige kümmerliche Exemplare des Dianthus barbatus, die in der Nähe des A. premdoplalames; fern von allen menschlichen Wohnstiitten, wuchsen, nu als Giartenflüchtlinge gedeutet werden konnten. - Herr Lehrer Lettau in Insterburg hatte teils in der Ungegend seines Wohnortes, teils in der Rominter Heile einige Untersuchungen angestellt. Von seinen höchst interessanten Funden mögen genannt 
werilen: Gymnadenia odoralissima Rich. im Königl. Forst-Revier Nassawen, Belauf Pelkawen Jagen 62, Kr. Goldap, ne u für Nordost de ut schland, G. conopea in demselben Forstrevier, ferner T'ofieldia calyculata Whlbg.,

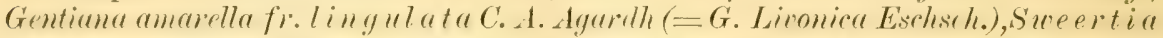
prenennis, Carex flaca $\times$ Oederi, Microstylis momoplyyllus und Coniosel in um I'aturicum, das Herr Lettau an Wasserwerke des Königl. Jagdschlosses Rominten, sowie auch bei Insterburg an zwei Stellen konstatiert hat. In der Umgebung letzterer Stadt sammelte Herr L e tta u eine grössere Anzahl von Hieracien, sowie Bastarde von Geum urbanum, G. vivale und G. strictum Ait. in verschiedenen Formen. - Herr Dr. Hilbert in Sensburg entrleckte nen für Ostpreussen Seneciocampester b) aurantiacus hei Collogienen und Kruttinen, Kr. Sensburg und gab an die Versammelten aus: Campomula bononiensis aus Ostpreussen, speziell aus Masuren von neuen Fundorten, ferner einige Farbenabänderungen, sowie Abnormitäten. Herr Lehrer Kalkreuth in Elbing hatte $\mathrm{mm}$ Elbing und im Kreise Pr. Stargard botanisiert. Von bemerkenswerten Funden, die er dort konstatierte, mögen erwähnt werden: Silene dichotoma Ehrh., Archangelica officinalis, Lilium martagon fir. albiflora, Nasturtium armoracioides und Euphorbia virguta, sowie Juncus temuis als Adventivpflanzen. Um Elbing hatte Herr K. u. a. Sisymbrium Loesclii als neue Adventivpflanze heobachtet mnd Nuphar luteum b) muboptalum Casp. im frischen Haff gesammelt. - Herr Dr. med. Hilbert in Senshurg hielt hierauf einen Vortrag über sprungweise Variation und Atavismus in der Pflanzenwelt anknüpfend an die bekannten Beobachtungen Krašans und erläuterte seine Ausführungen unter Bezugnahme auf Blattformen bei Populus und Ficus. - Herr Oberlandesgerichts-Sekretar Scholz in Marienwerder sprach eingehender über die Wechselbeziehungen zwischen Blütenpflanzen und Insekten, sowie ïber Modehlumen. Beide Themata vereinigte der Vortragende in iusserst geschickter Weise zu einem Ganzen unter Vorführung zahlreicher Beispiele aus dem überaus weiten Gehiet seines anregenden Vortrages. Hierauf gab Herr Dr. Georg T'ischler in Heidelberg einen kurzen Abriss des Entwickelungsganges der Botanik von den Zeiten des Altertums his auf die Gegenwart, doch beschränkte sich der Vortragende nur auf den Entwickelungsgang der Systematik und beleuchtete die Hauptphasen der Entwickelung dieses sehr wichtigen Zweiges der Botanik. - In der geschäftlichen Sitzung wurde 11. a. beschlossen, zur Pfingstzeit eine gemeinsame Exkursion anzustellen, ferner die Kreise Ragnit und Tilsit, sowie Marienwerder und Rosenberg ergänzend botanisch untersuchen zu lassen, event. die Untersuchung einiger anderer geeigneter Gebietsteile in Angriff zu nehmen. Als Ort für die nächste Jahresversammlung wurde Angerburg auserwählt. Nach einer kurzen Frühstückspause wurden eine grosse Zahl vorzüglich ausgeführter Zeichnungen von Pilzen und Prïparaten, besonders Hymenomyceten, von Herm Realschullehrer K a $u$ fmann in Elbing angefertigt, in Augenschein genommen. Herr $\mathrm{Ka} u \mathrm{fm}$ ann hat bereits seit vielen Jahren die Pilze der waldreichen Umgegend Elbings beobachtet, gesammelt und gezeichnet, so dass er sie ganz vorzüglich kennt und hierin als Autoritat bekannt ist. Sodann wurden hemerkenswertere Pflanzen an die Anwesenden rerselienkt, kurze Bemerkungen an kritische Formen geknüpft und die Sitzung gegen 4 Uhr gesohlossen. Am folgenden Tage wurde ein Ausflug nach dem nahe gelegenen Vogelsang unternommen. Ortskundige Botaniker, wie die Herren Rektor Kallmuss und Kaufmann hatten freundlichst die Führung in dem bergigen und schluchtenreichen Gelände übernommen. Die Phanerogamenflora war bereits vielfach im Erlöschen begriffen. Es gelang aber democh, die eine oder andere bemerkenswerte Pflanze zu erspähen. An den hewaldeteten Hängen des Albertssteges standen in Frucht mehrere Exemplare der seltenen und hier wohl noch nicht beobachteten $E$ pipactis sessitifolia Peterm. Hoose und mancherlei Pilze boten sich den Teilnehmern an der Excursion in den Rothbuchen- und Mischbeständen dar. Heerdenweise war Galinm Schultesii I'est. in den verschiedensten Abänderungen zu finden, während V'ronica montana in Gesellschaft von Galeobdolon lutem die feuchteren Rimusale einnahm. An einer Stelle, die Herrn 
Rektor Kalmuss bekannt ist, wächst dort das im Gebiet seltene Plagiothecium latebricola. An Abhängen unfern Wilhelmshöhe waren noch Reste von I'marospermum Austriacum, Petasites albus, Equisetum maximum, Bromus Brneloni u. m. a. zu bemerken.

Thüringer botan. Tauschverein. Die diesjührige 1t. Offertenliste ist 24 Seiten stark und enthïl die P'flanzen in alphabetischer Reihenfolge. Eine Anzahl Pflanzen aus dem Kaukasus und ans Australien sind p. 23 u. 24 besonders aufgeführt. Die Zahl der angebotenen Phanerogamen und Pteridophyten dürfte sich auf über 4000 belaufen. Der Kaufwert ist in Einheitsziffern den einzelnen Namen nachgesetzt. Besonders reich vertretene Gattungen sind: Achilleu, Astrogalus, Carex, Dianthus, Euphorbia, Hievacium, Potentilla, Rosa, Rubus, Silene, Tiola. Recht interessant sind die vom Herausgeber H. Prof. Sagorski in Pforta in Thüringen auf seiner Reise nach Dalmatien und Montenegro gesammelten Pflanzen, sowie die aus Frankreich, Unteritalien, Spanien, Algier, Persien und Südrussland stammenden Arten.

Berliner bot. Tauschverein. Wie der Leiter des Vereins, Herr Seminaroberlehrer Otto Leonhardt in Nossen (Kgr. Sachsen), mitteilt, wird der diesjïhrige sehr reichhaltige Katalog am 18. Dez. zur Versendung gelangen. Die Besprechung wird später erfolgen. Die bis jetzt gedruckten 24 Seiten sind reich an Raritäten.

Sehultz, Paul F. F., Tauschvermittlung far Herbarpflanzen. Am 15. Dez. erscheint bei Paul F. F. Schultz in Berlin No. Virchowstr. 9 der nene Katalog der "Tauschvermittlung für Herbarpflanzen". Terselhe bietet ausser einer grossen Zahl seltener Tauschpflanzen auch e irca 750 s pecies a us M a dagaskar und Wesindien, welche zu dem ausserordentlich niedrigen Preise von 25, bezw. $20 \mathrm{Pf}$. per Stück abgegeben werden. Die Pflanzen sind sehr gut prïpariert und fast sämtlich in 10, 20 und mehr Exemplaren vorhanden. Es befinden sich darunter vornehmlich viele Glumaceen und Filices, Der Versand des Katalogs erfolgt auch an solche Reflektanten, welche nicht Ilitglieder der Tauschgesellschaft sind, gratis und franko, sohald der Unterzeichnete rechtzeitig (möglichst umgehend) mit der Zustellung betraut wird.

Herbarium normale, Centurie XL. Die Pflanzen der 40. Centurie verteilen sich auf 29 Genera und wurden von 38 . Witarheitern geliefert. Das Genus Asperula ist z. B. durch 8, Crocus durch 4 , Gatium durch 9, Hieracium durch 17, Lathraea durch 3, Lotus durch t, Potentilla durch 11, Putmonaria durch 7, Salix durch 12 Nummern vertreten. Die Schedice sind 28 Seiten stark und enthalten kritische Bemerkungen von Dörfler, M. Hellweger, J. Murr, Kupffer und Teploukhoff. Die Präparation und Auflage der Pflanzen ist in jeder Hinsicht eine musterhafte. Die Adresse des verdienstvollen Herausgebers ist J. Dörfler in Wien III, Barichgasse 36, Leiter der Wiener bot. Tauschanstalt.

Bänitz, Dr. C., Herbarium Europaeum, Prospekt 1901. Der langjährige Herausgeber des "Herhariums Europaeum", Herr Dr. Bainitz in Breslau. Marienstrasse $1 \mathrm{f}$., hat sich num entschlosien, die Herausgahe dieses Werkes mit Lief. $122 \mathrm{zu}$ beschliessen und wird voraussichtlich, dasselhe einer anderen Kraft übertragen, so dass das „Herharium Europarum“ im nächsten Jahre höchstwahrscheinlich weiter erscheinen kam. Alis diesem Grumde sind lie Preise für die Pflanzen aus Prospekt 1901 sehr ermïssigt (at 10, 20 od. 30 P'f.). Ein weiterer Prospekt enthält das Verzeichnis der ersten Lieferumg des von Bän itz hegonnenen "Herbarium dendrologicum". Interessenten seien auf die reichhaltigen Prospekte selbst verwiesen.

Association Pyrénéenne. Liste génerale des doubles. 1900/1901. Die ca. 3500 alphabetisch georineten Pflanzennamen sind mit Einheitsziffern versehen. Die Einheit wird im Kauf mit 6, bezw. 5 Cts. berechnet; Beitrag der Mitglieder 
ist jährl. 4 Fres. Die "Association Pyrénéenne* ist, wie schon früher erwähnt wurde, hauptsächlich eine Bezugsquelle für Pflanzen aus Süd- und Westeuropa. Die Pflanzen sind im ganzen nicht hoch bewertet. Der Herausgeber ist Monsieur Giraudias, 5 rue de l'Arche de Noë à Orleans (Loiret) France.

Reverchon. Elisée, Catalogue de 1900. Der bekannte botan. Reisende E. Reverchon, 8 rue de l'Etoile d'Alaï in Lyon (St. Just) France, versendet sein diesjähriges Verzeichnis von Pflanzen aus Spanien, Algier ete. Für aus. gewïhlte Pflanzen berechnet er die Centurie mit 30 Frcs., bei Abnahme aller Pflanzen aus Spanien und Algier mit 25 Fres. und bei Abnahme aller Pflanzen des Katalogs mit 10 Fres. Die Pflanzen sind gut präpariert und reichlich aufgelegt; es wurde aus diesem Grunde für die "Glumaceae exsiccatae" von Reverchon für 84 Fres. Material erworben.

Haglund, Arvid u. Kållström, Joh., Katalog getrockneter Pflanzen aus Skadinavien. Interessenten seien auf diese Bezugsquelle skandinavischer Pflanzen aufmerksam gemacht; Adresse: Falum in Schweden. Die mit verschiedenen Ziffern bezeichneten Pflanzen werden zum Preise für je 5, 10, 15, 20, 25, 30, 35, 40, 45, 50, 75 und $100 \mathrm{Pfg}$. etc. abgegeben. Die Versendung geschieht nur gegen Voreinzahlung, bezw. gegen Nachnahme des Betrags.

Ortlepp, Karl, Gesuch um Uebersendung von Labiatensamen. Karl Ortlepp in Got ha (Thüringen) Ohrdruferstrasse 14 richtet an alle Botaniker das ergebenste Ersuchen, ihm behufs Abfassung einer Arbeit üher das Keimlingsstadium der Labiaten Samen von genau bestimmten Pflanzen dieser Familie, soweit möglich, freundlichst zukommen lassen zu wollen. Auch genau bestimmte Keimpflänzchen werden mit Dank entgegengenommen.

\section{Personalnachrichten.}

Todesfälle: Wagner, Prof. Dr. med., in Königshütte. - Prot. Dr. Serg. Iwan. Korshinsky, seit 1 Jahre Direktor des bot. Museums der kais. Akad. der Wissenschaften in S. Petersburg, am 1. Dez. $1900 \mathrm{im}$ Alter v. $40 \mathrm{~J}$.

\section{Mitteilung.}

Auf die in Nr. 9 dieser Zeitschrift p. 196 erlassene öffentliche Anfrage erhielt der Unterzeichnete teils von Professoren der Botanik an Universitaten, die sich in der Beantwortung der Anfrage kompetent hielten, teils von Heraus. gebern botanischer Exsiccatenwerke und von solchen Floristen, die aut dem Gebiet der Ptlanzenpräparation langjährige Erfahrung besitzen, zahlreiche Gutachten. Nach Vorlage dieser im Widerspruch mit der Erklïrung des , wissenschaftlich gebildeten Sachverständigen" stehenden Zuschriften sah sich die Oberpostdirektion in Karlsruhe veranlasst, ihren auf jene Erklärung sich stuitzenden ablehnenden Entscheid aufzuheben und die für das grösstenteils verdorbene Material der Poa Badensis geforderte volle Entschädigung zur Auszahlung anzuweisen. Den Eincendern der verschiedenen Gutachten sage ich an dieser Stelle meinen verbindlichsten Dank.

Karlsruhe, im Dez. 1900.

A. Kneucker.

Druck von J. J. Reiff in Karlsruhe. 




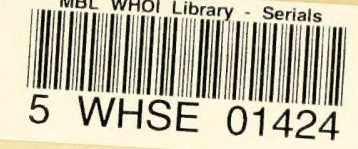


